\begin{tabular}{|l|l|}
\hline $\begin{array}{l}\text { 2. To: (Receiving Organization) } \\
\text { Distribution }\end{array}$ & $\begin{array}{l}\text { 3. From: (Originating Organization) } \\
\text { NHC }\end{array}$ \\
\hline 5. Proj./Prog./Dept./Div.: & 6. Design Authority/ Design Agent/Cog \\
TWRS/Process Development & Engr.: \\
\hline
\end{tabular}

8. Originator Remarks:

This documents the "Feed Tank Fill Status Study" referred to in Issue 19D,

"Feed Tank Fill Status Issue" of Interface Control Document Between DOE and the PHMC, Low-Activity Waste Feed, HNF-SP-1223 (ICD-19), PHMC 1997.

11. Receiver Remarks: 11A. Design Baseline Document? [] Yes [X] No

4. Related EDT No.:
NA
7. Purchase order No.:
NA

9. Equip./Component No.: NA

10. System/Bldg./Facility: NA

12. Major Assm. Dwg. No.: NA

13. Permit/Permit Application No.: NA

14. Required Response Date:

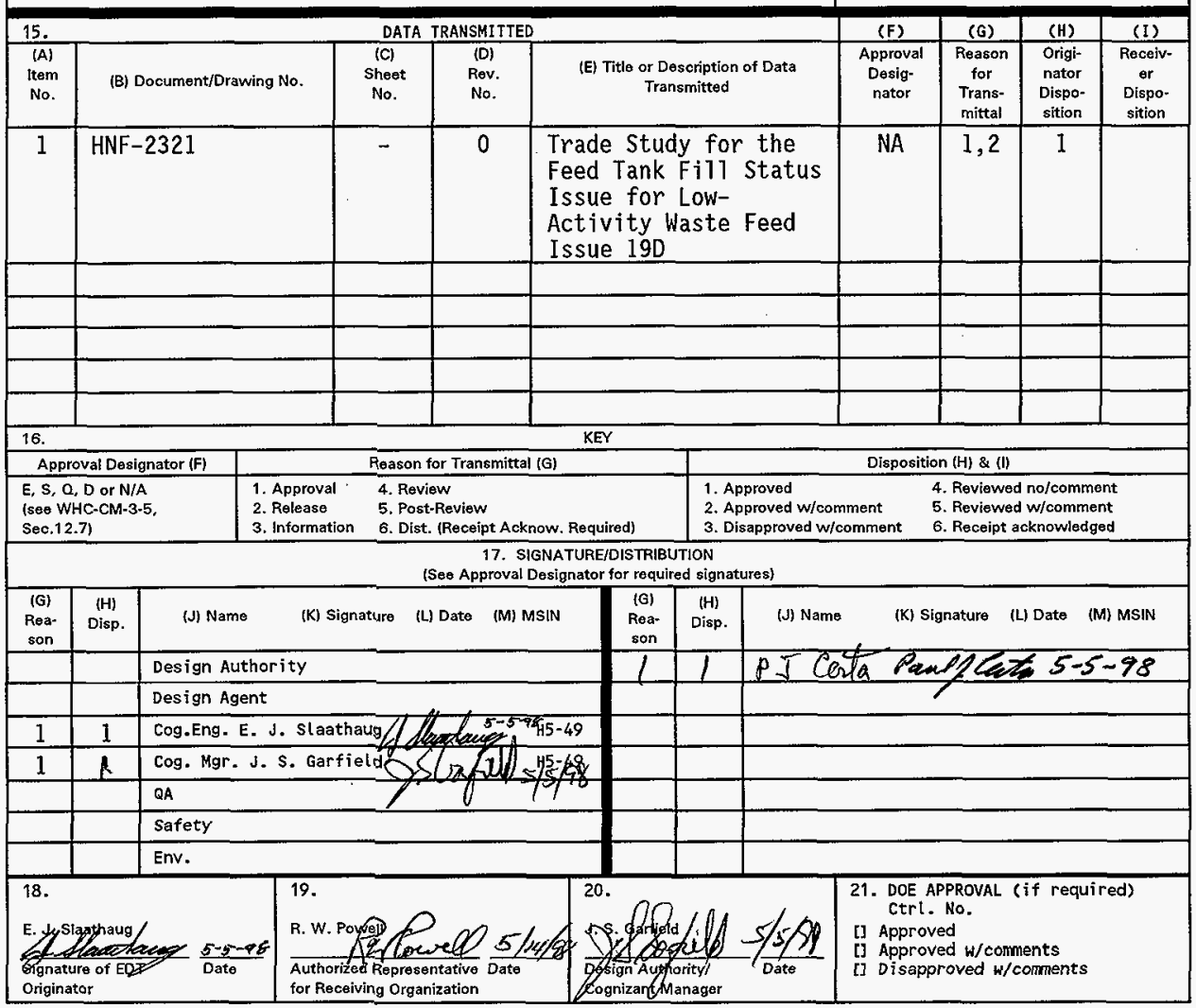




\title{
Trade Study for the Feed Tank Fill Status Issue for Low-Activity Waste Feed Issue 19D
}

\author{
E. J. Slaathaug
}

Numatec Hanford Corporation, Richland, WA 99352

U.S. Department of Energy Contract DE-AC06-96RL.13200

EDT:10: 622710

Org Code: $8 \mathrm{C} 451$

BQR Code: EW3130010
UC: 721

Charge Code: D2D44

Total Pages: 259

Key Words: 10w-activity waste, feed

Abstract: This document identifies and evaluates alternatives that will provide DOE-RL sufficient information from which a decision can be negotiated regarding the Project Hanford Management Contractor team's use of tanks 241-AP-106/-108 versus the private contractors need to upgrade them for their purposes.

TRADEMARK DISCLAIMER. Reference herein to any specific commercial product, process, or service by trade name, trademark, manufacturer, or otherwise, does not necessarily constitute or imply its endorsement, recommendation, or favoring by the United States Governitient or any agency thereof or its contractors or subcontractors.

Printed in the United States of America. To obtain copies of this document, contact: Document Control Services, P.0. Box 950, Nailstop H6-08, Richland WA 99352, Phone (509) 372-2420; Fax (509) 376-4989.
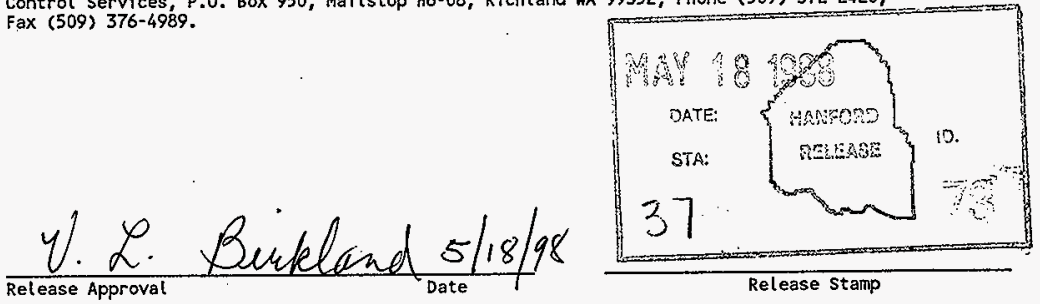
HNF-2321

Revision 0

\title{
TRADE STUDY FOR THE FEED TANK FILL STATUS ISSUE FOR LOW-ACTIVITY WASTE FEED -.ISSUE 19D
}

\author{
April 1998 \\ E. J. Slaathaug \\ Numatec Hanford Corporation \\ Richland, Washington \\ Prepared for \\ U.S. Department of Energy \\ Richland, Washington
}


HNF-2321

Revision 0

This page intentionally left blank. 
HNF-2321

Revision 0

\section{EXECUTIVE SUMMARY}

The scope of this study is to identify and evaluate a set of alternatives and measures that will provide the U.S. Department of Energy - Richland Operations Office (RL) sufficient information from which a decision can be negotiated regarding the Project Hanford Management Contract (PHMC) Team's use of tanks 241-AP-106 and 241-AP-108 versus the Private Contractors ( $P C s)$ need to upgrade them. The desired alternatives to be evaluated and the measures for comparison were selected in a separate meeting with the customer (RL). These are defined in the sections that follow.

The following summarizes the results of this study. More detailed explanations of the results can be found later in this summary and in Section 6.0 of the document.

1. Relinquishing the use of tanks early increases the programmatic risk when compared to the baseline via the following areas:

- Tank Space. The amount of usable tank space decreases. This also impacts the amount of spare and contingency space available.

- Waste Transfer Complexity. The complexity of tank farm transfers increases. As double-shell tank (DST) space becomes limited, the number and interdependency of waste transfers increases.

- Float. Float time for low-activity waste (LAW) feed staging operations decreases.

- Waste Segregation. The segregation of tank wastes (specifically dilute noncomplexed/dilute complexed wastes) may be violated. 
HNF-2321

Revision 0

- Programmatic risk tends to decrease when the early relinquishment is coupled with a delay in saltwell liquid retrieval. However, this decrease does not offset the increase defined above.

- The programmatic risk for Phase $1 B$ operations decreases if the number of LAW contractors decreases.

- The one-contractor scenario negatively impacts Phase 2 operations.

Overall, it is concluded that the results of this study demonstrate that the PHMC Team cannot guarantee delivery of Phase IB feed if the use of tanks 24I-AP-106 and 241-AP-108 are relinquished early and empty for the two-contractor cases. It can also be concluded that the programmatic risk associated with the one-contractor cases is less than the two-contractor cases based upon the metrics and assumptions used in this study. However, since these cases are not developed to the extent of the two-contractor cases (in regards to impact on Tri-Party Agreement milestones, projects, etc.) these results should be considered as preliminary. Additional work will need to be performed to further develop these cases before final conclusions can be drawn.

Based upon the results listed above, the following recommendations are made:

1. Examine alternatives to emptying and allocating tank 241-AP-107 for accumulation of the PCs returns.

2. Evaluate an option that allows the first feed batch (or a newly defined first feed batch) to be present in the tanks at the time that their use by the PHMC Team is relinquished.

3. Conduct a separate study that examines a case(s) that includes a further delay of saltwell liquid retrieval or a delay of the start of Phase 1 operations for both the oneand two-contractor scenarios. 
HNF-2321

Revision 0

4. Update the modeling assumptions in the Hanford Tank Waste Operations Simulator (HTWOS) that affect LAW and high-level waste (HLW) feed staging and evaporator operations. Also update the model to integrate the Operational Waste Volume Projection (OWVP) completely with other operations and include an algorithm (if available) to account for the reliability, availability, and maintainability of equipment.

These recommendations are more fully defined in Section 7.0

\section{Definition of Alternatives Examined}

Figure ES-1 shows the complete range of and the relationships between the parameters under consideration for this study. A brief description of the parameters follows after

Figure ES-1. More detailed descriptions of the parameters can be found in Section 2.1.

Figure ES-1. Screening of Alternatives.

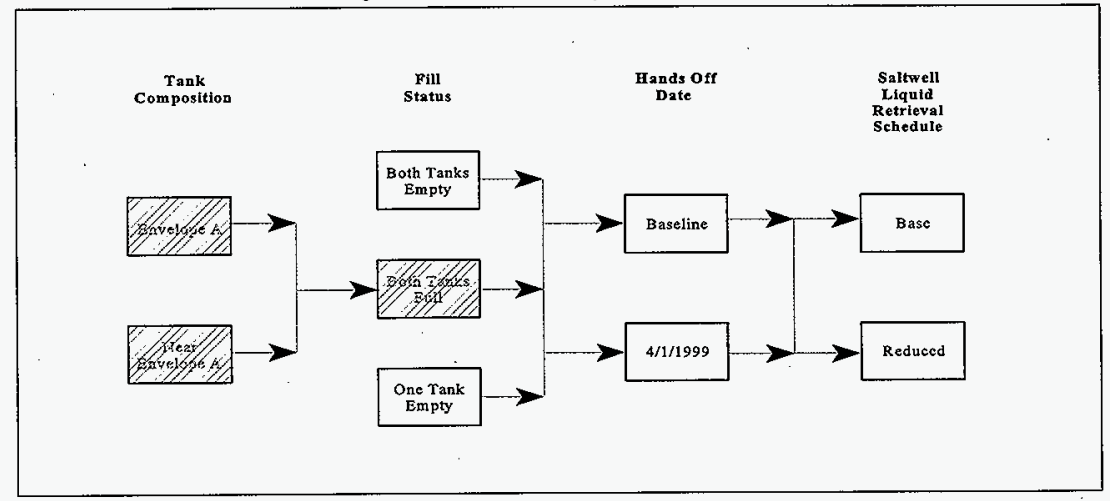

Tank Composition. Composition of the waste in $241-A P-106$ and $241-A P-108$ at the time the use of these tanks is relinquished by the PHMC Team ("Hands-Off" date). 
HNF-2321

Revision 0

Fill Status. Status of 241-AP-106 and 241-AP-108 at "Hands-Off" date. "One Tank Empty" pertains to a scenario in which there is only one $L A W P C$.

"Hands-Off" Date. Date when the use of tanks 241-AP-106 and 241-AP-108 is relinquished by the PHMC Team. "Baseline" corresponds to the dates in the Tank Waste Remediation System Operation and Utilization Plan (TWRSO\&UP) (Kirkbride et al. 1997) when the tanks are no longer used by tank farm operations (10/2000 for 24I-AP-106 and 4/2001 for 241-AP-108)

Saltwell Liquid Retrieval Schedule. Schedule for saltwell liquid retrieval "Base" corresponds to the same schedule as used in the TWRSO\&UP (Kirkbride et al. 1997). "Reduced" corresponds to a hypothesized schedule that includes a three-year delay. This schedule is detailed in Section 2.1.4.

The crosshatched parameters in Figure ES-1 were deleted from analysis in this study per direction from $R L$. Also it was stated by the customer $(R L)$ that the "Baseline" "hands-off" date would only use the "Base" saltwell liquid retrieval schedule. Table ES-1 defines the cases evaluated for this study.

Table ES-1. Definition of Alternatives Under Consideration.

\begin{tabular}{|c|c|c|c|}
\hline Case & Fill status & "Hands-off" date & $\begin{array}{c}\text { Saltwell liquid } \\
\text { retrieval schedule }\end{array}$ \\
\hline 1 & Both Tanks Empty & Baseline & Base \\
\hline 2 & Both Tanks Empty & $4 / 1 / 1999$ & Base \\
\hline 3 & Both Tanks Empty & $4 / 1 / 1999$ & Reduced \\
\hline 4 & One Tank Empty & Baseline & Base \\
\hline 5 & One Tank Empty & $4 / 1 / 1999$ & Base \\
\hline 6 & One Tank Empty & $4 / 1 / 1999$ & Reduced \\
\hline
\end{tabular}

'. Dates are 10/2000 for tank 241-AP-106 and 4/2001 for tank 241-AP-108.

\section{Metrics for Comparison}

The data for comparison were obtained by employing the most current version of the HTWOS. The HTWOS model was used in the TWRSO\&UP to model the interactions between Phase 1 HLW and LAW feed staging operations, Phase 2 SST retrieval operations, and daily 
HNF-2321

Revision 0

tank farm operations. The metrics defined as needed for the comparisons are given and defined below:

Synopsis of Model Changes. Changes made to the HTWOS model since the release of the TWRSO\&UP, for specific contractor scenarios (two-or one-contractor), and for specific cases.

Tank Transfer History. Tank-to-tank transfers, evaporator campaigns, and waste additions to the DST system. Evaluations of LAW and HLW feed staging will be made here.

Evaporator Schedule. Evaporator usage schedule.

Vendor Feed Tank Fill History. Volume history of 24l-AP-108 and possibly 241-AP-106 depending on scenario (two-or one-contractor).

PHMC Staging Tank Fill History. Volume history of 241-AP-102 and 241-AP-104.

DST System Fill History. History of total DST volume and amount of "usable" volume available.

Phase 2 SST Retrieval. Summation of volume of SST waste retrieved and number of SSTs retrieved from.

The data obtained for each of the cases can be found in Section 4.0.

The following table (Table ES-2) summarizes the results. The scores for each case are based upon Case 1 being the standard. The relative score for each case for each parameter is calculated based on its performance when compared to the standard. Section 6.0 defines the logic behind the scoring in more detail. 
HNF-2321

Revision 0

Table ES-2. Summary of Results.

\begin{tabular}{|c|c|c|c|c|c|c|c|c|}
\hline \multirow{3}{*}{ Metric } & \multicolumn{6}{|c|}{ Tank transfers } & \multirow{3}{*}{$\begin{array}{c}\text { Evapor- } \\
\text { ator } \\
\text { usage }\end{array}$} & \multirow{3}{*}{$\begin{array}{c}\text { Vendor } \\
\text { tanks } \\
\text { "hands } \\
\text { off" } \\
\text { on time }\end{array}$} \\
\hline & \multicolumn{2}{|c|}{$\begin{array}{c}\text { High-level waste } \\
\text { staging }\end{array}$} & \multicolumn{2}{|c|}{$\begin{array}{c}\text { Low-activity } \\
\text { waste staging }\end{array}$} & \multicolumn{2}{|c|}{$\begin{array}{c}\text { Waste } \\
\text { transfers }\end{array}$} & & \\
\hline & Delivery & Float & Delivery & Float & Volume & Number & & \\
\hline \multicolumn{9}{|l|}{ Case } \\
\hline 1 & Met & 0 & Met & 0 & 0 & 0 & 0 & Met \\
\hline 2 & Met & 0 & Met & -2 & 0 & 0 & -1 & Met \\
\hline 3 & Met & +1 & Met & -1 & 0 & 0 & 0 & Met \\
\hline 4 & Met & +1 & Met & +3 & +1 & +1 & 0 & Met \\
\hline 5 & Met & 0 & Met & +1 & +1 & +1 & -1 & Met \\
\hline 6 & Met & +1 & Met & +2 & +1 & +1 & 0 & Met \\
\hline \multirow{2}{*}{ Metric } & \multirow{2}{*}{$\begin{array}{c}\text { Staging } \\
\text { tanks } \\
\text { usage }\end{array}$} & \multicolumn{4}{|c|}{ Total double-shell tank system } & \multicolumn{3}{|c|}{$\begin{array}{c}\text { Phase } 2 \text { single-shell tank } \\
\text { retrieval }\end{array}$} \\
\hline & & $\begin{array}{c}\text { Total } \\
\text { volume }\end{array}$ & $\begin{array}{r}\text { Free } \\
\text { volum }\end{array}$ & & $\begin{array}{l}\text { DN/DC } \\
\text { egregation }\end{array}$ & Tanks & & olume \\
\hline \multicolumn{9}{|l|}{ Case } \\
\hline 1 & 0 & 0 & 0 & & 0 & 0 & & 0 \\
\hline 2 & -2 & 0 & -1 & & -1 & 0 & & 0 \\
\hline 3 & -2 & -1 & -1 & & -1 & 0 & & 0 \\
\hline 4 & 0 & -1 & +1 & & 0 & -1 & & $-I$ \\
\hline 5 & -1 & -1 & 0 & & -1 & -1 & & $-I$ \\
\hline 6 & -1 & -2 & 0 & & -1 & -1 & & -1 \\
\hline
\end{tabular}

As can be seen from the results, the early "hands-off" date decreases the overall score of the case regardless of the contractor scenario being modeled. However, the scores tend to increase when the early release is coupled with a delay in saltwell liquid retrieval, but not so much as to equal the base cases (Cases 1 or 4 ).

The scores for the "sister" cases (where the only difference is the number of contractors) tend to favor the one-contractor scenarios for all metrics except for total volume and Phase 2 SST retrieval. These two parameters reflect negatively on the one-contractor scenarios because 
HNF-2321

Revision 0

these cases do not remove as much overall waste from the DST system as the two-contractor cases. Therefore, the ending DST volume is higher and fewer SSTs can be retrieved.

The data demonstrate that for each case, based upon the constraints within the model, feed delivery can be accomplished by the PHMC Team on schedule. However, the data also demonstrate that tank farm operations (tank-to-tank waste transfers, waste generator transfers, evaporator operations) are very complex and interdependent before and during the early years of Phase 1. This is caused by the usable DST space limitation and is true regardless of the number of LAW contractors or which saltwell liquid retrieval schedule is used. Common sense tells us that as the complexity and interdependency of tank farm operations increase, the impact a specific transfer has upon the entire system increases and therefore the risk of failure increases. Thus it is concluded that for the early "hands-off" cases (Cases 2, 3, 5, and 6) the PHMC Team can not guarantee delivery of LAW and HLW feed to the PCs solely based upon the output of this study. Another study should be undertaken that includes reliability, availability, and maintainability (RAM) algorithms of tank farm components (pumps, valves, piping, etc.) to better simulate "reality." The results of the RAM analyses are intended to be included in a future revision of the HTWOS model.

The study also shows that the saltwell liquid retrieval schedule should either be accelerated so that it completes in 1999/2000, or include an additional two-year or so delay (2000 to 2002) or reduction (i.e., less salt well liquid retrieved during this time frame). The early completion of saltwell liquid retrieval may remove the potential conflict between it and feed staging operations, but this scenario is unlikely. The retrieval of saltwell liquid is presently behind the "base" schedule and therefore a delay/reduction is already expected in the completion date. A two-year or so delay or reduction in the retrieval operations has the potential to move the bulk of the volume conflict away from the time period at which tank space is extremely tight, but this option needs to be examined in more detail. A too lengthy delay may detrimentally impact the two-contractor cases because (at present) they process waste that results from the concentration of retrieved saltwell liquid (241-AP-101, 241-AW-104, and 241-AN-106). Also, Tri-Party Agreement milestones may need to be renegotiated and the risk of leaving salt well liquid in SSTs for longer periods of time needs to be evaluated. 
HNF-2321

Revision 0

This page intentionally left blank. 
HNF-2321

Revision 0

CONTENTS

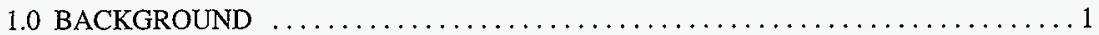

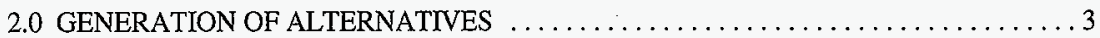

2.1 ALTERNATIVES GENERATION PROCESS $\ldots \ldots \ldots \ldots \ldots \ldots \ldots \ldots \ldots \ldots$

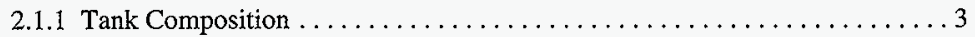

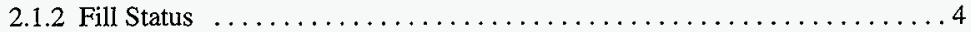

2.1 .3 "Hands-Off" Date . . . . . . . . . . . . . . . . . . . . . . . 4

2.1.4 Saltwell Liquid Retrieval Schedule . . . . . . . . . . . . . . . . 5

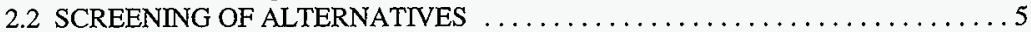

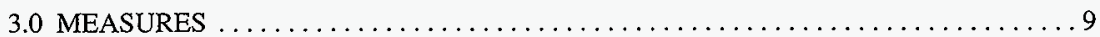

3.1 SYNOPSIS OF MODEL CHANGES $\ldots \ldots \ldots \ldots \ldots \ldots \ldots \ldots, \ldots \ldots, \ldots \ldots$

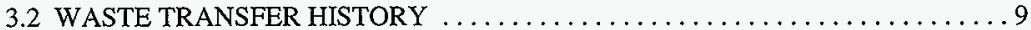

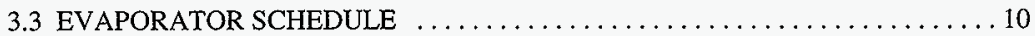

3.4 VENDOR FEED TANK FLL HISTORY $\ldots \ldots \ldots \ldots \ldots \ldots \ldots \ldots \ldots \ldots \ldots$

3.5 PROJECT HANFORD MANAGEMENT CONTRACTOR

STAGING TANKS FILL HISTORY $\ldots \ldots \ldots \ldots \ldots \ldots \ldots \ldots \ldots \ldots \ldots$

3.6 DOUBLE-SHELL TANK SYSTEM FILL HISTORY $\ldots \ldots \ldots \ldots \ldots \ldots \ldots \ldots$

3.7 PHASE 2 SINGLE-SHELL TANK RETRIEVAL $\ldots \ldots \ldots \ldots \ldots \ldots \ldots \ldots$

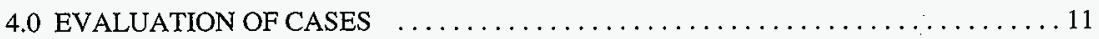

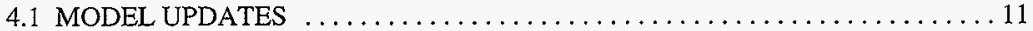

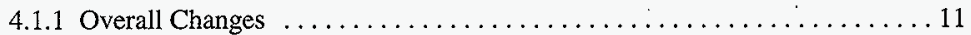

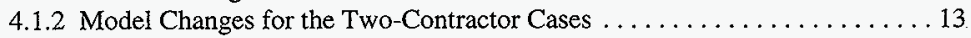

4.1.3 Model Changes for the One-Contractor Cases $\ldots \ldots \ldots \ldots \ldots \ldots \ldots$

4.1.4 Technical Approach for Evaluation $\ldots \ldots \ldots \ldots \ldots \ldots \ldots \ldots \ldots \ldots \ldots$

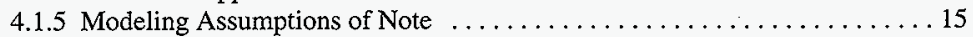

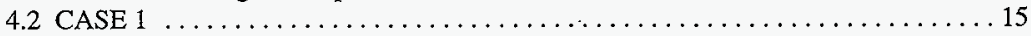

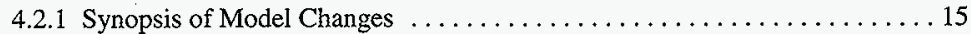

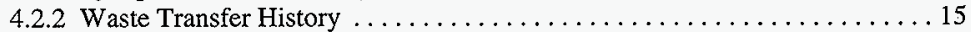

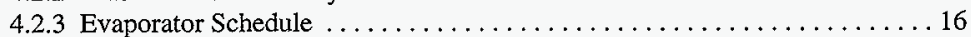

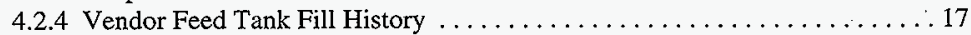

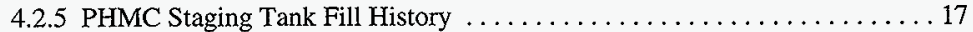

4.2.6 Double-Shell Tank System Fill History $\ldots \ldots \ldots \ldots \ldots \ldots \ldots \ldots \ldots$

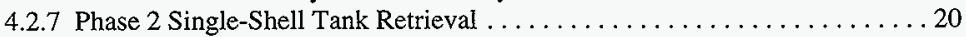

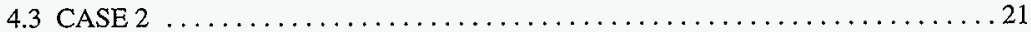

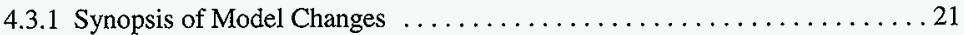

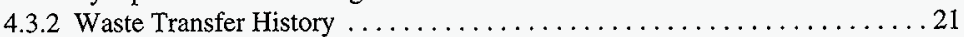

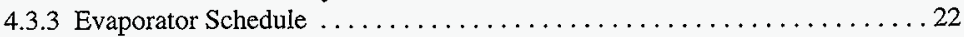

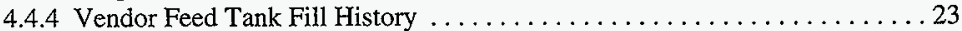

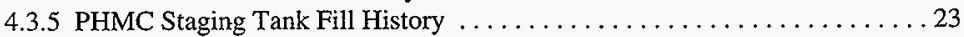

4.3.6 Total Double-Shell Tank System Fill History $\ldots \ldots \ldots \ldots \ldots \ldots \ldots \ldots 26$

4.3.7 Phase 2 Single-Shell Tank Retrieval . . . . . . . . . . . . . . . . 26

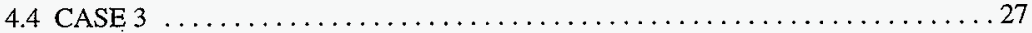


HNF-2321

Revision 0

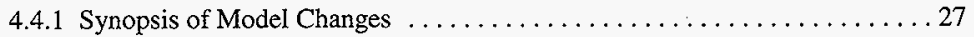

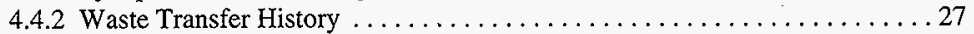

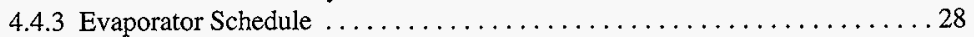

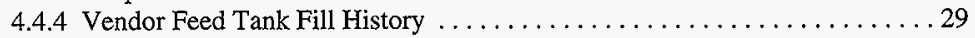

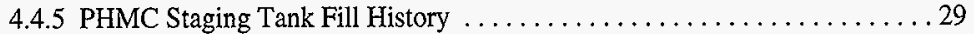

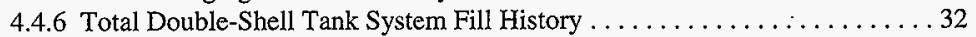

4.4.7 Phase 2 Single-Shell Tank Retrieval $\ldots \ldots \ldots \ldots \ldots \ldots \ldots \ldots \ldots \ldots \ldots \ldots \ldots \ldots \ldots \ldots$

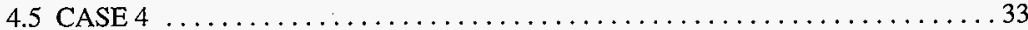

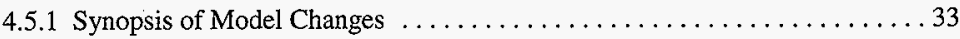

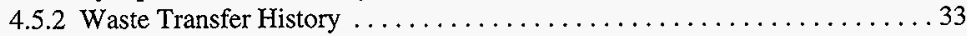

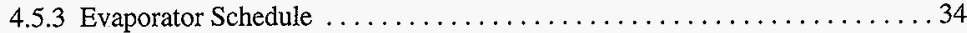

4.5 .4 Vendor Feed Tank Fill History .......................... 35

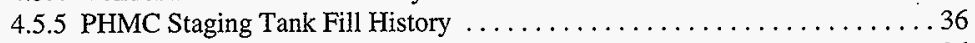

4.5.6 Total Double-Shell Tank System Fill History . . . . . . . . . . . . . . 36

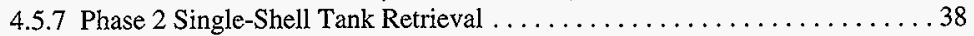

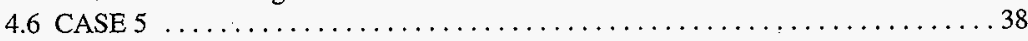

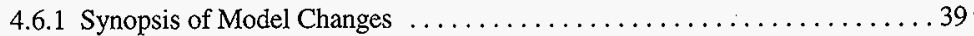

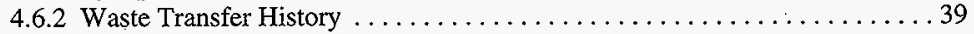

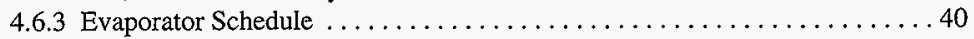

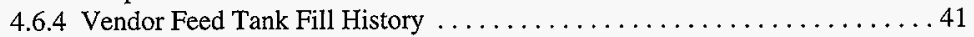

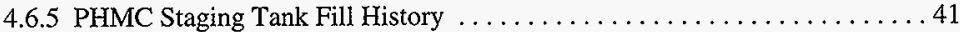

4.6.6 Total Double-Shell Tank System Fill History . . . . . . . . . . . . . 43

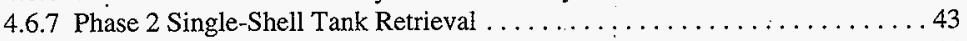

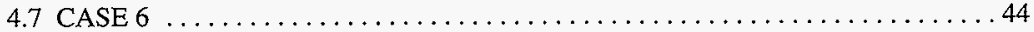

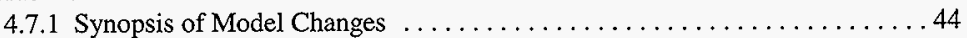

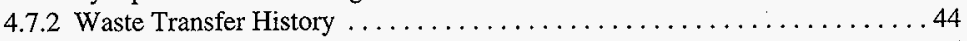

4.7.3 Evaporator Schedule ................................. 45

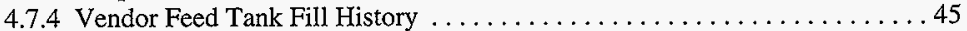

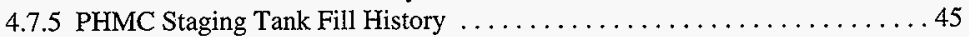

4.7.6 Total Double-Shell Tank System Fill History . . . . . . . . . . . . . . 48

4.7.7 Phase 2 Single-Shell Tank Retrieval . . . . . . . . . . . . . . . 48

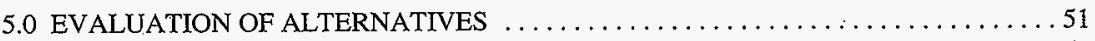

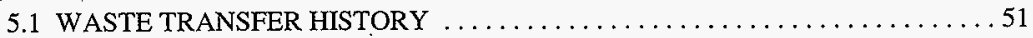

5.1.1 Low-Activity Waste and High-Level Waste Feed Staging Transfers . . . . . 51

5.1 .2 Number and Volume of Tank Transfers $\ldots \ldots \ldots \ldots \ldots \ldots \ldots \ldots \ldots$

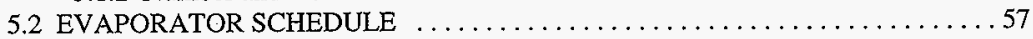

5.3 TOTAL DOUBLE-SHELL TANK SYSTEM FILL HISTORY $\ldots \ldots \ldots \ldots \ldots \ldots 6$

5.4 PHASE 2 SINGLE-SHELL TANK RETRIEVAL $\ldots \ldots \ldots \ldots \ldots \ldots \ldots \ldots 6$

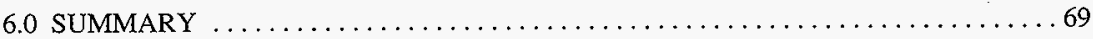

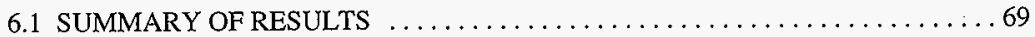

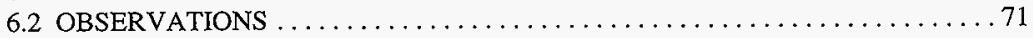

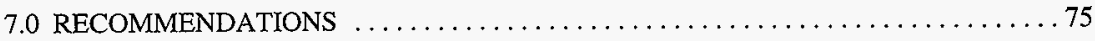


HNF-2321

Revision 0

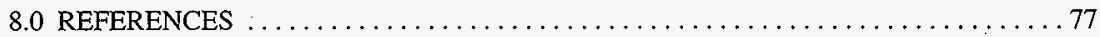

APPENDIX A - TRANSFER LIST $\ldots \ldots \ldots \ldots \ldots \ldots \ldots \ldots \ldots \ldots \ldots \ldots \ldots \ldots \ldots \ldots \ldots \ldots \ldots$ 
HNF-2321

Revision 0

\section{LIST OF FIGURES}

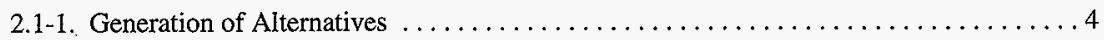

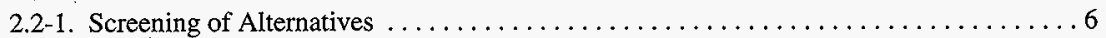

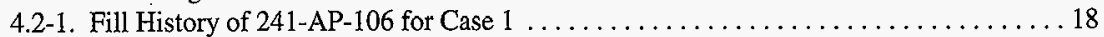

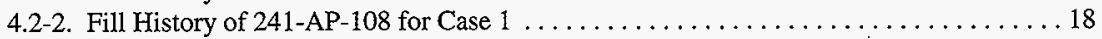

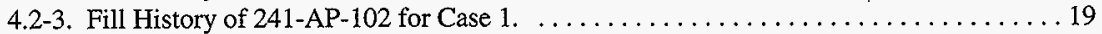

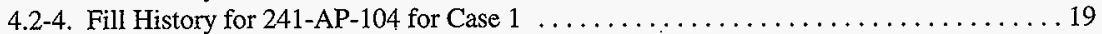

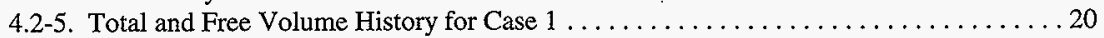

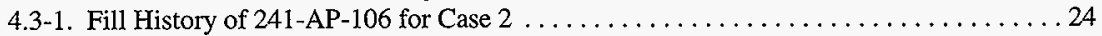

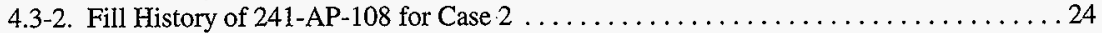

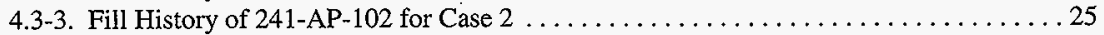

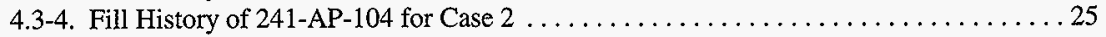

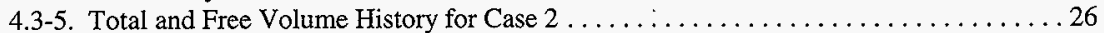

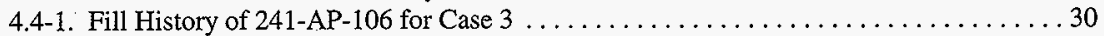

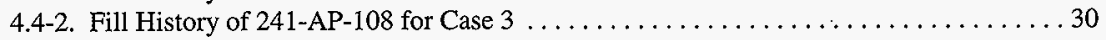

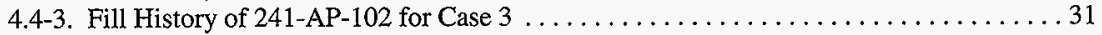

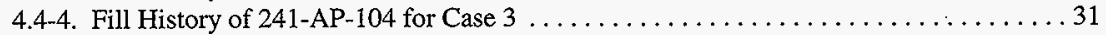

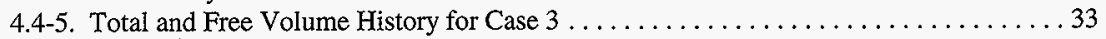

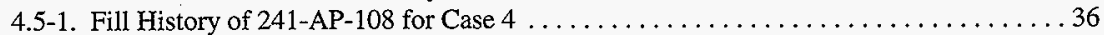

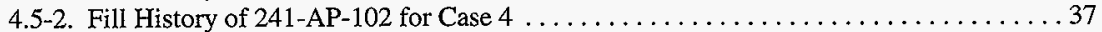

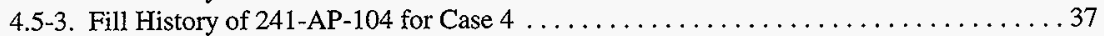

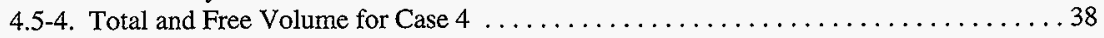

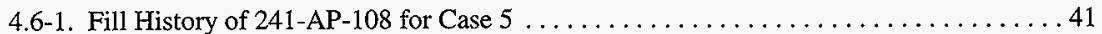

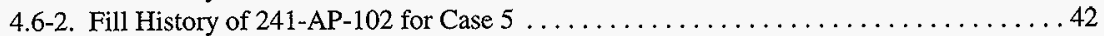

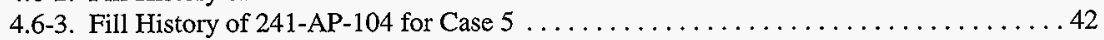

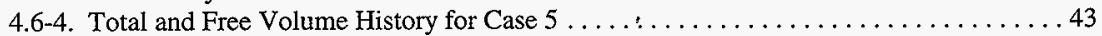

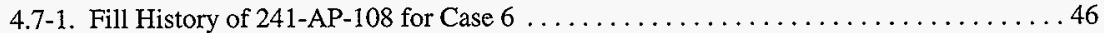

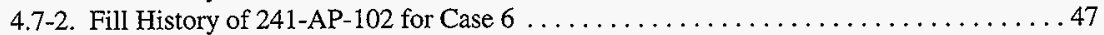

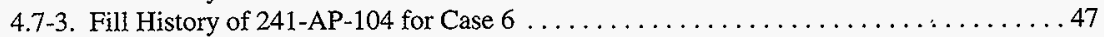

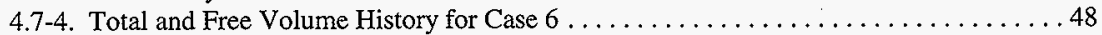

5.1-1. Total Volume of Tank Transfers per Calendar Year Two-Contractor Cases . . . . . . . . 55

5.1-2. Total Number of Tank Transfers per Calendar Year Two-Contractor Cases . . . . . . . . 56

5.1-3. Total Volume of Tank Transfers per Calendar Year One-Contractor Cases . . . . . . . . 56

5.1-4. Total Number of Tank Transfers per Calendar Year One-Contractor Cases. . . . . . . . . 57

5.3-1. Total Volume Comparison for Two-Contractor Cases .....................61 61

5.3-2. Free Volume Comparison for Two-Contractor Cases $\ldots \ldots \ldots \ldots \ldots \ldots \ldots \ldots \ldots \ldots \ldots \ldots \ldots$

5.3-3. Total Volume Comparison for One-Contractor Cases .................... 62

5.3-4. Free Volume Comparison for One-Contractor Cases . . . . . . . . . . . . . . . 62

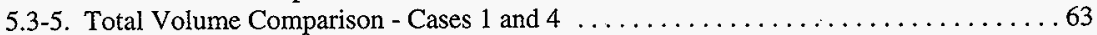

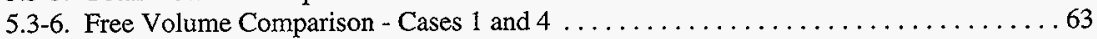

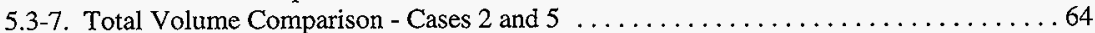

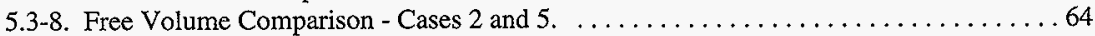

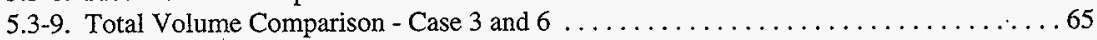

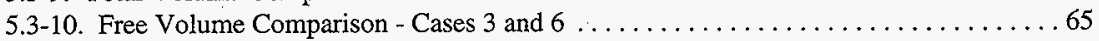


HNF-2321

Revision 0

\section{LIST OF TABLES}

2.1-1. Saltwell Liquid Retrieval Schedules. .......................... 5

2.2-1. Definition of Alternatives Under Consideration . . . . . . . . . . . . . . . . . .

4.2-1. Low-Activity Waste Feed Delivery Schedule for Case $1 \ldots \ldots \ldots \ldots \ldots \ldots \ldots \ldots$

4.2-2. High-Level Waste Feed Delivery Schedule for Case $1 \ldots \ldots \ldots \ldots \ldots \ldots \ldots \ldots \ldots \ldots$

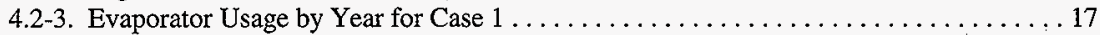

4.2-4. Phase 2 Single-Shell Tank Retrieval Status for Case $1 \ldots \ldots \ldots \ldots \ldots \ldots \ldots \ldots \ldots \ldots$

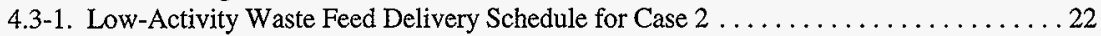

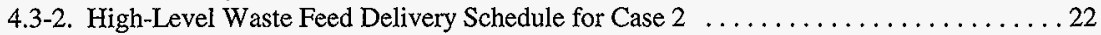

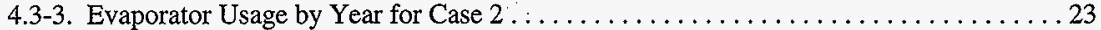

4.3-4. Phase 2 Single-Shell Tank Retrieval Status for Case $2 \ldots \ldots \ldots \ldots \ldots \ldots \ldots \ldots 27$

4.4-1. Low-Activity Waste Feed Delivery Schedule for Case $3 \ldots \ldots \ldots \ldots \ldots \ldots \ldots$

4.4-2. High-Level Waste Feed Delivery Schedule for Case $3 \ldots \ldots \ldots \ldots \ldots \ldots \ldots 28$

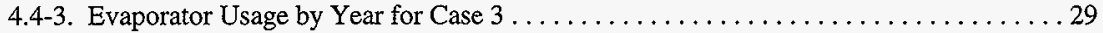

4.4-4. Phase 2 Single-Shell Tank Retrieval Status for Case $3 \ldots \ldots \ldots \ldots \ldots \ldots \ldots \ldots \ldots \ldots$

4.5-1. Low-Activity Waste Feed Delivery Schedule for Case $4 \ldots \ldots \ldots \ldots \ldots \ldots \ldots \ldots$

4.5-2. High-Level Waste Feed Delivery Schedule for Case $4 \ldots \ldots \ldots \ldots \ldots \ldots \ldots \ldots$

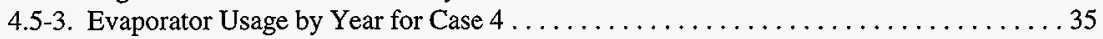

4.5-4. Phase 2 Single-Shell Tank Retrieval Status for Case $4 \ldots \ldots \ldots \ldots \ldots \ldots \ldots$

4.6-1. Low-Activity Waste Feed Delivery Schedule for Case $5 \ldots \ldots \ldots \ldots \ldots \ldots \ldots$

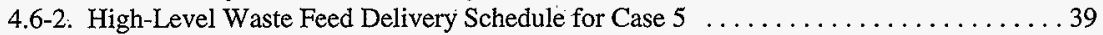

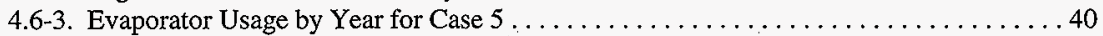

4.6-4. Phase 2 Single-Shell Tank Retrieval Status for Case $5 \ldots \ldots \ldots \ldots \ldots \ldots \ldots . \ldots 4$

4.7-1. Low-Activity Waste Feed Delivery Schedule for Case $6 \ldots \ldots \ldots \ldots \ldots \ldots \ldots$

4.7-2. High-Level Waste Feed Delivery Schedule for Case $6 \ldots \ldots \ldots \ldots \ldots \ldots \ldots \ldots$

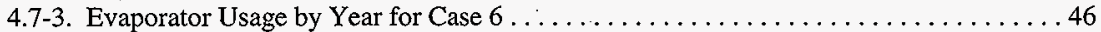

4.7-4. Phase 2 Single-Shell Tank Retrieval Status for Case $6 \ldots \ldots \ldots \ldots \ldots \ldots \ldots . \ldots 49$

5.1-1. Low-Activity Waste Time for Sampling--Two-Contractor Cases . . . . . . . . . 52

5.1-2. Low-Activity Waste Time for Sampling--One-Contractor Cases $\ldots \ldots \ldots \ldots \ldots \ldots . \ldots 2$

5.1-3. High-Level Waste Time for Sampling--Two-Contractor Cases . . . . . . . . . . . . 53

5.1-4. High-Level Waste Time for Sampling--One-Contractor Cases $\ldots \ldots \ldots \ldots \ldots \ldots \ldots 5$

$5.2-1$. Evaporator Usage for Two-Contractor Cases $\ldots \ldots \ldots \ldots \ldots \ldots \ldots \ldots \ldots \ldots$

5.2-2. Evaporator Usage for One-Contractor Cases $\ldots \ldots \ldots \ldots \ldots \ldots \ldots \ldots \ldots \ldots \ldots$

5.4-1. Single-Shell Tank Retrieval Status by Case $\ldots \ldots \ldots \ldots \ldots \ldots \ldots \ldots \ldots \ldots$

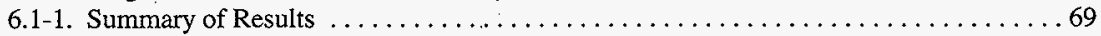


HNF-2321

Revision 0

\section{LIST OF TERMS}

$\begin{array}{ll}\text { AGA } & \text { Alternatives Generation and Analysis } \\ \text { DC } & \text { Dilute complexed } \\ \text { DN } & \text { Dilute non-complexed } \\ \text { DOE } & \text { U.S. Department of Energy } \\ \text { DRT } & \text { Dilute receiver tank } \\ \text { FY } & \text { Fiscal year } \\ \text { HLW } & \text { High-level waste } \\ \text { HTWOS } & \text { Hanford Tank Waste Operation Simulator } \\ \text { ICD } & \text { Interface Control Document } \\ \text { LMAES } & \text { Lockheed Martin Advanced Environmental Services } \\ \text { LMHC } & \text { Lockheed Martin Hanford Corporation } \\ \text { NHC } & \text { Numatec Hanford Corporation } \\ \text { OWVP } & \text { Operational Waste Volume Projection } \\ \text { PC } & \text { Private Contractor } \\ \text { PHMC } & \text { Project Hanford Management Contractor } \\ \text { LAW } & \text { Low-Activity Waste } \\ \text { RL } & \text { U.S. Department of Energy-Richland Operations Office } \\ \text { SST } & \text { Single-shell tank } \\ \text { SWL } & \text { Saltwell liquid } \\ \text { TWRS } & \text { Tank Waste Remediation System } \\ \text { TWRSO\&UP } & \text { Tank Waste Remediation System Operation and Utilization Plan }\end{array}$


HNF-2321

Revision 0

\section{TRADE STUDY FOR THE FEED TANK FILL STATUS ISSUE FOR LOW-ACTIVITY WASTE FEED - ISSUE 19D}

\subsection{BACKGROUND}

The privatization contracts (DOE 1997a,b) state that each private contractor (PC) will modify their assigned feed tank (241-AP-106 or 241-AP-108) and supporting systems to meet their specific needs for processing operations. However, due to double-shell tank (DST) space limitations, the current Operational Waste Volume Projection (OWVP) (Rev. 23) (Strode and Boyles 1997) and the Tank Waste Remediation System Operation and Utilization Plan (TWRSO\&UP) (Kirkbride et al. 1997) continue to use these tanks for waste management activities during the same time frame that the PCs are expected to be modifying them. Therefore, the U.S. Department of Energy-Richland Operations Office (RL) needs to decide if the Project Hanford Management Contract (PHMC) Team can continue to use 241-AP-106 and 241-AP-108 as currently assumed. If so, the PHMC Team and the PCs need to coordinate the PHMC Team's continued use of these tanks with permitting, tank upgrade, and turnover activities. If not, the PHMC Team will need to perform a study to determine how to conduct feed staging and other tank farm operations without the use of these tanks. This study is the "if not" study.

The scope of this study is to identify and evaluate a set of alternatives and measures that will provide RL sufficient information from which a decision can be negotiated regarding the PHMC Team's use of these tanks versus the PC's need to upgrade them. The desired alternatives to be evaluated and the measures for comparison were selected in a separate meeting with the customer (RL) and are defined in Section 2.0. 
HNF-2321

Revision 0

This page intentionally left blank. 
HNF-2321

Revision 0

\subsection{GENERATION OF ALTERNATIVES}

The range of alternatives were originally defined in the "Feed Tank Fill Status Issue" activity description. This activity description stated that the study will evaluate up to 6 cases that consist of "yet to be determined" combinations of the following parameters:

- Deșired Tank Fill Status

- Two empty tanks

- Two tanks full of waste

- One tank empty for single LAW contractor

- Date tanks are no longer available for use by the PHMC ("Hands-Off" Date)

- Required Waste Composition

- Envelope A

- "Near" Envelope A

The customer (RL) further defined another parameter that they wanted included:

- Saltwell Liquid Retrieval Sequence

- $\quad$ As defined in OWVP Rev. 23 (Strode and Boyles1997)

- Delayed an additional three years

The following sections define fully what these parameters are, the gambit of alternatives that could be modeled, and how/why some alternatives were excluded from consideration.

\subsection{ALTERNATIVES GENERATION PROCESS}

As stated previously, the parameters that would be varied for this alternatives generation and analysis (AGA) have been defined. Figure 2.1-1 illustrates the full range of alternatives that could be considered. The following sections define each parameter.

\subsubsection{Tank Composition}

As can be seen, tank composition is only viable if the tanks are turned over with waste in them. The two possible states for this parameter are the composition of the waste in the feed tanks already meet the Envelope A requirements or it does not. However it is assumed that if the waste does not meet Envelope A that it will be "near" to Envelope A. 
Figure 2.1-1. Generation of Alternatives.

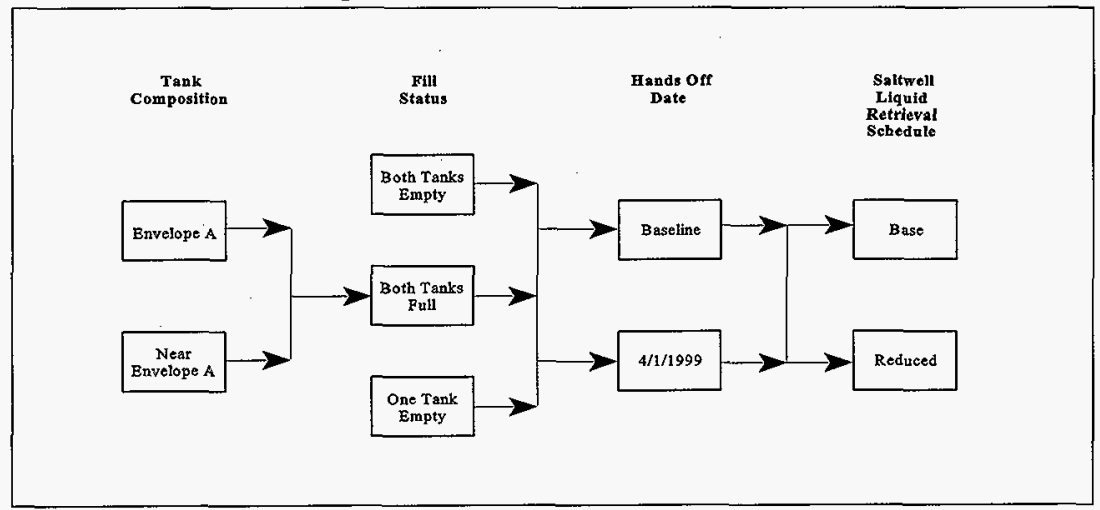

\subsubsection{Fill Status}

Fill status is the crux of the entire AGA. The "Two Empty" state is both tanks empty at the given date. The "Two Full State" is both tanks containing waste at the "hands-off" date having the composition as defined by Tank Composition. The "One Empty" state is for a special case in which there is only one contractor. In this case, only one tank (241-AP-108) will need to be empty by the specified "hands-off" date.

\subsection{3 "Hands-Off" Date}

The "hands-off" date is the date at which the PHMC will no longer use the vendor feed tank(s) for waste storage. Only one date is under consideration at this time (4/1/1999). This date was specified by the customer (RL) as its preferred date. The second "date" (baseline) is the same dates as were used in the TWRSO\&UP (241-AP-106 - 10/2000, 241-AP-108 4/2001). These dates will only be used to develop "baseline" cases to compare against the early "hands-off" cases. 
HNF-2321

Revision 0

\subsubsection{Saltwell Liquid Retrieval Schedule}

Saltwell liquid is removed from the SSTs in order to stabilize them to reduce the impact of leaks. The TWRSO\&UP used the "Base" saltwell liquid retrieval schedule (identical to what was modeled in OWVP Rev. 23 [Strode and Boyles 1997]) which completes in fiscal year (FY) 2000. The "Reduced" saltwell liquid retrieval schedule does not complete until FY 2003. The schedules for these two parameters are given in Table 2.1-1. Only the "Base" schedule will be used with the "Baseline" "hands-off" date since this date was defined by RL as only to be used for defining the baseline cases.

Table 2.1-1. Saltwell Liquid Retrieval Schedules.

(Volume in Kgals)

\begin{tabular}{|c|c|c|c|c|c||c|c|c|c|c|}
\hline \multirow{2}{*}{$\begin{array}{c}\text { Fiscal } \\
\text { year }\end{array}$} & \multicolumn{5}{|c||}{ Base schedule } & \multicolumn{5}{|c|}{ Reduced schedule } \\
\cline { 2 - 11 } & \multicolumn{2}{|c|}{200 East } & \multicolumn{2}{|c|}{200 West } & & 200 East & \multicolumn{2}{|c|}{200 West } & \\
\cline { 2 - 11 } & DN & DC & DN & DC & Total & DN & DC & DN & DC & Total \\
\hline 1997 & 71 & 84 & 285 & 0 & 440 & 23 & 0 & 140 & 0 & 163 \\
\hline 1998 & 314 & 546 & 1,592 & 72 & 2,524 & 28 & 203 & 140 & 0 & 371 \\
\hline 1999 & 6 & 226 & 1,016 & 585 & 1,833 & 324 & 277 & 318 & 0 & 919 \\
\hline 2000 & 0 & 56 & 273 & 190 & 519 & 33 & 331 & 1,084 & 37 & 1,485 \\
\hline 2001 & & & & & & 0 & 85 & 1,245 & 48 & 1,378 \\
\hline 2002 & & & & & & 0 & 16 & 226 & 604 & 846 \\
\hline 2003 & & & & & & 0 & 0 & 0 & 158 & 158 \\
\hline Total & 391 & 912 & 3,166 & 847 & 5,316 & 408 & 912 & 3,153 & 847 & 5,320 \\
\hline
\end{tabular}

$\mathrm{DC}=$ Dilute complexed

$\mathrm{DN}=$ Dilute non-complexed .

There are slight differences between the overall totals for some of the waste originations, but they are minor and will not affect the results of this study.

\subsection{SCREENING OF ALTERNATIVES}

Figure 2.2-1 presents the parameters selected for further study and the rejected parameters. 
HNF-2321

Revision 0

Figure 2.2-1. Screening of Alternatives.

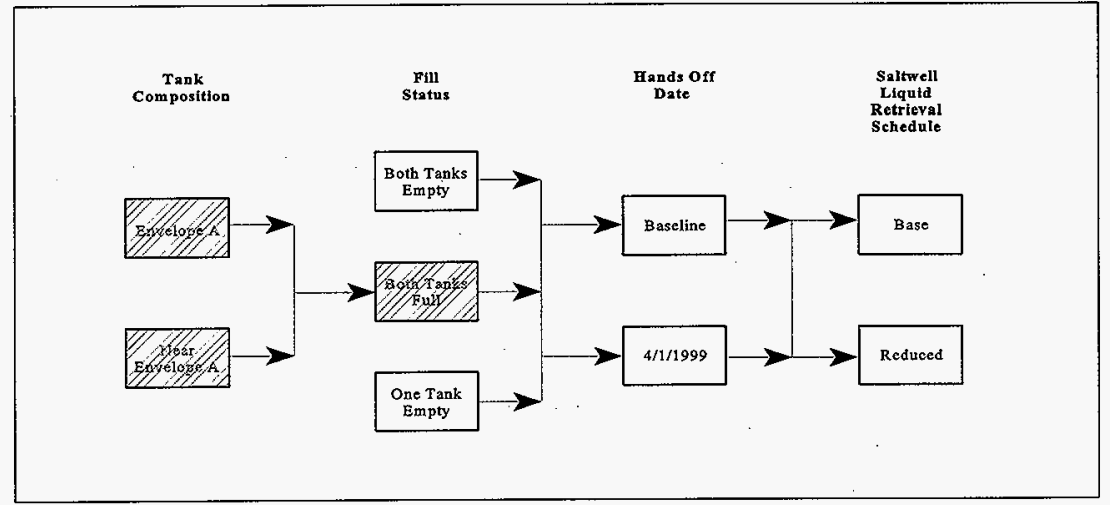

The "Two Full" state was removed at the request of the customer (RL). It was not believed that this is a viable option based upon impacts it would have on project schedules and contract requirements. This may need to be considered, however, as a "fallback" option. If this study demonstrates that the PHMC is not able to relinquish use of the tanks at the desired date, attempts may be made to obtain a date at which it is possible. If no possible date exists, then the only other option is to turn the tanks over full.

Since the "Two Full" state is removed then the composition parameters, which are only applicable to the "Two Full" state, can also be dropped.

This leaves a possibility for eight different cases. However as was stated in Section 2.1.4, the "Baseline" "hands-off date" will only be used for the "Base" saltwell liquid retrieval schedule. Therefore, two additional cases can be dropped leaving a total of six cases: two baseline cases and four alternative cases. All six cases are defined in Table 2.2-1. 
HNF-2321

Revision 0

Table 2.2-1. Definition of Alternatives Under Consideration.

\begin{tabular}{|c|c|c|c|}
\hline Case & Fill status & $\begin{array}{c}\text { "Hands-off" } \\
\text { date }\end{array}$ & $\begin{array}{c}\text { Saltwell liquid retrieval } \\
\text { schedule }\end{array}$ \\
\hline 1 & Both Tanks Empty & Baseline $^{\mathrm{a}}$ & Base \\
\hline 2 & Both Tanks Empty & $4 / 1 / 1999$ & Base \\
\hline 3 & Both Tanks Empty & $4 / 1 / 1999$ & Reduced \\
\hline 4 & One Tank Empty & Baseline ${ }^{\mathrm{a}}$ & Base \\
\hline 5 & One Tank Empty & $4 / 1 / 1999$ & Base \\
\hline 6 & One Tank Empty & $4 / 1 / 1999$ & Reduced \\
\hline
\end{tabular}

${ }^{\mathrm{a}}$ Baseline dates are 10/2000 for tank 241-AP-106 and 4/2001 for tank 241-AP-108. 
HNF-2321

Revision 0

This page intentionally left blank. 
HNF-2321

Revision 0

\subsection{MEASURES}

The measures used for evaluation of the cases defined in Section 2.2 were decided upon via a meeting with the customer (RL) and are given below:

- Synopsis of Model Changes

- Tank Transfer History

- Evaporator Schedule

- Vendor Feed Tank Fill History (241-AP-108 and possibly 241-AP-106)

- PHMC Staging Tank Fill History (241-AP-102 and 241-AP-104)

- DST System Fill History

- Phase 2 SST Retrieval.

The following sections give the definitions of the measures and the format in which the measures will be presented.

\subsection{SYNOPSIS OF MODEL CHANGES}

The different cases will be modeled using the most current Hanford Tank Waste Operation Simulator (HTWOS). HTWOS is a dynamic computer simulation (programmed in $\mathrm{G}^{1}$ ) that models the operation of the tank farm systems withing the 200 East and West areas from present day until the conclusion of Phase 2 operations. HTWOS simulates operational waste volume projections, low level feed staging, high level feed staging, Phase 1 single-shell tank (SST) retrieval, and Phase 2 SST retrieval providing a common assumption basis for all activities, as well as showing operational impacts of each activity on the other activities. Its primary purpose is to support the TWRSO\&UP.

The output from this model was employed to obtain all the data used for the comparisons (Section 4.0). Descriptions of any alterations made to the HTWOS since the version used for the TWRSO\&UP Revision OA are given in Section 4.1. These changes include changes made to the overall model (regardless of case) and specific changes relating to the scenario selected (one- or two-contractor). Specific changes made for each case will be included in this section.

\subsection{WASTE TRANSFER HISTORY}

Waste transfer history will include every transfer that occurs in the model. This includes tank to tank transfers, evaporator runs, and incoming waste from waste generators. The data will include the tank/waste source transferred from, tank transferred to, starting transfer date, ending transfer date, liquid volume transferred, and solid volume transferred. Important transfers pertaining to Phase $1 \mathrm{HLW}$ and LAW feed staging will be broken out and listed on separate

${ }^{1} \mathrm{G} 2$ is a registered trademark of Gensym Corporation, Cambridge, MA. 
HNF-2321

Revision 0

tables. These data show delays in staging transfers (if any), complexity of bootstrap transfers, density of tank to tank transfers, and evaporator usage.

\subsection{EVAPORATOR SCHEDULE}

Evaporator schedule includes every evaporator campaign. This data shows how much volume is removed from the tank system over time and the modeled usage of the evaporator. This data will be presented in a tabular form containing the number of evaporator campaigns per calendar year and the volume evaporated. More elaborate descriptions of evaporator operations can be found in the Waste Transfer History tables.

\subsection{VENDOR FEED TANK FILL HISTORY}

This measure presents the fill volume of the vendor feed tanks over time. Depending on the alternative, this measure shows either when the tanks become empty and are turned over or shows the estimated usage of the tanks by operations during upgrade. This information will be presented as a graphic.

\subsection{PROJECT HANFORD MANAGEMENT CONTRACTOR STAGING TANKS FILL HISTORY}

This measure presents the fill volume of the staging tanks over time. Its purpose is to show the estimated usage of these tanks by operations during the PHMC Team's upgrade operations. This information will be presented as a graphic.

\subsection{DOUBLE-SHELL TANK SYSTEM FILL HISTORY}

This measure presents the total volume of the DST system over time plus the calculated "free" space over time. The free space is the unfilled tank space not belonging to tanks used for Phase $1 \mathrm{HLW}$ or LAW operations. This information will be presented as a graphic.

\subsection{PHASE 2 SINGLE-SHELL TANK RETRIEVAL}

This measure presents the total amount of Phase 2 SST waste retrieved (retrieved volumes), number of SSTs in which retrieval is completed, and number of SSTs that are being retrieved through Phase 1. This information will be presented in a tabular form. More detailed information can be found in the Waste Transfer History tables. 
HNF-2321

Revision 0

\subsection{EVALUATION OF CASES}

This section individually evaluates each of the six cases previously defined in Section 2.0 (Section 5.0 will compare the alternatives to one another). As defined in Section 3.0, the evaluation criteria will include the following:

- Synopsis of Model Changes

- Waste Transfer History

- Evaporator Schedule

- Vendor Feed Tank Fill History (241-AP-108 and possibly 241-AP-106)

- PHMC Staging Tank Fill History (241-AP-102 and 241-AP-104)

- Total DST System Fill History

- Phase 2 SST Retrieval.

Overall model changes (i.e., changes made to HTWOS since the release of the TWRSO\&UP) will be defined in Section 4.1.1. These changes are applicable to all alternatives. Changes pertinent to the two-contractor cases or the one-contractor cases will be defined in Sections 4.1 .2 and 4.1 .3 respectively.

Section 4.1.4 describes the technical approach that was taken to evaluate the alternatives and Section 4.1.5 lists several important modeling assumptions. The modeling assumptions are identical to what was used in the TWRSO\&UP, but are important to understand when examining the cases.

\subsection{MODEL UPDATES}

This section describes changes to the baseline model (TWRSO\&UP) since the release of the TWRSO\&UP. It is divided into three parts. The first part deals with changes to the overall model that apply to both the two- and one-contractor scenarios, the second part deals with changes specific only to the two-contractor cases (Cases 1-3), and the third describes changes pertinent only to the one-contractor cases (Cases 4-6).

\subsubsection{Overall Changes}

The changes described in this section came about because of the continuing effort to maintain/update/refine the HTWOS model so that it better simulates what is expected to occur in tank farms in the future. These changes will be further documented in and incorporated into the next revision of the TWRSO\&UP.

The previous version of HTWOS lumped similar OWVP transfers together. For example, if $1,000 \mathrm{~L}$ (204 gal) of saltwell liquid were added to 241-AN-101 every month for a year, this would be represented as $12,000 \mathrm{~L}$ ( $3,170 \mathrm{gal})$ being added at the end of the year. This was done because running the model with all the transfers would have made the overall run time 
HNF-2321

Revision 0

unmanageable (in excess of 16 hours). However, the model has been updated/refined and now runs times have been reduced to 1 to 2 hours even with monthly transfers.

Evaporator campaigns are now generated on an as-needed basis. The previous HTWOS had evaporator campaigns running every six months wether they were necessary or not. Now campaigns are initiated when the dilute receiver tank (DRT) has $3,000 \mathrm{~m}^{3}(800 \mathrm{Kgal})$ of liquid or a total volume of $3,800 \mathrm{~m}^{3}(1 \mathrm{Mgal})$. The six month sampling period is still present, however the sampling period is based on the last transfer into the DRT. What this means is if the DRT contains a "batch," but it cannot be transferred into the evaporator feed tank (the evaporator is running, the evaporator feed tank already contains a batch that is sampling, etc.) then the sampling time is assumed to start at that date as long as no other additions are made to the DRT. It is important to note that these "smart" evaporator campaigns are only initiated after the date the tanks are turned over to the PCs. Until then the evaporator campaigns given in the OWVP are used.

The "smart" evaporator campaigns also cycle the waste through the evaporator. The previous HTWOS would "evaporate" the feed until the bottoms achieved a SpG of 1.4. This would occur in one pass with some removals being in excess of 90 percent. After discussions with evaporator employees, it was found that the evaporator operates based on a constant feed rate, holdup time, and heat input and that in order to achieve a desired bottoms $\mathrm{SpG}$ several passes were sometimes necessary. Therefore a maximum boiloff rate of $189 \mathrm{~L}(50 \mathrm{gal} / \mathrm{min})$ (based on a feed rate of $530 \mathrm{~L} / \mathrm{min}[140 \mathrm{gal} / \mathrm{min}]$ ) was used. If the bottoms did not achieve the desired SpG after one pass, it would be pumped back to the evaporator feed tank and (after a 14 day delay) re-evaporated. This cycle would continue until the bottoms achieved the $\mathrm{SpG}$ desired or the bottoms volume was less than $946 \mathrm{~m}^{3}(250 \mathrm{Kgal})$. The importance of this change is it more accurately models operations and it also demonstrates the need for keeping the evaporator bottoms tank empty.

A source of additional HLW feed was added (241-C-104) and the overall processing operations were changed. The additional feed was necessary to prove that the PHMC could provide the maximum feed order quantity and the change in operations was necessary to allow for the retrieval of 241-C-104. The partial retrieval of 241-C-104 (approximately 20 percent) into $241-\mathrm{AY}-102$ is initiated in 8/2004. Before this, retrieved $241-\mathrm{C}-106$ and mobilized 241-AY-102 are transferred to 241-AZ-101 to be pretreated. This transfer is done after the waste in $241-\mathrm{AZ}-101$ is processed. After the waste in $241-\mathrm{AZ}-102$ is processed, the retrieved $241-\mathrm{C}$ 104 is transferred into it and pretreated.

The storage of SST dilute complexed (DC) and dilute non-complexed (DN) saitwell liquid is not necessarily segregated for all cases. The OWVP Rev. 23 used 241-AP-106 as the DN east area receiver. However, the removal of this tank from the PHMC's usable volume restricts the volume available for storage and may therefore cause mixing of DC and DN. 
HNF-2321

Revision 0

\subsubsection{Model Changes for the Two-Contractor Cases}

A twelfth LAW feed batch was added. This batch was added so that it could be shown that the PHMC could provided the PCs with the minimum feed order quantity of Envelope $A$ and the maximum feed order quantity. This batch conșists of 241-AP-101, a fraction of 241-AW-104, and shim caustic. This batch is inserted as batch 5 .

Tank 241-AP-107 is used as the east area DRT until 241-AN-105 is emptied. The previous version used 241-AW-105 as the DRT, but the solids slurry volume in 241-AW-105 reduces the usable volume of the tank. This causes more "smart" evaporator campaigns to be initiated and therefore underestimates the rate at which volume could be freed up within the DST system.

\subsubsection{Model Changes for the One-Contractor Cases}

Since there is only one contractor, the amount of feed that needs to be retrieved and processed is cut in half. Therefore a different feed order must be used. The DST feed order is changed to (Table 4.1-1):

Table 4.1-1. Double-Shell Tank Processing Order for One-Contractor Cases.

\begin{tabular}{|l|c|c|}
\hline Tank From & Envelope & Percent Retrieved \\
\hline $241-\mathrm{AN}-105$ & $\mathrm{~A}$ & $100 \%$ Solids and Liquids \\
\hline $241-\mathrm{AN}-104$ & $\mathrm{~A}$ & $100 \%$ Solids and Liquids \\
\hline $241-\mathrm{AW}-101$ & $\mathrm{~A}$ & $100 \%$ Solids and Liquids \\
\hline $241-\mathrm{AY}-101$ & $\mathrm{~B}$ & $\sim 120 \mathrm{MT} \mathrm{Na}$ \\
\hline $241-\mathrm{AN}-107$ & $\mathrm{C}$ & $100 \%$ Liquids \\
\hline $241-\mathrm{AN}-102$ & $\mathrm{C}$ & $\sim 50 \%$ Liquids \\
\hline $241-\mathrm{AN}-103$ & $\mathrm{~A}$ & $100 \%$ Solids and Liquids \\
\hline
\end{tabular}

This sequence uses tanks that are also slated to be retrieved for the two-contractor cases. However, unlike the two-contractor cases, all of the tanks are full as of today (their Phase 1B compositions are set) and no cross-site transfers are necessary. The 50 percent 241-AN-102 batch was added to demonstrate that the PHMC could supply the contractor with the maximum order quantity of feed. Further work is required to refine this sequence to the extent that the twocontractor scenario has been (change order, impact on schedules, etc), but that is outside the scope of this study: For all intents and purposes, this sequence is adequate. 
HNF-2321

Revision 0

The following bullets define changes to the tank allocation used in the TWRSO\&UP HTWOS:

- Tank 241-AP-106 is used as the DRT throughout the entire mission. Since 241-AP106 is not used for Phase 1 activities, there is no need to switch it from its current usage.

- Tank 241-AP-108 serves as the only PC feed tank.

- Tank 241-AN-105 stores the waste that is in 241-AP-107. In the two-contractor cases, 241-AN-104 stores 241-AP-107. Tank 241-AN-105 is used because 241-AN-104 is not empty by the time the PCs begin processing.

- Tank 241-AN-104 will serve as the SE quadrant receipt tank in support of Phase 2 SST retrieval after AW-101 is retrieved. Tank 241-AN-104 is needed to serve as the evaporator bottoms accumulation tank until 241-AW-101 is free due to DST space limitations. In the two-contractor case, $241-\mathrm{AN}-103$ serves as the SE quadrant receipt tank. Tank 241-AN-103 could be used as the SE quadrant receipt tank, but it is not empty until near the end of the mission. In order for the two scenarios to be comparable in Phase 2, SST retrieval effort was made to allocate space for SE quadrant retrieval as early as possible.

- Tank 241-AN-103 will serve as the transfer-site receiver tank after it is retrieved. In the two-contractor case, tank $241-\mathrm{AN}-102$ serves in this role. Tank 241-AN-103 is used because 241-AN-102 is not retrieved completely in this scenario and since 241 -AN-103 is retrieved fully (solids and liquids) it was deemed to be more appropriate than 241-AN-107.

\subsubsection{Technical Approach for Evaluation}

Whenever possible, the HTWOS model was employed to initiate feed staging and pretreatment transfers and/or evaporator campaigns. However not all waste transfers could be modeled in this fashion. Operational tank-to-tank transfers that were made outside of OWVP Rev. 23 (all tank-to-tank transfers that occur after the date 241-AP-108 and 241-AP-106 are turned over or after the first change to the saltwell liquid retrieval sequence) had to be programmed manually. This was accomplished by running the model, determining where the volume or timing pinch is, and altering the waste transfer sequence. This procedure was iterated over until the model achieved the desired results. Integrating OWVP and HTWOS completely (as is recommended in Section 7.0) will eventually alleviate this bothersome task, but at the present time, this iteration is necessary.

Also the timing and volume of all waste generator streams were kept equal to what is in the OWVP Rev. 23 (except for the reduced saltwell liquid retrieval), but for some the destinations had to be altered. East area waste additions that are not originally routed to 241-AN-101 are now routed to the DRT as are cross-site transfers. Also, completed evaporator bottoms are routed to a 
HNF-2321

Revision 0

single DST (241-AW-101 for two-contractor scenario and 241-AN-104 or 241-AW-101 for one) for storage.

\subsubsection{Modeling Assumptions of Note}

The modeling assumptions below are identical to what was used in the TWRSO\&UP, but are important to note when comparing alternatives:

- All cases assume HLW operations

- Phase 1 LAW facilities process waste at $2 \mathrm{MT} /$ day (both two- and one-contractor scenarios)

- The Phase $1 \mathrm{HLW}$ facility processes waste at 0.164 MT non-volatile oxides less Na and $\mathrm{Si} /$ day

- Qualifying/sampling times are equal to 114 days for LAW and 315 days for HLW

- Evaporator bottoms are spec'ed to a SpG of 1.4 .

\subsection{CASE 1}

This case is the basecase alternative for the two-contractor scenario. It is necessary to define a new basecase that includes all the changes defined in Sections 4.1.1 and 4.1.2.

\subsubsection{Synopsis of Model Changes}

Other than the changes outlined in Sections 4.1.1 and 4.1.2, the assumptions for this case match what was given in the TWRSO\&UP.

\subsubsection{Waste Transfer History}

The complete list of tank/waste transfers, the LAW specific transfers, and the HLW specific transfers are included in Appendix A. Table 4.2-1 and Table 4.2-2 below give the schedule for LAW and HLW feed delivery from the PC feed tanks to the facilities. As can be seen in Table 4.2-1 and 4.2-2, no delay in feed delivery is encountered. Small differences between pump end and the next pump start account for the filling of the feed tanks. 
HNF-2321

Revision 0

Table 4.2-1. Low-Activity Waste Feed Delivery Schedule for Case 1.

\begin{tabular}{|l|c|c|c|}
\hline & Envelope & Pump Start & Pump End \\
\hline \multirow{3}{*}{$\begin{array}{l}\text { Contractor 1 } \\
\text { To Process }\end{array}$} & A & $6 / 1 / 02$ & $12 / 31 / 05$ \\
\cline { 2 - 4 } & B & $1 / 2 / 06$ & $3 / 11 / 06$ \\
\cline { 2 - 4 } $\begin{array}{l}\text { Contractor 2 } \\
\text { To Process }\end{array}$ & C & $3 / 12 / 06$ & $5 / 25 / 09$ \\
\cline { 2 - 4 } & A & $6 / 1 / 02$ & $3 / 6 / 06$ \\
\cline { 2 - 4 } & B & $3 / 8 / 06$ & $5 / 14 / 06$ \\
\hline
\end{tabular}

Table 4.2-2. High-Level Waste Feed Delivery Schedule for Case 1.

\begin{tabular}{|c|c|c|c|}
\hline & Envelope & Pump Start & Pump End \\
\hline \multirow{4}{*}{$\begin{array}{c}\text { High-level waste } \\
\text { contractor } \\
\text { feed tank } \\
\text { to process }\end{array}$} & D & $6 / 1 / 02$ & $2 / 21 / 03$ \\
\cline { 2 - 4 } & D & $2 / 22 / 03$ & $11 / 14 / 03$ \\
\cline { 2 - 4 } & D & $11 / 14 / 03$ & $8 / 7 / 04$ \\
\cline { 2 - 4 } & D & $8 / 8 / 04$ & $5 / 2 / 05$ \\
\cline { 2 - 4 } & D & $5 / 2 / 05$ & $11 / 16 / 05$ \\
\cline { 2 - 4 } & D & $11 / 17 / 05$ & $6 / 2 / 06$ \\
\cline { 2 - 4 } & D & $6 / 3 / 06$ & $12 / 18 / 06$ \\
\cline { 2 - 4 } & D & $12 / 18 / 06$ & $7 / 4 / 07$ \\
\cline { 2 - 4 } & D & $7 / 5 / 07$ & $1 / 18 / 08$ \\
\hline & D & $1 / 19 / 08$ & $12 / 22 / 08$ \\
\hline
\end{tabular}

\subsubsection{Evaporator Schedule}

Table 4.2-3 defines the number of evaporator campaigns per year and the total amount removed from the DST system. More elaborate details on evaporator campaigns can be found in the tank transfer table in Appendix A. 
HNF-2321

Revision 0

Table 4.2-3. Evaporator Usage by Year for Case 1.

\begin{tabular}{|c|c|c|c|}
\hline \multirow{2}{*}{$\begin{array}{c}\text { Calendar } \\
\text { year }\end{array}$} & \multirow{2}{*}{$\begin{array}{c}\text { Number of } \\
\text { campaigns }\end{array}$} & \multicolumn{2}{|c|}{ Volume removed } \\
\cline { 3 - 4 } & 2 & L & gal \\
\hline 1997 & 3 & $8,755,650$ & $1,256,300$ \\
\hline 1998 & 4 & $6,034,977$ & $2,122,600$ \\
\hline 1999 & 2 & $6,562,441$ & $1,767,568$ \\
\hline 2000 & 2 & $3,278,949$ & $8,733,600$ \\
\hline 2001 & 1 & $3,111,633$ & 822,000 \\
\hline 2002 & 0 & 0 & 0 \\
\hline 2003 & 2 & $6,167,636$ & $1,629,305$ \\
\hline 2004 & 1 & $2,879,097$ & 760,571 \\
\hline 2005 & 2 & $3,785,820$ & $1,000,100$ \\
\hline 2006 & 1 & $4,078,813$ & $1,077,500$ \\
\hline 2007 & 0 & 0 & 0 \\
\hline 2008 & 0 & 0 & 0 \\
\hline 2009 & 1 & $2,979,142$ & 787,000 \\
\hline 2010 & 0 & 0 & 0 \\
\hline 2011 & 21 & $52,325,181$ & $13,822,743$ \\
\hline Total & & & \\
\hline
\end{tabular}

\subsubsection{Vendor Feed Tank Fill History}

The following figures (4.2-1 and 4.2-2) illustrate the fill status of the vendor feed tanks (tanks 241-AP-106 and 241-AP-108 respectively). As can be seen from the figures, 241-AP-106 and 241-AP-108 continue to be used by the PHMC nearly up until the delivery of the first feed batch.

\subsubsection{PHMC Staging Tank Fill History}

The following figures (4.2-3 and 4.2-4) illustrate the fill status of the PHMC staging tanks (tanks 241-AP-102 and 241-AP-104 respectively). Tank 241-AP-102 is used for waste storage until 1/2000 and AP-104 until 6/2000, after which time they remain empty. They are not slated to receive waste from waste generators like 241-AP-106 and 241-AP-108. 
HNF-2321

Revision 0

Figure 4.2-1. Fill History of 241-AP-106 for Case 1.

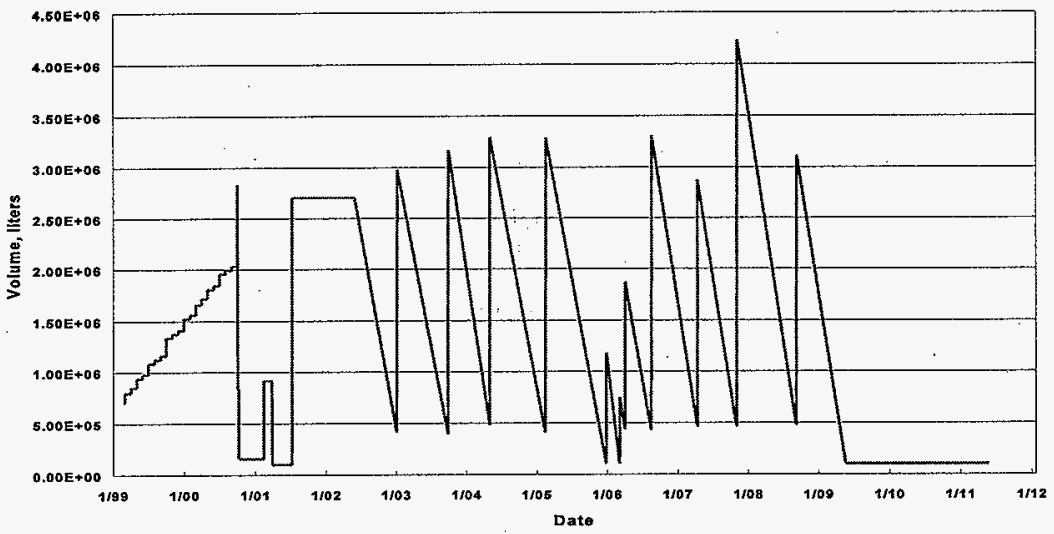

Figure 4.2-2. Fill History of 241-AP-108 for Case 1.

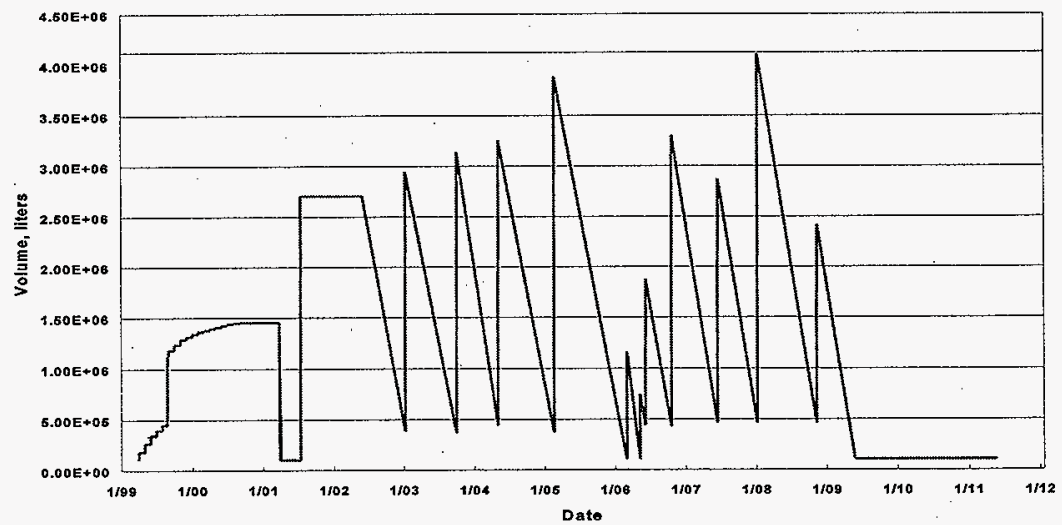


HNF-2321

Revision 0

Figure 4.2-3. Fill History of 241-AP-102 for Case 1.

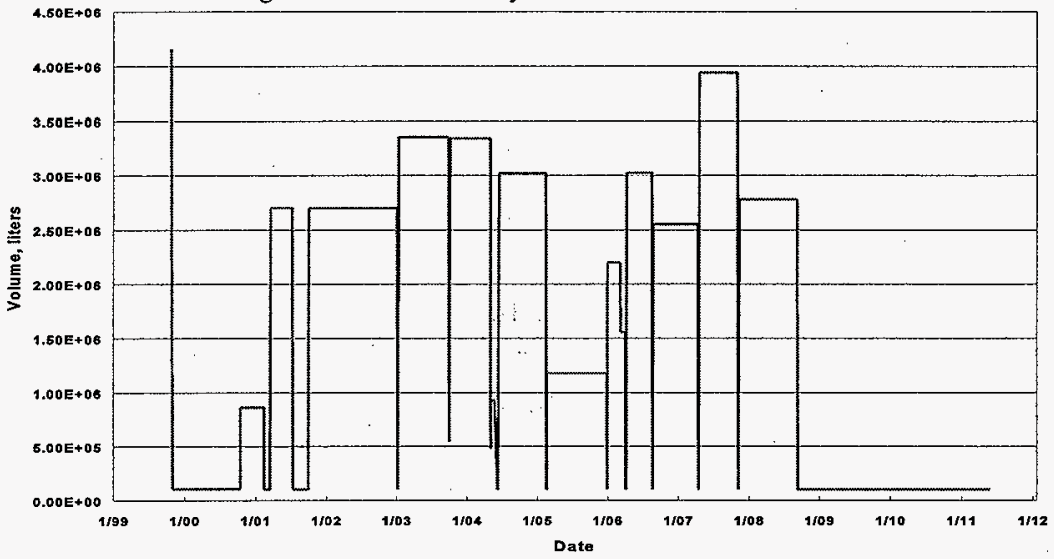

Figure 4.2-4. Fill History for 241-AP-104 for Case 1.

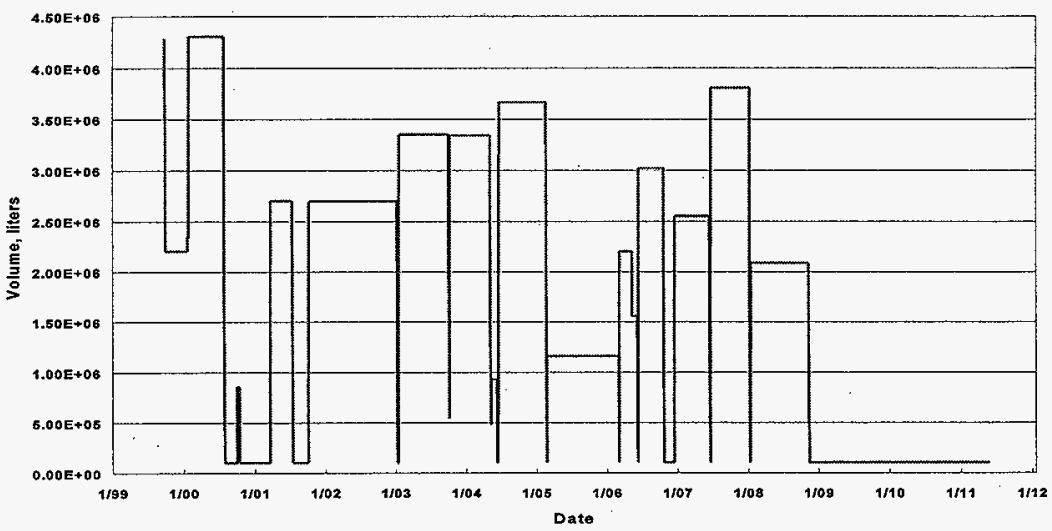


HNF-2321

Revision 0

\subsubsection{Double-Shell Tank System Fill History}

Figure 4.2-5 shows the total volume of the DST system as well as the amount of "free" volume in the DST system through Phase 1. "Free" volume is defined as headspace above tanks that are not used for Phase 1 activities. This excludes tanks 241-AP-102,-104,-106, and -108 after initiation of Phase 1 LAW retrieval; tanks 241-AZ-101, -102 and 241-AY-102; 241-AP-107 after the start of Phase 1 processing; and all DSTs that will be used for Phase 1 LAW feed and in the feed staging activities. After a DST has been retrieved for Phase $1 \mathrm{LAW}$ activities and it is not used for other retrieval operations, its volume becomes "free." Total volume and "free" volume are shown with and without the impact of SST retrieval.

Figure 4.2-5. Total and Free Volume History for Case 1.

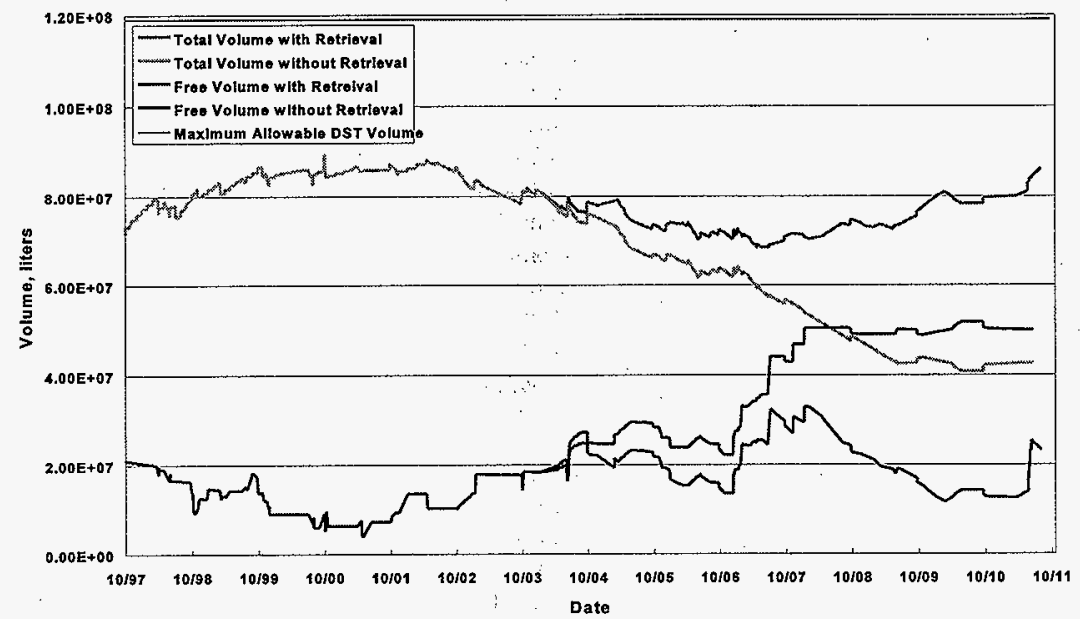

As can be seen in the figure, the "free" volume becomes extremely tight from 10/1999 until approximately 10/2002. After 10/2002 Phase 1 processing exceeds the influx of new DST waste (saltwell liquid [SWL], HLW pretreatment, waste generators).

\subsubsection{Phase 2 Single-Shell Tank Retrieval}

Table 4.2-4 shows the status of SST retrieval for Case 1 at 10/2011 (the start of Phase 2 LAW operations). 
HNF-2321

Revision 0

Table 4.2-4. Phase 2 Single-Shell Tank Retrieval Status for Case 1.

\begin{tabular}{|l|c|c|}
\hline & $\begin{array}{c}\text { Number of } \\
\text { single-shell tanks }\end{array}$ & $\begin{array}{c}\text { Volume retrieved } \\
\text { L (Mgal) }\end{array}$ \\
\hline Retrieval Completed & 36 & $35.2(9.3)$ \\
\hline Retrieval Initiated & 10 & $15.5(4.1)$ \\
\hline Total & 46 & $50.7(13.4)$ \\
\hline
\end{tabular}

No attempt was made to optimize the retrieval sequence. The sequence used was the base case outlined in the TWRSO\&UP.

\subsection{CASE 2}

This case determines what effect emptying the PC tanks (241-AP-106 and 241-AP-108) by 4/1/1999 has on the overall system for the two-contractor scenario.

\subsubsection{Synopsis of Model Changes}

Major changes, other than those outlined in Sections 4.1.1 and 4.1.2, include delaying Phase 1 retrieval activities until 1/2002 (Case 1 - 3/2001) and using 241-AP-104 and 241-AP-103 to stage evaporator waste (until 7/2001 and 10/2001 respectively).

\subsubsection{Waste Transfer History}

The complete list of tank/waste transfers; the LAW specific transfers, and the HLW specific transfers are included in Appendix A: 'Tables 4.3-1 and 4.3-2 give the schedule for LAW and HLW feed delivery from the PC staging tanks to the facilities. As can be seen in Tables 4.31 and 4.3-2, no delay in feed delivery is encountered. Small differences between pump end and the next pump start account for the filling of the feed tanks. 
HNF-2321

Revision 0

Table 4.3-1. Low-Activity Waste Feed Delivery Schedule for Case 2.

\begin{tabular}{|c|c|c|c|}
\hline & Envelope & Pump Start & Pump End \\
\hline \multirow{3}{*}{$\begin{array}{c}\text { Contractor 1 } \\
\text { to process }\end{array}$} & $\mathrm{A}$ & $6 / 1 / 02$ & $11 / 30 / 05$ \\
\cline { 2 - 4 } & $\mathrm{B}$ & $12 / 2 / 05$ & $2 / 8 / 06$ \\
\cline { 2 - 4 } & $\mathrm{C}$ & $2 / 9 / 06$ & $5 / 20 / 09$ \\
\hline \multirow{3}{*}{$\begin{array}{c}\text { Contractor 2 } \\
\text { to process }\end{array}$} & $\mathrm{A}$ & $6 / 1 / 02$ & $1 / 27 / 06$ \\
\cline { 2 - 4 } & $\mathrm{B}$ & $1 / 28 / 06$ & $4 / 7 / 06$ \\
\cline { 2 - 4 } & $\mathrm{C}$ & $4 / 8 / 06$ & $5 / 21 / 09$ \\
\hline
\end{tabular}

Table 4.3-2. High-Level Waste Feed Delivery Schedule for Case 2.

\begin{tabular}{|c|c|c|c|}
\hline \multirow{4}{*}{} & Envelope & $\begin{array}{c}\text { Pump } \\
\text { start }\end{array}$ & $\begin{array}{c}\text { Pump } \\
\text { end }\end{array}$ \\
\hline \multirow{4}{*}{$\begin{array}{c}\text { High-level waste } \\
\text { contractor } \\
\text { feed tank } \\
\text { to process }\end{array}$} & $\mathrm{D}$ & $6 / 1 / 02$ & $2 / 21 / 03$ \\
\cline { 2 - 4 } & $\mathrm{D}$ & $2 / 21 / 03$ & $11 / 14 / 03$ \\
\cline { 2 - 4 } & $\mathrm{D}$ & $11 / 14 / 03$ & $8 / 7 / 04$ \\
\cline { 2 - 4 } & $\mathrm{D}$ & $8 / 8 / 04$ & $5 / 2 / 05$ \\
\cline { 2 - 4 } & $\mathrm{D}$ & $5 / 2 / 05$ & $11 / 16 / 05$ \\
\cline { 2 - 4 } & $\mathrm{D}$ & $11 / 17 / 05$ & $6 / 2 / 06$ \\
\cline { 2 - 4 } & $\mathrm{D}$ & $6 / 3 / 06$ & $12 / 18 / 06$ \\
\cline { 2 - 4 } & $\mathrm{D}$ & $12 / 18 / 06$ & $7 / 4 / 07$ \\
\cline { 2 - 4 } & $\mathrm{D}$ & $7 / 5 / 07$ & $1 / 18 / 08$ \\
\cline { 2 - 4 } & $\mathrm{D}$ & $1 / 19 / 08$ & $12 / 22 / 08$ \\
\hline
\end{tabular}

\subsubsection{Evaporator Schedule}

Table 4.3-3 defines the number of evaporator campaigns per year and the total amount removed from the DST system. More elaborate details on evaporator campaigns can be found in the tank transfer table in Appendix A. 
HNF-2321

Revision 0

Table 4.3-3. Evaporator Usage by Year for Case 2.

\begin{tabular}{|c|c|c|c|}
\hline \multirow{2}{*}{$\begin{array}{c}\text { Calendar } \\
\text { year }\end{array}$} & \multirow{2}{*}{$\begin{array}{c}\text { Number of } \\
\text { campaigns }\end{array}$} & \multicolumn{2}{|c|}{ Volume removed } \\
\cline { 3 - 4 } & 2 & $4,755,650$ & gal \\
\hline 1997 & 3 & $8,034,977$ & $2,1256,300$ \\
\hline 1998 & 2 & $4,347,849$ & $1,148,571$ \\
\hline 1999 & 2 & $3,878,185$ & $1,024,500$ \\
\hline 2000 & 3 & $5,873,783$ & $1,551,677$ \\
\hline 2001 & 1 & $2,346,595$ & 619,900 \\
\hline 2002 & 1 & $3,154,719$ & 833,382 \\
\hline 2003 & 3 & $6,790,160$ & $1,793,757$ \\
\hline 2004 & 1 & $4,231,196$ & $1,117,755$ \\
\hline 2005 & 2 & $4,489,155$ & $1,185,900$ \\
\hline 2006 & 1 & $2,189,878$ & 578,500 \\
\hline 2007 & 1 & $2,297,763$ & 607,000 \\
\hline 2008 & 0 & 0 & 0 \\
\hline 2009 & 1 & $2,734,661$ & 722,415 \\
\hline 2010 & 0 & 0 & 0 \\
\hline 2011 & 23 & $55,124,570$ & $14,562,258$ \\
\hline & & & \\
\hline & & &
\end{tabular}

\subsubsection{Vendor Feed Tank Fill History}

The following figures (4.3-1 and 4.3-2) illustrate the fill status of the vendor feed tanks (tanks 241-AP-106 and 241-AP-108 respectivelý). As can be seen from the figures, 241-AP-106 and 241-AP-108 are empty by $4 / 1999$. The small addition prior to $1 / 2002$ is flush water.

\subsubsection{PHMC Staging Tank Fill History}

The following figures (4.3-3 and 4.3-4) illustrate the fill status of the PHMC staging tanks (tanks AP-102 and AP-104 respectively). AP-102 is used for waste storage until 10/2001 and AP-104 until 7/2001, after which they are quickly flushed and then immediately receive the first batch of LAW feed. Waste transfer operations are very tight for this case. 
HNF-2321

Revision 0

Figure 4.3-1. Fill History of 241-AP-106 for Case 2.

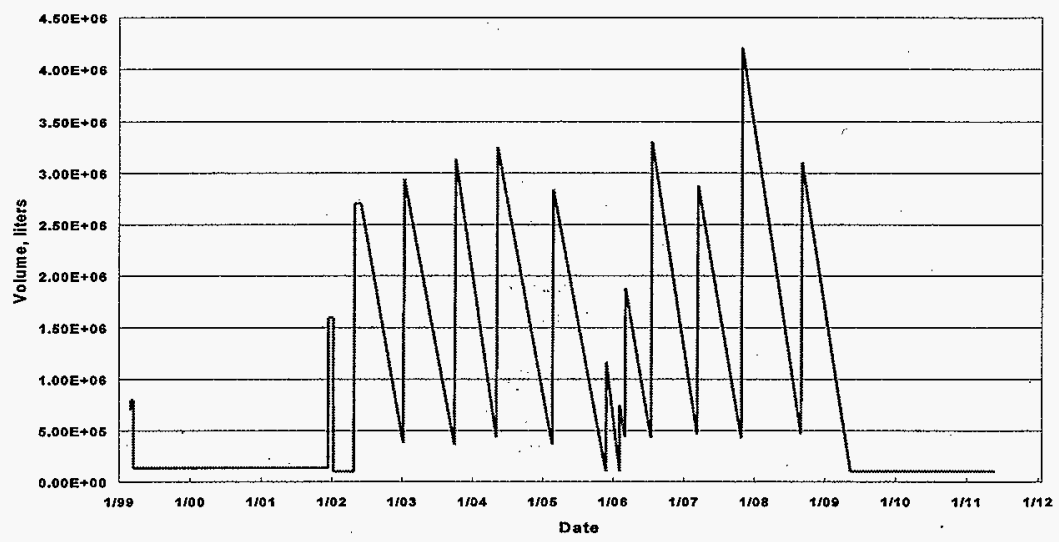

Figure 4.3-2. Fill History of 241-AP-108 for Case 2.

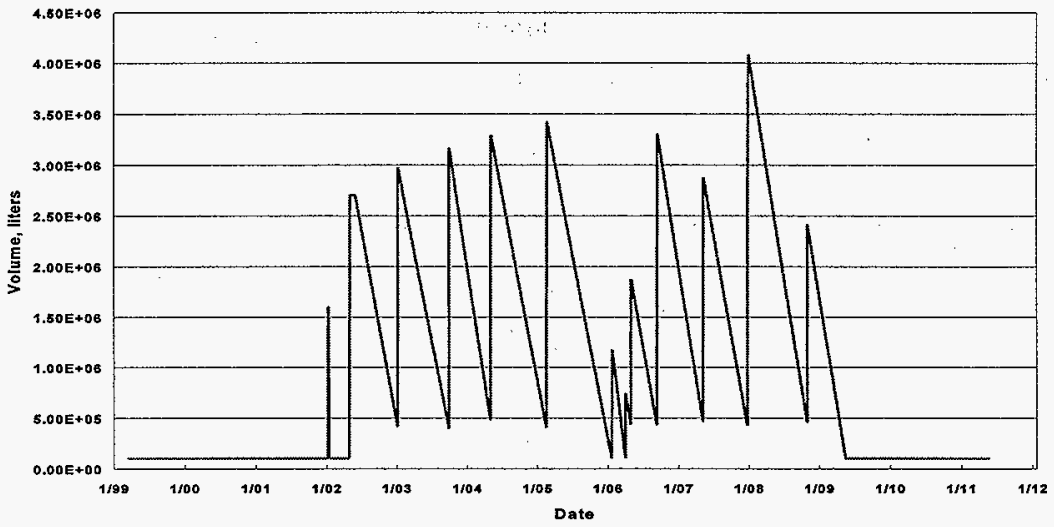


HNF-2321

Revision 0

Figure 4.3-3. Fill History of 241-AP-102 for Case 2.

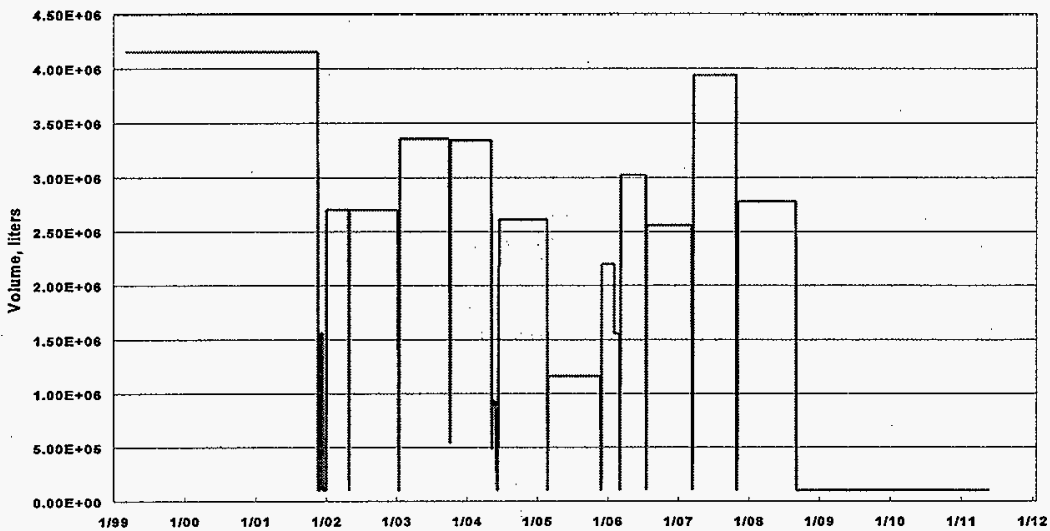

Figure 4.3-4. Fill History of 241-AP-104 for Case 2.

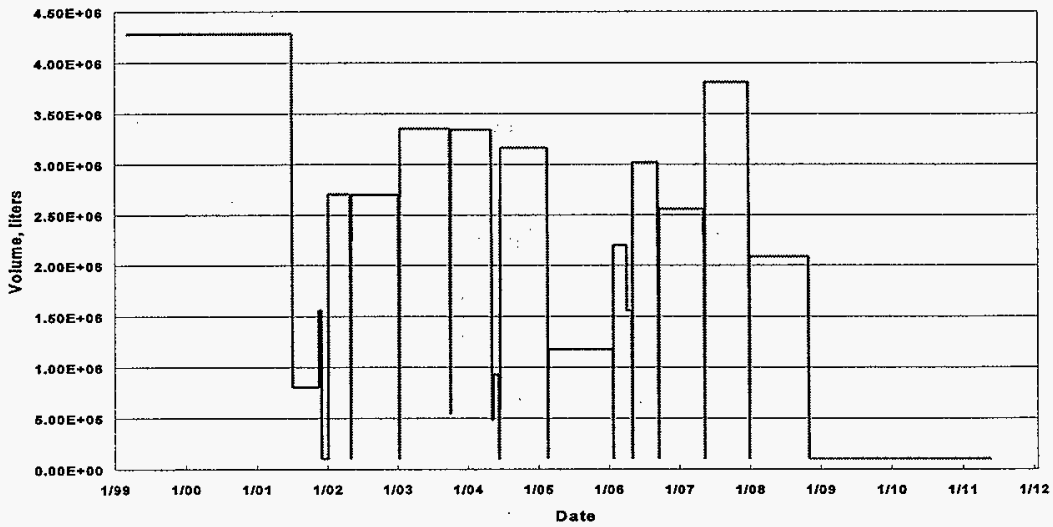


HNF-2321

Revision 0

\subsubsection{Total Double-Shell Tank System Fill History}

Figure 4.3-5 shows the total volume of the DST system as well as the amount of "free" volume in the DST system through Phase 1. "Free" volume is defined as headspace above tanks that are not used for Phase 1 activities. This excludes tanks 241-AP-102,-104,-106, and -108 after initiation of Phase 1 LAW retrieval; tanks 241-AZ-101, -102 and 241-AY-102; 241-AP-107 after the start of Phase 1 processing; and all DSTs that will be used for Phase 1 LAW feed and in the feed staging activities. After a DST has been retrieved for Phase $1 \mathrm{LAW}$ activities and it is not used for other retrieval operations, its volume becomes "free." Total volume and "free" volume are shown with and without the impact of SST retrieval.

As can be seen in the figure, the "free" volume becomes extremely tight from 10/1998 until approximately $1 / 2003$. After $1 / 2003$ Phase 1 processing exceeds the influx of new DST waste (SWL, HLW pretreatment, waste generators).

Figure 4.3-5. Total and Free Volume History for Case 2.

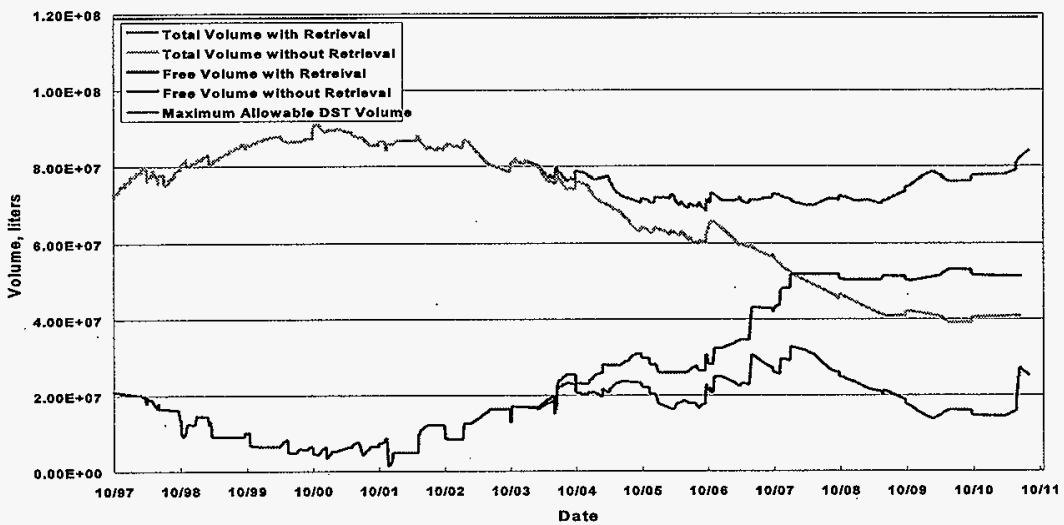

\subsubsection{Phase 2 Single-Shell Tank Retrieval}

Table 4.3-4 below shows the status of SST retrieval for Case 2 at 10/2011 (the start of Phase 2 LAW operations). The results are identical to the results for Case 1. This will be examined in Section 5.0. 
HNF-2321

Revision 0

iable 4.3-4. Phase 2 Single-Shell Tank Retrieval Status for Case 2.

\begin{tabular}{|l|c|c|}
\hline etrieval Completed & $\begin{array}{c}\text { Number of } \\
\text { single-shell tanks }\end{array}$ & $\begin{array}{c}\text { Volume retrieved } \\
\text { ML (Mgals) }\end{array}$ \\
\hline Retrieval Initiated & 36 & $35.2(9.3)$ \\
\hline Total & 10 & $15.5(4.1)$ \\
\hline
\end{tabular}

\subsection{CASE 3}

This case determines what effects emptying the PC tanks (241-AP-106 and 241-AP-108) by 4/1/1999 and delaying the saltwell liquid retrieval operations (until FY 2003) has on the DST system for the two-contractor scenario.

\subsubsection{Synopsis of Model Changes}

Major changes, other than those outlined in Section 4.1.1 and 4.1.2, include delaying Phase 1 retrieval activities until 8/2001 (Case 1 - 3/2001) and using 241-AP-104 and 241-AP-102 to stage evaporator waste (until 5/2001 and 2/2001 respectively). The delay in Phase 1 retrieval activities is not as great as for Case 2 because the effects of the reduced saltwell liquid retrieval schedule appear later in the simulation.

\subsubsection{Waste Transfer History}

The complete list of tank/waste transfers, the LAW specific transfers, and the HLW specific transfers are included in Appendix A. Table 4.4-1 and Table 4.4-2 below give the schedule for LAW and HLW feed delivery from the PC staging tanks to the facilities. As can be seen in Table 4.4-1 and 4.4-2, no delay in feed delivery is encountered. Small differences between pump end and the next pump start account for the filling of the feed tanks. 
HNF-2321

Revision 0

Table 4.4-1. Low-Activity Waste Feed Delivery Schedule for Case 3.

\begin{tabular}{|l|c|c|c|}
\hline & Envelope & Pump start & Pump end \\
\hline \multirow{2}{*}{$\begin{array}{l}\text { Contractor } 1 \\
\text { To Process }\end{array}$} & $\mathrm{A}$ & $6 / 1 / 02$ & $12 / 9 / 05$ \\
\cline { 2 - 4 } & $\mathrm{B}$ & $12 / 11 / 05$ & $2 / 16 / 06$ \\
\cline { 2 - 4 } & $\mathrm{C}$ & $2 / 17 / 06$ & $5 / 25 / 09$ \\
\hline Contractor 2 & $\mathrm{A}$ & $6 / 1 / 02$ & $2 / 6 / 06$ \\
\cline { 2 - 4 } & $\mathrm{B}$ & $2 / 8 / 06$ & $4 / 16 / 06$ \\
\hline & $\mathrm{C}$ & $4 / 17 / 06$ & $5 / 18 / 09$ \\
\hline
\end{tabular}

Table 4.4-2. High-Level Waste Feed Delivery Schedule for Case 3.

\begin{tabular}{|c|c|c|c|}
\hline & Envelope & Pump start & Pump end \\
\hline \multirow{4}{*}{$\begin{array}{c}\text { High-leyel waste } \\
\text { contractor feed tank to } \\
\text { process }\end{array}$} & $\mathrm{D}$ & $6 / 1 / 02$ & $2 / 21 / 03$ \\
\cline { 2 - 4 } & $\mathrm{D}$ & $2 / 22 / 03$ & $11 / 14 / 03$ \\
\cline { 2 - 4 } & $\mathrm{D}$ & $11 / 14 / 03$ & $8 / 7 / 04$ \\
\cline { 2 - 4 } & $\mathrm{D}$ & $8 / 8 / 04$ & $5 / 2 / 05$ \\
\cline { 2 - 4 } & $\mathrm{D}$ & $5 / 2 / 05$ & $11 / 16 / 05$ \\
\cline { 2 - 4 } & $\mathrm{D}$ & $11 / 17 / 05$ & $6 / 2 / 06$ \\
\cline { 2 - 4 } & $\mathrm{D}$ & $6 / 3 / 06$ & $12 / 18 / 06$ \\
\cline { 2 - 4 } & $\mathrm{D}$ & $12 / 18 / 06$ & $7 / 4 / 07$ \\
\cline { 2 - 4 } & $\mathrm{D}$ & $7 / 5 / 07$ & $1 / 18 / 08$ \\
\cline { 2 - 4 } & $\mathrm{D}$ & $1 / 19 / 08$ & $12 / 22 / 08$ \\
\hline
\end{tabular}

\subsubsection{Evaporator Schedule}

Table 4.4-3 below defines the number of evaporator campaigns per year and the total amount removed from the DST system. More elaborate details on evaporator campaigns can be found in the tank transfer table in Appendix A. 
HNF-2321

Revision 0

Table 4.4-3. Evaporator Usage by Year for Case 3.

\begin{tabular}{|c|c|c|c|}
\hline \multirow{2}{*}{$\begin{array}{c}\text { Calendar } \\
\text { year }\end{array}$} & \multirow{2}{*}{$\begin{array}{c}\text { Number of } \\
\text { campaigns }\end{array}$} & \multicolumn{2}{|c|}{ Volume removed } \\
\cline { 3 - 4 } & 2 & $4,755,650$ & gal \\
\hline 1997 & 2 & $4,893,440$ & $1,256,300$ \\
\hline 1998 & 2 & $3,765,757$ & 994,700 \\
\hline 1999 & 1 & $2,145,340$ & 566,734 \\
\hline 2000 & 3 & $6,176,326$ & $1,631,600$ \\
\hline 2001 & 2 & $4,647,972$ & $1,227,855$ \\
\hline 2002 & 2 & $5,268,199$ & $1,391,700$ \\
\hline 2003 & 2 & $5,225,936$ & $1,380,536$ \\
\hline 2004 & 2 & $4,701,666$ & $1,242,039$ \\
\hline 2005 & 1 & $2,833,988$ & 748,654 \\
\hline 2006 & 1 & $3,177,121$ & 839,300 \\
\hline 2007 & 1 & $2,793,656$ & 738,000 \\
\hline 2008 & 0 & 0 & 0 \\
\hline 2009 & 0 & 0 & 0 \\
\hline 2010 & 1 & $3,160,086$ & 834,800 \\
\hline 2011 & 22 & $53,545,135$ & $14,145,018$ \\
\hline & & & \\
\hline & & & \\
\hline & & & \\
\hline
\end{tabular}

\subsubsection{Vendor Feed Tank Fill History}

The following figures (4.4-1 and 4.4-2) illustrate the fill status of the vendor feed tanks (tanks 241-AP-106 and 241-AP-108 respectively). As can be seen from the figures, 241-AP-106 and 241-AP-108 are empty by 4/1999. The small additions around 7/2001 are flush water.

\subsubsection{PHMC Staging Tank Fill History}

The following figures (4.4-3 and 4.4-4) illustrate the fill status of the PHMC staging tanks (tanks 241-AP-102 and 241-AP-104 respectively). Tank 241-AP-102 is used for waste storage until 10/2001 and 241-AP-104 until 7/2001, after which they are quickly flushed and then immediately receive the first batch of LAW feed. Waste transfer operations are very tight for this case. 
HNF-2321

Revision 0

Figure 4.4-1. Fill History of 241-AP-106 for Case 3.

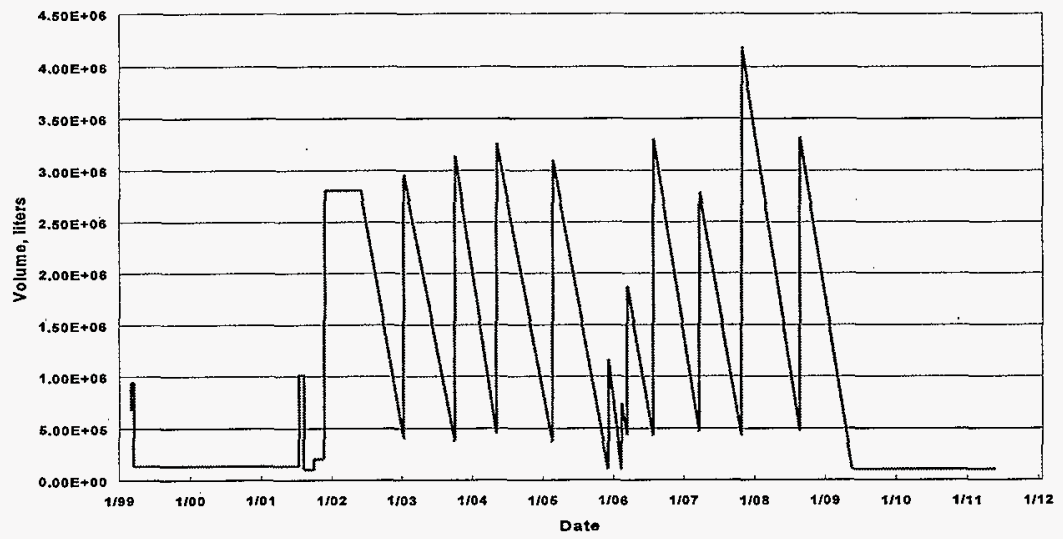

Figure 4.4-2. Fill History of 241-AP-108 for Case 3.

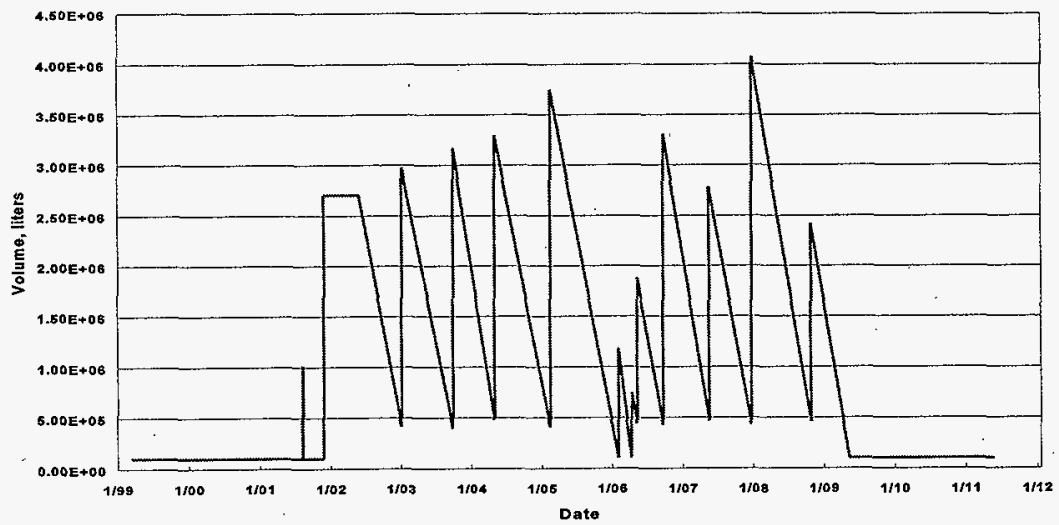


HNF-2321

Revision 0

Figure 4.4-3. Fill History of 241-AP-102 for Case 3.

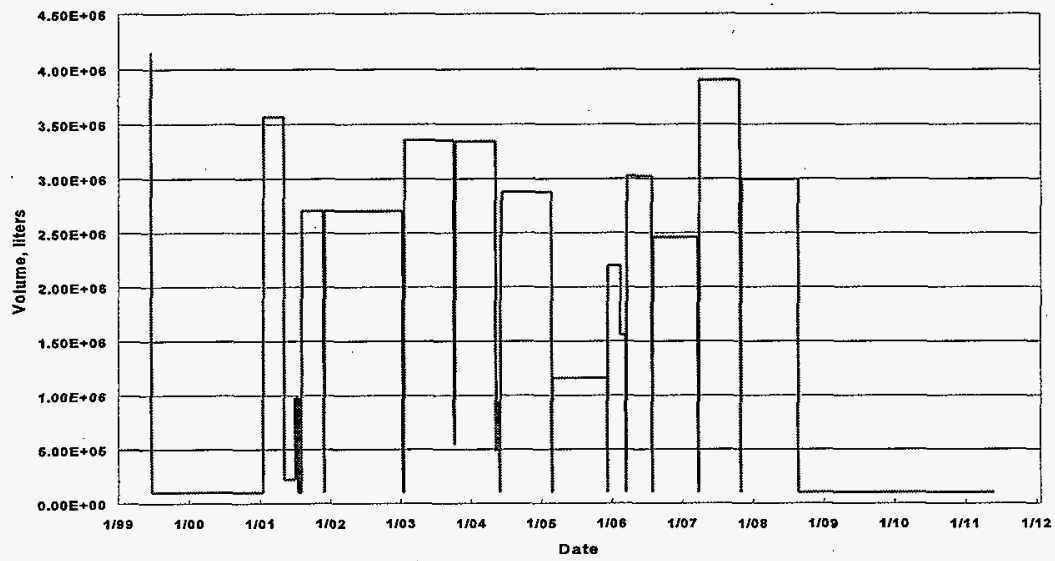

Figure 4.4-4. Fill History of 241-AP-104 for Case 3.

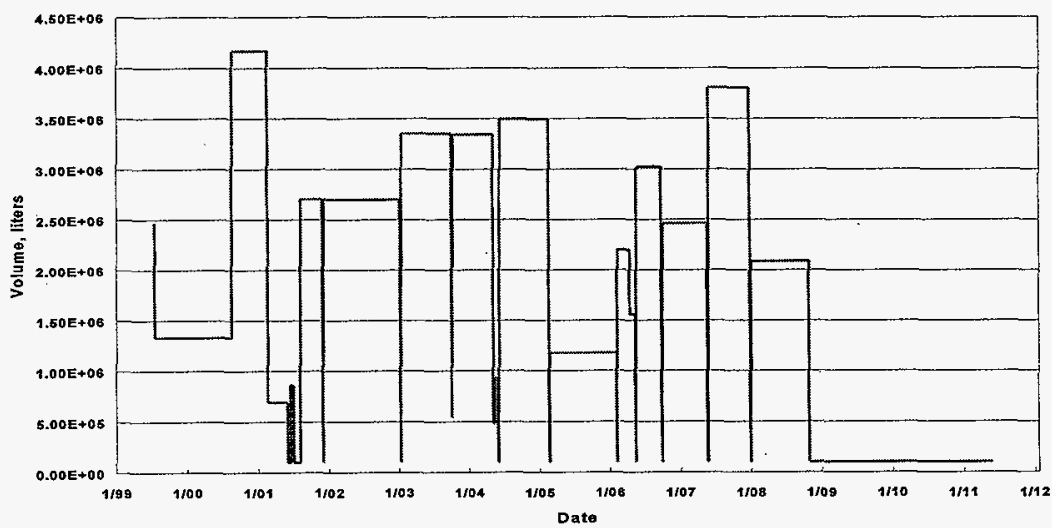




\subsubsection{Total Double-Shell Tank System Fill History}

Figure 4.4-5 shows the total volume of the DST system as well as the amount of "free" volume in the DST system through Phase 1. "Free" volume is defined as headspace above tanks that are not used for Phase 1 activities. This excludes tanks 241-AP-102,-104,-106, and -108 after initiation of Phase 1 LAW retrieval; tanks 241-AZ-101, -102 and 241-AY-102; 241-AP-107 after the start of Phase 1 processing; and all DSTs that will be used for Phase 1 LAW feed and in the feed staging activities. After a DST has been retrieved for Phase $1 \mathrm{LAW}$ activities and it is not used for other retrieval operations, its volume becomes "free." Total volume and "free" volume are shown with and without the impact of SST retrieval.

As can be seen in the figure, the "free" volume becomes extremely tight from 10/1999 until approximately $1 / 2004$. After $1 / 2003$ Phase 1 processing exceeds the influx of new DST waste (SWL, HLW pretreatment, waste generators).

\subsubsection{Phase 2 Single-Shell Tank Retrieval}

Table 4.4-4 below shows the status of SST retrieval for the alternative at 10/2011 (the start of Phase 2 LAW operations). The total number of SSTs retrieved from is greater than alternatives 1 and 2 . This will be addressed in Section 5.0.

Table 4.4-4. Phase 2 Single-Shell Tank Retrieval Status for Case 3.

\begin{tabular}{|l|c|c|}
\hline & $\begin{array}{c}\text { Number of } \\
\text { single-shell tanks }\end{array}$ & $\begin{array}{c}\text { Volume retrieved } \\
\text { ML (Mgal) }\end{array}$ \\
\hline Retrieval Completed & 38 & $39.0(10.3)$ \\
\hline Retrieval Initiated & 10 & $11.4(3.0)$ \\
\hline Total & 48 & $50.4(13.3)$ \\
\hline
\end{tabular}


HNF-2321

Revision 0

Figure 4.4-5. Total and Free Volume History for Case 3.

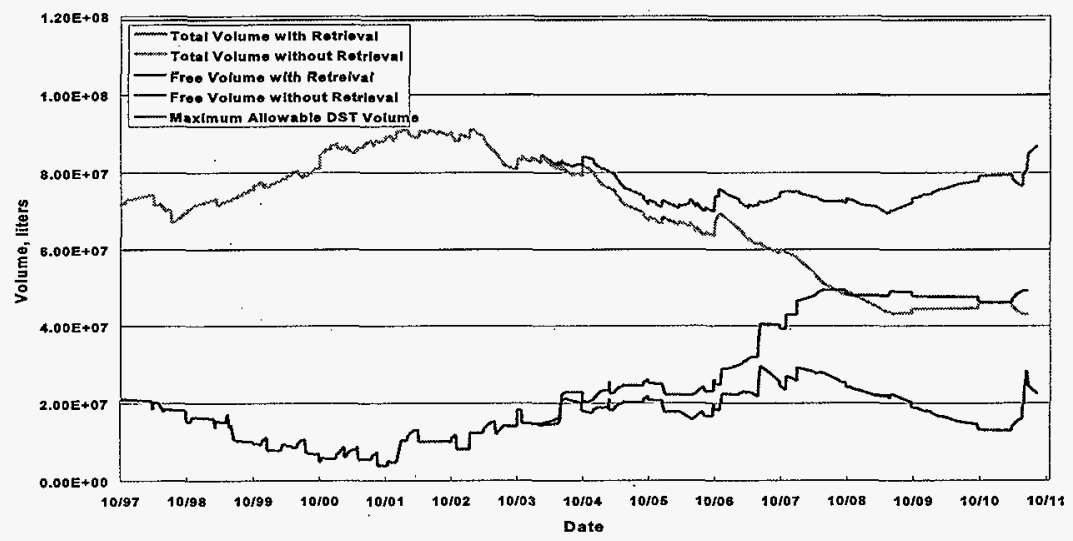

\subsection{CASE 4}

This case is the basecase for the one-contractor alternatives.

\subsubsection{Synopsis of Model Changes}

No major changes, other than those outlined in Section 4.1.1 and 4.1.3, were made to the model for this case.

\subsubsection{Waste Transfer History}

The complete list of tank/waste transfers, the LAW specific transfers, and the HLW specific transfers are included in Appendix A. Table 4.5-1 and Table 4.5-2 below give the schedule for LAW and HLW feed delivery from the PC staging tanks to the facilities. 
HNF-2321

Revision 0

Table 4.5-1. Low-Activity Waste Feed Delivery Schedule for Case 4.

\begin{tabular}{|c|c|c|c|}
\hline & Envelope & Pump start & Pump end \\
\hline \multirow{3}{*}{$\begin{array}{c}\text { Contractor 1 } \\
\text { to process }\end{array}$} & A & $6 / 1 / 02$ & $6 / 12 / 06$ \\
\cline { 2 - 4 } & B & $6 / 14 / 06$ & $8 / 12 / 06$ \\
\cline { 2 - 4 } & C & $8 / 14 / 06$ & $4 / 10 / 08$ \\
\cline { 2 - 4 } & A & $4 / 14 / 08$ & $11 / 28 / 09$ \\
\hline
\end{tabular}

Table 4.5-2. High-Level Waste Feed Delivery Schedule for Case 4.

\begin{tabular}{|c|c|c|c|}
\hline & Envelope & Pump start & Pump end \\
\hline \multirow{4}{*}{$\begin{array}{c}\text { High-level waste } \\
\text { contractor feed tank to } \\
\text { process }\end{array}$} & $\mathrm{D}$ & $6 / 1 / 02$ & $2 / 21 / 03$ \\
\cline { 2 - 4 } & $\mathrm{D}$ & $2 / 22 / 03$ & $11 / 14 / 03$ \\
\cline { 2 - 4 } & $\mathrm{D}$ & $11 / 14 / 03$ & $8 / 7 / 04$ \\
\cline { 2 - 4 } & $\mathrm{D}$ & $8 / 8 / 04$ & $5 / 2 / 05$ \\
\cline { 2 - 4 } & $\mathrm{D}$ & $5 / 2 / 05$ & $11 / 16 / 05$ \\
\cline { 2 - 4 } & $\mathrm{D}$ & $11 / 17 / 05$ & $6 / 2 / 06$ \\
\cline { 2 - 4 } & $\mathrm{D}$ & $6 / 3 / 06$ & $12 / 18 / 06$ \\
\cline { 2 - 4 } & $\mathrm{D}$ & $12 / 18 / 06$ & $7 / 4 / 07$ \\
\cline { 2 - 4 } & $\mathrm{D}$ & $7 / 5 / 07$ & $1 / 18 / 08$ \\
\cline { 2 - 4 } & $\mathrm{D}$ & $1 / 19 / 08$ & $12 / 22 / 08$ \\
\hline & $\mathrm{D}$ & $12 / 22 / 08$ & $11 / 25 / 09$ \\
\hline
\end{tabular}

As can be seen in Table 4.5-1 and 4.5-2, no delay in feed delivery is encountered. Small differences between pump end and the next pump start account for the filling of the feed tanks. For LAW operations, the switch from Envelope $\mathrm{C}$ to $\mathrm{A}$ takes 4 days because of the large volume involved. For the previous envelope switches, small volumes (approximately $120 \mathrm{MT}$ worth of Na) were transferred.

\subsubsection{Evaporator Schedule}

Table 4.5-3 below defines the number of evaporator campaigns per year and the total amount removed from the DST system. More elaborate details on evaporator campaigns can be found in the tank transfer table in Appendix A. 
HNF-2321

Revision 0

Table 4.5-3. Evaporator Usage by Year for Case 4.

\begin{tabular}{|c|c|c|c|}
\hline \multirow{2}{*}{$\begin{array}{c}\text { Calendar } \\
\text { year }\end{array}$} & Number of & \multicolumn{2}{|c|}{ Volume removed } \\
\cline { 3 - 4 } campaigns & L & gal \\
\hline 1997 & 2 & $4,755,650$ & $1,256,300$ \\
\hline 1998 & 3 & $8,034,977$ & $2,122,600$ \\
\hline 1999 & 4 & $6,691,023$ & $1,767,568$ \\
\hline 2000 & 2 & $6,562,441$ & $1,733,600$ \\
\hline 2001 & 1 & $2,748,987$ & 726,200 \\
\hline 2002 & 1 & $2,937,502$ & 776,000 \\
\hline 2003 & 1 & $2,795,427$ & 738,468 \\
\hline 2004 & 1 & $2,961,797$ & 782,418 \\
\hline 2005 & 2 & $5,328,766$ & $1,407,700$ \\
\hline 2006 & 1 & $1,317,334$ & 348,000 \\
\hline 2007 & 1 & $3,911,024$ & $1,033,175$ \\
\hline 2008 & 0 & 0 & 0 \\
\hline 2009 & 1 & $2,356,059$ & 622,400 \\
\hline 2010 & 0 & 0 & 0 \\
\hline 2011 & 1 & $2,776,724$ & 733,527 \\
\hline & 21 & $53,177,711$ & $14,047,956$ \\
\hline
\end{tabular}

\subsubsection{Vendor Feed Tank Fill History}

The following figure (4.5-1) illustrates the fill status of the vendor feed tank (tank 241-AP108). As in Case 1, 241-AP-108 continues to be used to waste storage operations up until delivery of the first feed batch to 241-AP-102 and 241-AP-104. The small spike after 241-AP108 is emptied is flush water. 
HNF-2321

Revision 0

Figure 4.5-1. Fill History of 241-AP-108 for Case 4.

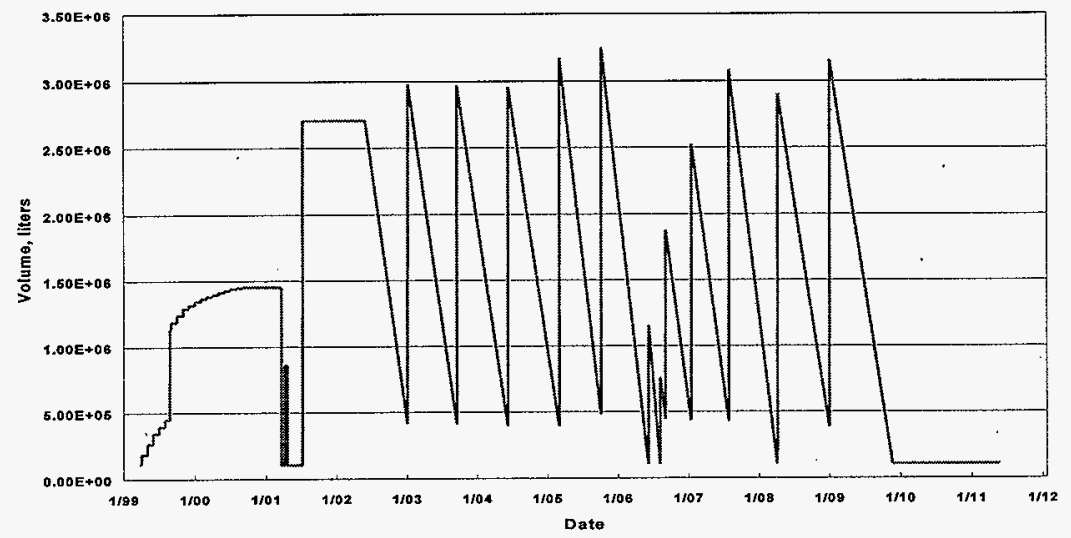

\subsubsection{PHMC Staging Tank Fill History}

The following figures (4.5-2 and 4.5-3) illustrate the fill status of the PHMC staging tanks (tanks 241-AP-102 and 241-AP-104 respectively). Tank 241-AP-104 is used for waste storage until 6/2000; 241-AP-102 is empty 10/1999.

\subsubsection{Total Double-Shell Tank System Fill History}

Figure 4.5-4 shows the total volume of the DST system as well as the amount of "free" volume in the DST system through Phase 1. "Free" volume is defined as headspace above tanks that are not used for Phase 1 activities. This excludes tanks 241-AP-102,-104, and -108 after initiation of Phase 1 LAW retrieval; tanks 241-AZ-101,-102 and 241-AY-102; 241-AP-107 after the start of Phase 1 processing; and all DSTs that will be used for Phase 1 LAW feed and in the feed staging activities. After a DST has been retrieved for Phase $1 \mathrm{LAW}$ activities and it is not used for other retrieval operations, its volume becomes "free." Total volume and "free" volume are shown with and without the impact of SST retrieval. 
HNF-2321

Revision 0

Figure 4.5-2. Fill History of 241-AP-102 for Case 4.

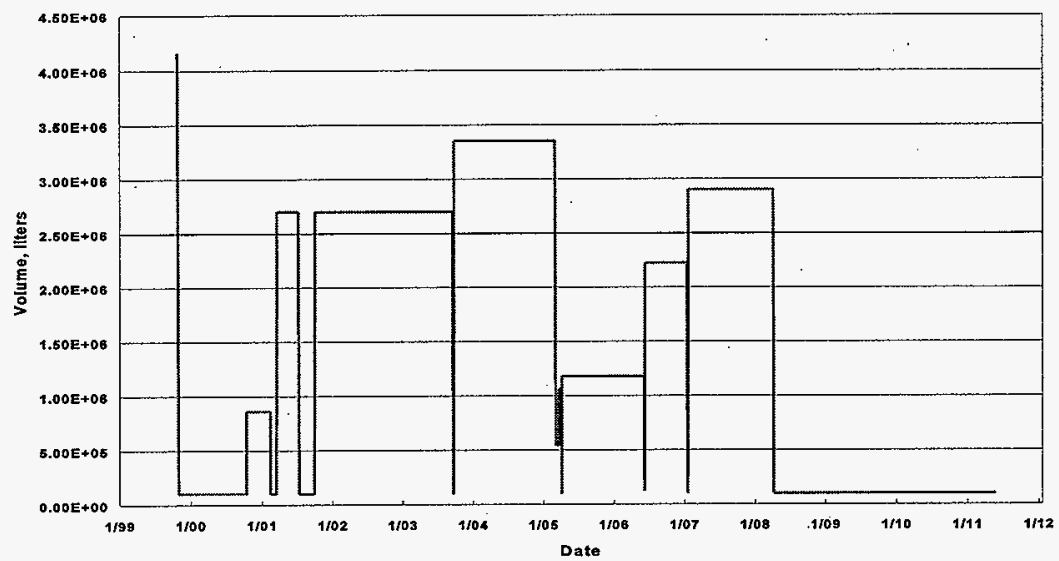

Figure 4.5-3. Fill History of 241-AP-104 for Case 4.

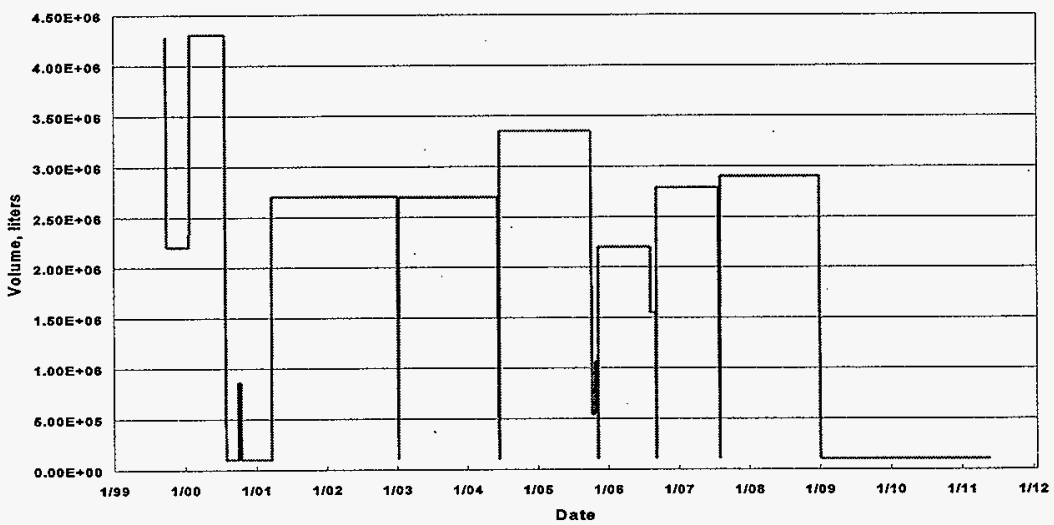


HNF-2321

Revision 0

Figure 4.5-4. Total and Free Volume for Case 4.

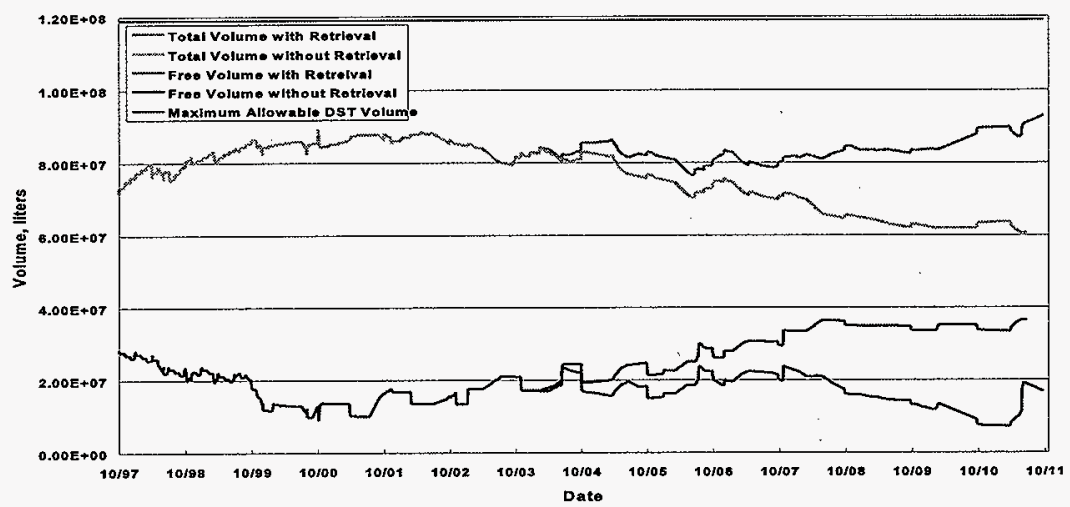

As can be seen in the figure, the "free" volume becomes extremely tight from 10/1999 until approximately $1 / 2002$. After $1 / 2002$ Phase 1 processing exceeds the influx of new DST waste (SWL, HLW pretreatment, waste generators).

\subsubsection{Phase 2 Single-Shell Tank Retrieval}

Table 4.5-4 below shows the status of SST retrieval for the alternative at 10/2011 (the start of Phase 2 LAW operations). As is expected, the number and volume of SSTs retrieved is quite smaller than all of the two-contractor cases (Cases 1-3).

Table 4.5-4. Phase 2 Single-Shell Tank Retrieval Status for Case 4.

\begin{tabular}{|l|c|c|}
\hline & $\begin{array}{c}\text { Number of } \\
\text { single-shell tanks }\end{array}$ & $\begin{array}{c}\text { Volume retrieved } \\
\text { ML (Mgals) }\end{array}$ \\
\hline Retrieval Completed & 27 & $30.3(8.0)$ \\
\hline Retrieval Initiated & 10 & $7.9(2.1)$ \\
\hline Total & 37 & $38.2(10.1)$ \\
\hline
\end{tabular}

\subsection{CASE 5}

This case is the one-contractor, early "hands-off" (4/1999 - 241-AP-108 only) case. 
HNF-2321

Revision 0

\subsubsection{Synopsis of Model Changes}

Major changes, other than those outlined in Section 4.1.1 and 4.1.3, include staging waste to the evaporator from 241-AP-103 (5/2000), 241-AP-107 (7/2000), and 241-AW-105 (7/2001). Also the start of LAW feed staging operations is delayed until 11/2001 (Case 4 is 3/2001).

\subsubsection{Waste Transfer History}

The complete list of tank/waste transfers, the LAW specific transfers, and the HLW specific transfers are included in Appendix A. Tables 4.6-1 and 4.6-2 give the schedule for LAW and HLW feed delivery from the PC staging tanks to the facilities.

Table 4.6-1. Low-Activity Waste Feed Delivery Schedule for Case 5.

\begin{tabular}{|l|c|c|c|}
\hline \multirow{2}{*}{$\begin{array}{l}\text { Contractor } 1 \\
\text { to process }\end{array}$} & Envelope & Pump start & Pump end \\
\cline { 2 - 4 } & A & $6 / 1 / 02$ & $6 / 13 / 06$ \\
\cline { 2 - 4 } & B & $6 / 15 / 06$ & $8 / 13 / 06$ \\
\cline { 2 - 4 } & C & $8 / 15 / 06$ & $4 / 11 / 08$ \\
\hline
\end{tabular}

Table 4.6-2. High-Level Waste Feed Delivery Schedule for Case 5.

\begin{tabular}{|c|c|c|c|}
\hline & Envelope & Pump start & Pump end \\
\hline \multirow{4}{*}{$\begin{array}{c}\text { High-level waste } \\
\text { contractor feed tank } \\
\text { to process }\end{array}$} & $\mathrm{D}$ & $6 / 1 / 02$ & $2 / 21 / 03$ \\
\cline { 2 - 4 } & $\mathrm{D}$ & $2 / 22 / 03$ & $11 / 14 / 03$ \\
\cline { 2 - 4 } & $\mathrm{D}$ & $11 / 14 / 03$ & $8 / 9 / 04$ \\
\cline { 2 - 4 } & $\mathrm{D}$ & $8 / 10 / 04$ & $5 / 6 / 05$ \\
\cline { 2 - 4 } & $\mathrm{D}$ & $5 / 7 / 05$ & $11 / 21 / 05$ \\
\cline { 2 - 4 } & $\mathrm{D}$ & $11 / 21 / 05$ & $6 / 7 / 06$ \\
\cline { 2 - 4 } & $\mathrm{D}$ & $6 / 8 / 06$ & $12 / 22 / 06$ \\
\cline { 2 - 4 } & $\mathrm{D}$ & $12 / 23 / 06$ & $7 / 9 / 07$ \\
\cline { 2 - 4 } & $\mathrm{D}$ & $7 / 9 / 07$ & $1 / 23 / 08$ \\
\cline { 2 - 4 } & $\mathrm{D}$ & $1 / 24 / 08$ & $12 / 26 / 08$ \\
\hline
\end{tabular}

As can be seen in Tables 4.6-1 and 4.6-2, no delay in feed delivery is encountered. Small differences between pump end and the next pump start account for the filling of the feed tanks. For LAW operations, the switch from Envelope $\mathrm{C}$ to $\mathrm{A}$ takes 4 days because of the large volume 
HNF-2321

Revision 0

involved. For the previous envelope switches, small volumes (approximately $120 \mathrm{MT}$ worth of $\mathrm{Na}$ ) were transferred:

\subsubsection{Evaporator Schedule}

Table 4.6-3 below defines the number of evaporator campaigns per year and the total amount removed from the DST system. More elaborate details on evaporator campaigns can be found in the tank transfer table in Appendix A.

Table 4.6-3. Evaporator Usage by Year for Case 5.

\begin{tabular}{|c|c|c|c|}
\hline Calendar year & Number of campaigns & \multicolumn{2}{|c|}{ Volume removed } \\
\cline { 3 - 4 } & & L & gal \\
\hline 1997 & 2 & $4,755,650$ & $1,256,300$ \\
\hline 1998 & 3 & $8,034,977$ & $2,122,600$ \\
\hline 1999 & 2 & $5,200,947$ & $1,373,934$ \\
\hline 2000 & 3 & $4,326,002$ & $1,142,800$ \\
\hline 2001 & 3 & $6,879,287$ & $1,817,301$ \\
\hline 2002 & 2 & $6,394,733$ & $1,689,297$ \\
\hline 2003 & 0 & 0 & 0 \\
\hline 2004 & 2 & $5,947,413$ & $1,571,128$ \\
\hline 2005 & 1 & $3,040,050$ & 803,090 \\
\hline 2006 & 1 & $2,094,641$ & 553,341 \\
\hline 2007 & 1 & $2,336,374$ & 617,200 \\
\hline 2008 & 1 & $2,335,996$ & 617,100 \\
\hline 2009 & 0 & 0 & 0 \\
\hline 2010 & 1 & $2,741,955$ & 724,342 \\
\hline 2011 & 0 & 0 & 0 \\
\hline & 22 & $54,088,026$ & $14,288,434$ \\
\hline
\end{tabular}

\subsubsection{Vendor Feed Tank Fill History}

The following figure (4.6-1) illustrates the fill status of the vendor feed tank (tank 241-AP108). Tank $241-\mathrm{AP}-108$ is empty by $4 / 1999$. The small spike after $241-\mathrm{AP}-108$ is emptied $(11 / 2001)$ is flush water. 
HNF-2321

Revision 0

\subsubsection{PHMC Staging Tank Fill History}

The following figures (4.6-2 and 4.6-3) illustrate the fill status of the PHMC staging tanks (tanks 241-AP-102 and 241-AP-104 respectively). Tank 241-AP-104 is used for waste storage until $10 / 2001 ; 241$-AP-102 is empty 5/2000.

Figure 4.6-1. Fill History of 241-AP-108 for Case 5.

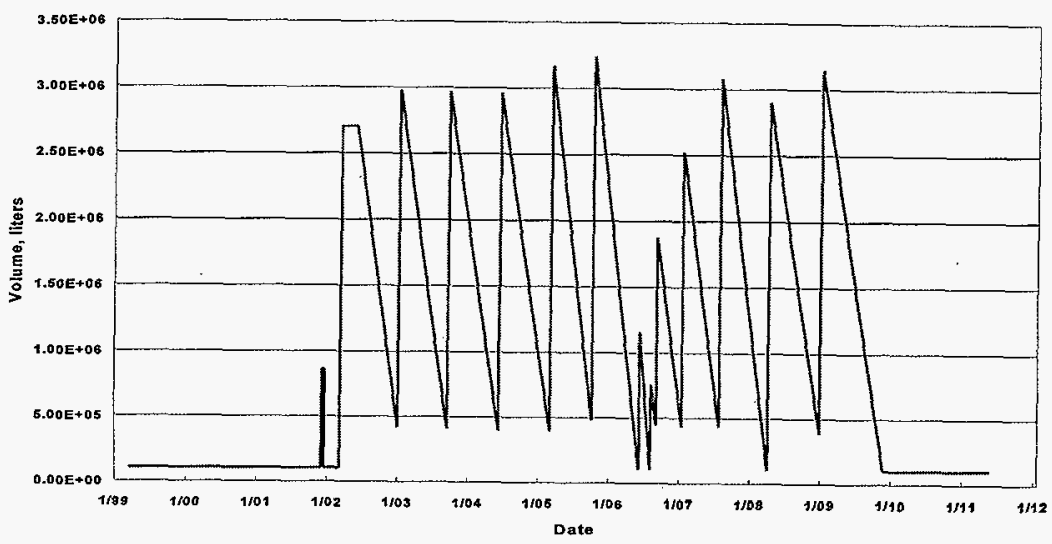


HNF-2321

Revision 0

Figure 4.6-2. Fill History of 241-AP-102 for Case 5.

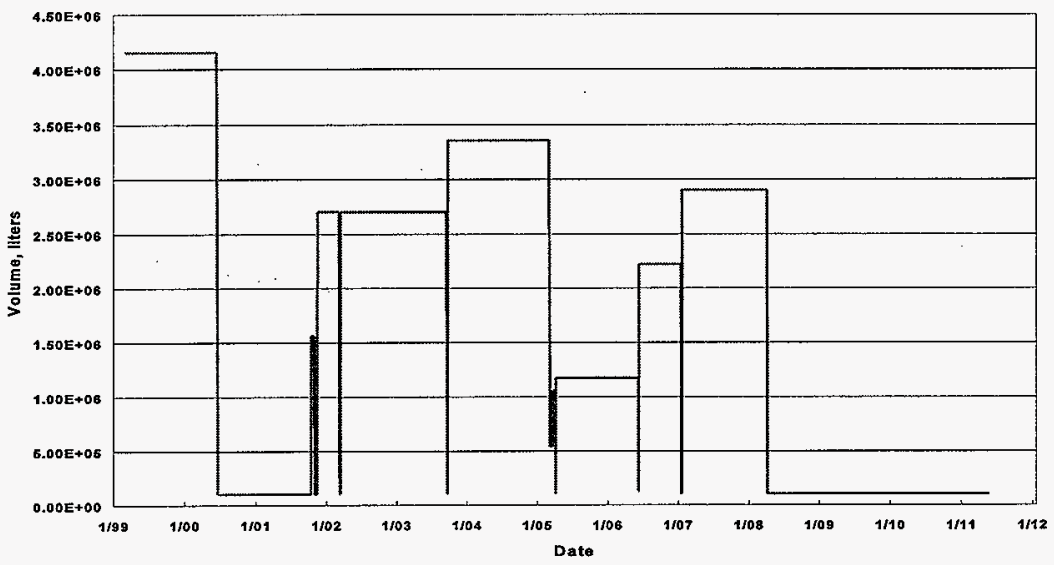

Figure 4.6-3. Fill History of 241-AP-104 for Case 5.

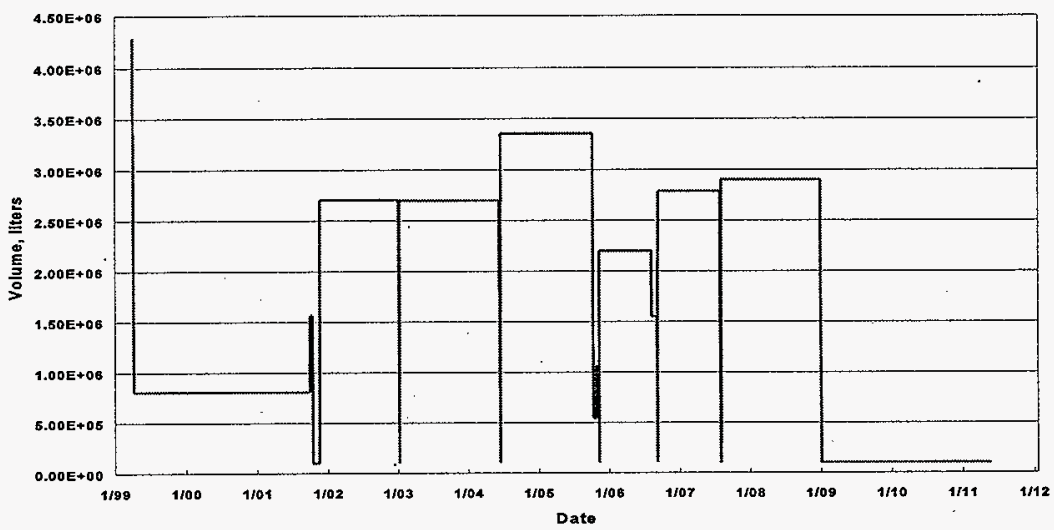


HNF-2321

Revision 0

\subsubsection{Total Double-Shell Tank System Fill History}

Figure 4.6-4 shows the total volume of the DST system as well as the amount of "free" volume in the DST system through Phase 1. "Free" volume is defined as headspace above tanks that are not used for Phase 1 activities. This excludes tanks 241-AP-102,-104, and -108 after initiation of Phase 1 LAW retrieval; tanks 241-AZ-101, -102 and 241-AY-102; 241-AP-107 after the start of Phase 1 processing; and all DSTs that will be used for Phase $1 \mathrm{LAW}$ feed and in the feed staging activities. After a DST has been retrieved for Phase $1 \mathrm{LAW}$ activities and it is not used for other retrieval operations, its volume becomes "free." Total volume and "free" volume are shown with and without the impact of SST retrieval.

Figure 4.6-4. Total and Free Volume History for Case 5.

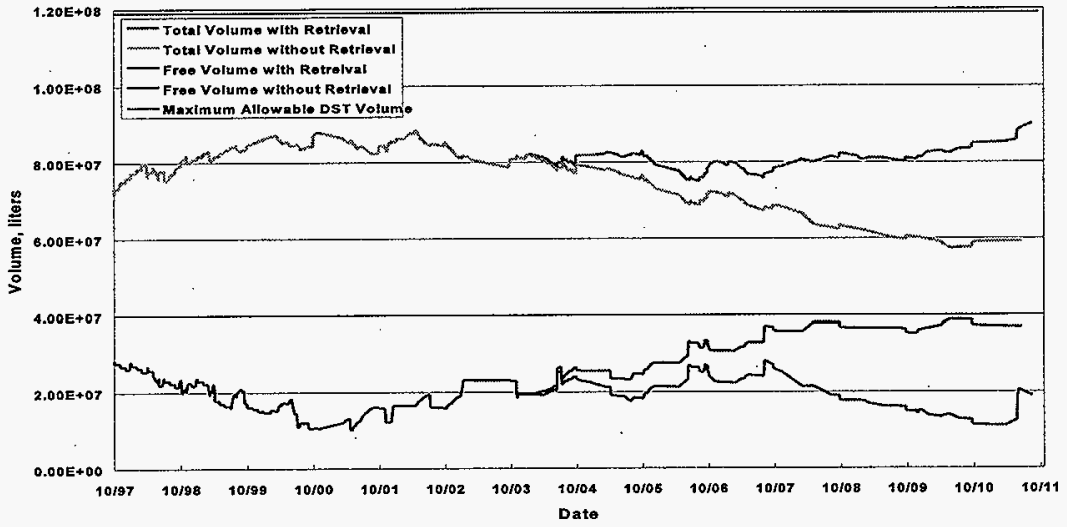

As can be seen in the figure, the "free" volume becomes extremely tight from $8 / 2000$ until approximately 10/2001. After 10/2001 Phase 1 processing exceeds the influx of new DST waste (SWL, HLW pretreatment, waste generators).

\subsubsection{Phase 2 Single-Shell Tank Retrieval}

Table 4.6-4 shows the status of SST retrieval for Case 5 at 10/2011 (the start of Phase 2 LAW operations). As is expected, the number and volume of SSTs retrieved is quite smaller than the two-contractor cases (Cases 1-3). 
HNF-2321

Revision 0

Table 4.6-4. Phase 2 Single-Shell Tank Retrieval Status for Case 5.

\begin{tabular}{|l|c|c|}
\hline & $\begin{array}{c}\text { Number of } \\
\text { single-shell tanks }\end{array}$ & $\begin{array}{c}\text { Volume retrieved } \\
\text { ML (Mgal) }\end{array}$ \\
\hline Retrieval Completed & 26 & $22.0(5.8)$ \\
\hline Retrieval Initiated & 10 & $15.5(4.1)$ \\
\hline Total & 36 & $37.5(9.9)$ \\
\hline
\end{tabular}

\subsection{CASE 6}

This case is the one-contractor, early "hands-off" (4/1999 - 241-AP-108 only), reduced saltwell liquid retrieval schedule case.

\subsubsection{Synopsis of Model Changes}

Major changes, other than those outlined in Section 4.1.1 and 4.1.3, include staging waste to the evaporator from 241-AP-107 (7/2000 and 5/2001), 241-AP-104 (7/2001), 241-AN-105 (6/2003), and 241-AW-105 (7/2004). Also the start of LAW feed staging operations is delayed until $9 / 2001$ (basecase is $3 / 2001$ ).

\subsubsection{Waste Transfer History}

The complete list of tank/waste transfers, the LAW specific transfers, and the HLW specific transfers are included in Appendix A. Table 4.7-1 and Table 4.7-2 below give the schedule for LAW and HLW feed delivery from the PC staging tanks to the facilities.

Table 4.7-1. Low-Activity Waste Feed Delivery Schedule for Case 6.

\begin{tabular}{|c|c|c|c|}
\hline & Envelope & Pump start & Pump end \\
\hline \multirow{3}{*}{$\begin{array}{c}\text { Contractor 1 to } \\
\text { process }\end{array}$} & A & $6 / 1 / 02$ & $6 / 11 / 06$ \\
\cline { 2 - 4 } & B & $6 / 12 / 06$ & $8 / 11 / 06$ \\
\cline { 2 - 4 } & C & $8 / 12 / 06$ & $4 / 9 / 08$ \\
\cline { 2 - 4 } & A & $4 / 13 / 08$ & $11 / 27 / 09$ \\
\hline
\end{tabular}


HNF-2321

Revision 0

Table 4.7-2. High-Level Waste Feed Delivery Schedule for Case 6.

\begin{tabular}{|c|c|c|c|}
\hline & Envelope & Pump start & Pump end \\
\hline \multirow{4}{*}{$\begin{array}{c}\text { High-level waste } \\
\text { contractor feed tank } \\
\text { to process }\end{array}$} & $\mathrm{D}$ & $6 / 1 / 02$ & $2 / 21 / 03$ \\
\cline { 2 - 4 } & $\mathrm{D}$ & $2 / 22 / 03$ & $11 / 14 / 03$ \\
\cline { 2 - 4 } & $\mathrm{D}$ & $11 / 14 / 03$ & $8 / 7 / 04$ \\
\cline { 2 - 4 } & $\mathrm{D}$ & $8 / 8 / 04$ & $5 / 2 / 05$ \\
\cline { 2 - 4 } & $\mathrm{D}$ & $5 / 2 / 05$ & $11 / 16 / 05$ \\
\cline { 2 - 4 } & $\mathrm{D}$ & $11 / 17 / 05$ & $6 / 2 / 06$ \\
\cline { 2 - 4 } & $\mathrm{D}$ & $6 / 3 / 06$ & $12 / 18 / 06$ \\
\cline { 2 - 4 } & $\mathrm{D}$ & $12 / 18 / 06$ & $7 / 4 / 07$ \\
\cline { 2 - 4 } & $\mathrm{D}$ & $7 / 5 / 07$ & $1 / 18 / 08$ \\
\cline { 2 - 4 } & $\mathrm{D}$ & $1 / 19 / 08$ & $12 / 22 / 08$ \\
\hline
\end{tabular}

As can be seen in Table 4.7-1 and 4.7-2, no delay in feed delivery is encountered. Small differences between pump end and the next pump start account for the filling of the feed tanks. For LAW operations, the switch from Envelope $\mathrm{C}$ to $\mathrm{A}$ takes 4 days because of the large volume involved. For the previous envelope switches, small volumes (approximately $120 \mathrm{MT}$ worth of Na) were transferred.

\subsubsection{Evaporator Schedule}

Table 4.7-3 below defines the number of evaporator campaigns per year and the total amount removed from the DST system. More elaborate details on evaporator campaigns can be found in the tank transfer table in Appendix A.

\subsubsection{Vendor Feed Tank Fill History}

The following figure (4.7-1) illustrates the fill status of the vendor feed tank (tank 241-AP108). Tank 241-AP-108 is empty by 4/1999. The small spike after 241-AP-108 is emptied $(11 / 2001)$ is flush water.

\subsubsection{PHMC Staging Tank Fill History}

The following figures (4.7-2 and 4.7-3) illustrate the fill status of the PHMC staging tanks (tanks 241-AP-102 and 241-AP-104 respectively). Tanks 241-AP-104 is used for waste storage until 10/2001, 241-AP-102 is empty 5/2000. 
HNF-2321

Revision 0

Table 4.7-3. Evaporator Usage by Year for Case 6.

\begin{tabular}{|c|c|c|c|}
\hline Calendar year & Number of campaigns & \multicolumn{2}{|c|}{ Volume removed } \\
\cline { 3 - 4 } & & $\mathrm{L}$ & gal \\
\hline 1997 & 2 & $4,755,650$ & $1,256,300$ \\
\hline 1998 & 2 & $4,893,440$ & $1,292,700$ \\
\hline 1999 & 2 & $3,372,450$ & 890,900 \\
\hline 2000 & 2 & $4,609,532$ & $1,217,700$ \\
\hline 2001 & 3 & $5,844,759$ & $1,544,010$ \\
\hline 2002 & 2 & $6,172,162$ & $1,630,500$ \\
\hline 2003 & 2 & $3,963,652$ & $1,047,078$ \\
\hline 2004 & 3 & $6,526,984$ & $1,724,233$ \\
\hline 2005 & 1 & $3,590,870$ & 948,600 \\
\hline 2006 & 1 & $2,337,131$ & 617,400 \\
\hline 2007 & 1 & $2.335,617$ & 617,000 \\
\hline 2008 & 1 & $2,336,374$ & 617,200 \\
\hline 2009 & 0 & 0 & 0 \\
\hline 2010 & 1 & $2,742,294$ & 724,432 \\
\hline 2011 & 0 & 0 & 0 \\
\hline & 23 & $53,480,913$ & $14,128,053$ \\
\hline
\end{tabular}

Figure 4.7-1. Fill History of 241-AP-108 for Case 6.

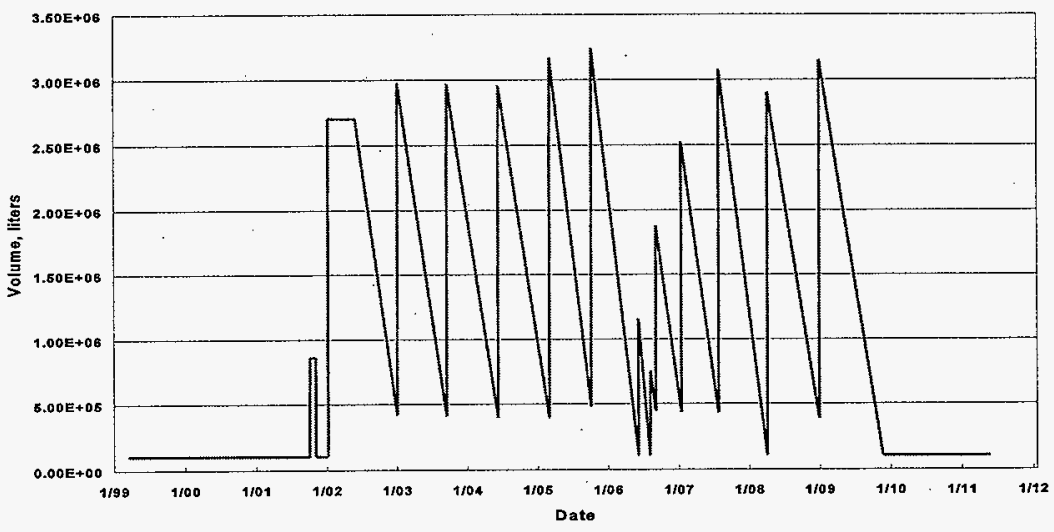


HNF-2321

Revision 0

Figure 4.7-2. Fill History of 241-AP-102 for Case 6.

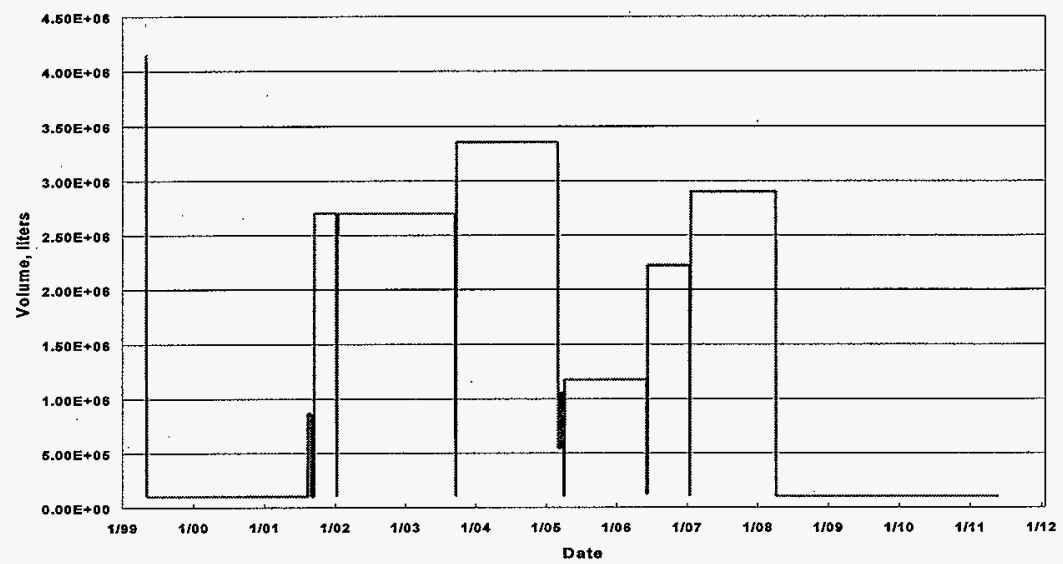

Figure 4.7-3. Fill History of 241-AP-104 for Case 6.

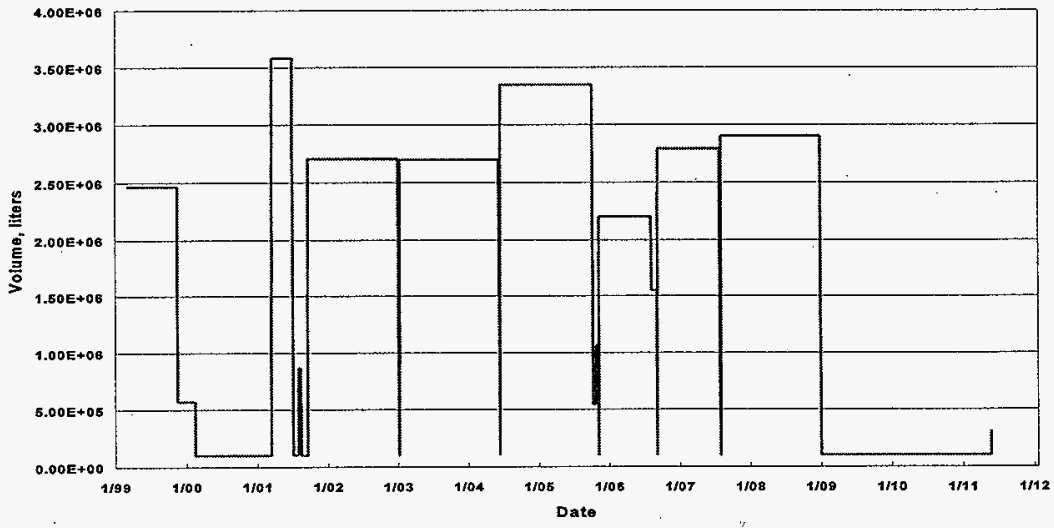


HNF-2321

Revision 0

\subsubsection{Total Double-Shell Tank System Fill History}

Figure 4.7-4 shows the total volume of the DST system as well as the amount of "free" volume in the DST system through Phase 1. "Free" volume is defined as headspace above tanks that are not used for Phase 1 activities. This excludes tanks 241-AP-102,-104, and -108 after initiation of Phase 1 LAW retrieval; tanks 241-AZ-101, -102 and 241-AY-102; 241-AP-107 after the start of Phase 1 processing; and all DSTs that will be used for Phase 1 LAW feed and in the feed staging activities. After a DST has been retrieved for Phase 1 LAW activities and it is not used for other retrieval operations, its volume becomes "free." Total volume and "free" volume are shown with and without the impact of SST retrieval.

Figure 4.7-4. Total and Free Volume History for Case 6.

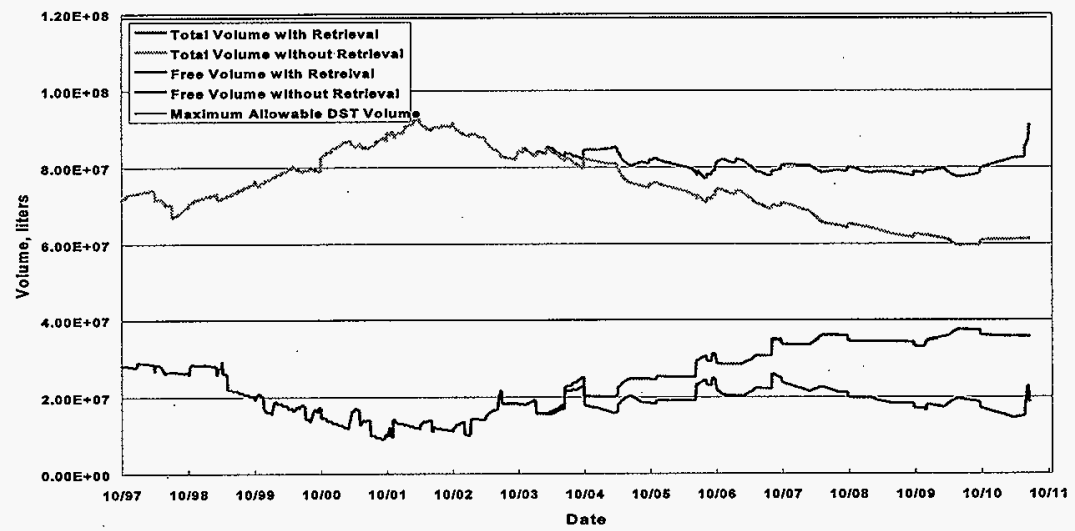

As can be seen in the figure, the "free" volume becomes extremely tight from $8 / 2000$ until approximately $4 / 2002$. After $4 / 2002$ Phase 1 processing exceeds the influx of new DST waste (SWL, HLW pretreatment, waste generators).

\subsubsection{Phase 2 Single-Shell Tank Retrieval}

Table 4.7-4 shows the status of SST retrieval for the alternative at 10/2011 (the start of Phase 2 LAW operations). As is expected, the number and volume of SSTs retrieved is quite smaller than the two-contractor cases. 
HNF-2321

Revision 0

Table 4.7-4. Phase 2 Single-Shell Tank Retrieval Status for Case 6.

\begin{tabular}{|l|c|c|}
\hline & $\begin{array}{c}\text { Number of } \\
\text { single-shell tanks }\end{array}$ & $\begin{array}{c}\text { Volume retrieved } \\
\text { ML (Mgal) }\end{array}$ \\
\hline Retrieval Completed & 30 & $21.6(5.7)$ \\
\hline Retrieval Initiated & 10 & $16.3(4.3)$ \\
\hline Total & 40 & $37.9(10.0)$ \\
\hline
\end{tabular}


HNF-2321

Revision 0

This page intentionally left blank. 
HNF-2321

Revision 0

\subsection{EVALUATION OF ALTERNATIVES}

The evaluation of alternatives will focus on the following parameters that were examined on a case by case basis in Section 4.0:

- Waste Transfer History

- Evaporator Schedule

- Total DST System Fill History

- Phase 2 SST Retrieval.

The vendor feed tank fill history and the PHMC staging tank fill history will not be evaluated since all alternatives stages LAW and HLW feed to the contractors on time without schedule interruption.

The evaluations will be between the two-contractor cases (Cases 1-3) and the one-contractor cases (Cases 4-6) separately. "Sister" cases (Cases 1 and 4, 2 and 5, 3 and 6, where the difference is whether there is one or two contractors) may also be compared.

\subsection{WASTE TRANSFER HISTORY}

The waste transfer history tables (Appendix A) can not be compared on a one-to-one basis with one another. However, what can be compared is pertinent transfers relating to the staging of LAW and HLW feed and the number of tank to tank transfers and the volume of waste transferred per year. The following sections contain the comparisons.

\subsubsection{Low-Activity Waste and High-Level Waste Feed Staging Transfers}

The following tables show the number of days between when a batch of LAW (Table 5.1-1 and 5.1-2) or HLW (Table 5.1-3 and 5.1-4) has been staged/pretreated and when it is transferred to the $\mathrm{PC}$ for processing.

The data in Table 5.1-1 shows that for all batches except 7 and 8 the duration between staging and delivering is greater than or equal to the 114 days modeled as necessary for sampling/qualifying. Batches 7 and 8 are staged into the PHMC feed tanks at the same time. Batch 7 is the delivery of the first batch of Envelope $C$ which follows the delivery of the first batch of Envelope B. Since these first batches for Envelope B and C are to be approximately 120 MT of sodium, processing these batches will take approximately 60 days. Therefore the qualifying time for these two batches will need to be reduced (e.g., the model ignored the time constraint for these batches). This anomaly has been previously noted (TWRSO\&UP) and will be addressed in the next revision of the TWRSO\&UP. 
HNF-2321

Revision 0

Table 5.1-1. Low-Activity Waste Time for Sampling--Two-Contractor Cases.

\begin{tabular}{|c|c|c|c|c|c|c|}
\hline \multirow{3}{*}{ Batch } & \multicolumn{3}{|c|}{ 241-AP-102 } & \multicolumn{3}{|c|}{ 241-AP-104 } \\
\hline & \multicolumn{3}{|c|}{ Case } & \multicolumn{3}{|c|}{ Case } \\
\hline & 1 & 2 & 3 & 1 & 2 & 3 \\
\hline 1 & 114 & 114 & 114 & 114 & 114 & 114 \\
\hline 2 & $\dot{458}$ & 252 & 404 & 458 & 245 & 396 \\
\hline 3 & 258 & 257 & 257 & 257 & 259 & 258 \\
\hline 4 & 210 & 210 & 209 & 209 & 210 & 209 \\
\hline 5 & 244 & 252 & 259 & 245 & 246 & 254 \\
\hline 6 & 310 & 274 & 284 & 371 & 336 & 347 \\
\hline 7 & 66 & 65 & 64 & 64 & 66 & 65 \\
\hline 8 & 27 & 27 & 27 & 27 & 27 & 27 \\
\hline 9 & 129 & 129 & 129 & 129 & 129 & 129 \\
\hline 10 & 232 & 232 & 233 & 181 & 232 & 232 \\
\hline 11 & 196 & 220 & 209 & 197 & 221 & 211 \\
\hline 12 & 299 & 301 & 293 & 300 & 302 & 300 \\
\hline
\end{tabular}

Table 5.1-2. Low-Activity Waste Time for Sampling--One-Contractor Cases.

\begin{tabular}{|c|c|c|c|c|}
\hline \multirow{2}{*}{ Batch } & \multicolumn{4}{|c|}{ Case } \\
\cline { 2 - 5 } & 4 & 5 & 6 & Tank \\
\hline 1 & 114 & 114 & 114 & 241-AN-105 \\
\hline 2 & $\mathbf{6 5 2}$ & $\mathbf{4 0 8}$ & $\mathbf{4 6 9}$ & 241-AN-105 \\
\hline 3 & $\mathbf{7 1 5}$ & $\mathbf{5 5 0}$ & $\mathbf{6 1 0}$ & $\mathbf{2 4 1 - A N - 1 0 4}$ \\
\hline 4 & 516 & 518 & 516 & $241-\mathrm{AN}-104$ \\
\hline 5 & 526 & 526 & 526 & $241-\mathrm{AW}-101$ \\
\hline 6 & 477 & 477 & 477 & $241-\mathrm{AW}-101$ \\
\hline 7 & 428 & 428 & 428 & $241-\mathrm{AY}-101$ \\
\hline 8 & 270 & 270 & 271 & $241-\mathrm{AN}-107$ \\
\hline 9 & 27 & 27 & 27 & $241-\mathrm{AN}-107$ \\
\hline 10 & 218 & 218 & 218 & $241-\mathrm{AN}-107$ \\
\hline 11 & 322 & 322 & 322 & $241-\mathrm{AN}-102$ \\
\hline 12 & 439 & 439 & 440 & $241-\mathrm{AN}-103$ \\
\hline 13 & 513 & 513 & 513 & $241-\mathrm{AN}-103$ \\
\hline
\end{tabular}


HNF-2321

Revision 0

Table 5.1-3. High-Level Waste Time for Sampling--Two-Contractor Cases.

\begin{tabular}{|c|c|c|c|}
\hline \multirow{2}{*}{ Batch } & \multicolumn{3}{|c|}{ Case } \\
\cline { 2 - 4 } & 1 & 2 & 3 \\
\hline $241-\mathrm{AZ}-101$ & 479 & 462 & 479 \\
\hline $241-\mathrm{AZ}-102$ & 518 & $\mathbf{3 4 0}$ & 426 \\
\hline $241-\mathrm{AY}-102 / 241-\mathrm{C}-106$ & $\mathbf{3 1 8}$ & $\mathbf{3 2 1}$ & 440 \\
\hline $241-\mathrm{C}-104$ & $\mathbf{3 3 8}$ & $\mathbf{4 5 2}$ & 465 \\
\hline
\end{tabular}

Table 5.1-4. High-Level Waste Time for Sampling--One-Contractor Cases.

\begin{tabular}{|c|c|c|c|}
\hline \multirow{2}{*}{ Batch } & \multicolumn{3}{|c|}{ Case } \\
\cline { 2 - 4 } & 4 & 5 & 6 \\
\hline $241-\mathrm{AZ}-101$ & 479 & $\mathbf{3 3 4}$ & 479 \\
\hline $241-\mathrm{AZ}-102$ & 518 & 484 & 466 \\
\hline $241-\mathrm{AY}-102 / 241-\mathrm{C}-106$ & 440 & $\mathbf{3 2 1}$ & 418 \\
\hline $241-\mathrm{C}-104$ & 460 & 466 & 466 \\
\hline
\end{tabular}

The differences in Table 5.1-1 for Batch 2 for the cases can be attributed to the different starting times for LAW Phase 1 feed staging. The values for Cases 2 and 3 are not optimized, but the do show that changes to the baseline will affect the amount of time available for staging/qualifying Batch 2. The differences for Batch 6 between 241-AP-102 and 241-AP-104 (approximately 60 days) is due to the addition of the new feed batch. The amount of sodium is different for the two contractors for this batch. However the sodium fed to each contractor balances out by the conclusion of Batch 12. The differences across the board for Batch 6 is due to 241-AP-101 and 241-AW-104 having different compositions for the different cases. This is because 241-AP-101 (and to a lesser extent 241-AW-104) contains evaporator bottoms. As the evaporator campaigns and tank transfers are changed, different compositions can result in 241AP-101 and therefore result in different processing times. The delta between Batch 10, Case 1 for 241-AP-102 and 241-AP-104 is due to a model conflict between LAW and HLW staging. However, this has been determined to be an artificial conflict and the delta should be ignored. This can be validated by examining Figure 4.2-4. The duration for both tanks should be 232 days. The differences across the board for Batch 11 are due to 241-AN-106 having different compositions for each case. This situation is identical to the Batch 6 differences defined above.

Table 5.1-2 shows that for the one-contractor cases every batch except for 9 has the necessary duration between staging and delivering. However, unlike the two-contractor cases, the one-contractor cases do not require modification of the sampling/qualifying time allotment. This is because Batch 9 is the delivery of the remainder of Batch 8 . Batch 8 is the delivery of the first batch of Envelope $C$ and Batch 9 follows when the PC feed tank contains 30 days of feed. 
HNF-2321

Revision 0

Since Batches $8 / 9$ are essentially the same batch, the sampling/qualifying time for Batch 9 is 270 days (duration for Batch 8 ).

The differences in Batches 2 and 3 for the one-contractor cases is due to the differences in the starting times for LAW Phase 1 feed staging. Two batches are affected instead of one (like the two-contractor cases) because of the two staging tanks, one-contractor tank configuration.

As can be seen from Tables 5.1-1 and 5.1-2, the one-contractor cases allow nearly twice the amount of time to sample/qualify the LAW feed. This is because the two-contractor cases have a 1:1 ratio between PHMC staging tanks and private contractor feed tanks while the one-contractor cases have a 2:1 ratio. This higher ratio essentially allows for lag storage prior to delivery and therefore higher sampling/qualifying durations. Also since the one-contractor cases retrieve waste from DST's that are not scheduled to receive any additional waste, the processing durations for each batch are equal for each case.

Table 5.1-3 shows that for the two-contractor cases every batch of HLW has the necessary modeled duration between the end of pretreatment and the start of delivery (314 days). However, several batches are close to this duration. This is due to evaporator campaign conflicts. Since HLW pretreatment interacts greatly with the DST system (decanting supernate, wash solutions) conflicts with waste generator additions and ultimately evaporator campaigns can occur. These "close" durations should be noted, but should not be used as absolute discriminators.

Table 5.1-4 shows that for the one-contractor cases every batch of HLW has the necessary modeled duration between the end of pretreatment and the start of delivery (314 days). However, like the two-contractor cases, several batches are close to this duration. As stated previously, these "close" durations should be noted, but should not be used as absolute discriminators.

As can be seen from Table 5.1-3 and 5.1-4 there is essentially no difference in HLW feed staging between the one- and two-contractor cases. While it has been stated that HLW feed staging durations should not be a discriminator, it is interesting to note the results for Cases 3 and 6. Neither of these cases contain a duration that is near the modeled 314 day limit. This may be due to an effect of delay the retrieval of saltwell liquid or may be coincidence. Determining the cause will require further analysis but, since HLW durations are not discriminators, it is not necessary for this exercise. 
HNF-2321

Revision 0

\subsubsection{Number and Volume of Tank Transfers}

Figures 5.1-1 and 5.1-2 show the total volume and the number of tank to tank transfers for the two-contractor cases per calendar year. Figures 5.1-3 and 5.1-4 show the total volume and the number of tank to tank transfers per calendar year for the one-contractor cases. The important years to focus on are 1999 through 2004. These years are when tank space becomes critical and after 2004 waste processing begins to have more effect on freeing up additional tank space.

Figures 5:1-1 and 5.1-2 show that turning over 241-AP-106 and 241-AP-108 early push transfers and volumes out into 2002 - 2003. This is critical, because during this time HLW pretreatment is occurring and available tank space is minimal. The figures also demonstrate the impact delaying the saltwell liquid retrieval schedule has on operations. A large amount of additional waste (when compared to Cases 1 and 2) is transferred in the $2001-2003$ time frame. This corresponds to the delay in saltwell pumping operations. This can complicate LAW and HLW feed staging operations because they will be occurring simultaneously with saltwell liquid retrieval.

Figure 5.1-1. Total Volume of Tank Transfers per Calendar Year Two-Contractor Cases.

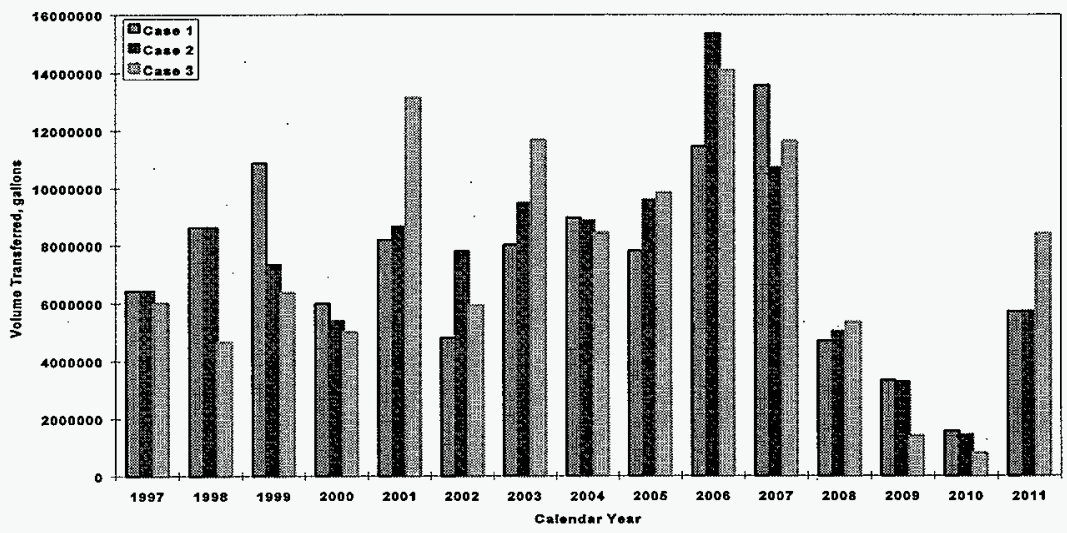


HNF-2321

Revision 0

Figure 5.1-2. Total Number of Tank Transfers per Calendar Year

Two-Contractor Cases.

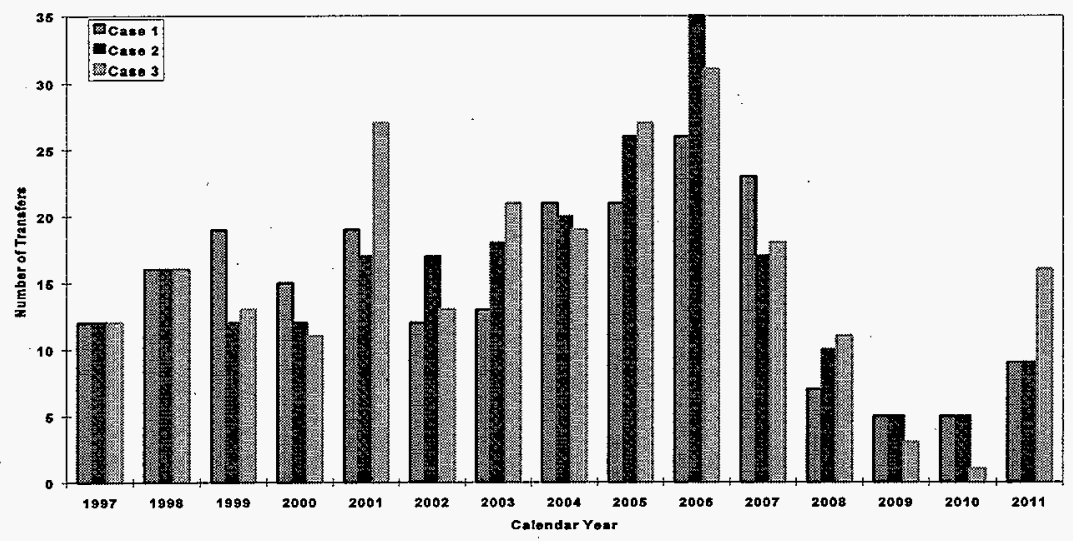

Figure 5.1-3. Total Volume of Tank Transfers per Calendar Year One-Contractor Cases.

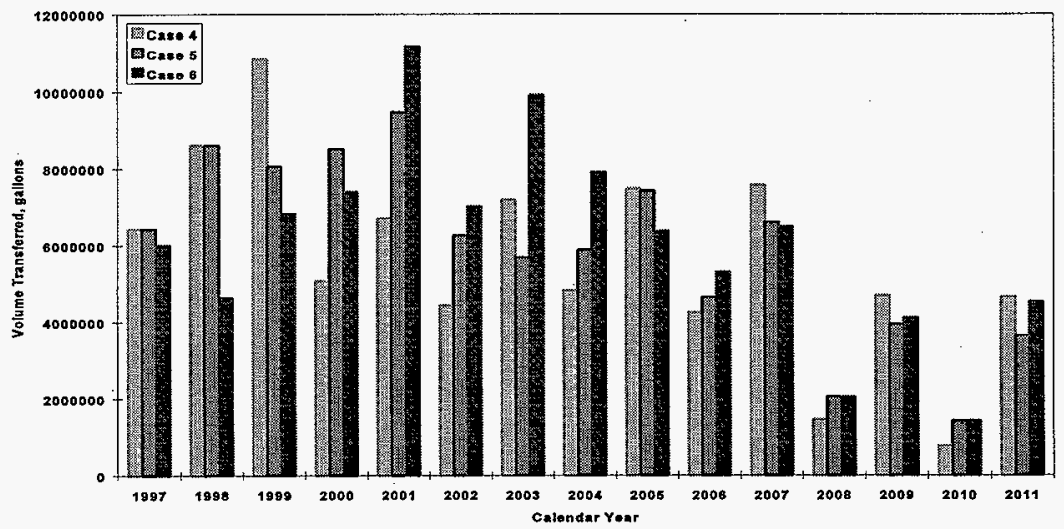


HNF-2321

Revision 0

Figure 5.1-4. Total Number of Tank Transfers per Calendar Year One-Contractor Cases.

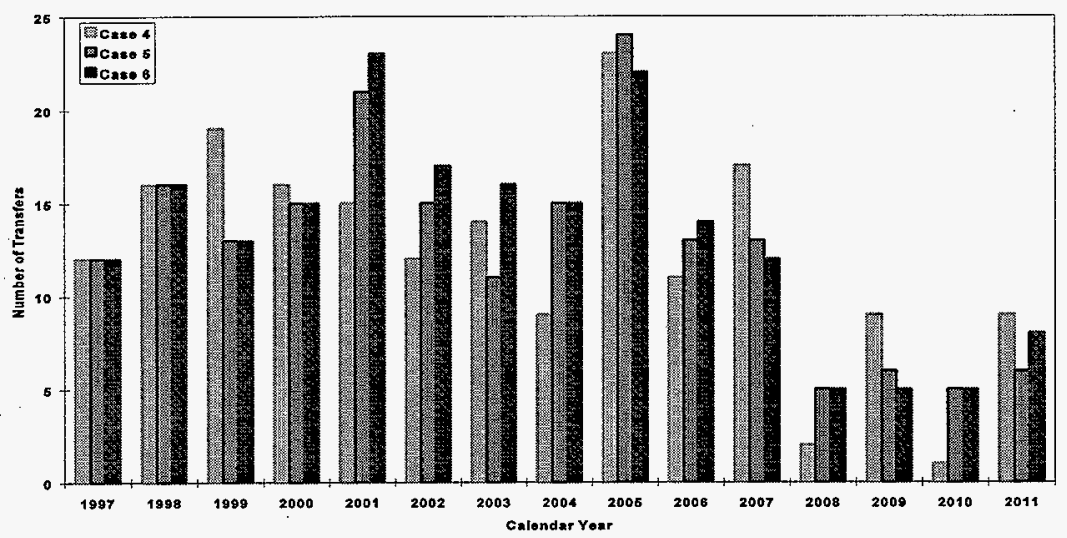

Figures 5.1-3 and 5.1-4 demonstrate the same results as for the two-contractor cases. The figures show that turning AP-108 early pushes transfers and volumes out into 2002 - 2003. The figures also demonstrate the same impact delaying the saltwell liquid retrieval schedule has on operations as the two-contractor cases.

The total volume transferred and the number of transfers per year for the one-contractor cases are much smaller than that for the two-contractor cases. This is to be expected since the cases process different amounts of waste over the same time period. However, the complexity of tank farm operations is not reduced by 50 percent, a large number of tank transfers still need to occur to manage the DST system.

\subsection{EVAPORATOR SCHEDULE}

Tables 5.2-1 and 5.2-2 below summarize the evaporator schedule per calendar year for the two and one-contractor cases respectively. As can be gathered from the tables, each case requires approximately the same number of evaporator campaigns (approximately 22) and results in the same DST volume reduction (approximately $14 \mathrm{Mgals}$ ). 
Table 5.2-1. Evaporator Usage for Two-Contractor Cases.

\begin{tabular}{|c|c|c|c|c|c|c|c|c|c|}
\hline \multirow{3}{*}{ Calendar year } & \multicolumn{3}{|c|}{1} & \multicolumn{3}{|c|}{2} & \multicolumn{3}{|c|}{3} \\
\hline & \multirow{2}{*}{ Number } & \multicolumn{2}{|c|}{ Volume removed } & \multirow{2}{*}{ Number } & \multicolumn{2}{|c|}{ Volume removed } & \multirow{2}{*}{ Number } & \multicolumn{2}{|c|}{ Volume removed } \\
\hline & & $\mathrm{L}$ & gal & & $\mathbf{L}$ & gal & & $\mathrm{L}$ & $\mathrm{gal}$ \\
\hline 1997 & 2 & $4,755,650$ & $1,256,300$ & 2 & $4,755,650$ & $1,256,300$ & 2 & $4,755,650$ & $1,256,300$ \\
\hline 1998 & 3 & $8,034,977$ & $2,122,600$ & 3 & $8,034,977$ & $2,122,600$ & 2 & $4,893,440$ & $1,292,700$ \\
\hline 1999 & 4 & $6,691,023$ & $1,767,568$ & 2 & $4,347,849$ & $1,148,571$ & 2 & $3,765,757$ & 994,800 \\
\hline 2000 & 2 & $6,562,441$ & $1,733,600$ & 2 & $3,878,185$ & $1,024,500$ & 1 & $2,145,340$ & 566,734 \\
\hline 2001 & 2 & $3,278,949$ & 866,200 & 3 & $5,873,783$ & $1,551,677$ & 3 & $6,176,326$ & $1,631,600$ \\
\hline 2002 & 1 & $3,111,633$ & 822,000 & 1 & $2,346,595$ & 619,900 & 2 & $4,647,972$ & $1,227,855$ \\
\hline 2003 & 0 & 0 & 0 & 1 & $3,154,719$ & 833,382 & 2 & $5,268,199$ & $1,391,700$ \\
\hline 2004 & 2 & $6,167,636$ & $1,629,305$ & 3 & $6,790,160$ & $1,793,757$ & 2 & $5,225,936$ & $1,380,536$ \\
\hline 2005 & 1 & $2,879,097$ & 760,571 & 1 & $4,231,196$ & $1,117,755$ & 2 & $4,701,666$ & $1,242,039$ \\
\hline 2006 & 2 & $3,785,820$ & $1,000,100$ & 2 & $4,489,155$ & $1,185,900$ & 1 & $2,833,988$ & 748,655 \\
\hline 2007 & 1 & $4,078,813$ & $1,077,500$ & 1 & $2,189,878$ & 578,500 & 1 & $3,177,121$ & 839,300 \\
\hline 2008 & 0 & 0 & 0 & 1 & $2,297,763$ & 607,000 & 1 & $2,793,656$ & 738,000 \\
\hline 2009 & 0 & 0 & 0 & 0 & 0 & 0 & 0 & 0 & 0 \\
\hline 2010 & 1 & $2,979,142$ & 787,000 & 1 & $2,734,661$ & 722,415 & 0 & 0 & 0 \\
\hline 2011 & 0 & 0 & 0 & 0 & 0 & 0 & 1 & $3,160,086$ & 834,800 \\
\hline Total & 21 & $52,425,181$ & $13,822,743$ & 23 & $55,124,570$ & $14,562,258$ & 22 & $53,545,135$ & $14,145,018$ \\
\hline
\end{tabular}


Table 5.2-2. Evaporator Usage for One-Contractor Cases.

\begin{tabular}{|c|c|c|c|c|c|c|c|c|c|}
\hline \multirow{3}{*}{$\begin{array}{c}\text { Case } \\
\begin{array}{c}\text { Calendar } \\
\text { year }\end{array}\end{array}$} & \multicolumn{3}{|c|}{4} & \multicolumn{3}{|c|}{5} & \multicolumn{3}{|c|}{6} \\
\hline & \multirow{2}{*}{ Number } & \multicolumn{2}{|c|}{ Volume removed } & \multirow{2}{*}{ Number } & \multicolumn{2}{|c|}{ Volume removed } & \multirow{2}{*}{ Number } & \multicolumn{2}{|c|}{ Volume removed } \\
\hline & & $\mathrm{L}$ & gal & & $\mathrm{L}$ & gal & & $\mathrm{L}$ & gal \\
\hline 1997 & 2 & $4,755,650$ & $1,256,300$ & 2 & $4,755,650$ & $1,256,300$ & 2 & $4,755,650$ & $1,256,300$ \\
\hline 1998 & 3 & $8,034,977$ & $2,122,600$ & 3 & $8,034,977$ & $2,122,600$ & 2 & $4,893,440$ & $1,292,700$ \\
\hline 1999 & 4 & $6,691,023$ & $1,767,568$ & 2 & $5,200,947$ & $1,373,934$ & 2 & $3,372,450$ & 890,900 \\
\hline 2000 & 2 & $6,562,441$ & $1,733,600$ & 3 & $4,326,002$ & $1,142,800$ & 2 & $4,609,532$ & $1,217,700$ \\
\hline 2001 & 1 & $2,748,987$ & 726,200 & 3 & $6,879,287$ & $1,817,301$ & 3 & $5,844,759$ & $1,544,010$ \\
\hline 2002 & 1 & $2,937,502$ & 776,000 & 2 & $6,394,733$ & $1,689,297$ & 2 & $6,172,162$ & $1,630,500$ \\
\hline 2003 & 1 & $2,795,427$ & 738,468 & 0 & 0 & 0 & 2 & $3,963,652$ & $1,047,078$ \\
\hline 2004 & 1 & $2,961,797$ & 782,418 & 2 & $5,947,413$ & $1,571,128$ & 3 & $6,526,984$ & $1,724,233$ \\
\hline 2005 & 2 & $5,328,766$ & $1,407,700$ & 1 & $3,040,050$ & 803,090 & 1 & $3,590,870$ & 948,600 \\
\hline 2006 & 1 & $1,317,334$ & 348,000 & 1 & $2,094,641$ & 553,341 & 1 & $2,337,131$ & 617,400 \\
\hline 2007 & 1 & $3,911,024$ & $1,033,175$ & 1 & $2,336,374$ & 617,200 & 1 & $2,335,617$ & 617,000 \\
\hline 2008 & 0 & 0 & 0 & 1 & $2,335,996$ & 617,100 & 1 & $2,336,374$ & 617,200 \\
\hline 2009 & 1 & $2,356,059$ & 622,400 & 0 & 0 & 0 & 0 & 0 & 0 \\
\hline 2010 & 0 & 0 & 0 & 1 & $2,741,995$ & 724,342 & 1 & $2,742,294$ & 724,432 \\
\hline 2011 & 1 & $2,776,724$ & 733,527 & 0 & 0 & 0 & 0 & 0 & 0 \\
\hline Total & 21 & $53,177,711$ & $14,047,956$ & 22 & $54,088,026$ & $14,288,434$ & 23 & $53,480,913$ & $14,128,053$ \\
\hline
\end{tabular}


HNF-2321

Revision 0

As with the tank transfers, the important years to focus on are 1999 through 2004. A portion of the evaporator need in the $2000-2002$ time frame can be attributed to HLW pretreatment, but it is primarily in support of concentrating retrieved saltwell liquid from east and west area. As can be seen from Cases 3 and 6, delaying saltwell liquid retrieval may have an impact on the complexity of LAW and HLW feed staging operations during the $2000-2002$ time frame. However there appears to be no discrimination between having two or one LAW contractor.

\subsection{TOTAL DOUBLE-SHELL TANK SYSTEM FILL HISTORY}

The following figures (5.3-1 to -10) show the total DST system volume and calculated free volume for the two-contractor cases, the one-contractor cases, and each "sister" case as defined in Section 5.0.

Figures 5.3-1 and 5.3-3 demonstrate the impact delaying saltwell liquid retrieval (Cases 3 and 6 respectively) has on the total volume of the DST system. The total volume of the DST system is lower for the early years (1997 - 2000), but increases to a maximum higher than the other cases in the $2001-2003$ time frame. As stated previously, this time frame corresponds to the start of Phase 1 staging operations. The potential for tank space conflict is greater for these cases. In the free volume figures (Figures 5.3-2 and 5.3-4) the minimum free volume for Cases 3 and 6 are also shifted to the later years, but they are not smaller than the other cases. This means that the minimum will occur at a time that corresponds to the start of Phase 1 staging operations, but its effect does not appear to be greater than the other cases. 
HNF-2321

Revision 0

Figure 5.3-1. Total Volume Comparison for Two-Contractor Cases.

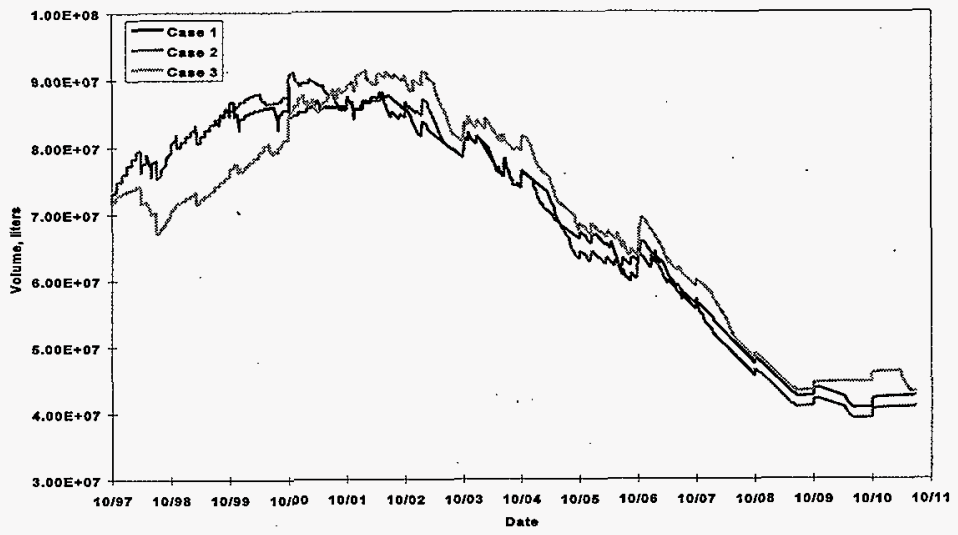

Figure 5.3-2. Free Volume Comparison for Two-Contractor Cases.

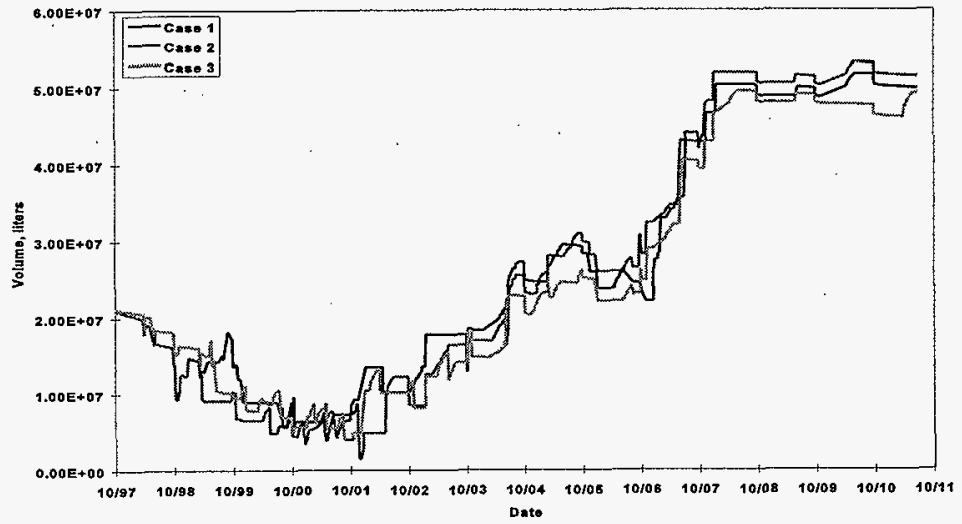


HNF-2321

Revision 0

Figure 5.3-3. Total Volume Comparison for One-Contractor Cases.

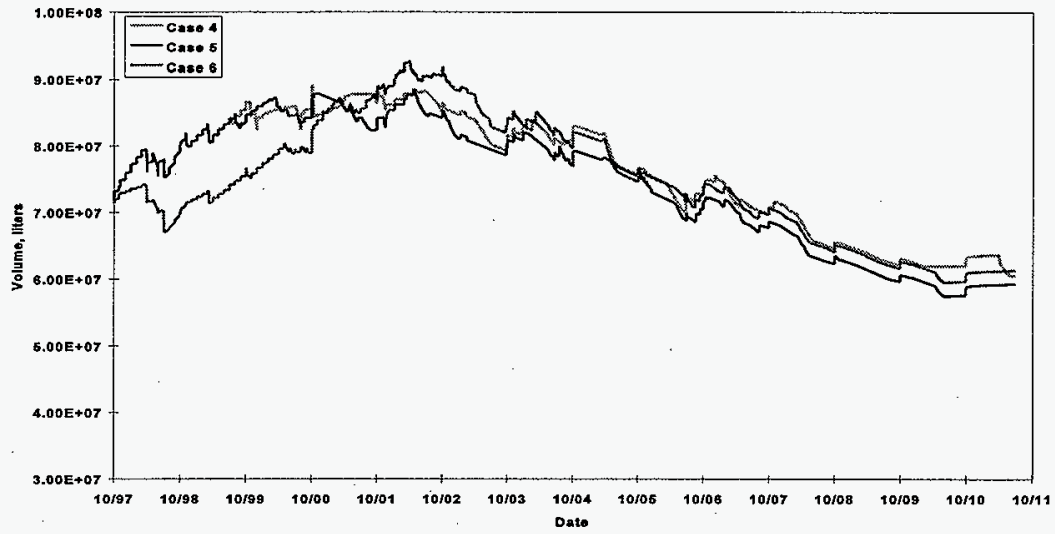

Figure 5.3-4. Free Volume Comparison for One-Contractor Cases.

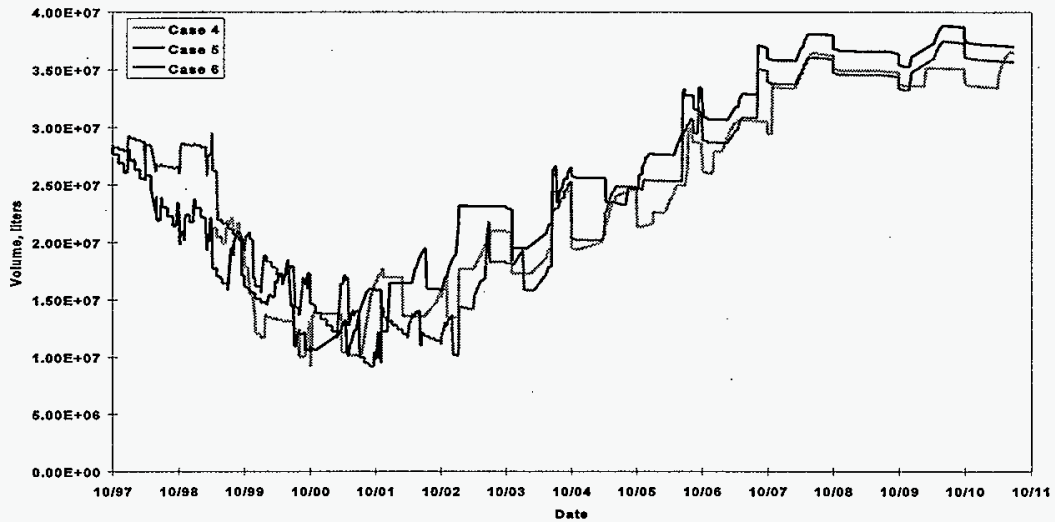


HNF-2321

Revision 0

Figure 5.3-5. Total Volume Comparison - Cases 1 and 4.

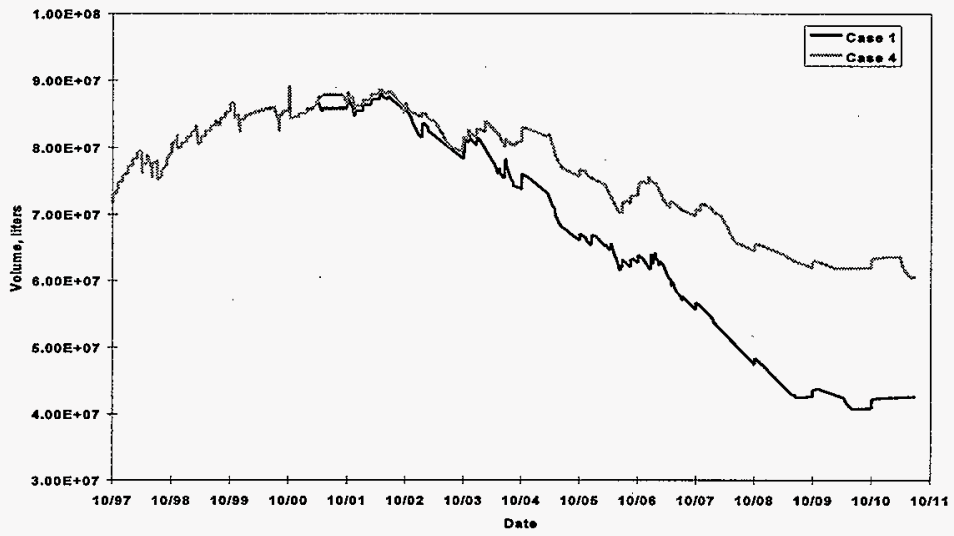

Figure 5.3-6. Free Volume Comparison - Cases 1 and 4.

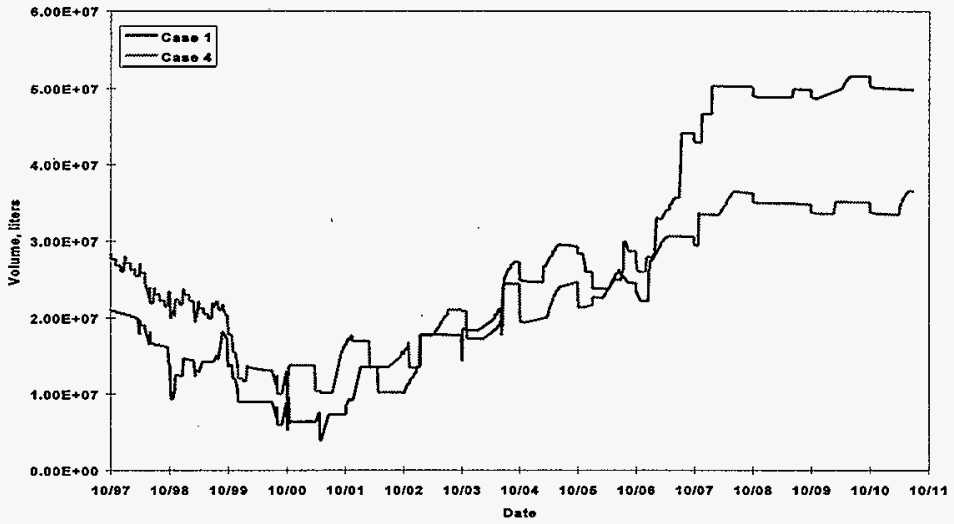


HNF-2321

Revision 0

Figure 5.3-7. Total Volume Comparison - Cases 2 and 5.

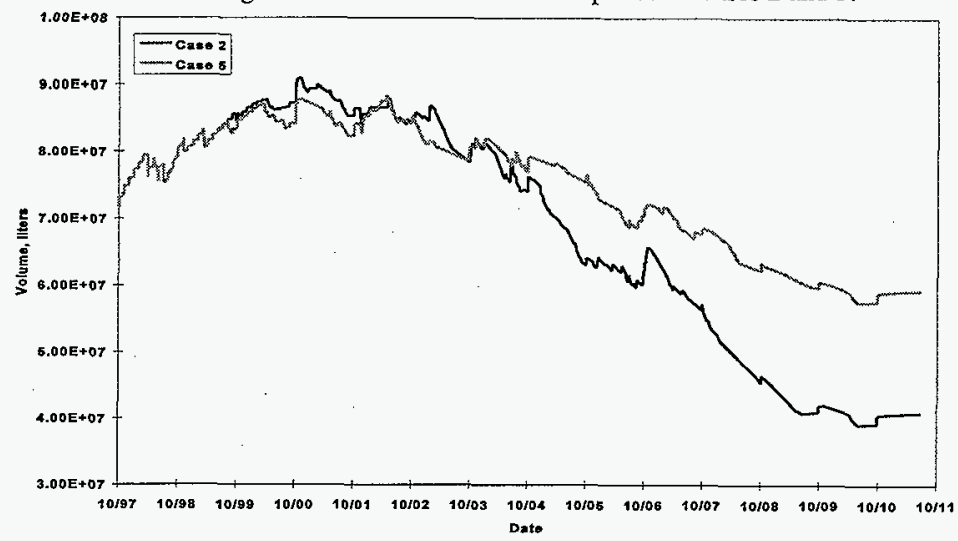

Figure 5.3-8. Free Volume Comparison - Cases 2 and 5,

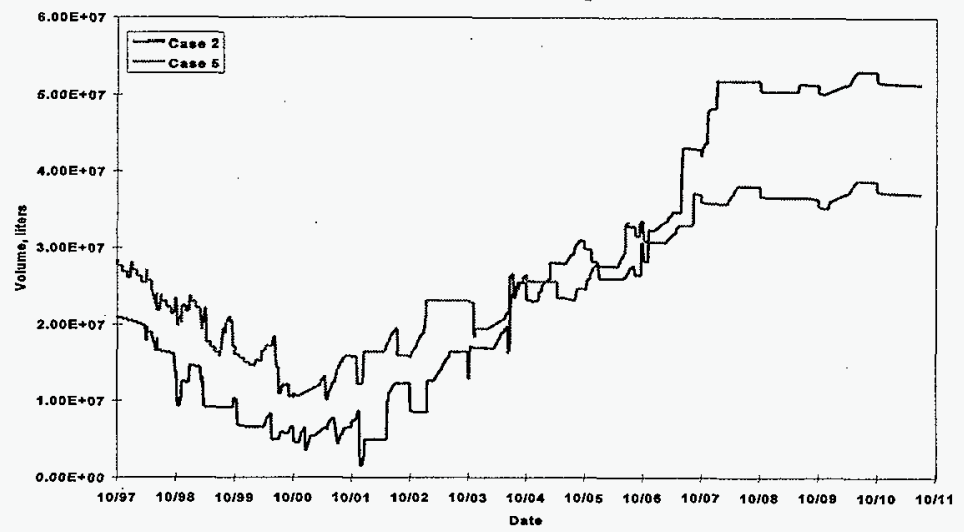


HNF-2321

Revision 0

Figure 5.3-9. Total Volume Comparison - Case 3 and 6.

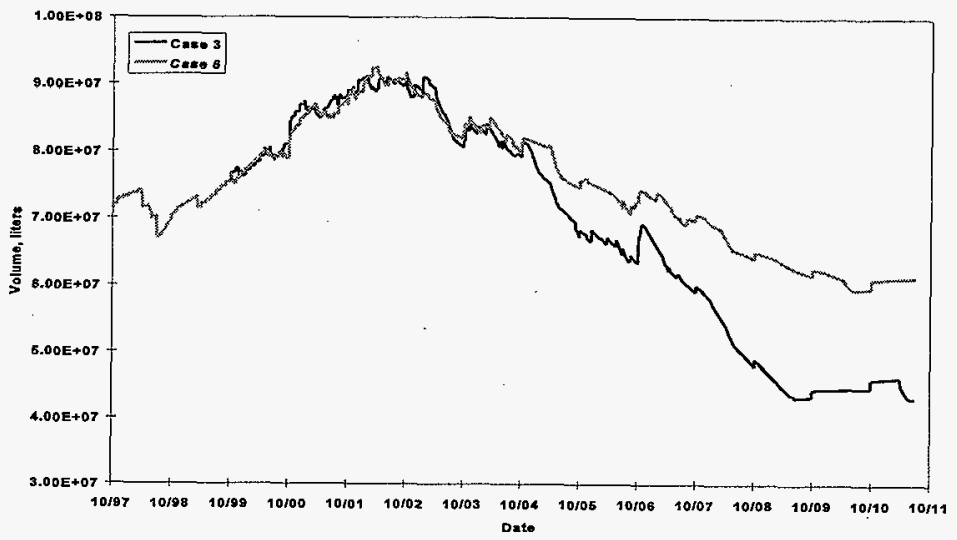

Figure 5.3-10. Free Volume Comparison - Cases 3 and 6.

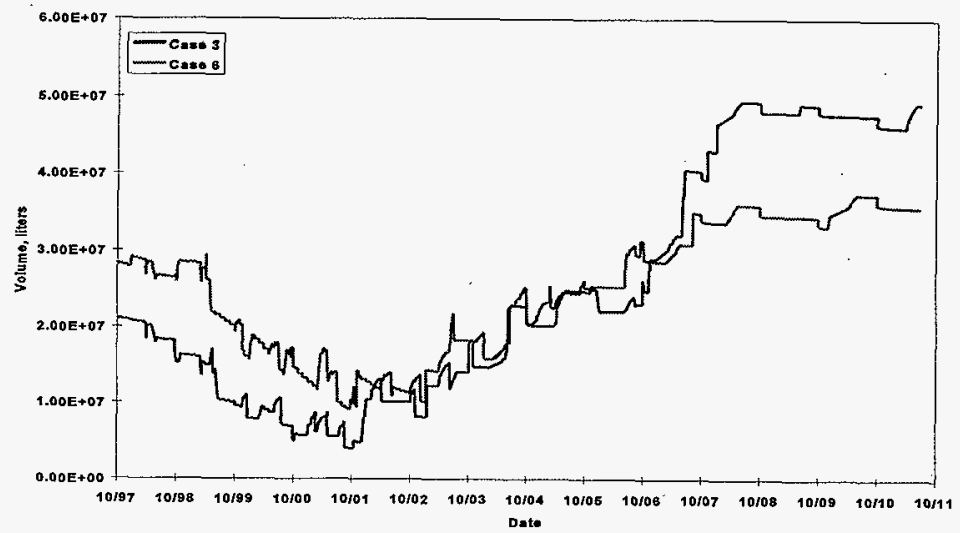


HNF-2321

Revision 0

Figures 5.3-1 and 5.3-2 also demonstrate the impact turning over AP-106 and AP-108 early has on the total volume of the DST system and the free DST system volume. From early 1999 until 2001, the two-contractor, early release case (Case 2) lags behind the base case (Case 1) in both total system yolume and free space. After 2001 the cases tend to balance out. For the onecontractor, early release case (Case 5) the same effect is noticed (Figures 5.3-3 and 5.3-4), but the magnitude is smaller and the recovery is quicker. This is because only one tank is turned over (241-AP-108) thereby leaving an additional tank for tank farm operations use.

By examining the total volume figures for the "sister" cases (Figures 5.3-5, -7 , and -9) it can be deduced that the effect of having two contractors instead of one on total volume becomes prevalent 10/2003 for the base cases (Cases 1 and 4) and 10/2004 for the early reiease cases. At this time the lines begin to diverge at a faster rate. The free volume figures (Figures 5.3-6, -8 , and -10) do not diverge significantly until after 10/2006 and they show a large disparity from start until 10/2001. The late divergence and the degree of disparity may be partly caused by how free volume was calculated. As stated previously, free volume excludes tanks 241-AP-102,-104, and -108 (and 241-AP-106 depending on the case) after initiation of Phase 1 LAW retrieval; tanks 241-AZ-101, -102 and 241-AY-102; 241-AP-107 after the start of Phase 1 processing; and all DSTs that will be used for Phase $1 \mathrm{LAW}$ feed and in the feed staging activities. This last part is where the problem occurs. For the two-contractor cases, 241-SY-102 is used in the retrieval of 241-SY-101 and 241-SY-103 ("feed staging activities"). Therefore, the volume in 241-SY-102 is not considered "free" until after these tanks are retrieved (approximately 10/2007). Tank 241-SY-102 should be considered "free" up until the time it is needed for SY retrieval, but it is not. Therefore the free volume differences between the "sister" cases should be considered smaller than pictured. However, there is still at least a one tank difference between the volumes since the one-contractor cases do not relinquish the use of 241-AP-106.

\subsection{PHASE 2 SINGLE-SHELL TANK RETRIEVAL}

Table 5.4-1 summarizes the state of Phase 2 SST retrieval operations for each of the cases. The table lists the number of tanks in which retrieval has been completed (by 10/2011), the number of tanks being retrieved from, and the total volume retrieved on an as-retrieved basis (includes retrieval water). 
HNF-2321

Revision 0

Table 5.4-1. Single-Shell Tank Retrieval Status by Case.

\begin{tabular}{|l|c|c|c|c|c|c|}
\hline Case & 1 & 2 & 3 & 4 & 5 & 6 \\
\hline $\begin{array}{l}\text { Single-shell tanks } \\
\text { retrieved }\end{array}$ & 36 & 36 & 38 & 27 & 26 & 30 \\
\hline $\begin{array}{l}\text { Single-shell tanks being } \\
\text { retrieved }\end{array}$ & 10 & 10 & 10 & 10 & 10 & 10 \\
\hline $\begin{array}{l}\text { Total volume retrieved } \\
\text { ML (Mgals) }\end{array}$ & $\begin{array}{c}50.7 \\
(13.4)\end{array}$ & $\begin{array}{c}50.7 \\
(13.4)\end{array}$ & $\begin{array}{c}50.3 \\
(13.3)\end{array}$ & $\begin{array}{c}38.2 \\
(10.1)\end{array}$ & $\begin{array}{c}37.5 \\
(9.9)\end{array}$ & $\begin{array}{c}37.9 \\
(10.0)\end{array}$ \\
\hline
\end{tabular}

It should be noted that the same SST retrieval schedule that was used in Rev. 0 of the TWRSO\&UP was used for each case. No attempt was made to optimize the retrieval sequence. In any event, Table 5.4-1 shows that the two-contractor cases have the potential for retrieving an additional 8 to 10 SSTs compared to the one-contractor cases. This is due to the fact that more DST waste is being processed in the two-contractor cases and therefore more DST spaced is freed up to be used for SST retrieval operations. The two-contractor cases all meet the driver associated with the complete retrieval of AX tank farm prior to 2011 - none of the one-contractor cases meet this goal. However, this result is based upon a sequence that was developed around a two-contractor case. The 2011 date for the complete retrieval of AX farm could be met for the one-contractor cases, but the retrieval sequence would have to be completely reworked and potentially fewer SSTs would have retrieval completed. This may impact up-front milestones and current planning/projects. 
HNF-2321

Revision 0

This page intentionally left blank. 
HNF-2321

Revision 0

\subsection{SUMMARY}

This section is divided up into two sections. The first section summarizes the results of this study. The second lists general observations that were made while running the HTWOS model and collecting data.

\subsection{SUMMARY OF RESULTS}

Table 6.1-1 summarizes the results of this study using all the metrics defined in Section 3.0. The scores for the cases are based relative to the TWRSO\&UP "base case" (Case 1). Case 1 is assumed to have a score of 0 for all metrics. The results for the other cases are then scored based upon their relation to Case 1 and each other.

Table 6.1-1. Summary of Results.

\begin{tabular}{|c|c|c|c|c|c|c|c|c|}
\hline \multirow{3}{*}{ Metric } & \multicolumn{6}{|c|}{ Tank transfers } & \multirow{3}{*}{$\begin{array}{l}\text { Evap- } \\
\text { orator } \\
\text { usage }\end{array}$} & \multirow{3}{*}{$\begin{array}{l}\text { Vendor } \\
\text { tanks } \\
\text { "hands- } \\
\text { off" } \\
\text { on time }\end{array}$} \\
\hline & \multicolumn{2}{|c|}{ High-level waste staging } & \multicolumn{2}{|c|}{$\begin{array}{c}\text { Low-activity waste } \\
\text { staging }\end{array}$} & \multicolumn{2}{|c|}{$\begin{array}{l}\text { Waste } \\
\text { transfers }\end{array}$} & & \\
\hline & Delivery & Float & Delivery & Float & Volume & Number & & \\
\hline \multicolumn{9}{|l|}{ Case } \\
\hline 1 & Met & 0 & Met & 0 & 0 & 0 & 0 & Met \\
\hline 2 & Met & 0 & Met & -2 & 0 & 0 & -1 & Met \\
\hline 3 & Met & +1 & Met & -1 & 0 & 0 & 0 & Met \\
\hline 4 & Met & +1 & Met & +3 & +1 & +1 & 0 & Met \\
\hline 5 & Met & 0 & Met & +1 & +1 & +1 & -1 & Met \\
\hline 6 & Met & +1 & Met & +2 & +1 & +1 & 0 & Met \\
\hline \multirow[b]{2}{*}{ Metric } & \multirow{2}{*}{$\begin{array}{l}\text { Staging } \\
\text { tanks } \\
\text { usage }\end{array}$} & \multicolumn{4}{|c|}{ Total double-shell tank system } & \multicolumn{3}{|c|}{ Phase 2 single-shell tank retrieval } \\
\hline & & $\begin{array}{c}\text { Total } \\
\text { volume }\end{array}$ & $\begin{array}{l}\text { Pree } \\
\text { volume }\end{array}$ & & $\begin{array}{c}\text { DN/DC } \\
\text { segregation }\end{array}$ & Tanks & & lume \\
\hline \multicolumn{9}{|l|}{ Case } \\
\hline 1 & 0 & 0 & \multicolumn{2}{|l|}{0} & 0 & 0 & \multicolumn{2}{|c|}{0} \\
\hline 2 & -2 & 0 & \multicolumn{2}{|l|}{-1} & -1 & 0 & \multicolumn{2}{|c|}{0} \\
\hline 3 & -2 & -1 & \multicolumn{2}{|l|}{-1} & -1 & 0 & \multicolumn{2}{|c|}{0} \\
\hline 4 & 0 & -1 & \multicolumn{2}{|l|}{+1} & 0 & -1 & \multicolumn{2}{|c|}{-1} \\
\hline 5 & -1 & -1 & \multicolumn{2}{|l|}{0} & -1 & -1 & \multicolumn{2}{|c|}{-1} \\
\hline 6 & -1 & -2 & \multicolumn{2}{|l|}{0} & -1 & -1 & \multicolumn{2}{|c|}{-1} \\
\hline
\end{tabular}

$\mathrm{DC}=$ Dilute complexed

DN = Dilute non-complexed

The following paragraphs describe how the scoring was arrived at for each metric. 
HNF-2321

Revision 0

HLW Staging. All cases meet the delivery schedule for HLW. The scores for float are based upon the information presented in Section 5.1.1 (Tables 5.1-3 and -4). Cases 3, 4, and 6 were given scores of +1 because they do not have durations that approach the minimum of 314 days. However, as stated in Section 5.1.1, HLW staging should not be used as an absolute discriminator because it interacts directly with the DST system (OWVP operations) which does not operate "smart" at this time.

LAW Staging. All cases meet the delivery schedule for LAW. The scores for float are based upon the information presented in Section 5.1.1 (Tables 5.1-1 and -2). Case 2 has less float time than Case 3 and both have less than Case 1. Cases 4,5, and 6 all have greater float time than Case 1 with 4 having more than 5 and 5 having more than 6 .

Waste Transfers. Cases 1, 2, and 3 have approximately the same number of transfers and the same total volume of waste transferred. Cases 4,5 , and 6 have fewer transfers and transfer less volume than Case 1, but each have approximately the same number of transfers and the same total volume of waste transferred.

Evaporator Usage. Each case, except Cases 2 and 5, are approximately equal in volume evaporated and number of evaporator campaigns. Cases 2 and 5 were given scores of -1 because they require larger amounts of evaporation.

Vendor Tanks "Hands-Off" on Time. This metric summarizes if each specific case met its "hands-off" date. All cases meet this metric.

Staging Tank Usage. This metric examines the usage of 241-AP-102 and 241-AP-104 for tank farm operations up until retrieval of the first feed batch. Case 4 usage for these tanks is identical to Case 1 so its value is 0 . Cases 5 and 6 use 241-AP-102 and 241-AP-104 past what they are used in Case 1, but do not interfere with LAW feed staging operations. Therefore they are given a score of -1 . Cases 2 and 3 also use 241-AP-102 and 241-AP-104 past Case 1, but they are also used past Cases 5 and 6 . They are given a score of -2 . However, like Cases 5 and 6 , they do not interfere with LAW feed staging operations.

Total Volume. The total volume for Case 3 peaks higher than for Cases 1 and 2 (Figure 5.3-1) and therefore is given a score of -1 . Cases 4 and 5 do not peak higher than their "sister" cases (Figures 5.3-5 and -7 respectively), but they do end up with higher total volumes. They receive a score of -1 . Case 6 peaks higher than Cases 4 and 5 (Figure 5.3-3) and ends up with a higher total volume (Figure 5.3-9) and is given a score of -2 .

Free Volume. Cases 2 and 3 have lower free volumes than Case 1 because they relinquish the right to use tanks 241-AP-106 and 241-AP-108 for tank farm operations sooner. However, it appears that delaying saltwell liquid retrieval does not have a large impact on the amount of free space available (Figure 5.3-2). Therefore they are scored as -1 . Since Case 4 is identical to Case 1 , except that the use of 241-AP-106 is not relinquished, its free space is greater and it receives a score of +1 . This is subject to debate because even though Case 4 has more free space in the early years, it has less in the later years (Figure 5.3-6). Like Case 4, Cases 5 and 6 do not 
HNF-2321

Revision 0

relinquish 241-AP-106 and have less volume in the later years, but they do relinquish use of 241AP-108 earlier. Therefore they are scored as 0.

DN/DC Segregation. DN/DC segregation of retrieved saltwell liquid was used in the OWVP Rev. 23 and, therefore, was used for Cases 1 and 4. However Cases 2, 3, 5, and 6 did not follow the tank-to-tank transfers as outlined in the OWVP rev 23 after the "hands-off" date. Therefore the segregation of DN/DC were not rigorously followed. Because of this, these cases were given a score of -1 .

SST Tanks Retrieved. Cases 1,2, and 3 effectively complete retrieval from the same number of SSTs and retrieve the same total volume of SST waste (Table 5.4-1). Cases 4, 5, 6 retrieve far less (Table 5.4-1) than the two-contractor cases, but they are effectively equal to one another. Therefore they are scored as -1 .

As can be seen from the results, relinquishing the use of tanks early decreases the overall score of the case regardless of the contractor scenario being modeled. However, the scores tend to increase when the early release is coupled with a delay in saltwell liquid retrieval, but not so much as to equal the base cases (Cases 1 or 4 ).

The scores for the "sister" cases (as defined in Section 5.0) tend to favor the one-contractor scenarios except for total volume and Phase 2 SST retrieval. Both parameters reflect negatively on the one-contractor scenarios because these cases do not remove as much waste from the DST system as the two-contractor cases. Therefore the ending DST volume is higher (see definition of Total Volume above) and fewer SSTs can be retrieved.

\subsection{OBSERVATIONS}

General observations are summarized below - more detailed descriptions of important observations follow.

1. Usable tank space will become limited in the $2000-2002$ time frame for all cases

- Turning over any tank early to the PC(s) will compound this problem.

- Delaying saltwell liquid retrieval so that the bulk of the remaining volume is retrieved during this time frame will further compound the problem.

- The one-contractor scenario lessens the effect of early "hands-off" since one tank instead of two is turned over.

- This early effect is lessened by the greater processing of two contractors by $2003 / 2004$.

- SST retrieval in support of Phase 2 is delayed by the lack of free DST space. 
HNF-2321

Revision 0

- The number of LAW contractors does not affect the HLW pretreatment operations which have a large impact on available DST volume

2. HLW feed staging/pretreatment operations are more susceptible to delays caused by DST space limitations. HLW pretreatment operations add more waste to the system and therefore directly impact the amount of free space available, evaporator operations, and waste transfer timing.

3. Turning over tanks early to the $\mathrm{PC}(\mathrm{s})$ and therefore limiting the amount of usable space makes the interdependence of tank transfers more intricate. With the amount of usable space shrinking, transfers become more dependant on earlier transfers occurring. This can greatly increase the risk of failure.

4. All cases can meet the feed requirements for LAW and HLW staging based upon the assumptions made in the model,

- However, model is too simplistic at this point to guarantee delivery

- No RAM analysis

- Piping/valving not modeled

- Model assumes AP-102 and AP-104 remain usable

- Some schedules contain near zero float

- Production ramp factor of 1.0 assumed for Phase 1 processing

5. All cases employ evaporator operations rather heavily until the 2007/2008 time frame

The data in Section 4.0 (specifically Sections 4.X.2, X = 2-7) demonstrate that for each case, based upon the constraints within the model, feed delivery can be accomplished by the PHMC Team on schedule. However, the data also demonstrate that tank farm operations (tankto-tank waste transfers, waste generator transfers, evaporator operations) are very complex and interdependent before and during the early years of Phase 1. This is caused by the usable DST space limitation and is true regardless of wether there are two LAW contractors or which saltwell liquid retrieval schedule is used. Common sense tells us that as the complexity and interdependency of tank farm operations increase, the impact a specific transfer has upon the entire system increases and therefore the risk of failure increases. Thus it is concluded that for the early "hands-off" cases (Cases 2, 3, 5,and 6) the PHMC Team can not guarantee delivery of LAW and HLW feed to the PCs solely based upon the output of this study. Another study should be undertaken that includes reliability, availability, and maintainability (RAM) algorithms of tank farm components (pumps, valves, piping, etc.) to better simulate "reality." The addition of RAM analyses is intended to be included in a future revision of the HTWOS model.

The study shows that the saltwell liquid retrieval schedule should either be accelerated so that it completes in 1999/2000 or include an additional 2 year or so delay (2000 - 2002) or reduction (i.e., less salt well liquid retrieved during this time frame). The early completion of saltwell liquid retrieval may remove the potential conflict between it and feed staging operations, but this scenario is unlikely. The retrieval of saltwell liquid is presently behind the "base" 
HNF-2321

Revision 0

schedule and therefore a delay/reduction is already expected in the completion date. A two-year or so delay or reduction in the retrieval operations has the potential to move the bulk of the volume conflict away from the time period at which tank space is extremely tight, but this option needs to be examined in more detail. A too lengthy delay may detrimentally impact the twocontractor cases because they process waste that results from the concentration of retrieved saltwell liquid (241-AP-101, 241-AW-104, and 241-AN-106). Also, Tri-Party Agreement milestones may need to be renegotiated and the risk of leaving salt well liquid in leaking SST's for longer periods of time needs to be evaluated. 
HNF-2321

Revision 0

This page intentionally left blank. 
HNF-2321

Revision 0

\subsection{RECOMMENDATIONS}

The following bullets outline recommended modeling work/studies/assumption revisions that should be undertaken to support the ICD 19D issue - "Feed Tank Fill Status." Impacts of changes resulting from the below work may result in this study being revised.

1. Conduct a separate study that examines a case(s) which "decouples" Phase 1 operations and SWL retrieval. This may include delaying saltwell liquid retrieval or delaying the start of Phase 1 operations for both the one and two-contractor scenarios. This evaluation should be initiated after the next revision of the TWRSO\&UP to allow the HTWOS model to be further updated/refined. The reason for waiting is that the updating will include changes to operations as well as possible inventory changes (DSTs and saltwell liquid). These changes will directly affect the results.

2. In HTWOS, schedule the HLW pretreatment operations around saltwell liquid retrieval (or visa versa) in order to avoid conflicts. Currently the HLW pretreatment is scheduled on an as-needed/when-allowable basis. This scheduling may need to be done manually at first, but the HTWOS model should be able to be programmed to set it.

3. Re-examine the assumptions used for modeling the evaporator. This should be accomplished by the next revision of the TWRSO\&UP. Higher allowable boiloff rates and/or shorter sampling times may reduce tank space conflicts.

4. Re-evaluate the necessary sampling/qualification time for the LAW and HLW feed.

5. Revisit the assumptions for handling the contractor returns. Presently the model assumes that the contractor will accumulate returns (entrained solids and $\mathrm{TRU} / \mathrm{Sr} / \mathrm{Tc}$ for the LAW contractor w/o HLW) until its volume reaches approximately $34 \mathrm{~m}^{3}$ (9,000 gal). The slurry is then pumped into a DST (presently 241-AP-107) that was emptied prior to the start of Phase 1 processing $(6 / 1 / 2002)$. The first transfer of returns is not scheduled to occur until $1 / 2003$. If this holds true, then the PHMC Team may be allowed to use 241-AP-107 for waste storage operations after the start of Phase 1. Also, if the PC returns accumulation tank is greater than approximately $38 \mathrm{~m}^{3}(10,000 \mathrm{gal})$, the PHMC may be allowed to use $241-\mathrm{AP}-107$ past $1 / 2003$ and possibly up to the time at which tank space is no longer limited. Another possibility is to reserve space in 241-AP-107 (or another DST) to receive contractor returns, but not empty the entire tank. These options need to be discussed further, but do warrant consideration.

6. Meld the OWVP and HTWOS models together completely. Currently the OWVP section of the model is hard coded in. Dates are given for specific transfers and they do not take into account LAW and HLW feed staging operations; they are completely independent. 
HNF-2321

Revision 0

7. Incorporate the results of the RAM analysis into the HTWOS model. This may be included in the next version of the TWRSO\&UP depending on when the analysis is complete and the complexity of incorporating it.

8. Revisit the reasoning behind why, in Section 2.2, the "Both Full" "Fill Status" parameter was removed from consideration. As stated in Section 2.2, this may need to be considered as an option if the other parameters result in scenarios that do not meet the desired objective(s). The need to do this may be dependant on the results of the other work defined above and may result in revising this document. 
HNF-2321

Revision 0

\subsection{REFERENCES}

DOE-RL, 1997a, TWRS Privatization, Contract Number DE-RP06-96RL13308 and 13309, U.S. Department of Energy, Richland, Washington.

DOE-RL, 1997b, TWRS Privatization, Contract Number DE-RP06-96RL13309, U.S. Department of Energy, Richland, Washington.

Ecology, EPA, and DOE, 1994, Hanford Federal Facility Agreement and Consent Order, as amended, Washington State Department of Ecology, U.S. Environmental Protection Agency, and the U.S. Department of Energy, Olympia, Washington.

Kirkbride, R. A., G. K. Allen, P. J. Certa, A. F. Manuel, R. M. Orme, L. W. Shelton, E. J. Slaathaug, R. S. Wittman, and G. T. MacLean and D. L. Penwell (SESC), 1997, Tank Waste Remediation System Operation and Utilization Plan, HNF-SD-WM-SP-012, Rev. 0A, Vol. I and II, Numatec Hanford Corporation, Richland, Washington.

Strode, J. N., and V. C. Boyles, 1997, Operational Waste Volume Projection, HNF-SD-WM-ER-029, Rev. 23, Lockheed Martin Hanford Corporation, Richland, Washington. 
HNF-2321

Revision 0

This page intentionally left blank. 
HNF-2321

Revision 0

APPENDIX A

\section{TRANSFER LIST}

A-1 
HNF-2321

Revision 0

This page intentionally left blank. 
HNF-2321

Revision 0

\section{APPENDIX A}

\section{TRANSFER LIST}

This appendix contains a transfer list that contains every transfer (tank-to-tank, waste generators, Phase 1 feed staging, evaporator campaigns) for each case evaluated. Also included are tables of transfers that are pertinent to Phase 1 LAW and HLW feed staging operations. The List of Tables below guides the reader to the appropriate table:

\section{List of Tables}

A.0-1. Index for Waste Transfer Tables

A.1-1. Complete Transfer List for Case 1

A.1-2. Phase $1 \mathrm{LAW}$ Feed Staging Transfers for Case 1

A.1-3. Phase $1 \mathrm{HLW}$ Feed Staging Transfers for Case 1

A.2-1. Complete Transfer List for Case 2

A.2-2. Phase 1 LAW Feed Staging Transfers for Case 2

A.2-3. Phase $1 \mathrm{HLW}$ Feed Staging Transfers for Case 2

A.3-1. Complete Transfer List for Case 3

A.3-2. Phase 1 LAW Feed Staging Transfers for Case 3

A.3-3. Phase $1 \mathrm{HLW}$ Feed Staging Transfers for Case 3

A.4-1. Complete Transfer List for Case 4

A.4-2. Phase 1 LAW Feed Staging Transfers for Case 4

A.4-3. Phase $1 \mathrm{HLW}$ Feed Staging Transfers for Case 4

A.5-1. Complete Transfer List for Case 5

A.5-2. Phase 1 LAW Feed Staging Transfers for Case 5

A.6-1. Complete Transfer List for Case 6

For the complete list of transfers, the data are presented in the following format:

\begin{tabular}{|c|c|c|c|c|c|}
\hline From & To & Start Date & End Date & $\begin{array}{c}\text { Liquid } \\
\text { (gallons) }\end{array}$ & $\begin{array}{c}\text { Solid } \\
\text { (gallons) }\end{array}$ \\
\hline
\end{tabular}

where:

From

To

Start Date

End Date

Liquid

Solid the tank/equipment/waste source/facility pumping from the tank/equipment/facility pumping to the date pumping was initiated the date pumping was completed the gallons of liquid pumped the gallons of solid pumped 
HNF-2321

Revision 0

For the Phase $1 \mathrm{LAW}$ and HLW feed staging transfers, the data are presented in the following format:

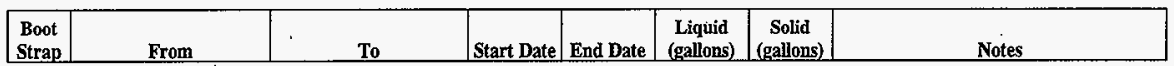

where:

Boot Strap defines a transfer as a necessary boot strap transfer for feed staging

From

To

Start Date

End Date

Liquid

Solid

Notes the tank/equipment/waste source/facility pumping from

the tank/equipment/facility pumping to

the date pumping was initiated

the date pumping was completed

the gallons of liquid pumped

the gallons of solid pumped

brief description of what transfer is or why was performed

The transfer files contain transfers that are from and to SSTs and DSTs. However, there are a large number of transfers that include equipment/sources that are not existing as SSTs or DSTs. These include the 242-A evaporator, PC facilities and feed tank, annex tanks used for Phase 2 retrieval, and waste generator sources. The definitions for these sources are in Table A.0-1 below.

Most of the transfers in the tables are simple tank to tank or waste source to tank transfers, but some are more complicated. The more elaborate transfers are described separately in the paragraphs below.

Evaporator Runs. Evaporator passes consist of three lines in the transfer tables. The first line gives the transfer from the evaporator feed tank (AW-102) to 242-A, the second line gives the transfer from 242-A to the evaporator bottoms catch tank (AW-106), and the third line gives the transfer from 242-A to the condensate catch tank (defined as LERF in the model). The reader is reminded that an evaporator campaign can consist of several passes.

In-Line Dilution Transfers. For some of the transfers in support of LAW feed staging, DST contents will be retrieved and transferred using in-line dilution. In-line dilution consists of pumping (and mixing if desired) the DST contents as they exist and adding dilution/transfer water in the line. No water is added directly to the tank. Also if any dissolution/precipitation reactions are to occur they will occur in the line. Therefore, in order to be accurate, this transfer needed to be described in three steps: what is removed from the source tank, the amount of water added, and what is added to the destination tank. 
Table A.0-1. Index for Waste Transfer Tables.

\begin{tabular}{|c|c|}
\hline Name & Definition \\
\hline 1FL96 & 105-F,105-H, and 100-N Liquid TCO Waste \\
\hline 1KL96 & 100-K Liquid TCO Waste \\
\hline 1NS96 & 100-Area Solid TCO Waste \\
\hline $242-\mathrm{A}$ & 242-A Evaporator \\
\hline $34 \mathrm{~L} 87$ & 300/400 Area Lab Waste \\
\hline BA-? & Annex Tanks for NE Quadrant \\
\hline BPTCO & B Plant TCO Waste \\
\hline EVAPF & Evaporator Flush and Tank Farm Water \\
\hline HLW-CONTRACTOR & HLW Contractor Processing Facility \\
\hline LAW-CONTRACTOR-? & LAW Contractor Processing Facility \\
\hline LERF & Evaporator Overheads Accumulation \\
\hline P1HLW_FEED_TANK & Phase 1 HLW Contractor Feed Tank \\
\hline PXTCO & PUREX TCO Waste \\
\hline RETURN_? & Phase 1 LAW Contractor Returns Tanks \\
\hline SPN87 & S Plant Dilute Non-Complexed Waste \\
\hline TA-? & Annex Tanks for NW Quadrant \\
\hline TAL88 & T Plant Supernate \\
\hline TNS88 & T Plant Solids \\
\hline UA-? & Annex Tanks for U Farm \\
\hline UNKN & Unknown Addition (Water) \\
\hline WASH-CAUSTIC & Caustic Solution for Enhanced Wash \\
\hline WASH-WATER & Dilute Caustic Solution for Solids Washing \\
\hline WATER & Water \\
\hline WCE88 & East Area Complexed Salt Well Liquid \\
\hline WCW88 & West Area Complexed Salt Well Liquid \\
\hline WESF & WESF Waste \\
\hline WNE88 & East Area Dilute, Non-Complexed Salt Well Liquid \\
\hline WNW88 & West Area Dilute, Non-Complexed Salt Well Liquid \\
\hline ZNL87 & Combined PFP Waste Stream \\
\hline
\end{tabular}


The in-line dilution transfers can be found in the transfer table by examining the "To" column to find an entry that is "name of a DST"-PUMP. This entry is for the addition of the dilution water. The entry before this one gives the transfer out of the source tank and the entry after gives the transfer to the destination tank. For the some of the transfers one can see that the solids transferred out of the source tank do not equal the solids transferred into the destination tank. This is due to the dissolution/precipitation reactions that occur in-line. 
HNF-2321

Revision 0

Table A.1-1. Complete Transfer List for Case 1.

\begin{tabular}{|c|c|c|c|c|c|}
\hline From & To & Start Date & End Date & $\begin{array}{c}\text { Liquid } \\
\text { (gallons) }\end{array}$ & $\begin{array}{c}\text { Solid } \\
\text { (gallons) }\end{array}$ \\
\hline 1FL96 & AW-105 & $10 / 1 / 96$ & $10 / 1 / 96$ & 13,000 & 0 \\
\hline $1 N 596$ & AW-105 & $10 / 1 / 96$ & $10 / 1 / 96$ & 750 & 250 \\
\hline $34 \mathrm{~L} 87$ & $\mathrm{AP}-106$ & $10 / 1 / 96$ & $10 / 1 / 96$ & 10,000 & 0 \\
\hline BPTCO & AP-106 & $10 / 1 / 96$ & $10 / 1 / 96$ & 11,000 & 0 \\
\hline EVAPF & AP-106 & $10 / 1 / 96$ & $10 / 1 / 96$ & 10,000 & 0 \\
\hline SPN87 & AP-106 & $10 / 1 / 96$ & $10 / 1 / 96$ & 7,000 & 0 \\
\hline TAL88 & $\mathrm{AP}-106$ & $10 / 1 / 96$ & $10 / 1 / 96$ & 4,000 & 0 \\
\hline TNS88 & AP-106 & $10 / 1 / 96$ & $10 / 1 / 96$ & 750 & 250 \\
\hline WATER & SY-102 & $10 / 1 / 96$ & $10 / 1 / 96$ & 5,000 & 0 \\
\hline WESF & AP-106 & $10 / 1 / 96$ & $10 / 1 / 96$ & 5,000 & 0 \\
\hline WNE88 & AP-106 & $10 / 1 / 96$ & $10 / 1 / 96$ & 1,000 & 0 \\
\hline WNW88 & SY-102 & $10 / 1 / 96$ & $10 / 1 / 96$ & 19,000 & 0 \\
\hline ZNL87 & SY-102 & $10 / 1 / 96$ & $10 / 1 / 96$ & 2,000 & 0 \\
\hline WATER & AP-106 & $.10 / 2 / 96$ & $10 / 2 / 96$ & 3,000 & 0 \\
\hline WATER & SY-102 & $10 / 2 / 96$ & $10 / 2 / 96$ & 5,000 & 0 \\
\hline WATER & AW-105 & $10 / 2 / 96$ & $10 / 2 / 96$ & 5,000 & 0 \\
\hline WATER & AP-106 & $10 / 2 / 96$ & $10 / 2 / 96$ & 5,000 & 0 \\
\hline WATER & $\mathrm{AP}-106$ & $10 / 2 / 96$ & $10 / 2 / 96$ & 5,000 & 0 \\
\hline WATER & AP-106 & $10 / 2 / 96$ & $10 / 2796$ & 12,000 & 0 \\
\hline BPTCO & $\mathrm{AP}-106$ & $11 / 1 / 96$ & $11 / 1 / 96$ & 10,000 & 0 \\
\hline EVAPF & $\mathrm{AP}-106$ & $11 / 1 / 96$ & $11 / 1 / 96$ & 10,000 & 0 \\
\hline WATER & SY-102 & $11 / 1 / 96$ & $11 / 1 / 96$ & 3,000 & 0 \\
\hline WNE\&8 & AP-106 & $11 / 1 / 96$ & $11 / 1 / 96$ & 1,000 & 0 \\
\hline WNW88 & SY-102 & $11 / 1 / 96$ & $11 / 1 / 96$ & 14,000 & 0 \\
\hline BPTCO & $\mathrm{AP}-106$ & $12 / 1 / 96$ & $12 / 1 / 96$ & 11,000 & 0 \\
\hline EVAPF & AP-106 & $12 / 1 / 96$ & $12 / 1 / 96$ & 10,000 & 0 \\
\hline WATER & SY-102 & $12 / 1 / 96$ & $12 / 1 / 96$ & 3,000 & 0 \\
\hline WNE88 & AP-106 & $12 / 1 / 96$ & $12 / 1 / 96$ & 1,000 & 0 \\
\hline WNW88 & $S Y-102$ & $12 / 1 / 96$ & $12 / 1 / 96$ & 14,000 & 0 \\
\hline $34 \mathrm{~L} 87$ & $\mathrm{AP}-106$ & $1 / 1 / 97$ & $1 / 1 / 97$ & 10,000 & 0 \\
\hline BPTCO & AP-106 & $1 / 1 / 97$ & $1 / 1 / 97$ & 10,000 & 0 \\
\hline EVAPF & $\mathrm{AP}-106$ & $1 / 1 / 97$ & $1 / 1 / 97$ & 10,000 & 0 \\
\hline SPN87 & AP-106 & $1 / 1 / 97$ & $1 / 1 / 97$ & 6,000 & 0 \\
\hline TAL88 & $\mathrm{AP}-106$ & $1 / 1 / 97$ & $1 / 1 / 97$ & 4,000 & 0 \\
\hline WATER & AP-106 & $1 / 1 / 97$ & $1 / 1 / 97$ & 4,000 & 0 \\
\hline WATER & $S Y-102$ & $1 / 1 / 97$ & $1 / 1 / 97$ & 4,000 & 0 \\
\hline WESF & $\mathrm{AP}-106$ & $1 / 1 / 97$ & $1 / 1 / 97$ & 5,000 & 0 \\
\hline WNE88 & AP-106 & $1 / 1 / 97$ & $1 / 1 / 97$ & 1,000 & 0 \\
\hline WNW88 & SY-102 & $1 / 1 / 97$ & $1 / 1 / 97$ & 14,000 & 0 \\
\hline AW-106 & $\mathrm{AP}-101$ & $1 / 4 / 97$ & $1 / 4 / 97$ & 69,511 & 3 \\
\hline AN-106 & AP-108 & $1 / 11 / 97$ & $1 / 12 / 97$ & 177,000 & 7 \\
\hline BPTCO & AP-106 & $2 / 1 / 97$ & $2 \pi 1 / 97$ & 10,000 & 0 \\
\hline EVAPF & $\mathrm{AP}-106$ & $2 / 1 / 97$ & $2 / 1 / 97$ & 10,000 & 0 \\
\hline WATER & $S Y-102$ & $2 / 1 / 97$ & $2 / 1 / 97$ & 4,000 & 0 \\
\hline WNW88 & $S Y-102$ & $2 / 1 / 97$ & $2 / 1 / 97$ & 14,000 & 0 \\
\hline EVAPF & $\mathrm{AW}-102$ & $2 / 20 / 97$ & $2 / 20 / 97$ & 50,000 & 0 \\
\hline$\overline{A P}-105$ & $\mathrm{AW}-102$ & $2 / 20 / 97$ & $2 / 24 / 97$ & 942,000 & 39 \\
\hline 34187 & AP-106 & $3 / 1 / 97$ & $3 / 1 / 97$ & 10,000 & 0 \\
\hline BPTCO & $\mathrm{AP}-106$ & $3 / 1 / 97$ & $3 / 1 / 97$ & 11,000 & 0 \\
\hline EVAPF & $\mathrm{AP}-106$ & $3 / 1 / 97$ & $3 / 1 / 97$ & 10,000 & 0 \\
\hline PXTCO & $\mathrm{AP}-106$ & $3 / 1 / 97$ & $3 / 1 / 97$ & 5,000 & 0 \\
\hline WATER & SY-102 & $3 / 1 / 97$ & $3 / 1 / 97$ & 4,000 & 0 \\
\hline WATER & AP-106 & $3 / 1 / 97$ & $3 / 1 / 97$ & 5,000 & 0 \\
\hline WNW88 & $S Y-102$ & $3 / 1 / 97$ & $3 / 1 / 97$ & 16,000 & 0 \\
\hline 242-A & LERF & $2 / 26 / 97$ & $3 / 2 / 97$ & 405,000 & 0 \\
\hline $242-A$ & AW-106 & $2 / 26 / 97$ & $3 / 2 / 97$ & 622,000 & 42 \\
\hline $\mathrm{AW}-102$ & 242-A & $2 / 26 / 97$ & $3 / 2 / 97$ & $1,027,000$ & 42 \\
\hline EVAPF: & $\mathrm{AW}-102$ & $3 / 3 / 97$ & $3 / 3 / 97$ & 35,000 & 0 \\
\hline BPTCO & AP-106 & $4 / 1 / 97$ & $4 / 1 / 97$ & 10,000 & 0 \\
\hline EVAPF & AP-106 & $4 / 1 / 97$ & $4 / 1 / 97$ & 10,000 & 0 \\
\hline SRN87 & AP-106 & $4 / 1 / 97$ & $4 / 1 / 97$ & 6,000 & 0 \\
\hline TAL88 & AP-106 & $4 / 1 / 97$ & $4 / 1 / 97$ & 4,000 & 0 \\
\hline WATER & SY-102 & $4 / 1 / 97$ & $4 / 1 / 97$ & 6,000 & 0 \\
\hline WESF & AP-106 & $4 / 1 / 97$ & $4 / 1 / 97$ & 5,000 & 0 \\
\hline WNE88 & AP-106 & $4 / 1 / 97$ & $4 / 1 / 97$ & 1,000 & 0 \\
\hline WNW88 & $S Y-102$ & $4 \longdiv { 1 / 9 7 }$ & $4 / 1 / 97$ & 22,000 & 0 \\
\hline AW-106 & AP-105 & $4 / 25 / 97$ & $4 / 28 / 97$ & 617,000 & 29 \\
\hline $\mathrm{AY}-101$ & AP-108 & $4 / 25 / 97$ & $4 / 29 / 97$ & 809,000 & 30 \\
\hline $34 \mathrm{~L} 87$ & AP-106 & $5 / 1 / 97$ & $5 / 1 / 97$ & 10,000 & 0 \\
\hline BPTCO & AP-106 & $5 / 1 / 97$ & $5 / 1 / 97$ & 11,000 & 0 \\
\hline
\end{tabular}


HNF-2321

Revision 0

Table A.1-1. Complete Transfer List for Case 1.

\begin{tabular}{|c|c|c|c|c|c|}
\hline From & To & Start Date & End Date & $\begin{array}{c}\text { Liquid } \\
\text { (gallons) }\end{array}$ & $\begin{array}{c}\text { Solid } \\
\text { (gallons) }\end{array}$ \\
\hline EVAPF & AP-106 & $5 / 1 / 97$ & $5 / 1 / 97$ & 10,000 & 0 \\
\hline WATER & AP-106 & $5 / 1 / 97$ & $5 / 1 / 97$ & 4,000 & 0 \\
\hline WATER & SY-102 & $5 / 1 / 97$ & $5 / 1 / 97$ & 5,000 & 0 \\
\hline WNE88 & AP-106 & $5 / 1 / 97$ & $5 / 1 / 97$ & 1,000 & 0 \\
\hline WNW88 & $\$ Y-102$ & $5 / 1 / 97$ & $5 / 1 / 97$ & 19,000 & 0 \\
\hline BPTCO & $\mathrm{AP}-106$ & $6 / 1 / 97$ & $6 / 1 / 97$ & 10,000 & 0 \\
\hline EVAPF & AP-106 & $6 / 1 / 97$ & $6 / 1 / 97$ & 10,000 & 0 \\
\hline WATER & SY-102 & $6 / 1 / 97$ & $6 / 1 / 97$ & 4,000 & 0 \\
\hline WNE88 & $\mathrm{AP}-106$ & $6 / 1 / 97$ & $6 / 1 / 97$ & 1,000 & 0 \\
\hline WNW88 & $S Y-102$ & $6 / 1 / 97$ & $6 / 1 / 97$ & 16,000 & 0 \\
\hline $34 \mathrm{~L} 87$ & $A P-106$ & $7 / 1 / 97$ & $7 / 1 / 97$ & 10,000 & 0 \\
\hline BPTCO & $\mathrm{AP}-106$ & $7 / 1 / 97$ & $7 / 1 / 97$ & 11,000 & 0 \\
\hline EVAPF & $\mathrm{AP}-106$ & $7 / 1 / 97$ & $7 / 1 / 97$ & 10,000 & 0 \\
\hline SPN87 & AP-106 & $7 / 1 / 97$ & $7 / 1 / 97$ & 6,000 & 0 \\
\hline TAL 88 & AP-106 & $7 / 1 / 97$ & $7 / 1 / 97$ & 4,000 & 0 \\
\hline WATER & AP-106 & $7 / 1 / 97$ & $7 / 1 / 97$ & 4,000 & 0 \\
\hline WATER & AN-101 & $7 / 1 / 97$ & $7 / 1 / 97$ & 5,000 & 0 \\
\hline WATER & $5 Y-102$ & $7 / 1 / 97$ & $7 / 1 / 97$ & 6,000 & 0 \\
\hline WCE 88 & AN-101 & $7 / 1 / 97$ & $7 / 1 / 97$ & 20,000 & 0 \\
\hline WESF & AP-106 & $7 / 1 / 97$ & $7 / 1 / 97$ & 5,000 & 0 \\
\hline WNE88 & AP-106 & $7 / 1 / 97$ & $7 / 1 / 97$ & 1,000 & 0 \\
\hline WNW88 & SY-102 & $7 / 1 / 97$ & $7 / 1 / 97$ & 24,000 & 0 \\
\hline$A N-106$ & AP-108 & $7 / 25 / 97$ & $7 / 26 / 97$ & 194,000 & 7 \\
\hline AP-108 & AW-102 & $7 / 25 / 97$ & $7 / 31 / 97$ & $1,198,000$ & 42 \\
\hline BPTCO & $A P-106$ & $8 / 1 / 97$ & $8 / 1 / 97$ & 10,000 & 0 \\
\hline EVAPF & AP-106 & $8 / 1 / 97$ & $8 / 1 / 97$ & 10,000 & 0 \\
\hline WATER & AP-106 & $8 / 1 / 97$ & $8 / 1 / 97$ & 4,000 & 0 \\
\hline WATER & AN-101 & $8 / 1 / 97$ & $8 / 1 / 97$ & 6,000 & 0 \\
\hline WATER & $S Y-102$ & $8 / 1 / 97$ & $8 / 1 / 97$ & 13,000 & 0 \\
\hline WCE88 & AN-101 & $8 / 1 / 97$ & $8 / 1 / 97$ & 25,000 & 0 \\
\hline WNE88 & AP-106 & $8 / 1 / 97$ & $8 / 1 / 97$ & 16,000 & 0 \\
\hline WNW88 & $S Y-102$ & $8 / 1 / 97$ & $8 / 1 / 97$ & 51,000 & 0 \\
\hline $242-A$ & AW-106 & $7 / 28 / 97$ & $8 / 2 / 97$ & 346,700 & 39 \\
\hline $242-A$ & LERF & $7 / 28 / 97$ & $8 / 2 / 97$ & 851,300 & 0 \\
\hline AW-102 & $242-\mathrm{A}$ & $7 / 28 / 97$ & $8 / 2 / 97$ & $1,198,000$ & 39 \\
\hline EVAPF & AW-102 & $8 / 3 / 97$ & $8 / 3 / 97$ & 25,000 & 0 \\
\hline WATER & AP-107 & $8 / 20 / 97$ & $8 / 20 / 97$ & 35,000 & 0 \\
\hline AW-106 & $\mathrm{AN}-106$ & $8 / 25 / 97$ & $8 / 26 / 97$ & 347,000 & 16 \\
\hline SY-102 & AP-107 & $8 / 25 / 97$ & $8 / 27 / 97$ & 520,000 & 20 \\
\hline $\mathrm{BPTCO}$ & $\mathrm{AP}-106$ & $9 / 1 / 97$ & $9 / 1 / 97$ & 10,000 & 0 \\
\hline EVAPF & AP-106 & $9 / 1 / 97$ & $9 / 1 / 97$ & 10,000 & 0 \\
\hline WATER & $\mathrm{AN}-101$ & $9 / 1 / 97$ & $9 / 1 / 97$ & 10,000 & 0 \\
\hline WATER & AP-106 & $9 / 1 / 97$ & $9 / 1 / 97$ & 12,000 & 0 \\
\hline WATER & $5 Y-102$ & $9 / 1 / 97$ & $9 / 1 / 97$ & 15,000 & 0 \\
\hline WCE88 & AN-101 & $9 / 1 / 97$ & $9 / 1 / 97$ & 40,000 & 0 \\
\hline WNE88 & $A P-106$ & $9 / 1 / 97$ & $9 / 1 / 97$ & 46,000 & 0 \\
\hline WNW88 & $S Y-102$ & $9 / 1 / 97$ & $9 / 1 / 97$ & 61,000 & 0 \\
\hline 34287 & $\mathrm{AP}-106$ & $10 / 1 / 97$ & $10 / 1 / 97$ & 7,000 & 0 \\
\hline $34 \mathrm{~L} 87$ & AP-106 & $10 / 1 / 97$ & $10 / 1 / 97$ & 10,000 & 0 \\
\hline BPTCO & AP-106 & $10 / 1 / 97$ & $10 / 1 / 97$ & $11, \infty \infty$ & 0 \\
\hline EVAPF & AP-106 & $10 / 1 / 97$ & $10 / 1 / 97$ & 10,000 & 0 \\
\hline PXTCO & AP-106 & $10 / 1 / 97$ & $10 / 1 / 97$ & 5,000 & 0 \\
\hline SPN87 & $\mathrm{SY}-102$ & $10 / 1 / 97$ & $10 / 1 / 97$ & 7,000 & 0 \\
\hline TAL88 & AP-106 & $10 / 1 / 97$ & $10 / 1 / 97$ & 4,000 & 0 \\
\hline TNS88 & $A P-106$ & $10 / 1 / 97$ & $10 / 1 / 97$ & 750 & 250 \\
\hline WATER & AN-101 & $10 / 1 / 97$ & $10 / 1 / 97$ & 10,000 & 0 \\
\hline WATER & AP.106 & $10 / 1 / 97$ & $10 / 1 / 97$ & 12,000 & 0 \\
\hline WATER & $\mathrm{SY}-102$ & $10 / 1 / 97$ & $10 / 1 / 97$ & 28,000 & 0 \\
\hline WCE88 & $\mathrm{AN}-101$ & $10 / 1 / 97$ & $10 / 1 / 97$ & 40,000 & 0 \\
\hline WESF & AP-106 & $10 / 1 / 97$ & $10 / 1 / 97$ & 5,000 & 0 \\
\hline WNE88 & AP-106 & $10 / 1 / 97$ & $10 / 1 / 97$ & 46,000 & 0 \\
\hline WNW88 & SY-102 & $10 / 1 / 97$ & $10 / 1 / 97$ & 111,000 & 0 \\
\hline ZNL87 & SY-102 & $10 / 1 / 97$ & $10 / 1 / 97$ & 4,000 & 0 \\
\hline WASH-CAUSTIC & AN-107 & $10 / 2 / 97$ & $10 / 2 / 97$ & 66,000 & 0 \\
\hline WATER & SY-102 & $10 / 2 / 97$ & $10 / 2 / 97$ & 1,000 & 0 \\
\hline WATER & $\mathrm{AP}-10 \mathrm{6}$ & $10 / 2 / 97$ & $10 / 2 / 97$ & 3,000 & 0 \\
\hline WATER & $\mathrm{AP}-106$ & $10 / 2 / 97$ & $10 / 2 / 97$ & 3,000 & 0 \\
\hline WATER & SY -102 & $10 / 2 / 97$ & $10 / 2 / 97$ & 5,000 & 0 \\
\hline WATER & $\mathrm{AP}-106$ & $10 / 2 / 97$ & $10 / 2 / 97$ & 5,000 & 0 \\
\hline WATER & AP-106 & $10 / 2 / 97$ & $10 / 2 / 97$ & 5.000 & 0 \\
\hline
\end{tabular}


HNF-2321

Revision 0

Table A.1-1. Complete Transfer List for Case 1.

\begin{tabular}{|c|c|c|c|c|c|}
\hline From & To & Start Date & End Date & $\begin{array}{l}\text { Liquid } \\
\text { (gallons) }\end{array}$ & $\begin{array}{c}\text { Solid } \\
\text { (gallons) }\end{array}$ \\
\hline$\overline{A Y}-102$ & AP-106 & $10 / 25 / 97$ & $.10 / 25 / 97$ & 35,999 & 1 \\
\hline WATER & AY-102 & $10 / 26 / 97$ & $10 / 27 / 97$ & 176,000 & 0 \\
\hline EVAPि & AP-106 & $11 / 1 / 97$ & $11 / 1 / 97$ & 10,000 & 0 \\
\hline WATER & AN-101 & $11 / 1 / 97$ & $11 / 1 / 97$ & 10,000 & 0 \\
\hline WATER & AP-106 & $11 / 1 / 97$ & $11 / 1 / 97$ & 12,000 & 0 \\
\hline WATER & SY-102 & $11 / 1 / 97$ & $11 / 1 / 97$ & 30,000 & 0 \\
\hline WCE 88 & AN-101 & $11 / 1 / 97$ & $11 / 1 / 97$ & 40,000 & 0 \\
\hline WNE88 & AP-106 & $11 / 1 / 97$ & $11 / 1 / 97$ & 46,000 & 0 \\
\hline WNW88 & SY-102 & $11 / 1 / 97$ & $11 / 1 / 97$ & 119,000 & 0 \\
\hline EVAPF & $A P-106$ & $12 / 1 / 97$ & $12 / 1 / 97$ & 10,000 & 0 \\
\hline WATER & AN-101 & $12 / 1 / 97$ & $12 / 1 / 97$ & 10,000 & 0 \\
\hline WATER & AP-106 & $12 / 1 / 97$ & $12 / 1 / 97$ & 11,000 & 0 \\
\hline WATER & $S Y-102$ & $12 / 1 / 97$ & $12 / 1 / 97$ & 33,000 & 0 \\
\hline WCE 88 & AN-101 & $12 / 1 / 97$ & $12 / 1 / 97$ & 40,000 & 0 \\
\hline WNE88 & $A P-106$ & $12 / 1 / 97$ & $12 / 1 / 97$ & 42,000 & 0 \\
\hline WNW88 & $S Y-102$ & $12 / 1 / 97$ & $12 / 1 / 97$ & 131,000 & 0 \\
\hline WATER & AP-107 & $12 / 20 / 97$ & $12 / 20 / 97$ & 35,000 & 0 \\
\hline$S Y-102$ & AP-107 & $12 / 26 / 97$ & $12 / 28 / 97$ & 520,000 & 21 \\
\hline AP-106 & AP-104 & $12 / 25 / 97$ & $12 / 30 / 97$ & 996,600 & 35 \\
\hline $34 \mathrm{~L} 87$ & AP-106 & $1 / 1 / 98$ & $1 / 1 / 98$ & 10,000 & 0 \\
\hline BPTCO & $\mathrm{AP}-106$ & $1 / 1 / 98$ & $1 / 1 / 98$ & 11,000 & 0 \\
\hline EVAPF & AP-106 & $1 / 1 / 98$ & $1 / 1 / 98$ & 10,000 & 0 \\
\hline SPN87 & $S Y-102$ & $1 / 1 / 98$ & $1 / 1 / 98$ & 6,000 & 0 \\
\hline TAL8B & AP-106 & $1 / 1 / 98$ & $1 / 1 / 98$ & 4,000 & 0 \\
\hline WATER & $\mathrm{AP}-106$ & $1 / 1 / 98$ & $1 / 1 / 98$ & 4,000 & 0 \\
\hline WATER & AN-101 & $1 / 1 / 98$ & $1 / 1 / 98$ & 10,000 & 0 \\
\hline WATER & $\mathrm{AP}-106$ & $1 / 1 / 98$ & $1 / 1 / 98$ & 10,000 & 0 \\
\hline WATER & $\$ Y-102$ & $1 / 1 / 98$ & $1 / 1 / 98$ & 37,000 & 0 \\
\hline WCE88 & AN-101 & $1 / 1 / 98$ & $1 / 1 / 98$ & 40,000 & 0 \\
\hline WNE88 & AP-106 & $1 / 1 / 98$ & $1 / 1 / 98$ & 38,000 & 0 \\
\hline WNW88 & $S Y-102$ & $1 / 1 / 98$ & $1 / 1 / 98$ & 146,000 & 0 \\
\hline EVAPF & AP-106 & $2 / 1 / 98$ & $2 / 1 / 98$ & 10,000 & 0 \\
\hline WATER & AP-106 & $2 / 1 / 98$ & $2 / 1 / 98$ & 8,000 & 0 \\
\hline WATER & $\mathrm{AN}-101$ & $2 / 1 / 98$ & $2 / 1 / 98$ & 10,000 & 0 \\
\hline WATER & $S Y-102$ & $2 / 1 / 98$ & $2 / 1 / 98$ & 35,000 & 0 \\
\hline WCE88 & AN-101 & $2 / 1 / 98$ & $2 / 1 / 98$ & 40,000 & 0 \\
\hline WNE88 & AP-106 & $2 / 1 / 98$ & $2 / 1 / 98$ & 33,000 & 0 \\
\hline WNW88 & SY-102 & $2 / 1 / 98$ & $2 / 1 / 98$ & 140,000 & 0 \\
\hline $34 L 87$ & AP-106 & $3 / 1 / 98$ & $3 / 1 / 98$ & 10,000 & 0 \\
\hline BPTCO & AP-106 & $3 / 1 / 98$ & $3 / 1 / 98$ & 11,000 & 0 \\
\hline EVAPF & AP-106 & $3 / 1 / 98$ & $3 / 1 / 98$ & 10,000 & 0 \\
\hline WATER & $A P-106$ & $3 / 1 / 98$ & $3 / 1 / 98$ & 5,000 & 0 \\
\hline WATER & AP-106 & $3 / 1 / 98$ & $3 / 1 / 98$ & 7,000 & 0 \\
\hline WATER & AN-101 & $3 / 1 / 98$ & $3 / 1 / 98$ & 10,000 & 0 \\
\hline WATER & $S \bar{Y}-102$ & $3 / 1 / 98$ & $3 / 1 / 98$ & 31,000 & 0 \\
\hline WCE88 & AN-101 & $3 / 1 / 98$ & $3 / 1 / 98$ & 40,000 & 0 \\
\hline WNE88 & AP-106 & $3 / 1 / 98$ & $3 / 1 / 98$ & 26,000 & 0 \\
\hline WNW88 & SY-102 & $3 / 1 / 98$ & $3 / 1 / 98$ & 124,000 & 0 \\
\hline WATER & AP-104 & $3 / 20 / 98$ & $3 / 20 / 98$ & 35,000 & 0 \\
\hline SY-102 & AP-104 & $3 / 25 / 98$ & $3 / 27 / 98$ & 520,000 & 21 \\
\hline AP-104 & AW-102 & $3 / 25 / 98$ & $3 / 30 / 98$ & 998,000 & 33 \\
\hline $242-A$ & $A W-106$ & $3 / 28 / 98$ & $4 / 1 / 98$ & 180,100 & 31 \\
\hline $242-A$ & LERF & $3 / 28 / 98$ & $4 / 1 / 98$ & 877,800 & 0 \\
\hline AW-102 & $242-A$ & $3 / 28 / 98$ & $4 / 1 / 98$ & $1,058,000$ & 31 \\
\hline EVAPF & Ap-106 & $4 / 1 / 98$ & $4 / 1 / 98$ & 10,000 & 0 \\
\hline SPN87 & SY-102 & $4 / 1 / 98$ & $4 / 1 / 98$ & 6,000 & 0 \\
\hline TAL88 & $\mathrm{AP}-106$ & $4 / 1 / 98$ & $4 / 1 / 98$ & 4,000 & 0 \\
\hline WATER & Aि-106 & $4 / 1 / 98$ & $4 / 1 / 98$ & 5,000 & 0 \\
\hline WATER & AN-101 & $4 / 1 / 98$ & $4 / 1 / 98$ & 25,000 & 0 \\
\hline WATER & SY-102 & $4 / 1 / 98$ & $4 / 1 / 98$ & 36,000 & 0 \\
\hline WCER8 & AN-101 & $4 / 1 / 98$ & $4 / 1 / 98$ & 101,000 & 0 \\
\hline WNE88 & AP-106 & $4 / 1 / 98$ & $4 / 1 / 98$ & 20,000 & 0 \\
\hline WNW88 & $S Y-102$ & $4 / 1 / 98$ & $4 / 2 / 98$ & 146,000 & 0 \\
\hline $34 \mathrm{~L} 87$ & AP-106 & $5 / 1 / 98$ & $5 / 1 / 98$ & 10,000 & 0 \\
\hline BPTCO & AP-106 & $5 / 1 / 98$ & $5 / 1 / 98$ & 11,000 & 0 \\
\hline EVAPF & AP-106 & $5 / 1 / 98$ & $5 / 1 / 98$ & 10,000 & 0 \\
\hline WATER & $A P-106$ & $5 / 1 / 98$ & $5 / 1 / 98$ & 4,000 & 0 \\
\hline WATER & AP-106 & $5 / 1 / 98$ & $5 / 1 / 98$ & 5,000 & 0 \\
\hline WATER & SY-102 & $5 / 1 / 98$ & $5 / 1 / 98$ & 5,000 & 0 \\
\hline WATER & AN-101 & $5 / 1 / 98$ & $5 / 1 / 98$ & 24,000 & 0 \\
\hline
\end{tabular}


HNF-2321

Revision 0

Tạble A.1-1. Complete Transfer List for Case 1.

\begin{tabular}{|c|c|c|c|c|c|}
\hline From & To & Start Date & End Date & $\begin{array}{c}\text { Liquid } \\
\text { (gallons) }\end{array}$ & $\begin{array}{c}\text { Solid } \\
\text { (gallons) }\end{array}$ \\
\hline WATER & $\mathbf{S Y}-102$ & $5 / 1 / 98$ & $5 / 1 / 98$ & 37,000 & 0 \\
\hline WCE 88 & AN-101 & $5 / 1 / 98$ & $5 / 1 / 98$ & 95,000 & 0 \\
\hline WCW88 & SY-102 & $5 / 1 / 98$ & $5 / 1 / 98$ & 19,000 & 0 \\
\hline WNE88 & AP-106 & $5 / 1 / 98$ & $5 / 1 / 98$ & 20,000 & 0 \\
\hline WNW88 & SY-102 & $5 / 1 / 98$ & $5 / 2 / 98$ & 147,000 & 0 \\
\hline $\mathrm{AP}-107$ & $A W-102$ & $5 / 25 / 98$ & $5 / 30 / 98$ & $1,104,000$ & 40 \\
\hline $242-A$ & AW-106 & $5 / 28 / 98$ & $6 / 1 / 98$ & 448,700 & 39 \\
\hline $242-\mathrm{A}$ & LERF & $5 / 28198$ & $6 / 1 / 98$ & 655,200 & 0 \\
\hline $\mathrm{AW}-102$ & $242-A$ & $5 / 28 / 98$ & $6 / 1 / 98$ & $1,104,000$ & 39 \\
\hline EVAPF & AP-106 & $6 / 1 / 98$ & $6 / 1 / 98$ & 10,000 & 0 \\
\hline WATER & AP-106 & $6 / 1 / 98$ & $6 / 1 / 98$ & 4,000 & 0 \\
\hline WATER & $S Y-102$ & $6 / 1 / 98$ & $6 / 1 / 98$ & 5,000 & 0 \\
\hline WATER & $\mathrm{AN}-10 \mathrm{I}$ & $6 / 1 / 98$ & $6 / 1 / 98$ & 7,000 & 0 \\
\hline WATER & $S Y-102$ & $6 / 1 / 98$ & $6 / 1 / 98$ & 36,000 & 0 \\
\hline WCE88 & AN-101 & $6 / 1 / 98$ & $6 / 1 / 98$ & 28,000 & 0 \\
\hline WCW88 & SY-102 & $6 / 1 / 98$ & $6 / 1 / 98$ & 19,000 & 0 \\
\hline WNE88 & AP-106 & $6 / 1 / 98$ & $6 / 1 / 98$ & 16,000 & 0 \\
\hline WNW88 & SY-102 & $6 / 1 / 98$ & $6 / 2198$ & 142,000 & 0 \\
\hline EVAPF & $A W-102$ & $6 / 3 / 98$ & $6 / 3 / 98$ & 35,000 & 0 \\
\hline WATER & AP-104 & $6 / 20 / 98$ & $6 / 20 / 98$ & 35,000 & 0 \\
\hline AW-106 & AP-105 & $6 / 25 / 98$ & $6 / 26 / 98$ & 341,000 & 17 \\
\hline$S Y-102$ & AP-104 & $6 / 25 / 98$ & $6 / 27 / 98$ & 520,000 & 21 \\
\hline $34 \mathrm{~L} 87$ & AP-106 & $7 / 1 / 98$ & $7 / 1 / 98$ & 10,000 & 0 \\
\hline EVAPF & AP-106 & $7 / 1 / 98$ & $7 / 1 / 98$ & 10,000 & 0 \\
\hline SPN87 & SY-102 & $7 / 1 / 98$ & $7 / 1 / 98$ & 6,000 & 0 \\
\hline TAL88 & AP-106 & $7 / 1 / 98$ & $7 / 1 / 98$ & 4,000 & 0 \\
\hline WATER & $S Y-102$ & $7 / 1 / 98$ & $7 / 1 / 98$ & 3,000 & 0 \\
\hline WATER & AP-106 & $7 / 1 / 98$ & $7 / 1 / 98$ & 3,000 & 0 \\
\hline WATER & AP-106 & $7 / 1 / 98$ & $7 / 1 / 98$ & 4,000 & 0 \\
\hline WATER & AN-101 & $7 / 1 / 98$ & $7 / 1 / 98$ & 7.000 & 0 \\
\hline WATER & $S Y-102$ & $7 / 1 / 98$ & $7 / 1 / 98$ & 32,000 & 0 \\
\hline WCE 88 & AN-101 & $7 / 1 / 98$ & $7 / 1 / 98$ & 28,000 & 0 \\
\hline WCW88 & $S Y-102$ & $7 / 1 / 98$ & $7 / 1 / 98$ & 13,000 & 0 \\
\hline WNE88 & AP-106 & $7 / 1 / 98$ & $7 / 1198$ & 13,000 & 0 \\
\hline WNW88 & $5 Y-102$ & $7 / 1 / 98$ & $7 / 2 / 98$ & 127,000 & 0 \\
\hline $\mathrm{AY}-102$ & C-106 & $7 / 1 / 98$ & $7 / 5 / 98$ & 920,700 & 31 \\
\hline BPTCO & AP-106 & $7 / 11 / 98$ & $7 / 11 / 98$ & 11,000 & 0 \\
\hline EVAPF & $\mathrm{AP}-106$ & $8 / 1 / 98$ & $8 / 1 / 98$ & 10,000 & 0 \\
\hline WATER & AP-106 & $8 / 1 / 98$ & $8 / 1 / 98$ & 2,000 & 0 \\
\hline WATER & $S Y-102$ & $8 / 1 / 98$ & $8 / 1 / 98$ & 3,000 & 0 \\
\hline WATER & AN-101 & $8 / 1 / 98$ & $8 / 1 / 98$ & 7,000 & 0 \\
\hline WATER & SY-102 & $8 / 1 / 98$ & $8 / 1 / 98$ & 30,000 & 0 \\
\hline WCE88 & $A N-101$ & $8 / 1 / 98$ & $8 / 1 / 98$ & 28,000 & 0 \\
\hline WCW88 & $5 Y-102$ & $8 / 1 / 98$ & $8 / 1 / 98$ & 13,000 & 0 \\
\hline WNE88 & AP-I06 & $8 / 1 / 98$ & $8 / 1 / 98$ & 7,000 & 0 \\
\hline WNW88 & $S Y-102$ & $8 / 1 / 98$ & $8 / 1 / 98$ & 121,000 & 0 \\
\hline EVAPF & AP-106 & $9 / 1 / 98$ & $9 / 1 / 98$ & 10,000 & 0 \\
\hline WATER & $\mathrm{AP}-106$ & $9 / 1 / 98$ & $9 / 1 / 98$ & 2,000 & 0 \\
\hline WATER & $S Y-102$ & $9 / 1 / 98$ & $9 / 1 / 98$ & 2,000 & 0 \\
\hline WATER & AN-101 & $9 / 1 / 98$ & $9 / 1 / 98$ & 7,000 & 0 \\
\hline WATER & $\$ Y-102$ & $9 / 1 / 98$ & $9 / 1 / 98$ & 34,000 & 0 \\
\hline WCE88 & $A N-101$ & $9 / 1 / 98$ & $9 / 1 / 98$ & 28,000 & 0 \\
\hline WCW88 & $5 Y-102$ & $9 / 1 / 98$ & $9 / 1 / 98$ & 8,000 & 0 \\
\hline WNE88 & AP-106 & $9 / 1 / 98$ & $9 / 1 / 98$ & 7,000 & 0 \\
\hline WNW88 & $S Y-102$ & $9 / 1 / 98$ & $9 / 2 / 98$ & 137,000 & 0 \\
\hline WATER & AP-107 & $9 / 20 / 98$ & $9 / 20 / 98$ & 35,000 & 0 \\
\hline SY-102 & AP-107 & $9 / 25 / 98$ & $9 / 27 / 98$ & 520,000 & 21 \\
\hline $\mathrm{AP}-101$ & AW-103 & $9 / 25 / 98$ & $9 / 28 / 98$ & 625,000 & 27 \\
\hline 34L87 & AP-106 & $10 / 1 / 98$ & $10 / 1 / 98$ & 10,000 & 0 \\
\hline EVAPF & AP-106 & $10 / 1 / 98$ & $10 / 1 / 98$ & 10,000 & 0 \\
\hline PXTCO & $A P-106$ & $10 / 1 / 98$ & $10 / 1 / 98$ & 5,000 & 0 \\
\hline SPN87 & SY-102 & $10 / 1 / 98$ & $10 / 1 / 98$ & 7,000 & 0 \\
\hline TAl 88 & AP-106 & $10 / 1 / 98$ & $10 / 1 / 98$ & 4,000 & 0 \\
\hline TNS88 & AP-106 & $10 / 1 / 98$ & $10 / 1 / 98$ & 750 & 250 \\
\hline WATER & AP-106 & $10 / 1 / 98$ & $10 / 1 / 98$ & 1,000 & 0 \\
\hline WATER & SY-102 & $10 / 1 / 98$ & $10 / 1 / 98$ & 2,000 & 0 \\
\hline WATER & AN-101 & $10 / 1 / 98$ & $10 / 1 / 98$ & 7,000 & 0 \\
\hline WATER & SY-102 & $10 / 1 / 98$ & $10 / 1 / 98$ & 30,000 & 0 \\
\hline WCE 88 & AN-101 & $10 / 1 / 98$ & $10 / 1 / 98$ & 28,000 & 0 \\
\hline WCW88 & $\mathrm{SY}-102$ & $10 / 1 / 98$ & $10 / 1 / 98$ & 8,000 & 0 \\
\hline
\end{tabular}


HNF-2321

Revision 0

Table A.1-1. Complete Transfer List for Case 1.

\begin{tabular}{|c|c|c|c|c|c|}
\hline From & To & Start Date & End Date & $\begin{array}{l}\text { Liquid } \\
\text { (gallons) }\end{array}$ & $\begin{array}{c}\text { Solid } \\
\text { (gallons) }\end{array}$ \\
\hline WESF & $\mathrm{AP}-106$ & $10 / 1 / 98$ & $10 / 1 / 98$ & 5,000 & 0 \\
\hline WNE88 & AP-106 & $10 / 1 / 98$ & $10 / 1 / 98$ & 3,000 & 0 \\
\hline WNW88 & $5 Y-102$ & $10 / 1 / 98$ & $10 / 1 / 98$ & 121,000 & 0 \\
\hline ZNL87 & $5 Y-102$ & $10 / 1 / 98$ & $10 / 1 / 98$ & 4,000 & 0 \\
\hline WATER & $A \mathrm{P}-106$ & $10 / 2 / 98$ & $10 / 2 / 98$ & 3,000 & 0 \\
\hline WATER & $5 Y-102$ & $10 / 2 / 98$ & $10 / 2 / 98$ & 5,000 & 0 \\
\hline WATER & AP-106 & $10 / 2 / 98$ & $10 / 2 / 98$ & 5,000 & 0 \\
\hline EVAPF & AW-102 & $10 / 4 / 98$ & $10 / 4 / 98$ & 50,000 & 0 \\
\hline AW-102 & AW-106 & $10 / 9 / 98$ & $10 / 9 / 98$ & 150,000 & 6 \\
\hline AP-104 & AW-102 & $10 / 5 / 98$ & $10 / 11 / 98$ & $1,107,000$ & 43 \\
\hline AW-104 & AP-107 & $10 / 9 / 98$ & $10 / 11 / 98$ & 540,000 & 18 \\
\hline AW-104 & AP-104 & $10 / 18 / 98$ & $10 / 18 / 98$ & 164,000 & 6 \\
\hline AP- 101 & AW-104 & $10 / 21 / 98$ & $10 / 23 / 98$ & 460,000 & 20 \\
\hline AW-106 & AP-101 & $10 / 29 / 98$ & $10 / 30 / 98$ & 289,000 & 13 \\
\hline EVAPF & $A P-106$ & $11 / 1 / 98$ & $11 / 1 / 98$ & 10,000 & 0 \\
\hline WATER & SY-102 & $11 / 1 / 98$ & $11 / 1 / 98$ & 1,000 & 0 \\
\hline WATER & AN-101 & $11 / 1 / 98$ & $11 / 1 / 98$ & 7,000 & 0 \\
\hline WATER & SY-102 & $11 / 1 / 98$ & $11 / 1 / 98$ & 30,000 & 0 \\
\hline WCE88 & $A N-101$ & $11 / 1 / 98$ & $11 / 1 / 98$ & 28,000 & 0 \\
\hline WCW88 & SY-102 & $11 / 1 / 98$ & $11 / 1 / 98$ & 3,000 & 0 \\
\hline WNE88 & AP-106 & $11 / 1 / 98$ & $11 / 1 / 98$ & 2,000 & 0 \\
\hline WNW88 & $S Y-102$ & $11 / 1 / 98$ & $11 / 1 / 98$ & 120,000 & 0 \\
\hline AW-106 & AW-102 & $11 / 5 / 98$ & $11 / 5 / 98$ & 150,000 & 9 \\
\hline $242-A$ & LERF & $11 / 4 / 98$ & $11 / 9 / 98$ & 589,600 & 0 \\
\hline $242-A$ & $A W-106$ & $11 / 4 / 98$ & $11 / 9 / 98$ & 602,900 & 46 \\
\hline$A W-102$ & $242-\mathrm{A}$ & $11 / 4 / 98$ & $11 / 9 / 98$ & $1,193,000$ & 46 \\
\hline C-106 & AY-102 & $7 / 15 / 98$ & $11 / 10 / 98$ & 809,000 & 15,169 \\
\hline EVAPF & AP-106 & $12 / 1 / 98$ & $12 / 1 / 98$ & 10,000 & 0 \\
\hline WATER & SY-102 & $12 / 1 / 98$ & $12 / 1 / 98$ & 1,000 & 0 \\
\hline WATER & AN-101 & $12 / 1 / 98$ & $12 / 1 / 98$ & 6,000 & 0 \\
\hline WATER & SY-102 & $12 / 1 / 98$ & $12 / 1 / 98$ & 29,000 & 0 \\
\hline WCE88 & AN-101 & $12 / 1 / 98$ & $12 / 1 / 98$ & 23,000 & 0 \\
\hline WCW88 & SY-102 & $12 / 1 / 98$ & $12 / 1 / 98$ & 3,000 & 0 \\
\hline WNE88 & AP- 106 & $.12 / 1 / 98$ & $12 / 1 / 98$ & 1,000 & 0 \\
\hline WNW88 & $S Y-102$ & $12 / 1 / 98$ & $12 / 1 / 98$ & 116,000 & 0 \\
\hline WATER & AP-104 & $12 / 20 / 98$ & $12 / 20 / 98$ & 35,000 & 0 \\
\hline SY-102 & AP-104 & $12 / 25 / 98$ & $12 / 27 / 98$ & 520,000 & 21 \\
\hline AW-106 & AP-I01 & $12 / 25 / 98$ & $12 / 28 / 98$ & 605,000 & 27 \\
\hline $34 \mathrm{~L} 87$ & AP-106 & $1 / 1 / 99$ & $1 / 1 / 99$ & 10,000 & 0 \\
\hline EVAPF & AP-106 & $1 / 1 / 99$ & $1 / 1 / 99$ & 10,000 & 0 \\
\hline SPN87 & $S Y-102$ & $1 / 1 / 99$ & $1 / 1 / 99$ & 6,000 & 0 \\
\hline TAL88 & AP- 106 & $1 / 1 / 99$ & $1 / 1 / 99$ & 4,000 & 0 \\
\hline WATER & AP-106 & $1 / 1 / 99$ & $1 / 1199$ & 4,000 & 0 \\
\hline WATER & AN-101 & $1 / 1 / 99$ & $1 / 1 / 99$ & 6,000 & 0 \\
\hline WATER & SY-102 & $1 / 1 / 99$ & $1 / 1 / 99$ & 6,000 & 0 \\
\hline WATER & SY-102 & $1 / 1 / 99$ & $1 / 1 / 99$ & 25,000 & 0. \\
\hline WCE88 & $A N-101$ & $1 / 1 / 99$ & $1 / 1 / 99$ & 23,000 & 0 \\
\hline WCW88 & $\$ Y-102$ & $1 / 1 / 99$ & $1 / 1 / 99$ & 23,000 & 0 \\
\hline WNW88 & $\$ Y-102$ & $1 / 1 / 99$ & $1 / 1 / 99$ & 102,000 & 0 \\
\hline AP-106 & $\mathrm{AP}-104$ & $1 / 25 / 99$ & $1 / 27 / 99$ & 385,000 & 14 \\
\hline EVAPF & $\mathrm{AP}-106$ & $2 / 1 / 99$ & $2 / 1 / 99$ & 10,000 & 0 \\
\hline WATER & AN-101 & $2 / 1 / 99$ & $2 / 1 / 99$ & 6,000 & 0 \\
\hline WATER & SY-102 & $2 / 1 / 99$ & $2 / 1199$ & 11,000 & 0 \\
\hline WATER & SY-102 & $2 / 1 / 99$ & $2 / 1 / 99$ & 23,000 & 0 \\
\hline WCE88 & $A N-101$ & $2 / 1 / 99$ & $2 / 1 / 99$ & 23,000 & 0 \\
\hline WCW88 & $S Y-102$ & $2 / 1 / 99$ & $2 / 1 / 99^{\circ}$ & 45,000 & 0 \\
\hline WNW88 & SY-102 & $2 / 1 / 99$ & $2 / 1 / 99$ & 91,000 & 0 \\
\hline $34 L 87$ & AP-106 & $3 / 1 / 99$ & $3 / 1 / 99$ & 10,000 & 0 \\
\hline EVAPF & $\mathrm{AP}-106$ & $3 / 1 / 99$ & $3 / 1 / 99$ & 10,000 & 0 \\
\hline WATER & AN-101 & $3 / 1 / 99$ & $3 / 1 / 99$ & 4,000 & 0 \\
\hline WATER & AP-106 & $3 / 1 / 99$ & $3 / 1 / 99$ & 5,000 & 0 \\
\hline WATER & SY-102 & $3 / 1 / 99$ & $3 / 1 / 99$ & 17,000 & 0 \\
\hline WATER & SY-102 & $3 / 1 / 99$ & $3 / 1 / 99$ & 21,000 & 0 \\
\hline WCE88 & $A N-101$ & $3 / 1 / 99$ & $3 / 1 / 99$ & 17,000 & 0 \\
\hline WCW88 & $S Y-102$ & $3 / 1 / 99$ & $3 / 1 / 99$ & 67,000 & 0 \\
\hline WNW88 & SY-102 & $3 / 1 / 99$ & $3 / 1 / 99$ & 82,000 & 0 \\
\hline AN-101 & AP-103 & $3 / 5 / 99$ & $3 / 10 / 99$ & $1,024,000$ & 0 \\
\hline AP-107 & $\mathrm{AW}-102$ & $3 / 5 / 99$ & $3 / 10 / 99$ & $1,095,000$ & 40 \\
\hline $242-A$ & AW-106 & $3 / 8 / 99$ & $3 / 12 / 99$ & 353,000 & 38 \\
\hline $242-\mathrm{A}$ & LERF & $3 / 8 / 99$ & $3 / 12 / 99$ & 741,900 & 0 \\
\hline
\end{tabular}




\section{HNF-2321 \\ Revision 0}

Table A.1-1. Complete Transfer List for Case 1.

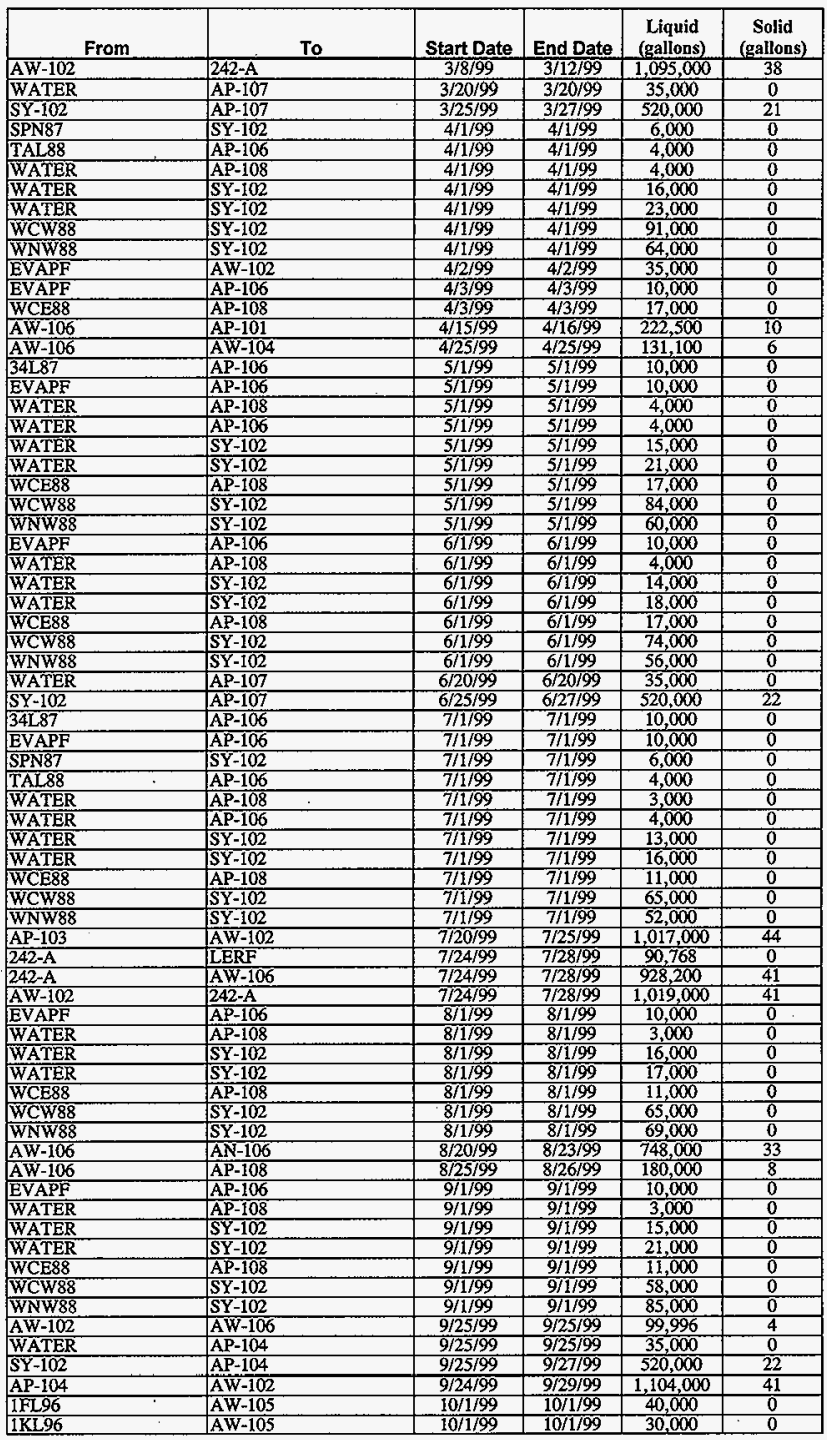


HNF-2321

Revision 0

Table A.1-1. Complete Transfer List for Case 1.

\begin{tabular}{|c|c|c|c|c|c|}
\hline From & To & Start Date & End Date & $\begin{array}{c}\text { Liquid } \\
\text { (gallons) }\end{array}$ & $\begin{array}{c}\text { Solid } \\
\text { (gallons) }\end{array}$ \\
\hline INS96 & AW-105 & $10 / 1 / 99$ & $10 / 1 / 99$ & 750 & 250 \\
\hline $34 \mathrm{~L} 87$ & AP-106 & $10 / 1 / 99$ & $10 / 1 / 99$ & 10,000 & 0 \\
\hline EVAPF. & AP-106 & $10 / 1 / 99$ & $10 / 1 / 99$ & 10,000 & 0 \\
\hline PXTCO & $\mathrm{AP}-106$ & $10 / 1 / 99$ & $10 / 1 / 99$ & 5,000 & 0 \\
\hline SPN87 & SY-102 & $10 / 1 / 99$ & $10 / 1 / 99$ & 7,000 & 0 \\
\hline TAL88 & AP-106 & $10 / 1 / 99$ & $10 / 1 / 99$ & 6,000 & 0 \\
\hline TNS88 & AP-106 & $10 / 1 / 99$ & $10 / 1 / 99$ & 750 & 250 \\
\hline WATER & AP-108 & $10 / 1 / 99$ & $10 / 1 / 99$ & 3,000 & 0 \\
\hline WATER & $S Y-102$ & $10 / 1 / 99$ & $10 / 1 / 99$ & 11,000 & 0 \\
\hline WATER & SY-102 & $10 / 1 / 99$ & $10 / 1 / 99$ & 15,000 & 0 \\
\hline WCE88 & AP-108 & $10 / 1 / 99$ & $10 / 1 / 99$ & 11,000 & 0 \\
\hline WCW88 & SY-102 & $10 / 1 / 99$ & $10 / 1 / 99$ & 43,000 & 0 \\
\hline WESF & \begin{tabular}{|l|} 
AP-106 \\
\end{tabular} & $10 / 1 / 99$ & $10 / 1 / 99$ & 5,000 & 0 \\
\hline WNW88 & SY-102 & $10 / 1 / 99$ & $10 / 1 / 99$ & 62,000 & 0 \\
\hline ZNL87 & SY-102 & $10 / 1 / 99$ & $10 / 1 / 99$ & 4,000 & 0 \\
\hline WATER & AP-106 & $10 / 2 / 99$ & $10 / 2 / 99$ & 5,000 & 0 \\
\hline WATER & AP-106 & $10 / 2 / 99$ & $10 / 2 / 99$ & 5,000 & 0 \\
\hline WATER & SY-102 & $10 / 2 / 99$ & $10 / 2 / 99$ & 5,000 & 0 \\
\hline WATER & AW-10S & $10 / 2 / 99$ & $10 / 2 / 99$ & 13,000 & 0 \\
\hline WATER & AW-105 & $10 / 2 / 99$ & $10 / 2 / 99$ & 17,000 & 0 \\
\hline $\mathrm{AW}-106$ & AW-104 & $10 / 20 / 99$ & $10 / 20 / 99$ & 100,200 & 4 \\
\hline AW-102 & AW-106 & $10 / 25 / 99$ & $10 / 25 / 99$ & 99,996 & 4 \\
\hline AW-106 & AN-101 & $10 / 26 / 99$ & $10 / 26 / 99$ & 159,200 & 6 \\
\hline $\mathrm{AP}-102$ & AP-103 & $10 / 25 / 99$ & $10 / 30 / 99$ & $1,070,000$ & 0 \\
\hline $242-A$ & $A W-106$ & $10 / 26 / 99$ & $10 / 30 / 99$ & 361,400 & 37 \\
\hline $242-\mathrm{A}$ & LERF & $10 / 26 / 99$ & $10 / 30 / 99$ & 576,100 & 0 \\
\hline $\mathrm{AW}-102$ & $242-\mathrm{A}$ & $10 / 26 / 99$ & $10 / 30 / 99$ & 937,500 & 37 \\
\hline EVAPF & AP-106 & [1/1/99 & $11 / 1 / 99$ & 10,000 & 0 \\
\hline WATER & AP-108 & {$[1 / 1 / 99$} & $11 / 1 / 99$ & 3,000 & 0 \\
\hline WATER & $\$ Y-102$ & $11 / 1 / 99$ & $11 / 1 / 99$ & 10,000 & 0 \\
\hline WATER & SY-102 & $11 / 1 / 99$ & $11 / 1 / 99$ & 12,000 & 0 \\
\hline WCE88 & AP-108 & $11 / 1 / 99$ & $11 / 1 / 99$ & 11,000 & 0 \\
\hline WCW88 & $S Y-102$ & $11 / 1 / 99$ & $11 / 1 / 99$ & 39,000 & 0 \\
\hline WNW88 & $S Y-102$ & $11 / 1 / 99$ & $11 / 1 / 99$ & 50,000 & 0 \\
\hline EVAPF & $\mathrm{AW}-102$ & $11 / 17 / 99$ & $11 / 17 / 99$ & 35,000 & 0 \\
\hline AP- 107 & AW-102 & $11 / 25 / 99$ & $11 / 30 / 99$ & $1,110,000$ & 43 \\
\hline EVAPF & AP-106 & $12 / 1 / 99$ & $12 / 1 / 99$ & 10,000 & 0 \\
\hline WATER & AP-108 & $12 / 1 / 99$ & $12 / 1 / 99$ & 1,000 & 0 \\
\hline WATER & SY-102 & $12 / 1 / 99$ & $12 / 2 / 99$ & 10,000 & 0 \\
\hline WATER & SY-102 & $12 / 1 / 99$ & $12 / 1 / 99$ & 11,000 & 0 \\
\hline WCE88 & AP-108 & $12 / 1 / 99$ & $12 / 1 / 99$ & 6,000 & 0 \\
\hline WCW88 & $\$ Y-102$ & $12 / 1 / 99$ & $12 / 1 / 99$ & 39,000 & 0 \\
\hline WNW88 & SY -102 & $12 / 1 / 99$ & $12 / 1 / 99$ & 43,000 & 0 \\
\hline $242-A$ & LERF & $11 / 28 / 99$ & $12 / 2 / 99$ & 358,800 & 0 \\
\hline $242-\mathrm{A}$ & AW-106 & $11 / 28 / 99$ & $12 / 2 / 99$ & 751,100 & 40 \\
\hline AW-102 & $242-\mathrm{A}$ & $11 / 28 / 99$ & $12 / 2 / 99$ & $1,110,000$ & 40 \\
\hline EVAPF & $\mathrm{AW}-102$ & $12 / 3 / 99$ & $12 / 3 / 99$ & 35,000 & 0 \\
\hline AW-106 & AN-101 & $12 / 25 / 99$ & $12 / 28 / 99$ & 750,000 & 34 \\
\hline $34 \mathrm{~L} 87$ & AP-106 & 1/1100 & $1 / 1 / 00$ & 10,000 & 0 \\
\hline EVAPF & $\mathrm{AP}-106$ & $1 / 1 / 00$ & $1 / 1 / 00$ & 10,000 & 0 \\
\hline SPN87 & $S Y-102$ & $1 / 1 / 00$ & $1 / 1 / 00$ & 6,000 & 0 \\
\hline TAL88 & AP-105 & $1 / 1 / 00$ & $1 / 1 / 00$ & 6,000 & 0 \\
\hline WATER & AP-108 & $1 / 1 / 00$ & $1 / 1 / 00$ & 1,000 & 0 \\
\hline WATER & AP- 106 & $1 / 1 / 00$ & $1 / 1 / 00$ & 4,000 & 0 \\
\hline WATER & SY-102 & $1 / 1 / 00$ & $1 / 1 / 00$ & 9,000 & 0 \\
\hline WATER & $\$ Y-102$ & $1 / 1 / 00$ & $1 / 1 / 00$ & 9,000 & 0 \\
\hline WCE88 & AP-108 & $1 / 1 / 00$ & $1 / 1 / 00$ & 6,000 & 0 \\
\hline WCW88 & $\$ Y-102$ & $1 / 1 / 00$ & $1 / 1 / 00$ & 26,000 & 0 \\
\hline WNW88 & SY-102 & $1 / 1 / 00$ & $1 / 1 / 00$ & 37,000 & 0 \\
\hline WATER & AP-104 & $1 / 20 / 00$ & $1 / 20 / 00$ & 35,000 & 0 \\
\hline$S Y-102$ & AP-104 & $1 / 25 / 00$ & $1 / 27 / 00$ & $\$ 20,000$ & 22 \\
\hline EVAPF & AP-106 & $2 / 1 / 00$ & $2 / 1 / 00$ & 10,000 & 0 \\
\hline WATER & $A P-108$ & $2 / 1 / 00$ & $2 / 1 / 00$ & 1,000 & 0 \\
\hline WATER & $S Y-102$ & $2 / 1 / 00$ & $2 / 1 / 00$ & 4,000 & 0 \\
\hline WATER & SY-102 & $2 / 1 / 00$ & $2 / 1 / 00$ & 8,000 & 0 \\
\hline WCE 88 & AP-108 & $2 / 1 / 00$ & $2 / 1100$ & 5,000 & 0 \\
\hline WCCW 88 & SY-102 & 271100 & $2 / 1 / 00$ & 17,000 & 0 \\
\hline WNW88 & SY-102 & $2 / 1 / 00$ & $2 / 1 / 00$ & 30,000 & 0 \\
\hline $34 \mathrm{~L} 87$ & AP-106 & $3 / 1 / 00$ & $3 / 1 / 00$ & 10,000 & 0 \\
\hline EVAPF & AP-106 & $3 / 1 / 00$ & $3 / 1 / 00$ & 10,000 & 0 \\
\hline
\end{tabular}


HNF-2321

Revision 0

Table A.1-1. Complete Transfer List for Case 1.

\begin{tabular}{|c|c|c|c|c|c|}
\hline From & To & Start Date & End Date. & $\begin{array}{c}\text { Liquid } \\
\text { (gallons) }\end{array}$ & $\begin{array}{c}\text { Solid } \\
\text { (gallons) }\end{array}$ \\
\hline WATER & $\mathrm{AP}-108$ & $3 / 1 / \infty 0$ & $3 / 1 / 00$ & 1,000 & 0 \\
\hline WATER & $\$ Y-102$ & $3 / 1 / 100$ & $3 / 1 / 00$ & 3,000 & 0 \\
\hline WATER & SY-102 & $3 / 1 / \infty 0$ & $3 / 1 / 00$ & 4,000 & 0 \\
\hline WATER & AP-106 & $3 / 1 / 00$ & $3 / 1 / 00$ & 5,000 & 0 \\
\hline WCE88 & $\mathrm{AP}-108$ & $3 / 1 / 00$ & $3 / 1 / 00$ & 3,000 & 0 \\
\hline WCW88 & $S Y-102$ & $3 / 1 / 00$ & $3 / 1 / 00$ & 13,000 & 0 \\
\hline WNW88 & $S Y-102$ & $3 / 1 / 00$ & $3 / 1 / 00$ & 17,000 & 0 \\
\hline EVAPF & AP-106 & $4 / 1 / 00$ & $4 / 1 / 00$ & 10,000 & 0 \\
\hline SPN87 & $S Y-102$ & $4 / 1 / 00$ & $4 / 1 / 100$ & 6,000 & 0 \\
\hline TAL88 & $\mathrm{AP}-106$ & $4 / 1 / 00$ & $4 / 1 / 00$ & 6,000 & 0 \\
\hline WATER & AP-108 & $4 / 1 / 00$ & $4 / 1 / 00$ & 1,000 & 0 \\
\hline WATER & $5 Y-102$ & $4 / 1 / 00$ & $4 / 1 / 00$ & 2,000 & 0 \\
\hline WATER & SY-102 & $4 / 1 / 00$ & $4 / 1100$ & 3,000 & 0 \\
\hline WCE 88 & $\mathrm{AP}-108$ & $4 / 1 / 00$ & $4 / 1 / 00$ & 3,000 & 0 \\
\hline WCW88 & $S Y-102$ & $4 / 1 / 00$ & $4 / 1 / 00$ & 9,000 & 0 \\
\hline WNW88 & $\$ Y-102$ & $4 / 1 / 00$ & $4 / 1 / 00$ & 13,000 & 0 \\
\hline $34 \mathrm{~L} 87$ & AP-106 & $5 / 1 / 00$ & $5 / 1 / 00$ & 10,000 & 0 \\
\hline EVAPF & AP-106 & $5 / 1 / 00$ & $5 / 1 / 00$ & 10,000 & 0 \\
\hline WATER & AP-108 & $5 / 1 / 00$ & $5 / 1 / 00$ & 1,000 & 0 \\
\hline WATER & SY-102 & $5 / 1 / 00$ & $5 / 1 / 00$ & 1,000 & 0 \\
\hline WATER & $\$ Y-102$ & $5 / 1 / 00$ & $5 / 1 / 00$ & 3,000 & 0 \\
\hline WATER & $A \mathrm{P}-106$ & $5 / 1 / 00$ & $5 / 1 / 00$ & 4,000 & 0 \\
\hline WCE88 & $\mathrm{AP}-108$ & $5 / 1 / 00$ & $5 / 1 / 00$ & 3,000 & 0 \\
\hline WCW88 & $S \bar{Y}-10 \tilde{Q}$ & $5 / 1 / 100$ & $5 / 1 / 100$ & 6,000 & 0 \\
\hline WNW88 & SY-102 & $5 / 1 / 00$ & $5 / 1 / 00$ & 11,000 & 0 \\
\hline EVAPF & AP-106 & $6 / 1 / 00$ & $6 / 1 / 00$ & 10,000 & 0 \\
\hline WATER & AP-108 & $6 / 1 / 00$ & $6 / 1 / 00$ & 1,000 & 0 \\
\hline WATER & $5 Y-102$ & $6 / 1 / 00$ & $6 / 1 / 100$ & 1,000 & 0 \\
\hline WCE 88 & $\mathrm{AP}-108$ & $6 / 1 / 00$ & $6 / 1 / 00$ & 3,000 & 0 \\
\hline WNW88 & SY-102 & $6 / 1 / 00$ & $6 / 1 / 00$ & 4,000 & 0 \\
\hline $34 \mathrm{~L} 87$ & AP-106 & $7 / 1 / 00$ & $7 / 1 / 100$ & 10,000 & 0 \\
\hline EVAPE & AP-106 & $7 / 1 / 00$ & $7 / 1 / 00$ & 10,000 & 0 \\
\hline SPN87 & $S Y-102$ & $7 / 1 / 00$ & $7 / 1 / 00$ & 6,000 & 0 \\
\hline TAL 88 & AP-106 & $7 / 1 / 100$ & $7 / 1 / 00$ & 5,000 & 0 \\
\hline WATER & AP-108 & $7 / 1 / 00$ & $7 / 1 / 00$ & 1,000 & 0 \\
\hline WATER & $S Y-102$ & $7 / 1 / 100$ & $7 / 1 / 00$ & 1,000 & 0 \\
\hline WATER & AP-106 & $7 / 1 / 00$ & $7 / 1 / 00$ & 4,000 & 0 \\
\hline WCE 88 & $\mathrm{AP}-108$ & $7 / 1 / 00$ & $7 / 1 / 00$ & 3,000 & 0 \\
\hline WNW88 & SY-102 & $7 / 1 / 00$ & $7 / 1 / 00$ & 4,000 & 0 \\
\hline AP-104 & AW-102 & $7 / 25 / 00$ & $7 / 30 / 00$ & $1,110,000$ & 43 \\
\hline EVAPF & AP-106 & $8 / 1 / 00$ & $8 / 1100$ & 10,000 & 0 \\
\hline WATER & SY-102 & $8 / 1 / 00$ & $8 / 1 / 00$ & 1,000 & 0 \\
\hline WCE88 & $\mathrm{AP}-108$ & $8 / 1 / 00$ & $8 / 1100$ & 2,000 & 0 \\
\hline WNW88 & $\$ Y-102$ & $8 / 1 / 00$ & $8 / 1 / 00$ & 4,000 & 0 \\
\hline $242-A$ & LERF & $7 / 28 / 00$ & $8 / 2 / 00$ & 342,600 & 0 \\
\hline $242-A$ & AW-106 & $7 / 28 / 00$ & $8 / 2 / 00$ & 802,300 & 39 \\
\hline $\mathrm{AW}-102$ & $242-\mathrm{A}$ & $7 / 28 / 00$ & $8 / 2 / 00$ & $1,145,000$ & 39 \\
\hline EVAPF & AW-102 & $8 / 3 / 00$ & $8 / 3 / 00$ & 35,000 & 0 \\
\hline AZ-101 & AY-101 & $8 / 17 / 00$ & $8 / 20 / 00$ & 685,000 & 0 \\
\hline AZ-101 & $A P-107$ & $8 / 20 / 00$ & $8 / 20 / 00$ & 0 & 0 \\
\hline WASH-WATER & AZ-101 & $8 / 20 / 00$ & $8 / 21 / 00$ & 146,000 & 0 \\
\hline EVAPF & AP-106 & $9 / 1 / 00$ & $9 / 1 / 00$ & 10,000 & 0 \\
\hline WCE88 & AP-108 & $9 / 1 / 00$ & $9 / 1 / 00$ & 1,000 & 0 \\
\hline AW-106 & AP-107 & $9 / 25 / 00$ & $9 / 29 / 00$ & 804,000 & 35 \\
\hline 1 NS96 & AW-105 & $10 / 1 / 00$ & $10 / 1 / 00$ & 13,502 & 4,498 \\
\hline $34 \mathrm{~L} 87$ & AP-106 & $10 / 1 / 00$ & $10 / 1 / 00$ & 7,000 & 0 \\
\hline $34 \mathrm{~L} 87$ & AP-106 & $10 / 1 / 00$ & $10 / 1 / 00$ & 50,000 & 0 \\
\hline EVAPF & AP-106 & $10 / 1 / 00$ & $10 / 1 / 00$ & 120,000 & 0 \\
\hline PXTCO & AP-106 & $10 / 1 / 00$ & $10 / 1 / 00$ & 5,000 & 0 \\
\hline SPN87 & SY-102 & $10 / 1 / 00$ & $10 / 1 / 00$ & 25,000 & 0 \\
\hline TAL88 & AP-106 & $10 / 1 / 00$ & $10 / 1 / 100$ & 23,000 & 0 \\
\hline TNS88 & AP-106 & $10 / 1 / 00$ & $10 / 1 / 00$ & 750 & 250 \\
\hline WESF & AP-106 & $10 / 1 / 00$ & $10 / 1 / 00$ & 5,000 & 0 \\
\hline ZNL87 & SY-102 & $10 / 1 / 00$ & $10 / 1 / 00$ & 4,000 & 0 \\
\hline $1 \mathrm{KL} 96$ & AW-105 & $10 / 1 / 00$ & $10 / 2 / 00$ & 302,000 & 0 \\
\hline WASH-WATER & AP-104 & $10 / 1 / 00$ & $10 / 2 / 00$ & 200,000 & 0 \\
\hline WATER & AP-106 & $10 / 2 / 00$ & $10 / 2 / 00$ & 5,000 & 0 \\
\hline WATER & AW-105 & $10 / 2 / 00$ & $10 / 2 / 00$ & 7,000 & 0 \\
\hline WATER & AW-105 & $10 / 2 / 00$ & $10 / 2 / 00$ & 132,000 & 0 \\
\hline WATER & AP-106 & $10 / 2 / 00$ & $10 / 3 / 00$ & 22,000 & 0 \\
\hline
\end{tabular}


HNF-2321

Revision 0

Table A.1-1. Complete Transfer List for Case 1.

\begin{tabular}{|c|c|c|c|c|c|}
\hline From & To & Start Date & End Date & $\begin{array}{c}\text { Liquid } \\
\text { (gallons) }\end{array}$ & $\begin{array}{c}\text { Solid } \\
\text { (gallons) }\end{array}$ \\
\hline WATER & SY-102 & $10 / 3 / 00$ & $10 / 3 / 00$ & 1,000 & 0 \\
\hline WATER & $A P-106$ & $10 / 3 / 00$ & $10 / 3 / 00$ & 3,000 & 0 \\
\hline WATER & SY-102 & $10 / 3 / 00$ & $10 / 3 / 00$ & 5,000 & 0 \\
\hline$A P-106$ & AW-102 & $10 / 2 / 00$ & $10 / 4 / 00$ & 474,000 & 16 \\
\hline EVAPF & AW-102 & $10 / 4 / 00$ & $10 / 4 / 00$ & 50,000 & 0 \\
\hline$\overline{\mathrm{AP}}-106$ & $\mathrm{AW}-102$ & $10 / 5 / 00$ & $10 / 5 / 00$ & 10,000 & 0 \\
\hline AP-106 & $A W-102$ & $10 / 6 / 00$ & $10 / 6 / 00$ & 71,998 & 2 \\
\hline AP-106 & $A W-102$ & $10 / 7 / 00$ & $10 / 7 / 00$ & 180,300 & 6 \\
\hline AW-105 & $\mathrm{AW}-102$ & $10 / 6 / 00$ & $10 / 8 / 00$ & 414,000 & 14 \\
\hline AP-106 & $A W-102$ & $10 / 8 / 00$ & $10 / 8 / 00$ & 0 & 0 \\
\hline AW-105 & AW-102 & $10 / 9 / 00$ & $10 / 10 / 00$ & 213,200 & 7 \\
\hline AZ -101 & AP-107 & $10 / 9 / 00$ & $10 / 10 / 00$ & 143,200 & 0 \\
\hline $242-\mathrm{A}$ & $A W-106$ & $10 / 5 / 00$ & $10 / 12 / 00$ & 92,234 & 52 \\
\hline $242-\mathrm{A}$ & LERF & $10 / 5 / 00$ & $10 / 12 / 00$ & $1,391,000$ & 0 \\
\hline AW-102 & $242-\dot{A}$ & $10 / 5 / 00$ & $10 / 12 / 00$ & $1,483,000$ & 52 \\
\hline WASH-WATER & $A Z-101$ & $10 / 11 / 00$ & $10 / 12 / 00$ & 146,000 & 0 \\
\hline AP-104 & AP-102 & $10 / 15 / 00$ & $10 / 16 / 00$ & 200,000 & 1 \\
\hline$A P-107$ & $A W-102$ & $10 / 13 / 00$ & $10 / 17 / 00$ & 917,400 & 0 \\
\hline AW-106 & $\mathrm{AP}-107$ & $10 / 25 / 00$ & $10 / 25100$ & 110,000 & 5 \\
\hline EVAPF & $\mathrm{AW}-102$ & $11 / 3 / 00$ & $11 / 3 / 00$ & 35,000 & 0 \\
\hline $\mathrm{AZ}-10 \mathrm{I}$ & $\mathrm{AP}-107$ & $11 / 30 / 00$ & $12 / 1 / 00$ & 140,200 & 0 \\
\hline WASH-WATER & A2-101 & $12 / 2 / 00$ & $12 / 2 / 00$ & 146,000 & 0 \\
\hline AW-106 & AW-105: & $1 / 1 / 01$ & $1 / 2 / 01$ & 283,200 & 13 \\
\hline$A Z-101$ & AP-107 & $1 / 21 / 01$ & $1 / 21 / 01$ & 142,900 & 0 \\
\hline WASH WATER & $A Z-10 \mathrm{I}$ & $1 / 22 / 01$ & $1 / 23 / 01$ & 138,200 & 0 \\
\hline AP-102 & AP-106 & $2 / 15 / 01$ & $2 / 16 / 01$ & 200,500 & 1 \\
\hline AN-105 & AP-102 & $3 / 17 / 01$ & $3 / 20 / 01$ & 542,400 & 7,895 \\
\hline AN-105 & AP-102 & $3 / 17 / 01$ & $3 / 20 / 01$ & 686,500 & 294 \\
\hline LERF & AN-105-PUMP & $3 / 17 / 01$ & $3 / 20 / 01$ & 154,100 & 0 \\
\hline AN-10S & AP-104 & $3 / 21 / 01$ & $3 / 24 / 01$ & 542,400 & 7,895 \\
\hline AN-IOS & AP-104 & $3 / 21 / 01$ & $3 / 24 / 01$ & 686,500 & 294 \\
\hline LERF & AN-105-PUMP & $3 / 21 / 01$ & $3 / 24 / 01$ & 154,100 & 0 \\
\hline AP-107 & $A N-105$ & $3 / 24 / 01$ & $3 / 26 / 01$ & 423,200 & 16 \\
\hline$\overline{\mathrm{AP}}-108$ & AN.105 & $3 / 27 / 01$ & $3 / 28 / 01$ & 356.500 & 8 \\
\hline AP-106 & AP-108 & $3 / 29 / 01$ & $3 / 30 / 01$ & 214,000 & 1,044 \\
\hline AP-108 & AN-105 & $3 / 31 / 01$ & $4 / 1 / 01$ & 214,200 & 926 \\
\hline $242-A$ & LERF & $4 / 17 / 01$ & $4 / 21 / 01$ & 321,500 & 0 \\
\hline $242-A$ & AW-106 & $4 / 17 / 01$ & $4 / 21 / 01$ & 630,800 & 40 \\
\hline $\mathrm{AW}-102$ & $242-\mathrm{A}$ & $4 / 17 / 01$ & $4 / 21 / 01$ & 952,300 & 40 \\
\hline $\mathrm{AN}-105$ & AW-102 & $4 / 22 / 01$ & $4 / 27 / 01$ & 917,400 & 0 \\
\hline WATER & $A W-102$ & $5 / 1 / 01$ & $5 / 1 / 01$ & 35,000 & 0 \\
\hline EVAPF & AW-102 & $5 / 3 / 01$ & $5 / 3 / 01$ & 35,000 & 0 \\
\hline AW-105 & AP-107 & $5 / 15 / 01$ & $5 / 16 / 01$ & 283,200 & 12 \\
\hline$A W-106$ & AP-107 & $6 / 15 / 01$ & $6 / 18 / 01$ & 630,700 & 29 \\
\hline AP-102 & AP-106 & $7 / 10 / 01$ & $7 / 14 / 01$ & 686,500 & 282 \\
\hline$A P-104$ & AP-108 & $7 / 14 / 01$ & $7 / 18 / 01$ & 686,500 & 282 \\
\hline 34L87 & AN-105 & $10 / 1 / 01$ & $10 / 1 / 01$ & 50,000 & 0 \\
\hline EVAPF & $\mathrm{AN}-105$ & $10 / 1 / 01$ & $10 / 1 / 01$ & 120,000 & 0 \\
\hline SPN87 & $S Y-102$ & $10 / 1 / 01$ & $10 / 1 / 01$ & 25,000 & 0 \\
\hline TAL 88 & AN-105 & $10 / 1 / 01$ & $10 / 1 / 01$ & 23,000 & 0 \\
\hline TNS88 & $A N-105$ & $10 / 1 / 01$ & $10 / 1 / 01$ & 750 & 250 \\
\hline WATER & AN-105 & $10 / 1 / 01$ & $10 / 1 / 01$ & 35,000 & 0 \\
\hline WESF & AN-105 & $10 / 1 / 01$ & $10 / 1 / 01$ & 5,000 & 0 \\
\hline ZNL87 & SY-102 & $10 / 1 / 01$ & $10 / 1 / 01$ & 4,000 & 0 \\
\hline WATER & $S Y-102$ & $10 / 2 / 01$ & $10 / 2 / 01$ & 5,000 & 0 \\
\hline WATER & AN-105 & $10 / 2 / 01$ & $10 / 2 / 01$ & 5,000 & 0 \\
\hline WATER & AN-105 & $10 / 2 / 01$ & $10 / 2 / 01$ & 22,000 & 0 \\
\hline AN-104 & AP- 102 & $10 / 1 / 01$ & $10 / 4 / 01$ & 502,200 & 12,513 \\
\hline AN-104 & AP-102 & $10 / 1 / 01$ & $10 / 4 / 01$ & 684,700 & 702 \\
\hline LERF & AN-104-PUMP & $10 / 1 / 01$ & $10 / 4 / 01$ & 200,800 & 0 \\
\hline $242-A$ & LERF & $10 / 3 / 01$ & $10 / 8 / 01$ & 354,000 & 0 \\
\hline $242-\mathrm{A}$ & AW-106 & $10 / 3 / 01$ & $10 / 8 / 01$ & 633,600 & 39 \\
\hline$A W-102$ & $242-\mathrm{A}$ & $10 / 3 / 01$ & $10 / 8 / 01$ & 987,600 & 39 \\
\hline AN-104 & AP-104 & $10 / 5 / 01$ & $10 / 8 / 01$ & 502,200 & 12,513 \\
\hline AN-104 & AP-104 & $10 / 5 / 01$ & $10 / 8 / 01$ & 684,700 & 702 \\
\hline LERF & AN-104-PUMP & $10 / 5 / 01$ & $10 / 8 / 01$ & 200,800 & 0 \\
\hline$A W-106$ & AW-102 & $10 / 8 / 0 \mathrm{~L}$ & $10 / 12 / 01$ & 633,500 & 27 \\
\hline $242-A$ & LEKF & $10 / 26 / 01$ & $10 / 29701$ & 190,700 & 0 \\
\hline 242-A & AW-106 & $10 / 26 / 01$ & $10 / 29 / 01$ & 442,700 & 27 \\
\hline AW-102 & $242-\mathrm{A}$ & $10 / 26 / 01$ & $10 / 29 / 01$ & 633,500 & 27 \\
\hline
\end{tabular}


HNF-2321

Revision 0

Table A.1-1. Complete Transfer List for Case 1.

\begin{tabular}{|c|c|c|c|c|c|}
\hline From & To & Start Date & End Date & $\begin{array}{c}\text { Liquid } \\
\text { (gallons) }\end{array}$ & $\begin{array}{c}\text { Solid } \\
\text { (gallons) }\end{array}$ \\
\hline EVAPF & $A W-102$ & $11 / 3 / 01$ & $11 / 3 / 01$ & 35,000 & 0 \\
\hline$A Z-102$ & $\mathrm{AY}-101$ & $11 / 14 / 01$ & $11 / 15 / 01$ & 204,000 & 0 \\
\hline$A Z-102$ & $\mathrm{AP}-107$ & $11 / 16 / 01$ & $11 / 16 / 01$ & 181,800 & 0 \\
\hline WASH-WATER & AZ-102 & $11 / 17 / 01$ & $11 / 18 / 01$ & 213,000 & 0 \\
\hline $\mathrm{AP}-107$ & $\mathrm{AN}-104$ & $1 / 1 / 02$ & $1 / 6 / 02$ & $1,096,000$ & 65 \\
\hline$A Z-102$ & AN-105 & $1 / 6 / 02$ & $1 / 6 / 02$ & 127,700 & 0 \\
\hline WASH-WATER & $A Z-102$ & $1 / 8 / 02$ & $1 / 9 / 02$ & 213,000 & 0 \\
\hline AZ-102 & $A N-105$ & $2 / 27 / 02$ & $2 / 28 / 02$ & 210,300 & 0 \\
\hline WASH-WATER & $A Z-102$ & $3 / 1 / 02$ & $3 / 2 / 02$ & 213,000 & 0 \\
\hline $\mathrm{AZ}-102$ & AN-105 & $4 / 20 / 02$ & $4 / 21 / 02$ & 211,800 & 0 \\
\hline WASH-WATER & $A Z-102$ & $4 / 22 / 02$ & $4 / 23 / 02$ & 213,000 & 0 \\
\hline AN-105 & $\mathrm{AW}-102$ & $4 / 21 / 02$ & $4 / 26 / 02$ & 870,200 & 0 \\
\hline AZ-101 & PIHLW FEED TANK & $5 / 17 / 02$ & $5 / 17 / 02$ & 134,300 & 5,079 \\
\hline AZ-102 & AN-105 & $6 / 12 / 02$ & $6 / 13 / 02$ & 212,500 & 0 \\
\hline WASH-WATER & $A Z-102$ & $6 / 14 / 02$ & $6 / 14 / 02$ & 110,000 & 0 \\
\hline AW-106 & AW-105 & $7 / 15 / 02$ & $7 / 17 / 02$ & 442,700 & 21 \\
\hline $34\lfloor 87$ & $A N-105$ & $10 / 1 / 02$ & $10 / 1 / 02$ & 50,000 & 0 \\
\hline EVAPF & AN-105 & $10 / 1 / 02$ & $10 / 1 / 02$ & 120,000 & 0 \\
\hline SPN87 & SY-102 & $10 / 1 / 02$ & $10 / 1 / 02$ & 25,000 & 0 \\
\hline TAL 88 & AN-105 & $10 / 1 / 02$ & $10 / 1 / 02$ & 24,000 & 0 \\
\hline TNS88 & $A N-105$ & $10 / 1 / 02$ & $10 / 1 / 02$ & 750 & 250 \\
\hline WATER & AN-105 & $10 / 1 / 02$ & $10 / 1 / 02$ & 35,000 & 0 \\
\hline WESF & $A N-105$ & $10 / 1 / 02$ & $10 / 1 / 02$ & 5,000 & 0 \\
\hline ZNL87 & $\mathrm{SY}-102$ & $10 / 1 / 02$ & $10 / 1 / 02$ & 3,000 & 0 \\
\hline WATER & SY-102 & $10 / 2 / 02$ & $10 / 2 / 02$ & 1,000 & 0 \\
\hline WATER & $A N-105$ & $10 / 2 / 02$ & $10 / 2 / 02$ & 5,000 & 0 \\
\hline WATER & SY-102 & $10 / 2 / 02$ & $10 / 2 / 02$ & 5,000 & 0 \\
\hline WATER & $A N-105$ & $10 / 2 / 02$ & $10 / 2 / 02$ & 22,000 & 0 \\
\hline EVAPF & $\bar{A} W-102$ & $10 / 4 / 02$ & $10 / 4 / 02$ & 50,000 & 0 \\
\hline $242-A$ & LERF & $10 / 24 / 02$ & $10 / 29 / 02$ & 342,000 & 0 \\
\hline $242-A$ & AW-106 & $10 / 24 / 02$ & $10 / 29 / 02$ & 613,500 & 33 \\
\hline AW-102 & 242-A & $10 / 24 / 02$ & $10 / 29 / 02$ & 955,500 & 33 \\
\hline AW-106 & $A W-102$ & $10 / 29 / 02$ & $11 / 1 / 02$ & 613,300 & 22 \\
\hline EVAPF & AW-102 & $11 / 3 / 02$ & $11 / 3 / 02$ & 35,000 & 0 \\
\hline $242-A$ & LERF & $11 / 15 / 02$ & $11 / 18 / 02$ & 234,000 & 0 \\
\hline $242-A$ & AW-106 & $11 / 15 / 02$ & $11 / 18 / 02$ & 414,300 & 23 \\
\hline $\mathrm{AW}-102$ & $242-\bar{A}$ & $11 / 15 / 02$ & $11 / 18 / 02$ & 648,300 & 23 \\
\hline AW-106 & $A W-102$ & $11 / 18 / 02$ & $11 / 20 / 02$ & 414,200 & 15 \\
\hline RETURN 1 & $\mathrm{AP}-107$ & $12 / 5 / 02$ & $12 / 5 / 02$ & 8,702 & 299 \\
\hline $242-A$ & LERF & $12 / 4 / 02$ & $12 / 6 / 02$ & 150,000 & 0 \\
\hline $242-\mathrm{A}$ & AW-106 & $12 / 4 / 02$ & $12 / 6 / 02$ & 264,300 & 15 \\
\hline $\mathrm{AW} \cdot 102$ & $242-A$ & $12 / 4 / 02$ & $12 / 6 / 02$ & 414,300 & 15 \\
\hline AW-106 & $A W-102$ & $12 / 6 / 02$ & $12 / 8 / 02$ & 264,200 & 10 \\
\hline $242-\mathrm{A}$ & LERF & $12 / 22 / 02$ & $12 / 23 / 02$ & 96,000 & 0 \\
\hline $242-A$ & AW-106 & $12 / 22 / 02$ & $12 / 23 / 02$ & 168,300 & 10 \\
\hline $\mathrm{AW}-102$ & $242-A$ & $12 / 22 / 02$ & $12 / 23 / 02$ & 264,300 & 10 \\
\hline$A W-106$ & $A W-102$ & $12 / 23 / 02$ & $12 / 24 / 02$ & 168,300 & 7 \\
\hline $\mathrm{AW}-102$ & AN-105 & $12 / 25 / 02$ & $12 / 26 / 02$ & 168,400 & 7 \\
\hline RETURN 2 & AP-107 & $1 / 3 / 03$ & $1 / 3 / 03$ & 8,671 & 331 \\
\hline$A P-102$ & $\mathrm{AP}-106$ & $1 / 5 / 03$ & $1 / 9 / 03$ & 684,700 & 686 \\
\hline $\mathrm{AP}-104$ & AP-108 & $1 / 9 / 03$ & $1 / 12 / 03$ & 684,700 & 686 \\
\hline AW-101 & AP-102 & $1 / 10 / 03$ & $1 / 14 / 03$ & 537,200 & 13,013 \\
\hline AW-101 & $\mathrm{AP}-102$ & $1 / 10 / 03$ & $1 / 14 / 03$ & 845,400 & 13,013 \\
\hline LERF & AW-101-PUMP & $1 / 10 / 03$ & $1 / 14 / 03$ & 308,100 & 0 \\
\hline AW-101 & AP-104 & $1 / 15 / 03$ & $1 / 19 / 03$ & 537,200 & 13,013 \\
\hline AW-101 & AP-104 & $1 / 15 / 03$ & $1 / 19 / 03$ & 845,400 & 13,013 \\
\hline LERF & AW-101-PUMP & $1 / 15 / 03$ & $1 / 19 / 03$ & 308,100 & 0 \\
\hline AW-105 & AW-101 & $2 / 15 / 03$ & $2 / 17 / 03$ & 442,700 & 20 \\
\hline P1HLW FEED TANK & HLW-CONTRACTOR & $6 / 1 / 02$ & $2 / 21 / 03$ & 134,300 & 5,079 \\
\hline AZ-101 & PIHLW FEED TANK & $2 / 21 / 03$ & $2 / 21 / 03$ & 134,300 & 5,079 \\
\hline AY-102 & $\mathrm{AZ}-101$ & $2 / 24 / 03$ & $3 / 1 / 03$ & 819,600 & 16,032 \\
\hline RETURN 1 & AP-107 & $6 / 28 / 03$ & $6 / 28 / 03$ & 8,677 & 326 \\
\hline RETURN 2 & AP-107 & $8 / 7 / 03$ & $8 / 7 / 03$ & 8,671 & 332 \\
\hline $34 \mathrm{LB7}$ & AN-10S & $10 / 1 / 03$ & $10 / 1 / 03$ & 7,000 & 0 \\
\hline $34 \mathrm{~L} 87$ & AN-10S & $10 / 1 / 03$ & $10 / 1 / 03$ & 50,000 & 0 \\
\hline EVAPF & $\mathrm{AN}-105$ & $10 / 1 / 03$ & $10 / 1 / 03$ & 120,000 & 0 \\
\hline SPN87 & $\$ Y-102$ & $10 / 1 / 03$ & $10 / 1 / 03$ & 25,000 & 0 \\
\hline TAL88 & AN-105 & $10 / 1 / 03$ & $10 / 1 / 03$ & 24,000 & 0 \\
\hline TNSS88 & AN-105 & $10 / 1 / 03$ & $10 / 1 / 03$ & 750 & 250 \\
\hline WATER & AN-105 & $10 / 1 / 03$ & $10 / 1 / 03$ & 35,000 & 0 \\
\hline
\end{tabular}




\section{HNF-2321}

Revision 0

Table A.1-1. Complete Transfer List for Case 1.

\begin{tabular}{|c|c|c|c|c|c|}
\hline From & To & Start Date & End Date & $\begin{array}{c}\text { Liquid } \\
\text { (gallons) }\end{array}$ & $\begin{array}{c}\text { Solid } \\
\text { (gallons) }\end{array}$ \\
\hline WESF & AN-105 & $10 / 1 / 03$ & $10 / 1 / 03$ & 5,000 & 0 \\
\hline ZNL87 & SY-102 & $10 / 1 / 03$ & $10 / 1 / 03$ & 3,000 & 0 \\
\hline WATER & $A N-105$ & $10 / 2 / 03$ & $10 / 2 / 03$ & 3,000 & 0 \\
\hline WATER & $A N-10 S$ & $10 / 2 / 03$ & $10 / 2 / 03$ & 5.000 & 0 \\
\hline WATER & SY-102 & $10 / 2 / 03$ & $10 / 2 / 03$ & 5,000 & 0 \\
\hline WATER & AN-105 & $10 / 2 / 03$ & $10 / 2 / 03$ & 22,000 & 0 \\
\hline $\mathrm{AP}-102$ & AP-106 & $9 / 29 / 03$ & $10 / 3 / 03$ & 740,300 & 1,581 \\
\hline AN-105 & AW-102 & $10 / 1 / 03$ & $10 \% 6 / 03$ & 911,300 & 270 \\
\hline AP-104 & $\mathrm{AP}-108$ & $10 / 3 / 03$ & $10 / 7 / 03$ & 740,300 & 1,581 \\
\hline AN-103 & $\mathrm{AP}-102$ & $10 / 4 / 03$ & $10 / 7 / 03$ & 436,600 & 28,133 \\
\hline $\mathrm{AN}-103$ & AP- 102 & $10 / 4 / 03$ & $10 / 7 / 03$ & 738,300 & 549 \\
\hline LERF & AN-103-PUMP & $10 / 4 / 03$ & $10 / 7 / 03$ & 264,900 & 0 \\
\hline $\mathrm{AN}-103$ & AP-104 & $10 / 8 / 03$ & $10 / 11 / 03$ & 436,600 & 28,133 \\
\hline AN-103 & AP-104 & $10 / 8 / 03$ & $10 / 11 / 03$ & 738,300 & 549 \\
\hline LERF & AN-103-PUMP & $10 / 8 / 03$ & $10 / 11 / 03$ & 264,900 & 0 \\
\hline AZ-101 & AN-105 & $10 / 28 / 03$ & $10 / 31 / 03$ & 622,700 & 0 \\
\hline WASH-WATER & $\mathrm{AZ}-101$ & $10 / 31 / 03$ & $11 / 2 / 03$ & 324,000 & 0 \\
\hline EVAPF & $\mathrm{AW}-102$ & $11 / 3 / 03$ & $11 / 3 / 03$ & 35,000 & 0 \\
\hline P1HLW FEED TANK & HLW-CONTRACTOR & $2 / 22 / 03$ & $11 / 14 / 03$ & 134,300 & 5,079 \\
\hline$A Z-102$ & P1HLW FEED TANK & $11 / 14 / 03$ & $11 / 14 / 03$ & 123,900 & 5,202 \\
\hline RETURN 1 & AP-107 & $12 / 14 / 03$ & $12 / 14 / 03$ & 8,670 & 331 \\
\hline AZ-101 & AN-105 & $12 / 21 / 03$ & $12 / 22 / 03$ & 320,000 & 0 \\
\hline WASH-WATER & AZ-101 & $12 / 24 / 03$ & $12 / 25 / 03$ & 324,000 & 0 \\
\hline RETURN 2 & AP-107 & $1 / 11 / 04$ & $1 / 11 / 04$ & 8,672 & 330 \\
\hline $242-A$ & LERF & $4 / 4 / 04$ & $4 / 9 / 04$ & 338,100 & 0 \\
\hline $242-\mathrm{A}$ & AW-106 & $4 / 4 / 04$ & $4 / 9 / 04$ & 605,100 & 33 \\
\hline AW-102 & $242-A$ & $4 / 4 / 04$ & $4 / 9 / 04$ & 943,100 & 33 \\
\hline AW-106 & AW-102 & $4 / 9 / 04$ & $4 / 12 / 04$ & 605,000 & 22 \\
\hline RETURN 1 & AP-107 & $4 / 28 / 04$ & $4 / 28 / 04$ & 8,672 & 330 \\
\hline $242-A$ & LERF & $4 / 26 / 04$ & $4 / 29 / 04$ & 216,300 & 0 \\
\hline $242-\mathrm{A}$ & AW-106 & $4 / 26 / 04$ & $4 / 29 / 04$ & 388,800 & 22 \\
\hline AW-102 & $242-\mathrm{A}$ & $4 / 26 / 04$ & $4 / 29 / 04$ & 605,100 & 22 \\
\hline AW-106 & AW-102 & $4 / 29 / 04$ & $5 / 1 / 04$ & 388,800 & 15 \\
\hline AP-102 & $\mathrm{AP}-106$ & $5 / 4 / 04$ & $5 / 7 / 04$ & 753,200 & 1,687 \\
\hline WASTH-WATER & AP-102 & $5 / 8 / 04$ & $5 / 8 / 04$ & 118,100 & 0 \\
\hline AP-104 & $\mathrm{AP}-108$ & $5 / 7 / 04$ & $5 / 11 / 04$ & 753,200 & 1,687 \\
\hline WASH-WATER & $A P-104$ & $5 / 12 / 04$ & $5 / 12 / 04$ & 118,100 & 0 \\
\hline RETURN 2 & AP-107 & $5 / 14 / 04$ & $5 / 14 / 04$ & 8,676 & 330 \\
\hline $242-\mathrm{A}$ & LERF & $5 / 15 / 04$ & $5 / 17 / 04$ & 139,700 & 0 \\
\hline $242-A$ & AW-106 & $5 / 15 / 04$ & $5 / 17 / 04$ & 249,200 & 15 \\
\hline AW-102 & $242-A$ & $5 / 15 / 04$ & $5 / 17 / 04$ & 388,900 & 15 \\
\hline AW-106 & AW-102 & $5 / 17 / 04$ & $5 / 18 / 04$ & 249,100 & 10 \\
\hline AX-103 & AN-103 & $1 / 10 / 04$ & $5 / 28 / 04$ & 310,200 & 2,846 \\
\hline $242-A$ & LERF & $6 / 1 / 04$ & $6 / 3 / 04$ & 90,000 & 0 \\
\hline $242-\mathrm{A}$ & AW-106 & $6 / 1 / 04$ & $6 / 3 / 04$ & 159,200 & 10 \\
\hline AW-102 & $242-\mathrm{A}^{-}$ & $6 / 1 / 04$ & $6 / 3 / 04$ & 249,200 & 10 \\
\hline AW-106 & AW-102 & $6 / 3 / 04$ & $6 / 4 / 04$ & 159,200 & 7 \\
\hline $\mathrm{AW}-102$ & AW-106 & $6 / 5 / 04$ & $6 / 6 / 04$ & 159,300 & 7 \\
\hline AP-102 & AN-105 & $5 / 27 / 04$ & $6 / 11 / 04$ & 209,500 & 9,168 \\
\hline AN-105 & AW-102 & $6 / 6 / 04$ & $6 / 11 / 04$ & 915,200 & 0 \\
\hline AP-104 & AN-105 & $6 / 12 / 04$ & $6 / 13 / 04$ & 209,500 & 9,168 \\
\hline WASH-CAUSTIC & $\mathrm{AP}-102$ & $6 / 13 / 04$ & $6 / 13 / 04$ & 103,400 & 0 \\
\hline WASH-CAUSTIC & AP-104 & $6 / 14 / 04$ & $6 / 14 / 04$ & 126,400 & 0 \\
\hline AZ-101 & AN-105 & $6 / 13 / 04$ & $6 / 15 / 04$ & 322,300 & 0 \\
\hline$\overline{\mathrm{AP}}-101$ & AP-102 & $6 / 15 / 04$ & $6 / 17 / 04$ & 502,400 & 23 \\
\hline AP-101 & AP-102 & $6 / 15 / 04$ & $6 / 17 / 04$ & 557,700 & 23 \\
\hline LERF & AP-101-PUMP & $6 / 15 / 04$ & $6 / 17 / 04$ & 55,267 & 0 \\
\hline WASH-WATER & AZ-101 & $6 / 16 / 04$ & $6 / 18 / 04$ & 433,600 & $\overline{0}$ \\
\hline AW-104 & $\mathrm{AP}-102$ & $6 / 19 / 04$ & $6 / 19 / 04$ & 109,000 & 5 \\
\hline $\mathrm{AP}-101$ & AP-104 & $6 / 18 / 04$ & $6 / 21 / 04$ & 614,000 & 28 \\
\hline AP-101 & AP-104 & $6 / 18 / 04$ & $6 / 21 / 04$ & 681,600 & 28 \\
\hline LERF & AP-101-PUMP & $6 / 18 / 04$ & $6 / 21 / 04$ & 67,548 & 0 \\
\hline RETURN 1 & AP-107 & $6 / 21 / 04$ & $6 / 21 / 04$ & 8,676 & 331 \\
\hline AW-104 & AP-104 & $6 / 22 / 04$ & $6 / 22 / 04$ & 133,200 & 6 \\
\hline $242-\mathrm{A}$ & LERF & $6 / 26 / 04$ & $7 / 1 / 04$ & 330,000 & 0 \\
\hline $242-\mathrm{A}$ & AW-106 & $6 / 26 / 04$ & $7 / 1 / 04$ & 585,100 & 32 \\
\hline AW-102 & $242-\mathrm{A}$ & $6 / 26 / 04$ & $7 / 1 / 04$ & 915,100 & 32 \\
\hline RETURN 2 & $\mathrm{AP}-107$ & $7 / 3 / 04$ & $7 / 3 / 04$ & 8,674 & 331 \\
\hline AW-106 & AW-102 & $7 / 1 / 04$ & $7 / 5 / 04$ & 744,300 & 28 \\
\hline $242-\mathrm{A}$ & LERF & $7 / 19 / 04$ & $7 / 23 / 04$ & 267,400 & 0 \\
\hline
\end{tabular}


HNF-2321

Revision 0

Table A.1-1. Complete Transfer List for Case 1.

\begin{tabular}{|c|c|c|c|c|c|}
\hline From & To & Start Date & End Date & $\begin{array}{c}\text { Liquid } \\
\text { (gallons) }\end{array}$ & $\begin{array}{c}\text { Solid } \\
\text { (gallons) }\end{array}$ \\
\hline $242-\mathrm{A}$ & $\mathrm{AW}-106$ & $7 / 19 / 04$ & $7 / 23 / 04$ & 476,900 & 28 \\
\hline$A W-102$ & $242-\mathrm{A}$ & $7 / 19 / 04$ & $7 / 23 / 04$ & 744,400 & 28 \\
\hline AW-106 & $A W-102$ & $7 / 23 / 04$ & $7 / 25 / 04$ & 476,800 & 19 \\
\hline P1HLW FEED TANK & HLW-CONTRACTOR & $11 / 14 / 03$ & $8 / 7 / 04$ & 123,900 & 5,202 \\
\hline$A Z-102$ & P1HLW FEED TANK & $8 / 7 / 04$ & $8 / 8 / 04$ & 123,900 & 5,202 \\
\hline RETURN 1 & AP-107 & $8 / 10 / 04$ & $8 / 10 / 04$ & 8,675 & 331 \\
\hline $\mathrm{C}-103$ & $\mathrm{AN}-103$ & $5 / 28 / 04$ & $8 / 11 / 04$ & 271,800 & 10,423 \\
\hline $242-\mathrm{A}$ & LERF & $8 / 8 / 04$ & $8 / 11 / 04$ & 171,200 & 0 \\
\hline $242-A$ & AW-106 & $8 / 8 / 04$ & $8 / 11 / 04$ & 305,700 & 19 \\
\hline AW-102 & $242-\mathrm{A}$ & $8 / 8 / 04$ & $8 / 11 / 04$ & 477,000 & 19 \\
\hline AW-106 & AW-102 & $8 / 11 / 04$ & $8 / 12 / 04$ & 305,700 & 13 \\
\hline RETURN 2 & AP-107 & $8 / 22 / 04$ & $8 / 22 / 04$ & 8,674 & 331 \\
\hline $242-\mathrm{A}$ & LERF & $8 / 26 / 04$ & $8 / 28 / 04$ & 76,605 & 0 \\
\hline $242-\mathrm{A}$ & $A W-106$ & $8 / 26 / 04$ & $8 / 28 / 04$ & 229,100 & 13 \\
\hline AW-102 & $242-A$ & $8 / 26 / 04$ & $8 / 28 / 04$ & 305,800 & 13 \\
\hline AW-106 & AW-101 & $8 / 28 / 04$ & $8 / 29 / 04$ & 229,100 & 11 \\
\hline $\mathrm{C}-104$ & AY-102 & $8 / 1 / 04$ & $9 / 8 / 04$ & 279,100 & 9,724 \\
\hline AW-101 & AW-104 & $9 / 15 / 04$ & $9 / 16 / 04$ & 276,800 & 13 \\
\hline RETURN 1 & AP-107 & $9 / 30 / 04$ & $9 / 30 / 04$ & 8,675 & 331 \\
\hline $34 L 87$ & AN-105 & $10 / 1 / 04$ & $10 / 1 / 04$ & 50,000 & 0 \\
\hline EVAPF & $A N-105$ & $10 / 1 / 04$ & $10 / 1 / 04$ & 120,000 & 0 \\
\hline SPN87 & SY-102 & $10 / 1 / 04$ & $10 / 1 / 04$ & 2,000 & 0 \\
\hline SPN87 & SY-102 & $10 / 1 / 04$ & $10 / 1 / 04$ & 23,000 & 0 \\
\hline TAL 88 & AN-105 & $10 / 1 / 04$ & $10 / 1 / 04$ & 2,000 & 0 \\
\hline TAL88 & AN-105 & $10 / 1 / 04$ & $10 / 1 / 04$ & 22,000 & 0 \\
\hline TNS88 & AN-105 & $10 / 1 / 04$ & $10 / 1 / 04$ & 750 & 250 \\
\hline WATER & $A N-105$ & $10 / 1 / 04$ & $10 / 1 / 04$ & 35,000 & 0 \\
\hline WESF & $A N-105$ & $10 / 1 / 04$ & $10 / 1 / 04$ & 5,000 & 0 \\
\hline ZNL87 & $\$ Y-102$ & $10 / 1 / 04$ & $10 / 1 / 04$ & 3,000 & 0 \\
\hline $1 F L 96$ & AW-105 & $10 / 1 / 04$ & $10 / 2704$ & 200,000 & 0 \\
\hline WATER & AN-105 & $10 / 2 / 04$ & $10 / 2 / 04$ & 4,000 & 0 \\
\hline WATER & SY-I02 & $10 / 2 / 04$ & $10 / 2 / 04$ & 5,000 & 0 . \\
\hline WATER & AN-105 & $10 / 2 / 04$ & $10 / 2 / 04$ & 22,000 & 0 \\
\hline WATER & $A W=105$ & $10 / 2 / 04$ & $10 / 2 / 04$ & 88,000 & 0 \\
\hline WATER & SY-102 & $10 / 2 / 04$ & $10 / 3 / 04$ & 1,000 & 0 \\
\hline WATER & AN-105 & $10 / 3 / 04$ & $10 / 3 / 04$ & 1,000 & 0 \\
\hline EVAPF & $A W-102$ & $10 / 4 / 04$ & $10 / 4 / 04$ & 50,000 & 0 \\
\hline $\mathrm{AN}-105$ & $\mathrm{AW}-102$ & $10 / 1 / 04$ & $10 / 5 / 04$ & 831,800 & 267 \\
\hline RETURN 2 & AP-107 & $10 / 11 / 04$ & $10 / 11 / 04$ & 8,674 & 331 \\
\hline EVAPF & AW-102 & $11 / 3 / 04$ & $11 / 3 / 04$ & 35,000 & 0 \\
\hline RETURN I & $A P-107$ & $11 / 19 / 04$ & $11 / 19 / 04$ & 8,675 & 331 \\
\hline$A-102$ & $\mathrm{AN}-103$ & $8 / 11 / 04$ & $11 / 30 / 04$ & 100,600 & 744 \\
\hline RETURN 2 & AP-107 & $11 / 30 / 04$ & $11 / 30 / 04$ & 8,674 & 331 \\
\hline RETURN 1 & $\mathrm{AP}-107$ & $1 / 8 / 05$ & $1 / 9705$ & 8,675 & 331 \\
\hline $\mathrm{AN}-103$ & $A W-103$ & $1 / 19 / 05$ & $1 / 19 / 05$ & 3,896 & 104 \\
\hline AN-103 & AP-105 & $1 / 19 / 05$ & $1 / 19 / 05$ & 3,897 & 104 \\
\hline AN-103 & AN-104 & $1 / 19 / 05$ & $1 / 19 / 05$ & 19,895 & 529 \\
\hline RETURN 2 & $A P-107$ & $1 / 19 / 05$ & $1 / 19 / 05$ & 8,674 & 331 \\
\hline $\mathrm{AN}-103$ & $\mathrm{AP}-103$ & $1 / 19 / 05$ & $1 / 20 / 05$ & 43,355 & 1,152 \\
\hline AN-103. & $A N-101$ & $1 / 20 / 05$ & $1 / 21 / 05$ & 169,300 & 4,495 \\
\hline AN-103 & $A P-101$ & $1 / 21 / 05$ & $1 / 25 / 05$ & 878,900 & 23,322 \\
\hline AP-102 & $\mathrm{AP}-106$ & $2 / 18 / 05$ & $2 / 22 / 05$ & 769,000 & $1,140^{\circ}$ \\
\hline$A Y-101$ & $\mathrm{AP}-102$ & $2 / 23 / 05$ & $2 / 24 / 05$ & 283,600 & 12 \\
\hline $\mathrm{AP}-104$ & AP-108 & $2 / 22 / 05$ & $2 / 26 / 05$ & 940,100 & 1,153 \\
\hline $\mathrm{AY}-101$ & AP-104 & $2 / 27 / 05$ & $2 / 28 / 05$ & 279,700 & 11 \\
\hline RETURN 1 & $A \mathrm{P}-107$ & $3 / 1 / 05$ & $3 / 1 / 05$ & 8.679 & 331 \\
\hline RETURN 2 & AP-107 & $3 / 13 / 05$ & $3 / 13 / 05$ & 8,676 & 331 \\
\hline C-105 & $A N-103$ & $11 / 30 / 04$ & $3 / 24 / 05$ & 827,500 & 31,875 \\
\hline $242-A$ & LERF & $4 / 4 / 05$ & 4/9/05 & 329,800 & 0 \\
\hline $242-A$ & AW-106 & $4 / 4 / 05$ & $4 / 9 / 105$ & 584,100 & 32 \\
\hline AW-102 & $242-\mathrm{A}$ & $4 / 4 / 05$ & $4 / 9 / 05$ & 914,000 & 32 \\
\hline AW-106 & AW-102 & $4 / 9 / 05$ & $4 / 12 / 05$ & 584,000 & 21 \\
\hline RETURN 1 & AP-107 & $4 / 27 / 05$ & $4 / 27 / 05$ & 8,670 & 330 \\
\hline $242-\mathrm{A}$ & LERF & $4 / 26 / 05$ & $4 / 29 / 05$ & 210,000 & 0 \\
\hline $242-\mathrm{A}$ & AW-106 & $4 / 26 / 05$ & $4 / 29 / 05$ & 374,200 & 21 \\
\hline AW-102 & $242-\mathbf{A}$ & $4 / 26 / 05$ & $4 / 29 / 05$ & 584,200 & 21 \\
\hline AW-106 & $\mathrm{AW}-102$ & $4 / 29 / 05$ & $5 / 1 / 05$ & 374,100 & 14 \\
\hline P1HLW FEED TANK & HLW-CONTRACTOR & $8 / 8 / 04$ & $5 / 2 / 05$ & 123,900 & 5,202 \\
\hline$A Z-101$ & PIHLW FEED TANK & $5 / 2 / 05$ & $5 / 2 / 05$ & 127,200 & 3,269 \\
\hline RETURN 2 & $\mathrm{AP}-107$ & $5 / 9 / 05$ & $5 / 9 / 05$ & 8,679 & 331 \\
\hline
\end{tabular}


HNF-2321

Revision 0

Table A.1-1. Complete Transfer List for Case 1.

\begin{tabular}{|c|c|c|c|c|c|}
\hline From & To & Start Date & End Date & $\begin{array}{c}\text { Liquid } \\
\text { (gallons) }\end{array}$ & $\begin{array}{c}\text { Solid } \\
\text { (gallons) }\end{array}$ \\
\hline $242-\mathbf{A}$ & LERF & $5 / 15 / 05$ & $5 / 17 / 05$ & 134,900 & 0 \\
\hline $242-\mathbf{A}$ & $A W-106$ & $5 / 15 / 05$ & $5 / 17 / 05$ & 239,300 & 14 \\
\hline AW-102 & $242-A$ & $5 / 15 / 05$ & $5 / 17 / 05$ & 374,200 & 14 \\
\hline AW-106 & AW-102 & $5 / 17 / 05$ & $5 / 18 / 05$ & 239,300 & 10 \\
\hline $242-A$ & LERF & $6 / 2 / 05$ & $6 / 3 / 05$ & 85,871 & 0 \\
\hline $242-\mathrm{A}$ & AW-106 & $6 / 2 / 05$ & $6 / 3 / 05$ & 153,500 & 10 \\
\hline $\mathrm{AW}-102$ & $242-A$ & $6 / 2 / 05$ & $6 / 3 / 05$ & 239,300 & 10 \\
\hline AW-106 & AW-102 & $6 / 3 / 05$ & $6 / 4 / 05$ & 153,400 & 7 \\
\hline AW-102 & AN-105 & $6 / 5 / 05$ & $6 / 6 / 05$ & 153,600 & 7 \\
\hline RETURN 1 & $\mathrm{AP}-107$ & $6 / 23 / 05$ & $6 / 23 / 05$ & 8,670 & 330 \\
\hline A-104 & AN-103 & $3 / 24 / 05$ & $7 / 3 / 05$ & 29,827 & 832 \\
\hline RETURN 2 & AP-107 & $7 / 5 / 05$ & $7 / 5 / 05$ & 8,679 & 331 \\
\hline$C-204$ & $\mathrm{AN}-103$ & $7 / 3 / 05$ & $7 / 10 / 05$ & 3,775 & 55 \\
\hline C-201 & $\mathrm{AN}-103$ & $7 / 10 / 05$ & $7 / 16 / 05$ & 2,464 & 59 \\
\hline AY-102 & $\mathrm{AZ}-102$ & $8 / 1 / 05$ & $8 / 3 / 05$ & 279,400 & 9,360 \\
\hline RETURN 1 & AP-107 & $8 / 19 / 05$ & $8 / 19 / 05$ & 8,670 & 330 \\
\hline AX-104 & $\mathrm{AN}-103$ & $7 / 16 / 05$ & $8 / 20 / 05$ & 18,516 & 572 \\
\hline RETURN 2 & $A P-107$ & $8 / 31 / 05$ & $8 / 31 / 05$ & 8,679 & 331 \\
\hline $34 L 87$ & AN-105 & $10 / 1 / 05$ & $10 / 1 / 05$ & 50,000 & 0 \\
\hline EVAPF & $A N-105$ & $10 / 1 / 05$ & $10 / 1 / 05$ & 120,000 & 0 \\
\hline SPN87 & SY-102 & $10 / 1 / 05$ & $10 / 1 / 05$ & 25,000 & 0 \\
\hline TAL88 & $A N-105$ & $10 / 1 / 05$ & $10 / 1 / 05$ & 24,000 & 0 \\
\hline TNS88 & $\mathrm{AN}-105$ & $10 / 1 / 05$ & $10 / 1 / 05$ & 750 & 250 \\
\hline WATER & AN-105 & $10 / 1 / 05$ & $10 / 1 / 05$ & 35,000 & 0 \\
\hline WESF & $\mathrm{AN}-105$ & $10 / 1 / 05$ & $10 / 1 / 05$ & 5,000 & 0 \\
\hline ZNL87 & $S Y-102$ & $10 / 1 / 05$ & $10 / 1 / 05$ & 3,000 & 0 \\
\hline WATER & SY-102 & $10 / 2 / 05$ & $10 / 2 / 05$ & 1,000 & 0 \\
\hline WATER & $\mathrm{AN}-105$ & $10 / 2 / 05$ & $10 / 2 / 05$ & 5,000 & 0 \\
\hline WATER & SY-102 & $10 / 2 / 05$ & $10 / 2 / 05$ & 5,000 & 0 \\
\hline WATER & $\mathrm{AN}-105$ & $10 / 2 / 05$ & $10 / 2 / 05$ & 22,000 & 0 \\
\hline RETURN 1 & $\mathrm{AP}-107$ & $10 / 15 / 05$ & $10 / 15 / 05$ & 8,670 & 330 \\
\hline RETURN 2 & AP-107 & $10 / 27 / 05$ & $10 / 27 / 05$ & 8,679 & 331 \\
\hline AW-105 & AN-105 & $11 / 1 / 05$ & $11 / 2 / 05$ & 288,100 & 10 \\
\hline EVAPF & AW-102 & $11 / 3 / 05$ & $11 / 3 / 05$ & 35,000 & 0 \\
\hline P1HLW FEED TANK & HLW-CONTRACTOR & $5 / 2 / 05$ & $11 / 16 / 05$ & 127,200 & 3,269 \\
\hline SY-102 & AW-105 & $11 / 15 / 05$ & $11 / 17 / 05$ & 573,800 & 22 \\
\hline AZ-101 & P1HLW FEED TANK & $11 / 16 / 05$ & $11 / 17 / 05$ & 127,200 & 3,269 \\
\hline AX-102 & AN-103 & $8 / 20 / 05$ & $12 / 8 / 05$ & 87,597 & 897 \\
\hline RETURN 1 & AP-107 & $12 / 11 / 05$ & $12 / 11 / 05$ & 8,670 & 330 \\
\hline WATER & $5 Y-102$ & $12 / 15 / 05$ & $12 / 17 / 05$ & 426,000 & 0 \\
\hline AN-10S & AW-102 & $12 / 17 / 05$ & $12 / 21 / 05$ & 874,800 & 0 \\
\hline AW-105 & AN-105 & $12 / 15 / 05$ & $12 / 23 / 05$ & 573,800 & 22 \\
\hline RETURN 2 & $\mathrm{AP}-107$ & $12 / 23 / 05$ & $12 / 23 / 05$ & 8,679 & 331 \\
\hline AP-106 & LAW-CONTRACTOR-1 & $6 / 1 / 02$ & $12 / 31 / 05$ & $3,634,000$ & 5,468 \\
\hline $\mathrm{AP}-102$ & AP-106 & $12 / 31 / 05$ & $1 / 2 / 06$ & 283,500 & 48 \\
\hline$S Y-102$ & AW-105 & $1 / 1 / 06$ & $1 / 3 / 06$ & 535,600 & 22,049 \\
\hline $\mathrm{AN}-107$ & $\mathrm{AP}-102$ & $1 / 2 / 06$ & $1 / 4 / 06$ & 481,700 & 22 \\
\hline $\mathrm{AN}-107$ & $A P-102$ & $1 / 2 / 06$ & $1 / 4 / 06$ & $\$ 54,000$ & 22 \\
\hline LERF & AN-107-PUMP & $1 / 2 / 06$ & $1 / 4 / 06$ & 72,259 & 0 \\
\hline RETURN 2 & AP-107 & $2 / 18 / 06$ & $2 / 18 / 06$ & 8,679 & 331 \\
\hline AW-105 & AN-105 & $3 / 1 / 06$ & $3 / 2 / 06$ & 280,600 & 10 \\
\hline AP-108 & LAW-CONTRACTOR-2 & $6 / 1 / 02$ & $3 / 6 / 06$ & $3,805,000$ & 5,473 \\
\hline AP-104 & AP-108 & $3 / 6 / 06$ & $3 / 8 / 06$ & 279,600 & 41 \\
\hline AP-106 & LAW-CONTRACTOR-1 & $1 / 2 / 06$ & $3 / 11 / 06$ & 283,500 & 83 \\
\hline AN-107 & AP-104 & $3 / 9 / 06$ & $3 / 11 / 06$ & 481,700 & 22 \\
\hline AN-107 & $\mathrm{AP}-104$ & $3 / 9 / 06$ & $3 / 11 / 06$ & 554,000 & 22 \\
\hline LERF & AN-107-PUMP & $3 / 9 / 06$ & $3 / 11 / 06$ & 72,260 & 0 \\
\hline $\mathrm{AP}-102$ & $\mathrm{AP}-106$ & $3 / 11 / 06$ & $3 / 12 / 06$ & 169,400 & 8 \\
\hline $\mathrm{AN}-103$ & $\mathrm{AP}-101$ & $3 / 16 / 06$ & $3 / 17 / 06$ & 210,200 & 4,029 \\
\hline $\mathrm{AN}-103$ & AW-104 & $3 / 17 / 06$ & $3 / 17 / 06$ & 0 & 0 \\
\hline $\mathrm{AN}-103$ & AW-105 & $4 / 1 / 06$ & $4 / 3 / 06$ & 466,400 & 8,930 \\
\hline AP-102 & AP-106 & $4 / 8 / 06$ & $4 / 10 / 06$ & 384,600 & 18 \\
\hline $\mathrm{AN}-102$ & $\mathrm{AP}-102$ & $4 / 11 / 06$ & $4 / 14 / 06$ & 481,700 & 23 \\
\hline $\mathrm{AN}-102$ & AP-102 & $4 / 11 / 06$ & $4 / 14 / 06$ & 770,700 & 23 \\
\hline LERF & AN-102-PUMP & $4 / 11 / 06$ & $4 / 14 / 06$ & 289,000 & 0 \\
\hline $242-A$ & LERF & $5 / 6 / 06$ & $5 / 1106$ & 326,000 & 0 \\
\hline $242-\mathrm{A}$ & AW-106 & $5 / 6 / 06$ & $5 / 11 / 06$ & 583,800 & 33 \\
\hline$A W-102$ & $242-A$ & $5 / 6 / 06$ & $5 / 11 / 06$ & 909,800 & 33 \\
\hline $\mathrm{AP}-108$ & LAW-CONTRACTOR-2 & $3 / 8 / 06$ & $5 / 14 / 06$ & 279,600 & 70 \\
\hline AW-106 & $A W-102$ & $5 / 11 / 06$ & $5 / 14 / 06$ & 583,700 & 23 \\
\hline
\end{tabular}




\section{HNF-2321 \\ Revision 0}

Table A.1-1. Complete Transfer List for Case 1.

\begin{tabular}{|c|c|c|c|c|c|}
\hline From & To & Start Date & End Date & $\begin{array}{c}\text { Liquid } \\
\text { (gallons) }\end{array}$ & $\begin{array}{c}\text { Solid } \\
\text { (gallons) }\end{array}$ \\
\hline AP-104 & AP-108 & $5 / 14 / 06$ & $5 / 15 / 06$ & 169,400 & 8 \\
\hline $242-\mathrm{A}$ & LERF & $5 / 28 / 06$ & $5 / 31 / 06$ & 210,000 & 0 \\
\hline $242-\mathrm{A}$ & AW-106 & $5 / 28 / 06$ & $5 / 31 / 06$ & 373,900 & 23 \\
\hline AW-102 & $242-\hat{A}$ & $5 / 28 / 06$ & $5 / 31 / 06$ & 583,900 & 23 \\
\hline PIHLW FEED TANK & HLW-CONTRACTOR & $11 / 17 / 05$ & $6 / 2 / 06$ & 127,200 & 3,269 \\
\hline AW-105 & AW-102 & $5 / 31 / 06$ & $6 / 2 / 06$ & 373,800 & 16 \\
\hline$A Z-101$ & P1HLW FEED TANK & $6 / 2 / 06$ & $6 / 3 / 06$ & 127,200 & 3,269 \\
\hline AP-104 & AP- 108 & $6 / 11 / 06$ & $6 / 13 / 06$ & 384,600 & 17 \\
\hline AN-102 & AP-104 & $6 / 14 / 06$ & $6 / 17 / 06$ & 481,700 & 23 \\
\hline$A N-102$ & AP-104 & $6 / 14 / 06$ & $6 / 17 / 06$ & 770,700 & 23 \\
\hline LERF & AN-102-PUMP & $6 / 14 / 06$ & $6 / 17 / 06$ & 289,000 & 0 \\
\hline $242-A$ & LERF & $6 / 16 / 06$ & $6 / 18 / 06$ & 133,700 & 0 \\
\hline $242-\mathrm{A}$ & AW-106 & $6 / 16 / 06$ & $6 / 18 / 06$ & 240,200 & 15 \\
\hline AW-102 & $242-\mathrm{A}$ & $6 / 16 / 06$ & $6 / 18 / 06$ & 373,900 & 15 \\
\hline AW-106 & AW-101 & $6 / 18 / 06$ & $6 / 19 / 06$ & 240,200 & $\frac{11}{11}$ \\
\hline AZ-102 & AN-105 & $6 / 23 / 06$ & $6 / 24 / 06$ & 159,500 & 0 \\
\hline WASH-WATER & $A Z-102$ & $6 / 24 / 06$ & $6 / 25 / 06$ & 324,000 & 0 \\
\hline AN-105 & $A W-102$ & $6 / 24 / 06$ & $6 / 28 / 06$ & 839,300 & 0 \\
\hline$A Z-102$ & AÑ-105 & $8 / 13 / 06$ & $8 / 15 / 06$ & 320,100 & 0 \\
\hline WASH-WATER & $A Z-102$ & $8 / 16 / 06$ & $8 / 18 / 06$ & 324,000 & 0 \\
\hline AP-102 & $\mathrm{AP}-106$ & $8 / 21 / 06$ & $8 / 25 / 06$ & 770,700 & 23 \\
\hline AN-106 & AP-102 & $8 / 26 / 06$ & $8 / 29 / 06$ & 547,800 & 24 \\
\hline AN-105 & $A P-102$ & $8 / 26 / 06$ & $8 / 29 / 06$ & 646,400 & 24 \\
\hline LERF & AN-106-PUMP & $8 / 26 / 06$ & $8 / 29 / 06$ & 98,603 & 0 \\
\hline $34 \mathrm{~L} 87$ & AN-105 & $10 / 1 / 06$ & $10 / 1 / 06$ & 7,000 & 0 \\
\hline $34 L 87$ & AN-105 & $10 / 1 / 06$ & $10 / 1 / 06$ & 50,000 & 0 \\
\hline EVAPF & AN-105 & $10 / 1 / 06$ & $10 / 1 / 06$ & 120,000 & 0 \\
\hline SPN87 & SY-102 & $.10 / 1 / 06$ & $10 / 1 / 06$ & 25,000 & 0 \\
\hline TAL88 & AN-105 & $10 / 1 / 06$ & $10 / 1 / 06$ & 25,000 & 0 \\
\hline TNS88 & AN-105 & $10 / 1 / 06$ & $10 / 1 / 06$ & 750 & 250 \\
\hline WATER & $A N-105$ & $10 / 1 / 06$ & $10 / 1 / 06$ & 35,000 & 0 \\
\hline WESF & AN-105 & $10 / 1 / 06$ & $10 / 1 / 06$ & 5,000 & 0 \\
\hline WATER & $A N-105$ & $10 / 2 / 06$ & $10 / 2 / 06$ & 3,000 & 0 \\
\hline WATER & AN-105 & $10 / 2 / 06$ & $10 / 2 / 06$ & 5,000 & 0 \\
\hline WATER & SY-102 & $10 / 2 / 06$ & $10 / 2 / 06$ & 5,000 & 0 \\
\hline WATER & AN -105 & $10 / 2 / 06$ & $10 / 2 / 06$ & 22,000 & 0 \\
\hline EVAPF & AW-102 & $10 / 4 / 06$ & $10 / 4 / 06$ & 50,000 & 0 \\
\hline $\bar{A} \mathbf{P}-104$ & AP- 108 & $10 / 24 / 06$ & $10 / 28 / 06$ & 770,700 & 23 \\
\hline EVAPF & AW-102 & $11 / 3 / 06$ & $11 / 3 / 06$ & 35,000 & 0 \\
\hline PIHLW FEED TANK & HLW-CONTRACTOR & $6 / 3 / 06$ & $12 / 18 / 06$ & 127,200 & 3,269 \\
\hline $\mathrm{AZ}-101$ & P1HLW FEED TANK & $12 / 18 / 06$ & $12 / 18 / 06$ & 127,200 & 3,269 \\
\hline$A N-106$ & AP-104 & $12 / 18 / 06$ & $12 / 22 / 06$ & 547,800 & 24 \\
\hline$A N-106$ & AP-104 & $12 / 18 / 06$ & $12 / 22 / 06$ & 646,400 & 24 \\
\hline LERF & AN-106-PUMP & $12 / 18 / 06$ & $12 / 22 / 06$ & 98,603 & 0 \\
\hline LERF & SY-101-PUMP & $12 / 19 / 06$ & $12 / 24 / 06$ & 532,400 & 0 \\
\hline SY-101 & SY -102 & $12 / 19 / 06$ & $12 / 24 / 06$ & 537,800 & 5,486 \\
\hline SY-101 & SY- 102 & $12 / 19 / 06$ & $12 / 24 / 06$ & $1,082,000$ & 611 \\
\hline $242-\mathrm{A}$ & LERF & $12 / 26 / 06$ & $12 / 31 / 06$ & 330,400 & 0 \\
\hline $242-A$ & AW-106 & $12 / 26 / 06$ & $12 / 31 / 06$ & 594,100 & 34 \\
\hline AW-102 & $242-A$ & $12 / 26 / 06$ & $12 / 31 / 06$ & 924,500 & 34 \\
\hline AW-106 & $\mathrm{AW}-102$ & $12 / 3 \mathrm{~L} / 06$ & $1 / 3 / 07$ & 594,000 & 23 \\
\hline SY-102 & $A N-102$ & $1 / 1 / 07$ & $1 / 4 / 07$ & $1,027,000$ & 1,532 \\
\hline LERF & SY-101-PUMP & $1 / 12 / 07$ & $1 / 17 / 07$ & 532,400 & 0 \\
\hline SY-101 & $S Y=102$ & $1 / 12 / 07$ & $1 / 17 / 07$ & 537,800 & 5,486 \\
\hline SY-101 & SY -102 & $1 / 12 / 07$ & $1 / 17 / 07$ & $1,082,000$ & 611 \\
\hline $242-\mathrm{A}$ & LERF & $1 / 17 / 07$ & $1 / 20 / 07$ & 213,700 & 0 \\
\hline $242-\mathrm{A}$ & AW-106 & $1 / 17 / 07$ & $1 / 20 / 07$ & 380,400 & 23 \\
\hline AW-102 & $242-A$ & $1 / 17 / 07$ & $1 / 20 / 07$ & 594,100 & 23 \\
\hline$A W-106$ & AW-102 & $1 / 20 / 07$ & $1 / 22 / 07$ & 380,300 & 16 \\
\hline SY-102 & AN-107 & $1 / 25 / 07$ & $1 / 28 / 07$ & 983,800 & 641 \\
\hline SY-101 & SY -102 & $2 / 5 / 07$ & $2 / 5 / 07$ & 0 & 0 \\
\hline $242-\mathrm{A}$ & LERF & $2 / 5 / 07$ & $2 / 7 / 07$ & 136,500 & 0 \\
\hline $242-\hat{A}$ & AW-106 & $2 / 5 / 07$ & $2 / 7 / 07$ & 244,000 & 16 \\
\hline AW-102 & $242-A$ & $2 / 5 / 07$ & $2 / 7 / 07$ & 380,400 & 16 \\
\hline AW-106 & AW-101 & $2 / 7 / 07$ & $2 / 8 / 07$ & 243,900 & 11 \\
\hline$A Z-102$ & AN-105 & $10 / 6 / 06$ & $2 / 12 / 07$ & 322,700 & 0 \\
\hline AN-105 & AW-102 & $2 / 8 / 07$ & $2 / 12 / 07$ & 871,400 & 0 \\
\hline WASH-WATER & $A Z-102$ & $2 / 13 / 07$ & $2 / 14 / 07$ & 150,500 & 0 \\
\hline RETURN 1 & AP-107 & $3 / 9 / 07$ & $3 / 9 / 07$ & 8,732 & 269 \\
\hline AN-103 & AN-106 & $3 / 22 / 07$ & $3 / 24 / 07$ & 424,700 & 8,123 \\
\hline
\end{tabular}


HNF-2321
Revision 0

Table A.1-1. Complete Transfer List for Case 1.

\begin{tabular}{|c|c|c|c|c|c|}
\hline From , & To & Start Date & End Date & $\begin{array}{c}\text { Liquid } \\
\text { (gallons) }\end{array}$ & $\begin{array}{c}\text { Solid } \\
\text { (gallons) }\end{array}$ \\
\hline $242-\mathrm{A}$ & LERF & $4 / 5 / 07$ & $4 / 10 / 07$ & 312,000 & 0 \\
\hline $242-A$ & AW-106 & $4 / 5 / 07$ & $4 / 10 / 07$ & 559,500 & 30 \\
\hline $\mathrm{AW}-102$ & $242-A$ & $4 / 5 / 07$ & $4 / 10 / 07$ & 871,500 & 30 \\
\hline AW-106 & $A W-102$ & $4 / 10 / 07$ & $4 / 13 / 07$ & 559,400 & 20 \\
\hline AP-102 & AP-106 & $4 / 18 / 07$ & $4 / 21 / 07$ & 646,400 & 24 \\
\hline AN-102 & AP-102 & $4 / 22 / 07$ & $4 / 27 / 07$ & $1,013,000$ & 44 \\
\hline $242-\mathrm{A}$ & LERF & $4 / 27 / 07$ & $4 / 30 / 07$ & 202,400 & 0 \\
\hline $242-\bar{A}$ & $A W-106$ & $4 / 27 / 07$ & $4 / 30 / 07$ & 357,000 & 20 \\
\hline$A W-102$ & $242-\mathrm{A}$ & $4 / 27 / 07$ & $4 / 30 / 07$ & 559,500 & 20 \\
\hline AW-106 & $A W-102$ & $4 / 30 / 07$ & $5 / 2 / 07$ & 356,300 & 13 \\
\hline LERF & SY-103-PUMP & $4 / 30 / 07$ & $5 / 2 / 07$ & 183,500 & 0 \\
\hline SY-103 & SY-102 & $4 / 30 / 07$ & $5 / 2 / 07$ & 350,000 & 9,759 \\
\hline$S Y-103$ & SY-102 & $4 / 30 / 07$ & $5 / 2 / 07$ & 537,500 & 1,449 \\
\hline$S Y-102$ & $A N-102$ & $5 / 10 / 07$ & $5 / 12 / 07$ & 720,700 & 1,528 \\
\hline $242-A$ & LERF & $5 / 16 / 07$ & $5 / 18 / 07$ & 128,900 & 0 \\
\hline $242-A$ & AW-106 & $5 / 16 / 07$ & $5 / 18 / 07$ & 227,500 & 13 \\
\hline $\mathrm{AW}-102$ & 242-A & $5 / 16 / 07$ & $5 / 18 / 07$ & 356,400 & 13 \\
\hline $\mathrm{AW}-106$ & AW-102 & $5 / 18 / 07$ & $5 / 19 / 07$ & 227,500 & 8 \\
\hline $242-\mathrm{A}$ & LERF & $6 / 2 / 07$ & $6 / 3 / 07$ & 84,000 & 0 \\
\hline $242-A$ & $A W-106$ & $6 / 2 / 07$ & $6 / 3 / 07$ & 143,500 & 8 \\
\hline AW-102 & $242-\mathrm{A}$ & $6 / 2 / 07$ & $6 / 3 / 07$ & 227,500 & 8 \\
\hline AW-106 & AW-102 & $6 / 3 / 07$ & $6 / 4 / 07$ & 143,500 & 6 \\
\hline $\mathrm{AW}-102$ & AN-105 & $6 / 5 / 07$ & $6 / 6 / 07$ & 143,600 & 6 \\
\hline $\mathrm{AP}-104$ & AP-108 & $6 / 21 / 07$ & $6 / 24 / 07$ & 646,400 & 24 \\
\hline $\mathrm{AN}-107$ & AP-104 & $6 / 25 / 07$ & $6 / 29 / 07$ & 978,400 & 43 \\
\hline PIHLW FEED TANK & HLW-CONTRACTOR. & $12 / 18 / 06$ & $7 / 4 / 07$ & 127,200 & 3,269 \\
\hline LERF & SY-103-PUMP & $7 / 3 / 07$ & $7 / 5 / 07$ & 183,500 & 0 \\
\hline SY-103 & SY-102 & $7 / 3 / 07$ & $7 / 5 / 07$ & 350,000 & 9,759 \\
\hline SY-103 & SY -102 & $7 / 3 / 07$ & $7 / 5 / 07$ & 537,500 & 1,449 \\
\hline$A Z-101$ & PLHLW FEED TANK & $7 / 4 / 07$ & $7 / 5 / 07$ & 127,200 & 3,269 \\
\hline$\$ Y-102$ & AN-107 & $7 / 13 / 07$ & $7 / 14 / 07$ & 537,500 & 1,434 \\
\hline 34 IS 87 & AN-105 & $10 / 1 / 07$ & $10 / 1 / 07$ & 50,000 & 0 \\
\hline EVAPF & AN-105 & $10 / 1 / 07$ & $10 / 1 / 07$ & 120,000 & 0 \\
\hline SPN87 & SY-102 & $10 / 1 / 07$ & $10 / 1 / 07$ & 25,000 & 0 \\
\hline TAL88 & AN-105 & $10 / 1 / 07$ & $10 / 1 / 07$ & 25,000 & 0 \\
\hline TNS88 & AN-105 & $10 / 1 / 07$ & $10 / 1 / 07$ & 750 & 250 \\
\hline WATER & AN-105 & $10 / 1 / 07$ & $10 / 1 / 07$ & 35,000 & 0 \\
\hline WESF & AN-105 & $10 / 1 / 07$ & $10 / 1 / 07$ & 5,000 & 0 \\
\hline WATER & AN-105 & $10 / 2 / 07$ & $10 / 2 / 07$ & 5,000 & 0 \\
\hline WATER & SY-102 & $10 / 2 / 07$ & $10 / 2 / 07$ & 5,000 & 0 \\
\hline WATER & AN-105 & $10 / 2 / 07$ & $10 / 2 / 07$ & 22,000 & 0 \\
\hline S-103 & $S Y-101$ & $5 / 9 / 07$ & $10 / 31 / 07$ & 712,300 & 1,011 \\
\hline EVAPF & $A W-102$ & $11 / 3 / 07$ & $11 / 3 / 07$ & 35,000 & 0 \\
\hline $\mathrm{AN}-103$ & AN-106 & $11 / 7 / 07$ & $11 / 11 / 07$ & 664,600 & 2,116 \\
\hline$A P-102$ & AP-106 & $11 / 9 / 07$ & $11 / 14 / 07$ & $1,013,000$ & 44 \\
\hline AN-102 & AP-102 & $11 / 15 / 07$ & $11 / 18 / 07$ & 707,100 & 31 \\
\hline $\mathrm{SX}-113$ & SY-103 & $10 / 6 / 07$ & $1 / 11 / 08$ & 10,322 & 399 \\
\hline$A P-104$ & $\mathrm{AP}-108$ & $1 / 12 / 08$ & $1 / 17 / 08$ & 978,400 & 43 \\
\hline P1HLW FEED TANK & HLW-CONTRACTOR & $7 / 5 / 07$ & $1 / 18 / 08$ & 127,200 & 3,269 \\
\hline$\overline{A Z}-102$ & P1HLW FEED TANK & $1 / 18 / 08$ & $1 / 19 / 08$ & 137,700 & 4,774 \\
\hline$A N-107$ & AP-104 & $1 / 18 / 08$ & $1 / 20 / 08$ & 524,700 & 23 \\
\hline$S Y=101$ & $\mathrm{AN}-102$ & $2 / 16 / 08$ & $2 / 20 / 08$ & 996,600 & 1,676 \\
\hline$A N-103$ & AN-107 & $4 / 19 / 08$ & $4 / 22 / 08$ & 458,500 & 1,458 \\
\hline $\mathrm{AN}-102$ & AN-107 & $4 / 22 / 08$ & $4 / 24 / 08$ & 500,300 & 3,978 \\
\hline SX-112 & $S Y-103$ & $1 / 11 / 08$ & $5 / 22 / 08$ & 397,100 & 16,406 \\
\hline$\overline{A X}-101$ & $\mathrm{AN}-103$ & $12 / 8 / 05$ & $8 / 15 / 08$ & $2,149,000$ & 6,072 \\
\hline $\mathrm{C}-202$ & AN-103 & $8 / 15 / 08$ & $8 / 20 / 08$ & 1,181 & 28 \\
\hline $\mathrm{AP}-102$ & AP-106 & $9 / 12 / 08$ & $9 / 16 / 08$ & 707,100 & 31 \\
\hline$\$ X-110$ & SY -103 & $5 / 22 / 08$ & $9 / 24 / 08$ & 212,700 & 8,909 \\
\hline $34 L 87$ & AN-105 & $10 / 1 / 08$ & $10 / 1 / 08$ & 50,000 & 0 \\
\hline EVAPF & $A N-10 S$ & $10 / 1 / 08$ & $10 / 1 / 08$ & 120,000 & 0 \\
\hline SPN87 & SY-102 & $10 / 1 / 08$ & $10 / 1 / 08$ & 25,000 & 0 \\
\hline TAL88 & AN-10S & $10 / 1 / 08$ & $10 / 1 / 08$ & 25,000 & 0 \\
\hline TNS88 & $A N-105$ & $10 / 1 / 08$ & $10 / 1 / 08$ & 750 & 250 \\
\hline WATER & $A N-10 S$ & $10 / 1 / 08$ & $10 / 1 / 08$ & 35,000 & 0 \\
\hline WESF & AN-10S & $10 / 1 / 08$ & $10 / 1 / 08$ & 5,000 & 0 \\
\hline WATER & $A N-105$ & $10 / 2 / 08$ & $10 / 2 / 08$ & 5,000 & 0 \\
\hline WATER & $\$ Y-102$ & $10 / 2 / 08$ & $10 / 2 / 08$ & 5,000 & 0 \\
\hline WATER & AN-105 & $10 / 2 / 08$ & $10 / 2 / 08$ & 22,000 & 0 \\
\hline EVAPF & $A W-102$ & $10 / 4 / 08$ & $10 / 4 / 08$ & 50,000 & 0 \\
\hline
\end{tabular}


HNF-2321

Revision 0

Table A.1-1. Complete Transfer List for Case 1.

\begin{tabular}{|c|c|c|c|c|c|}
\hline From & To & Start Date & End Date & $\begin{array}{c}\text { Liquid } \\
\text { (gallons) }\end{array}$ & $\begin{array}{c}\text { Solid } \\
\text { (gallons) }\end{array}$ \\
\hline EVAPF & $A W-102$ & $11 / 3 / 08$ & $11 / 3 / 08$ & 35,000 & 0 \\
\hline A-105 & AN-103 & $8 / 20 / 08$ & $11 / 12 / 08$ & 50,372 & 1,545 \\
\hline $\mathrm{C}-203$ & AN-103 & $11 / 12 / 08$ & $11 / 18 / 08$ & 6,307 & 150 \\
\hline AP-104 & AP-108 & $11 / 15 / 08$ & $11 / 18 / 08$ & 524,700 & 23 \\
\hline SX-115 & $S Y-103$ & $9 / 24 / 08$ & $11 / 25 / 08$ & 42,100 & 1,556 \\
\hline PIHLW FEED TANK & HLW-CONTRACTOR & $1 / 19 / 08$ & $12 / 22 / 08$ & 137,700 & 4,774 \\
\hline$A \bar{Z}-102$ & P1HLW FEED TANK & $12 / 22 / 08$ & $12 / 22 / 08$ & 137,700 & 4,774 \\
\hline U-202 & $\widehat{\mathrm{UA}-2}$ & $1 / 3 / 09$ & $1 / 10 / 09$ & 27,411 & 1,061 \\
\hline $\mathrm{U}-203$ & UA-2 & $1 / 10 / 09$ & $1 / 16 / 09$ & 13,895 & 537 \\
\hline C-108 & AN-103 & $11 / 18 / 08$ & $2 / 3 / 09$ & 83,934 & 3,840 \\
\hline $\mathrm{U}-110$ & UA-2 & $1 / 16 / 09$ & $3 / 10 / 09$ & 99,290 & 4,252 \\
\hline UA-2 & $S Y-102$ & $3 / 11 / 09$ & $3 / 12 / 09$ & 173,900 & 5,847 \\
\hline $\mathrm{C}-111$ & $\mathrm{AN}-103$ & $2 / 3 / 09$ & $4 / 17 / 09$ & 176,500 & 7,080 \\
\hline AP-106 & LAW-CONTRACTOR-1 & $3 / 12 / 06$ & $5 / 25 / 09$ & $3,692,000$ & 155 \\
\hline RETURN 1 & AP-107 & $5 / 26 / 09$ & $5 / 26 / 09$ & 2,727 & 98 \\
\hline $\mathrm{U}-110$ & UA-1 & $3 / 10 / 09$ & $5 / 27 / 09$ & 143,800 & 6,160 \\
\hline UA-1 & SY -102 & $5 / 28 / 09$ & $5 / 29 / 09$ & 186,800 & 6,157 \\
\hline $\mathrm{U}-110$ & UA-2 & $5 / 27 / 09$ & $5 / 30 / 09$ & 4,829 & 207 \\
\hline AP-108 & LAW-CONTRACTOR-2 & $5 / 15 / 06$ & $6 / 1 / 09$ & $3,474,000$ & 144 \\
\hline RETURN 2 & AP-107 & $6 / 2 / 09$ & $6 / 2 / 09$ & 8,391 & 319 \\
\hline AP-107 & AZ-101 & $6 / 12 / 09$ & $6 / 13 / 09$ & 289,700 & 9,938 \\
\hline AN-102 & AP-107 & $6 / 22 / 09$ & $6 / 25 / 09$ & 608,400 & 4,834 \\
\hline SY-101 & AN-102 & $6 / 25 / 09$ & $6 / 29 / 09$ & $1,115,000$ & 1,795 \\
\hline AN-102 & AP-107 & $6 / 29 / 09$ & $7 / 2 / 09$ & 502,400 & 885 \\
\hline S-105 & SY-101 & $10 / 31 / 07$ & $7 / 23 / 09$ & $1,518,000$ & 2,459 \\
\hline $\mathrm{U}-101$ & $\mathrm{UA}-2$ & $5 / 30 / 09$ & $7 / 30 / 09$ & 27,955 & 668 \\
\hline 34 L87 & AN-105 & $10 / 1 / 09$ & $10 / 1 / 09$ & 7,000 & 0 \\
\hline $34 \mathrm{LB} 7$ & AN-105 & $10 / 1 / 09$ & $10 / 1 / 09$ & 50,000 & 0 \\
\hline EVAPF & AN-105 & $10 / 1 / 09$ & $10 / 1 / 09$ & 120,000 & 0 \\
\hline SPN87 & SY -102 & $10 / 1 / 09$ & $10 / 1 / 09$ & 25,000 & 0 \\
\hline TAL88 & AN-105 & $10 / 1 / 09$ & $10 / 1 / 09$ & 26,000 & 0 \\
\hline TNS88 & AN-105 & $10 / 1 / 09$ & $10 / 1 / 09$ & 750 & 250 \\
\hline WATER & $\mathrm{AN}-105$ & $10 / 1 / 09$ & $10 / 1 / 09$ & 35,000 & 0 \\
\hline WESF & AN-105 & $10 / 1 / 09$ & $10 / 1 / 09$ & 5,000 & 0 \\
\hline WATER & $\mathrm{AN}-105$ & $10 / 2 / 09$ & $10 / 2 / 09$ & 3,000 & 0 \\
\hline WATER & AN-105 & $10 / 2 / 09$ & $10 / 2 / 09$ & 5,000 & 0 \\
\hline WATER & SY-102 & $10 / 2 / 09$ & $10 / 2 / 09$ & 5,000 & 0 \\
\hline WATER & AN-105 & $10 / 2 / 09$ & $10 / 2 / 09$ & 22,000 & 0 \\
\hline AN-10S & $A W-102$ & $10 / 1 / 09$ & $10 / 5 / 09$ & 793,700 & 245 \\
\hline EVAPF & AW-102 & $11 / 3 / 09$ & $11 / 3 / 09$ & 35,000 & 0 \\
\hline U-104 & UA-2 & $7 / 30 / 09$ & $11 / 9 / 09$ & 50,908 & 1,221 \\
\hline P1HLW FEED TANK & HLW-CONTRACTOR & $12 / 22 / 08$ & $11 / 25 / 09$ & 137,700 & 4,774 \\
\hline $\mathrm{U}-112$ & UA-2 & $11 / 9 / 09$ & $12 / 5 / 09$ & 58,521 & 2330 \\
\hline UA-2 & $S Y-102$ & $12 / 5 / 09$ & $12 / 6 / 09$ & 154,400 & 4,426 \\
\hline U-112 & UA-1 & $12 / 5 / 09$ & $1 / 17 / 10$ & 102,000 & 4,061 \\
\hline $\mathrm{U}-204$ & $\mathrm{UA-1}$ & $1 / 17 / 10$ & $1 / 23 / 10$ & 13,004 & 503 \\
\hline $\mathrm{U}-201$ & $\mathrm{UA}-1$ & $1 / 23 / 10$ & $1 / 27 / 10$ & 13,912 & 538 \\
\hline $\mathrm{UA-1}$ & SY-102 & $1 / 28 / 10$ & $1 / 28 / 10$ & 146,500 & 5,102 \\
\hline U-201 & UA-2 & $1 / 27 / 10$ & $1 / 30 / 10$ & 13,499 & 522 \\
\hline U-106 & UA-2 & $1 / 30 / 10$ & $3 / 1 / 10$ & 134,400 & 338 \\
\hline UA-2 & $S Y-102$ & $3 / 2 / 10$ & $3 / 3 / 10$ & 151,500 & 862 \\
\hline U-106 & $\mathrm{UA}-1$ & $3 / 1 / 10$ & $4 / 4 / 10$ & 149,500 & 376 \\
\hline $242-\mathrm{A}$ & LERF & $4 / 4 / 10$ & $4 / 9 / 10$ & 340,700 & 0 \\
\hline $242-\mathrm{A}$ & AW-106 & $4 / 4 / 10$ & $4 / 9 / 10$ & 605,100 & 32 \\
\hline $\mathrm{AW}-102$ & $242-A$ & $4 / 4 / 10$ & $4 / 9 / 10$ & 945,800 & 32 \\
\hline AW-106 & AW-102 & $4 / 9 / 10$ & $4 / 12 / 10$ & 605,000 & 21 \\
\hline $242-\mathrm{A}$ & LERF & $4 / 26 / 10$ & $4 / 29 / 10$ & 216,300 & 0 \\
\hline $242-\mathrm{A}$ & AW-106 & $4 / 26 / 10$ & $4 / 29 / 10$ & 388,800 & 21 \\
\hline$A W-102$ & 242-A & $4 / 26 / 10$ & $4 / 29 / 10$ & 605,200 & 21 \\
\hline AW-106 & $\overline{A W}-102$ & $4 / 29 / 10$ & $5 / 1 / 10$ & 388,700 & 14 \\
\hline U. 106 & $\mathrm{UA-2}$ & $4 / 4 / 10$ & $5 / 7 / 10$ & 149,500 & 376 \\
\hline $242-\mathrm{A}$ & LERF & $5 / 15 / 10$ & $5 / 17 / 10$ & 140,000 & 0 \\
\hline $242-\mathrm{A}$ & $\sqrt{A W}-106$ & $5 / 15 / 10$ & $5 / 17 / 10$ & 248,900 & 14 \\
\hline$A W-102$ & $242-A$ & $5 / 15 / 10$ & $5 / 17 / 10$ & 388,800 & 14 \\
\hline AW-106 & AW-102 & $5 / 17 / 10$ & $5 / 19 / 10$ & 248,800 & 9 \\
\hline $242-A$ & LERF & $6 / 2 / 10$ & $6 / 3 / 10$ & 90,000 & 0 \\
\hline $242-A$ & $A W-106$ & $6 / 2 / 10$ & $6 / 3 / 10$ & 158,900 & 9 \\
\hline AW-102 & $242-\mathrm{A}$ & $6 / 2 / 10$ & $6 / 3 / 10$ & 248,900 & 9 \\
\hline AW-106 & AW-102 & $6 / 3 / 10$ & $6 / 4 / 10$ & 158,800 & 6 \\
\hline AW-102 & AN-105 & $6 / 5 / 10$ & $6 / 6 / 10$ & 159,000 & 6 \\
\hline
\end{tabular}


HNF-2321

Revision 0

Table A.1-1. Complete Transfer List for Case 1.

\begin{tabular}{|c|c|c|c|c|c|}
\hline From & To & Start Date & End Date & $\begin{array}{c}\text { Liquid } \\
\text { (gallons) }\end{array}$ & $\begin{array}{c}\text { Solid } \\
\text { (gallons) }\end{array}$ \\
\hline $34 \mathrm{~L} 87$ & $A N-105$ & $10 / 1 / 10$ & $10 / 1 / 10$ & 50,000 & 0 \\
\hline SPN87 & SY -102 & $10 / 1 / 10$ & $10 / 1 / 10$ & 25,000 & 0 \\
\hline TAL88 & $A N-105$ & $10 / 1 / 10$ & $10 / 1 / 10$ & 26,000 & 0 \\
\hline TNS88 & AN-10S & $10 / 1 / 10$ & $10 / 1 / 10$ & 750 & 250 \\
\hline WATER & $A N-105$ & $10 / 1 / 10$ & $10 / 1 / 10$ & 25,000 & 0 \\
\hline WATER & AN-105 & $10 / 1 / 10$ & $10 / 1 / 10$ & 35,000 & 0 \\
\hline WESF & AN-105 & $10 / 1 / 10$ & $10 / 1 / 10$ & 5,000 & 0 \\
\hline EVAPF & AN-10S & $10 / 1 / 10$ & $10 / 2 / 10$ & 120,000 & 0 \\
\hline WATER & $A N-105$ & $10 / 2 / 10$ & $10 / 2 / 10$ & 5,000 & 0 \\
\hline WATER & $S Y-102$ & $10 / 2 / 10$ & $10 / 2 / 10$ & 5,000 & 0 \\
\hline WATER & AN-105 & $10 / 2 / 10$ & $10 / 2 / 10$ & 22,000 & 0 \\
\hline EVAPF & $A W-102$ & $10 / 4 / 10$ & $10 / 4 / 10$ & 50,000 & 0 \\
\hline EVAPF & $A W-102$ & $11 / 3 / 10$ & $11 / 3 / 10$ & 35,000 & 0 \\
\hline WATER & AN-105 & $1 / 1 / 11$ & $1 / 1 / 11$ & 25,000 & 0 \\
\hline BY-110. & $\mathrm{BA}-3$ & $1 / 3 / 11$ & $2 / 8 / 11$ & 147,400 & 2,633 \\
\hline TX-109 & TA-5 & $1 / 3 / 11$ & $2 / 10 / 11$ & 144,100 & 5,925 \\
\hline TY-101 & TA-4 & $1 / 3 / 11$ & $2 / 22 / 11$ & 146,500 & 3,494 \\
\hline $\mathrm{T}-106$ & TA-6 & $1 / 3 / 11$ & $3 / 2 / 11$ & 74,648 & 2,929 \\
\hline B-112 & $\mathrm{BA}-\mathrm{I}$ & $1 / 3 / 11$ & $3 / 9 / 11$ & 75,213 & 1,083 \\
\hline BY -110 & $\mathrm{BA}-4$ & $2 / 8 / 11$ & $3 / 16 / 11$ & 147,400 & 2,633 \\
\hline TX-109 & $\mathrm{TA}-3$ & $2 / 10 / 11$ & $3 / 19 / 11$ & 144,100 & 5,925 \\
\hline$\overline{\mathrm{T}}-101$ & TA-6 & $3 / 2 / 11$ & $3 / 25 / 11$ & 61,377 & 2,346 \\
\hline WATER & AN-105 & $4 / 1 / 11$ & $4 / 1 / 11$ & 25,000 & 0 \\
\hline $\mathrm{BX}-102$ & BA-2 & $1 / 3 / 11$ & $4 / 4 / 11$ & 52,548 & 2,443 \\
\hline $\mathrm{B}-111$ & $\mathrm{BA}-1$ & $3 / 9 / 11$ & $4 / 9 / 11$ & 72,630 & 2,383 \\
\hline TY-101 & $\mathrm{TA}-2$ & $2 / 22 / 11$ & $4 / 13 / 11$ & 146,500 & 3,494 \\
\hline $\mathrm{BY}-110$ & BA-5 & $3 / 16 / 11$ & $4 / 21 / 11$ & 147,400 & 2,633 \\
\hline TX-109. & TA-1 & $3 / 19 / 1$ & $4 / 26 / 11$ & 144,100 & 5,925 \\
\hline $\mathrm{BX}-112$ & BA-2 & $4 / 4 / 11$ & $5 / 2 / 11$ & 67,370 & 2,937 \\
\hline BA-3 & AP-104 & $6 / 1 / 11$ & $6 / 2 / 11$ & 155,600 & 2,631 \\
\hline $\mathrm{BA}-4$ & AP-108 & $6 / 1 / 11$ & $6 / 2 / 11$ & 155,600 & 2,631 \\
\hline $\mathrm{BA}-1$ & AP-104 & $6 / 2 / 11$ & $6 / 2 / 11$ & 157,300 & 3,464 \\
\hline $\mathrm{BA}-2$ & AP-104 & $6 / 2 / 11$ & $6 / 3 / 11$ & 174,500 & 5,377 \\
\hline BA-5 & AP-108 & $6 / 2 / 11$ & $6 / 3 / 11$ & 155,600 & 2,631 \\
\hline $\mathrm{AN}-102$ & AP-102 & $6 / 1 / 11$ & $6 / 4 / 11$ & 613,100 & 1,079 \\
\hline$A N-103$ & AP-106 & $6 / 1 / 11$ & $6 / 6 / 11$ & $1,099,000$ & 17,131 \\
\hline $\mathrm{AN}-103$ & AP-102 & $6 / 6 / 11$ & $6 / 6 / 11$ & 4,514 & 69 \\
\hline SY-103 & $A N-102$ & $6 / 4 / 11$ & $6 / 8 / 11$ & $1,081,000$ & 35,223 \\
\hline B-111 & BA-6 & $4 / 9 / 11$ & $6 / 11 / 11$ & 145,200 & 4,764 \\
\hline $\mathrm{AN}-102$ & AP-102 & $6 / 8 / 11$ & $6 / 11 / 11$ & 482,400 & 15,338 \\
\hline BA-6 & AP-108 & $6 / 11 / 11$ & $6 / 12 / 11$ & 159,400 & 4,762 \\
\hline AN-102 & AP-104 & $6 / 11 / 11$ & $6 / 14 / 11$ & 598,600 & 19,020 \\
\hline$A N-102$ & AP-108 & $6 / 14 / 11$ & $6 / 14 / 11$ & 2,579 & 81 \\
\hline SY-101 & $A N-102$ & $6 / 14 / 11$ & $6 / 18 / 11$ & $1,114,000$ & 2,136 \\
\hline$A N-102$ & AP-108 & $6 / 18 / 11$ & $6 / 22 / 11$ & 631,600 & 1,643 \\
\hline WATER & AN-105 & $7 / 1 / 11$ & $7 / 1 / 11$ & 25,000 & 0 \\
\hline$B Y-110$ & $\mathrm{BA}-4$ & $6 / 2 / 11$ & $7 / 8 / 11$ & 147,300 & 2,631 \\
\hline $\mathrm{BX}-112$ & BA-3 & $6 / 2 / 11$ & $8 / 2 / 11$ & 143,600 & 6,262 \\
\hline A-106 & AN-103 & $4 / 17 / 09$ & $8 / 8 / 11$ & 253,300 & 5,796 \\
\hline $\mathrm{B}-111$ & $\mathrm{BA}-1$ & $6 / 11 / 11$ & $8 / 12 / 11$ & 145,100 & 4,761 \\
\hline SX-111 & SY-103 & $11 / 25 / 08$ & $8 / 13 / 11$ & 451,500 & 19,767 \\
\hline BY-110 & BA-5 & $7 / 8 / 11$ & $8 / 13 / 11$ & 147,300 & 2,631 \\
\hline B-111 & BA-6 & $8 / 12 / 1$ & $8 / 13 / 11$ & 719 & 24 \\
\hline BX-112 & $\mathrm{BA}-2$ & $8 / 2 / 11$ & $9 / 4 / 11$ & 78,662 & 3,430 \\
\hline BY-110 & $\mathrm{BA}-6$ & $8 / 13 / 11$ & $9 / 18 / 11$ & 146,500 & 2,618 \\
\hline B-103 & $\mathrm{BA}-2$ & $9 / 4 / 11$ & $9 / 22 / 11$ & 41,507 & 78 \\
\hline
\end{tabular}


Table A.1-2. Phase 1 Low-Activity Waste Feed Staging Transfers for Case 1.

\begin{tabular}{|c|c|c|c|c|c|c|c|}
\hline $\begin{array}{c}\text { Boot } \\
\text { Strap }\end{array}$ & From & $T_{0}$ & Start Date & End Date & $\begin{array}{c}\text { Liquid } \\
\text { (gallons) }\end{array}$ & $\begin{array}{c}\text { Solid } \\
\text { (gallons) }\end{array}$ & Notes \\
\hline $\mathrm{X}$ & AP-102 & AP-103 & $10 / 25 / 99$ & $10 / 30 / 99$ & $1,070,000$ & 0 & Empty 2AP \\
\hline $\mathrm{X}$ & $\mathrm{AP}-104$ & AW-102 & $7 / 25 / 00$ & $7 / 30 / 00$ & $1,110,000$ & 43 & Empty 4AP \\
\hline \multirow[t]{2}{*}{$\mathrm{X}$} & $A Z-101$ & AY-101 & $8 / 17 / 00$ & $8 / 20 / 00$ & 685,000 & 0 & Pre-Stage Envelope B \\
\hline & WASH-WATER & AP-104 & $10 / 1 / 00$ & $10 / 2 / 00$ & 200,000 & 0 & Filush Water to 4AP \\
\hline $\mathrm{X}$ & AP-106 & AW-102 & $10 / 2 / 00$ & $10 / 4 / 00$ & 474,000 & 16 & Empty 6AP \\
\hline $\mathrm{X}$ & AP-106 & AW-102 & $10 / 5 / 00$ & $10 / 5 / 00$ & 10,000 & 0 & Empty 6AP \\
\hline$x$ & $\mathrm{AP}-106$ & AW-102 & $10 / 6 / 00$ & $10 / 6 / 00$ & 71,998 & 2 & Empty 6AP \\
\hline \multirow[t]{9}{*}{$\mathrm{x}$} & AP-106 & AW-102 & $10 / 7 / 00$ & $10 / 7 / 00$ & 180,300 & 6 & Empty 6AP \\
\hline & AP-104 & AP-102 & $10 / 15 / 00$ & $10 / 16 / 00$ & 200,000 & 1 & Flush 4AP \\
\hline & AP-102 & AP-106 & $2 / 15 / 01$ & $2 / 16 / 01$ & 200,500 & 1 & Flush 2AP \\
\hline & AN-105. & AP-102 & $3 / 17 / 01$ & $3 / 20 / 01$ & 542,400 & 7,895 & Retrieve/Stage LAW Batch 1, Contractor 1, Envelope A \\
\hline & LERF & AN-105-PUMP & $3 / 17 / 01$ & $3 / 20 / 01$ & 154,100 & 0 & Dilution Water \\
\hline & AN-105 & AP-102 & $3 / 17 / 0 \mathrm{I}$ & $3 / 20 / 01$ & 686,500 & 294 & As-received Ghost \\
\hline & AN-105 & AP-104 & $3 / 21 / 01$ & $3 / 24 / 01$ & 542,400 & 7,895 & Retrieve/Stage LAW Batch 1, Contractor 2, Envelope A \\
\hline & LERF & AN-105-PUMP & $3 / 21 / 01$ & $3 / 24 / 01$ & 154,100 & 0 & Dilution Water \\
\hline & AN-105 & A.P-104 & $3 / 21 / 01$ & $3 / 24 / 01$ & 686,500 & 294 & As-received Ghost \\
\hline \multirow[t]{11}{*}{$\mathrm{X}$} & AP-108 & AN-105 & $3 / 27 / 01$ & $3 / 28 / 01$ & 356,500 & 8 & Empty 8AP \\
\hline & AP-106 & AP-108 & $3 / 29 / 01$ & $3 / 30 / 01$ & 214,000 & 1,044 & Flush 6AP \\
\hline & AP-108 & AN-105 & $3 / 31 / 01$ & $4 / 1 / 01$ & 214,200 & 926 & Fush 8AP \\
\hline & AP-102 & AP-106 & $7 / 10 / 01$ & $7 / 14 / 01$ & 686,500 & 282 & Deliver LAW Batch 1, Contractor 1 \\
\hline & AP-104 & AP-108 & $7 / 14 / 01$ & $7 / 18 / 01$ & 686,500 & 282 & Deliver LAW Batch 1, Contractor 2 \\
\hline & AN-104 & AP-102 & $10 / 1 / 01$ & $10 / 4 / 01$ & 502,200 & 12,513 & Retrieve/Stage LAW Batch 2, Contractor 1, Envelope A \\
\hline & LERF & AN-104-PUMP & $10 / 1 / 01$ & $10 / 4 / 01$ & 200,800 & 0 & Dilution Water \\
\hline & AN-104 & AP-102 & $10 / 1 / 01$ & $10 / 4 / 01$ & 684,700 & 702 & As-received Ghost \\
\hline & AN-104 & AP-104 & $10 / 5 / 01$ & $10 / 8 / 01$ & 502,200 & 12,513 & Retrieve/Stage LAW Batch 2, Contractor 2, Envelope A \\
\hline & LERF & AN-104-PUMP & $10 / 5 / 01$ & $10 / 8 / 01$ & 200,800 & 0 & Dilution Water \\
\hline & AN-104 & AP-104 & $10 / 5 / 01$ & $10 / 8 / 01$ & 684,700 & 702 & As-received Ghost \\
\hline \multirow[t]{29}{*}{$\mathrm{X}$} & $A z-102$ & AY-10I & $11 / 14 / 01$ & $11 / 15 / 01$ & 204,000 & 0 & Pre-Stage Envelope B \\
\hline & RETURN_1 & AP-107 & $12 / 5 / 02$ & $12 / 5 / 02$ & 8,702 & 299 & Returns From Contractor 1 \\
\hline & RETURN_2 & AP- 107 & $1 / 3 / 03$ & $1 / 3 / 03$ & 8,671 & 331 & Returns From Contractor 2 \\
\hline & $\mathrm{AP}-102$ & AP-106 & $1 / 5 / 03$ & $1 / 9 / 03$ & 684,700 & 686 & Deliver LAW Batch 2, Contractor 1 \\
\hline & AP-104 & AP-108 & $1 / 9 / 03$ & $1 / 12 / 03$ & 684,700 & 686 & Deliver LAW Batch 2, Contractor 2 \\
\hline & AW-101 & AP-102 & $1 / 10 / 03$ & $1 / 14 / 03$ & 537,200 & 13,013 & Retrieve/Stage LAW Batch 3, Contractor 1, Envelope A \\
\hline & LERF & AW-101-PUMP & $1 / 10 / 03$ & $1 / 14 / 03$ & 308,100 & 0 & Dilution Water \\
\hline & AW-101 & AP-102 & $1 / 10 / 03$ & $1 / 14 / 03$ & 845,400 & 13,013 & As-received Ghost \\
\hline & AW-101 & AP-104 & $1 / 15 / 03$ & $1 / 19 / 03$ & 537,200 & 13,013 & Retrieve/Stage LAW Batch 3, Contractor 2, Envelope A \\
\hline & LERF & AW-101-PUMP & $1 / 15 / 03$ & $1 / 19 / 03$ & 308,100 & 0 & Dilution Water \\
\hline & AW-101 & AP-104 & $1 / 15 / 03$ & $1 / 19 / 03$ & 845,400 & 13,013 & As-received Ghost \\
\hline & RETURN_1 & AP-107 & $6 / 28 / 03$ & $6 / 28 / 03$ & 8,677 & 326 & Returns From Contractor 1 \\
\hline & RETURN_2 & AP-107 & $8 / 7 / 03$ & $8 / 7 / 03$ & 8,671 & 332 & Returns From Contractor 2 \\
\hline & $\mathrm{AP}-102$ & AP-106 & $9 / 29 / 03$ & $10 / 3 / 03$ & 740,300 & 1,581 & Deliver LAW Batch 3, Contractor 1 \\
\hline & AP-104 & AP-I08 & $10 / 3 / 03$ & $10 / 7 / 03$ & 740,300 & 1,581 & Deliver LAW Batch 3, Contractor 2 \\
\hline & AN-103 & AP-102 & $10 / 4 / 03$ & $10 / 7 / 03$ & 436,600 & 28,133 & Retrieve/Stage LAW Batch 4, Contractor 1, Envelope A \\
\hline & LERF & AN-103-PUMP & $10 / 4 / 03$ & $10 / 7 / 03$ & 264,900 & 0 & Dilution Water \\
\hline & AN-103 & AP-102 & $10 / 4 / 03$ & $10 / 7 / 03$ & 738,300 & 549 & As-received Ghost \\
\hline & AN-103 & AP-104 & $10 / 8 / 03$ & $10 / 11 / 03$ & 436,600 & 28,133 & Retrieve/Stage LAW Batch 4, Contractor 2, Envelope A \\
\hline & LERF & AN-103-PUMP & $10 / 8 / 03$ & $10 / 11 / 03$ & 264,900 & 0 & Dilution Water \\
\hline & AN-103 & AP-104 & $10 / 8 / 03$ & $10 / 11 / 03$ & 738,300 & 549 & As-received Ghost \\
\hline & RETURN_1 & AP-107 & $12 / 14 / 03$ & $12 / 14 / 03$ & 8,670 & 331 & Returns From Contractor 1 \\
\hline & RETURN_2 & AP-107 & $1 / 11 / 04$ & $1 / 11 / 04$ & 8,672 & 330 & Returns From Contractor 2 \\
\hline & RETURN_I & AP-107 & $4 / 28 / 04$ & $4 / 28 / 04$ & 8,672 & 330 & Returns From Contractor 1 \\
\hline & AP-102 & AP-106 & $5 / 4 / 04$ & $5 / 7 / 04$ & 753,200 & 1,687 & Deliver LAW Batch 4, Contractor 1 \\
\hline & WASH-WATER & AP-102 & $5 / 8 / 04$ & $5 / 8 / 04$ & 118,100 & 0 & Flush Water to $2 \mathrm{AP}$ \\
\hline & AP-104 & AP-108 & $5 / 7 / 04$ & $5 / 11 / 04$ & 753,200 & 1,687 & Deliver LAW Batch 4, Contractor 2 \\
\hline & WASH-WATER & $\mathrm{AP}-104$ & $5 / 12 / 04$ & $5 / 12 / 04$ & 118,100 & 0 & Flush Water to 4AP \\
\hline & RETURN_2 & AP-107 & $5 / 14 / 04$ & $5 / 14 / 04$ & 8,676 & 330 & Returns From Contractor 2 \\
\hline
\end{tabular}


HNF-2321

Revision 0

Table A.1-2. Phase 1 Low-Activity Waste Feed Staging Transfers for Case 1.

\begin{tabular}{|c|c|c|c|c|c|c|c|}
\hline $\begin{array}{l}\text { Boot } \\
\text { Strap }\end{array}$ & From & To & Start Date & End Date & $\begin{array}{c}\text { Liquid } \\
\text { (gallons) }\end{array}$ & $\begin{array}{c}\text { Solid } \\
\text { (gallons) }\end{array}$ & Notes \\
\hline & AP-102 & AN-105 & $5 / 27 / 04$ & $6 / 11 / 04$ & 209,500 & 9,168 & Flush 2AP \\
\hline & AP-104 & AN-105 & $6 / 12 / 04$ & $6 / 13 / 04$ & 209,500 & 9,168 & Flush 4AP \\
\hline & WASH-CAUSTIC & $A P=102$ & $6 / 13 / 04$ & $6 / 13 / 04$ & 103,400 & 0 & Shim Caustic for Batch 5, Contractor 1 \\
\hline & WASH-CAUSTIC & AP-104 & $6 / 14 / 04$ & $6 / 14 / 04$ & 126,400 & 0 & Shim Caustic for Batch 5, Contractor 2 \\
\hline & AP-101 & AP-102 & $6 / 15 / 04$ & $6 / 17 / 04$ & 502,400 & 23 & Retrieve/Stage LAW Batch 5, Contractor 1, Envelope A \\
\hline & LERF & AP-101-PUMP & $6 / 15 / 04$ & $6 / 17 / 04$ & 55,267 & 0 & Dilution Water \\
\hline & AP-101 & AP -102 & $6 / 15 / 04$ & $6 / 17 / 04$ & 557,700 & 23 & As-received Ghost \\
\hline & AW-104 & AP-102 & $6 / 19 / 04$ & $6 / 19 / 04$ & 109,000 & 5 & $\begin{array}{l}\text { Complete Retrieve/Stage LAW Batch 5, Contractor 1, } \\
\text { Envelope A }\end{array}$ \\
\hline & AP-101 & AP-104 & $6 / 18 / 04$ & $6 / 21 / 04$ & 614,000 & 28 & Retrieve/Stage LAW Batch 5, Contractor 2, Envelope A \\
\hline & LERF & AP-101-PUMP & $6 / 18 / 04$ & $6 / 21 / 04$ & 67,548 & 0 & Dilution Water \\
\hline & AP-101 & AP-104 & $6 / 18 / 04$ & $6 / 21 / 04$ & 681,600 & 28 & As-received Ghost \\
\hline & RETURN_1 & AP-107 & $6 / 21 / 04$ & $6 / 21 / 04$ & 8,676 & 331 & Returns From Contractor 1 \\
\hline & AW-104 & AP-104 & $6 / 22 / 04$ & $6 / 22 / 04$ & 133,200 & 6 & $\begin{array}{l}\text { Complete Retrieve/Stage LAW Batch 5, Contractor 2, } \\
\text { Envelope A }\end{array}$ \\
\hline & RETURN_2 & AP-107 & $7 / 3 / 04$ & $7 / 3 / 04$ & 8,674 & 331 & Returns From Contractor 2 \\
\hline & RETURN_1 & AP-107 & $8 / 10 / 04$ & $8 / 10 / 04$ & 8,675 & 331 & Returns From Contractor 1 \\
\hline & RETURN_2 & AP-107 & $8 / 22 / 04$ & $8 / 22 / 04$ & 8,674 & 331 & Returns From Contractor 2 \\
\hline & RETURN_1 & AP-107 & $9 / 30 / 04$ & $9 / 30 / 04$ & 8,675 & 331 & Returns From Contractor 1 \\
\hline & RETURN_2 & AP-107 & $10 / 11 / 04$ & $10 / 11 / 04$ & 8,674 & 331 & Returns From Contractor 2 \\
\hline & RETURN_1 & AP-107 & $\pm 1 / 19 / 04$ & $11 / 19 / 04$ & 8,675 & 331 & Returns From Contractor 1 \\
\hline & RETURN_2 & AP-107 & $11 / 30 / 04$ & $11 / 30 / 04$ & 8,674 & 331 & Reurns From Contractor 2 \\
\hline & RETURN_1 & AP-107 & $1 / 8 / 05$ & $1 / 9 / 05$ & 8,675 & $331^{\circ}$ & Returns From Contractor 1 \\
\hline & RETURN_2 & AP-107 & $1 / 19 / 05$ & $1 / 19 / 05$ & 8,674 & 331 & Returns From Contractox 2 \\
\hline & AP-102 & AP-106 & $2 / 18 / 05$ & $2 / 22 / 05$ & 769,000 & 1,140 & Deliver LAW Batch 5, Contractor 1 \\
\hline & $A Y-101$ & AP-102 & $2 / 23 / 05$ & $2 / 24 / 05$ & 283,600 & 12 & Stage LAW Batch 6, Contractor 1, Envelope B \\
\hline & AP-104 & AP-108 & $2 / 22 / 05$ & $2 / 26 / 05$ & 940,100 & 1,153 & Deliver LAW Batch 5, Contractor 2 \\
\hline & $A Y-101$ & $\mathrm{AP}-104$ & $2 / 27 / 05$ & $2 / 28 / 05$ & 279,700 & 11 & Stage LAW Batch 6, Contractor 2, Envelope B \\
\hline & RETURN_1 & AP-107 & $3 / 1 / 05$ & $3 / 1 / 05$ & 8,679 & 331 & Returns From Contractor 1 \\
\hline & RETURN_2 & AP-107 & $3 / 13 / 05$ & $3 / 13 / 05$ & 8,676 & 331 & Returns From Contractor 2 \\
\hline & RETURN_1 & AP-107 & $4 / 27 / 05$ & $4 / 27 / 05$ & 8,670 & 330 & Returns From Contractor 1 \\
\hline & RETURN_2 & AP-107 & $5 / 9 / 05$ & $5 / 9 / 05$ & 8,679 & 331 & Returns From Contractor 2 \\
\hline & RETURN_1 & AP-107. & $6 / 23 / 05$ & $6 / 23 / 05$ & 8,670 & 330 & Returns From Contractor 1 \\
\hline & RETURN_2 & AP-107 & $7 / 5 / 05$ & $7 / 5 / 05$ & 8,679 & 331 & Returns From Contractor 2 \\
\hline & RETURN_1 & AP-107 & $8 / 19 / 05$ & $8 / 19 / 05$ & 8,670 & 330 & Returns From Contractor I \\
\hline & RETURN_2 & AP-107 & $8 / 31 / 05$ & $8 / 3 \mathrm{~L} / 05$ & 8,679 & 331 & Returns From Contractor 2 \\
\hline & RETURN_1 & AP-107 & $10 / 15 / 05$ & $10 / 15 / 05$ & 8,670 & 330 & Returns From Contractor 1 \\
\hline & RETURN_2 & $\mathrm{AP}-107$ & $10 / 27 / 05$ & $10 / 27 / 05$ & 8,679 & 331 & Reums From Contractor 2 \\
\hline $\mathrm{X}$ & $S Y-102$ & AW-105 & $11 / 15 / 05$ & $11 / 17 / 05$ & 573,800 & 22 & Decant Supernate from SY-102 \\
\hline & RETURN_1 & AP-107 & $12 / 11 / 05$ & $12 / 11 / 05$ & 8,670 & 330 & Returns From Contractor 1 \\
\hline $\mathrm{X}$ & WATER & SY-102 & $12 / 15 / 05$ & $12 / 17 / 05$ & 426,000 & 0 & Flush Water for $2 \mathrm{SY}$ Solids Cleanout \\
\hline & RETURN_2 & AP-107 & $12 / 23 / 05$ & $12 / 23 / 05$ & 8,679 & 331 & Returns From Contractor 2 \\
\hline & AP-106 & LAW-CONTRACTOR-1 & $6 / 1 / 02$ & $12 / 31 / 05$ & $3,634,000$ & 5,468 & Complete Processing Envelope A, Contractor 1 \\
\hline & AP-102 & AP-106 & $12 / 31 / 05$ & $1 / 2 / 06$ & 283,500 & 48 & Deliver LAW Batch 6, Contractor 1 \\
\hline $\mathrm{X}$ & SY-102 & AW-105 & $1 / 1 / 06$ & $1 / 3 / 06$ & 535,600 & 22,049 & Cleanout Solids 2SY \\
\hline & AN-107 & AP-102 & $1 / 2 / 06$ & $1 / 4 / 06$ & 481,700 & 22 & Retrieve/Stage LAW Batch 7/8, Contractor 1, Envelope \\
\hline & LERF & AN-107-PUMP & $1 / 2 / 06$ & $1 / 4 / 06$ & 72,259 & 0 & Dilution Water \\
\hline & AN-107 & AP-102 & $1 / 2 / 06$ & $1 / 4 / 06$ & 554,000 & 22 & As-received Ghost \\
\hline & RETURN_2 & $\mathrm{AP}-107$ & $2 / 18 / 06$ & $2 / 18 / 06$ & 8,679 & 331 & Returns From Contractor 2 \\
\hline & AP-108 & LAW-CONTRACTOR-2 & $6 / 1 / 02$ & $3 / 6 / 06$ & $3,805,000$ & 5,473 & Complete Processing Envelope A, Contractor 2 \\
\hline & AP-104 & AP-108 & $3 / 6 / 06$ & $3 / 8 / 06$ & 279,600 & 41 & Deliver LAW Batch 6 , Contractor 2 \\
\hline & AP-106 & LAW-CONTRACTOR-1 & $1 / 2 / 06$ & $3 / 11 / 06$ & 283,500 & 83 & Complete Processing Envelope B, Contractor 1 \\
\hline & AN-107 & AP-104 & $3 / 9 / 06$ & $3 / 11 / 06$ & 481,700 & 22 & Retrieve/Stage LAW Batch 7/8, Contractor 2, Envelope \\
\hline & LERP & AN-107-PUMP & $3 / 9 / 06$ & $3 / 11 / 06$ & 72,260 & 0 & Dilution Water \\
\hline & AN-107 & AP-104 & $3 / 9 / 06$ & $3 / 11 / 06$ & 554,000 & 22 & As-received Ghost \\
\hline & AP-102 & AP-106 & $3 / 11 / 06$ & $3 / 12 / 06$ & 169,400 & 8 & Deliver LAW Batch 7, Contractor 1 \\
\hline
\end{tabular}


HNF-2321

Revision 0

Table A.1-2. Phàse 1 Low-Activity Waste Feed Staging Transfers for Case 1.

\begin{tabular}{|c|c|c|c|c|c|c|c|}
\hline $\begin{array}{l}\text { Boot } \\
\text { Strap }\end{array}$ & From & To & Start Date & End Date & $\begin{array}{c}\text { Liquid } \\
\text { (gallons) }\end{array}$ & $\begin{array}{c}\text { Solid } \\
\text { (gallons) }\end{array}$ & Notes \\
\hline & AP-102 & AP-106 & $4 / 8 / 06$ & $4 / 10 / 06$ & 384,600 & 18 & Deliver LAW Batch 8 , Contractor 1 \\
\hline & AN-102 & AP-102 & $4 / 11 / 06$ & $4 / 14 / 06$ & 481,700 & 23 & Retrieve/Stage LAW Batch 9, Contractor 1, Envelope C \\
\hline & LERF & AN-102-PUMP & $4 / 11 / 06$ & $4 / 14 / 06$ & 289,000 & 0 & Dilution Water \\
\hline & $\mathrm{AN}-102$ & AP-102 & $4 / 11 / 06$ & $4 / 14 / 06$ & 770,700 & 23 & As-received Ghost \\
\hline & AP-108 & LAW-CONTRACTOR-2 & $3 / 8 / 06$ & $5 / 14 / 06$ & 279,600 & 70 & Complete Processing Envelope B, Contractor 2 \\
\hline & AP-104 & AP-108 & $5 / 14 / 06$ & $5 / 15 / 06$ & 169,400 & 8 & Deliver LAW Batch 7, Contractor 2. \\
\hline & AP-104 & AP-108 & $6 / 11 / 06$ & $6 / 13 / 06$ & 384,600 & 17 & Deliver LAW Batch 8 , Contractor 2 \\
\hline & AN-102 & AP-104 & $6 / 14 / 06$ & $6 / 17 / 06$ & 481,700 & 23 & Retrieve/Stage LAW Batch 9, Contractor 2, Envelope C \\
\hline & LERF & AN-102-PUMP & $6 / 14 / 06$ & $6 / 17 / 06$ & 289,000 & 0 & Dilution Water \\
\hline & AN-102 & AP-104 & $6 / 14 / 06$ & $6 / 17 / 06$ & 770,700 & 23 & As-received Ghost \\
\hline & AP-102 & AP-106 & $8 / 21 / 06$ & $8 / 25 / 06$ & 770,700 & 23 & Deliver LAW Batch 9, Contractor I \\
\hline & AN-106 & AP-102 & $8 / 26 / 06$ & $8 / 29 / 06$ & 547,800 & 24 & Retrieve/Stage LAW Batch 10, Contractor 1, Envelope C \\
\hline & LERF & AN-106-PUMP & $8 / 26 / 06$ & $8 / 29 / 06$ & 98,603 & 0 & Dilution Water \\
\hline & AN-106 & AP-102 & $8 / 26 / 06$ & $8 / 29 / 06$ & 646,400 & 24 & As-received Ghost \\
\hline & $\mathrm{AP}-104$ & AP-108 & $10 / 24 / 06$ & $10 / 28 / 06$ & 770,700 & 23 & Deliver LAW Batch 9, Contractor 2 \\
\hline & AN-106 & AP-104 & $12 / 18 / 06$ & $12 / 22 / 06$ & 547,800 & 24 & Retrieve/Stage LAW Batch 10, Contractor 2, Envelope C \\
\hline & LERF & AN-106-PUMP & $12 / 18 / 06$ & $12 / 22 / 06$ & 98,603 & 0 & Dilution Water \\
\hline & AN-106 & AP-104 & $12 / 18 / 06$ & $12 / 22 / 06$ & 646,400 & 24 & As-received Ghost \\
\hline & SY-101 & $5 Y-102$ & $12 / 19 / 06$ & $12 / 24 / 06$ & 537,800 & 5,486 & Retrieve LAW Batch 11, Envelope C \\
\hline & LERF & SY-101-PUMP & $12 / 19 / 06$ & $12 / 24 / 06$ & 532,400 & 0 & Dilution Water \\
\hline & $S Y-101$ & $S Y-102$ & $12 / 19 / 06$ & $12 / 24 / 06$ & $1,082,000$ & 611 & As-received Ghost \\
\hline & SY-102 & AN-102 & $1 / 1 / 07$ & $1 / 4 / 07$ & $1,027,000$ & 1,532 & Pre-Stage LAW Batch 11, Envelope C \\
\hline & SY-101 & SY-102 & $1 / 12 / 07$ & $1 / 17 / 07$ & 537,800 & 5,486 & Retrieve LAW Batch II, Envelope C \\
\hline & LERF & SY-101-PUMP & $1 / 12 / 07$ & $1 / 17 / 07$ & 532,400 & 0 & Dilution Water \\
\hline & SY-101 & SY-102 & $1 / 12 / 07$ & $1 / 17 / 07$ & $1,082,000$ & 611 & As-received Ghost \\
\hline & SY-102 & AN-107 & $1 / 25 / 07$ & $1 / 28 / 07$ & 983,800 & 641 & Pre-Stage LAW Batch 11, Envelope C \\
\hline & RETURN_1 & AP-107 & $3 / 9 / 07$ & $3 / 9 / 07$ & 8,732 & 269 & Returns From Contractor 1 \\
\hline & AP-102 & AP-106 & $4 / 18 / 07$ & $4 / 21 / 07$ & 646,400 & 24 & Deliver LAW Batch 10, Contractor 1 \\
\hline & AN-102 & AP-102 & $4 / 22 / 07$ & $4 / 27 / 07$ & $1,013,000$ & 44 & Stage LAW Batch 11, Contractor 1, Envelope C \\
\hline & SY-103 & SY-102 & $4 / 30 / 07$ & $5 / 2 / 07$ & 350,000 & 9,759 & Retrieve LAW Batch 12, Envelope C \\
\hline & LERF & SY-103-PUMP & $4 / 30 / 07$ & $5 / 2 / 07$ & 183,500 & 0 & Dilution Water \\
\hline & SY-103 & $S Y-102$ & $4 / 30 / 07$ & $5 / 2 / 07$ & 537,500 & 1,449 & As-received Chost \\
\hline & $S Y-102$ & $\mathrm{AN}-102$ & $5 / 10 / 07$ & $5 / 12 / 07$ & 720,700 & 1,528 & Pre-Stage LAW Batch 12, Envelope C \\
\hline & AP-104 & AP-108 & $6 / 21 / 07$ & $6 / 24 / 07$ & 646,400 & 24 & Deliver LAW Batch 10 , Contractor 2 \\
\hline & AN-107 & $\mathrm{AP}=104$ & $6 / 25 / 07$ & $6 / 29 / 07$ & 978,400 & 43 & Stage LAW Batch 11, Contractor 2, Envelope C \\
\hline & SY-103 & SY-102 & $7 / 3 / 07$ & $7 / 5 / 07$ & 350,000 & 9,759 & Retrieve LAW Batch 12, Envelope C \\
\hline & LERF & SY-103-PUMP & $7 / 3 / 07$ & $7 / 5 / 07$ & 183,500 & 0 & Dilution Water \\
\hline & $S Y-103$ & $S Y-102$ & $7 / 3 / 07$ & $7 / 5 / 07$ & 537,500 & 1,449 & As-received Ghost \\
\hline & SY-102 & AN-107 & $7 / 13 / 07$ & $7 / 14 / 07$ & 537,500 & $\square, 434$ & Pre-Stage LAW Batch 12, Envelope C \\
\hline & AP-102 & AP-106 & $11 / 9 / 07$ & $11 / 14 / 07$ & $1,013,000$ & 44 & Deliver LAW Batch 11 , Contractor 1 \\
\hline & AN-102 & AP- 102 & $11 / 15 / 07$ & $11 / 18 / 07$ & 707,100 & 31 & Stage LAW Batch 12, Contractor 1, Envelope C \\
\hline & AP-104 & AP-108 & $1 / 12 / 08$ & $1 / 17 / 08$ & 978,400 & 43 & Deliver LAW Batch 11, Contractor 2 \\
\hline & AN-107 & AP-104 & $1 / 18 / 08$ & $1 / 20 / 08$ & 524,700 & 23 & Stage LAW Batch 12, Contractor 2, Envelope C \\
\hline & AP-102 & AP-106 & $9 / 12 / 08$ & $9 / 16 / 08$ & 707,100 & 31 & Deliver LAW Batch 12 , Contractor I \\
\hline & AP-104 & AP-108 & $11 / 15 / 08$ & $11 / 18 / 08$ & 524,700 & 23 & Deliver LAW Batch 12 , Contractor 2 \\
\hline & AP-106 & LAW-CONTRACTOR-1 & $3 / 12 / 06$ & $5 / 25 / 09$ & $3,692,000$ & 155 & Complete Processing Envelope $\mathrm{C}_{3}$ Contractor 1 \\
\hline & RETURN_L & AP-107 & $5 / 26 / 09$ & $5 / 26 / 09$ & 2,727 & 98 & Returns From Contractor 1 \\
\hline & AP-108 & LAW-CONTRACTOR-2 & $5 / 15 / 06$ & $6 / 1 / 09$ & $3,474,000$ & 144 & Complete Processing Envelope C, Contractor 2 \\
\hline & RETURN_2 & AP-107 & $6 / 2 / 09$ & $6 / 2 / 09$ & 8,391 & 319 & Returns From Contractor 2 \\
\hline $\mathrm{X}$ & AP-107 & $A Z-101$ & $6 / 12 / 09$ & $6 / 13 / 09$ & 289,700 & 9,938 & Transfer Returns to AZ-101 (Phase 2 HLW Tank) \\
\hline
\end{tabular}


HNF-2321

Revision 0

Table A.1-3. Phase 1 High-Level Waste Feed Staging Transfers for Case 1.

\begin{tabular}{|c|c|c|c|c|c|c|c|}
\hline $\begin{array}{l}\text { Boot } \\
\text { Strap }\end{array}$ & From & To & Start Date & End Date & $\begin{array}{c}\text { Liquid } \\
\text { (gallons) }\end{array}$ & $\begin{array}{c}\text { Solid } \\
\text { (gallons) }\end{array}$ & Notes \\
\hline & $A Y-102$ & C-106 & $7 / 1 / 98$ & $7 / 5 / 98$ & 920,700 & 31 & $\begin{array}{l}\text { Sluice "Water" for C-106 Retrieval - Lumped } \\
\text { (see Below) }\end{array}$ \\
\hline & C-106 & AY-102 & $7 / 15 / 98$ & $11 / 10 / 98$ & 809,000 & 15,169 & C-106 Retrieval (Project W-320) \\
\hline \multirow[t]{8}{*}{$\mathrm{x}$} & $A Z-101$ & AY-101 & $8 / 17 / 00$ & $8 / 20 / 00$ & 685,000 & 0 & Initial Decant $1 \mathrm{AZ}$ \\
\hline & WASH-WATER & $\mathrm{AZ}-101$ & $8 / 20 / 00$ & $8 / 21 / 00$ & 146,000 & 0 & First Wash $1 \mathrm{AZ}$ \\
\hline & AZ-101 & AP-107 & $10 / 9 / 00$ & $10 / 10 / 00$ & 143,200 & 0 & First Decant 1AZ \\
\hline & WASH-WATER & AZ-101 & $10 / 11 / 00$ & $10 / 12 / 00$ & 146,000 & 0 & Second Wash $1 \mathrm{AZ}$ \\
\hline & $\mathrm{AZ-101}$ & AP-107 & $11 / 30 / 00$ & $12 / 1 / 00$ & 140,200 & 0 & Second Decant $1 \mathrm{AZ}$ \\
\hline & WASH-WATER & AZ-101 & $12 / 2 / 00$ & $12 / 2 / 00$ & 146,000 & $\mathbf{0}$ & Third Wash $1 \mathrm{AZ}$ \\
\hline & $\mathrm{AZ}-101$ & AP-107 & $1 / 21 / 01$ & $1 / 21 / 01$ & 142,900 & 0 & Third Decant 1AZ \\
\hline & WASH-WATER & AZ-101 & $1 / 22 / 01$ & $1 / 23 / 01$ & 138,200 & $\mathbf{0}$ & Transfer Water Add 1AZ \\
\hline \multirow[t]{14}{*}{$\mathrm{x}$} & $\mathrm{AZ}-102$ & AY-101 & $11 / 14 / 01$ & $11 / 15 / 01$ & 204,000 & $\mathbf{0}$ & Initial Decant $2 \mathrm{AZ}$ \\
\hline & $\overline{\mathrm{AZ}-102}$ & AP-107 & $11 / 16 / 01$ & $11 / 16 / 01$ & 181,800 & 0 & Complete Initial Decant 2AZ \\
\hline & WASH-WATER & AZ-102 & $11 / 17 / 01$ & $11 / 18 / 01$ & 213,000 & 0 & First Wash $2 \mathrm{AZ}$ \\
\hline & $\mathrm{AZ}-102$ & AN-105 & $1 / 6 / 02$ & $1 / 6 / 02$ & 127,700 & 0 & First Decant 2AZ \\
\hline & WASH-WATER & $\mathrm{AZ}-102$ & $1 / 8 / 02$ & $1 / 9 / 02$ & 213,000 & 0 & Second Wash $2 \mathrm{AZ}$ \\
\hline & AZ-102 & AN-105 & $2 / 27 / 02$ & $2 / 28 / 02$ & 210,300 & 0 & Second Decant $2 \mathrm{AZ}$ \\
\hline & WASH-WATER & AZ-102 & $3 / 1 / 02$ & $3 / 2 / 02$ & 213,000 & 0 & Third Wash 2AZ \\
\hline & $\mathrm{AZ}-102$ & AN-105 & $4 / 20 / 02$ & $4 / 21 / 02$ & 211,800 & 0 & Third Decant 2AZ \\
\hline & WASH-WATER & AZ-102 & $4 / 22 / 02$ & $4 / 23 / 02$ & 213,000 & 0 & Fourth Wash $2 \mathrm{AZ}$ \\
\hline & AZ-101 & P1HLW_FEED_TANK & $5 / 17 / 02$ & $5 / 17 / 02$ & 134,300 & 5,079 & 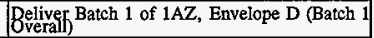 \\
\hline & $A Z-102$ & AN-105 & $6 / 12 / 02$ & $6 / 13 / 02$ & 212,500 & 0 & Fourth Decant $2 \mathrm{AZ}$ \\
\hline & WASH-WATER & AZ-102 & $6 / 14 / 02$ & $6 / 14 / 02$ & 110,000 & 0 & Transfer Water Add $2 \mathrm{AZ}$ \\
\hline & P1HLW_FEED_TANK & HLW-CONTRACTOR & $6 / 1 / 02$ & $2 / 21 / 03$ & 134,300 & 5,079 & 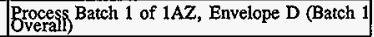 \\
\hline & AZ-101 & P1HLW_FEED_TANK & $2 / 21 / 03$ & $2 / 21 / 03$ & 134,300 & 5,079 & Beliveraf) Batch 2 of $1 \mathrm{AZ}$, Envelope D (Batch 2 \\
\hline \multirow[t]{24}{*}{$\mathrm{x}$} & AY-102 & AZ-101 & $2 / 24 / 03$ & $3 / 1 / 03$ & 819,600 & 16,032 & $\begin{array}{l}\text { Transfer } 2 \mathrm{AY} / 6 \mathrm{C} \\
\text { Pretreatment }\end{array}$ \\
\hline & AZ-101 & AN-105 & $10 / 28 / 03$ & $10 / 31 / 03$ & 622,700 & 0 & Initial Decant 2AY/6C Slurry from $1 \mathrm{AZ}$ \\
\hline & WASH-WATER & AZ-101 & $10 / 31 / 03$ & $11 / 2 / 03$ & 324,000 & 0 & First Wash $2 \mathrm{AY} / 6 \mathrm{C}$ Slurry in $1 \mathrm{AZ}$ \\
\hline & P1HLW_FEED_TANK & HLW-CONTRACTOR & $2 / 22 / 03$ & $11 / 14 / 03$ & 134,300 & 5,079 & $\begin{array}{l}\text { Process } \\
\text { Ovexall) }\end{array}$ Batch 2 of $1 \mathrm{AZ}$, Envelope D (Batch 2 \\
\hline & AZ-102 & P1HLW_FEED_TANK & $11 / 14 / 03$ & $11 / 14 / 03$ & 123,900 & 5,202 & 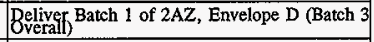 \\
\hline & AZ-101 & AN-105 & $12 / 21 / 03$ & $12 / 22 / 03$ & 320,000 & 0 & First Decant 2AY/6C Slurry from 1AZ \\
\hline & WASH-WATER & AZ-101 & $12 / 24 / 03$ & $12 / 25 / 03$ & 324,000 & 0 & Second Wash $2 \mathrm{AY} / 6 \mathrm{C}$ Slurry in $1 \mathrm{AZ}$ \\
\hline & AZ-101 & AN-105 & $6 / 13 / 04$ & $6 / 15 / 04$ & 322,300 & 0 & Second Decant $2 \mathrm{AY} / 6 \mathrm{C}$ Slurry from $1 \mathrm{AZ}$ \\
\hline & WASH-WATER & AZ-101 & $6 / 16 / 04$ & $6 / 18 / 04$ & 433,600 & 0 & Transfer Water Add 1AZ for 2AY/6C Slurry \\
\hline & P1HLW_FEED_TANK & HLW-CONTRACTOR & $11 / 14 / 03$ & $8 / 7 / 04$ & 123,900 & 5,202 & $\begin{array}{l}\text { Process } \\
\text { Overal) }\end{array}$ Batch 1 of 2AZ, Envelope D (Batch 3 \\
\hline & AZ-102 & P1HLW_FEED_TANK & $8 / 7 / 04$ & $8 / 8 / 04$ & 123,900 & 5,202 & Beliverfl Batch 2 of $2 \mathrm{AZ}$, Envelope D (Batch 4 \\
\hline & C-104 & $\mathrm{AY}-102$ & $8 / 1 / 04$ & 9/8/04 & 279,100 & 9,724 & C-104 Retrieval (Project W-???) \\
\hline & PIHLW_FEED_TANK & HLW-CONTRACTOR & $8 / 8 / 04$ & $5 / 2 / 05$ & 123,900 & 5,202 & $\begin{array}{l}\text { Brocess Batch } 2 \text { of } 2 A Z \text {, Envelope D (Batch } 4 \\
\text { Overall) }\end{array}$ \\
\hline & AZ-101 & P1HLW_FEED_TANK & $5 / 2 / 05$ & $5 / 2 / 05$ & 127,200 & 3,269 & 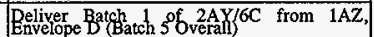 \\
\hline & AY -102 & AZ-102 & $8 / 1 / 05$ & $8 / 3 / 05$ & 279,400 & 9,360 & Transfer $4 \mathrm{C}$ Slurry to $2 \mathrm{AZ}$ for Pretreatment \\
\hline & P1HLW_FEED_TANK & HLW-CONTRACTOR & $5 / 2 / 05$ & $11 / 16 / 05$ & 127,200 & 3,269 & $\begin{array}{l}\text { Process Batch } 1 \text { of } 2 \mathrm{AY} / 6 \mathrm{C} \text {, Envelope D (Batch } \\
5 \text { Verall) }\end{array}$ \\
\hline & AZ-101 & P1HLW_FEED_TANK & $11 / 16 / 05$ & $11 / 17 / 05$ & 127,200 & 3,269 & $\begin{array}{l}\text { Deliver Batch } 2 \text { of } 2 \mathrm{AY} / 6 \mathrm{C} \text { from } 1 \mathrm{AZ} \text {, } \\
\text { Envelope } \mathrm{D} \text { (Batch } 6 \text { V verall) }\end{array}$ \\
\hline & P1HLW_FEED_TANK & HLW-CONTRACTOR & $11 / 17 / 05$ & $6 / 2 / 06$ & 127,200 & 3,269 & $\begin{array}{l}\text { Process Batch } 2 \text { of } 2 \mathrm{AY} / 6 \mathrm{C} \text {, Envelope D (Batch } \\
\text { 60verafl) }\end{array}$ \\
\hline & AZ-101 & P1HLW_FEED_TANK & $6 / 2 / 06$ & $6 / 3 / 06$ & 127,200 & 3,269 & Reliver Batch 3 of $2 \mathrm{AY} / 6 \mathrm{C}$ from $1 \mathrm{AZ}$, \\
\hline & $A Z-102$ & AN-105 & $6 / 23 / 06$ & $6 / 24 / 06$ & 159,500 & 0 & Initial Decant $4 \mathrm{C}$ Slurry from $2 \mathrm{AZ}$ \\
\hline & WASH-WATER & $A Z-102$ & $6 / 24 / 06$ & $6 / 25 / 05$ & 324,000 & 0 & First Wash $4 \mathrm{C}$ Slurry in $2 \mathrm{AZ}$ \\
\hline & $A Z-102$ & AN=105 & $8 / 13 / 06$ & $8 / 15 / 06$ & 320,100 & 0 & First Decant 4C Slurry from $2 \mathrm{AZ}$ \\
\hline & WASH-WATER & $\mathrm{AZ}-102$ & $8 / 16 / 06$ & $8 / 18 / 05$ & 324,000 & 0 & Second Wash 4C Slurry in $2 \mathrm{AZ}$ \\
\hline & P1HLW_FEED_TANK & HLW-CONTRACTOR & $6 / 3 / 06$ & $12 / 18 / 06$ & 127,200 & 3,269 & 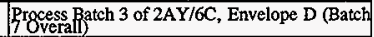 \\
\hline
\end{tabular}


HNF-2321

Revision 0

Table A.1-3. Phase 1 High-Level Waste Feed Staging Transfers for Case 1.

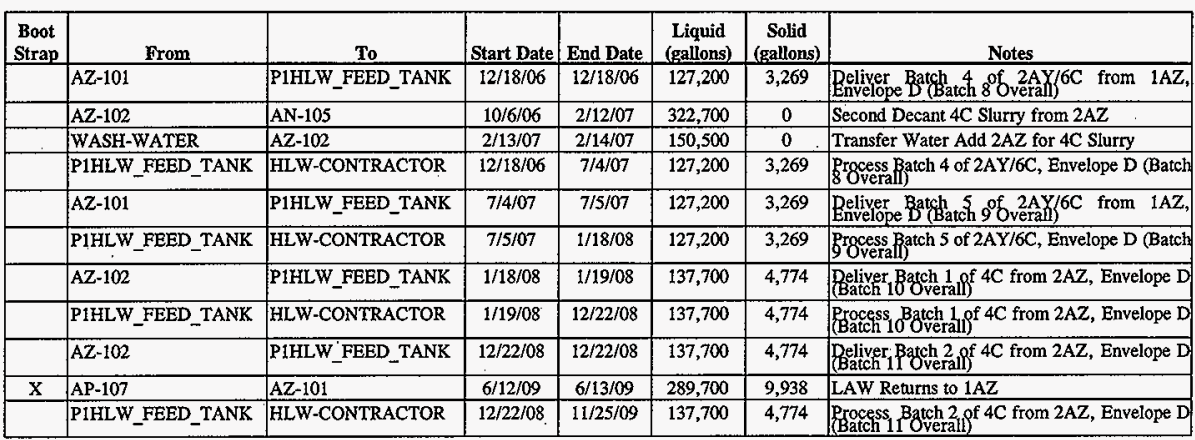


HNF-2321

Revision 0

Table A.2-1. Complete Transfer List for Case 2.

\begin{tabular}{|c|c|c|c|c|c|}
\hline From & To & Start Date & End Date & $\begin{array}{c}\text { Liquid } \\
\text { (gallons) }\end{array}$ & $\begin{array}{c}\text { Solid } \\
\text { (gallons) }\end{array}$ \\
\hline BPTCO & AP-106 & $10 / 1 / 96$ & $10 / 1 / 96$ & 11,000 & 0 \\
\hline $34 亡 87$ & AP-106 & $10 / 1 / 96$ & $10 / 1 / 96$ & 10,000 & 0 \\
\hline WNE88 & AP-106 & $10 / 1 / 96$ & $10 / 1 / 96$ & 1,000 & 0 \\
\hline TNS88 & $\overline{\mathrm{AP}-106}$ & $10 / 1 / 96$ & $10 / 1 / 96$ & 750 & 250 \\
\hline TAL88 & AP-106 & $10 / 1 / 96$ & $10 / 1 / 96$ & 4,000 & 0 \\
\hline 1 FL96 & AW-105 & $10 / 1 / 96$ & $10 / 1 / 96$ & 13,000 & 0 \\
\hline EVAPF & AP-106 & $10 / 1 / 96$ & $10 / 1 / 96$ & 10,000 & 0 \\
\hline 1NS96 & AW-105 & $10 / 1 / 96$ & $10 / 1 / 96$ & 750 & 250 \\
\hline SPN87 & AP-106 & $10 / 1 / 96$ & $10 / 1 / 96$ & 7,000 & 0 \\
\hline WESF & AP-106 & $10 / 1 / 96$ & $10 / 1 / 96$ & 5,000 & 0 \\
\hline WATER & SY-102 & $10 / 1 / 96$ & $10 / 1 / 96$ & 5,000 & 0 \\
\hline ZNL87 & SY-102 & $10 / 1 / 96$ & $10 / 1 / 96$ & 2,000 & 0 \\
\hline \begin{tabular}{|l} 
WNW88 \\
\end{tabular} & SY-102 & $10 / 1 / 96$ & $10 / 1 / 96$ & 19,000 & 0 \\
\hline WATER & AP-106 & $10 / 2 / 96$ & $10 / 2 / 96$ & 5,000 & 0 \\
\hline WATER & $\mathrm{AP}-106$ & $10 / 2 / 96$ & $10 / 2 / 96$ & 3,000 & 0 \\
\hline WATER & SY-102 & $10 / 2 / 96$ & $10 / 2 / 96$ & 5,000 & 0 \\
\hline \begin{tabular}{|l} 
WATER \\
\end{tabular} & AW-105 & $10 / 2 / 96$ & $10 / 2 / 96$ & 5,000 & 0 \\
\hline WATER & AP-106 & $10 / 2 / 96$ & $10 / 2 / 96$ & 5,000 & 0 \\
\hline WATER & AP-106 & $10 / 2 / 96$ & $10 / 2 / 96$ & 12,000 & 0 \\
\hline EVAPF & AP-106 & $11 / 1 / 96$ & $11 / 1 / 96$ & 10,000 & 0 \\
\hline WNE88 & AP-106 & $11 / 1 / 96$ & $\$ 1 / 1 / 96$ & 1,000 & 0 \\
\hline BPTCO & AP-106 & $11 / 1 / 96$ & $11 / 1 / 96$ & 10,000 & 0 \\
\hline WATER & SY-102 & $11 / 1 / 96$ & $11 / 1 / 96$ & 3,000 & 0 \\
\hline WNW88 & SY-102 & $11 / 1 / 96$ & $11 / 1 / 96$ & 14,000 & 0 \\
\hline EVAPF & AP-106 & $12 / 1 / 96$ & $12 / 1 / 96$ & 10,000 & 0 \\
\hline WNW88 & $S Y-102$ & $12 / 1 / 96$ & $12 / 1 / 96$ & 14,000 & 0 \\
\hline WATER & SY-102 & $12 / 1 / 96$ & $12 / 1 / 96$ & 3,000 & 0 \\
\hline WNE88 & AP-106 & $12 / 1 / 96$ & $12 / 1 / 96$ & 1,000 & 0 \\
\hline BPTCO & AP-106 & $12 / 1 / 96$ & $12 / 1 / 96$ & 11,000 & 0 \\
\hline EVAPF & AP-106 & $1 / 1 / 97$ & $1 / 1 / 97$ & 10,000 & 0 \\
\hline WNE88 & AP-106 & $1 / 1 / 97$ & $1 / 1 / 97$ & 1,000 & 0 \\
\hline WESF & AP-106 & $1 / 1 / 97$ & $1 / 1 / 97$ & 5,000 & 0 \\
\hline SPN87 & AP-106. & $1 / 1 / 97$ & $1 / 1 / 97$ & 6,000 & 0 \\
\hline \begin{tabular}{|l|} 
WNW88 \\
\end{tabular} & SY-102 & $1 / 1 / 97$ & $1 / 1 / 97$ & 14,000 & 0 \\
\hline WATER & SY-102 & $1 / 1 / 97$ & $1 / 1 / 97$ & 4,000 & 0 \\
\hline TAL88 & AP-106 & $1 / 1 / 97$ & $1 / 1 / 97$ & 4,000 & 0 \\
\hline BPTCO & AP-106 & $1 / 1 / 97$ & $1 / 1 / 97$ & 10,000 & 0 \\
\hline 34L87 & AP-106 & $1 / 1 / 97$ & $1 / 1 / 97$ & 10,000 & 0 \\
\hline WATER & AP-106 & $1 / 1 / 97$ & $1 / 1 / 97$ & 4,000 & 0 \\
\hline AW-106 & AP-101 & $1 / 4 / 97$ & $1 / 4 / 97$ & 69,511 & 3 \\
\hline AN-106 & AP-108 & $1 / 11 / 97$ & $1 / 12 / 97$ & 177,000 & 7 \\
\hline EVAPF & AP-106 & $2 / 1 / 97$ & $2 / 1 / 97$ & 10,000 & 0 \\
\hline BPTCO & AP-106 & $2 / 1 / 97$ & $2 / 1 / 97$ & 10,000 & 0 \\
\hline WATER & SY-102 & $2 / 1 / 97$ & $2 / 1 / 97$ & 4,000 & 0 \\
\hline WNW88 & SY-102 & $2 / 1 / 97$ & $2 / 1 / 97$ & 14,000 & 0 \\
\hline EVAPF & AW-102 & $2 / 20 / 97$ & $2 / 20 / 97$ & 50,000 & 0 \\
\hline AP-105 & AW-102 & $2 / 20 / 97$ & $2 / 24 / 97$ & 942,000 & 39 \\
\hline PXTCO & AP-106 & $3 / 1 / 97$ & $3 / 1 / 97$ & 5,000 & 0 \\
\hline WNW88 & SY-102 & $3 / 1 / 97$ & $3 / 1 / 97$ & 16,000 & 0 \\
\hline WATER & SY-102 & $3 / 1 / 97$ & $3 / 1 / 97$ & 4,000 & 0 \\
\hline WATER & AP-106 & $3 / 1 / 97$ & $3 / 1 / 97$ & 5,000 & 0 \\
\hline $34 \mathrm{~L} 87$ & AP-106 & $3 / 1 / 97$ & $3 / 1 / 97$ & 10,000 & 0 \\
\hline BPTCO & AP-106 & $3 / 1 / 97$ & $3 / 1 / 97$ & 11,000 & 0 \\
\hline EVAPF & AP-106 & $3 / 1 / 97$ & $3 / 1 / 97$ & 10,000 & 0 \\
\hline AW-102 & 242-A & $2 / 26 / 97$ & $3 / 2 / 97$ & $1,027,000$ & 42 \\
\hline 242-A & $A W-106$ & $2 / 26 / 97$ & $3 / 2 / 97$ & 622,000 & 42 \\
\hline
\end{tabular}


HNF-2321

Revision 0

Table A.2-1. Complete Transfer List for Case 2.

\begin{tabular}{|c|c|c|c|c|c|}
\hline From & To. & Start Date & End Date & $\begin{array}{c}\text { Liquid } \\
\text { (gallons) }\end{array}$ & $\begin{array}{c}\text { Solid } \\
\text { (gallons) }\end{array}$ \\
\hline $242-\mathrm{A}$ & LERF & $2 / 26 / 97$ & $3 / 2 / 97$ & 405,000 & 0 \\
\hline EVAPF & AW-102 & $3 / 3 / 97$ & $3 / 3 / 97$ & 35,000 & 0 \\
\hline EVAPF & AP-106 & $4 / 1 / 97$ & $4 / 1 / 97$ & 10,000 & 0 \\
\hline WESF & AP-106 & $4 / 1 / 97$ & $4 / 1 / 97$ & 5,000 & 0 \\
\hline TAL88 & AP-106 & $4 / 1 / 97$ & $4 / 1 / 97$ & 4,000 & 0 \\
\hline WATER & SY-102 & $4 / 1 / 97$ & $4 / 1 / 97$ & 6,000 & 0 \\
\hline BPTCO & AP-106 & $4 / 1 / 97$ & $4 / 1 / 97$ & 10,000 & 0 \\
\hline WNE88 & AP-106 & $4 / 1 / 97$ & $4 / 1 / 97$ & 1,000 & 0 \\
\hline SPN87 & AP-106 & $4 / 1 / 97$ & $4 / 1 / 97$ & 6,000 & 0 \\
\hline WNW88 & SY-102 & $4 / 1 / 97$ & $4 / 1 / 97$ & 22,000 & 0 \\
\hline \begin{tabular}{|l|} 
AW-106 \\
\end{tabular} & AP-105 & $4 / 25 / 97$ & $4 / 28 / 97$ & 617,000 & 29 \\
\hline AY-101 & AP-108 & $4 / 25 / 97$ & $4 / 29 / 97$ & 809,000 & 30 \\
\hline EVAPF & AP-106 & $5 / 1 / 97$ & $5 / 1 / 97$ & 10,000 & 0 \\
\hline 34L87 & AP-106 & $5 / 1 / 97$ & $5 / 1 / 97$ & 10,000 & 0 \\
\hline WNE88 & AP-106 & $5 / 1 / 97$ & $5 / 1 / 97$ & 1,000 & 0 \\
\hline BPTCO & AP-106 & $5 / 1 / 97$ & $5 / 1 / 97$ & 11,000 & 0 \\
\hline WATER & AP-106 & $5 / 1 / 97$ & $5 / 1 / 97$ & 4,000 & 0 \\
\hline WNW88 & SY-102 & $5 / 1 / 97$ & $5 / 1 / 97$ & 19,000 & 0 \\
\hline WATER & SY-102 & $5 / 1 / 97$ & $5 / 1 / 97$ & 5,000 & 0 \\
\hline BPTCO & AP-106 & $6 / 1 / 97$ & $6 / 1 / 97$ & 10,000 & 0 \\
\hline WATER & SY-102 & $6 / 1 / 97$ & $6 / 1 / 97$ & 4,000 & 0 \\
\hline WNW88 & SY-102 & $6 / 1 / 97$ & $6 / 1 / 97$ & 16,000 & 0 \\
\hline WNE88 & AP-105 & $6 / 1 / 97$ & $6 / 1 / 97$ & 1,000 & 0 \\
\hline EVAPF & AP-106 & $6 / 1 / 97$ & $6 / 1 / 97$ & 10,000 & 0 \\
\hline $34 \mathrm{~L} 87$ & AP-106 & $7 / 1 / 97$ & $7 / 1 / 97$ & 10,000 & 0 \\
\hline WESF & AP-106 & $7 / 1 / 97$ & $7 / 1 / 97$ & 5,000 & 0 \\
\hline WATER & AN-101 & $7 / 1 / 97$ & 7/1/97 & 5,000 & 0 \\
\hline \begin{tabular}{|l|} 
WNE88 \\
\end{tabular} & AP-106 & $7 / 1 / 97$ & $7 / 1 / 97$ & 1,000 & 0 \\
\hline EVAPF & AP-106 & $7 / 1 / 97$ & $7 / 1 / 97$ & 10,000 & 0 \\
\hline WNW88 & SY-102 & $7 / 1 / 97$ & $7 / 1 / 97$ & 24,000 & 0 \\
\hline WATER & SY-102 & $7 / 1 / 97$ & $7 / 1 / 97$ & 6,000 & 0 \\
\hline WATER & AP-106 & $7 / 1 / 97$ & $7 / 1 / 97$ & 4,000 & 0 \\
\hline SPN87 & AP-106 & $7 / 1 / 97$ & $7 / 1 / 97$ & 6,000 & 0 \\
\hline TAL88 & AP-106 & $7 / 1 / 97$ & $7 / 1 / 97$ & 4,000 & 0 \\
\hline BPTCO & A.P-106 & $7 / 1 / 97$ & $7 / 1 / 97$ & 11,000 & 0 \\
\hline WCE88 & AN-101 & $7 / 1 / 97$ & $7 / 1 / 97$ & 20,000 & 0 \\
\hline AN-106 & AP-108 & $7 / 25 / 97$ & $7 / 26 / 97$ & 194,000 & 7 \\
\hline AP-108 & AW-102 & $7 / 25 / 97$ & $7 / 31 / 97$ & $1,198,000$ & 42 \\
\hline BPTCO & AP-106 & $8 / 1 / 97$ & $8 / 1 / 97$ & 10,000 & 0 \\
\hline WATER & SY-102 & $8 / 1 / 97$ & $8 / 1 / 97$ & 13,000 & 0 \\
\hline EVAPF & AP-106 & $8 / 1 / 97$ & $8 / 1 / 97$ & 10,000 & 0 \\
\hline WATER & AP-106 & $8 / 1 / 97$ & $8 / 1 / 97$ & 4,000 & 0 \\
\hline WCE88 & AN-101 & $8 / 1 / 97$ & $8 / 1 / 97$ & 25,000 & 0 \\
\hline WATER & AN-101 & $8 / 1 / 97$ & $8 / 1 / 97$ & 6,000 & 0 \\
\hline WNE88 & AP-106 & $8 / 1 / 97$ & $8 / 1 / 97$ & 16,000 & 0 \\
\hline WNW88 & SY-102 & $8 / 1 / 97$ & $8 / 1 / 97$ & 51,000 & 0 \\
\hline$A W-102$ & $242-A$ & $7 / 28 / 97$ & $8 / 2 / 97$ & $1,198,000$ & 39 \\
\hline $242-\mathrm{A}$ & AW-106 & $7 / 28 / 97$ & $8 / 2 / 97$ & 346,700 & 39 \\
\hline $242-\mathrm{A}$ & LERF & $7 / 28 / 97$ & $8 / 2 / 97$ & 851,300 & 0 \\
\hline EVAPF & AW-102 & $8 / 3 / 97$ & $8 / 3 / 97$ & 25,000 & 0 \\
\hline WATER & AP-107 & $8 / 20 / 97$ & $8 / 20 / 97$ & 35,000 & 0 \\
\hline AW-106 & $\mathrm{AN}-106$ & $8 / 25 / 97$ & $8 / 26 / 97$ & 347,000 & 16 \\
\hline SY-102 & AP-107 & $8 / 25 / 97$ & $8 / 27 / 97$ & 520,000 & 20 \\
\hline EVAPF & AP-106 & $9 / 1 / 97$ & $9 / 1 / 97$ & 10,000 & 0 \\
\hline WATER & SY-102 & $9 / 1 / 97$ & $9 / 1 / 97$ & 15,000 & 0 \\
\hline WATER & AP-106 & $9 / 1 / 97$ & $9 / 1 / 97$ & 12,000 & 0 \\
\hline
\end{tabular}


HNF-2321

Revision 0

Table A.2-1. Complete Transfer List for Case 2.

\begin{tabular}{|c|c|c|c|c|c|}
\hline From & To & Start Date & End Date & $\begin{array}{c}\text { Liquid } \\
\text { (gallons) }\end{array}$ & $\begin{array}{c}\text { Solid } \\
\text { (gallous) }\end{array}$ \\
\hline BPTCO & AP-106 & $9 / 1 / 97$ & $9 / 1 / 97$ & 10,000 & 0 \\
\hline WCE88 & AN-101 & 9/1/97 & $9 / 1 / 97$ & 40,000 & 0 \\
\hline WNE88 & AP-106 & $9 / 1 / 97$ & $9 / 1 / 97$ & 46,000 & 0 \\
\hline WATER & AN-101 & $9 / 1 / 97$ & $9 / 1 / 97$ & 10,000 & 0 \\
\hline WNW88 & SY-102 & $9 / 1 / 97$ & $9 / 1 / 97$ & 61,000 & 0 \\
\hline WATER & AP-106 & $10 / 1 / 97$ & $10 / 1 / 97$ & 12,000 & 0 \\
\hline WESF & AP-106 & $10 / 1 / 97$ & $10 / 1 / 97$ & 5,000 & 0 \\
\hline $34 \mathrm{L87}$ & AP-106 & $10 / 1 / 97$ & $10 / 1 / 97$ & 10,000 & 0 \\
\hline TNS88 & AP-106 & $10 / 1 / 97$ & $10 / 1 / 97$ & 750 & 250 \\
\hline TAL88 & AP-106 & $10 / 1 / 97$ & $10 / 1 / 97$ & 4,000 & 0 \\
\hline EVAPF & AP-106 & $10 / 1 / 97$ & $10 / 1 / 97$ & 10,000 & 0 \\
\hline WATER & AN-101 & $10 / 1 / 97$ & $10 / 1 / 97$ & 10,000 & 0 \\
\hline $34 L 87$ & AP-106 & $10 / 1 / 97$ & $10 / 1 / 97$ & 7,000 & 0 \\
\hline BPTCO & AP-106 & $10 / 1 / 97$ & $10 / 1 / 97$ & 11,000 & 0 \\
\hline SPN87 & SY-102 & $10 / 1 / 97$ & $10 / 1 / 97$ & 7,000 & 0 \\
\hline WCE88 & $\mathrm{AN}-101$ & $10 / 1 / 97$ & $10 / 1 / 97$ & 40,000 & 0 \\
\hline ZNL87 & SY-102 & $10 / 1 / 97$ & $10 / 1 / 97$ & 4,000 & 0 \\
\hline PXTCO & AP-106 & $10 / 1 / 97$ & $10 / 1 / 97$ & 5,000 & 0 \\
\hline WNE88 & AP-106 & $10 / 1 / 97$ & $10 / 1 / 97$ & 46,000 & 0 \\
\hline WATER & SY-102 & $10 / 1 / 97$ & $10 / 1 / 97$ & 28,000 & 0 \\
\hline WNW88 & SY -102 & $10 / 1 / 97$ & $10 / 1 / 97$ & 111,000 & 0 \\
\hline WATER & SY-102 & $10 / 2 / 97$ & $10 / 2 / 97$ & 5,000 & 0 \\
\hline WATER & AP-106 & $10 / 2 / 97$ & $10 / 2 / 97$ & 5,000 & 0 \\
\hline WATER & SY-102 & $10 / 2 / 97$ & $10 / 2 / 97$ & 1,000 & 0 \\
\hline WASH-CAUSTIC & AN-107 & $10 / 2 / 97$ & $10 / 2 / 97$ & 66,000 & 0 \\
\hline WATER & AP-106 & $10 / 2 / 97$ & $10 / 2 / 97$ & 3,000 & 0 \\
\hline WATER & AP-106 & $10 / 2 / 97$ & $10 / 2 / 97$ & 3,000 & 0 \\
\hline WATER & AP-106 & $10 / 2 / 97$ & $10 / 2 / 97$ & 5,000 & 0 \\
\hline AY-102 & AP-106 & $10 / 25 / 97$ & $10 / 25 / 97$ & 35,999 & 1 \\
\hline WATER & $A Y-102$ & $10 / 26 / 97$ & $10 / 27 / 97$ & 176,000 & 0 \\
\hline EVAPF & AP-106 & $11 / 1 / 97$ & $11 / 1 / 97$ & 10,000 & 0 \\
\hline WATER & SY-102 & $11 / 1 / 97$ & $11 / 1 / 97$ & 30,000 & 0 \\
\hline WNE88 & AP-106 & $11 / 1 / 97$ & $11 / 1 / 97$ & 46,000 & 0 \\
\hline WATER & AN-101 & $11 / 1 / 97$ & $11 / 1 / 97$ & 10,000 & 0 \\
\hline WATER & $A P-106$ & $11 / 1 / 97$ & $11 / 1 / 97$ & 12,000 & 0 \\
\hline WCE 88 & AN-101 & $11 / 1 / 97$ & $11 / 1 / 97$ & 40,000 & 0 \\
\hline WNW88 & SX-102 & $11 / 1 / 97$ & $11 / 1 / 97$ & 119,000 & 0 \\
\hline EVAPF & AP-106 & $12 / 1 / 97$ & $12 / 1 / 97$ & 10,000 & 0 \\
\hline WATER & SY -102 & $12 / 1 / 97$ & $12 / 1 / 97$ & 33,000 & 0 \\
\hline WNE88 & AP-106 & $12 / 1 / 97$ & $12 / 1 / 97$ & 42,000 & 0 \\
\hline WCE 88 & AN-101 & $12 / 1 / 97$ & $12 / 1 / 97$ & 40,000 & 0 \\
\hline WATER & AN-101 & $12 / 1 / 97$ & $12 / 1 / 97$ & 10,000 & 0 \\
\hline WATER & AP-106 & $12 / 1 / 97$ & $12 / 1 / 97$ & 11,000 & 0 \\
\hline WNW88 & $\$ Y-102$ & $12 / 1 / 97$ & $12 / 1 / 97$ & 131,000 & 0 \\
\hline WATER & AP-107 & $12 / 20 / 97$ & $12 / 20 / 97$ & 35,000 & 0 \\
\hline SY-102 & AP-107 & $12 / 26 / 97$ & $12 / 28 / 97$ & 520,000 & 21 \\
\hline AP-106 & AP-104 & $12 / 25 / 97$ & $12 / 30 / 97$ & 996,600 & 35 \\
\hline $34 L 87$ & AP-106 & $1 / 1 / 98$ & $1 / 1 / 98$ & 10,000 & 0 \\
\hline TAL88 & AP-106 & $1 / 1 / 98$ & $1 / 1 / 98$ & 4,000 & 0 \\
\hline WATER & AP-106 & $1 / 1 / 98$ & $1 / 1 / 98$ & 10,000 & 0 \\
\hline WATER & $\mathrm{AP}-106$ & $1 / 1 / 98$ & $1 / 1 / 98$ & 4,000 & 0 \\
\hline EVAPF & AP-106 & $1 / 1 / 98$ & $1 / 1 / 98$ & 10,000 & 0 \\
\hline SPN87 & SY-102 & $1 / 1 / 98$ & $1 / 1 / 98$ & 6,000 & 0 \\
\hline BPTCO & AP-106 & $1 / 1 / 98$ & $1 / 1 / 98$ & 11,000 & 0 \\
\hline WATER & AN-101 & $1 / 1 / 98$ & $1 / 1 / 98$ & 10,000 & 0 \\
\hline WCE 88 & AN-101 & $1 / 1 / 98$ & $1 / 1 / 98$ & 40,000 & 0 \\
\hline
\end{tabular}


Table A.2-1. Complete Transfer List for Case 2.

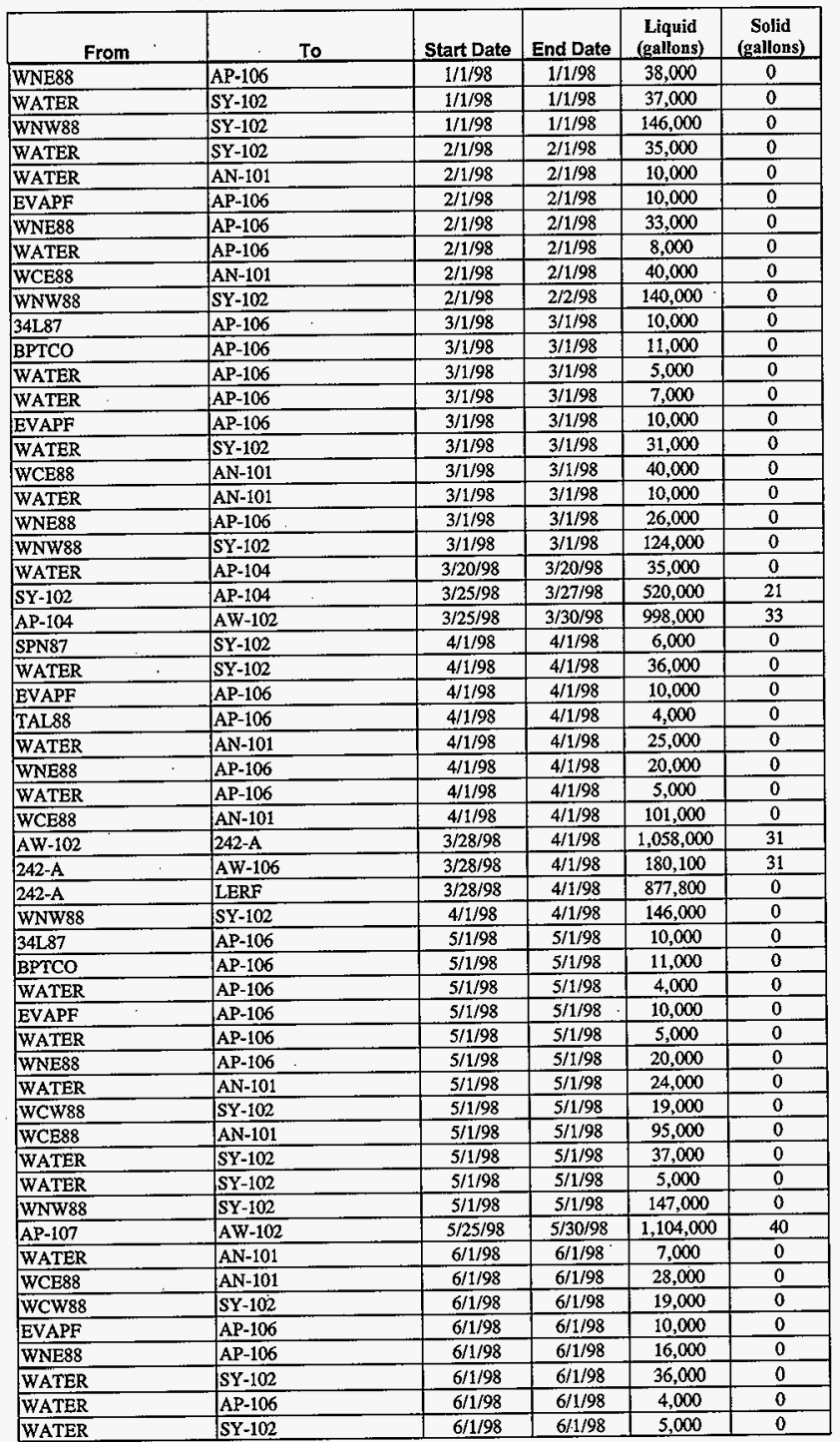


HNF-2321

Revision 0

Table A.2-1. Complete Transfer List for Case 2.

\begin{tabular}{|c|c|c|c|c|c|}
\hline From & To & Start Date & End Date & $\begin{array}{c}\text { Liquid } \\
\text { (gallons) }\end{array}$ & $\begin{array}{c}\text { Solid } \\
\text { (gallons) }\end{array}$ \\
\hline AW-102 & $242-A$ & $5 / 28 / 98$ & $6 / 1 / 98$ & $1,104,000$ & 39 \\
\hline $242-\mathrm{A}$ & AW-106 & $5 / 28 / 98$ & $6 / 1 / 98$ & 448,700 & 39 \\
\hline $242-\mathrm{A}$ & LERF & $5 / 28 / 98$ & $6 / 1 / 98$ & 655,200 & 0 \\
\hline WNW88 & $5 Y-102$ & $6 / 1 / 98$ & $6 / 2 / 98$ & 142,000 & 0 \\
\hline EVAPF & AW-102 & $6 / 3 / 98$ & $6 / 3 / 98$ & 35,000 & 0 \\
\hline WATER & AP-104 & $6 / 20 / 98$ & $6 / 20 / 98$ & 35,000 & 0 \\
\hline AW-106 & $\mathrm{AP}-105$ & $6 / 25 / 98$ & $6 / 26 / 98$ & 341,000 & 17 \\
\hline SY-102 & AP-104 & $6 / 25 / 98$ & $6 / 27 / 98$ & 520,000 & 21 \\
\hline EVAPF & AP-106 & $7 / 1 / 98$ & $7 / 1 / 98$ & 10,000 & 0 \\
\hline WNE88 & AP-106 & $7 / 1 / 98$ & $7 / 1 / 98$ & 13,000 & 0 \\
\hline WATER & AN-101 & $7 / 1 / 98$ & $7 / 1 / 98$ & 7,000 & 0 \\
\hline WCE 88 & AN-101 & $7 / 1 / 98$ & $7 / 1 / 98$ & 28,000 & 0 \\
\hline \begin{tabular}{|l} 
WATER \\
\end{tabular} & SY-102 & $7 / 1 / 98$ & $7 / 1 / 98$ & 3,000 & 0 \\
\hline \begin{tabular}{|l} 
WCW88 \\
\end{tabular} & SY-102 & $7 / 1 / 98$ & $7 / 1 / 98$ & 13,000 & 0 \\
\hline \begin{tabular}{|l} 
WATER \\
\end{tabular} & SY-102 & $7 / 1 / 98$ & $7 / 1 / 98$ & 32,000 & 0 \\
\hline WATER & AP-106 & $7 / 1 / 98$ & $7 / 1 / 98$ & 4,000 & 0 \\
\hline \begin{tabular}{|l|} 
SPN87 \\
\end{tabular} & SY-102 & $7 / 1 / 98$ & $7 / 1 / 98$ & 6,000 & 0 \\
\hline 34L_87 & AP-106 & $7 / 1 / 98$ & $7 / 1 / 98$ & 10,000 & 0 \\
\hline WATER & AP-106 & $7 / 1 / 98$ & $7 / 1 / 98$ & 3,000 & 0 \\
\hline TAL88 & AP-106 & $7 / 1 / 98$ & $7 / 1 / 98$ & 4,000 & 0 \\
\hline WNW88 & SY-102 & $7 / 1 / 98$ & $7 / 2 / 98$ & 127,000 & 0 \\
\hline $\mathrm{AY}-102$ & $\mathrm{C}-106$ & $7 / 1 / 98$ & $7 / 5 / 98$ & 920,700 & 31 \\
\hline BPTCO & AP-106 & $7 / 11 / 98$ & $7 / 11 / 98$ & 11,000 & 0 \\
\hline \begin{tabular}{|l} 
WATER \\
\end{tabular} & AN-101 & $8 / 1 / 98$ & $8 / 1 / 98$ & 7,000 & 0 \\
\hline EVAPF & AP-106 & $8 / 1 / 98$ & $8 / 1 / 98$ & 10,000 & 0 \\
\hline WCE88 & AN-101 & $8 / 1 / 98$ & $8 / 1 / 98$ & 28,000 & 0 \\
\hline WATER & $S Y-102$ & $8 / 1 / 98$ & $8 / 1 / 98$ & 30,000 & 0 \\
\hline WATER & AP-106 & $8 / 1 / 98$ & $8 / 1 / 98$ & 2,000 & 0 \\
\hline WNE88 & AP-106 & $8 / 1 / 98$ & $8 / 1 / 98$ & 7,000 & 0 \\
\hline WCW88 & SY-102 & $8 / 1 / 98$ & $8 / 1 / 98$ & 13,000 & 0 \\
\hline WATER & SY -102 & $8 / 1 / 98$ & $8 / 1 / 98$ & 3,000 & 0 \\
\hline \begin{tabular}{|l} 
WNW88 \\
\end{tabular} & SY-102 & $8 / 1 / 98$ & $8 / 2 / 98$ & 121,000 & 0 \\
\hline \begin{tabular}{|l} 
WATER \\
\end{tabular} & SY-102 & $9 / 1 / 98$ & $9 / 1 / 98$ & 2,000 & 0 \\
\hline \begin{tabular}{|l|} 
WNES8 \\
\end{tabular} & AP-106 & $9 / 1 / 98$ & $9 / 1 / 98$ & 7,000 & 0 \\
\hline \begin{tabular}{|l} 
EVAPF \\
\end{tabular} & AP-106 & $9 / 1 / 98$ & 9/1/98 & 10,000 & 0 \\
\hline \begin{tabular}{|l|} 
WATER \\
\end{tabular} & AP-106 & $9 / 1 / 98$ & $9 / 1 / 98$ & 2,000 & 0 \\
\hline WCE88 & $\mathrm{AN}-101$ & $9 / 1 / 98$ & $9 / 1 / 98$ & 28,000 & 0 \\
\hline WATER & AN-101 & $9 / 1 / 98$ & $9 / 1 / 98$ & 7,000 & 0 \\
\hline WCW88 & SY-102 & $9 / 1 / 98$ & $9 / 1 / 98$ & 8,000 & 0 \\
\hline \begin{tabular}{|l} 
WATER \\
\end{tabular} & SY-102 & $9 / 1 / 98$ & $9 / 1 / 98$ & 34,000 & 0 \\
\hline \begin{tabular}{|l|} 
WNW88 \\
\end{tabular} & SY-102 & $9 / 1 / 98$ & $9 / 1 / 98$ & 137,000 & 0 \\
\hline WATER & AP-107 & $9 / 20 / 98$ & $9 / 20 / 98$ & 35,000 & 0 \\
\hline SY:102 & AP-107 & $9 / 25 / 98$ & $9 / 27 / 98$ & 520,000 & 21 \\
\hline $\mathrm{AP}-101$ & AW-103 & $9 / 25 / 98$ & $9 / 28 / 98$ & 625,000 & 27 \\
\hline WNE88 & AP-106 & $10 / 1 / 98$ & $10 / 1 / 98$ & 3,000 & 0 \\
\hline TAL88 & AP-106 & $10 / 1 / 98$ & $10 / 1 / 98$ & 4,000 & 0 \\
\hline TNS88 & AP-106 & $10 / 1 / 98$ & $10 / 1 / 98$ & 750 & 250 \\
\hline WESF & AP-106 & $10 / 1 / 98$ & $10 / 1 / 98$ & 5,000 & 0 \\
\hline EVAPF & AP-106 & $10 / 1 / 98$ & $10 / 1 / 98$ & 10,000 & 0 \\
\hline PXTCO & AP-106 & $10 / 1 / 98$ & $10 / 1 / 98$ & 5,000 & 0 \\
\hline 34L87 & AP-106 & $10 / 1 / 98$ & $10 / 1 / 98$ & 10,000 & 0 \\
\hline \begin{tabular}{|l|} 
SPN87 \\
\end{tabular} & SY-102 & $10 / 1 / 98$ & $10 / 1 / 98$ & 7,000 & 0 \\
\hline WATER & SY-102 & $10 / 1 / 98$ & $10 / 1 / 98$ & 2,000 & 0 \\
\hline WATER & AP-106 & $10 / 1 / 98$ & $10 / 1 / 98$ & 1,000 & 0 \\
\hline ZNL87 & SY-102 & $10 / 1 / 98$ & $10 / 1 / 98$ & 4,000 & 0 \\
\hline WCW88 & SY-102 & $10 / 1 / 98$ & $10 / 1 / 98$ & 8,000 & 0 \\
\hline
\end{tabular}


Table A.2-1. Complete Transfer List for Case 2.

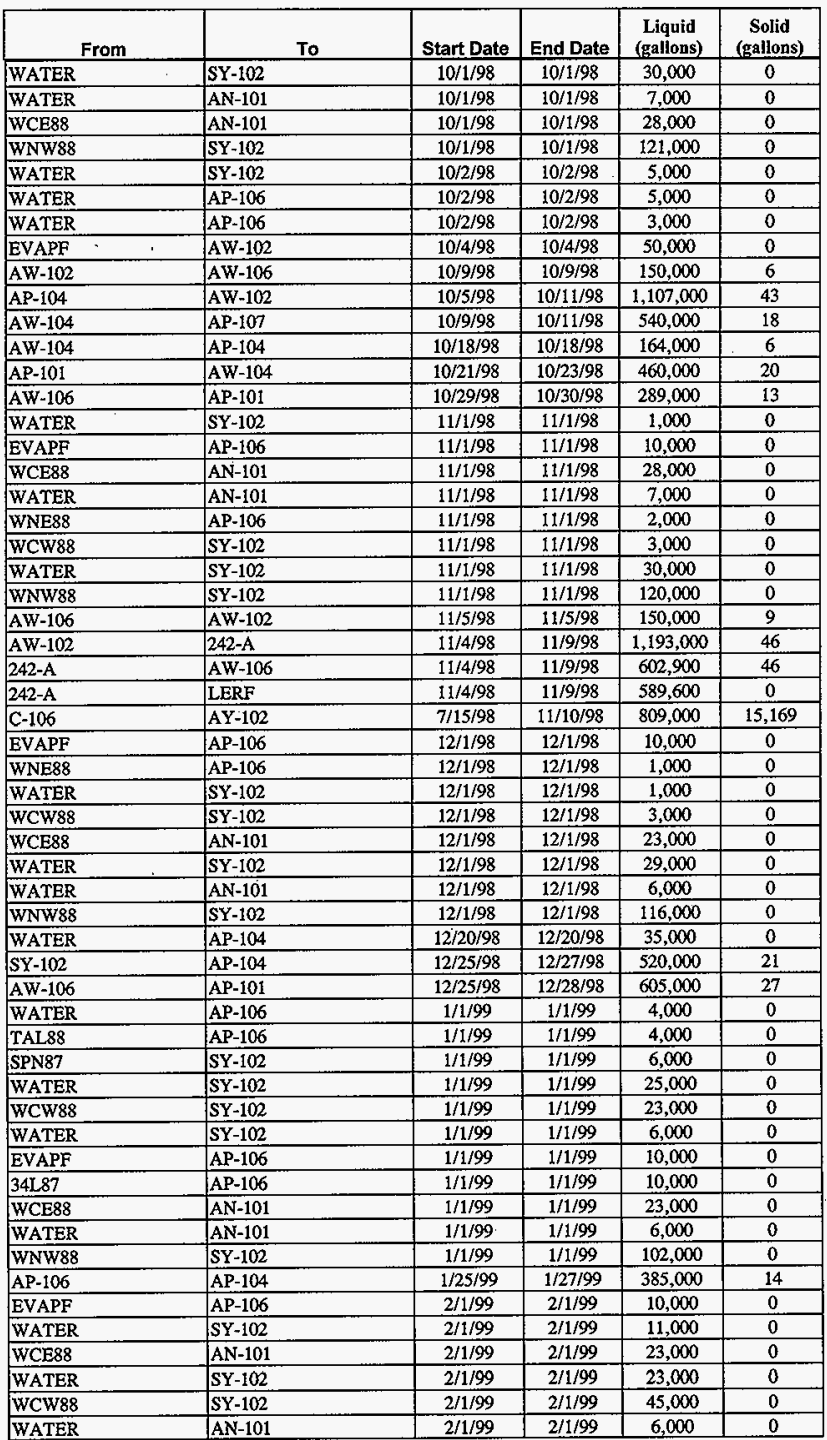


Table A.2-1. Complete Transfer List for Case 2.

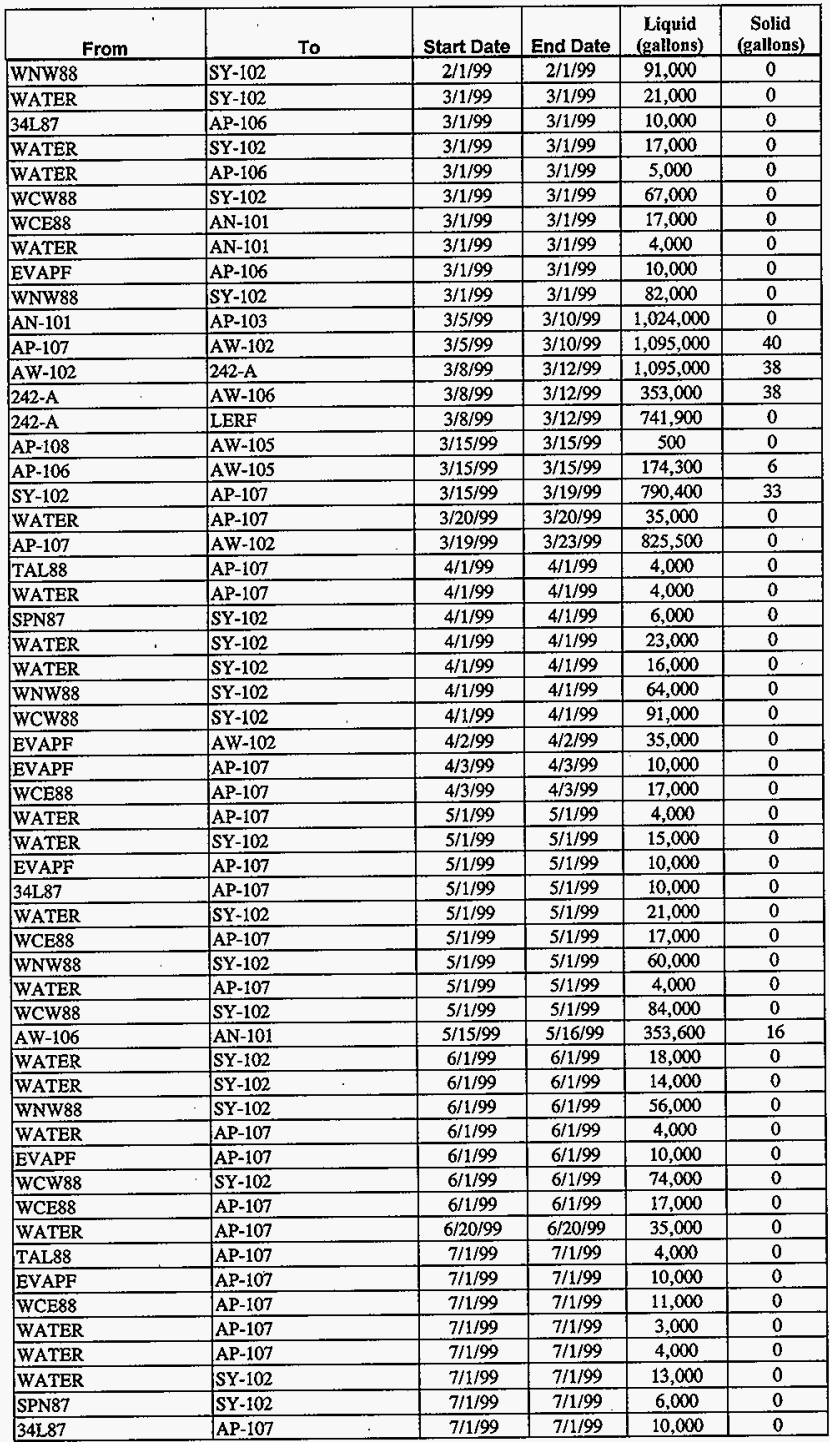


HNF-2321

Revision 0

Table A.2-1. Complete Transfer List for Case 2.

\begin{tabular}{|c|c|c|c|c|c|}
\hline From & To & Start Date & End Date & $\begin{array}{c}\begin{array}{c}\text { Liquid } \\
\text { (gallons) }\end{array} \\
\end{array}$ & $\begin{array}{c}\text { Solid } \\
\text { (gallons) }\end{array}$ \\
\hline WCW88 & SY-102 & $7 / 1 / 99$ & $7 / 1 / 99$ & 65,000 & 0 \\
\hline WATER & SY-102 & $7 / 1 / 99$ & $7 / 1 / 99$ & 16,000 & 0 \\
\hline WNW88 & SY-102 & $7 / 1 / 99$ & $7 / 1 / 99$ & 52,000 & 0 \\
\hline EVAPF & AP-107 & $8 / 1 / 99$ & $8 / 1 / 99$ & 10,000 & 0 \\
\hline WCE88 & AP-107 & $8 / 1 / 99$ & $8 / 1 / 99$ & 11,000 & 0 \\
\hline WATER & $S Y-102$ & $8 / 1 / 99$ & $8 / 1 / 99$ & 17,000 & 0 \\
\hline WATER & AP-107 & $8 / 1 / 99$ & $8 / 1 / 99$ & 3,000 & 0 \\
\hline WCW88 & SY-102 & $8 / 1 / 99$ & $8 / 1 / 99$ & 65,000 & 0 \\
\hline WATER & SY-102 & $8 / 1 / 99$ & $8 / 1 / 99$ & 16,000 & 0 \\
\hline WNW88 & SY-102 & $8 / 1 / 99$ & $8 / 1 / 99$ & 69,000 & 0 \\
\hline SY-102 & AP-107 & $8 / 15 / 99$ & $8 / 18 / 99$ & 750,000 & 32 \\
\hline WATER & SY-102 & $9 / 1 / 99$ & $9 / 1 / 99$ & 21,000 & 0 \\
\hline \begin{tabular}{|l} 
WATER \\
\end{tabular} & AP-107 & $9 / 1 / 99$ & $9 / 1 / 99$ & 3,000 & 0 \\
\hline \begin{tabular}{|l|} 
WCE88 \\
\end{tabular} & AP-107 & $9 / 1 / 99$ & 9/1/99 & 11,000 & 0 \\
\hline \begin{tabular}{|l} 
WATER \\
\end{tabular} & SY-102 & 9/1/99 & 9/1/99 & 15,000 & 0 \\
\hline EVAPF & AP-107 & $9 / 1 / 99$ & $9 / 1 / 99$ & 10,000 & 0 \\
\hline WCW88 & SY-102 & $9 / 1 / 99$ & $9 / 1 / 99$ & 58,000 & 0 \\
\hline WNW88 & SY-102 & $9 / 1 / 99$ & $9 / 1 / 99$ & 85,000 & 0 \\
\hline WATER & AP-107 & $9 / 20 / 99$ & $9 / 20 / 99$ & 35,000 & 0 \\
\hline AW-102 & $242-\mathrm{A}$ & $9 / 20 / 99$ & $9 / 24 / 99$ & 860,500 & 35 \\
\hline $242-\mathrm{A}$ & AW-106 & $9 / 20 / 99$ & $9 / 24 / 99$ & 552,800 & 35 \\
\hline $242-\mathrm{A}$ & LERF & $9 / 20 / 99$ & $9 / 24 / 99$ & 307,700 & 0 \\
\hline AW-106 & AW-102 & $9 / 25 / 99$ & $9 / 28 / 99$ & 552,700 & 25 \\
\hline $1 \mathrm{NS96}$ & AW-105 & $10 / 1 / 99$ & $10 / 1 / 99$ & 750 & 250 \\
\hline WATER & SY-102 & $10 / 1 / 99$ & $10 / 1 / 99$ & 15,000 & 0 \\
\hline SPN87 & SY-102 & $10 / 1 / 99$ & $10 / 1 / 99$ & 7,000 & 0 \\
\hline $1 \mathrm{KL} 96$ & AW-105 & $10 / 1 / 99$ & $10 / 1 / 99$ & 30,000 & 0 \\
\hline \begin{tabular}{|l} 
WATER \\
\end{tabular} & SY-102 & $10 / 1 / 99$ & $10 / 1 / 99$ & 11,000 & 0 \\
\hline TNS88 & AP-107 & $10 / 1 / 99$ & $10 / 1 / 99$ & 750 & 250 \\
\hline WESF & AP-107 & $10 / 1 / 99$ & $10 / 1 / 99$ & 5,000 & 0 \\
\hline WCE 88 & AP-107 & $10 / 1 / 99$ & $10 / 1 / 99$ & 11,000 & 0 \\
\hline \begin{tabular}{|l} 
WCW88 \\
\end{tabular} & SY-102 & $10 / 1 / 99$ & $10 / 1 / 99$ & 43,000 & 0 \\
\hline 1FL96 & AW-105 & $10 / 1 / 99$ & $10 / 1 / 99$ & 40,000 & 0 \\
\hline WATER & AP-107 & $10 / 1 / 99$ & $10 / 1 / 99$ & 3,000 & 0 \\
\hline ZNL87 & SY-102 & $10 / 1 / 99$ & $10 / 1 / 99$ & 4,000 & 0 \\
\hline TAL88 & AP-107 & $10 / 1 / 99$ & $10 / 1 / 99$ & 6,000 & 0 \\
\hline PXTCO & AP-107 & $10 / 1 / 99$ & $10 / 1 / 99$ & 5,000 & 0 \\
\hline EVAPF & AP-107 & $10 / 1 / 99$ & $10 / 1 / 99$ & 10,000 & 0 \\
\hline $34 \mathrm{~L} 87$ & AP-107 & $10 / 1 / 99$ & $10 / 1 / 99$ & 10,000 & 0 \\
\hline \begin{tabular}{|l|} 
WNW88 \\
\end{tabular} & SY-102 & $10 / 1 / 99$ & $10 / 1 / 99$ & 62,000 & 0 \\
\hline WATER & SY-102 & $10 / 2 / 99$ & $10 / 2 / 99$ & 5,000 & 0 \\
\hline WATER & AW-105 & $10 / 2 / 99$ & $10 / 2 / 99$ & 17,000 & 0 \\
\hline WATER & $A P-107$ & $10 / 2 / 99$ & $10 / 2 / 99$ & 5,000 & 0 \\
\hline WATER & AW-105 & $10 / 2 / 99$ & $10 / 2 / 99$ & 13,000 & 0 \\
\hline WATER & AP-107 & $10 / 2 / 99$ & $10 / 2 / 99$ & 5,000 & 0 \\
\hline AW-102 & 242-A & $10 / 12 / 99$ & $10 / 15 / 99$ & 552,700 & 24 \\
\hline $242-\mathrm{A}$ & AW-106 & $10 / 12 / 99$ & $10 / 15 / 99$ & 453,700 & 24 \\
\hline $242-\mathrm{A}$ & LERF & $10 / 12 / 99$ & $10 / 15 / 99$ & 98,971 & 0 \\
\hline AP-107 & $\mathrm{AW}-102$ & $10 / 15 / 99$ & $10 / 20 / 99$ & 917,400 & 0 \\
\hline WATER & SY-102 & $11 / 1 / 99$ & $11 / 1 / 99$ & 12,000 & 0 \\
\hline WATER & SY-102 & $11 / 1 / 99$ & $11 / 1 / 99$ & 10,000 & 0 \\
\hline EVAPF & AP-107 & $11 / 1 / 99$ & $11 / 1 / 99$ & 10,000 & 0 \\
\hline WCW88 & SY-102 & $11 / 1 / 99$ & $11 / 1 / 99$ & 39,000 & 0 \\
\hline \begin{tabular}{|l} 
WNW88 \\
\end{tabular} & SY-102 & $11 / 1 / 99$ & $11 / 1 / 99$ & 50,000 & 0 \\
\hline WATER & AP-107 & $11 / 1 / 99$ & $11 / 1 / 99$ & 3,000 & 0 \\
\hline WCE88 & AP-107 & $11 / 1 / 99$ & $11 / 1 / 99$ & 11,000 & 0 \\
\hline
\end{tabular}


HNF-2321

Revision 0

Table A.2-1. Complete Transfer List for Case 2.

\begin{tabular}{|c|c|c|c|c|c|}
\hline From & To & Start Date & End Date & $\begin{array}{c}\text { Liquid } \\
\text { (gallons) }\end{array}$ & $\begin{array}{c}\text { Solid } \\
\text { (gallons) }\end{array}$ \\
\hline AW-106 & AN-101 & $11 / 15 / 99$ & $11 / 17 / 99$ & 453,700 & 21 \\
\hline EVAPF & AW-102 & $11 / 17 / 99$ & $11 / 17 / 99$ & 35,000 & 0 \\
\hline WATER & SY-102 & $12 / 1 / 99$ & $12 / 1 / 99$ & 10,000 & 0 \\
\hline WCE88 & AP-107 & $12 / 1 / 99$ & $12 / 1 / 99$ & 6,000 & 0 \\
\hline WATER & AP-107 & $12 / 1 / 99$ & $12 / 1 / 99$ & 1,000 & 0 \\
\hline EVAPF & AP-107 & $12 / 1 / 99$ & $12 / 1 / 99$ & 10,000 & 0 \\
\hline WNW88 & SY-102 & $12 / 1 / 99$ & $12 / 1 / 99$ & 43,000 & 0 \\
\hline WATER & SY-102 & $12 / 1 / 99$ & $12 / 1 / 99$ & 11,000 & 0 \\
\hline WCW88 & SY-102 & $12 / 1 / 99$ & $12 / 1 / 99$ & 39,000 & 0 \\
\hline EVAPF & AW-102 & $12 / 3 / 99$ & $12 / 3 / 99$ & 35,000 & 0 \\
\hline WATER & $S Y-102$ & $1 / 1 / 00$ & $1 / 1 / 00$ & 9,000 & 0 \\
\hline WATER & SY-102 & $1 / 1 / 00$ & $1 / 1 / 00$ & 9,000 & 0 \\
\hline SPN87 & SY-102 & $1 / 1 / 00$ & $1 / 1 / 00$ & 6,000 & 0 \\
\hline EVAPF & AP-107 & $1 / 1 / 00$ & $1 / 1 / 00$ & 10,000 & 0 \\
\hline WCE 88 & AP- 107 & $1 / 1 / 00$ & $1 / 1 / 00$ & 6,000 & $\overline{0}$ \\
\hline WCW88 & SY-102 & $1 / 1 / 00$ & $1 / 1 / 00$ & 26,000 & 0 \\
\hline WATER & AP- 107 & $1 / 1 / 00$ & $1 / 1 / 00$ & 1,000 & 0 \\
\hline $34 \mathrm{~L} 87$ & AP-107 & $1 / 1 / 00$ & $1 / 1 / 00$ & 10,000 & 0 \\
\hline WNW88 & SY-102 & $1 / 1 / 00$ & $1 / 1 / 00$ & 37,000 & 0 \\
\hline WATER & AP-107 & $1 / 1 / 00$ & $1 / 1 / 00$ & 4,000 & 0 \\
\hline TAL88 & AP- 107 & $1 / 1 / 00$ & $1 / 1 / 00$ & 6,000 & 0 \\
\hline WATER & AP-107 & $1 / 20 / 00$ & $1 / 20 / 00$ & 35,000 & 0 \\
\hline WATER & SY-102 & $2 / 1 / 00$ & $2 / 1 / 00$ & 4,000 & 0 \\
\hline WATER & SY-102 & $2 / 1 / 00$ & $2 / 1 / 00$ & 8,000 & 0 \\
\hline WNW88 & SY-102 & $2 / 1 / 00$ & $2 / 1 / 00$ & 30,000 & 0 \\
\hline WCW88 & SY-102 & $2 / 1 / 00$ & $2 / 1 / 00$ & 17,000 & 0 \\
\hline WATER & AP-107 & $2 / 1 / 00$ & $2 / 1 / 00$ & 1,000 & 0 \\
\hline EVAPF & AP-107 & $2 / 1 / 00$ & $2 / 1 / 00$ & 10,000 & 0 \\
\hline WCE 88 & AP-107 & $2 / 1 / 00$ & $2 / 1 / 00$ & 5,000 & 0 \\
\hline WATER & SY-102 & $3 / 1 / 00$ & $3 / 1 / 00$ & 3,000 & 0 \\
\hline $34 \mathrm{~L} 87$ & AP-107 & $3 / 1 / 00$ & $3 / 1 / 00$ & 10,000 & 0 \\
\hline WATER & AP-107 & $3 / 1 / 00$ & $3 / 1 / 00$ & 5,000 & 0 \\
\hline EVAPF & AP-107 & $3 / 1 / 00$ & $3 / 1 / 00$ & 10,000 & 0 \\
\hline WATER & AP-107 & $3 / 1 / 00$ & $3 / 1 / 00$ & 1,000 & 0 \\
\hline WCE 88 & AP-107 & $3 / 1 / 00$ & $3 / 1 / 00$ & 3,000 & 0 \\
\hline WCW88 & SY-102 & $3 / 1 / 00$ & $3 / 1 / 00$ & 13,000 & 0 \\
\hline WNW88 & SY-102 & $3 / 1 / 00$ & $3 / 1 / 00$ & 17,000 & 0 \\
\hline WATER & SY-102 & $3 / 1 / 00$ & $3 / 1 / 00$ & 4,000 & 0 \\
\hline SY-102 & AP- 107 & $3 / 15 / 00$ & $3 / 18 / 00$ & 700,000 & 29 \\
\hline WCE88 & AP- 107 & $4 / 1 / 00$ & $4 / 1 / 00$ & 3,000 & 0 \\
\hline WATER & AP-107 & $4 / 1 / 00$ & $4 / 1 / 00$ & 1,000 & 0 \\
\hline EVAPF & AP-107 & $4 / 1 / 00$ & $4 / 1 / 00$ & 10,000 & 0 \\
\hline TAL88 & AP-107 & $4 / 1 / 00$ & $4 / 1 / 00$ & 6,000 & 0 \\
\hline SPN87 & SY-102 & $4 / 1 / 00$ & $4 / 1 / 00$ & 6,000 & 0 \\
\hline WATER & SY-102 & $4 / 1 / 00$ & $4 / 1 / 00$ & 2,000 & 0 \\
\hline WNW88 & $S Y-102$ & $4 / 1 / 00$ & $4 / 1 / 00$ & 13,000 & 0 \\
\hline WATER & SY-102 & $4 / 1 / 00$ & $4 / 1 / 00$ & 3,000 & 0 \\
\hline WCW88 & SY-102 & $4 / 1 / 00$ & $4 / 1 / 00$ & 9,000 & 0 \\
\hline $\mathrm{AW}-102$ & $242-A$ & $4 / 18 / 00$ & $4 / 23 / 00$ & 987,600 & 40 \\
\hline $242-A$ & AW-106 & $4 / 18 / 00$ & $4 / 23 / 00$ & 633,600 & 40 \\
\hline $242-A$ & LERF & $4 / 18 / 00$ & $4 / 23 / 00$ & 354,000 & 0 \\
\hline AW-106 & AW-102 & $4 / 23 / 00$ & $4 / 26 / 00$ & 633,500 & 28 \\
\hline WATER & SY-102 & $5 / 1 / 00$ & $5 / 1 / 00$ & 3,000 & 0 \\
\hline WATER & AP-107 & $5 / 1 / 00$ & $5 / 1 / 00$ & 4,000 & 0 \\
\hline WATER & SY-102 & $5 / 1 / 00$ & $5 / 1 / 00$ & 1,000 & 0 \\
\hline WNW88 & SY-102 & $5 / 1 / 00$ & $5 / 1 / 00$ & 11,000 & 0 \\
\hline
\end{tabular}


HNF-2321

Revision 0

Table A.2-1. Complete Transfer List for Case 2.

\begin{tabular}{|c|c|c|c|c|c|}
\hline From & To & Start Date & End Date & $\begin{array}{c}\text { Liquid } \\
\text { (gallons) }\end{array}$ & $\begin{array}{c}\text { Solid } \\
\text { (gallons) }\end{array}$ \\
\hline WCWW8 & SY-102 & $5 / 1 / 00$ & $5 / 1 / 00$ & 6,000 & 0 \\
\hline WATER & AP-107 & $5 / 1 / 00$ & $5 / 1 / \infty 0$ & 1,000 & 0. \\
\hline WCE88 & AP-107 & $5 / 1 / 00$ & $5 / 1 / 00$ & 3,000 & 0 \\
\hline $34 \mathrm{~L} 87$ & $\mathrm{AP}-107$ & $5 / 1 / 00$ & $5 / 1 / 00$ & 10,000 & 0 \\
\hline EVAPF & AP-107 & $5 / 1 / 00$ & $5 / 1 / 00$ & 10,000 & 0 \\
\hline AW-102 & $242-\mathrm{A}$ & $5 / 10 / 00$ & $5 / 14 / 00$ & 633,500 & 28 \\
\hline $242-\mathrm{A}$ & AW-106 & $5 / 10 / 00$ & $5 / 14 / 00$ & 504,500 & 28 \\
\hline $242-\mathrm{A}$ & LERF & $5 / 10 / 00$ & $5 / 14 / 00$ & 128,900 & 0 \\
\hline AP-107 & AW-102 & $5 / 14 / 00$ & $5 / 19 / 00$ & 917,600 & 0 \\
\hline WNW88 & SY-102 & $6 / 1 / 00$ & $6 / 1 / 00$ & 4,000 & 0 \\
\hline WATER & SY-102 & $6 / 1 / 00$ & $6 / 1 / 00$ & 1,000 & .0 \\
\hline EVAPF & $A P-107$ & $6 / 1 / 00$ & $6 / 1 / 00$ & 10,000 & 0 \\
\hline WCE 88 & AP-107 & $6 / 1 / 00$ & $6 / 1 / 00$ & 3,000 & 0 \\
\hline WATER & AP-107 & $6 / 1 / 00$ & $6 / 1 / 00$ & 1,000 & 0 \\
\hline AW-106 & AN-101 & $6 / 15 / 00$ & $6 / 16 / 00$ & 250,000 & 12 \\
\hline WCE 88 & AP-107 & $7 / 1 / 00$ & $7 / 1 / 00$ & 3,000 & 0 \\
\hline \begin{tabular}{|l} 
WATER \\
\end{tabular} & AP-107 & $7 / 1 / 00$ & $7 / 1 / 00$ & 1,000 & 0 \\
\hline EVAPF & AP-107 & $7 / 1 / 00$ & $7 / 1 / 00$ & 10,000 & 0 \\
\hline TAL88 & AP-107 & $7 / 1 / 00$ & $7 / 1 / 00$ & 5,000 & 0 \\
\hline SPN87 & SY-102 & $7 / 1 / 00$ & $7 / 1 / 00$ & 6,000 & 0 \\
\hline $34 \mathrm{~L} 87$ & AP-107 & $7 / 1 / 00$ & $7 / 1 / 00$ & 10,000 & 0 \\
\hline WATER & AP-107 & $7 / 1 / 00$ & $7 / 1 / 00$ & 4,000 & 0 \\
\hline WATER & SY-102 & $7 / 1 / 00$ & $7 / 1 / 00$ & 1,000 & 0 \\
\hline WNW88 & SY-102 & $7 / 1 / 00$ & $7 / 1 / 00$ & 4,000 & 0 \\
\hline AW-106 & AN-106 & $7 / 15 / 00$ & $7 / 16 / 00$ & 254,500 & 12 \\
\hline WATER & SY-102 & $8 / 1 / 00$ & $8 / 1 / 00$ & 1,000 & 0 \\
\hline EVAPF & AP-107 & $8 / 1 / 00$ & $8 / 1 / 00$ & 10,000 & 0 \\
\hline WCE88 & AP-107 & $8 / 1 / 00$ & $8 / 1 / 00$ & 2,000 & 0 \\
\hline WNW88 & SY-102 & $8 / 1 / 00$ & $8 / 1 / 00$ & 4,000 & 0 \\
\hline EVAPF & AW-102 & $8 / 3 / 00$ & $8 / 3 / 00$ & 35,000 & 0 \\
\hline AZ-101 & AY-101 & $8 / 17 / 00$ & $8 / 20 / 00$ & 685,000 & 0 \\
\hline$A Z-101$ & AP-107 & $8 / 20 / 00$ & $8 / 20 / 00$ & 0 & 0 \\
\hline WASH-WATER & AZ-101 & $8 / 20 / 00$ & $8 / 21 / 00$ & 146,000 & 0 \\
\hline EVAPF & AP-107 & $9 / 1 / 00$ & $9 / 1 / 00$ & 10,000 & 0 \\
\hline WCE88 & AP- 107 & $9 / 1 / 00$ & $9 / 1 / 00$ & 1,000 & 0 \\
\hline AW-105 & AP-107 & $9 / 15 / 00$ & $9 / 16 / 00$ & 200,000 & 7 \\
\hline WESF & AP- 107 & $10 / 1 / 00$ & $10 / 1 / 00$ & 5,000 & 0 \\
\hline TNS88 & AP-107 & $10 / 1 / 00$ & $10 / 1 / 00$ & 750 & 250 \\
\hline 1NS96 & AW-105 & $10 / 1 / 00$ & $10 / 1 / 00$ & 13,502 & 4,498 \\
\hline TAL88 & AP-107 & $10 / 1 / 00$ & $10 / 1 / 00$ & 23,000 & 0 \\
\hline $34 \mathrm{~L} 87$ & AP-107 & $10 / 1 / 00$ & $10 / 1 / 00$ & 50,000 & 0 \\
\hline $34 L 87$ & AP- 107 & $10 / 1 / 00$ & $10 / 1 / 00$ & 7,000 & 0 \\
\hline \begin{tabular}{|l|} 
ZNL87 \\
\end{tabular} & SY- 102 & $10 / 1 / 00$ & $10 / 1 / 00$ & 4,000 & 0 \\
\hline PXTCO & AP-107 & $10 / 1 / 00$ & $10 / 1 / 00$ & 5,000 & 0 \\
\hline SPN87 & SY-102 & $10 / 1 / 00$ & $10 / 1 / 00$ & 25,000 & 0 \\
\hline EVAPF & AP-107 & $10 / 1 / 00$ & $10 / 1 / 00$ & 120,000 & 0 \\
\hline WATER & AP- 107 & $10 / 2 / 00$ & $10 / 2 / 00$ & 3,000 & 0 \\
\hline WATER & $S Y-102$ & $10 / 2 / 00$ & $10 / 2 / 00$ & 5,000 & 0 \\
\hline WATER & AW-105 & $10 / 2 / 00$ & $10 / 2 / 00$ & 7,000 & 0 \\
\hline WATER & SY-102 & $10 / 2 / 00$ & $10 / 2 / 00$ & 1,000 & 0 \\
\hline $1 \mathrm{KL} 96$ & AW-105 & $10 / 1 / 00$ & $10 / 2 / 00$ & 302,000 & 0 \\
\hline WATER & AW-105 & $10 / 2 / 00$ & $10 / 3 / 00$ & 132,000 & 0 \\
\hline WATER & AP-107 & $10 / 3 / 00$ & $10 / 3 / 00$ & 22,000 & 0 \\
\hline WATER & AP-107 & $10 / 3 / 00$ & $10 / 3 / 00$ & 5,000 & 0 \\
\hline EVAPF & $\mathrm{AW}-102$ & $10 / 4 / 00$ & $10 / 4 / 00$ & 50,000 & 0 \\
\hline $\mathrm{AZ}-101$ & AP-107 & $10 / 9 / 00$ & $10 / 10 / 00$ & 143,200 & 0 \\
\hline
\end{tabular}


HNF-2321

Revision 0

Table A.2-1. Complete Transfer List for Case 2.

\begin{tabular}{|c|c|c|c|c|c|}
\hline From & To & Start Date & End Date & $\begin{array}{c}\text { Liquid } \\
\text { (gallons) }\end{array}$ & $\begin{array}{c}\text { Solid } \\
\text { (gallons) }\end{array}$ \\
\hline WASH-WATER & AZ-101 & $10 / 11 / 00$ & $10 / 12 / 00$ & 146,000 & 0 \\
\hline EVAPF & AW-102 & $11 / 3 / 00$ & $11 / 3 / 00$ & 35,000 & 0 \\
\hline AW-102 & $242-A$ & $11 / 16 / 00$ & $11 / 21 / 00$ & $1,038,000$ & 41 \\
\hline $242-\mathrm{A}$ & AW-106 & $11 / 16 / 00$ & $11 / 21 / 00$ & $.665,900$ & 41 \\
\hline $242-\mathrm{A}$ & LERF & $11 / 16 / 00$ & $11 / 21 / 00$ & 372,000 & 0 \\
\hline AW-106 & $A W=102$ & $11 / 22 / 00$ & $11 / 25 / 00$ & 665,800 & 29 \\
\hline AW-102 & $242-A$ & $12 / 9 / 00$ & $12 / 13 / 00$ & 665,700 & 29 \\
\hline $242-A$ & AW-106 & $12 / 9 / 00$ & $12 / 13 / 00$ & 496,100 & 29 \\
\hline $242-\mathrm{A}$ & LERF & $12 / 9 / 00$ & $12 / 13 / 00$ & 169,600 & 0 \\
\hline \begin{tabular}{|l|} 
AP-107 \\
\end{tabular} & AW-102 & $12 / 13 / 00$ & $12 / 17 / 00$ & 800,700 & 0 \\
\hline AZ-101 & AP-107 & $12 / 17 / 00$ & $12 / 18 / 00$ & 140,200 & 0 \\
\hline WASH-WATER & AZ-101 & $12 / 19 / 00$ & $12 / 20 / 00$ & 146,000 & 0 \\
\hline AW-106 & AN-106 & $1 / 15 / 01$ & $1 / 17 / 01$ & 496,000 & 23 \\
\hline $\mathrm{AZ}-101$ & AP-107 & $2 / 7 / 01$ & $2 / 7 / 01$ & 142,900 & 0 \\
\hline WASH-WATER & $\mathrm{AZ}-101$ & $2 / 8 / 01$ & $2 / 9 / 01$ & 138,200 & 0 \\
\hline $\mathrm{AW}-102$ & $242-\mathrm{A}$ & $4 / 13 / 01$ & $4 / 17 / 01$ & 800,900 & 29 \\
\hline $242-\mathrm{A}$ & AW-106 & $4 / 13 / 01$ & $4 / 17 / 01$ & 512,900 & 29 \\
\hline $242-\mathrm{A}$ & LERF & $4 / 13 / 01$ & $4 / 17 / 01$ & 288,000 & 0 \\
\hline AW-106 & AW-102 & $4 / 18 / 01$ & $4 / 20 / 01$ & 512,800 & 20 \\
\hline WATER & AW-102 & $5 / 1 / 01$ & $5 / 1 / 01$ & 35,000 & 0 \\
\hline EVAPF & AW-102 & $5 / 3 / 01$ & $5 / 3 / 01$ & 35,000 & 0 \\
\hline AW-102 & $242-A$ & $5 / 5 / 01$ & $5 / 7 / 01$ & 582,800 & 22 \\
\hline $242-\mathrm{A}$ & AW-106 & $5 / 5 / 01$ & $5 / 7 / 01$ & 372,800 & 22 \\
\hline 242-A & LERF & $5 / 5 / 01$ & $5 / 7 / 01$ & 210,000 & 0 \\
\hline AW-106 & AW-102 & $5 / 7 / 01$ & $5 / 9 / 01$ & 372,700 & 15 \\
\hline AW-102 & $242-\mathrm{A}$ & $5 / 23 / 01$ & $5 / 25 / 01$ & 372,800 & 15 \\
\hline $242-A$ & AW-106 & $5 / 23 / 01$ & $5 / 25 / 01$ & 239,200 & 15 \\
\hline 242-A & LERF & $5 / 23 / 01$ & $5 / 25 / 01$ & 133,600 & 0 \\
\hline AW-106 & $\mathrm{AW}-102$ & $5 / 25 / 01$ & $5 / 27 / 01$ & 239,100 & 10 \\
\hline AW-102 & $242-\mathrm{A}$ & $6 / 10 / 01$ & $6 / 11 / 01$ & 239,200 & 10 \\
\hline $242-\mathrm{A}$ & AW-106 & $6 / 10 / 01$ & $6 / 11 / 01$ & 173,000 & 10 \\
\hline $242-\mathrm{A}$ & LERF & $6 / 10 / 01$ & $6 / 11 / 01$ & 66,177 & 0 \\
\hline AP-104 & AW-102 & $7 / 2 / 01$ & $7 / 6 / 01$ & 918,300 & 0 \\
\hline AW-102 & $242-A$ & $7 / 21 / 01$ & $7 / 26 / 01$ & 918,300 & 35 \\
\hline 242-A & AW-105 & $7 / 21 / 01$ & $7 / 26 / 01$ & 588,300 & 35 \\
\hline $242-\mathrm{A}$ & LERF & $7 / 21 / 01$ & $7 / 26 / 01$ & 330,000 & 0 \\
\hline AW-106 & AW-102 & $7 / 26 / 01$ & $7 / 30 / 01$ & 761,200 & 32 \\
\hline$A W-102$ & $242-A$ & $8 / 13 / 01$ & $8 / 17 / 01$ & 761,100 & 32 \\
\hline $242-\mathrm{A}$ & AW-106 & $8 / 13 / 01$ & $8 / 17 / 01$ & 509,100 & 32 \\
\hline $242-\mathrm{A}$ & LERF & $8 / 13 / 01$ & $8 / 17 / 01$ & 252,000 & 0 \\
\hline ZNL87 & $S Y-102$ & $10 / 1 / 01$ & $10 / 1 / 01$ & 4,000 & 0 \\
\hline TNS88 & AP-107 & $10 / 1 / 01$ & $10 / 1 / 01$ & 750 & 250 \\
\hline \begin{tabular}{|l} 
WESF \\
\end{tabular} & AP-107 & $10 / 1 / 01$ & $10 / 1 / 01$ & 5,000 & 0 \\
\hline SPN87 & SY-102 & $10 / 1 / 01$ & $10 / 1 / 01$ & 25,000 & 0 \\
\hline TAL 88 & AP-107 & $10 / 1 / 01$ & $10 / 1 / 01$ & 23,000 & 0 \\
\hline $34 L 87$ & $\mathrm{AP}-107$ & $10 / 1 / 01$ & $10 / 1 / 01$ & 50,000 & 0 \\
\hline WATER & AP-107 & $10 / 1 / 01$ & $10 / 1 / 01$ & 35,000 & 0 \\
\hline EVAPF & AP-107 & $10 / 1 / 01$ & $10 / 1 / 01$ & 120,000 & 0 \\
\hline WATER & SY-102 & $10 / 2 / 01$ & $10 / 2 / 01$ & 5,000 & 0 \\
\hline AW-106 & AW-104 & $10 / 1 / 01$ & $10 / 2 / 01$ & 250,000 & 12 \\
\hline WATER & AP- 107 & $10 / 2 / 01$ & $10 / 2 / 01$ & 22,000 & 0 \\
\hline WATER & AP-107 & $10 / 2 / 01$ & $10 / 2 / 01$ & 5,000 & 0 \\
\hline AP-103 & AW-102 & $10 / 16 / 01$ & $10 / 20 / 01$ & 918,200 & 0 \\
\hline AP-103 & $\mathrm{AP}-107$ & $11 / 1 / 01$ & $11 / 1 / 01$ & 98,859 & 4 \\
\hline EVAPF & AW-102 & $11 / 3 / 01$ & $11 / 3 / 01$ & 35,000 & 0 \\
\hline AW-102 & $242-\mathrm{A}$ & $11 / 4 / 01$ & $11 / 9 / 01$ & 907,200 & 39 \\
\hline
\end{tabular}


HNF-2321

Revision 0

Table A.2-1. Complete Transfer List for Case 2.

\begin{tabular}{|c|c|c|c|c|c|}
\hline From & To & Start Date & End Date & $\begin{array}{c}\text { Liquid } \\
\text { (gallons) }\end{array}$ & $\begin{array}{c}\text { Solid } \\
\text { (gallons) }\end{array}$ \\
\hline $242-A$ & $A W-106$ & $11 / 4 / 01$ & $11 / 9 / 01$ & 635,300 & 39 \\
\hline $242-\mathrm{A}$ & LERF & $11 / 4 / 01$ & $11 / 9 / 01$ & 271,900 & 0 \\
\hline $\mathrm{AZ}-102$ & $A Y-101$ & $11 / 14 / 01$ & $11 / 15 / 01$ & 204,000 & 0 \\
\hline AZ-102 & AP-107 & $11 / 16 / 01$ & $11 / 16 / 01$ & 181,800 & 0 \\
\hline WASH-WATER & AZ-102 & $11 / 17 / 01$ & $11 / 18 / 01$ & 213,000 & 0 \\
\hline WASH-WATER & AP-104 & $11 / 19 / 01$ & $11 / 20 / 01$ & 200,000 & 0 \\
\hline AP-107 & AW-102 & $11 / 16 / 01$ & $11 / 21 / 01$ & 821,600 & 0 \\
\hline AP-102 & AP-103 & $11 / 17 / 01$ & $11 / 22 / 01$ & $1,070,000$ & 0 \\
\hline AP-104 & AP-102 & $12 / 1 / 01$ & $12 / 3 / 01$ & 385,700 & 39 \\
\hline AP-102 & AP-106 & $12 / 15 / 01$ & $12 / 17 / 01$ & 385,700 & 37 \\
\hline AW-106 & AP-107 & $12 / 15 / 01$ & $12 / 19 / 01$ & 894,200 & 42 \\
\hline AN-105 & AP-102 & $1 / 1 / 02$ & $1 / 4 / 02$ & 542,400 & 7,895 \\
\hline LERF & AN-105-PUMP & $1 / 1 / 02$ & $1 / 4 / 02$ & 154,100 & 0 \\
\hline AN-105 & AP-102 & $1 / 1 / 02$ & $1 / 4 / 02$ & 686,500 & 294 \\
\hline AN-105 & AP-104 & $1 / 5 / 02$ & $1 / 8 / 02$ & 542,400 & 7,895 \\
\hline LERF & AN-105-PUMP & $1 / 5 / 02$ & $1 / 8 / 02$ & 154,100 & 0 \\
\hline AN-105 & AP-104 & $1 / 5 / 02$ & $1 / 8 / 02$ & 686,500 & 294 \\
\hline AP-108 & AN-105 & $1 / 9 / 02$ & $1 / 9 / 02$ & 0 & 0 \\
\hline AP-106 & AP-108 & $1 / 10 / 02$ & $1 / 12 / 02$ & 393,700 & 684 \\
\hline AP-108 & AN-105 & $1 / 13 / 02$ & $1 / 15 / 02$ & 393,700 & 641 \\
\hline AP-102 & AP-106 & $4 / 26 / 02$ & $4 / 30 / 02$ & 686,500 & 285 \\
\hline AN-104 & AP-102 & $4 / 30 / 02$ & $5 / 3 / 02$ & 502,200 & 12,513 \\
\hline LERF & AN-104-PUMP & $4 / 30 / 02$ & $5 / 3 / 02$ & 200,800 & 0 \\
\hline AN-104 & AP-102 & $4 / 30 / 02$ & $5 / 3 / 02$ & 684,700 & 702 \\
\hline AP-104 & AP-108 & $4 / 30 / 02$ & $5 / 4 / 02$ & 686,500 & 285 \\
\hline AN-104 & AP-104 & $5 / 4 / 02$ & $5 / 7 / 02$ & 502,200 & 12,513 \\
\hline LERF & AN-104-PUMP & $5 / 4 / 02$ & $5 / 7 / 02$ & 200,800 & 0 \\
\hline AN-104 & AP-104 & $5 / 4 / 02$ & $5 / 7 / 02$ & 684,700 & 702 \\
\hline AP-107 & AN-104 & $5 / 8 / 02$ & $5 / 12 / 02$ & 903,400 & 871 \\
\hline$A Z-101$ & P1HLW FEED TANK & $5 / 17 / 02$ & $5 / 17 / 02$ & 134,300 & 5,079 \\
\hline $\mathrm{AW}-102$ & $242-\mathrm{A}$ & $5 / 21 / 02$ & $5 / 25 / 02$ & 868,200 & 32 \\
\hline $242-A$ & AW-106 & $5 / 21 / 02$ & $5 / 25 / 02$ & 556,200 & 32 \\
\hline $242-\mathrm{A}$ & LERF & $5 / 21 / 02$ & $5 / 25 / 02$ & 312,000 & 0 \\
\hline AW-106 & AW-102 & $5 / 26 / 02$ & $5 / 29 / 02$ & 556,100 & 22 \\
\hline AW-102 & $242-\mathrm{A}$ & $6 / 12 / 02$ & $6 / 14 / 02$ & 556,100 & 22 \\
\hline $242-\mathrm{A}$ & AW-106 & $6 / 12 / 02$ & $6 / 14 / 02$ & 357,200 & 22 \\
\hline $242-\mathrm{A}$ & LERF & $6 / 12 / 02$ & $6 / 14 / 02$ & 198,900 & 0 \\
\hline AW-106 & AW-102 & $6 / 14 / 02$ & $6 / 16 / 02$ & 357,100 & 15 \\
\hline $\mathrm{AW}-102$ & $242-\mathrm{A}$ & $6 / 30 / 02$ & $7 / 2 / 02$ & 357,200 & 15 \\
\hline $242-\mathrm{A}$ & AW-106 & $6 / 30 / 02$ & $7 / 2 / 02$ & 248,200 & 15 \\
\hline $242-A$ & LERF & $6 / 30 / 02$ & $7 / 2 / 02$ & 109,000 & 0 \\
\hline $\mathrm{AZ}-102$ & AN-105 & $7 / 3 / 02$ & $7 / 3 / 02$ & 127,700 & 0 \\
\hline WASH-WATER & $A Z-102$ & $7 / 4 / 02$ & $7 / 5 / 02$ & 213,000 & 0 \\
\hline AZ-102 & AN-105 & $8 / 23 / 02$ & $8 / 25 / 02$ & 210,300 & 0 \\
\hline WASH-WATER & $A Z-102$ & $8 / 26 / 02$ & $8 / 27 / 02$ & 213,000 & 0 \\
\hline WESF & AN-105 & $10 / 1 / 02$ & $10 / 1 / 02$ & 5,000 & 0 \\
\hline ZNL87 & SY-102 & $10 / 1 / 02$ & $10 / 1 / 02$ & 3,000 & 0 \\
\hline TNS88 & $A N-105$ & $10 / 1 / 02$ & $10 / 1 / 02$ & 750 & 250 \\
\hline TAL88 & AN-105 & $10 / 1 / 02$ & $10 / 1 / 02$ & 24,000 & 0 \\
\hline SPN87 & SY-102 & $10 / 1 / 02$ & $10 / 1 / 02$ & 25,000 & 0 \\
\hline WATER & AN-105 & $10 / 1 / 02$ & $10 / 1 / 02$ & 35,000 & 0 \\
\hline $34 \mathrm{~L} 87$ & AN-105 & $10 / 1 / 02$ & $10 / 1 / 02$ & 50,000 & 0 \\
\hline EVAPF & AN-105 & $10 / 1 / 02$ & $10 / 1 / 02$ & 120,000 & 0 \\
\hline WATER & AN-105 & $10 / 2 / 02$ & $10 / 2 / 02$ & 22,000 & 0 \\
\hline WATER & SY-102 & $10 / 2 / 02$ & $10 / 2 / 02$ & 5,000 & 0 \\
\hline WATER & AN-105 & $10 / 2 / 02$ & $10 / 2 / 02$ & 5,000 & 0 \\
\hline
\end{tabular}


HNF-2321

Revision 0

Table A.2-1. Complete Transfer List for Case 2.

\begin{tabular}{|c|c|c|c|c|c|}
\hline From & To & Start Date & End Date & $\begin{array}{c}\text { Liquid } \\
\text { (gallons) }\end{array}$ & $\begin{array}{c}\text { Solid } \\
\text { (gallons) }\end{array}$ \\
\hline WATER & SY-102 & $10 / 2 / 02$ & $10 / 2 / 02$ & 1,000 & 0 \\
\hline EVAPF & AW-102 & $10 / 4 / 02$ & $10 / 4 / 02$ & 50,000 & 0 \\
\hline AN-105 & AW-102 & $10 / 1 / 02$ & $10 / 5 / 02$ & 918,600 & 257 \\
\hline $\mathrm{AZ}-102$ & $\mathrm{AN}-105$ & $10 / 15 / 02$ & $10 / 16 / 02$ & 211,800 & 0 \\
\hline WASH-WATER & AZ-102 & $10 / 17 / 02$ & $10 / 18 / 02$ & 213,000 & 0 \\
\hline EVAPF & AW-102 & $11 / 3 / 02$ & $11 / 3 / 02$ & 35,000 & 0 \\
\hline $\mathrm{AZ}-102$ & AN-105 & $12 / 6 / 02$ & $12 / 7 / 02$ & 212,500 & 0 \\
\hline WASH-WATER & $A Z-102$ & $12 / 9 / 02$ & $12 / 9 / 02$ & 110,000 & 0 \\
\hline AP-104 & AP-108 & $1 / 7 / 03$ & $1 / 10 / 03$ & 684,700 & 686 \\
\hline AP-102 & AP-106 & $1 / 10 / 03$ & $1 / 13 / 03$ & 684,700 & 686 \\
\hline AW-101 & AP-104 & $1 / 11 / 03$ & $1 / 15 / 03$ & 537,200 & 13,013 \\
\hline LERF & AW-101-PUMP & $1 / 11 / 03$ & $1 / 15 / 03$ & 308,100 & 0 \\
\hline AW-101 & AP-104 & $1 / 11 / 03$ & $1 / 15 / 03$ & 845,400 & 13,013 \\
\hline RETURN 1 & AP-107 & $1 / 17 / 03$ & $1 / 17 / 03$ & 8,709 & 292 \\
\hline $\mathrm{AW}-101$ & AP-102 & $1 / 16 / 03$ & $1 / 20 / 03$ & 537,200 & 13,013 \\
\hline LERF & AW-101-PUMP & $1 / 16 / 03$ & $1 / 20 / 03$ & 308,100 & 0 \\
\hline AW-101 & AP-102 & $1 / 16 / 03$ & $1 / 20 / 03$ & 845,400 & 13,013 \\
\hline RETURN 2 & AP-107 & $2 / 19 / 03$ & $2 / 19 / 03$ & $.8,669$ & 331 \\
\hline AW-106 & AW-101 & $2 / 19 / 03$ & $2 / 20 / 03$ & 248,100 & 11 \\
\hline P1HLW FEED TANK & HLW-CONTRACTOR & $6 / 1 / 02$ & $2 / 21 / 03$ & 134,300 & 5,079 \\
\hline \begin{tabular}{|l|}
$\mathrm{AZ}-101$ \\
\end{tabular} & P1HLW FEED TANK & $2 / 21 / 03$ & $2 / 21 / 03$ & 134,300 & 5,079 \\
\hline $\mathrm{AY}-102$ & $\mathrm{AZ}-101$ & $2 / 22 / 03$ & $2 / 26 / 03$ & 819,600 & 16,032 \\
\hline $\mathrm{AW}-102$ & $242-\mathrm{A}$ & $4 / 5 / 03$ & $4 / 10 / 03$ & $1,001,000$ & 35 \\
\hline $242-\mathrm{A}$ & AW-106 & $4 / 5 / 03$ & $4 / 10 / 03$ & 640,800 & 35 \\
\hline $242-\mathrm{A}$ & LERF & $4 / 5 / 03$ & $4 / 10 / 03$ & 360,000 & 0 \\
\hline AW-106 & $\mathrm{AW}-102$ & $4 / 11 / 03$ & $4 / 14 / 03$ & 640,700 & 24 \\
\hline AW-102 & $242-\mathrm{A}$ & $4 / 28 / 03$ & $5 / 1 / 03$ & 640,900 & 23 \\
\hline $242-\mathrm{A}$ & AW-106 & $4 / 28 / 03$ & $5 / 1 / 03$ & 411,000 & 23 \\
\hline $242-\mathrm{A}$ & LERF & $4 / 28 / 03$ & $5 / 1 / 03$ & 229,900 & 0 \\
\hline AW-106 & $\mathrm{AW}-102$ & $5 / 1 / 03$ & $5 / 3 / 03$ & 410,900 & 16 \\
\hline AW-102 & $242-A$ & $5 / 17 / 03$ & $5 / 19 / 03$ & 411,000 & 16 \\
\hline $242-\mathrm{A}$ & $\mathrm{AW}-106$ & $5 / 17 / 03$ & $5 / 19 / 03$ & 262,100 & 16 \\
\hline $242-\mathrm{A}$ & LERF & $5 / 17 / 03$ & $5 / 19 / 03$ & 148,900 & 0 \\
\hline AW-106 & AW-102 & $5 / 19 / 03$ & $5 / 21 / 03$ & 262,100 & 11 \\
\hline AW-102 & $242-\mathrm{A}$ & $6 / 4 / 03$ & $6 / 5 / 03$ & 262,100 & 11 \\
\hline $242-\mathrm{A}$ & AW-106 & $6 / 4 / 03$ & $6 / 5 / 03$ & 167,500 & 11 \\
\hline $242-\mathrm{A}$ & LERF & $6 / 4 / 03$ & $6 / 5 / 03$ & 94,582 & 0 \\
\hline AW-106 & AW-102 & $6 / 5 / 03$ & $6 / 6 / 03$ & 167,500 & 7 \\
\hline AW-102 & AN-105 & $6 / 7 / 03$ & $6 / 8 / 03$ & 167,600 & 7 \\
\hline $34 \mathrm{~L} 87$ & AN-105 & $10 / 1 / 03$ & $10 / 1 / 03$ & 7,000 & 0 \\
\hline TNS88 & AN-105 & $10 / 1 / 03$ & $10 / 1 / 03$ & 750 & 250 \\
\hline WESF & $\mathrm{AN}-105$ & $10 / 1 / 03$ & $10 / 1 / 03$ & 5,000 & 0 \\
\hline ZNL87 & SY-102 & $10 / 1 / 03$ & $10 / 1 / 03$ & 3,000 & 0 \\
\hline SPN87 & SY-102 & $10 / 1 / 03$ & $10 / 1 / 03$ & 25,000 & 0 \\
\hline TAL88 & AN-10S & $10 / 1 / 03$ & $10 / 1 / 03$ & 24,000 & 0 \\
\hline WATER & AN-105 & $10 / 1 / 03$ & $10 / 1 / 03$ & 35,000 & 0 \\
\hline $34 L 87$ & AN-105 & $10 / 1 / 03$ & $10 / 1 / 03$ & 50,000 & 0 \\
\hline EVAPF & $A N-105$ & $10 / 1 / 03$ & $10 / 1 / 03$ & 120,000 & 0 \\
\hline WATER & $A N-105$ & $10 / 2 / 03$ & $10 / 2 / 03$ & 22,000 & 0 \\
\hline WATER & SY-102 & $10 / 2 / 03$ & $10 / 2 / 03$ & 5,000 & 0 \\
\hline WATER & AN-105 & $10 / 2 / 03$ & $10 / 2 / 03$ & 3,000 & 0 \\
\hline \begin{tabular}{|l} 
WATER \\
\end{tabular} & AN-10S & $10 / 2 / 03$ & $10 / 2 / 03$ & 5,000 & 0 \\
\hline AP-104 & AP-108 & $10 / 1 / 03$ & $10 / 4 / 03$ & 740,300 & 1,581 \\
\hline AN-105 & AW-102 & $10 / 1 / 03$ & $10 / 5 / 03$ & 916,100 & 271 \\
\hline RETURN 1 & AP-107 & $10 / 5 / 03$ & $10 / 6 / 03$ & 8,671 & 332 \\
\hline AP-102 & AP-106 & $10 / 4 / 03$ & $10 / 8 / 03$ & 740,300 & 1,581 \\
\hline
\end{tabular}


HNF-2321

Revision 0

Table A.2-1. Complete Transfer List for Case 2.

\begin{tabular}{|c|c|c|c|c|c|}
\hline From & To & Start Date & End Date & $\begin{array}{c}\text { Liquid } \\
\text { (gallons) }\end{array}$ & $\begin{array}{c}\text { Solid } \\
\text { (gallons) }\end{array}$ \\
\hline AN-103 & AP-104 & $10 / 5 / 03$ & $10 / 8 / 03$ & 436,600 & 28,133 \\
\hline LERF & AN-103-PUMP & $10 / 5 / 03$ & $10 / 8 / 03$ & 264,900 & 0 \\
\hline AN-103 & AP-104 & $10 / 5 / 03$ & $10 / 8 / 03$ & 738,300 & 549 \\
\hline AN-103 & AP-102 & $10 / 9 / 03$ & $10 / 12 / 03$ & 436,600 & 28,133 \\
\hline LERF & AN-103-PUMP & $10 / 9 / 03$ & $10 / 12 / 03$ & 264,900 & 0 \\
\hline AN-103 & $A P-102$ & $10 / 9 / 03$ & $10 / 12 / 03$ & 738,300 & 549 \\
\hline RETURN 2 & AP-107 & $10 / 22 / 03$ & $10 / 22 / 03$ & 8,672 & 331 \\
\hline$A Z-101$ & AN-105 & $10 / 29 / 03$ & $11 / 1 / 03$ & 622,700 & 0 \\
\hline WASH-WATER & AZ-101 & $11 / 1 / 03$ & $11 / 2 / 03$ & 324,000 & 0 \\
\hline EVAPF & AW-102 & $11 / 3 / 03$ & $11 / 3 / 03$ & 35,000 & 0 \\
\hline PIHLW FEED TANK & HLW-CONTRACTOR & $2 / 21 / 03$ & $11 / 14 / 03$ & 134,300 & 5,079 \\
\hline $\mathrm{AZ}-102$ & P1HLW FEED TANK & $11 / 14 / 03$ & $11 / 14 / 03$ & 123,900 & 5,202 \\
\hline AZ-101 & $\mathrm{AN}-105$ & $12 / 22 / 03$ & $12 / 23 / 03$ & 320,000 & 0 \\
\hline WASH-WATER & AZ-101 & $12 / 24 / 03$ & $12 / 26 / 03$ & 324,000 & 0 \\
\hline RETURN 1 & AP-107 & $2 / 20 / 04$ & $2 / 20 / 04$ & 8,673 & 330 \\
\hline RETURN 2 & AP-107 & $3 / 8 / 04$ & $3 / 8 / 04$ & 8,672 & 330 \\
\hline AW-102 & $242-\mathrm{A}$ & $4 / 4 / 04$ & $4 / 8 / 04$ & 947,900 & 34 \\
\hline $242-\mathrm{A}$ & AW-106 & $4 / 4 / 04$ & $4 / 8 / 04$ & 606,100 & 34 \\
\hline $242-\mathrm{A}$ & LERF & $4 / 4 / 04$ & $4 / 8 / 04$ & 341,800 & 0 \\
\hline$A W-106$ & AW-102 & $4 / 9 / 04$ & $4 / 12 / 04$ & 605,900 & 23 \\
\hline AW-102 & $242-\mathrm{A}$ & $4 / 26 / 04$ & $4 / 29 / 04$ & 606,100 & 23 \\
\hline $242-\mathrm{A}$ & AW-106 & $4 / 26 / 04$ & $4 / 29 / 04$ & 389,200 & 23 \\
\hline $242-\mathrm{A}$ & LERF & $4 / 26 / 04$ & $4 / 29 / 04$ & 216,900 & 0 \\
\hline AW-106 & AW-102 & $4 / 29 / 04$ & $5 / 1 / 04$ & 389,100 & 15 \\
\hline AP-104 & AP-108 & $5 / 5 / 04$ & $5 / 9 / 04$ & 753,200 & 1,687 \\
\hline AP-102 & AP-106 & $5 / 9 / 04$ & $5 / 13 / 04$ & 753,200 & 1,687 \\
\hline WASHI-WATER & $\mathrm{AP}-102$ & $5 / 14 / 04$ & $5 / 14 / 04$ & 118,100 & 0 \\
\hline WASH-WATER & AP-104 & $5 / 15 / 04$ & $5 / 15 / 04$ & 118,100 & 0 \\
\hline AW-102 & 242-A & $5 / 15 / 04$ & $5 / 17 / 04$ & 389,200 & 15 \\
\hline $242-A$ & AW-106 & $5 / 15 / 04$ & $5 / 17 / 04$ & 249,600 & 15 \\
\hline $242-\mathrm{A}$ & LERF & $5 / 15 / 04$ & $5 / 17 / 04$ & 139,500 & 0 \\
\hline AW-106 & AW-102 & $5 / 17 / 04$ & $5 / 18 / 04$ & 249,600 & 11 \\
\hline RETURN 1 & AP-107 & $5 / 30 / 04$ & $5 / 30 / 04$ & 8,672 & 330 \\
\hline$A X-103$ & AN-103 & $1 / 15 / 04$ & $6 / 2 / 04$ & 310,200 & 2,846 \\
\hline AW-102 & $242-\mathrm{A}$ & $6 / 1 / 04$ & $6 / 3 / 04$ & 249,700 & 10 \\
\hline $242-\mathrm{A}$ & AW-106 & $6 / 1 / 04$ & $6 / 3 / 04$ & 163,200 & 10 \\
\hline $242-\mathrm{A}$ & LERF & $6 / 1 / 04$ & $6 / 3 / 04$ & 86,457 & 0 \\
\hline RETURN 2 & AP-107 & $6 / 4 / 04$ & $6 / 4 / 04$ & 8,678 & 331 \\
\hline AN-105 & AW-102 & $6 / 3 / 04$ & $6 / 8 / 04$ & 913,200 & 0 \\
\hline AP-102 & AN-105 & $5 / 30 / 04$ & $6 / 8 / 04$ & 209,500 & 9,168 \\
\hline AP-104 & AN-105 & $6 / 9 / 04$ & $6 / 10 / 04$ & 209,500 & 9,168 \\
\hline WASH-CAUSTIC & $\mathrm{AP}-\mathrm{LO2}$ & $6 / 10 / 04$ & $6 / 10 / 04$ & 103,400 & 0 \\
\hline WASH-CAUSTIC & AP-104 & $6 / 11 / 04$ & $6 / 11 / 04$ & 126,400 & 0 \\
\hline $\mathrm{AZ}-101$ & AN-105 & $6 / 10 / 04$ & $6 / 11 / 04$ & 322,300 & 0 \\
\hline AP-101 & $\mathrm{AP}-102$ & $6 / 12 / 04$ & $6 / 14 / 04$ & 402,300 & 18 \\
\hline LERF & AP-101-PUMP & $6 / 12 / 04$ & $6 / 14 / 04$ & 44,253 & 0 \\
\hline AP-101 & AP-102 & $6 / 12 / 04$ & $6 / 14 / 04$ & 446,500 & 18 \\
\hline WASH-WATER & $A Z-101$ & $6 / 13 / 04$ & $6 / 15 / 04$ & 433,600 & 0 \\
\hline AW-104 & AP-102 & $6 / 16 / 04$ & $6 / 16 / 04$ & 111,900 & 5 \\
\hline AP-101 & AP-104 & $6 / 15 / 04$ & $6 / 17 / 04$ & 491,700 & 22 \\
\hline LERF & AP-101-PUMP & $6 / 15 / 04$ & $6 / 17 / 04$ & 54,087 & 0 \\
\hline AP-101 & AP-104 & $6 / 15 / 04$ & $6 / 17 / 04$ & 545,800 & 22 \\
\hline AW-104 & AP-104 & $6 / 18 / 04$ & $6 / 18 / 04$ & 136,800 & 6 \\
\hline AW-102 & $242-\mathrm{A}$ & $6 / 25 / 04$ & $6 / 30 / 04$ & 913,300 & 32 \\
\hline $242-\mathrm{A}$ & AW-106 & $6 / 25 / 04$ & $6 / 30 / 04$ & 584,100 & 32 \\
\hline $242-\mathrm{A}$ & LERF & $6 / 25 / 04$ & $6 / 30 / 04$ & 329,200 & 0 \\
\hline
\end{tabular}


HNF-2321

Revision 0

Table A.2-1. Complete Transfer List for Case 2.

\begin{tabular}{|c|c|c|c|c|c|}
\hline From & To & Start Date & End Date & $\begin{array}{c}\text { Liquid } \\
\text { (gallons) }\end{array}$ & $\begin{array}{c}\text { Solid } \\
\text { (gallons) }\end{array}$ \\
\hline$A W-106$ & $A W-102$ & $6 / 30 / 04$ & $7 / 4 / 04$ & 747,200 & 29 \\
\hline RETURN 1 & AP-107 & $7 / 19 / 04$ & $7 / 19 / 04$ & 8,676 & 331 \\
\hline $\mathrm{AW}-102$ & $242-\mathrm{A}$ & $7 / 18 / 04$ & $7 / 22 / 04$ & 747,300 & 29 \\
\hline $242-\mathrm{A}$ & AW-106 & $7 / 18 / 04$ & $7 / 22 / 04$ & 478,500 & 29 \\
\hline $242-\mathrm{A}$ & LERF & $7 / 18 / 04$ & $7 / 22 / 04$ & 268,800 & 0 \\
\hline RETURN 2 & AP-107 & $7 / 24 / 04$ & $7 / 24 / 04$ & 8,682 & 331 \\
\hline AW-106 & AW-102 & $7 / 22 / 04$ & $7 / 24 / 04$ & 478,400 & 20 \\
\hline P1HLW FEED TANK & HLW-CONTRACTOR & $11 / 14 / 03$ & $8 / 7 / 04$ & 123,900 & 5,202 \\
\hline $\mathrm{AZ}-102$ & P1HLW FEED TANK & $8 / 7 / 04$ & $8 / 8 / 04$ & 123,900 & 5,202 \\
\hline AW-102 & $242-\mathrm{A}$ & $8 / 7 / 04$ & $8 / 10 / 04$ & 478,500 & 20 \\
\hline $242-\mathrm{A}$ & AW-106 & $8 / 7 / 04$ & $8 / 10 / 04$ & 307,400 & 20 \\
\hline $242-\mathrm{A}$ & LERF & $8 / 7 / 04$ & $8 / 10 / 04$ & 171,100 & 0 \\
\hline AW-106 & AW-101 & $8 / 10 / 04$ & $8 / 11 / 04$ & 307,300 & 14 \\
\hline AN-105 & $\mathrm{AW}-102$ & $8 / 10 / 04$ & $8 / 13 / 04$ & 583,600 & 0 \\
\hline C-103 & AN-103 & $6 / 2 / 04$ & $8 / 16 / 04$ & 271,800 & 10,423 \\
\hline RETURN_1 & AP-107 & $9 / 7 / 04$ & $9 / 7 / 04$ & 8,676 & 331 \\
\hline C-104 & $\mathrm{AY}-102$ & $8 / 1 / 04$ & $9 / 8 / 04$ & 279,100 & 9,724 \\
\hline RETURN 2 & AP-107 & $9 / 13 / 04$ & $9 / 13 / 04$ & 8,682 & 331 \\
\hline AW-105 & AN-105 & $9 / 15 / 04$ & $9 / 17 / 04$ & 500,000 & 17 \\
\hline WESF & AN-105 & $10 / 1 / 04$ & $10 / 1 / 04$ & 5,000 & 0 \\
\hline TNS88 & $\mathrm{AN}-105$ & $10 / 1 / 04$ & $10 / 1 / 04$ & 750 & 250 \\
\hline ZNL87 & SY-102 & $10 / 1 / 04$ & $10 / 1 / 04$ & 3,000 & 0 \\
\hline SPN87 & SY-102 & $10 / 1 / 04$ & $10 / 1 / 04$ & 2,000 & 0 \\
\hline TAL88 & AN-105 & $10 / 1 / 04$ & $10 / 1 / 04$ & 22,000 & 0 \\
\hline \begin{tabular}{|l} 
WATER \\
\end{tabular} & $A N-105$ & $10 / 1 / 04$ & $10 / 1 / 04$ & 35,000 & 0 \\
\hline $34 \mathrm{~L} 87$ & AN-105 & $10 / 1 / 04$ & $10 / 1 / 04$ & 50,000 & 0 \\
\hline SPN87 & SY-102 & $10 / 1 / 04$ & $10 / 1 / 04$ & 23,000 & 0 \\
\hline \begin{tabular}{|l|} 
TAL88 \\
\end{tabular} & AN-105 & $10 / 1 / 04$ & $10 / 1 / 04$ & 2,000 & 0 \\
\hline EVAPF & AN-105 & $10 / 1 / 04$ & $10 / 1 / 04$ & 120,000 & 0 \\
\hline 1FL96 & AW-105 & $10 / 1 / 04$ & $10 / 2 / 04$ & 200,000 & 0 \\
\hline \begin{tabular}{|l} 
WATER \\
\end{tabular} & AN-105 & $10 / 2 / 04$ & $10 / 2 / 04$ & 22,000 & 0 \\
\hline WATER & $\mathrm{AN}-105$ & $10 / 2 / 04$ & $10 / 2 / 04$ & 4,000 & 0 \\
\hline WATER & SY-102 & $10 / 2 / 04$ & $10 / 2 / 04$ & 1,000 & 0 \\
\hline WATER & AW-105 & $10 / 2 / 04$ & $10 / 2 / 04$ & 88,000 & 0 \\
\hline WATER & $S Y-102$ & $10 / 2 / 04$ & $10 / 2 / 04$ & 5,000 & 0 \\
\hline WATER & $\mathrm{AN}-105$ & $10 / 2 / 04$ & $10 / 3 / 04$ & 1,000 & 0 \\
\hline EVAPF & AW-102 & $10 / 4 / 04$ & $10 / 4 / 04$ & 50,000 & 0 \\
\hline RETURN 1 & AP-107 & $10 / 27 / 04$ & $10 / 27 / 04$ & 8,676 & 331. \\
\hline RETURN 2 & AP-107 & $11 / 2 / 04$ & $11 / 2 / 04$ & 8,682 & 331 \\
\hline EVAPF & AW-102 & $11 / 3 / 04$ & $11 / 3 / 04$ & 35,000 & 0 \\
\hline A -102 & AN-103 & $8 / 16 / 04$ & $12 / 5 / 04$ & 100,600 & 744 \\
\hline$A W-102$ & 242-A & $12 / 12 / 04$ & $12 / 15 / 04$ & 668,800 & 24 \\
\hline $242-\mathrm{A}$ & AW-106 & $12 / 12 / 04$ & $12 / 15 / 04$ & 428,800 & 24 \\
\hline $242-\mathrm{A}$ & LERF & $12 / 12 / 04$ & $12 / 15 / 04$ & 240,000 & 0 \\
\hline AW-101 & AW-104 & $12 / 15 / 04$ & $12 / 16 / 04$ & 250,000 & 12 \\
\hline RETURN 1 & AP-107 & $12 / 16 / 04$ & $12 / 16 / 04$ & 8,676 & 331 \\
\hline AW-106 & $\mathrm{AW}-102$ & $12 / 16 / 04$ & $12 / 18 / 04$ & 428,700 & 16 \\
\hline RETURN 2 & AP-107 & $12 / 23 / 04$ & $12 / 23 / 04$ & 8,682 & 331 \\
\hline AW-102 & $242-\mathrm{A}$ & $1 / 1 / 05$ & $1 / 3 / 05$ & 428,800 & 16 \\
\hline 242-A & AW-106 & $1 / 1 / 05$ & $1 / 3 / 05$ & 272,800 & 16 \\
\hline $242-\mathrm{A}$ & LERF & $1 / 1 / 05$ & $1 / 3 / 05$ & 156,000 & 0 \\
\hline AW-106 & AW-102 & $1 / 3 / 05$ & $1 / 5 / 05$ & 272,800 & 11 \\
\hline AW-102 & $242-\mathrm{A}$ & $1 / 19 / 05$ & 1/20/05 & 272,800 & 11 \\
\hline 242-A & AW-106 & $1 / 19 / 05$ & $1 / 20 / 05$ & 174,700 & 11 \\
\hline $242-A$ & LERF & $1 / 19 / 05$ & $1 / 20 / 05$ & 98,155 & 0 \\
\hline AW-106 & $A W-102$ & $1 / 20 / 05$ & $1 / 21 / 05$ & 174,700 & 7 \\
\hline
\end{tabular}


HNF-2321

Revision 0

Table A.2-1. Complete Transfer List for Case 2.

\begin{tabular}{|c|c|c|c|c|c|}
\hline From & To & Start Date & End Date & $\cdot \begin{array}{c}\text { Liquid } \\
\text { (gallons) }\end{array}$ & $\begin{array}{c}\text { Solid } \\
\text { (gallons) }\end{array}$ \\
\hline$A W-102$ & AW-106 & $1 / 22 / 05$ & $1 / 23 / 05$ & 174,800 & 7 \\
\hline AN-103 & AN-104 & $1 / 24 / 05$ & $1 / 25 / 05$ & 206,700 & 5,498 \\
\hline AN-103 & AW-103 & $1 / 25 / 05$ & $1 / 25 / 05$ & 3,896 & 104 \\
\hline AN-103 & AP-105 & $1 / 25 / 05$ & $1 / 25 / 05$ & 3,897 & 104 \\
\hline AN-103 & AP-103 & $1 / 25 / 05$ & $1 / 26 / 05$ & 42,472 & 1,128 \\
\hline AN-103 & AN-101 & $1 / 26 / 05$ & $1 / 26 / 05$ & 25,050 & 665 \\
\hline AN-105 & AW-102 & $1 / 24 / 05$ & $1 / 28 / 05$ & 884,300 & 0 \\
\hline AW-105 & AN-105 & $1 / 1 / 05$ & $1 / 29 / 05$ & 390,700 & 13 \\
\hline AN-103 & AP-101 & $1 / 26 / 05$ & $1 / 30 / 05$ & 837,200 & 22,207 \\
\hline RETURN 1 & AP-107 & $2 / 4 / 05$ & $2 / 4 / 05$ & 8,676 & 331 \\
\hline RETURN 2 & $\mathrm{AP}-107$ & $2 / 11 / 05$ & $2 / 11 / 05$ & 8,682 & 331 \\
\hline AP-104 & AP-108 & $2 / 19 / 05$ & $2 / 23 / 05$ & 807,800 & 1,143 \\
\hline AY-101 & AP-104 & $2 / 24 / 05$ & $2 / 25 / 05$ & 283,600 & 12 \\
\hline AP-102 & AP-106 & $2 / 23 / 05$ & $2 / 27 / 05$ & 660,800 & 1,129 \\
\hline $\mathrm{AY}-101$ & AP-102 & $2 / 28 / 05$ & $3 / 1 / 05$ & 279,700 & 11 \\
\hline C-105 & AN-103 & $12 / 5 / 04$ & $3 / 29 / 05$ & 827,500 & 31,875 \\
\hline RETURN 1 & AP-107 & $3 / 31 / 05$ & $3 / 31 / 05$ & 8,676 & 331 \\
\hline RETURN 2 & AP-107 & $4 / 8 / 05$ & $4 / 8 / 05$ & 8,673 & 331 \\
\hline AW-102 & $242-\mathrm{A}$ & $4 / 6 / 05$ & $4 / 11 / 05$ & 884,300 & 30 \\
\hline $242-\mathrm{A}$ & AW-106 & $4 / 6 / 05$ & $4 / 11 / 05$ & 566,300 & 30 \\
\hline $242-\mathrm{A}$ & LERF. & $4 / 6 / 05$ & $4 / 11 / 05$ & 318,000 & 0 \\
\hline $\mathrm{AW}-106$ & AW-102 & $4 / 11 / 05$ & $4 / 15 / 05$ & 741,000 & 27 \\
\hline P1HLW FEED TANK & HLW-CONTRACTOR & $8 / 8 / 04$ & $5 / 2 / 05$ & 123,900 & 5,202 \\
\hline AZ-101 & P1HLW FEED TANK & $5 / 2 / 05$ & $5 / 2 / 05$ & 127,200 & 3,269 \\
\hline AW-102 & $242-\mathrm{A}$ & $4 / 29 / 05$ & $5 / 3 / 05$ & 741,000 & 27 \\
\hline $242-A$ & AW-106 & $4 / 29 / 05$ & $5 / 3 / 05$ & 475,600 & 27 \\
\hline $242-\mathrm{A}$ & LERF & $4 / 29 / 05$ & $5 / 3 / 05$ & 265,400 & 0 \\
\hline AW-105 & AW-102 & $5 / 3 / 05$ & $5 / 5 / 05$ & 475,500 & 18 \\
\hline AW-102 & 242-A & $5 / 19 / 05$ & $5 / 22 / 05$ & 475,600 & 18 \\
\hline $242-\mathrm{A}$ & AW-106 & $5 / 19 / 05$ & $5 / 22 / 05$ & 304,200 & 18 \\
\hline $242-\mathrm{A}$ & LERF & $5 / 19 / 05$ & $5 / 22 / 05$ & 171,400 & 0 \\
\hline $\mathrm{AW}-106$ & AW-102 & $5 / 22 / 05$ & $5 / 23 / 05$ & 303,900 & 12 \\
\hline RETURN_1 & AP-107 & $5 / 27 / 05$ & $5 / 27 / 05$ & 8,675 & 331 \\
\hline RETURN 2 & AP-107 & $6 / 4 / 05$ & $6 / 4 / 05$ & 8,678 & 331 \\
\hline $\mathrm{AW}-102$ & 242-A & $6 / 6 / 05$ & $6 / 8 / 05$ & 304,000 & 12 \\
\hline $242-\mathrm{A}$ & AW-106 & $6 / 6 / 05$ & $6 / 8 / 05$ & 195,200 & 12 \\
\hline $242-\mathrm{A}$ & LERF & $6 / 6 / 05$ & $6 / 8 / 05$ & 108,800 & 0 \\
\hline AW-106 & AW-102 & $6 / 8 / 05$ & $6 / 9 / 05$ & 195,200 & 8 \\
\hline AW-102 & AN-105 & $6 / 10 / 05$ & $6 / 11 / 05$ & 195,300 & 8 \\
\hline A-104 & AN-103 & $3 / 29 / 05$ & $7 / 8 / 05$ & 29,827 & 832 \\
\hline C-204 & AN-103 & $7 / 8 / 05$ & $7 / 15 / 05$ & 3,775 & 55 \\
\hline C-201 & AN-103 & $7 / 15 / 05$ & $7 / 21 / 05$ & 2,464 & 59 \\
\hline RETURN I & AP-107 & $7 / 23 / 05$ & $7 / 23 / 05$ & 8,675 & 331 \\
\hline RETURN 2 & AP-107 & $7 / 31 / 05$ & $7 / 31 / 05$ & 8,678 & 331 \\
\hline $\mathrm{AY}-102$ & $A Z-102$ & $8 / 1 / 05$ & $8 / 2 / 05$ & 279,400 & 9,360 \\
\hline AX-104. & AN-103 & $7 / 21 / 05$ & $8 / 25 / 05$ & 18,516 & 572 \\
\hline RETURN 1 & AP-107 & $9 / 18 / 05$ & $9 / 18 / 05$ & 8,675 & 331 \\
\hline RETURN 2 & AP-107 & $9 / 26 / 05$ & $9 / 26 / 05$ & 8,678 & 331 \\
\hline TNS88 & $\mathrm{AN}-105$ & $10 / 1 / 05$ & $10 / 1 / 05$ & 750 & 250 \\
\hline WESF & AN-105 & $10 / 1 / 05$ & $10 / 1 / 05$ & 5,000 & 0 \\
\hline ZNL87 & SY-102 & $10 / 1 / 05$ & $10 / 1 / 05$ & 3,000 & 0 \\
\hline TAL88 & AN-105 & $10 / 1 / 05$ & $10 / 1 / 05$ & 24,000 & 0 \\
\hline SPN87 & SY-102 & $10 / 1 / 05$ & $10 / 1 / 05$ & 25,000 & 0 \\
\hline WATER & AN-105 & $10 / 1 / 05$ & $10 / 1 / 05$ & 35,000 & 0 \\
\hline $34 \mathrm{~L} 87$ & AN-105 & $10 / 1 / 05$ & $10 / 1 / 05$ & 50,000 & 0 \\
\hline EVAPF & AN-105 & $10 / 1 / 05$ & $10 / 1 / 05$ & 120,000 & 0 \\
\hline
\end{tabular}


HNF-2321

Revision 0

Table A.2-1. Complete Transfer List for Case 2.

\begin{tabular}{|c|c|c|c|c|c|}
\hline From & To & Start Date & End Date & $\begin{array}{c}\text { Liquid } \\
\text { (gallons) }\end{array}$ & $\begin{array}{c}\text { Solid } \\
\text { (gallons) }\end{array}$ \\
\hline WATER & SY-102 & $10 / 2 / 05$ & $10 / 2 / 05$ & 1,000 & 0 \\
\hline WATER & AN-10S & $10 / 2 / 05$ & $10 / 2 / 05$ & 22,000 & 0 \\
\hline WATER & AN-105 & $10 / 2 / 05$ & $10 / 2 / 05$ & 5,000 & 0 \\
\hline WATER & SY-102 & $10 / 2 / 05$ & $10 / 2 / 05$ & 5,000 & 0 \\
\hline AN-105 & $A W-102$ & $10 / 1 / 05$ & $10 / 4 / 05$ & 721,700 & 292 \\
\hline EVAPF & AW-102 & $11 / 3 / 05$ & $11 / 3 / 05$ & 35,000 & 0 \\
\hline RETURN 1 & AP-107 & $11 / 14 / 05$ & $11 / 14 / 05$ & 8,675 & 331 \\
\hline P1HLW FEED TANK & HLW-CONTRACTOR & $5 / 2 / 05$ & $11 / 16 / 05$ & 127,200 & 3,269 \\
\hline $\mathrm{AZ}-101$ & P1HLW FEED TANK & $11 / 16 / 05$ & $11 / 17 / 05$ & 127,200 & 3,269 \\
\hline SY-102 & AW-105 & {$[1 / 15 / 05$} & $11 / 17 / 05$ & 413,400 & 16 \\
\hline RETURN 2 & AP-107 & $11 / 22 / 05$ & $\$ 1 / 22 / 05$ & 8,678 & 331 \\
\hline AP-106 & LAW-CONTRACTOR-1 & $6 / 1 / 02$ & $11 / 30 / 05$ & $3,525,000$ & 5,368 \\
\hline AP-102 & AP-106 & $11 / 30 / 05$ & $12 / 2 / 05$ & 279,600 & 53 \\
\hline $\mathrm{AN}-107$ & AP-102 & $12 / 3 / 05$ & $12 / 5 / 05$ & 481,700 & 22 \\
\hline LERF & AN-107-PUMP & $12 / 3 / 05$ & $12 / 5 / 05$ & 72,259 & 0 \\
\hline AN-107 & AP-102 & $12 / 3 / 05$ & $12 / 5 / 05$ & 554,000 & 22 \\
\hline $\mathrm{AX}-102$ & AN-103 & $8 / 25 / 05$ & $12 / 13 / 05$ & 87,597 & 897 \\
\hline AW-105 & AN-105 & $12 / 15 / 05$ & $12 / 17 / 05$ & 413,400 & 16 \\
\hline WATER & SY-102 & $12 / 15 / 05$ & $12 / 17 / 05$ & 426,000 & 0 \\
\hline$S Y-102$ & AW-105 & $1 / 1 / 06$ & $1 / 3 / 06$ & 535,600 & 22,049 \\
\hline RETURN 2 & AP-107 & $1 / 18 / 06$ & $1 / 18 / 06$ & 8,678 & 331 \\
\hline AP-108 & LAW-CONTRACTOR-2 & $6 / 1 / 02$ & $1 / 27 / 06$ & $3,673,000$ & 5,386 \\
\hline AP-104 & AP-108 & $1 / 27 / 06$ & $1 / 28 / 06$ & 283,600 & 46 \\
\hline AN-107 & AP-104 & $1 / 29 / 06$ & $1 / 31 / 06$ & 481,700 & 22 \\
\hline LERF & AN-107-PUMP & $1 / 29 / 06$ & $1 / 31 / 06$ & 72,260 & 0 \\
\hline AN-107 & AP-104 & $1 / 29 / 06$ & $1 / 31 / 06$ & 554,000 & 22 \\
\hline AP-106 & LAW-CONTRACTOR-1 & $12 / 2 / 05$ & $2 / 8 / 06$ & 279,600 & 93 \\
\hline AP-102 & AP-106 & $2 / 8 / 06$ & $2 / 9 / 06$ & 169,400 & 8 \\
\hline AW-105 & AN-105 & $3 / 1 / 06$ & $3 / 2 / 06$ & 279,900 & 10 \\
\hline AP-102 & AP-106 & $3 / 8 / 06$ & $3 / 10 / 06$ & 384,600 & 18 \\
\hline AN-102 & AP-102 & $3 / 11 / 06$ & $3 / 14 / 06$ & 481,700 & 23 \\
\hline LERF & AN-102-PUMP & $3 / 11 / 06$ & $3 / 14 / 06$ & 289,000 & 0 \\
\hline AN-102 & AP-102 & $3 / 11 / 06$ & $3 / 14 / 06$ & 770,700 & 23 \\
\hline $\mathrm{AN}-103$ & AP-101 & $3 / 21 / 06$ & $3 / 22 / 06$ & 252,200 & 4,834 \\
\hline $\mathrm{AN}-103$ & AW-104 & $3 / 22 / 06$ & $3 / 22 / 06$ & 14,430 & 276 \\
\hline$A N-103$ & AW-105 & $4 / 1 / 06$ & $4 / 3 / 06$ & 466,300 & 8,927 \\
\hline AP-108 & LAW-CONTRACTOR-2 & $1 / 28 / 06$ & $4 / 7 / 06$ & 283,500 & 79 \\
\hline AW-102 & 242-A & $4 / 4 / 06$ & $4 / 7 / 06$ & 753,600 & 27 \\
\hline $242-\mathrm{A}$ & AW-106 & $4 / 4 / 06$ & $4 / 7 / 06$ & 483,600 & 27 \\
\hline $242-\mathrm{A}$ & LERF & $4 / 4 / 06$ & $4 / 7 / 06$ & 270,000 & 0 \\
\hline AP-104 & AP-108 & $4 / 7 / 06$ & $4 / 8 / 06$ & 169,400 & 8 \\
\hline AW-106 & $\mathrm{AW}-102$ & $4 / 8 / 06$ & $4 / 10 / 06$ & 483,500 & 18 \\
\hline AW-102 & $242-\mathrm{A}$ & $4 / 24 / 06$ & $4 / 26 / 06$ & 483,600 & 18 \\
\hline $242-\mathrm{A}$ & AW-106 & $4 / 24 / 06$ & $4 / 26 / 06$ & 309,600 & 18 \\
\hline $242-\mathrm{A}$ & LERF & $4 / 24 / 06$ & $4 / 26 / 06$ & 174,000 & 0 \\
\hline AW-106 & AW-102 & $4 / 26 / 06$ & $4 / 28 / 06$ & 309,500 & 12 \\
\hline AP-104 & AP-108 & $5 / 5 / 06$ & $5 / 7 / 06$ & 384,600 & 18 \\
\hline AN-102 & AP-104 & $5 / 8 / 06$ & $5 / 11 / 06$ & 481,700 & 23 \\
\hline LERF & AN-102-PUMP & $5 / 8 / 06$ & $5 / 11 / 06$ & 289,000 & 0 \\
\hline AN-102 & AP-104 & $5 / 8 / 06$ & $5 / 11 / 06$ & 770,700 & 23 \\
\hline$A W-102$ & $242-\mathrm{A}$ & $5 / 12 / 06$ & $5 / 14 / 06$ & 309,600 & 12 \\
\hline $242-A$ & AW-106 & $5 / 12 / 06$ & $5 / 14 / 06$ & 198,100 & 12 \\
\hline $242-A$ & LERF & $5 / 12 / 06$ & $5 / 14 / 06$ & 111,500 & 0 \\
\hline$A W-106$ & AW-102 & $5 / 14 / 06$ & $5 / 15 / 06$ & 198,100 & 9 \\
\hline AW-102 & AW-106 & $5 / 16 / 06$ & $5 / 17 / 06$ & 198,200 & 9 \\
\hline$A N-105$ & AW-102 & $5 / 17 / 06$ & $5 / 20 / 06$ & 693,100 & 0 \\
\hline
\end{tabular}


HNF-2321

Revision 0

Table A.2-1. Complete Transfer List for Case 2.

\begin{tabular}{|c|c|c|c|c|c|}
\hline From & To & Start Date & End Date & $\begin{array}{c}\text { Liquid } \\
\text { (gallons) }\end{array}$ & $\begin{array}{c}\text { Solid } \\
\text { (gallons) }\end{array}$ \\
\hline PIHLW FEED TANK & HLW-CONTRACTOR & $11 / 17 / 05$ & $6 / 2 / 06$ & 127,200 & 3,269 \\
\hline$A Z-101$ & P1HLW_FEED TANK & $6 / 2 / 06$ & $6 / 3 / 06$ & $.127,200$ & 3,269 \\
\hline$A Z-102$ & AN-105 & $6 / 24 / 06$ & $6 / 24 / 06$ & 159,500 & 0 \\
\hline WASH-WATER & AZ-102 & $6 / 24 / 06$ & $6 / 26 / 06$ & 324,000 & 0 \\
\hline $\mathrm{AP}-102$ & $\mathrm{AP}-106$ & $7 / 21 / 06$ & $7 / 25 / 06$ & 770,700 & 23 \\
\hline AN-106 & AP-102 & $7 / 26 / 06$ & $7 / 29 / 06$ & 549,100 & 25 \\
\hline LERF & AN-106-PUMP & $7 / 26 / 06$ & $7 / 29 / 06$ & 98,837 & 0 \\
\hline AN-106 & AP-102 & $7 / 26 / 06$ & $7 / 29 / 06$ & 647,900 & 25 \\
\hline$A Z-102$ & AN-105 & $8 / 14 / 06$ & $8 / 16 / 06$ & 320,100 & 0 \\
\hline WASH-WATER & AZ-102 & $8 / 17 / 06$ & $8 / 19 / 06$ & 324,000 & 0 \\
\hline AW-102 & $242-\mathrm{A}$ & $9 / 2 / 06$ & $9 / 6 / 06$ & 693,000 & 25 \\
\hline 242-A & AW-106 & $9 / 2 / 06$ & $9 / 6 / 06$ & 443,900 & 25 \\
\hline $242-\mathrm{A}$ & LERF & $9 / 2 / 06$ & $9 / 6 / 06$ & 249,200 & 0 \\
\hline AW-106 & AW-102 & $9 / 6 / 06$ & $9 / 10 / 06$ & 642,000 & 26 \\
\hline AP-104 & AP-108 & $9 / 17 / 06$ & $9 / 21 / 06$ & 770,700 & 23 \\
\hline AN-106 & AP-104 & $9 / 22 / 06$ & 9/25/06 & 549,100 & 25 \\
\hline LERF & AN-106-PUMP & $9 / 22 / 06$ & $9 / 25 / 06$ & 98,837 & 0 \\
\hline AN-106 & AP-104 & $9 / 22 / 06$ & $9 / 25 / 06$ & 647,900 & 25 \\
\hline AW-102 & $242-A$ & $9 / 24 / 06$ & $9 / 27 / 06$ & 642,000 & 26 \\
\hline $242-\mathrm{A}$ & AW-106 & $9 / 24 / 06$ & $9 / 27 / 06$ & 412,200 & 26 \\
\hline $242-\mathrm{A}$ & LERF & $9 / 24 / 06$ & $9 / 27 / 06$ & 229,800 & 0 \\
\hline SY-101 & SY-102 & $9 / 23 / 06$ & $9 / 28 / 06$ & 537,800 & 5,486 \\
\hline LERF & SY-101-PUMP & 9/23/06 & $9 / 28 / 06$ & 532,400 & 0 \\
\hline SY-101 & SY-102 & 9/23/06 & $9 / 28 / 06$ & $1,082,000$ & 611 \\
\hline$A W-106$ & AW-102 & $9 / 27 / 06$ & $9 / 29 / 06$ & 412,100 & 18 \\
\hline $34 \mathrm{~L} 87$ & AN-105 & $10 / 1 / 06$ & $10 / 1 / 06$ & $.7,000$ & 0 \\
\hline TNS88 & AN-105 & $10 / 1 / 06$ & $10 / 1 / 06$ & 750 & 250 \\
\hline WESF & AN-105 & $10 / 1 / 06$ & $10 / 1 / 06$ & 5,000 & 0 \\
\hline TAL88 & AN-105 & $10 / 1 / 06$ & $10 / 1 / 06$ & 25,000 & 0 \\
\hline SPN87 & SY-102 & $10 / 1 / 06$ & $10 / 1 / 06$ & 25,000 & 0 \\
\hline $34 \mathrm{~L} 87$ & AN-105 & $10 / 1 / 06$ & $10 / 1 / 06$ & 50,000 & 0 \\
\hline WATER & AN-105 & $10 / 1 / 06$ & $10 / 1 / 06$ & 35,000 & 0 \\
\hline EVAPF & AN-105 & $10 / 1 / 06$ & $10 / 1 / 06$ & 120,000 & 0 \\
\hline WATER & AN-105 & $10 / 2 / 06$ & $10 / 2 / 06$ & 3,000 & 0 \\
\hline WATER & AN-105 & $10 / 2 / 06$ & $10 / 2 / 06$ & 22,000 & 0 \\
\hline WATER & AN-105 & $10 / 2 / 06$ & $10 / 2 / 06$ & 5,000 & 0 \\
\hline WATER & $\$ Y-102$ & $10 / 2 / 06$ & $10 / 2 / 06$ & 5,000 & 0 \\
\hline EVAPF & AW-102 & $10 / 4 / 06$ & $10 / 4 / 06$ & 50,000 & 0 \\
\hline SY-102 & $\mathrm{AN}-102$ & $10 / 6 / 06$ & $10 / 9 / 06$ & $1,027,000$ & 1,532 \\
\hline$A W-102$ & 242-A & $10 / 13 / 06$ & $10 / 15 / 06$ & 462,200 & 20 \\
\hline $242-\hat{A}$ & AW-106 & $10 / 13 / 06$ & $10 / 15 / 06$ & 310,800 & 20 \\
\hline $242-\mathrm{A}$ & LERF & $10 / 13 / 06$ & $10 / 15 / 06$ & 151,400 & 0 \\
\hline AW-106 & AW-101 & $10 / 15 / 06$ & $10 / 17 / 06$ & 310,800 & 14 \\
\hline AN-10S & AW-102 & $10 / 16 / 06$ & $10 / 19 / 06$ & 749,400 & 0 \\
\hline AZ-102 & AN-105 & $10 / 20 / 06$ & $10 / 21 / 06$ & 322,700 & 0 \\
\hline SY-101 & SY-102 & $10 / 17 / 06$ & $10 / 22 / 06$ & 537,800 & 5,486 \\
\hline LERF & SY-101-PUMP & $10 / 17 / 06$ & $10 / 22 / 06$ & 532,400 & 0 \\
\hline SY-101 & SY-102 & $10 / 17 / 06$ & $10 / 22 / 06$ & $1,082,000$ & 611 \\
\hline WASH-WATER & $A Z-102$ & $10 / 22 / 06$ & $10 / 23 / 06$ & 150,500 & 0 \\
\hline SY-102 & AN-107 & $10 / 30 / 06$ & $11 / 2 / 06$ & 983,800 & 641 \\
\hline EVAPF & AW-102 & $11 / 3 / 06$ & $11 / 3 / 06$ & 35,000 & 0 \\
\hline SY-101 & SY-102 & $11 / 10 / 06$ & $11 / 10 / 06$ & 0 & 0 \\
\hline P1HLW FEED TANK & HLW-CONTRACTOR & $6 / 3 / 06$ & $12 / 18 / 06$ & 127,200 & 3,269 \\
\hline $\mathrm{AZ}-101$ & PIHLW FEED TANK & $12 / 18 / 06$ & $12 / 18 / 06$ & 127,200 & 3,269 \\
\hline AN-103 & AN-106 & $12 / 24 / 06$ & $12 / 26 / 06$ & 368,400 & 7,045 \\
\hline RETURN 1 & AP-107 & $2 / 25 / 07$ & $2 / 25 / 07$ & 8,735 & 265 \\
\hline
\end{tabular}


HNF-2321

Revision 0

Table A.2-1. Complete Transfer List for Case 2.

\begin{tabular}{|c|c|c|c|c|c|}
\hline From & To & Start Date & End Date & $\begin{array}{c}\text { Liquid } \\
\text { (gallons) }\end{array}$ & $\begin{array}{c}\text { Solid } \\
\text { (gallons) }\end{array}$ \\
\hline AP-102 & AP-106 & $3 / 18 / 07$ & $3 / 21 / 07$ & 647,900 & 25 \\
\hline AN-102 & AP-102 & $3 / 22 / 07$ & $3 / 27 / 07$ & $1,013,000$ & 44 \\
\hline SY-103 & SY-102 & $3 / 30 / 07$ & $4 / 1 / 07$ & 350,000 & 9,759 \\
\hline LERF & SY-103-PUMP & $3 / 30 / 07$ & $4 / 1 / 07$ & 183,500 & 0 \\
\hline SX-103 & SY-102 & $3 / 30 / 07$ & $4 / 1 / 07$ & 537,500 & 1,449 \\
\hline $\mathrm{AW}-102$ & $242-\mathrm{A}$ & $4 / 5 / 07$ & $4 / 8 / 07$ & 784,600 & 27 \\
\hline $242-A$ & AW-106 & $4 / 5 / 07$ & $4 / 8 / 07$ & 502,600 & 27 \\
\hline $242-\mathrm{A}$ & LERF & $4 / 5 / 07$ & $4 / 8 / 07$ & 282,000 & 0 \\
\hline$A \mathrm{~W}-106$ & AW-102 & $4 / 9 / 07$ & 4/11/07 & 502,500 & 18 \\
\hline SY-102 & AN-102 & $4 / 9 / 07$ & $4 / 11 / 07$ & 720,700 & 1,528 \\
\hline AW-102 & $242-\mathrm{A}$ & $4 / 25 / 07$ & $4 / 28 / 07$ & 502,600 & 18 \\
\hline $242-\mathrm{A}$ & AW-106 & $4 / 25 / 07$ & $4 / 28 / 07$ & 322,600 & 18 \\
\hline $242-\mathrm{A}$ & LERF & $4 / 25 / 07$ & $4 / 28 / 07$ & 180,000 & 0 \\
\hline AW-106 & AW-102 & $4 / 28 / 07$ & $4 / 29 / 07$ & 322,500 & 12 \\
\hline$A W-102$ & $242-\mathrm{A}$ & $5 / 13 / 07$ & $5 / 15 / 07$ & 322,600 & 12 \\
\hline $242-\mathrm{A}$ & AW-106 & $5 / 13 / 07$ & $5 / 15 / 07$ & 206,100 & 12 \\
\hline $242-\mathrm{A}$ & LERF & $5 / 13 / 07$ & $5 / 15 / 07$ & 116,500 & 0 \\
\hline AW-106 & AW-102 & $5 / 15 / 07$ & $5 / 16 / 07$ & 206,000 & 8 \\
\hline AW-102 & AN-105 & $5 / 17 / 07$ & $5 / 18 / 07$ & 206,200 & 8 \\
\hline AP-104 & AP-108 & $5 / 15 / 07$ & $5 / 18 / 07$ & 647,900 & 25 \\
\hline AN-107 & AP-104 & $5 / 19 / 07$ & $5 / 23 / 07$ & 978,400 & 43 \\
\hline SY-103 & SY-102 & $5 / 27 / 07$ & $5 / 29 / 07$ & 350,000 & 9,759 \\
\hline LERF & SY-103-PUMP & $5 / 27 / 07$ & $5 / 29 / 07$ & 183,500 & 0 \\
\hline SY-103 & SY-102 & $5 / 27 / 07$ & $5 / 29 / 07$ & 537,500 & 1,449 \\
\hline SY-102 & AN-107 & $6 / 6 / 07$ & $6 / 7 / 07$ & 537,500 & 1,434 \\
\hline P1HLW FEED TANK & HLW-CONTRACTOR & $12 / 18 / 06$ & $7 / 4 / 07$ & 127,200 & 3,269 \\
\hline $\mathrm{AZ}-101$ & PIHLW FEED TANK & $7 / 4 / 07$ & $7 / 5 / 07$ & 127,200 & 3,269 \\
\hline S-103 & SY-101 & $2 / 13 / 07$ & $8 / 7 / 07$ & 712,300 & 1,011 \\
\hline AN-103 & AN-106 & $8 / 11 / 07$ & $8 / 15 / 07$ & 721,800 & 2,298 \\
\hline WESF & AN-105 & $10 / 1 / 07$ & $10 / 1 / 07$ & 5,000 & 0 \\
\hline TNS88 & AN-105 & $10 / 1 / 07$ & $10 / 1 / 07$ & 750 & 250 \\
\hline TAL88 & AN-105 & $10 / 1 / 07$ & $10 / 1 / 07$ & 25,000 & 0 \\
\hline SPN87 & SY-102 & $10 / 1 / 07$ & $10 / 1 / 07$ & 25,000 & 0 \\
\hline $34 \mathrm{~L} 87$ & AN-105 & $10 / 1 / 07$ & $10 / 1 / 07$ & 50,000 & 0 \\
\hline WATER & AN-105 & $10 / 1 / 07$ & $10 / 1 / 07$ & 35,000 & 0 \\
\hline EVAPF & AN-105 & $10 / 1 / 07$ & $10 / 1 / 07$ & 120,000 & 0 \\
\hline WATER & AN-105 & $10 / 2 / 07$ & $10 / 2 / 07$ & 5,000 & 0 \\
\hline WATER & SY-102 & $10 / 2 / 07$ & $10 / 2 / 07$ & 5,000 & 0 \\
\hline WATER & AN-105 & $10 / 2 / 07$ & $10 / 2 / 07$ & 22,000 & 0 \\
\hline AN-105 & AW-102 & $10 / 1 / 07$ & $10 / 5 / 07$ & 791,500 & 314 \\
\hline EVAPF & AW-102 & $11 / 3 / 07$ & $11 / 3 / 07$ & 35,000 & 0 \\
\hline AP-102 & AP-106 & $11 / 2 / 07$ & $11 / 7 / 07$ & $1,013,000$ & 44 \\
\hline AN-102 & AP-102 & $11 / 8 / 07$ & $11 / 11 / 07$ & 707,100 & 31 \\
\hline $5 X-113$ & SY-103 & $9 / 1 / 07$ & $12 / 7 / 07$ & 10,322 & 399 \\
\hline AP-104 & AP-108 & $12 / 30 / 07$ & $1 / 4 / 08$ & 978,400 & 43 \\
\hline AN-107 & AP-104 & $1 / 5 / 08$ & $1 / 7 / 08$ & 524,700 & 23 \\
\hline P1HLW FEED TANK & HLW-CONTRACTOR & $7 / 5 / 07$ & $1 / 18 / 08$ & $\$ 27,200$ & 3,269 \\
\hline AZ-102 & P1HLW_FEED TANK & $1 / 18 / 08$ & $1 / 19 / 08$ & 137,700 & 4,774 \\
\hline SY-101 & AN-102 & $2 / 9 / 08$ & $2 / 13 / 08$ & 996,600 & 1,676 \\
\hline $\mathrm{AW}-102$ & $242-\mathrm{A}$ & $4 / 3 / 08$ & $4 / 7 / 08$ & 823,300 & 29 \\
\hline $242-\mathrm{A}$ & AW-106 & $4 / 3 / 08$ & $4 / 7 / 08$ & 529,200 & 29 \\
\hline $242-\mathrm{A}$ & LERF & $4 / 3 / 08$ & $4 / 7 / 08$ & 294,100 & 0 \\
\hline $\mathrm{AN}-103$ & AN-107 & $4 / 6 / 08$ & $4 / 8 / 08$ & 401,300 & 1,276 \\
\hline AW-106 & AW-102 & $4 / 8 / 08$ & $4 / 11 / 08$ & 529,100 & 19 \\
\hline AN-102 & AN-107 & $4 / 8 / 08$ & $4 / 11 / 08$ & 557,200 & 4,431 \\
\hline$S X-112$ & SY-103 & $12 / 7 / 07$ & $4 / 17 / 08$ & 397,100 & 16,406 \\
\hline
\end{tabular}


HNF-2321

Revision 0

Table A.2-1. Complete Transfer List for Case 2.

\begin{tabular}{|c|c|c|c|c|c|}
\hline From & To & Start Date & End Date & $\begin{array}{c}\text { Liquid } \\
\text { (gallons) }\end{array}$ & $\begin{array}{c}\text { Solid } \\
\text { (gallons) }\end{array}$ \\
\hline $\mathrm{AW}-102$ & $242-A$ & $4 / 25 / 08$ & $4 / 27 / 08$ & 529,200 & 19 \\
\hline $242-\mathrm{A}$ & AW-106 & $4 / 25 / 08$ & $4 / 27 / 08$ & 337,200 & 19 \\
\hline $242-\mathrm{A}$ & LERF & $4 / 25 / 08$ & $4 / 27 / 08$ & 192,000 & 0 \\
\hline AW-106 & AW-102 & $4 / 27 / 08$ & $4 / 29 / 08$ & 337,100 & 13 \\
\hline AW-102 & $242-\mathrm{A}$ & $5 / 13 / 08$ & $5 / 15 / 08$ & 337,200 & 13 \\
\hline $242-\mathrm{A}$ & AW-106 & $5 / 13 / 08$ & $5 / 15 / 08$ & 216,300 & 13 \\
\hline $242-A$ & LERF & $5 / 13 / 08$ & $.5 / 15 / 08$ & 120,900 & 0 \\
\hline AW-106 & $A W-102$ & $5 / 15 / 08$ & $5 / 16 / 08$ & 216,300 & 8 \\
\hline AW-102 & AN-105 & $5 / 17 / 08$ & $5 / 18 / 08$ & 216,500 & 8 \\
\hline$A X-101$ & AN-103 & $12 / 13 / 05$ & $8 / 2 / 08$ & $2,149,000$ & 6,072 \\
\hline C-202 & AN-103 & $8 / 2 / 08$ & $8 / 6 / 08$ & 1,181 & 28 \\
\hline$S X-110$ & SY-103 & $4 / 17 / 08$ & $8 / 20 / 08$ & 212,700 & 8,909 \\
\hline AP-102 & AP-106 & $9 / 7 / 08$ & $9 / 11 / 08$ & 707,100 & 31 \\
\hline WESF & AN-105 & $10 / 1 / 08$ & $10 / 1 / 08$ & 5,000 & 0 \\
\hline TNS88 & AN-105 & $10 / 1 / 08$ & $10 / 1 / 08$ & 750 & 250 \\
\hline SPN87 & SY-102 & $10 / 1 / 08$ & $10 / 1 / 08$ & 25,000 & 0 \\
\hline TAL88 & AN-105 & $10 / 1 / 08$ & $10 / 1 / 08$ & 25,000 & 0 \\
\hline \begin{tabular}{|l} 
WATER \\
\end{tabular} & AN-105 & $10 / 1 / 08$ & $10 / 1 / 08$ & 35,000 & 0 \\
\hline $34 \mathrm{~L} 87$ & AN-105 & $10 / 1 / 08$ & $10 / 1 / 08$ & 50,000 & 0 \\
\hline EVAPF & AN-105 & $10 / 1 / 08$ & $10 / 1 / 08$ & 120,000 & 0 \\
\hline WATER & AN-105 & $10 / 2 / 08$ & $10 / 2 / 08$ & 22,000 & 0 \\
\hline WATER & SY-102 & $10 / 2 / 08$ & $10 / 2 / 08$ & 5,000 & 0 \\
\hline WATER & $\mathrm{AN}-105$ & $10 / 2 / 08$ & $10 / 2 / 08$ & 5,000 & 0 \\
\hline EVAPF & $A W-102$ & $10 / 4 / 08$ & $10 / 4 / 08$ & 50,000 & 0 \\
\hline SX-115 & SY-103 & $8 / 20 / 08$ & $10 / 21 / 08$ & 42,100 & 1,556 \\
\hline $\mathrm{A}-105$ & AN-103 & $8 / 6 / 08$ & $10 / 29 / 08$ & 50,372 & 1,545 \\
\hline EVAPF & AW-102 & $11 / 3 / 08$ & $11 / 3 / 08$ & 35,000 & 0 \\
\hline C-203 & $\mathrm{AN}-103$ & $10 / 29 / 08$ & $11 / 5 / 08$ & 6,307 & 150 \\
\hline AP-104 & AP-108 & $11 / 4 / 08$ & $11 / 6 / 08$ & 524,700 & 23 \\
\hline P1HLW FEED TANK & HLW-CONTRACTOR & $1 / 19 / 08$ & $12 / 22 / 08$ & 137,700 & 4,774 \\
\hline AZ -102 & P1HLW FEED TANK & $12 / 22 / 08$ & $12 / 22 / 08$ & 137,700 & 4,774 \\
\hline $\mathrm{U}-202$ & UA-2 & $1 / 3 / 09$ & $1 / 10 / 09$ & 27,411 & 1,061 \\
\hline $\mathrm{U}-203$ & UA-2 & $1 / 10 / 09$ & $1 / 16 / 09$ & 13,895 & 537 \\
\hline C-108 & AN-103 & $11 / 5 / 08$ & $1 / 21 / 09$ & 83,934 & 3,840 \\
\hline$\hat{\mathbf{U}}-110$ & UA-2. & $1 / 16 / 09$ & $3 / 10 / 09$ & 99,290 & 4,252 \\
\hline UA-2 & $S Y-102$ & $3 / 11 / 09$ & $3 / 12 / 09$ & 173,900 & 5,847 \\
\hline C-111 & AN-103 & $1 / 21 / 09$ & $4 / 4 / 09$ & 176,500 & 7,080 \\
\hline AP-106 & LAW-CONTRACTOR-1 & $2 / 9 / 06$ & $5 / 20 / 09$ & $3,693,000$ & 158 \\
\hline RETURN 1 & AP-107 & $5 / 21 / 09$ & $5 / 21 / 09$ & 2,581 & 96 \\
\hline AP-108 & LAW-CONTRACTOR-2 & $4 / 8 / 06$ & $5 / 21 / 09$ & $3,476,000$ & 146 \\
\hline RETURN 2 & AP-107 & $5 / 22 / 09$ & $5 / 22 / 09$ & 7,544 & 287 \\
\hline $\mathrm{U}-110$ & UA-1 & $3 / 10 / 09$ & $5 / 27 / 09$ & 143,800 & 6,160 \\
\hline UA-1 & SY-102 & $5 / 28 / 09$ & $5 / 29 / 09$ & 186,800 & 6,157 \\
\hline $\mathrm{U}-110$ & UA-2 & $5 / 27 / 09$ & $5 / 30 / 09$ & 4,829 & 207 \\
\hline AP-107 & AZ-101 & $6 / 1 / 09$ & $6 / 2 / 09$ & 271,400 & 9,319 \\
\hline AN-102 & AP-107 & $6 / 11 / 09$ & $6 / 13 / 09$ & 551,400 & 4,381 \\
\hline SY-101 & AN-102 & $6 / 13 / 09$ & $6 / 17 / 09$ & $1,115,000$ & 1,795 \\
\hline AN-102 & AP-107 & $6 / 18 / 09$ & $6 / 21 / 09$ & 559,700 & 985 \\
\hline S-105 & SY-101 & $8 / 7 / 07$ & $7 / 12 / 09$ & $1,518,000$ & 2,459 \\
\hline U-101 & UA-2 & $5 / 30 / 09$ & $7 / 30 / 09$ & 27,955 & 668 \\
\hline TNS88 & AN-105 & $10 / 1 / 09$ & $10 / 1 / 09$ & 750 & 250 \\
\hline WESF & AN-I0S & $10 / 1 / 09$ & $10 / 1 / 09$ & 5,000 & 0 \\
\hline TAL88 & AN-10S & $10 / 1 / 09$ & $10 / 1 / 09$ & 26,000 & 0 \\
\hline $34 \mathrm{~L} 87$ & AN-105 & $10 / 1 / 09$ & $10 / 1 / 09$ & 50,000 & 0 \\
\hline WATER & AN-105 & $10 / 1 / 09$ & $10 / 1 / 09$ & 35,000 & 0 \\
\hline $34 \mathrm{~L} 87$ & AN-105 & $10 / 1 / 09$ & $10 / 1 / 09$ & 7,000 & 0 \\
\hline
\end{tabular}


HNF-2321

Revision 0

Table A.2-1. Complete Transfer List for Case 2.

\begin{tabular}{|c|c|c|c|c|c|}
\hline From & To & Start Date & End Date & $\begin{array}{c}\text { Liquid } \\
\text { (gallons) }\end{array}$ & $\begin{array}{c}\text { Solid } \\
\text { (gallons) }\end{array}$ \\
\hline SPN87 & $S Y-102$ & $10 / 1 / 09$ & $10 / 1 / 09$ & 25,000 & 0 \\
\hline EVAPF & AN-105 & $10 / 1 / 09$ & $10 / 1 / 09$ & 120,000 & 0 \\
\hline WATER & AN-105 & $10 / 2 / 09$ & $10 / 2 / 09$ & 5,000 & 0 \\
\hline WATER & AN-105 & $10 / 2 / 09$ & $10 / 2 / 09$ & 3,000 & 0 \\
\hline WATER & SY-102 & $10 / 2 / 09$ & $10 / 2 / 09$ & 5,000 & 0 \\
\hline WATER & AN-105 & $10 / 2 / 09$ & $10 / 2 / 09$ & 22,000 & 0 \\
\hline $\mathrm{AN}-105$ & $\mathrm{AW}-102$ & $10 / 1 / 09$ & $10 / 4 / 09$ & 750,100 & 325 \\
\hline EVAPF & AW-102 & $11 / 3 / 09$ & $11 / 3 / 09$ & 35,000 & 0 \\
\hline $\mathrm{U}-104$ & UA-2 & $7 / 30 / 09$ & $11 / 9 / 09$ & 50,908 & 1,221 \\
\hline PIHLW FEED TANK & HLW-CONTRACTOR & $12 / 22 / 08$ & $11 / 25 / 09$ & 137,700 & 4,774 \\
\hline $\mathrm{U}-112$ & UA-2 & $11 / 9 / 09$ & $12 / 5 / 09$ & 58,521 & 2,330 \\
\hline UA-2 & SY-102 & $12 / 5 / 09$ & $12 / 6 / 09$ & 154,400 & 4,426 \\
\hline $\mathrm{U}-112$ & UA-1 & $12 / 5 / 09$ & $1 / 17 / 10$ & 102,000 & 4,061 \\
\hline $\mathrm{U}-204$ & UA-1 & $1 / 17 / 10$ & $1 / 23 / 10$ & 13,004 & 503 \\
\hline U-201 & UA-1 & $1 / 23 / 10$ & $1 / 27 / 10$ & 13,912 & 538 \\
\hline $\mathrm{UA}-1$ & SY-102 & $1 / 28 / 10$ & $1 / 28 / 10$ & 146,500 & 5,102 \\
\hline U-201 & UA-2 & $1 / 27 / 10$ & $1 / 30 / 10$ & 13,499 & 522 \\
\hline U-106 & UA-2 & $1 / 30 / 10$ & $3 / 1 / 10$ & 134,400 & 338 \\
\hline UA-2 & SY-102 & $3 / 2 / 10$ & $3 / 3 / 10$ & 151,500 & 862 \\
\hline U-106 & UA-1 & $3 / 1 / 10$ & $4 / 4 / 10$ & 149,500 & 376 \\
\hline $\mathrm{AW}-102$ & $242-\mathrm{A}$ & $4 / 4 / 10$ & $4 / 8 / 10$ & 866,900 & 30 \\
\hline $242-\mathrm{A}$ & AW-106 & $4 / 4 / 10$ & $4 / 8 / 10$ & 554,900 & 30 \\
\hline $242-\mathrm{A}$ & LERF & $4 / 4 / 10$ & $4 / 8 / 10$ & 312,000 & 0 \\
\hline AW-106 & AW-102 & $4 / 9 / 10$ & $4 / 11 / 10$ & 554,800 & 20 \\
\hline $\mathrm{AW}-102$ & $242-A$ & $4 / 26 / 10$ & $4 / 28 / 10$ & 554,900 & 20 \\
\hline $242-\mathrm{A}$ & AW-106 & $4 / 26 / 10$ & $4 / 28 / 10$ & 356,500 & 20 \\
\hline $242-\mathrm{A}$ & LERF & $4 / 26 / 10$ & $4 / 28 / 10$ & 198,400 & 0 \\
\hline AW-106 & AW-102 & $4 / 28 / 10$ & $4 / 30 / 10$ & 356,400 & 13 \\
\hline U-106 & UA-2 & $4 / 4 / 10$ & $5 / 7 / 10$ & 149,500 & 376 \\
\hline AW-102 & $242-A$ & $5 / 14 / 10$ & $5 / 16 / 10$ & 356,500 & 13 \\
\hline $242-\mathrm{A}$ & AW-106 & $5 / 14 / 10$ & $5 / 16 / 10$ & 227,900 & 13 \\
\hline $242-\mathrm{A}$ & LERF & $5 / 14 / 10$ & $5 / 16 / 10$ & 128,600 & 0 \\
\hline AW-106 & AW-102 & $5 / 16 / 10$ & $5 / 17 / 10$ & 227,800 & 9 \\
\hline $\mathrm{AW}-102$ & $242-A$ & $5 / 31 / 10$ & $6 / 2 / 10$ & 227,900 & 9 \\
\hline $242-\mathrm{A}$ & AW-106 & $5 / 31 / 10$ & $6 / 2 / 10$ & 144,500 & 9 \\
\hline $242-\mathrm{A}$ & LERF & $5 / 31 / 10$ & $6 / 2 / 10$ & 83,415 & 0 \\
\hline AW-106 & AW-102 & $6 / 2 / 10$ & $6 / 2 / 10$ & 144,500 & 6 \\
\hline AW-102 & AN-105 & $6 / 4 / 10$ & $6 / 4 / 10$ & 144,600 & 6 \\
\hline TNS88 & AN-105 & $10 / 1 / 10$ & $10 / 1 / 10$ & 750 & 250 \\
\hline TAZ 88 & AN-105 & $10 / 1 / 10$ & $10 / 1 / 10$ & 26,000 & 0 \\
\hline WATER & AN-10S & $10 / 1 / 10$ & $10 / 1 / 10$ & 25,000 & 0 \\
\hline WESF & AN-105 & $10 / 1 / 10$ & $10 / 1 / 10$ & 5,000 & 0 \\
\hline SPN87 & SY-102 & $10 / 1 / 10$ & $10 / 1 / 10$ & 25,000 & 0 \\
\hline WATER & AN-105 & $10 / 1 / 10$ & $10 / 1 / 10$ & 35,000 & 0 \\
\hline $34 \mathrm{~L} 87$ & AN-105 & $10 / 1 / 10$ & $10 / 1 / 10$ & 50,000 & 0 \\
\hline EVAPF & AN-105 & $10 / 1 / 10$ & $10 / 1 / 10$ & 120,000 & 0 \\
\hline WATER & AN-105 & $10 / 2 / 10$ & $10 / 2 / 10$ & 5,000 & 0 \\
\hline WATER & SY-102 & $10 / 2 / 10$ & $10 / 2 / 10$ & 5,000 & 0 \\
\hline WATER & AN-105 & $10 / 2 / 10$ & $10 / 2 / 10$ & 22,000 & 0 \\
\hline EVAPF & AW-102 & $10 / 4 / 10$ & $10 / 4 / 10$ & 50,000 & 0 \\
\hline EVAPF & AW-102 & $11 / 3 / 10$ & $11 / 3 / 10$ & 35,000 & 0 \\
\hline WATER & AN-105 & $1 / 1 / 11$ & $1 / 1 / 11$ & 25,000 & 0 \\
\hline BY-110 & $\mathrm{BA}-3$ & $1 / 3 / 11$ & $2 / 8 / 11$ & 147,400 & 2,633 \\
\hline TX-109 & TA-5 & $1 / 3 / 11$ & $2 / 10 / 11$ & 144,100 & 5,925 \\
\hline TY-101 & $\mathrm{TA}-4$ & $1 / 3 / 11$ & $2 / 22 / 11$ & 146,500 & 3,494 \\
\hline$\Upsilon-106$ & TA-6 & $1 / 3 / 11$ & $3 / 2 / 11$ & 74,648 & 2,929 \\
\hline
\end{tabular}


HNF-2321

Revision 0

Table A.2-1. Complete Transfer List for Case 2.

\begin{tabular}{|c|c|c|c|c|c|}
\hline From & To & Start Date & End Date & $\begin{array}{c}\text { Liquid } \\
\text { (gallons) }\end{array}$ & $\begin{array}{c}\text { Solid } \\
\text { (gallons) }\end{array}$ \\
\hline B-112 & $\overline{\mathrm{BA}-1}$ & $1 / 3 / 11$ & $3 / 9 / 11$ & 75,213 & 1,083 \\
\hline BY-110 & $\mathrm{BA}-4$ & $2 / 8 / 11$ & $3 / 16 / 11$ & 147,400 & 2,633 \\
\hline TX-109 & TA-3 & $2 / 10 / 11$ & $3 / 19 / 11$ & 144,100 & 5,925 \\
\hline T-101 & $\mathrm{TA}-6$ & $3 / 2 / 11$ & $3 / 25 / 11$ & 61,377 & 2,346 \\
\hline WATER & $\mathrm{AN}-105$ & $4 / 1 / 11$ & $4 / 1 / 11$ & 25,000 & 0 \\
\hline$B X-102$ & $\mathrm{BA}-2$ & $1 / 3 / 11$ & $4 / 4 / 11$ & 52,548 & 2,443 \\
\hline B-111 & $B A-1$ & $3 / 9 / 11$ & $4 / 9 / 11$ & 72,630 & 2,383 \\
\hline TY-101 & TA-2 & $2 / 22 / 11$ & $4 / 13 / 11$ & 146,500 & 3,494 \\
\hline BY-110 & $\mathrm{BA}-5$ & $3 / 16 / 11$ & $4 / 21 / 11$ & 147,400 & 2,633 \\
\hline TX-109 & TA-1 & $3 / 19 / 11$ & $4 / 26 / 11$ & 144,100 & 5,925 \\
\hline$B X-112$ & $\mathrm{BA}-2$ & $4 / 4 / 11$ & $5 / 2 / 11$ & 67,370 & 2,937 \\
\hline BA-3 & AP-104 & $6 / 1 / 11$ & $6 / 2 / 11$ & 155,600 & 2,631 \\
\hline $\mathrm{BA}-4$ & AP-108 & $6 / 1 / 11$ & $6 / 2 / 11$ & 155,600 & 2,631 \\
\hline $\mathrm{BA}-1$ & AP-104 & $6 / 2 / 11$ & $6 / 2 / 11$ & 157,300 & 3,464 \\
\hline BA-5 & AP- 108 & $6 / 2 / 11$ & $6 / 3 / 11$ & 155,600 & 2,631 \\
\hline BA-2 & AP-104 & $6 / 2 / 11$ & $6 / 3 / 11$ & 174,500 & 5,377 \\
\hline AN-102 & AP-102 & $6 / 1 / 11$ & $6 / 3 / 11$ & 555,800 & 978 \\
\hline AN-103 & AP-106 & $6 / 1 / 11$ & $6 / 6 / 11$ & $1,099,000$ & 17,131 \\
\hline AN-103 & AP-102 & $6 / 6 / 11$ & $6 / 6 / 11$ & 4,514 & 69 \\
\hline SY-103 & $\mathrm{AN}-102$ & $6 / 3 / 11$ & $6 / 7 / 11$ & $1,081,000$ & 35,223 \\
\hline B-111 & BA-6 & $4 / 9 / 11$ & $6 / 11 / 11$ & 145,200 & 4,764 \\
\hline AN-102 & AP-102 & $6 / 8 / 11$ & $6 / 11 / 11$ & 538,000 & 17,107 \\
\hline BA- 6 & AP- 108 & $6 / 11 / 11$ & $6 / 12 / 11$ & 159,400 & 4,762 \\
\hline AN-102 & AP-104 & 6/11/11 & $6 / 14 / 11$ & 545,100 & 17,317 \\
\hline SY-101 & AN-102 & $6 / 14 / 11$ & $6 / 18 / 11$ & $1,114,000$ & 2,136 \\
\hline$A N-102$ & AP-104 & $6 / 18 / 11$ & $6 / 18 / 11$ & 55,096 & 144 \\
\hline AN-102 & AP-108 & $6 / 18 / 11$ & $6 / 22 / 11$ & 634,300 & 1,657 \\
\hline WATER & AN-105 & $7 / 1 / 11$ & $7 / 1 / 11$ & 25,000 & 0 \\
\hline BY-110 & $\mathrm{BA}-4$ & $6 / 2 / 11$ & $7 / 8 / 11$ & 147,300 & 2,631 \\
\hline $\mathrm{BX}-112$ & BA-3 & $6 / 2 / 11$ & $8 / 2 / 11$ & 143,600 & 6,262 \\
\hline A-106 & AN-103 & $4 / 4 / 09$ & $8 / 8 / 11$ & 253,300 & 5,796 \\
\hline B-111 & BA-1 & $6 / 11 / 11$ & $8 / 12 / 11$ & 145,100 & 4,761 \\
\hline$S X-111$ & SY-103 & $10 / 21 / 08$ & $8 / 13 / 11$ & 451,500 & 19,767 \\
\hline BY-110 & BA-5 & $7 / 8 / 11$ & $8 / 13 / 11$ & 147,300 & 2,631 \\
\hline B-111 & $\mathrm{BA}-6$ & $8 / 12 / 11$ & $8 / 13 / 11$ & 719 & 24 \\
\hline $\mathrm{BX}-112$ & $\mathrm{BA}-2$ & $8 / 2 / 11$ & $9 / 4 / 11$ & 78,662 & 3,430 \\
\hline$B Y-110$ & $\mathrm{BA}-6$ & $8 / 13 / 11$ & $9 / 18 / 11$ & 146,500 & 2,618 \\
\hline B-103 & BA-2 & $9 / 4 / 11$ & $9 / 22 / 11$ & 41,507 & 78 \\
\hline
\end{tabular}


HNF-2321

Revision 0

Table A.2-2. Phase 1 Low-Activity Waste Feed Staging Transfers for Case 2.

\begin{tabular}{|c|c|c|c|c|c|c|c|}
\hline $\begin{array}{l}\text { Boot } \\
\text { Strap }\end{array}$ & From & To & Start Date & End Date & $\begin{array}{c}\text { Liquid } \\
\text { (gallons) }\end{array}$ & $\begin{array}{c}\text { Solid } \\
\text { (gallons) }\end{array}$ & Notes. \\
\hline $\mathrm{x}$ & AP-106 & $\mathrm{AW}-105$ & $3 / 15 / 99$ & $3 / 15 / 99$ & 174,300 & 6 & Empty 6AP \\
\hline $\mathrm{X}$ & AP-108 & $A W-105$ & $3 / 15 / 99$ & $3 / 15 / 99$ & 500 & 0 & Empty 8AP \\
\hline $\mathrm{X}$ & $\mathrm{AZ}-101$ & AY-101 & $8 / 17 / 00$ & $8 / 20 / 00$ & 685,000 & 0 & Pre-Stage Envelope B \\
\hline \multirow[t]{2}{*}{$\mathrm{X}$} & AP-104 & AW-102 & $7 / 2 / 01$ & $7 / 6 / 01$ & 918,300 & 0 & Empty 4AP \\
\hline & WASH-WATER & AP-104 & $11 / 19 / 01$ & $11 / 20 / 01$ & 200,000 & 0 & Flush Water to 4AP \\
\hline \multirow[t]{19}{*}{$\mathrm{X}$} & AP-102 & AP-103. & $11 / 17 / 01$ & $11 / 22 / 01$ & $1,070,000$ & 0 & Empty 2AP \\
\hline & AP-104 & AP-102 & $12 / 1 / 01$ & $12 / 3 / 01$ & 385,700 & 39 & Flush 4AP \\
\hline & AP-102 & AP-106 & $12 / 15 / 01$ & $12 / 17 / 01$ & 385,700 & 37 & Flush $2 \mathrm{AP}$ \\
\hline & $\mathrm{AN}-\mathrm{LOS}$ & AP-102 & $1 / 1 / 02$ & $1 / 4 / 02$ & 542,400 & 7,895 & $\begin{array}{l}\text { Betrieve/Stage LAW Batch 1, Contractor } 1 \text {, } \\
\text { Envelope A }\end{array}$ \\
\hline & LERF & AN-105-PUMP & $1 / 1 / 02$ & $1 / 4 / 02$ & 154,100 & 0 & Dilution Water \\
\hline & $\mathrm{AN}-105$ & AP-102 & $1 / 1 / 02$ & $1 / 4 / 02$ & 686,500 & 294 & As-received Ghost \\
\hline & AN-105 & AP-104 & $1 / 5 / 02$ & $1 / 8 / 02$ & 542,400 & 7,895 & $\begin{array}{l}\text { Retrieve/Stage LAW Batch 1, Contractor 2, } \\
\text { Envelope A }\end{array}$ \\
\hline & LERF & AN-105-PUMP & $1 / 5 / 02$ & $1 / 8 / 02$ & 154,100 & 0 & Dilution Water \\
\hline & AN-IOS & AP-104 & $1 / 5 / 02$ & $1 / 8 / 02$ & 686,500 & 294 & As-received Ghost \\
\hline & AP-106 & AP-108 & $1 / 10 / 02$ & $1 / 12 / 02$ & 393,700 & 684 & Flush 6AP \\
\hline & AP-108 & AN-105 & $1 / 13 / 02$ & $1 / 15 / 02$ & 393,700 & 641 & Flush 8AP \\
\hline & $\mathrm{AP}-102$ & AP-106 & $4 / 26 / 02$ & $4 / 30 / 02$ & 686,500 & 285 & Deliver LAW Batch 1, Contractor 1 \\
\hline & AP-104 & AP-108 & $4 / 30 / 02$ & $5 / 4 / 02$ & 686,500 & 285 & Deliver LAW Batch 1, Contractor 2 \\
\hline & AN-104 & AP-102 & $4 / 30 / 02$ & $5 / 3 / 02$ & 502,200 & 12,513 & $\begin{array}{l}\text { Retrieve/Stage LAW Batch 2, Contractor 1, } \\
\text { Envelope A }\end{array}$ \\
\hline & LERF & AN-104-PUMP & $4 / 30 / 02$ & $5 / 3 / 02$ & 200,800 & 0 & Dilution Water \\
\hline & AN-104 & AP-102 & $4 / 30 / 02$ & $5 / 3 / 02$ & 684,700 & 702 & As-received Ghost \\
\hline & AN-104 & AP-104 & $5 / 4 / 02$ & $5 / 7 / 02$ & 502,200 & 12,513 & $\begin{array}{l}\text { Retrieve/Stage LAW Batch 2, Contractor 2, } \\
\text { Envelope A }\end{array}$ \\
\hline & LERF & AN-104-PUMP & $5 / 4 / 02$ & $5 / 7 / 02$ & 200,800 & 0 & Dilution Water \\
\hline & AN-104 & AP-104 & $5 / 4 / 02$ & $5 / 7 / 02$ & 684,700 & 702 & As-received Ghost \\
\hline \multirow[t]{28}{*}{$\mathrm{X}$} & $A Z-102$ & $\mathrm{AY}-101$ & $11 / 14 / 01$ & $11 / 15 / 01$ & 204,000 & 0 & Pre-Stage Envelope B \\
\hline & $\mathrm{AP}-104$ & AP-108 & $1 / 7 / 03$ & $1 / 10 / 03$ & 684,700 & 686 & Deliver LAW Batch 2, Contractor 2 \\
\hline & AP-102 & AP-106 & $1 / 10 / 03$ & $1 / 13 / 03$ & 684,700 & 686 & Deliver LAW Batch 2, Contractor 1 \\
\hline & $A W-101$ & AP-104 & $1 / 11 / 03$ & $1 / 15 / 03$ & 537,200 & 13,013 & $\begin{array}{l}\text { Retrieve/Stage LAW Batch 3, Contractor 2, } \\
\text { Envelope A }\end{array}$ \\
\hline & LERF & AW-101-PUMP & $1 / 11 / 03$ & $1 / 15 / 03$ & 308,100 & 0 & Dilution Water \\
\hline & AW-101 & AP-104 & $1 / 11 / 03$ & $1 / 15 / 03$ & 845,400 & 13,013 & As-received Ghost \\
\hline & RETURN 1 & AP-107 & $1 / 17 / 03$ & $1 / 17 / 03$ & 8,709 & 292 & Retums From Contractor 1 \\
\hline & AW-101 & AP-102 & $1 / 16 / 03$ & $1 / 20 / 03$ & 537,200 & 13,013 & $\begin{array}{l}\text { Retrieve/Stage LAW Batch 3, Contractor 1, } \\
\text { Envelope A }\end{array}$ \\
\hline & LERF & AW-101-PUMP & $1 / 16 / 03$ & $1 / 20 / 03$ & 308,100 & 0 & Dilution Water \\
\hline & AW-101 & AP-102 & $1 / 16 / 03$ & $1 / 20 / 03$ & 845,400 & 13,013 & As-received Ghost \\
\hline & RETURN 2 & AP-107 & $2 / 19 / 03$ & $2 / 19 / 03$ & 8,669 & 331 & Returns From Contractor 2 \\
\hline & AP-104 & AP-108 & $10 / 1 / 03$ & $10 / 4 / 03$ & 740,300 & 1,581 & Deliver LAW Batch 3, Contractor 2 \\
\hline & RETURN 1 & AP-107 & $10 / 5 / 03$ & $10 / 6 / 03$ & 8,671 & 332 & Returns From Contractor 1 \\
\hline & AN-103 & AP-104 & $10 / 5 / 03$ & $10 / 8 / 03$ & 436,600 & 28,133 & $\begin{array}{l}\text { Retrieve/Sfage LAW Batch 4, Contractor 2, } \\
\text { Envelope } A\end{array}$ \\
\hline & LERF & AN-103-PUMP & $10 / 5 / 03$ & $10 / 8 / 03$ & 264,900 & 0 & Dilution Water \\
\hline & AN-103 & AP-104 & $10 / 5 / 03$ & $10 / 8 / 03$ & 738,300 & 549 & As-received Ghost \\
\hline & AP-102 & AP-106 & $10 / 4 / 03$ & $10 / 8 / 03$ & 740,300 & 1,581 & Deliver LAW Batch 3, Contractor 1 \\
\hline & AN-103 & AP-102 & $10 / 9 / 03$ & $10 / 12 / 03$ & 436,600 & 28,133 & $\begin{array}{l}\text { Betrieve/Stage LAW Batch 4, Contractor 1, } \\
\text { Envelope A }\end{array}$ \\
\hline & LERF & AN-103-PUMP & $10 / 9 / 03$ & $10 / 12 / 03$ & 264,900 & 0 & Dilution Water \\
\hline & $\mathrm{AN}-103$ & AP-102 & $10 / 9 / 03$ & $10 / 12 / 03$ & 738,300 & 549 & As-received Ghost \\
\hline & RETURN_2 & AP-107 & $10 / 22 / 03$ & $10 / 22 / 03$ & 8,672 & 331 & Returns From Contractor 2 \\
\hline & RETURN 1 & AP-107 & $2 / 20 / 04$ & $2 / 20 / 04$ & 8,673 & 330 & Returns From Contractor 1 \\
\hline & RETURN_2 & AP-107 & $3 / 8 / 04$ & $3 / 8 / 04$ & 8,672 & 330 & Returns From Contractor 2 \\
\hline & AP-104 & AP-108 & $5 / 5 / 04$ & $5 / 9 / 04$ & 753,200 & 1,687 & Deliver LAW Batch 4, Contractor 2 \\
\hline & AP-102 & AP-106 & $5 / 9 / 04$ & $5 / 13 / 04$ & 753,200 & 1,687 & Deliver LAW Batch 4, Contractor 1 \\
\hline & WASH-WATER & AP-102 & $5 / 14 / 04$ & $5 / 14 / 04$ & 118,100 & 0 & Flush Water to 2AP \\
\hline & WASH-WATER & AP-104 & $5 / 15 / 04$ & $5 / 15 / 04$ & 118,100 & 0 & Flush Water to 4AP \\
\hline & RETURN 1. & AP-107 & $5 / 30 / 04$ & $5 / 30 / 04$ & 8,672 & 330 & Returns From Contractor 1 \\
\hline
\end{tabular}


HNF-2321

Revision 0

Table A.2-2. Phase 1 Low-Activity Waste Feed Staging Transfers for Case 2.

\begin{tabular}{|c|c|c|c|c|c|c|c|}
\hline $\begin{array}{l}\text { Boot } \\
\text { Strap } \\
\end{array}$ & From & To & Start Date & End Date & $\begin{array}{c}\text { Liquid } \\
\text { (gallons) }\end{array}$ & $\begin{array}{c}\text { Solid } \\
\text { (gallons) }\end{array}$ & Notes \\
\hline & RETURN 2 & AP-107 & \begin{tabular}{|l|l|}
$6 / 4 / 04$ \\
\end{tabular} & $6 / 4 / 04$ & 8,678 & 331 & Retums From Contractor 2 \\
\hline & AP-102 & AN-105 & $5 / 30 / 04$ & $6 / 8 / 04$ & 209,500 & 9,168 & Flush 2AP \\
\hline & WASH-CAUSTIC & AP-102 & $6 / 10 / 04$ & $6 / 10 / 04$ & 103,400 & 0 & Shim Caustic for Batch 5 , Contractor 1 \\
\hline & $\mathrm{AP}-104$ & $\mathrm{AN}-105$ & $6 / 9 / 04$ & $6 / 10 / 04$ & 209,500 & 9,168 & Flush 4AP \\
\hline & WASH-CAUSTIC & AP-104 & $6 / 11 / 04$ & $6 / 11 / 04$ & 126,400 & 0 & Shim Caustic for Batch 5, Contractor 2 \\
\hline & AP-10I & AP-102 & $6 / 12 / 04$ & $6 / 14 / 04$ & 402,300 & 18 & Betrieve/Stage LAW Batch 5, Contractor 1, \\
\hline & LERF & AP-101-PUMP & $6 / 12 / 04$ & $6 / 14 / 04$ & 44,253 & 0 & Dilution Water \\
\hline & AP-101 & AP-102 & $6 / 12 / 04$ & $6 / 14 / 04$ & 446,500 & 18 & As-received Ghost \\
\hline & AW-104 & AP-102 & $6 / 16 / 04$ & $6 / 16 / 04$ & 111,900 & 5 & \begin{tabular}{|l} 
Complete Retrieve/Stage LAW Batch 5 , \\
Contfactor 1, Envelope A
\end{tabular} \\
\hline & AP-101 & AP-104 & $6 / 15 / 04$ & $6 / 17 / 04$ & 491,700 & 22 & $\begin{array}{l}\text { Rerrieve/Stage LAW Batch 5, Contractor 2, } \\
\text { Envelope } A\end{array}$ \\
\hline & LERF & AP-101-PUMP & $6 / 15 / 04$ & $6 / 17 / 04$ & 54,087 & 0 & Dilution Water \\
\hline & AP-101 & AP-104 & $6 / 15 / 04$ & $6 / 17 / 04$ & 545,800 & .22 & As-received Ghost \\
\hline & AW-104 & AP-104 & $6 / 18 / 04$ & $6 / 18 / 04$ & 136,800 & 6 & \begin{tabular}{|llll} 
Complete Retrieve/Stage LAW Batch 5 , \\
Contfactor 2, Envelope
\end{tabular} \\
\hline & RETURN 1 & AP-107 & $7 / 19 / 04$ & $7 / 19 / 04$ & 8,676 & 331 & Returns From Contractor 1 \\
\hline & RETURN 2 & AP-107 & $7 / 24 / 04$ & $7 / 24 / 04$ & 8,682 & 331 & Returns From Contractor 2 \\
\hline & RETURN_ 1 & AP-107 & $9 / 7 / 04$ & $9 / 7 / 04$ & 8,676 & 331 & Returns From Contractor 1 \\
\hline & RETURN 2 & AP-107 & $9 / 13 / 04$ & $9 / 13 / 04$ & 8,682 & 331 & Returns From Contractor 2 \\
\hline & RETURN 1 & AP-107 & $10 / 27 / 04$ & $10 / 27 / 04$ & 8,676 & 331 & Returns From Contractor 1 \\
\hline & RETURN 2 & AP-107 & $11 / 2 / 04$ & $11 / 2 / 04$ & 8,682 & 331 & Retums From Contractor 2 \\
\hline & RETURN_I & AP-107 & $12 / 16 / 04$ & $12 / 16 / 04$ & 8,676 & 331 & Returns From Contractor 1 \\
\hline & RETURN 2 & AP-107 & $12 / 23 / 04$ & $12 / 23 / 04$ & 8,682 & 331 & Returns From Contractor 2 \\
\hline & RETURN 1 & AP-107 & $2 / 4 / 05$ & $2 / 4 / 05$ & 8,676 & 331 & Returns From Contractor 1 \\
\hline & RETURN 2 & AP-107 & $2 / 11 / 05$ & $2 / 11 / 05$ & 8,682 & 331 & Returns From Contractor 2 \\
\hline & $\mathrm{AP}-104$ & AP-108 & $2 / 19 / 05$ & $2 / 23 / 05$ & 807,800 & 1,143 & Deliver LAW Batch 5, Contractor 2 \\
\hline & AY-101 & AP-104 & $2 / 24 / 05$ & $2 / 25 / 05$ & 283,600 & 12 & Stage LAW Batch 6, Contractor 2, Envelope B \\
\hline & $\mathrm{AP}-102$ & AP-106 & $2 / 23 / 05$ & $2 / 27 / 05$ & 660,800 & 1,129 & Deliver LAW Batch 5 , Contractor 1 \\
\hline & AY-101 & AP-102 & $2 / 28 / 05$ & $3 / 1 / 05$ & 279,700 & 11 & Stage LAW Batch 6, Contractor 1, Envelope B \\
\hline & RETURN 1 & AP-107 & $3 / 31 / 05$ & $3 / 31 / 05$ & 8,676 & 331 & Returns From Contractor 1 \\
\hline & RETURN 2 & AP-107 & $4 / 8 / 05$ & $4 / 8 / 05$ & 8,673 & 331 & Returns From Contractor 2 \\
\hline & RETURN 1 & AP-107 & $5 / 27 / 05$ & $5 / 27 / 05$ & 8,675 & $33 !$ & Returns From Contractor 1 \\
\hline & RETURN 2 & AP-107 & $6 / 4 / 05$ & $6 / 4 / 05$ & 8,678 & 331 & Returns From Contractor 2 \\
\hline & RETURN 1 & AP-107 & $7 / 23 / 05$ & $7 / 23 / 05$ & 8,675 & 331 & Returns From Contractor 1 \\
\hline & RETURN 2 & AP-107 & $7 / 31 / 05$ & $7 / 31 / 05$ & 8,678 & 331 & Returns From Contractor 2 \\
\hline & RETURN 1 & AP-107 & 9/18/05 & $9 / 18 / 05$ & 8,675 & 331 & Returns From Contractor 1 \\
\hline & RETURN_2 & AP-107 & $9 / 26 / 05$ & $9 / 26 / 05$ & 8,678 & 331 & Retums From Contractor 2 \\
\hline & RETURN 1 & AP-107 & $11 / 14 / 05$ & $11 / 14 / 05$ & 8,675 & 331 & Returns From Contractor 1 \\
\hline \multirow[t]{7}{*}{$\mathrm{X}$} & $5 Y-102$ & AW-105 & $11 / 15 / 05$ & $11 / 17 / 05$ & 413,400 & 16 & Decant Supernate from SY-102 \\
\hline & RETURN_2 & \begin{tabular}{|l|l|} 
AP-107 \\
\end{tabular} & $11 / 22 / 05$ & $11 / 22 / 05$ & 8,678 & 331 & Returns From Contractor 2 \\
\hline & AP-106 & LAW-CONTRACTOR-1 & $6 / 1 / 02$ & $11 / 30 / 05$ & $3,525,000$ & 5,368 & Complete Processing Envelope A, Contractor 1 \\
\hline & AP-102 & AP-106 & $11 / 30 / 05$ & $12 / 2 / 05$ & 279,600 & 53 & Deliver LAW Batch 6, Contractor 1 \\
\hline & AN-107 & $\mathrm{AP}-102$ & $12 / 3 / 05$ & $12 / 5 / 05$ & 481,700 & 22 & $\begin{array}{l}\text { Retrieve/Stage LAW Batch 7/8, Contractor } 1 \text {, } \\
\text { Envelope C }\end{array}$ \\
\hline & LERF & AN-107-PUMP & $12 / 3 / 05$ & $12 / 5 / 05$ & 72,259 & 0 & Dilution Water \\
\hline & AN-107 & AP-102 & $12 / 3 / 05$ & $12 / 5 / 05$ & 554,000 & 22 & As-received Ghost \\
\hline $\mathbf{x}$ & WATER & SY-102 & $12 / 15 / 05$ & $12 / 17 / 05$ & 426,000 & 0 & Flush Water for 2SY Solids Cleanout \\
\hline \multirow[t]{9}{*}{$\mathbf{x}$} & SY-102 & AW-105 & $1 / 1 / 06$ & $1 / 3 / 06$ & 535,600 & 22,049 & Cleanout Solids 2SY \\
\hline & RETURN 2 & AP-107 & $1 / 18 / 06$ & $1 / 18 / 06$ & 8,678 & 331 & Returns From Contractor 2 \\
\hline & AP-108 & LAW-CONTRACTOR-2 & $6 / 1 / 02$ & $1 / 27 / 06$ & $3,673,000$ & 5,386 & Complete Processing Envelope A, Contractor 2 \\
\hline & AP-104 & AP-108 & $1 / 27 / 06$ & $1 / 28 / 06$ & 283,600 & 46 & Deliver LAW Batch 6, Contractor 2 \\
\hline & AN-107 & AP-104 & $1 / 29 / 06$ & $1 / 31 / 06$ & 481,700 & 22 & $\begin{array}{l}\text { Retrieve/Stage LAW Batch 7/8, Contractor 2, } \\
\text { Envelope C. }\end{array}$ \\
\hline & LERF & AN-107-PUMP & $1 / 29 / 06$ & $1 / 31 / 06$ & 72,260 & 0 & Dilution Water \\
\hline & AN-107 & AP-104 & $1 / 29 / 06$ & $1 / 31 / 06$ & 554,000 & 22 & As-received Ghost \\
\hline & AP-106 & LAW-CONTRACTOR-1 & $12 / 2 / 05$ & $2 / 8 / 06$ & 279,600 & 93 & Complete Processing Envelope B, Contractor 1 \\
\hline & AP-102 & AP-106. & $2 / 8 / 06$ & $2 / 9 / 06$ & 169,400 & 8 & Deliver LAW Batch 7, Contractor 1 \\
\hline
\end{tabular}


HNF-2321

Revision 0

Table A.2-2. Phase 1 Low-Activity Waste Feed Staging Transfers for Case 2.

\begin{tabular}{|c|c|c|c|c|c|c|c|}
\hline $\begin{array}{l}\text { Boot } \\
\text { Strap }\end{array}$ & From & To & Start Date & End Date & $\begin{array}{c}\text { Liquid } \\
\text { (gallons) }\end{array}$ & $\begin{array}{c}\text { Solid } \\
\text { (gallons) }\end{array}$ & Notes \\
\hline & AP-102 & AP-106 & $3 / 8 / 06$ & $3 / 10 / 06$ & 384,600 & 18 & Deliver LAW Batch 8 , Contractor 1 \\
\hline & AN-102 & AP-102 & $3 / 11 / 06$ & $3 / 14 / 06$ & 481,700 & 23 & $\begin{array}{l}\text { Retrieve/StSage LAW Batch 9, Contractor 1, } \\
\text { Envelope C }\end{array}$ \\
\hline & LERF & AN-102-PUMP & $3 / 11 / 06$ & $3 / 14 / 06$ & 289,000 & 0 & Dilution Water \\
\hline & AN-102 & AP-102 & $3 / 11 / 06$ & $3 / 14 / 05$ & 770,700 & 23 & As-received Ghost \\
\hline & AP-108 & LAW-CONTRACTOR-2 & $1 / 28 / 06$ & $4 / 7 / 06$ & 283,500 & 79 & Complete Processing Envelope B, Contractor 2 \\
\hline & AP-104 & AP- 108 & $4 / 7 / 06$ & $4 / 8 / 06$ & 169,400 & 8 & Deliver LAW Batch 7, Contractor 2 \\
\hline & AP-104 & AP-108 & $5 / 5 / 06$ & $5 / 7 / 06$ & 384,600 & 18 & Deliver LAW Batch 8, Contractor 2 \\
\hline & AN-102 & AP-104 & $5 / 8 / 06$ & $5 / 11 / 06$ & 481,700 & 23 & $\begin{array}{l}\text { Retrieve/Stage LAW Batch 9, Contractor 2, } \\
\text { Envelope c }\end{array}$ \\
\hline & LERF & AN-102-PUMP & $5 / 8 / 06$ & $5 / 11 / 06$ & 289,000 & 0 & Dilution Water \\
\hline & AN-102 & AP-104 & $5 / 8 / 06$ & $5 / 11 / 06$ & 770,700 & 23 & As-received Ghost \\
\hline & AP-102 & AP-106 & $7 / 21 / 06$ & $7 / 25 / 05$ & 770,700 & 23 & Deliver LAW Batch 9, Contractor 1 \\
\hline & AN-106 & AP-102 & $7 / 26 / 06$ & $7 / 29 / 06$ & 549,100 & 25 & $\begin{array}{l}\text { Retrieve/StSage LAW Batch 10, Contractor } 1 \text {, } \\
\text { Envelope C }\end{array}$ \\
\hline & LERF & AN-106-PUMP & $7 / 26 / 06$ & $7 / 29 / 06$ & 98,837 & 0 & Dilution Water \\
\hline & AN-106 & AP-102 & $7 / 26 / 06$ & $7 / 29 / 06$ & 647,900 & 25 & As-received Ghost \\
\hline & AP-104 & AP-108 & $9 / 17 / 06$ & $9 / 21 / 06$ & 770,700 & 23 & Deliver LAW Batch 9, Contractor 2 \\
\hline & AN-106 & AP-104 & $9 / 22 / 06$ & 9/25/06 & 549,100 & 25 & $\begin{array}{l}\text { Retrieve/Stsage LAW Batch } 10 \text {, Contractor } 2 \text {, } \\
\text { Envelope C }\end{array}$ \\
\hline & LERF & AN-106-PUMP & $9 / 22 / 06$ & $9 / 25 / 06$ & 98,837 & 0 & Dilution Water \\
\hline & AN-106 & AP-104 & $9 / 22 / 06$ & $9 / 25 / 06$ & 647,900 & 25 & As-received Ghost \\
\hline & SY-101 & SY- 102 . & $9 / 23 / 06$ & $9 / 28 / 06$ & 537,800 & 5,486 & Retrieve LAW Batch 11, Envelope C \\
\hline & LERF & SY-101-PUMP & $9 / 23 / 06$ & $9 / 28 / 06$ & 532,400 & 0 & Dilution Water \\
\hline & SY-101 & $S Y-102$ & $9 / 23 / 06$ & $9 / 28 / 06$ & $1,082,000$ & 611 & As-received Ghost \\
\hline & SY-102 & AN-102 & $10 / 6 / 06$ & $10 / 9 / 06$ & $1,027,000$ & 1,532 & Pre-Stage LAW Batch 11, Envelope C \\
\hline & SY-101 & SY-102 & $10 / 17 / 06$ & $10 / 22 / 06$ & 537,800 & 5,486 & Retrieve LAW Batch 11, Envelope C \\
\hline & LERF & SY-101-PUMP & $10 / 17 / 06$ & $10 / 22 / 06$ & 532,400 & 0 & Dilution Water \\
\hline . & SY-101 & SY-102 & $10 / 17 / 06$ & $10 / 22 / 06$ & $1,082,000$ & 611 & As-received Ghost \\
\hline & SY-102 & AN-107 & $10 / 30 / 06$ & $11 / 2 / 06$ & 983,800 & 641 & Pre-Stage LAW Batch 11, Envelope C \\
\hline & RETURN 1 & AP-107 & $2 / 25 / 07$ & $2 / 25 / 07$ & 8,735 & 265 & Returns From Contractor 1 \\
\hline & AP-102 & AP-106 & $3 / 18 / 07$ & $3 / 21 / 07$ & 647,900 & 25 & Deliver LAW Batch 10, Contractor 1 \\
\hline & AN-102 & AP-102 & $3 / 22 / 07$ & $3 / 27 / 07$ & $1,013,000$ & 44 & Stage LAW Batch 11, Contractor 1, Envelope \\
\hline & SY-103 & SY-102 & $3 / 30 / 07$ & $4 / 1 / 07$ & 350,000 & 9,759 & Retrieve LAW Batch 12, Envelope C \\
\hline & LERF & SY-103-PUMP & $3 / 30 / 07$ & $4 / 1 / 07$ & 183,500 & 0 & Dilution Water \\
\hline & SY-103 & SY-102 & $3 / 30 / 07$ & $4 / 1 / 07$ & 537,500 & 1,449 & As-received Ghost \\
\hline & SY-102 & AN-102 & $4 / 9 / 07$ & $4 / 11 / 07$ & 720,700 & 1,528 & Pre-Stage LAW Batch 12, Envelope C \\
\hline & AP-104 & AP-108 & $5 / 15 / 07$ & $5 / 18 / 07$ & 647,900 & 25 & Deliver LAW Batch 10, Contractor 2 \\
\hline & AN-107 & AP-104 & $5 / 19 / 07$ & $5 / 23 / 07$ & 978,400 & 43 & Stage LAW Batch 11, Contractor 2, Envelope \\
\hline & SY-103 & SY-102 & $5 / 27 / 07$ & $5 / 29 / 07$ & 350,000 & 9,759 & Retrieve LAW Batch 12, Envelope C \\
\hline & LERF & SY-103-PUMP & $5 / 27 / 07$ & $5 / 29 / 07$ & 183,500 & 0 & Dilution Water \\
\hline & SY-103 & SY-102 & $5 / 27 / 07$ & $5 / 29 / 07$ & 537,500 & 1,449 & As-received Ghost \\
\hline & $S Y-102$ & AN-107 & $6 / 6 / 07$ & $6 / 7 / 07$ & 537,500 & 1,434 & Pre-Stage LAW Batch 12, Envelope C \\
\hline & AP-102 & AP-106 & $11 / 2 / 07$ & $11 / 7 / 07$ & $1,013,000$ & 44 & Deliver LAW Batch 11, Contractor 1 \\
\hline & AN-102 & AP-102 & $11 / 8 / 07$ & $11 / 11 / 07$ & 707,100 & 31 & Stage LAW Batch 12, Contractor 1, Envelope \\
\hline & AP-104 & AP-108 & $12 / 30 / 07$ & $1 / 4 / 08$ & 978,400 & 43 & Deliver LAW Batch 11 , Contractor 2 \\
\hline & AN-107 & AP-104 & $1 / 5 / 08$ & $1 / 7 / 08$ & 524,700 & 23 & Stage LAW Batch 12, Contractor 2, Envelope \\
\hline & $\mathrm{AP}-102$ & AP-106 & $9 / 7 / 08$ & $9 / 11 / 08$ & 707,100 & 31 & Deliver LAW Batch 12, Contractor 1 \\
\hline & AP-104 & $\mathrm{AP}-108$ & $11 / 4 / 08$ & $11 / 6 / 08$ & 524,700 & 23 & Deliver LAW Batch 12, Contractor 2 \\
\hline & AP-106 & LAW-CONTRACTOR-1 & $2 / 9 / 06$ & $5 / 20 / 09$ & $3,693,000$ & 158 & Complete Processing Envelope C, Contractor 1 \\
\hline & AP-108 & LAW-CONTRACTOR-2 & $4 / 8 / 06$ & $5 / 21 / 09$ & $3,476,000$ & 146 & Complete Processing Envelope C, Contractor 2 \\
\hline & RETURN 1 & AP-107 & $5 / 21 / 09$ & $5 / 21 / 09$ & 2,581 & 96 & Returns From Contractor 1 \\
\hline & RETURN_2 & AP-107 & $5 / 22 / 09$ & $5 / 22 / 09$ & 7,544 & 287 & Returns From Contractor 2 \\
\hline $\mathrm{x}$ & AP-107 & AZ-10L & $6 / 1 / 09$ & $6 / 2 / 09$ & 271,400 & 9,319 & Transfer Returns to AZ-101 (Phase $2 \mathrm{HLW}$ \\
\hline
\end{tabular}


HNF-2321

Revision 0

Table A.2-3. Phase 1 High-Level Waste Feed Staging Transfers for Case 2.

\begin{tabular}{|c|c|c|c|c|c|c|c|}
\hline $\begin{array}{l}\text { Boot } \\
\text { Strap }\end{array}$ & From & To & Start Date & End Date & $\begin{array}{c}\text { Liquid } \\
\text { (gallons) }\end{array}$ & $\begin{array}{c}\text { Solid } \\
\text { (gallons) }\end{array}$ & Notes \\
\hline & $\mathrm{AY}-102$ & C-106 & $7 / 1 / 98$ & $7 / 5 / 98$ & 920,700 & 31 & $\begin{array}{l}\text { Sluice "Water" for C-106 Retrieval - Lumped } \\
\text { (See Below) }\end{array}$ \\
\hline & C-106 & $\mathrm{AY}-102$ & $7 / 15 / 98$ & $11 / 10 / 98$ & 809,000 & 15,169 & C-106 Retrieval (Project W-320) \\
\hline \multirow[t]{8}{*}{$\mathrm{X}$} & AZ-101 & $\mathrm{AY}-101$ & $8 / 17 / 00$ & $8 / 20 / 00$ & 685,000 & 0 & Initial Decant IAZ \\
\hline & WASH-WATER & AZ-101 & $8 / 20 / 00$ & $8 / 21 / 00$ & 146,000 & 0 & First Wash 1AZ \\
\hline & AZ-101 & AP-107 & $10 / 9 / 00$ & $10 / 10 / 00$ & 143,200 & 0 & First Decant $1 \mathrm{AZ}$ \\
\hline & WASH-WATER & AZ-101 & $10 / 11 / 00$ & $10 / 12 / 00$ & 146,000 & 0 & Second Wash IAZ \\
\hline & AZ-101 & AP-107 & $12 / 17 / 00$ & $12 / 18 / 00$ & 140,200 & 0 & Second Decant $1 \mathrm{AZ}$ \\
\hline & WASH-WATER & AZ-10I & $12 / 19 / 00$ & $12 / 20 / 00$ & 146,000 & 0 & Third Wash IAZ \\
\hline & $\mathrm{AZ}-101$ & AP-107 & $2 / 7 / 01$ & $2 / 7 / 01$ & 142,900 & 0 & Third Decant 1AZ \\
\hline & WASH-WATER & AZ-101 & $2 / 8 / 01$ & $2 / 9 / 01$ & 138,200 & 0 & Transfer Water Add IAZ \\
\hline \multirow[t]{14}{*}{$\mathrm{X}$} & $\mathrm{AZ}-102$ & AY-101 & $11 / 14 / 01$ & $11 / 15 / 01$ & 204,000 & 0 & Initial Decant 2AZ \\
\hline & AZ-102 & AP-107 & $11 / 16 / 01$ & $11 / 16 / 01$ & 181,800 & 0 & Complete Initial Decant $2 \mathrm{AZ}$ \\
\hline & WASH-WATER & AZ-102 & $11 / 17 / 01$ & $11 / 18 / 01$ & 213,000 & 0 & First Wash $2 \mathrm{AZ}$ \\
\hline & $\mathrm{AZ}-101$ & PIHLW_FEED_TANK & $5 / 17 / 02$ & $5 / 17 / 02$ & 134,300 & 5,079 & $\begin{array}{l}\text { Beliver Batch } 1 \text { of } 1 \mathrm{AZ} \text {, Envelope D (Batch } 1 \\
\text { Overal) }\end{array}$ \\
\hline & AZ-102 & AN-105 & $7 / 3 / 02$ & $7 / 3 / 02$ & 127,700 & 0 & First Decant 2AZ \\
\hline & WASH-WATER & AZ-102 & $7 / 4 / 02$ & $7 / 5 / 02$ & 213,000 & 0 & Second Wash $2 \mathrm{AZ}$ \\
\hline & $\mathrm{AZ}-102$ & AN-105 & $8 / 23 / 02$ & $8 / 25 / 02$ & 210,300 & 0 & Second Decant $2 \mathrm{AZ}$ \\
\hline & WASH-WATER & AZ-102 & $8 / 26 / 02$ & $8 / 27 / 02$ & 213,000 & 0 & Third Wash $2 \mathrm{AZ}$ \\
\hline & AZ-102 & AN-105 & $10 / 15 / 02$ & $10 / 16 / 02$ & 211,800 & 0 & Third Decant 2AZ \\
\hline & WASH-WATER & AZ-102 & $10 / 17 / 02$ & $10 / 18 / 02$ & 213,000 & 0 & Fourth Wash 2AZ \\
\hline & AZ-102 & AN-105 & $12 / 6 / 02$ & $12 / 7 / 02$ & 212,500 & 0 & Fourth Decant $2 \mathrm{AZ}$ \\
\hline & WASH-WATER & $A Z-102$ & $12 / 9 / 02$ & $12 / 9 / 02$ & 110,000 & 0 & Transfer Water Add 2AZ \\
\hline & P1HLW_FEED_TANK & HLW-CONTRACTOR & $6 / 1 / 02$ & $2 / 21 / 03$ & 134,300 & 5,079 & $\begin{array}{l}\text { frocess Batch } 1 \text { of } 1 \mathrm{AZ}, \text { Envelope D (Batch } 1 \\
\text { Overalh) }\end{array}$ \\
\hline & AZ-101 & PIHLW_FEED_TANK & $2 / 21 / 03$ & $2 / 21 / 03$ & 134,300 & 5,079 & 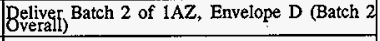 \\
\hline \multirow[t]{25}{*}{$\mathrm{X}$} & $\mathrm{AY}-102$ & AZ-101 & $2 / 22 / 03$ & $2 / 26 / 03$ & 819,600 & 16,032 & Transfer $2 \mathrm{AY} / 6 \mathrm{C}$ Slurry to $1 \mathrm{AZ}$ for Pretreatment \\
\hline & $\mathrm{AZ}-101$ & AN-105 & $10 / 29 / 03$ & $11 / 1 / 03$ & 622,700 & 0 & Initial Decant $2 \mathrm{AY} / 6 \mathrm{C}$ Slurry from $1 \mathrm{AZ}$ \\
\hline & WASH-WATER & AZ-101 & $11 / 1 / 03$ & $11 / 2 / 03$ & 324,000 & 0 & First Wash $2 \mathrm{AY} / 6 \mathrm{C}$ Slurry in $1 \mathrm{AZ}$ \\
\hline & P1HLW_FEED_TANK & HLW-CONTRACTOR & $2 / 21 / 03$ & $11 / 14 / 03$ & 134,300 & 5,079 & $\begin{array}{l}\text { Process } \\
\text { Overatif) }\end{array}$ \\
\hline & AZ-102 & PIHLW_FEED_TANK & $11 / 14 / 03$ & $11 / 14 / 03$ & 123,900 & 5,202 & Bveriver Batch 1 of $2 \mathrm{AZ}$, Envelope D (Batch 3 \\
\hline & AZ-101 & $\mathrm{AN}-105$ & $12 / 22 / 03$ & $12 / 23 / 03$ & 320,000 & 0 & First Decant 2AY/6C Slurry from 1AZ \\
\hline & WASH-WATER & AZ-101 & $12 / 24 / 03$ & $12 / 26 / 03$ & 324,000 & 0 & Second Wash 2AY/6C Slurry in 1AZ \\
\hline & $\mathrm{AZ}-101$ & AN-105 & $6 / 10 / 04$ & $6 / 11 / 04$ & 322,300 & 0 & Second Decant 2AY/6C Slurry from $1 \mathrm{AZ}$ \\
\hline & WASH-WATER & AZ-101 & $6 / 13 / 04$ & $6 / 15 / 04$ & 433,600 & 0 & Transfer Water Add $1 \mathrm{AZ}$ for $2 \mathrm{AY} / 6 \mathrm{C}$ Slurry \\
\hline & P1HLW_FEED_TANK & HLW-CONTRACTOR & $11 / 14 / 03$ & $8 / 7 / 04$ & 123,900 & $\overline{5,202}$ & $\begin{array}{l}\text { Process Batch } 1 \text { of } 2 \mathrm{AZ} \text {, Envelope D (Batch } 3 \\
\text { Overall) }\end{array}$ \\
\hline & $\mathrm{AZ}-102$ & P1HLW_FEED_TANK & $8 / 7 / 04$ & $8 / 8 / 04$ & 123,900 & 5,202 & Beliver Batch 2 of $2 \mathrm{AZ}$, Envelope D (Batch 4 \\
\hline & $\mathrm{C}-104$ & $\mathrm{AY}-102$ & $8 / 1 / 04$ & $9 / 8 / 04$ & 279,100 & 9,724 & C-104 Retrieval (Project W-???) \\
\hline & P1HLW_FEED_TANK & HLW-CONTRACTOR & $8 / 8 / 04$ & $5 / 2 / 05$ & 123,900 & 5,202 & $\begin{array}{l}\text { Brocess Batch } 2 \text { of } 2 \mathrm{AZ} \text {, Envelope D (Batch } 4 \\
\text { Overall) }\end{array}$ \\
\hline & AZ-101 & P1HLW_FEED_TANK & $5 / 2 / 05$ & $5 / 2 / 05$ & 127,200 & 3,269 & $\mathrm{~B}$ (Bater Batch 1 of $2 \mathrm{AY} / 6 \mathrm{C}$ from $1 \mathrm{AZ}$, Envelope \\
\hline & $A Y-102$ & $\mathrm{AZ}-102$ & $8 / 1 / 05$ & $8 / 2 / 05$ & 279,400 & 9,360 & Transfer $4 \mathrm{C}$ Slurry to $2 \mathrm{AZ}$ for Pretreatment \\
\hline & PIHLW_FEED_TANK & HLW-CONTRACTOR & $5 / 2 / 05$ & $11 / 16 / 05$ & 127,200 & 3,269 & $\begin{array}{l}\text { Process Batch } 1 \text { of } 2 \mathrm{AY} / 6 \mathrm{C} \text {, Envelope D (Batch } \\
5 \text { verall) }\end{array}$ \\
\hline & AZ-101 & P1HLW_FEED_TANK & $11 / 16 / 05$ & $11 / 17 / 05$ & 127,200 & 3,269 & $\mathrm{~B}$ (Batch 6 O Overalf) \\
\hline & P1HLW_FEED_TANK & HLW-CONTRACTOR & $11 / 17 / 05$ & $6 / 2 / 06$ & 127,200 & 3,269 & $\begin{array}{l}\text { Prgcess Batch } 2 \text { of } 2 \mathrm{AY} / 6 \mathrm{C} \text {, Envelope D (Batch } \\
6 \text { Overall) }\end{array}$ \\
\hline & AZZ-101 & P1HLW_FEED_TANK & $6 / 2 / 06$ & $6 / 3 / 06$ & 127,200 & 3,269 & $\mathrm{~B}$ (Batch 7 Overall) 3 of $2 \mathrm{AY} / 6 \mathrm{C}$ from $1 \mathrm{AZ}$, Envelope \\
\hline & AZ-102 & $\mathrm{AN}-105$ & $6 / 24 / 06$ & $6 / 24 / 06$ & 159,500 & 0 & Initial Decant $4 \mathrm{C}$ Slurry from $2 \mathrm{AZ}$ \\
\hline & WASH-WATER & AZ-102 & $6 / 24 / 06$ & $6 / 26 / 06$ & 324,000 & 0 & First Wash 4C Slurry in $2 \mathrm{AZ}$ \\
\hline & $A Z-102$ & $A N-105$ & $8 / 14 / 06$ & $8 / 16 / 05$ & 320,100 & 0 & First Decant 4C Slurry from $2 \mathrm{AZ}$ \\
\hline & WASH-WATER & AZ-102 & $8 / 17 / 06$ & $8 / 19 / 06$ & 324,000 & 0 & Second Wash $4 \mathrm{C}$ Slurry in $2 \mathrm{AZ}$ \\
\hline & $A Z-102$ & AN-105 & $10 / 20 / 06$ & $10 / 21 / 06$ & 322,700 & 0 & Second Decant $4 \mathrm{C}$ Slurry from $2 \mathrm{AZ}$ \\
\hline & WASH-WATER & $\mathrm{AZ}-102$ & $10 / 22 / 06$ & $10 / 23 / 06$ & 150,500 & 0 & Transfer Water Add 2AZ for 4C Slurry \\
\hline
\end{tabular}


HNF-2321

Revision 0

Table A.2-3. Phase 1 High-Level Waste Feed Staging Transfers for Case 2.

\begin{tabular}{|c|c|c|c|c|c|c|c|}
\hline $\begin{array}{l}\text { Boot } \\
\text { Strap }\end{array}$ & From & To & Start Date & End Date & $\begin{array}{c}\text { Liquid } \\
\text { (gallons) }\end{array}$ & $\begin{array}{c}\text { Solid } \\
\text { (gallons) }\end{array}$ & Notes \\
\hline & P1HLW_FEED_TANK & HLW-CONTRACTOR & $6 / 3 / 06$ & $12 / 18 / 06$ & 127,200 & 3,269 & $\begin{array}{l}\text { Process Batch } 3 \text { of } 2 \mathrm{AX} / 6 \mathrm{C}, \text { Envelope D (Batch } \\
7 \text { veralf) }\end{array}$ \\
\hline & AZ-101 & P1HLW_FEED_TANK & $12 / 18 / 06$ & $12 / 18 / 06$ & 127,200 & 3,269 & Beliver Batch 4 of $2 \mathrm{AY} / 6 \mathrm{C}$ from $1 \mathrm{AZ}$, Envelope \\
\hline & P1HLW_FEED_TANK & HLW-CONTRACTOR & $12 / 18 / 06$ & $7 / 4 / 07$ & 127,200 & 3,269 & 8 Overalf) \\
\hline & AZ-101 & P1HLW_FEED_TANK & $7 / 4 / 07$ & $7 / 5 / 07$ & 127,200 & 3,269 & $\mathrm{~B}$ (Biver Batch 5 of $2 \mathrm{AY} / 6 \mathrm{C}$ from $1 \mathrm{AZ}$, Envelope \\
\hline & P1HLW_FEED_TANK & HLW-CONTRACTOR & $7 / 5 / 07$ & $1 / 18 / 08$ & 127,200 & 3,269 & 9rocess Batch 5 of $2 \mathrm{AY} / 6 \mathrm{C}$, Envelope D (Batch \\
\hline & AZ-102 & PIHLW_FEED_TANK & $1 / 18 / 08$ & $1 / 19 / 08$ & 137,700 & 4,774 & Beliver Batch 1 of $4 \mathrm{C}$ from $2 \mathrm{AZ}$, Envelope $\mathrm{D}$ \\
\hline & P1HLW_FEED_TANK & HLW-CONTRACTOR & $1 / 19 / 08$ & $12 / 22 / 08$ & 137,700 & 4,774 & $\begin{array}{l}\text { Erocess Batch } 10 \text { of } 4 \mathrm{C} \text { from } 2 \mathrm{AZ} \text {, Envelope D } \\
\text { (Batch } 10 \text { Overall) }\end{array}$ \\
\hline & AZ-102 & P1HLW_FEED_TANK & $12 / 22 / 08$ & $12 / 22 / 08$ & 137,700 & 4,774 & Beliver Batch 2 of $4 \mathrm{C}$ from $2 \mathrm{AZ}$, Envelope $\mathrm{D}$ \\
\hline $\mathrm{x}$ & AP-107 & $A Z-101$ & $6 / 1 / 09$ & $6 / 2 / 09$ & 271,400 & 9,319 & LAW Returns to $\mathrm{LAZ}$ \\
\hline & P1HLW_FEED_TANK & HLW-CONTRACTOR & $12 / 22 / 08$ & $11 / 25 / 09$ & 137,700 & 4,774 & $\begin{array}{l}\text { Process Batch } 2 \text { of } 4 \mathrm{C} \text { from } 2 \mathrm{AZ} \text {, Envelope D } \\
\text { (Batch } 11 \text { Overail) }\end{array}$ \\
\hline
\end{tabular}


HNF-2321

Revision 0

Table A.3-1. Complete Transfer List for Case 3.

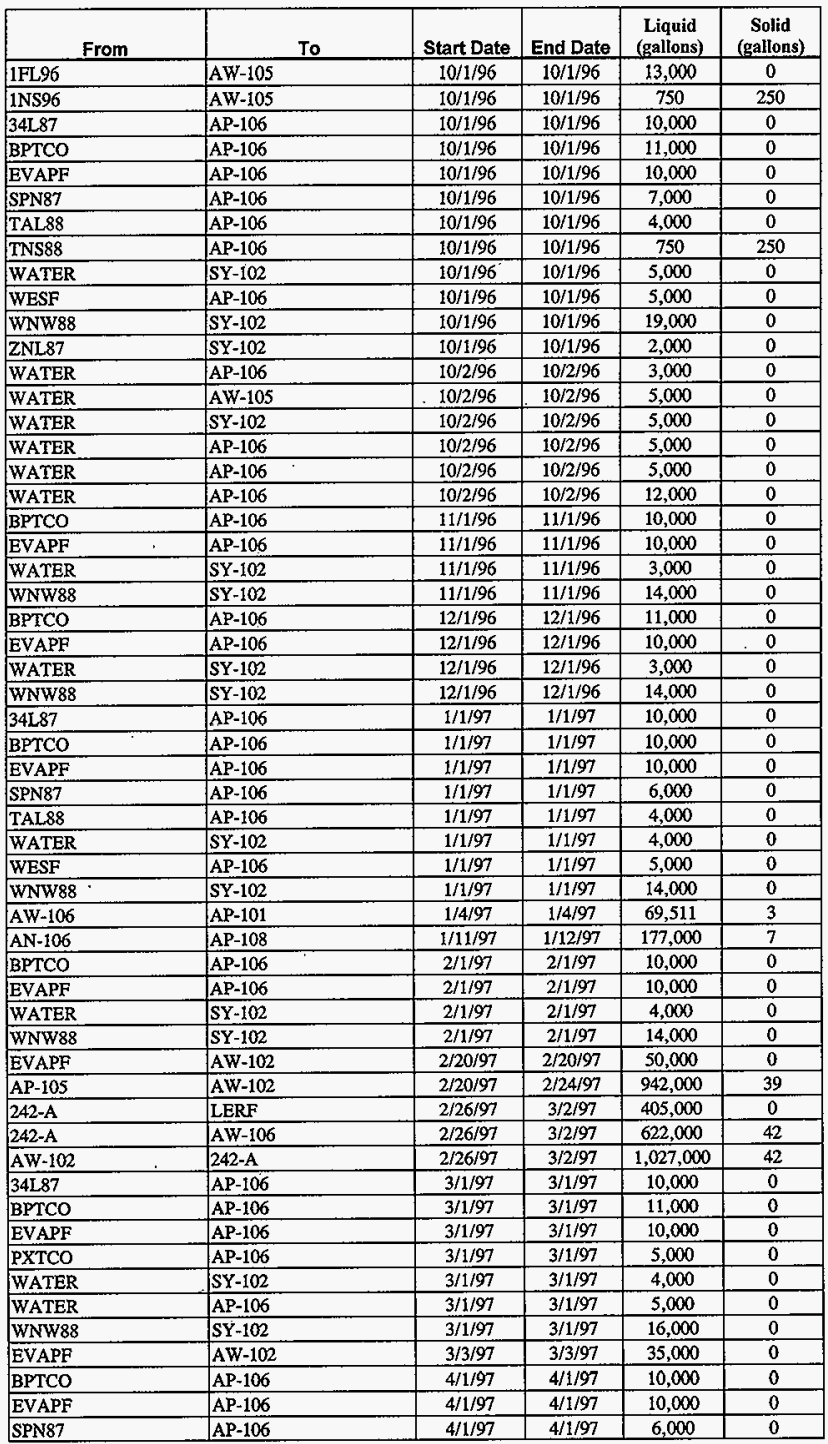


HNF-2321

Revision 0

Table A.3-1. Complete Transfer List for Case 3.

\begin{tabular}{|c|c|c|c|c|c|}
\hline From & To & Start Date & End Date & $\begin{array}{c}\text { Liquid } \\
\text { (gallons) }\end{array}$ & $\begin{array}{c}\text { Solid } \\
\text { (gallons) }\end{array}$ \\
\hline TAL88 & AP-106 & $4 / 1 / 97$ & $4 / 1 / 97$ & 4,000 & 0 \\
\hline WATER & SY-102 & $4 / 1 / 97$ & $4 / 1 / 97$ & 6,000 & 0 \\
\hline WESF & AP-106 & $4 / 1 / 97$ & $4 / 1 / 97$ & 5,000 & 0 \\
\hline WNW88 & SY-102 & $4 / 1 / 97$ & $4 / 1 / 97$ & 22,000 & 0 \\
\hline AW-106 & AP-105 & $4 / 25 / 97$ & $4 / 28 / 97$ & 617,000 & 29 \\
\hline AY-101 & AP-108 & $4 / 25 / 97$ & $4 / 29 / 97$ & 809,000 & 30 \\
\hline $34 L 87$ & AP-106 & $5 / 1 / 97$ & $5 / 1 / 97$ & 10,000 & 0 \\
\hline BPTCO & AP-106 & $5 / 1 / 97$ & $5 / 1 / 97$ & 11,000 & 0 \\
\hline EVAPF & AP-106 & $5 / 1 / 97$ & $5 / 1 / 97$ & 10,000 & 0 \\
\hline WATER & AP-106 & $5 / 1 / 97$ & $5 / 1 / 97$ & 4,000 & 0 \\
\hline WATER & SY-102 & $5 / 1 / 97$ & $5 / 1 / 97$ & 5,000 & 0 \\
\hline WNW88 & SY-102 & $5 / 1 / 97$ & $5 / 1 / 97$ & 19,000 & 0 \\
\hline BPTCO & AP-106 & $6 / 1 / 97$ & $6 / 1 / 97$ & 10,000 & 0 \\
\hline EVAPF & AP-106 & $6 / 1 / 97$ & $6 / 1 / 97$ & 10,000 & 0 \\
\hline WATER & SY-102 & $6 / 1 / 97$ & $6 / 1 / 97$ & 4,000 & 0 \\
\hline WNW88 & SY-102 & $6 / 1 / 97$ & $6 / 1 / 97$ & 16,000 & 0 \\
\hline $34 \mathrm{~L} 87$ & AP-106 & $7 / 1 / 97$ & $7 / 1 / 97$ & 10,000 & 0 \\
\hline BPTCO & AP-106 & $7 / 1 / 97$ & $7 / 1 / 97$ & 11,000 & 0 \\
\hline EVAPF & AP-106 & $7 / 1 / 97$ & $7 / 1 / 97$ & 10,000 & 0 \\
\hline SPN87 & AP-106 & $7 / 1 / 97$ & $7 / 1 / 97$ & 6,000 & 0 \\
\hline TAL88 & AP-106 & $7 / 1 / 97$ & $7 / 1 / 97$ & 4,000 & 0 \\
\hline WATER & AN-101 & $7 / 1 / 97$ & $7 / 1 / 97$ & 5,000 & 0 \\
\hline WATER & SY-102 & $7 / 1 / 97$ & $7 / 1 / 97$ & 6,000 & 0 \\
\hline WCE88 & AN-101 & $7 / 1 / 97$ & $7 / 1 / 97$ & 20,000 & 0 \\
\hline WESF & AP-106 & $7 / 1 / 97$ & $7 / 1 / 97$ & 5,000 & 0 \\
\hline WNW88 & $S Y-102$ & $7 / 1 / 97$ & $7 / 1 / 97$ & 24,000 & 0 \\
\hline AN-106 & AP-108 & $7 / 25 / 97$ & $7 / 26 / 97$ & 194,000 & 7 \\
\hline AP-108 & AW-102 & $7 / 25 / 97$ & $7 / 31 / 97$ & $1,198,000$ & 42 \\
\hline $242-A$ & AW-106 & $7 / 28 / 97$ & $8 / 2 / 97$ & 346,700 & 39 \\
\hline $242-\mathrm{A}$ & LERF & $7 / 28 / 97$ & $8 / 2 / 97$ & 851,300 & 0 \\
\hline AW-102 & $242-\mathrm{A}$ & $7 / 28 / 97$ & $8 / 2 / 97$ & $1,198,000$ & 39 \\
\hline BPTCO & AP-106 & $8 / 1 / 97$ & $8 / 1 / 97$ & 10,000 & 0 \\
\hline EVAPF & AP-106 & $8 / 1 / 97$ & $8 / 1 / 97$ & 10,000 & 0 \\
\hline WATER & AN-101 & $8 / 1 / 97$ & $8 / 1 / 97$ & 6,000 & 0 \\
\hline WATER & $S Y-102$ & $8 / 1 / 97$ & $8 / 1 / 97$ & 13,000 & 0 \\
\hline WCE88 & AN-101 & $8 / 1 / 97$ & $8 / 1 / 97$ & 25,000 & 0 \\
\hline WNW88 & SY-102 & $8 / 1 / 97$ & $8 / 1 / 97$ & 51,000 & 0 \\
\hline EVAPF & AW-102 & $8 / 3 / 97$ & $8 / 3 / 97$ & 25,000 & 0 \\
\hline WATER & AP-107 & $8 / 20 / 97$ & $8 / 20 / 97$ & 35,000 & 0 \\
\hline AW-106 & AN-106 & $8 / 25 / 97$ & $8 / 26 / 97$ & 347,000 & 16 \\
\hline SY-102 & AP-107 & $8 / 25 / 97$ & $8 / 27 / 97$ & 520,000 & 20 \\
\hline BPTCO & AP-106 & $9 / 1 / 97$ & 9/1/97 & 10,000 & 0 \\
\hline EVAPF & AP-106 & $9 / 1 / 97$ & $9 / 1 / 97$ & 10,000 & 0 \\
\hline WATER & AN-101 & $9 / 1 / 97$ & $9 / 1 / 97$ & 10,000 & 0 \\
\hline WATER & SY-102 & $9 / 1 / 97$ & $9 / 1 / 97$ & 15,000 & 0 \\
\hline WCE88 & AN-101 & $9 / 1 / 97$ & $9 / 1 / 97$ & 40,000 & 0 \\
\hline WNW88 & SY-102 & $9 / 1 / 97$ & $9 / 1 / 97$ & 61,000 & 0 \\
\hline 34 L87 & AP-106 & $10 / 1 / 97$ & $\cdot 10 / 1 / 97$ & 7,000 & 0 \\
\hline 34L87 & AP-106 & $10 / 1 / 97$ & $10 / 1 / 97$ & 10,000 & 0 \\
\hline BPTCO & AP-106 & $10 / 1 / 97$ & $10 / 1 / 97$ & 11,000 & 0 \\
\hline EVAPF & AP-106 & $10 / 1 / 97$ & $10 / 1 / 97$ & 10,000 & 0 \\
\hline PXTCO & AP-106 & $10 / 1 / 97$ & $10 / 1 / 97$ & 5,000 & 0 \\
\hline SPN87 & SY-102 & $10 / 1 / 97$ & $10 / 1 / 97$ & 7,000 & 0 \\
\hline TAL88 & AP-106 & $10 / 1 / 97$ & $10 / 1 / 97$ & 4,000 & 0 \\
\hline TNS88 & AP-106 & $10 / 1 / 97$ & $10 / 1 / 97$ & 750 & 250 \\
\hline WATER & AP-106 & $10 / 1 / 97$ & $10 / 1 / 97$ & 1,000 & 0 \\
\hline
\end{tabular}


HNF-2321

Revision 0

Table A.3-1. Complete Transfer List for Case 3.

\begin{tabular}{|c|c|c|c|c|c|}
\hline From & To & Start Date & End Date & $\begin{array}{c}\text { Liquid } \\
\text { (gallons) }\end{array}$ & $\begin{array}{c}\text { Solid } \\
\text { (gallons) }\end{array}$ \\
\hline WATER & AN.101 & $10 / 1 / 97$ & $10 / 1 / 97$ & 2,000 & 0 \\
\hline WATER & SY-102 & $10 / 1 / 97$ & $10 / 1 / 97$ & 4,000 & 0 \\
\hline WCE88 & AN-101 & $10 / 1 / 97$ & $10 / 1 / 97$ & 7,000 & 0 \\
\hline WESF & AP-106 & $10 / 1 / 97$ & $10 / 1 / 97$ & 5,000 & 0 \\
\hline WNE88 & AP-106 & $10 / 1 / 97$ & $10 / 1 / 97$ & 1,000 & 0 \\
\hline WNW88 & $S Y-102$ & $10 / 1 / 97$ & $10 / 1 / 97$ & 18,000 & 0 \\
\hline ZNL87 & SY-102 & $10 / 1 / 97$ & $10 / 1 / 97$ & 4,000 & 0 \\
\hline WASH-CAUSTIC & AN-107 & $10 / 2 / 97$ & $10 / 2 / 97$ & 66,000 & 0 \\
\hline WATER & $S Y-102$ & $10 / 2 / 97$ & $10 / 2 / 97$ & 1,000 & 0 \\
\hline WATER & AP-106 & $10 / 2 / 97$ & $10 / 2 / 97$ & 3,000 & 0 \\
\hline WATER & AP-106 & $10 / 2 / 97$ & $10 / 2 / 97$ & 3,000 & 0 \\
\hline WATER & SY-102 & $10 / 2 / 97$ & $10 / 2 / 97$ & 5,000 & 0 \\
\hline WATER & AP-106 & $10 / 2 / 97$ & $10 / 2 / 97$ & $.5,000$ & 0 \\
\hline WATER & AP-106 & $10 / 2 / 97$ & $10 / 2 / 97$ & 5,000 & 0 \\
\hline AY-102 & AP-106 & $10 / 25 / 97$ & $10 / 25 / 97$ & 35,999 & 1 \\
\hline WATER & $A Y-102$ & $10 / 26 / 97$ & $10 / 27 / 97$ & 176,000 & 0 \\
\hline EVAPF & AP-106 & $11 / 1 / 97$ & $11 / 1 / 97$ & 10,000 & 0 \\
\hline WATER & AP-106 & $11 / 1 / 97$ & $11 / 1 / 97$ & 1,000 & 0 \\
\hline WATER & $\$ Y-102$ & $11 / 1 / 97$ & $11 / 1 / 97$ & 4,000 & 0 \\
\hline WATER & AN-101 & $11 / 1 / 97$ & $11 / 1 / 97$ & 5,000 & 0 \\
\hline WCE88 & AN-101 & $11 / 1 / 97$ & $11 / 1 / 97$ & 20,000 & 0 \\
\hline WNE88 & AP-106 & $11 / 1 / 97$ & $11 / 1 / 97$ & 1,000 & 0 \\
\hline WNW88 & SY-102 & $11 / 1 / 97$ & $11 / 1 / 97$ & 16,000 & 0 \\
\hline EVAPF & AP- 106 & $12 / 1 / 97$ & $12 / 1 / 97$ & 10,000 & 0 \\
\hline WATER & AP-106 & $12 / 1 / 97$ & $12 / 1 / 97$ & 1,000 & 0 \\
\hline WATER & SY-102 & $12 / 1 / 97$ & $12 / 1 / 97$ & 3,000 & 0 \\
\hline WATER & AN-101 & $12 / 1 / 97$ & $12 / 1 / 97$ & 5,000 & 0 \\
\hline WCE88 & AN-101 & $12 / 1 / 97$ & $12 / 1 / 97$ & 20,000 & 0 \\
\hline WNE 88 & AP-106 & $12 / 1 / 97$ & $12 / 1 / 97$ & 1,000 & 0 \\
\hline WNW88 & SY-102 & $12 / 1 / 97$ & $12 / 1 / 97$ & 14,000 & 0 \\
\hline WATER & AP-107 & $12 / 20 / 97$ & $12 / 20 / 97$ & 35,000 & 0 \\
\hline AP-106 & AP-104 & $12 / 25 / 97$ & $12 / 28 / 97$ & 739,400 & 25 \\
\hline SY-102 & AP-107 & $12 / 26 / 97$ & $12 / 27 / 97$ & 351,700 & 14 \\
\hline $34 L .87$ & AP-106 & $1 / 1 / 98$ & $1 / 1 / 98$ & 10,000 & 0 \\
\hline BPTCO & AP-106 & $1 / 1 / 98$ & $1 / 1 / 98$ & 11,000 & 0 \\
\hline EVAPF & AP-106 & $1 / 1 / 98$ & $1 / 1 / 98$ & 10,000 & 0 \\
\hline SPN87 & SY-102 & $1 / 1 / 98$ & $1 / 1 / 98$ & 6,000 & 0 \\
\hline TAL88 & AP-106 & $1 / 1 / 98$ & $1 / 1 / 98$ & 4,000 & 0 \\
\hline WATER & AP-106 & $1 / 1 / 98$ & $1 / 1 / 98$ & 1,000 & 0 \\
\hline WATER & AP-106 & $1 / 1 / 98$ & $1 / 1 / 98$ & 4,000 & 0 \\
\hline WATER & $S Y-102$ & $1 / 1 / 98$ & $1 / 1 / 98$ & 4,000 & 0 \\
\hline WATER & AN-101 & $1 / 1 / 98$ & $1 / 1 / 98$ & 5,000 & 0 \\
\hline WCE88 & AN-101 & $1 / 1 / 98$ & $1 / 1 / 98$ & 20,000 & 0 \\
\hline WNES8 & AP-106 & $1 / 1 / 98$ & $1 / 1 / 98$ & 1,000 & 0 \\
\hline WNW88 & SY-102 & $1 / 1 / 98$ & $1 / 1 / 98$ & 14,000 & 0 \\
\hline EVAPF & AP-106 & $2 / 1 / 98$ & $2 / 1 / 98$ & 10,000 & 0 \\
\hline WATER & AP-106 & $2 / 1 / 98$ & $2 / 1 / 98$ & 1,000 & 0 \\
\hline WATER & SY-102 & $2 / 1 / 98$ & $2 / 1 / 98$ & 4,000 & 0 \\
\hline WATER & AN-101 & $2 / 1 / 98$ & $2 / 1 / 98$ & 5,000 & 0 \\
\hline WCE88 & AN-101 & $2 / 1 / 98$ & $2 / 1 / 98$ & 20,000 & 0 \\
\hline WNE88 & AP-106 & $2 / 1 / 98$ & $2 / 1 / 98$ & 1,000 & 0 \\
\hline WNW88 & SY-102 & $2 / 1 / 98$ & $2 / 1 / 98$ & 14,000 & 0 \\
\hline $34 \mathrm{~L} 87$ & AP-106 & $3 / 1 / 98$ & $3 / 1 / 98$ & 10,000 & 0 \\
\hline BPTCO & AP-106 & $3 / 1 / 98$ & $3 / 1 / 98$ & 11,000 & 0 \\
\hline EVAPF & AP-106 & $3 / 1 / 98$ & $3 / 1 / 98$ & 10,000 & 0 \\
\hline WATER & AP-106 & $3 / 1 / 98$ & $3 / 1 / 98$ & 1,000 & 0 \\
\hline
\end{tabular}


HNF-2321

Revision 0

Table A.3-1. Complete Transfer List for Case 3.

\begin{tabular}{|c|c|c|c|c|c|}
\hline From & To & Start Date & End Date & $\begin{array}{c}\text { Liquid } \\
\text { (gallons) }\end{array}$ & $\begin{array}{c}\text { Solid } \\
\text { (gallons) }\end{array}$ \\
\hline WATER & SY-102 & $3 / 1 / 98$ & $3 / 1 / 98$ & 3,000 & 0 \\
\hline WATER & AP-106 & $3 / 1 / 98$ & $3 / 1 / 98$ & 5,000 & 0 \\
\hline WATER & AN-101 & $3 / 1 / 98$ & $3 / 1 / 98$ & 5,000 & 0 \\
\hline WCE 88 & AN-101 & $3 / 1 / 98$ & $3 / 1 / 98$ & 20,000 & 0 \\
\hline WNE88 & AP-106 & $3 / 1 / 98$ & $3 / 1 / 98$ & 1,000 & 0 \\
\hline WNW88 & SY-102 & $3 / 1 / 98$ & $3 / 1 / 98$ & 10,000 & 0 \\
\hline WATER & AP-104 & $3 / 20 / 98$ & $3 / 20 / 98$ & 35,000 & 0 \\
\hline SY-102 & AP-104 & $3 / 25 / 98$ & $3 / 25 / 98$ & 55,010 & 2 \\
\hline AP-104 & AW-102 & $3 / 25 / 98$ & $3 / 29 / 98$ & 827,900 & 26 \\
\hline $242-\mathrm{A}$ & AW-106 & $3 / 28 / 98$ & $4 / 1 / 98$ & 151,300 & 24 \\
\hline $242-\mathrm{A}$ & LERF & $3 / 28 / 98$ & $4 / 1 / 98$ & 737,100 & 0 \\
\hline AW-102 & $242-\mathrm{A}$ & $3 / 28 / 98$ & $4 / 1 / 98$ & 888,400 & 24 \\
\hline EVAPF & AP-106 & $4 / 1 / 98$ & $4 / 1 / 98$ & 10,000 & 0 \\
\hline SPN87 & SY-102 & $4 / 1 / 98$ & $4 / 1 / 98$ & 6,000 & 0 \\
\hline TAL88 & AP-106 & $4 / 1 / 98$ & $4 / 1 / 98$ & 4,000 & 0 \\
\hline WATER & AP-106 & $4 / 1 / 98$ & $\cdot 4 / 1 / 98$ & 1,000 & 0 \\
\hline WATER & SY-102 & $4 / 1 / 98$ & $4 / 1 / 98$ & 3,000 & 0 \\
\hline WATER & AN-101 & $4 / 1 / 98$ & $4 / 1 / 98$ & 5,000 & 0 \\
\hline WCE88 & AN-101 & $4 / 1 / 98$ & $4 / 1 / 98$ & 20,000 & 0 \\
\hline WNE88 & AP-106 & $4 / 1 / 98$ & $4 / 1 / 98$ & 1,000 & 0 \\
\hline WNW88 & SY-102 & $4 / 1 / 98$ & $4 / 1 / 98$ & 10,000 & 0 \\
\hline $34 \mathrm{~L} 87$ & AP-106 & $5 / 1 / 98$ & $5 / 1 / 98$ & 10,000 & 0 \\
\hline BPTCO & AP-106 & $5 / 1 / 98$ & $5 / 1 / 98$ & 11,000 & 0 \\
\hline EVAPF & AP-106 & $5 / 1 / 98$ & $5 / 1 / 98$ & 10,000 & 0 \\
\hline WATER & AP-106 & $5 / 1 / 98$ & $5 / 1 / 98$ & 1,000 & 0 \\
\hline WATER & SY-102 & $5 / 1 / 98$ & $5 / 1 / 98$ & 3,000 & 0 \\
\hline WATER & AP-106 & $5 / 1 / 98$ & $5 / 1 / 98$ & 4,000 & 0 \\
\hline WATER & AN-101 & $5 / 1 / 98$ & $5 / 1 / 98$ & 5,000 & 0 \\
\hline WCE88 & AN-101 & $5 / 1 / 98$ & $5 / 1 / 98$ & 20,000 & 0 \\
\hline WNE88 & AP-106 & $5 / 1 / 98$ & $5 / 1 / 98$ & 1,000 & 0 \\
\hline WNW88 & SY-102 & $5 / 1 / 98$ & $5 / 1 / 98$ & 10,000 & 0 \\
\hline AP-107 & AW-102 & $5 / 25 / 98$ & $5 / 29 / 98$ & 936,200 & 33 \\
\hline $242-\mathrm{A}$ & AW-106 & $5 / 28 / 98$ & $6 / 1 / 98$ & 380,500 & 32 \\
\hline $242-\mathrm{A}$ & LERF & $5 / 28 / 98$ & $6 / 1 / 98$ & 555,600 & 0 \\
\hline AW-102 & $242-\mathrm{A}$ & $5 / 28 / 98$ & $6 / 1 / 98$ & 936,100 & 32 \\
\hline EVAPF & AP-106 & $6 / 1 / 98$ & $6 / 1 / 98$ & 10,000 & 0 \\
\hline WATER & AP-106 & $6 / 1 / 98$ & $6 / 1 / 98$ & 1,000 & 0 \\
\hline WATER & SY-102 & $6 / 1 / 98$ & $6 / 1 / 98$ & 3,000 & 0 \\
\hline WATER & AN-101 & $6 / 1 / 98$ & $6 / 1 / 98$ & 4,000 & 0 \\
\hline WCE88 & AN-101 & $6 / 1 / 98$ & $6 / 1 / 98$ & 15,000 & 0 \\
\hline WNE88 & AP-106 & $6 / 1 / 98$ & $6 / 1 / 98$ & 1,000 & 0 \\
\hline WNW88 & $S Y-102$ & $6 / 1 / 98$ & $6 / 1 / 98$ & 10,000 & 0 \\
\hline EVAPF & AW-102 & $6 / 3 / 98$ & $6 / 3 / 98$ & 35,000 & 0 \\
\hline WATER & AP-104 & $6 / 20 / 98$ & $6 / 20 / 98$ & 35,000 & 0 \\
\hline SY-102 & AP-104 & $6 / 25 / 98$ & $6 / 25 / 98$ & 45,008 & 2 \\
\hline AW-106 & AP-105 & $6 / 25 / 98$ & $6 / 26 / 98$ & 341,000 & 15 \\
\hline $34 \mathrm{~L} 87$ & AP-106 & $7 / 1 / 98$ & $7 / 1 / 98$ & 10,000 & 0 \\
\hline EVAPF & AP-106 & $7 / 1 / 98$ & $7 / 1 / 98$ & 10,000 & 0 \\
\hline \begin{tabular}{|l} 
SPN87 \\
\end{tabular} & SY-102 & $7 / 1 / 98$ & $7 / 1 / 98$ & 6,000 & 0 \\
\hline TAL88 & AP-106 & $7 / 1 / 98$ & $7 / 1 / 98$ & 4,000 & 0 \\
\hline WATER & SY-102 & $7 / 1 / 98$ & $7 / 1 / 98$ & 3,000 & 0 \\
\hline WATER & AP-106 & $7 / 1 / 98$ & $7 / 1 / 98$ & 4,000 & 0 \\
\hline WATER & AN-101 & $7 / 1 / 98$ & $7 / 1 / 98$ & 4,000 & 0 \\
\hline WCE88 & AN-101 & $7 / 1 / 98$ & $7 / 1 / 98$ & 14,000 & 0 \\
\hline WNW88 & SY-102 & $7 / 1 / 98$ & $7 / 1 / 98$ & 10,000 & 0 \\
\hline$A Y-102$ & $C-106$ & 7/1/98 & $7 / 5 / 98$ & 920,700 & 31 \\
\hline
\end{tabular}




\begin{tabular}{|c|c|c|c|c|c|}
\hline 0 & $000^{2} 9$ & $86 / \mathrm{I} / \mathrm{ZI}$ & $86 / \mathrm{L} / \mathrm{ZI}$ & ZOT-XS & $88 \mathrm{MNM}$ \\
\hline 0 & $000^{\circ} 6 z$ & $86 / \mathrm{I} / \mathrm{LI}$ & $86 / \mathrm{I} / \mathrm{ZI}$ & $90 \mathrm{I}-\mathrm{dV}$ & $88 \mathrm{AM}$ \\
\hline 0 & $000^{\circ} \pitchfork I$ & $86 / 1 / Z I$ & $86 / \mathrm{I} / \mathrm{ZL}$ & IOI-NV & $8830 M$ \\
\hline 0 & $0002 \mathrm{~L}$ & $86 / 1 / 21$ & $86 / \mathrm{L} / \mathrm{ZI}$ & $90 \mathrm{I}-\mathrm{dV}$ & dgIVM \\
\hline 0 & $000^{\circ} t$ & $86 / \mathrm{T} / \mathrm{ZI}$ & $86 / \mathrm{t} / \mathrm{Zl}$ & I0I-NV & \&G.LVM \\
\hline 0 & $000^{\circ} \mathrm{z}$ & $86 / \mathrm{l} / \mathrm{ZI}$ & $86 / \mathrm{L} / \mathrm{ZI}$ & $20 \mathrm{I}-\mathrm{XS}$ & 8GLYM \\
\hline 0 & $000^{\circ} 0 \mathrm{r}$ & $86 / \mathrm{L} / \mathrm{CI}$ & $86 / 1 / 2 I$ & $90 \mathrm{I}-\mathrm{dV}$ & HdV $\Lambda$ G \\
\hline$\tau$ & $\angle S 8^{\circ} S S$ & $86 / S / I I$ & $86 / 5 / 11$ & $20 \mathrm{I}-\mathrm{MV}$ & $90 \mathrm{I}-\mathrm{MV}$ \\
\hline 0 & $000^{\circ} 9$ & $86 / I / I 1$ & $86 / \mathrm{I} / \mathrm{II}$ & $20 \mathrm{I}-\mathrm{XS}$ & $88 \mathrm{MNM}$ \\
\hline 0 & $000^{2} z z$ & $86 / \mathrm{I} / \mathrm{tI}$ & $86 / \mathrm{l} / \mathrm{I} \mathrm{I}$ & $90 i-\mathrm{dv}$ & $88 \mathrm{ANM}$ \\
\hline 0 & $000^{2} \nabla I$ & $86 / \mathrm{T} / \mathrm{LI}$ & $86 / \mathrm{I} / \mathrm{II}$ & IOI-NV & $88 \mathrm{HOM}$ \\
\hline 0 & $000^{\circ} \mathrm{s}$ & $86 / \mathrm{T} / \mathrm{II}$ & $86 / 1 / 11$ & $90 \mathrm{I}-\mathrm{dV}$ & dGIVM \\
\hline 0 & $000^{\circ} t$ & $86 /$ /III & $86 / 1 / 11$ & I0I-NV & XG.LVM \\
\hline 0 & $000^{\circ} \mathrm{Z}$ & $86 / I / \mathrm{II}$ & $86 / / / \mathrm{ll}$ & $20 \mathrm{I}-\mathrm{xs}$ & צGLVM \\
\hline 0 & $000^{t} 0 \mathrm{I}$ & $86 / \mathrm{K} / \mathrm{II}$ & $86 / \mathrm{l} / \mathrm{tI}$ & $90 \mathrm{I}-\mathrm{dV}$ & AdV $\Lambda$ 更 \\
\hline $2 I$ & $000^{2} 68 \mathrm{Z}$ & $86 / 0 \mathrm{E} / \mathrm{OI}$ & $86 / 6 \mathrm{C} / 0 \mathrm{I}$ & I0I-dV & $90 \mathrm{I}-\mathrm{MV}$ \\
\hline $0 z$ & $000^{\circ} 090$ & $86 / \varepsilon Z / 0 \mathrm{I}$ & $86 / \mathrm{tZ} / 0 \mathrm{I}$ & t0I-MV & I0I-dV \\
\hline 9 & $000^{6}+91$ & $86 / 8 \% / 0 I$ & $86 / 8 \mathrm{I} / 0 \mathrm{I}$ & toI-dV & $t 0 I-M V$ \\
\hline $8 \mathrm{I}$ & $000^{\circ} \mathrm{OtS}$ & $86 / \mathrm{I} / \mathrm{TI}$ & $86 / 6 / 0 \mathrm{I}$ & $\angle 0 I-d V$ & $\$ 0 I-M V$ \\
\hline$s$ & $0000^{\circ} 051$ & $86 / 6 / 0 \mathrm{I}$ & $86 / 6 / 0 \mathrm{I}$ & $90 I-M V$ & $20 T-M V$ \\
\hline$z$ & $800^{6} 08$ & $86 / \mathrm{S} / 0 \mathrm{I}$ & $86 / 5 / 0 \mathrm{I}$ & $20 \mathrm{I}-\mathrm{MV}$ & toI-dV \\
\hline 0 & $000^{\circ} 05$ & $86 / t / 0 \mathrm{I}$ & $86 / 7 / 01$ & $20 \mathrm{I}-\mathrm{MV}$ & 배 $\forall \Lambda \mathrm{G}$ \\
\hline 0 & $000^{\circ} \mathrm{s}$ & $86 / Z / 0 \mathrm{I}$ & $86 / 2 / 0 \mathrm{I}$ & $z 0 \mathrm{I}-\mathrm{XS}$ & YGIVM \\
\hline 0 & $000^{t} s$ & $86 / 2 / 0 \mathrm{I}$ & $86 / \tau / 0 \mathrm{I}$ & $90 \mathrm{I}-\mathrm{dV}$ & dgIVM \\
\hline 0 & $000^{\circ} \varepsilon$ & $86 / 2 / 0 \mathrm{I}$ & $86 / 2 / 0 \mathrm{I}$ & $90 \mathrm{I}-\mathrm{dV}$ & \&GIVM \\
\hline 0 & $000^{\circ} t$ & $86 / \mathrm{L} / 0 \mathrm{I}$ & $86 / 7 / 0 \mathrm{I}$ & $20 \mathrm{I}-\lambda \mathrm{S}$ & $\angle 8 \mathrm{INZ}$ \\
\hline 0 & $000^{6} 9$ & $86 / \mathrm{L} / 0 \mathrm{I}$ & $86 / \mathrm{T} / 0 \mathrm{I}$ & $201-x S$ & $88 \mathrm{MNM}$ \\
\hline 0 & $000^{\prime} z \bar{z}$ & $86 / \mathrm{L} / 0 \mathrm{I}$ & $86 / \mathrm{T} / 0 \mathrm{I}$ & $90 \mathrm{I}-\mathrm{dV}$ & $88 \mathrm{GNM}$ \\
\hline 0 & $\infty 0^{\circ} \mathrm{s}$ & $86 / \mathrm{l} / 0 \mathrm{I}$ & $86 / \mathrm{T} / 0 \mathrm{I}$ & $90 \mathrm{r}-\mathrm{dV}$ & $\exists S I M$ \\
\hline 0 & $000^{\circ} \$ I$ & $86 / \mathrm{l} / 0 \mathrm{I}$ & $86 / \mathrm{L} / 0 \mathrm{I}$ & $\mathrm{IOI}-\mathrm{NV}$ & $889 \supset M$ \\
\hline 0 & $000^{\circ} \mathrm{s}$ & $86 / \mathrm{L} / 0 \mathrm{I}$ & $86 / \mathrm{L} / 0 \mathrm{I}$ & $90 I^{-d V}$ & \&GLVM \\
\hline 0 & $000^{\circ} t$ & $86 / \mathrm{h} / 0 \mathrm{I}$ & $86 / \mathrm{L} / 0 \mathrm{I}$ & I0I-NV & \&GLYM \\
\hline 0 & $000^{\prime} \mathrm{z}$ & $86 / \mathrm{I} / 0 \mathrm{I}$ & $86 / \mathrm{L} / 0 \mathrm{I}$ & $201-\pi s$ & \&gIVM \\
\hline OSZ & $O S L$ & $86 / \mathrm{l} / 0 \mathrm{I}$ & 86/1/0I & $90 \mathrm{I}-\mathrm{dV}$ & 88SNIL \\
\hline 0 & $000^{2} t$ & $86 / \mathrm{L} / 0 \mathrm{I}$ & $86 / \mathrm{I} / 0 \mathrm{I}$ & $90 \mathrm{I}-\mathrm{dV}$ & $88 \mathrm{TVL}$ \\
\hline 0 & $000^{\circ} \mathrm{L}$ & $86 / \mathrm{L} / 0 \mathrm{I}$ & $86 / \mathrm{l} / 0 \mathrm{I}$ & $20 \mathrm{I}-\lambda \mathrm{S}$ & $\angle 8 \mathrm{NdS}$ \\
\hline 0 & $000^{\circ} s$ & $86 / \mathrm{L} / 0 \mathrm{I}$ & $86 / \mathrm{L} / 0 \mathrm{~T}$ & $901-d V$ & OOLXd \\
\hline 0 & $000^{\circ} 0 \mathrm{I}$ & $86 / \mathrm{I} / 0 \mathrm{I}$ & $86 / \mathrm{L} / 0 \mathrm{I}$ & $90 \mathrm{l}-\mathrm{dV}$ & 매 $\Lambda \mathrm{Z}$ \\
\hline 0 & $000^{\circ} 0 \mathrm{I}$ & $86 / 1 / 0 \mathrm{I}$ & $86 / \mathrm{L} / 0 \mathrm{I}$ & $90 \mathrm{I}-\mathrm{dV}$ & $\angle 8 T t E$ \\
\hline$L z$ & $000^{\circ} \mathrm{s} 29$ & $86 / 82 / 6$ & $86 / S Z / 6$ & $E 0 I-M V$ & I0I-dV \\
\hline$z$ & $\angle 00^{2} 0 \mathrm{~b}$ & $86 / 52 / 6$ & $86 / \mathcal{S Z} / 6$ & $\angle 0 \mathrm{I}-\mathrm{dV}$ & $20 \mathrm{I}-\mathrm{\lambda S}$ \\
\hline 0 & $000^{t} S \mathcal{E}$ & $86 / 02 / 6$ & $86 / 02 / 6$ & $\angle O T-d V$ & \&gIVM \\
\hline 0 & $000^{\circ} 9$ & $86 / t / 6$ & $86 / \tau / 6$ & $201-x s$ & $88 \mathrm{MNM}$ \\
\hline 0 & $000^{2} z z$ & $86 / 1 / 6$ & $86 / \mathrm{I} / 6$ & $90 \mathrm{I}-\mathrm{dV}$ & $88 \mathrm{GNM}$ \\
\hline 0 & $000^{\circ} \nabla I$ & $86 / 1 / 6$ & $86 / t / 6$ & I0I-NV & 88BDM \\
\hline 0 & $000^{\circ} \mathrm{s}$ & $86 / 1 / 6$ & $86 / 1 / 6$ & $90 \mathrm{I}-\mathrm{dV}$ & dGIVM \\
\hline 0 & $000^{\circ} 7$ & $86 / 1 / 6$ & $86 / 1 / 6$ & I0I-NV & \&GJVM \\
\hline 0 & $000^{\circ} z$ & $86 / 1 / 6$ & $86 / \mathrm{L} / 6$ & $20 \mathrm{I}-\mathrm{XS}$ & ชด $\mathrm{V} \vee M$ \\
\hline 0 & $000^{\circ} 0 \mathrm{I}$ & $86 / \mathrm{t} / 6$ & $86 / \mathrm{L} / 6$ & $90 \mathrm{I}-\mathrm{dV}$ & ddV $A$ A \\
\hline 0 & $000^{\circ} 0 \mathrm{I}$ & $86 / 1 / 8$ & $86 / \mathrm{V} / 8$ & $20 \mathrm{I}-\mathrm{xs}$ & $88 \mathrm{MNM}$ \\
\hline 0 & $000^{\circ} \nabla I$ & $86 / 1 / 8$ & $86 / 1 / 8$ & I0I-NV & $88 \mathrm{AOM}$ \\
\hline 0 & $000^{\circ} t$ & $86 / \mathrm{t} / 8$ & $86 / \mathrm{T} / 8$ & I0I-NV & 8G.LVM \\
\hline 0 & $000^{t} \varepsilon$ & $86 / / / 8$ & $86 / 1 / 8$ & $20 \mathrm{I}-\mathrm{AS}$ & X尹ILVM \\
\hline 0 & $000^{\circ} 01$ & $86 / 1 / 8$ & $86 / \mathrm{L} / 8$ & $90 \mathrm{I}-\mathrm{dV}$ & $\exists d \forall \Lambda \Omega$ \\
\hline $69 I^{\circ} \mathrm{SI}$ & $000^{\prime} 608$ & $86 / 0 \mathrm{I} / \mathrm{II}$ & $86 / \varsigma L / L$ & $20 \mathrm{t}-\lambda \mathrm{XV}$ & $90 \mathrm{I}-\mathrm{O}$ \\
\hline 0 & $000^{\circ} 11$ & $86 / 1 \mathrm{~L} / \mathrm{L}$ & $86 / / / / L$ & $90 !-d V$ & ODIdG \\
\hline $\begin{array}{c}\text { (suo!lb3) } \\
\text { pt[0s }\end{array}$ & $\begin{array}{c}\text { (suo|fEa) } \\
\text { p!nb!t }\end{array}$ & әुе pug & गुलव मеन & 01 & uods \\
\hline
\end{tabular}

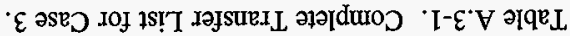

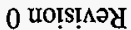 \\ IZEZ-UNH
}


HNF-2321

Revision 0

Table A.3-1. Complete Transfer List for Case 3.

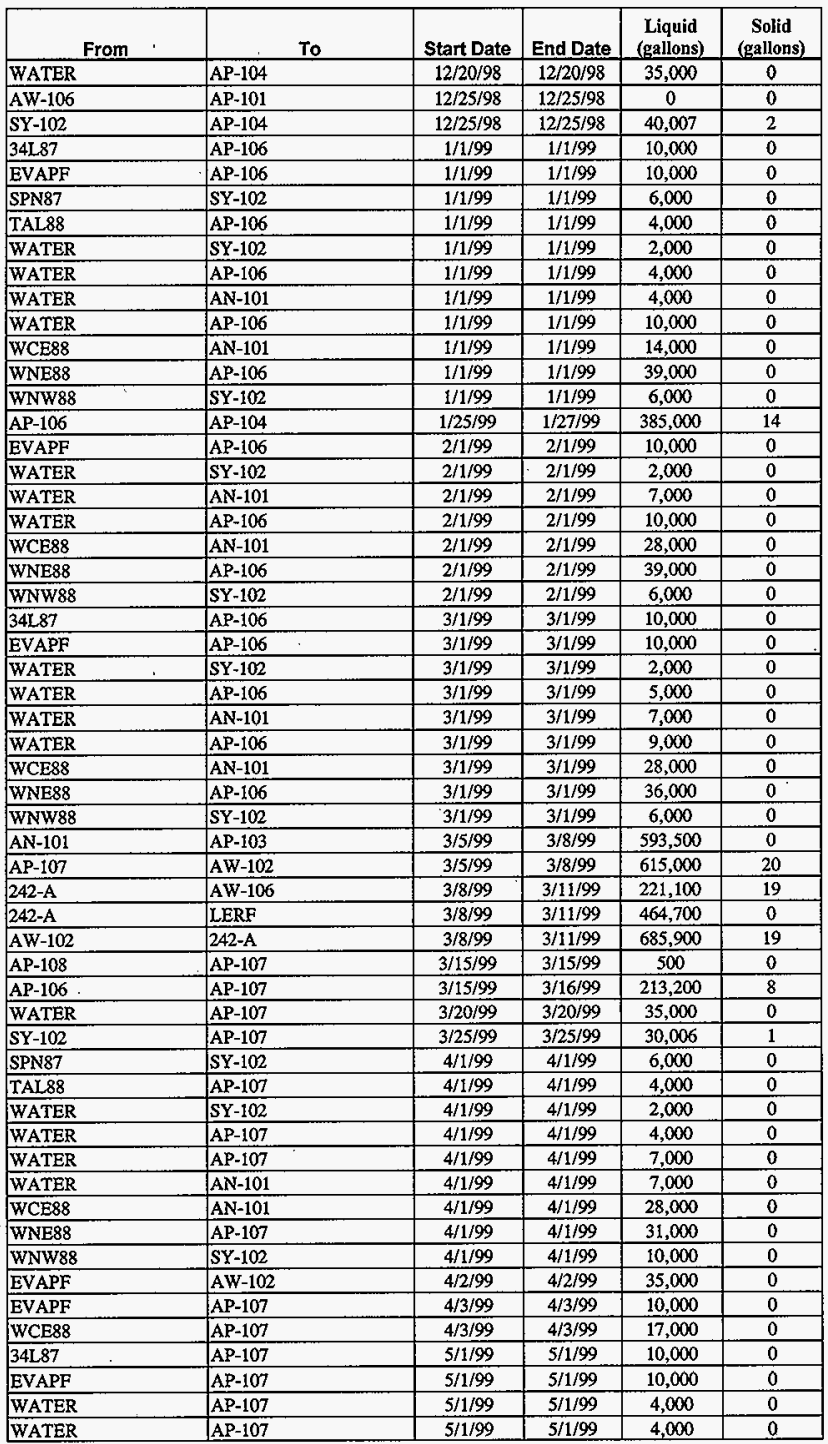


HNF-2321

Revision 0

Table A.3-1. Complete Transfer List for Case 3.

\begin{tabular}{|c|c|c|c|c|c|}
\hline From & To & Start Date & End Date & $\begin{array}{c}\text { Liquid } \\
\text { (gallons) }\end{array}$ & $\begin{array}{c}\text { Solid } \\
\text { (gallons) }\end{array}$ \\
\hline WATER & SY-102 & $5 / 1 / 99$ & $5 / 1 / 99$ & 5,000 & 0 \\
\hline WATER & AP-107 & $5 / 1 / 99$ & $5 / 1 / 99$ & 7,000 & 0 \\
\hline WATER & AN-101 & $5 / 1 / 99$ & $5 / 1 / 99$ & 7,000 & 0 \\
\hline WCE 88 & AP-107 & $5 / 1 / 99$ & $5 / 1 / 99$ & 17,000 & 0 \\
\hline WCE88 & AN-101 & $5 / 1 / 99$ & $5 / 1 / 99$ & 28,000 & 0 \\
\hline WNE88 & AP-107 & $.5 / 1 / 99$ & $5 / 1 / 99$ & 31,000 & 0 \\
\hline WNW88 & SY-102 & $5 / 1 / 99$ & $5 / 1 / 99$ & 20,000 & 0 \\
\hline AP-103 & AP-107 & $5 / 15 / 99$ & $5 / 17 / 99$ & 586,800 & 25 \\
\hline$\overline{A P-107}$ & AW-102 & $5 / 17 / 99$ & $5 / 22 / 99$ & 882,400 & 0 \\
\hline EVAPF & AP-107 & $6 / 1 / 99$ & $6 / 1 / 99$ & 10,000 & 0 \\
\hline WATER & AP-107 & $6 / 1 / 99$ & $6 / 1 / 99$ & 4,000 & 0 \\
\hline WATER & AP-107 & $6 / 1 / 99$ & $6 / 1 / 99$ & 6,000 & 0 \\
\hline WATER & AN-101 & $6 / 1 / 99$ & $6 / 1 / 99$ & 7,000 & 0 \\
\hline WATER & SY-102 & $6 / 1 / 99$ & $6 / 1 / 99$ & 10,000 & 0 \\
\hline WCE88 & AP-107 & $6 / 1 / 99$ & $6 / 1 / 99$ & 17,000 & 0 \\
\hline WCE88 & AN-101 & $6 / 1 / 99$ & $6 / 1 / 99$ & 28,000 & 0 \\
\hline WNE88 & AP-107 & $6 / 1 / 99$ & $6 / 1 / 99$ & 26,000 & 0 \\
\hline WNW88 & $S Y-102$ & $6 / 1 / 99$ & $6 / 1 / 99$ & 39,000 & 0 \\
\hline AW-106 & AW-104 & $6 / 1 / 99$ & $6 / 2 / 99$ & 221,000 & 8 \\
\hline AP-102 & AP-103 & $6 / 15 / 99$ & $6 / 20 / 99$ & $1,070,000$ & 0 \\
\hline WATER & AP-107 & $6 / 20 / 99$ & $6 / 20 / 99$ & 35,000 & 0 \\
\hline $34 \mathrm{L87}$ & AP-107 & $7 / 1 / 99$ & $7 / 1 / 99$ & 10,000 & 0 \\
\hline EVAPF & AP-107 & $7 / 1 / 99$ & $7 / 1 / 99$ & 10,000 & 0 \\
\hline SPN87 & SY-102 & $7 / 1 / 99$ & $7 / 1 / 99$ & 6,000 & 0 \\
\hline TAL88 & AP-107 & $7 / 1 / 99$ & $7 / 1 / 99$ & 4,000 & 0 \\
\hline WATER & AP-107 & $7 / 1 / 99$ & $7 / 1 / 99$ & 3,000 & 0 \\
\hline WATER & AP-107 & $7 / 1 / 99$ & $7 / 1 / 99$ & 4,000 & 0 \\
\hline WATER & AP-107 & $7 / 1 / 99$ & $7 / 1 / 99$ & 5,000 & 0 \\
\hline WATER & AN-101 & $7 / 1 / 99$ & $7 / 1 / 99$ & 7,000 & 0 \\
\hline WATER & SY-102 & $7 / 1 / 99$ & $7 / 1 / 99$ & 14,000 & 0 \\
\hline WCE88 & AP-107 & $7 / 1 / 99$ & $7 / 1 / 99$ & 11,000 & 0 \\
\hline WCE 88 & AN-101 & $7 / 1 / 99$ & $7 / 1 / 99$ & 28,000 & 0 \\
\hline WNE88 & AP-107 & $7 / 1 / 99$ & $7 / 1 / 99$ & 20,000 & 0 \\
\hline WNW88 & SY-102 & $7 / 1 / 99$ & $7 / 1 / 99$ & 57,000 & 0 \\
\hline AP-104 & AP-107 & $7 / 15 / 99$ & $7 / 16 / 99$ & 300,000 & 10 \\
\hline EVAPF & AP-107 & $8 / 1 / 99$ & $8 / 1 / 99$ & 10,000 & 0 \\
\hline WATER & AP-107 & $8 / 1 / 99$ & $8 / 1 / 99$ & 3,000 & 0 \\
\hline WATER & AP-107 & $8 / 1 / 99$ & $8 / 1 / 99$ & 3,000 & 0 \\
\hline WATER & AN-101 & $8 / 1 / 99$ & $8 / 1 / 99$ & 7,000 & 0 \\
\hline WATER & SY-102 & $8 / 1 / 99$ & $8 / 1 / 99$ & 20,000 & 0 \\
\hline WCE 88 & AP-107 & $8 / 1 / 99$ & $8 / 1 / 99$ & 11,000 & 0 \\
\hline WCE 88 & AN-101 & $8 / 1 / 99$ & $8 / 1 / 99$ & 28,000 & 0 \\
\hline WNE88 & AP-107 & $8 / 1 / 99$ & $8 / 1 / 99$ & 13,000 & 0 \\
\hline WNW88 & SY-102 & $8 / 1 / 99$ & $8 / 1 / 99$ & 82,000 & 0 \\
\hline EV:APF & AP-107 & $9 / 1 / 99$ & $9 / 1 / 99$ & 10,000 & 0 \\
\hline WATER & AP-107 & $9 / 1 / 99$ & 9/1/99 & 2,000 & 0 \\
\hline WATER & AP-107 & $9 / 1 / 99$ & $9 / 1 / 99$ & 3,000 & 0 \\
\hline WATER & AN-101 & $9 / 1 / 99$ & $9 / 1 / 99$ & 6,000 & 0 \\
\hline WATER & $S Y-102$ & $9 / 1 / 99$ & 9/1/99 & 19,000 & 0 \\
\hline WCE88 & $\mathrm{AP}-107$ & $9 / 1 / 99$ & $9 / 1 / 99$ & 11,000 & 0 \\
\hline WCE 88 & AN-101 & $9 / 1 / 99$ & 9/1/99 & 23,000 & 0 \\
\hline WNE88 & AP-107 & $9 / 1 / 99$ & $9 / 1 / 99$ & 13,000 & 0 \\
\hline WNW88 & SY-102 & $9 / 1 / 99$ & $9 / 1 / 99$ & 76,000 & 0 \\
\hline WATER & AP-107 & $9 / 25 / 99$ & $9 / 25 / 99$ & 35,000 & 0 \\
\hline 1FL96 & AW-105 & $10 / 1 / 99$ & $10 / 1 / 99$ & 40,000 & 0 \\
\hline $1 \mathrm{KL} 96$ & $A W-105$ & $10 / 1 / 99$ & $10 / 1 / 99$ & 30,000 & 0 \\
\hline
\end{tabular}


HNF-2321

Revision 0

Table A.3-1. Complete Transfer List for Case 3.

\begin{tabular}{|c|c|c|c|c|c|}
\hline From & To & Start Date & End Date & $\begin{array}{c}\text { Liquid } \\
\text { (gallons) }\end{array}$ & $\begin{array}{c}\text { Solid } \\
\text { (gallons) }\end{array}$ \\
\hline 1N\$96 & $A W-105$ & $10 / 1 / 99$ & $10 / 1 / 99$ & 750 & 250 \\
\hline $34 L 87$ & AP-107 & $10 / 1 / 99$ & $10 / 1 / 99$ & 10,000 & 0 \\
\hline EVAPF & AP- 107 & $10 / 1 / 99$ & $10 / 1 / 99$ & 10,000 & 0 \\
\hline PXTCO & AP-107 & $10 / 1 / 99$ & $10 / 1 / 99$ & 5,000 & 0 \\
\hline SPN87 & SY-102 & $10 / 1 / 99$ & $10 / 1 / 99$ & 7,000 & 0 \\
\hline TAL88 & AP- 107 & $10 / 1 / 99$ & $10 / 1 / 99$ & 6,000 & 0 \\
\hline TNS88 & AP-107 & $10 / 1 / 99$ & $10 / 1 / 99$ & 750 & 250 \\
\hline WATER & AP-107 & $10 / 1 / 99$ & $10 / 1 / 99$ & 2,000 & 0 \\
\hline WATER & AP-107 & $10 / 1 / 99$ & $10 / 1 / 99$ & 3,000 & 0 \\
\hline WATER & AN-101 & $10 / 1 / 99$ & $10 / 1 / 99$ & 6,000 & 0 \\
\hline WATER & SY-102 & $10 / 1 / 99$ & $10 / 1 / 99$ & 20,000 & 0 \\
\hline WCE 88 & AP-107 & $10 / 1 / 99$ & $10 / 1 / 99$ & 11,000 & 0 \\
\hline WCE 88 & AN-101 & $10 / 1 / 99$ & $10 / 1 / 99$ & 23,000 & 0 \\
\hline WESF & AP-107 & $10 / 1 / 99$ & $10 / 1 / 99$ & 5,000 & 0 \\
\hline WNE8 8 & AP-107 & $10 / 1 / 99$ & $10 / 1 / 99$ & 10,000 & 0 \\
\hline WNW88 & SY-102 & $10 / 1 / 99$ & $10 / 1 / 99$ & 79,000 & 0 \\
\hline ZNL87 & SY-102 & $10 / 1 / 99$ & $10 / 1 / 99$ & 4,000 & 0 \\
\hline WATER & SY-102 & $10 / 2 / 99$ & $10 / 2 / 99$ & 5,000 & 0 \\
\hline WATER & AP-107 & $10 / 2 / 99$ & $10 / 2 / 99$ & 5,000 & 0 \\
\hline WATER & AP-107 & $10 / 2 / 99$ & $10 / 2 / 99$ & 5,000 & 0 \\
\hline WATER & AW-105 & $10 / 2 / 99$ & $10 / 2 / 99$ & 13,000 & 0 \\
\hline WATER & AW-105 & $10 / 2 / 99$ & $10 / 2 / 99$ & 17,000 & 0 \\
\hline EVAPF & AP-107 & $11 / 1 / 99$ & $11 / 1 / 99$ & 10,000 & 0 \\
\hline WATER & AP-107 & $11 / 1 / 99$ & $11 / 1 / 99$ & 2,000 & 0 \\
\hline WATER & AP-107 & $11 / 1 / 99$ & $11 / 1 / 99$ & 3,000 & 0 \\
\hline WATER & AN-10I & $11 / 1 / 99$ & $11 / 1 / 99$ & 6,000 & 0 \\
\hline WATER & SY-102 & $11 / 1 / 99$ & $11 / 1 / 99$ & 23,000 & 0 \\
\hline WCE88 & AP-107 & $11 / 1 / 99$ & $11 / 1 / 99$ & 11,000 & 0 \\
\hline WCE 88 & AN-101 & $11 / 1 / 99$ & $11 / 1 / 99$ & 23,000 & 0 \\
\hline WNE88 & AP-107 & $11 / 1 / 99$ & $11 / 1 / 99$ & 10,000 & 0 \\
\hline WNW88 & SY-102 & $11 / 1 / 99$ & $11 / 1 / 99$ & 93,000 & 0 \\
\hline EVAPF & AW-102 & $11 / 17 / 99$ & $11 / 17 / 99$ & 35,000 & 0 \\
\hline $242-\mathrm{A}$ & LERF & $11 / 19 / 99$ & $11 / 24 / 99$ & 341,800 & 0 \\
\hline 242-A & AW-106 & $11 / 19 / 99$ & $11 / 24 / 99$ & 610,500 & 38 \\
\hline AW-102 & $242-A$ & $11 / 19 / 99$ & $11 / 24 / 99$ & 952,400 & 38 \\
\hline AW-106 & $\mathrm{AW}-102$ & $11 / 24 / 99$ & $11 / 27 / 99$ & 610,400 & 27 \\
\hline EVAPF & AP-107 & $12 / 1 / 99$ & $12 / 1 / 99$ & 10,000 & 0 \\
\hline WATER & AP-107 & $12 / 1 / 99$ & $12 / 1 / 99$ & 1,000 & 0 \\
\hline WATER & AP-107 & $12 / 1 / 99$ & $12 / 1 / 99$ & 1,000 & 0 \\
\hline WATER & AN-101 & $12 / 1 / 99$ & $12 / 1 / 99$ & 4,000 & 0 \\
\hline WATER & SY-102 & $12 / 1 / 99$ & $12 / 1 / 99$ & 26,000 & 0 \\
\hline WCE88 & AP-107 & $12 / 1 / 99$ & $12 / 1 / 99$ & 6,000 & 0 \\
\hline WCE 88 & AN-101 & $12 / 1 / 99$ & $12 / 1 / 99$ & 17,000 & 0 \\
\hline WNE8 8 & AP-107 & $12 / 1 / 99$ & $12 / 1 / 99$ & 3,000 & 0 \\
\hline WNW88 & SY-102 & $12 / 1 / 99$ & $12 / 1 / 99$ & 105,000 & 0 \\
\hline EVAPF & AW-102 & $12 / 3 / 99$ & $12 / 3 / 99$ & 35,000 & 0 \\
\hline $242-\mathrm{A}$ & LERF & $12 / 11 / 99$ & $12 / 15 / 99$ & 188,300 & 0 \\
\hline $242-\mathrm{A}$ & $\bar{A} \mathrm{~W}-106$ & $12 / 11 / 99$ & $12 / 15 / 99$ & 457,100 & 28 \\
\hline AW-102 & $242-\mathrm{A}$ & $12 / 11 / 99$ & $12 / 15 / 99$ & 645,400 & 28 \\
\hline AP-107 & AW-102 & $12 / 15 / 99$ & $12 / 19 / 99$ & 844,600 & 0 \\
\hline $34 \mathrm{~L} 87$ & AP-107 & $1 / 1 / 00$ & $1 / 1 / 00$ & 10,000 & 0 \\
\hline EVAPF & AP-107 & $1 / 1 / 00$ & $1 / 1 / 00$ & 10,000 & 0 \\
\hline SPN87 & SY-102 & $1 / 1 / 00$ & $1 / 1 / 00$ & 6,000 & 0 \\
\hline TAL88 & AP-107 & $1 / 1 / 00$ & $1 / 1 / 00$ & 6,000 & 0 \\
\hline WATER & AP-107 & $1 / 1 / 00$ & $1 / 1 / 00$ & 1,000 & 0 \\
\hline WATER & AP-107 & $1 / 1 / 00$ & $1 / 1 / 00$ & 1,000 & 0 \\
\hline
\end{tabular}


HNF-2321

Revision 0

Table A.3-1. Complete Transfer List for Case 3.

\begin{tabular}{|c|c|c|c|c|c|}
\hline From & To & Start Date & End Date & $\begin{array}{c}\text { Liquid } \\
\text { (gallons) }\end{array}$ & $\begin{array}{c}\text { Solid } \\
\text { (gallons) }\end{array}$ \\
\hline WATER & AP-107 & $1 / 1 / 00$ & $1 / 1 / 00$ & 4,000 & 0 \\
\hline WATER & AN-101 & $1 / 1 / 00$ & $1 / 1 / 00$ & 4,000 & 0 \\
\hline WATER & SY-102 & $1 / 1 / 00$ & $1 / 1 / 00$ & 24,000 & 0 \\
\hline WCE88 & AP-107 & $1 / 1 / 00$ & $1 / 1 / 00$ & 6,000 & 0 \\
\hline WCE88 & AN-101 & $1 / 1 / 00$ & $1 / 1 / 00$ & 17,000 & 0 \\
\hline WNE88 & AP-107 & $1 / 1 / 00$ & $1 / 1 / 00$ & 3,000 & 0 \\
\hline WNW88 & \$Y-102 & $1 / 1 / 00$ & $1 / 1 / 00$ & 98,000 & 0 \\
\hline WATER & AP-107 & $1 / 20 / 00$ & $1 / 20 / 00$ & 35,000 & 0 \\
\hline EVAPF & AP-107 & $2 / 1 / 00$ & $2 / 1 / 00$ & 10,000 & 0 \\
\hline WATER & AP-107 & $2 / 1 / 00$ & $2 / 1 / 00$ & 1,000 & 0 \\
\hline WATER & AP-107 & $2 / 1 / 00$ & $2 / 1 / 00$ & 1,000 & 0 \\
\hline WATER & AN-101 & $2 / 1 / 00$ & $2 / 1 / 00$ & 4,000 & 0 \\
\hline WATER & SY-102 & $2 / 1 / 00$ & $2 / 1 / 00$ & 22,000 & 0 \\
\hline WCE88 & AP- 107 & $2 / 1 / 00$ & $2 / 1 / 00$ & 5,000 & 0 \\
\hline WCE88 & AN-101 & $2 / 1 / 00$ & $2 / 1 / 00$ & 17,000 & 0 \\
\hline WNE88 & AP-107 & $2 / 1 / 00$ & $2 / 1 / 00$ & 3,000 & 0 \\
\hline WNW88 & SY-102 & $2 / 1 / 00$ & $2 / 1 / 00$ & 90,000 & 0 \\
\hline$S Y-102$ & AP-107 & $2 / 15 / 00$ & $2 / 18 / 00$ & 750,000 & 30 \\
\hline $34 \mathrm{~L} 87$ & AP-107 & $3 / 1 / 00$ & $3 / 1 / 00$ & 10,000 & 0 \\
\hline EVAPF & AP-107 & $3 / 1 / 00$ & $3 / 1 / 00$ & 10,000 & 0 \\
\hline WATER & $\mathrm{AP}-107$ & $3 / 1 / 00$ & $3 / 1 / 00$ & 1,000 & 0 \\
\hline WATER & AP-107 & $3 / 1 / 00$ & $3 / 1 / 00$ & 1,000 & 0 \\
\hline WATER & AN-101 & $3 / 1 / 00$ & $3 / 1 / 00$ & 4,000 & 0 \\
\hline WATER & AP-107 & $3 / 1 / 00$ & $3 / 1 / 00$ & $-5,000$ & 0 \\
\hline WATER & SY-102 & $3 / 1 / 00$ & $3 / 1 / 00$ & 21,000 & 0 \\
\hline WCE88 & AP-107 & $3 / 1 / 00$ & $3 / 1 / 00$ & 3,000 & 0 \\
\hline WCE88 & AN-101 & $3 / 1 / 00$ & $3 / 1 / 00$ & 17,000 & 0 \\
\hline WNE88 & AP-107 & $3 / 1 / 00$ & $3 / 1 / 00$ & 3,000 & 0 \\
\hline WNW88 & SY-102 & $3 / 1 / 00$ & $3 / 1 / 00$ & 82,000 & 0 \\
\hline$A W-106$ & AN-106 & $3 / 15 / 00$ & $3 / 17 / 00$ & 457,100 & 21 \\
\hline EVAPF & AP-107 & $4 / 1 / 00$ & $4 / 1 / 00$ & 10,000 & 0 \\
\hline SPN87 & SY-102 & $4 / 1 / 00$ & $4 / 1 / 00$ & 6,000 & 0 \\
\hline TAL88 & AP-107 & $4 / 1 / 00$ & $4 / 1 / 00$ & 6,000 & 0 \\
\hline WATER & AP-107 & $4 / 1 / 00$ & $4 / 1 / 00$ & 1,000 & 0 \\
\hline WATER & SY-102 & $4 / 1 / 00$ & $4 / 1 / 00$ & 20,000 & 0 \\
\hline WATER & AN-101 & $4 / 1 / 00$ & $4 / 1 / 00$ & 20,000 & 0 \\
\hline WCE88 & AP-107 & $4 / 1 / 00$ & $4 / 1 / 00$ & 3,000 & 0 \\
\hline WCE88 & AN-101 & $4 / 1 / 00$ & $4 / 1 / 00$ & 81,000 & 0 \\
\hline WNW88 & $5 Y-102$ & $4 / 1 / 00$ & $4 / 1 / 00$ & 79,000 & 0 \\
\hline 34 L87 & AP-107 & $5 / 1 / 00$ & $5 / 1 / 00$ & 10,000 & 0 \\
\hline EVAPF & AP-107 & $5 / 1 / 00$ & $5 / 1 / 00$ & 10,000 & 0 \\
\hline WATER & AP-107 & $5 / 1 / 00$ & $5 / 1 / 00$ & 1,000 & 0 \\
\hline WATER & AP-107 & $5 / 1 / 00$ & $5 / 1 / 00$ & 4,000 & 0 \\
\hline WATER & AN-101 & $5 / 1 / 00$ & $5 / 1 / 00$ & 20,000 & 0 \\
\hline WATER & SY -102 & $5 / 1 / 00$ & $5 / 1 / 00$ & 22,000 & 0 \\
\hline WCE88 & AP-107 & $5 / 1100$ & $5 / 1 / 00$ & 3,000 & 0 \\
\hline WCE88 & AN-101 & $5 / 1 / 00$ & $5 / 1 / 00$ & 81,000 & 0 \\
\hline WNW88 & SY -102 & $5 / 1 / 00$ & $5 / 1 / 00$ & 88,000 & 0 \\
\hline EVAPF & AP-107 & $6 / 1 / 00$ & $6 / 1 / 00$ & 10,000 & 0 \\
\hline WATER & AP-107 & $6 / 1 / 00$ & $6 / 1 / 00$ & 1,000 & 0 \\
\hline WATER & AN-101 & $6 / 1 / 00$ & $6 / 1 / 00$ & 4,000 & 0 \\
\hline WATER & SY-102 & $6 / 1 / 00$ & $6 / 1 / 00$ & 23,000 & 0 \\
\hline WCE88 & AP-107 & $6 / 1 / 00$ & $6 / 1 / 00$ & 3,000 & 0 \\
\hline WCE88 & AN-101 & $6 / 1 / 00$ & $6 / 1 / 100$ & 14,000 & 0 \\
\hline WNW88 & SY-102 & $6 / 1 / 00$ & $6 / 1 / 00$ & 91,000 & 0 \\
\hline $242-\mathrm{A}$ & LERF & $6 / 3 / 00$ & $6 / 7 / 00$ & 303,200 & 0 \\
\hline
\end{tabular}


Table A.3-1. Complete Transfer List for Case 3.

\begin{tabular}{|c|c|c|c|c|c|}
\hline From & To. & Start Date & End Date & $\begin{array}{c}\text { Liquid } \\
\text { (gallons) }\end{array}$ & $\begin{array}{c}\text { Solid } \\
\text { (gallons) }\end{array}$ \\
\hline $242-\mathrm{A}$ & AW-106 & $6 / 3 / 00$ & $6 / 7 / 00$ & 541,600 & 32 \\
\hline $\mathrm{AW}-102$ & 242-A & $6 / 3 / 00$ & $6 / 7 / 00$ & 844,900 & 32 \\
\hline AW-106 & AW-102 & $6 / 8 / 00$ & $6 / 11 / 00$ & 541,500 & 22 \\
\hline $242-\mathrm{A}$ & LERF & $6 / 25 / 00$ & $6 / 28 / 00$ & 193,800 & 0 \\
\hline $242-\mathrm{A}$ & AW-106 & $6 / 25 / 00$ & $6 / 28 / 00$ & 347,700 & 22 \\
\hline $\mathrm{AW}-102$ & $242-\mathrm{A}$ & $6 / 25 / 00$ & $6 / 28 / 00$ & 541,500 & 22 \\
\hline AW-106 & AW-102 & $6 / 28 / 00$ & $6 / 29 / 00$ & 347,600 & 15 \\
\hline $34 \mathrm{~L} 87$ & AP-107 & $7 / 1 / 00$ & $7 / 1 / 00$ & 10,000 & 0 \\
\hline EVAPF & AP-107 & $7 / 1 / 00$ & $7 / 1 / 00$ & 10,000 & 0 \\
\hline SPN87 & SY-102 & $7 / 1 / 00$ & $7 / 1 / 00$ & 6,000 & 0 \\
\hline \begin{tabular}{|l} 
TAL88 \\
\end{tabular} & AP-107 & $7 / 1 / 00$ & $7 / 1 / 00$ & 5,000 & 0 \\
\hline WATER & AP-107 & $7 / 1 / 00$ & $7 / 1 / 00$ & 1,000 & 0 \\
\hline WATER & AP-107 & $7 / 1 / 00$ & $7 / 1 / 00$ & 4,000 & 0 \\
\hline WATER & AN-101 & $7 / 1 / 00$ & $7 / 1 / 00$ & 4,000 & 0 \\
\hline WATER & SY-102 & $7 / 1 / 00$ & $7 / 1 / 00$ & 25,000 & 0 \\
\hline WCE88 & AP-107 & $7 / 1 / 00$ & $7 / 1 / 00$ & 3,000 & 0 \\
\hline WCE88 & $\mathrm{AN}-101$ & $7 / 1 / 00$ & $7 / 1 / 00$ & 14,000 & 0 \\
\hline WNW88 & SY-102 & $7 / 1 / 00$ & $7 / 1 / 00$ & 98,000 & 0 \\
\hline $242-\mathrm{A}$ & LERF & $7 / 14 / 00$ & $7 / 15 / 00$ & 69,734 & 0 \\
\hline $242-\mathrm{A}$ & AW-106 & $7 / 14 / 00$ & $7 / 15 / 00$ & 278,000 & 15 \\
\hline AW-102 & 242-A & $7 / 14 / 00$ & $7 / 15 / 00$ & 347,700 & 15 \\
\hline AP-107 & $A W-102$ & $7 / 16 / 00$ & $7 / 21 / 00$ & 917,800 & 0 \\
\hline EVAPF & AP- 107 & $8 / 1 / 00$ & $8 / 1 / 00$ & 10,000 & 0 \\
\hline WATER & AN-101 & $8 / 1 / 00$ & $8 / 1 / 00$ & 4,000 & 0 \\
\hline WATER & SY-102 & $8 / 1 / 00$ & $8 / 1 / 00$ & 5,000 & 0 \\
\hline WATER & SY-102 & $8 / 1 / 00$ & $8 / 1 / 00$ & 24,000 & 0 \\
\hline WCE88 & AP-107 & $8 / 1 / 00$ & $8 / 1 / 00$ & 2,000 & 0 \\
\hline WCE88 & AN-10L & $8 / 1 / 00$ & $8 / 1 / 00$ & 14,000 & 0 \\
\hline WCW88 & SY-102 & $8 / 1 / 00$ & $8 / 1 / 00$ & 19,000 & 0 \\
\hline WNW88 & SY-102 & $8 / 1 / 00$ & $8 / 1 / 00$ & 93,000 & 0 \\
\hline EVAPF & AW-102 & $8 / 3 / 00$ & $8 / 3 / 00$ & 35,000 & 0 \\
\hline SY-102 & AP-104 & $8 / 15 / 00$ & $8 / 18 / 00$ & 750,000 & 31 \\
\hline AZ-101 & AY-101 & $8 / 17 / 00$ & $8 / 20 / 00$ & 685,000 & 0 \\
\hline$A Z-101$ & AP-107 & $8 / 20 / 00$ & $8 / 20 / 00$ & 0 & 0 \\
\hline WASH-WATER & AZ-101 & $8 / 20 / 00$ & $8 / 21 / 00$ & 146,000 & 0 \\
\hline EVAPF & AP-107 & $9 / 1 / 00$ & $9 / 1 / 00$ & 10,000 & 0 \\
\hline WATER & AN-101 & $9 / 1 / 00$ & $9 / 1 / 00$ & 4,000 & 0 \\
\hline WATER & SY-102 & $9 / 1 / 00$ & $9 / 1 / 00$ & 5,000 & 0 \\
\hline WATER & $S Y-102$ & $9 / 1 / 00$ & $9 / 1 / 00$ & 22,000 & 0 \\
\hline WCE88 & AP-107 & $9 / 1 / 00$ & $9 / 1 / 00$ & 1,000 & 0 \\
\hline WCE88 & AN-101 & $9 / 1 / 00$ & $9 / 1 / 00$ & 14,000 & 0 \\
\hline WCW88 & SY-102 & $9 / 1 / 00$ & $9 / 1 / 00$ & 19,000 & 0 \\
\hline WNW88 & SY-102 & $9 / 1 / 00$ & $9 / 1 / 00$ & 88,000 & 0 \\
\hline 1NS96 & AW-105 & $10 / 1 / 00$ & $10 / 1 / 00$ & 13,502 & 4,498 \\
\hline PXTCO & AP-107 & $10 / 1 / 00$ & $10 / 1 / 00$ & 5,000 & 0 \\
\hline SPN87 & SX-102 & $10 / 1 / 00$ & $10 / 1 / 00$ & 25,000 & 0 \\
\hline TAL88 & AP-107 & $10 / 1 / \infty$ & $10 / 1 / 00$ & 23,000 & 0 \\
\hline TNS88 & AP-107 & $10 / 1 / 00$ & $10 / 1 / 00$ & 750 & 250 \\
\hline WATER & AN-101 & $10 / 1 / 00$ & $10 / 1 / 00$ & 2,000 & 0 \\
\hline WATER & SY-102 & $10 / 1 / 00$ & $10 / 1 / 00$ & 3,000 & 0 \\
\hline WATER & SX-102 & $10 / 1 / 00$ & $10 / 1 / 00$ & 103,000 & 0 \\
\hline WCE88 & AN-10I & $10 / 1 / 00$ & $10 / 1 / 00$ & 9,000 & 0 \\
\hline WCW88 & $S Y-102$ & $10 / 1 / 00$ & $10 / 1 / 00$ & 13,000 & 0 \\
\hline WESF & $\mathrm{AP}-10 \mathrm{~T}$ & $10 / 1 / 00$ & $10 / 1 / 00$ & 5,000 & 0 \\
\hline WNW88 & SY-102 & $10 / 1 / 00$ & $10 / 1 / 00$ & 26,000 & 0 \\
\hline ZNL87 & SY-102 & $10 / 1 / 00$ & $10 / 1 / 00$ & 4,000 & 0 \\
\hline
\end{tabular}


HNF-2321

Revision 0

Table A.3-1. Complete Transfer List for Case 3.

\begin{tabular}{|c|c|c|c|c|c|}
\hline From & To & Start Date & End Date & $\begin{array}{c}\text { Liquid } \\
\text { (gallons) }\end{array}$ & $\begin{array}{c}\text { Solid } \\
\text { (gallons) }\end{array}$ \\
\hline $1 \mathrm{KL} 96$ & $A W=105$ & $10 / 1 / 60$ & $10 / 2 / 00$ & 302,000 & 0 \\
\hline $34 \mathrm{~L} 87$ & AP-107 & $10 / 1 / 00$ & $10 / 2 / 00$ & 50,000 & 0 \\
\hline EVAPF & $\mathrm{AP}-107$ & $10 / 1 / 00$ & $10 / 2 / 00$ & 120,000 & 0 \\
\hline $34 \mathrm{~L} 87$ & AP- 107 & $10 / 2 / 00$ & $10 / 2 / 00$ & 7,000 & 0 \\
\hline WATER & SY-102 & $10 / 2 / 00$ & $10 / 2 / 00$ & 1,000 & 0 \\
\hline WATER & $S Y-102$ & $10 / 2 / 00$ & $10 / 2 / 00$ & 5,000 & 0 \\
\hline WATER & AW-105 & $10 / 2 / 00$ & $10 / 2 / 00$ & 132,000 & 0 \\
\hline WATER & AW-105 & $10 / 2 / 00$ & $10 / 3 / 00$ & 7,000 & 0 \\
\hline WATER & AP- 107 & $10 / 3 / 00$ & $10 / 3 / 00$ & 3,000 & 0 \\
\hline WATER & AP-107 & $10 / 3 / 00$ & $10 / 3 / 00$ & 5,000 & 0 \\
\hline \begin{tabular}{|l} 
WATER \\
\end{tabular} & AP-107. & $10 / 3 / 00$ & $10 / 3 / 00$ & 22,000 & 0 \\
\hline EVAPF & AW-102 & $10 / 4 / 00$ & $10 / 4 / 00$ & 50,000 & 0 \\
\hline AZ-101 & AP-107 & $10 / 9 / 00$ & $10 / 10 / 00$ & 143,200 & 0 \\
\hline WASH-WATER & $\mathrm{AZ}-101$ & $10 / 11 / 00$ & $10 / 12 / 00$ & 146,000 & 0 \\
\hline $\mathrm{AW}-106$ & AN-106 & $10 / 15 / 00$ & $10 / 16 / 00$ & 250,000 & 12 \\
\hline WATER & AN-101 & $11 / 1 / 00$ & $11 / 1 / 00$ & 2,000 & 0 \\
\hline WATER & SY-102 & $11 / 1 / 00$ & $11 / 1 / 00$ & 3,000 & 0 \\
\hline WATER & SY-102 & $11 / 1 / 00$ & $11 / 1 / 00$ & 28,000 & 0 \\
\hline WCE88 & $\mathrm{AN}-101$ & $11 / 1 / 00$ & $11 / 1 / 00$ & 9,000 & 0 \\
\hline WCW88 & SY-102 & $11 / 1 / 00$ & $11 / 1 / 00$ & 13,000 & 0 \\
\hline $\begin{array}{l}\text { WNW88 } \\
\end{array}$ & SY-102 & $11 / 1 / 00$ & $11 / 1 / 00$ & 111,000 & 0 \\
\hline EVAPF & AW-102 & $11 / 3 / 00$ & $11 / 3 / 00$ & 35,000 & 0 \\
\hline AZ-101 & $\mathrm{AP}-107$ & $11 / 30 / 00$ & $12 / 1 / 00$ & 140,200 & 0 \\
\hline WATER & AN-101 & $12 / 1 / 00$ & $12 / 1 / 00$ & 2,000 & 0 \\
\hline WATER & SY-102 & $12 / 1 / 00$ & $12 / 1 / 00$ & 2,000 & 0 \\
\hline \begin{tabular}{|l} 
WATER \\
\end{tabular} & SY-102 & $12 / 1 / 00$ & $12 / 1 / 00$ & 25,000 & 0 \\
\hline WCE88 & AN-101 & $12 / 1 / 00$ & $12 / 1 / 00$ & 9,000 & 0 \\
\hline WCW88 & SY-102 & $12 / 1 / 00$ & $12 / 1 / 00$ & 8,000 & 0 \\
\hline \begin{tabular}{|l|} 
WNW88 \\
\end{tabular} & SY-102 & $12 / 1 / 00$ & $12 / 1 / 00$ & 99,000 & 0 \\
\hline WASH-WATER & AZ-101 & $12 / 2 / 00$ & $12 / 2 / 00$ & 146,000 & 0 \\
\hline WATER & SY-102 & $1 / 1 / 01$ & $1 / 1 / 01$ & 2,000 & 0 \\
\hline WATER & AN-101 & $1 / 1 / 01$ & $1 / 1 / 01$ & 2,000 & 0 \\
\hline WATER & SY-102 & $1 / 1 / 01$ & $1 / 1 / 01$ & 26,000 & 0 \\
\hline WCE 88 & AN-101 & $1 / 1 / 01$ & $1 / 1 / 01$ & 9,000 & 0 \\
\hline \begin{tabular}{|l} 
WCW88 \\
\end{tabular} & SY-102 & $1 / 1 / 01$ & $1 / 1 / 01$ & 8,000 & 0 \\
\hline WNW88 & SY-102 & 1/1/0I & $1 / 1 / 01$ & 105,000 & 0 \\
\hline $242-A$ & LERF & $1 / 2 / 01$ & $1 / 8 / 01$ & 372,000 & 0 \\
\hline $242-\mathrm{A}$ & AW-106 & $1 / 2 / 01$ & $1 / 8 / 01$ & 665,900 & 40 \\
\hline AW-102 & $242-\mathrm{A}$ & $1 / 2 / 01$ & $1 / 8 / 01$ & $\$, 038,000$ & 40 \\
\hline AW-106 & AW-102 & $1 / 8 / 0 \mathrm{I}$ & $1 / 12 / 01$ & 693,700 & 29 \\
\hline SY-102 & $\mathrm{AP}-102$ & $1 / 15 / 01$ & $1 / 19 / 01$ & 914,500 & 37 \\
\hline $\mathrm{AZ}-101$ & AP- 107 & $1 / 20 / 01$ & $1 / 21 / 01$ & 142,900 & 0 \\
\hline WASH-WATER & AZ-10I & $1 / 22 / 01$ & $1 / 23 / 01$ & 138,200 & 0 \\
\hline $242-\mathrm{A}$ & LERF & $1 / 26 / 01$ & $1 / 30 / 01$ & 237,900 & 0 \\
\hline $242-\mathrm{A}$ & AW-106 & $1 / 26 / 01$ & $1 / 30 / 01$ & 455,700 & 29 \\
\hline AW-102 & 242-A & $1 / 26 / 01$ & $1 / 30 / 01$ & 693,700 & 29 \\
\hline WATER & SY-102 & $2 / 1 / 01$ & $2 / 1 / 01$ & 1,000 & 0 \\
\hline WATER & AN-101 & $2 / 1 / 01$ & $2 / 1 / 01$ & 2,000 & 0 \\
\hline WATER & $S Y-102$ & $2 / 1 / 01$ & $2 / 1 / 01$ & 23,000 & 0 \\
\hline WCE88 & AN-101 & $2 / 1 / 01$ & $2 / 1 / 01$ & 9,000 & 0 \\
\hline \begin{tabular}{|l} 
WCW88 \\
\end{tabular} & SY-102 & $2 / 1 / 01$ & $2 / 1 / 01$ & 3,000 & 0 \\
\hline WNW88 & SY-102 & $2 / 1 / 01$ & $2 / 1 / 01$ & 91,000 & 0 \\
\hline AW-106 & $\mathrm{AP}-101$ & $2 / 15 / 01$ & $2 / 17 / 01$ & 455,700 & 21 \\
\hline AP-104 & AW-102 & $2 / 16 / 01$ & $2 / 20 / 01$ & 917,800 & 0 \\
\hline WATER & SY-102 & $3 / 1 / 01$ & $3 / 1 / 01$ & 1,000 & 0 \\
\hline WATER & AN-101 & $3 / 1 / 01$ & $3 / 1 / 01$ & 2,000 & 0 \\
\hline
\end{tabular}


HNF-2321

Revision 0

Table A.3-1. Complete Transfer List for Case 3.

\begin{tabular}{|c|c|c|c|c|c|}
\hline From & To & Start Date & End Date & $\begin{array}{c}\text { Liquid } \\
\text { (gallons) }\end{array}$ & $\begin{array}{c}\text { Solid } \\
\text { (gallons) }\end{array}$ \\
\hline WATER & SY-102 & $3 / 1 / 01$ & $3 / 1 / 01$ & 28,000 & 0 \\
\hline WCE88 & $A N-101$ & $3 / 1 / 01$ & $3 / 1 / 01$ & 9,000 & 0 \\
\hline WCW88 & SY-102 & $3 / 1 / 01$ & $3 / 1 / 01$ & 2,000 & 0 \\
\hline WNW88 & SY-102 & $3 / 1 / 01$ & $3 / 1 / 01$ & 112,000 & 0 \\
\hline $242-\mathrm{A}$ & LERF & $3 / 7 / 01$ & $3 / 12 / 01$ & 330,000 & 0 \\
\hline $242-\mathrm{A}$ & AW-106 & $3 / 7 / 01$ & $3 / 12 / 01$ & 588,100 & 36 \\
\hline AW-102 & $242-A$ & $3 / 7 / 01$ & $3 / 12 / 01$ & 918,100 & 36 \\
\hline AW-106 & AW-102 & $3 / 12 / 01$ & $3 / 15 / 01$ & 588,000 & 25 \\
\hline $242-\mathrm{A}$ & LERF & $3 / 30 / 01$ & $4 / 1 / 01$ & 182,900 & 0 \\
\hline $242-\mathrm{A}$ & AW-106 & $3 / 30 / 01$ & $4 / 1 / 01$ & 405,100 & 25 \\
\hline AW-102 & $242-\mathrm{A}$ & $3 / 30 / 01$ & $4 / 1 / 01$ & 588,000 & 25 \\
\hline WATER & AN-101 & $4 / 1 / 01$ & $4 / 1 / 01$ & 2,000 & 0 \\
\hline WCE88 & AN-10I & $4 / 1 / 01$ & $4 / 1 / 01$ & 9,000 & 0 \\
\hline WATER & SY-102 & $4 / 15 / 01$ & $4 / 15 / 01$ & 27,000 & 0 \\
\hline WNW88 & SY-102 & $4 / 15 / 01$ & $4 / 15 / 01$ & 108,000 & 0 \\
\hline WATER & AP-107 & $5 / 1 / 01$ & $5 / 1 / 01$ & 2,000 & 0 \\
\hline WATER & SY-102 & $5 / 1 / 01$ & $5 / 1 / 01$ & 29,000 & 0 \\
\hline WATER & AW-102 & $5 / 1 / 01$ & $5 / 1 / 01$ & 35,000 & 0 \\
\hline WCE88 & AP-107 & $5 / 1 / 01$ & $5 / 1 / 01$ & 9,000 & 0 \\
\hline \begin{tabular}{|l} 
WNW88 \\
\end{tabular} & SY-102 & $5 / 1 / 01$ & $5 / 1 / 01$ & 118,000 & 0 \\
\hline AW-106 & AP-101 & $5 / 1 / 01$ & $5 / 2 / 01$ & 300,000 & 14 \\
\hline AP-102 & AW-102 & $5 / 2 / 01$ & $5 / 6 / 01$ & 883,100 & 0 \\
\hline EVAPF & AW-102 & $5 / 3 / 01$ & $5 / 3 / 01$ & 35,000 & 0 \\
\hline AP-104 & $\mathrm{AP}-107$ & $6 / 1 / 01$ & $6 / 1 / 01$ & 155,800 & 6 \\
\hline WATER & AP-107 & $6 / 1 / 01$ & $6 / 1 / 01$ & 2,000 & 0 \\
\hline WATER & SY-102 & $6 / 1 / 01$ & $6 / 1 / 01$ & 25,000 & 0 \\
\hline WCE88 & AP-107 & $6 / 1 / 01$ & $6 / 1 / 01$ & 9,000 & 0 \\
\hline WNW88 & SY-102 & $6 / 1 / 01$ & $6 / 1 / 01$ & 100,000 & 0 \\
\hline WASH-WATER & AP-104 & $6 / 15 / 01$ & $6 / 16 / 01$ & 200,000 & 0 \\
\hline WATER & AP-107 & $7 / 1 / 01$ & 7/1/01 & 1,000 & 0 \\
\hline WATER & SY-102 & $7 / 1 / 01$ & $7 / 1 / 01$ & 31,000 & 0 \\
\hline WCE88 & AP-107 & $7 / 1 / 01$ & $7 / 1 / 01$ & 3,000 & 0 \\
\hline WNW88 & SY-102 & $7 / 1 / 01$ & $7 / 1 / 01$ & 123,000 & 0 \\
\hline AP-104 & AP-102 & $7 / 1 / 01$ & $7 / 2 / 01$ & 200,400 & 32 \\
\hline AP-102 & AP-106 & $7 / 15 / 01$ & $7 / 16 / 01$ & 231,800 & 61 \\
\hline $242-\mathrm{A}$ & LERF & $7 / 23 / 01$ & $7 / 28 / 01$ & 342,000 & 0 \\
\hline $242-\mathrm{A}$ & AW-106 & $7 / 23 / 01$ & $7 / 28 / 01$ & 611,300 & 38 \\
\hline AW-102 & $242-\mathrm{A}$ & $7 / 23 / 01$ & $7 / 28 / 01$ & 953,300 & 38 \\
\hline AW-106 & AW-102 & $7 / 28 / 01$ & $8 / 1 / 01$ & 716,300 & 31 \\
\hline WATER & AP-107 & $8 / 1 / 01$ & $8 / 1 / 01$ & 1,000 & 0 \\
\hline WATER & SY-102 & $8 / 1 / 01$ & $8 / 1 / 01$ & 25,000 & 0 \\
\hline WCE88 & AP-107 & $8 / 1 / 01$ & $8 / 1 / 01$ & 3,000 & 0 \\
\hline WNW88 & SY- 102 & $8 / 1 / 01$ & $8 / 1 / 01$ & 101,000 & 0 \\
\hline $\mathrm{AN}-105$ & AP-102 & $8 / 1 / 01$ & $8 / 4 / 01$ & 542,400 & 7,895 \\
\hline AN-105 & AP-102 & $8 / 1 / 01$ & $8 / 4 / 01$ & 686,500 & 294 \\
\hline LERF & AN-105-PUMP & $8 / 1 / 01$ & $8 / 4 / 01$ & 154,100 & 0 \\
\hline AN-105 & AP-104 & $8 / 5 / 01$ & $8 / 8 / 01$ & 542,400 & 7,895 \\
\hline AN-105 & AP-104 & $8 / 5 / 01$ & $8 / 8 / 01$ & 686,500 & 294 \\
\hline LERF & AN-105-PUMP & $8 / 5 / 01$ & $8 / 8 / 01$ & 154,100 & 0 \\
\hline AP-108 & AN-105 & $8 / 9 / 01$ & $8 / 9 / 01$ & 0 & 0 \\
\hline AP-106 & AP-108 & $8 / 10 / 01$ & $8 / 11 / 01$ & 240,100 & 687 \\
\hline AP-108 & AN-105 & $8 / 12 / 01$ & $8 / 13 / 01$ & 240,200 & 618 \\
\hline $242-\mathrm{A}$ & LERF & $8 / 15 / 01$ & $8 / 18 / 01$ & 166,800 & 0 \\
\hline $242-\mathrm{A}$ & AW-106 & $8 / 15 / 01$ & $8 / 18 / 01$ & 549,400 & 31 \\
\hline AW-102 & $242-A$ & $8 / 15 / 01$ & $8 / 18 / 01$ & 716,200 & 31 \\
\hline AP-107 & $\mathrm{AW}-102$ & $8 / 19 / 01$ & $8 / 23 / 01$ & 918,300 & 0 \\
\hline
\end{tabular}


HNF-2321

Revision 0

Table A.3-1. Complete Transfer List for Case 3.

\begin{tabular}{|c|c|c|c|c|c|}
\hline From & To & Start Date & End Date & $\begin{array}{c}\text { Liquid } \\
\text { (gallons) }\end{array}$ & $\begin{array}{c}\text { Solid } \\
\text { (gallons) }\end{array}$ \\
\hline WATER & AN-105 & $9 / 1 / 01$ & 9/1/01 & $\$, 000$ & 0 \\
\hline WATER & SY-102 & $9 / 1 / 01$ & $9 / 1 / 01$ & 16,800 & 0 \\
\hline WCE88 & $\mathrm{AN}-105$ & 9/1/01 & 9/1/01 & 3,000 & 0 \\
\hline \begin{tabular}{|l|} 
WNW88 \\
\end{tabular} & SY-102 & 9/1/01 & 9/1/01 & 19,500 & 0 \\
\hline$S Y-102$ & AN-105 & 9/15/01 & 9/19/01 & 800,000 & 33 \\
\hline WATER & SY-102 & $9 / 19 / 01$ & $9 / 19 / 01$ & 2,200 & 0. \\
\hline WNW88 & SY-102 & 9/19/01 & $9 / 19 / 01$ & 54,500 & 0 \\
\hline $34 L 87$ & AN-105 & $10 / 1 / 01$ & $10 / 1 / 01$ & 16,800 & 0 \\
\hline EVAPF & AN-105 & $10 / 1 / 01$ & $10 / 1 / 01$ & 9,932 & 0 \\
\hline SPN87 & SY-102 & $10 / 1 / 01$ & $10 / 1 / 01$ & 25,000 & 0 \\
\hline TAL88 & AN-105 & $10 / 1 / 01$ & $10 / 1 / 01$ & 0 & 0 \\
\hline TNS88 & AN-105 & $10 / 1 / 01$ & $10 / 1 / 01$ & 750 & 250 \\
\hline WATER & AN-105 & $10 / 1 / 01$ & $10 / 1 / 01$ & 1,000 & 0 \\
\hline WATER & SY-102 & $10 / 1 / 01$ & $10 / 1 / 01$ & 6,000 & 0 \\
\hline WATER & SY-102 & $10 / 1 / 01$ & $10 / 1 / 01$ & 16,000 & 0 \\
\hline WATER & AN-105 & $10 / 1 / 01$ & $10 / 1 / 01$ & 35,000 & 0 \\
\hline WCE88 & AN-105 & $10 / 1 / 01$ & $10 / 1 / 01$. & 3,000 & 0 \\
\hline WCW88 & SY-102 & $10 / 1 / 01$ & $10 / 1 / 01$ & 23,000 & 0 \\
\hline WESF & AN-105 & $10 / 1 / 01$ & $10 / 1 / 01$ & 5,000 & 0 \\
\hline WNW88 & SY-102 & $10 / 1 / 01$ & $10 / 1 / 01$ & 62,000 & 0 \\
\hline ZNL87 & SY-102 & $10 / 1 / 01$ & $10 / 1 / 01$ & 4,000 & 0 \\
\hline \begin{tabular}{|l} 
WATER \\
\end{tabular} & SY-102 & $10 / 2 / 01$ & $10 / 2 / 01$ & 5,000 & 0 \\
\hline WATER & AP-106 & $10 / 2 / 01$ & $10 / 2 / 01$ & 5,000 & 0 \\
\hline WATER & AP-106 & $10 / 2 / 01$ & $10 / 2 / 01$ & 22,000 & 0 \\
\hline AN-105 & AN-101 & $10 / 15 / 01$ & $10 / 16 / 01$ & 300,000 & 12 \\
\hline AW-106 & AP-107 & $10 / 15 / 01$ & $10 / 17 / 01$ & 549,400 & 26 \\
\hline WATER & AN-105 & $11 / 1 / 01$ & $11 / 1 / 01$ & 1,000 & 0 \\
\hline WATER & SY-102 & 11/1/01 & $\mathrm{t} t / 1 / 01$ & 6,000 & 0 \\
\hline WATER & SY-102 & $11 / 1 / 01$ & $11 / 1 / 01$ & 12,000 & 0 \\
\hline WCE88 & AN-105 & $11 / 1 / 01$ & $11 / 1 / 01$ & 3,000 & 0 \\
\hline WCW88 & SY-102 & $11 / 1 / 01$ & $11 / 1 / 01$ & 23,000 & 0 \\
\hline WNW88 & SY-102 & $11 / 1 / 01$ & 11/1/01 & 47,000 & 0 \\
\hline EVAPF & AW-102 & $11 / 3 / 01$ & $11 / 3 / 01$ & 35,000 & 0 \\
\hline$A Z-102$ & $A Y-101$ & $11 / 14 / 01$ & $11 / 15 / 01$ & 204,000 & 0 \\
\hline AZ-102 & AP-107 & $11 / 15 / 01$ & $11 / 16 / 01$ & 181,800 & 0 \\
\hline WASH-WATER & AZ-102 & $11 / 16 / 01$ & $11 / 17 / 01$ & 213,000 & 0 \\
\hline $\mathrm{AP}-102$ & AP-106 & $11 / 24 / 01$ & $11 / 28 / 01$ & 686,500 & 289 \\
\hline AN-104 & AP-102 & $11 / 28 / 01$ & $12 / 1 / 01$ & 502,200 & 12,513 \\
\hline $\mathrm{AN}-104$ & AP-102 & $11 / 28 / 01$ & $12 / 1 / 01$ & 684,700 & 702 \\
\hline LERF & AN-104-PUMP & $11 / 28 / 01$ & $12 / 1 / 01$ & 200,800 & 0 \\
\hline AP-104 & AP-108 & $11 / 28 / 01$ & $12 / 2 / 01$ & 686,500 & 287 \\
\hline WATER & AN-105 & $12 / 1 / 01$ & $12 / 1 / 01$ & 1,000 & 0 \\
\hline WATER & SY-102 & $12 / 1 / 01$ & $12 / 1 / 01$ & 9,000 & 0 \\
\hline WATER & SY-102 & $12 / 1 / 01$ & $12 / 1 / 01$ & 12,000 & 0 \\
\hline WCE88 & AN-105 & $12 / 1 / 01$ & $12 / 1 / 01$ & 3,000 & 0 \\
\hline WCW 88 & $S Y-102$ & $12 / 1 / 01$ & $12 / 1 / 01$ & 46,000 & 0 \\
\hline \begin{tabular}{|l} 
WNW88 \\
\end{tabular} & SY-102 & $12 / 1 / 01$ & $12 / 1 / 01$ & 37,000 & 0 \\
\hline AN-104 & AP-104 & $12 / 2 / 01$ & $12 / 5 / 01$ & 502,200 & 12,513 \\
\hline $\mathrm{AN}-104$ & AP-104 & $12 / 2 / 01$ & $12 / 5 / 01$ & 684,700 & 702 \\
\hline LERF & AN-104-PUMP & $12 / 2 / 01$ & $12 / 5 / 01$ & 200,800 & 0 \\
\hline WATER & AN-105 & $1 / 1 / 02$ & $1 / 1 / 02$ & 1,000 & 0 \\
\hline WATER & SY-102 & $1 / 1 / 02$ & $1 / 1 / 02$ & 8,000 & 0 \\
\hline WATER & $\$ Y-102$ & $1 / 1 / 02$ & $1 / 1 / 02$ & 10,000 & 0 \\
\hline WCE88 & AN-105 & $1 / 1 / 02$ & $1 / 1 / 02$ & $3,000^{\circ}$ & 0 \\
\hline WCW88 & SY-102 & $1 / 1 / 02$ & $1 / 1 / 02$ & 39,000 & 0 \\
\hline WNW88 & SY-102 & $1 / 1 / 02$ & $1 / 1 / 02$ & 32,000 & 0 \\
\hline
\end{tabular}


HNF-2321

Revision 0

Table A.3-1. Complete Transfer List for Case 3.

\begin{tabular}{|c|c|c|c|c|c|}
\hline From & To & Start Date & End Date & $\begin{array}{c}\text { Liquid } \\
\text { (gallons) }\end{array}$ & $\begin{array}{c}\text { Solid } \\
\text { (gallons) }\end{array}$ \\
\hline AP-107 & AN-104 & $1 / 1 / 02$ & $1 / 4 / 02$ & 748,200 & 586 \\
\hline WATER & $\mathrm{AN}-105$ & $2 / 1 / 02$ & $2 / 1 / 02$ & 1,000 & 0 \\
\hline WATER & SY-102 & $2 / 1 / 02$ & $2 / 1 / 02$ & 4,000 & 0 \\
\hline WATER & SY -102 & $2 / 1 / 02$ & $2 / 1 / 02$ & 16,000 & 0 \\
\hline WCE 88 & AN-105 & $2 / 1 / 02$ & $2 / 1 / 02$ & 2,000 & 0 \\
\hline WCW88 & SY-102 & $2 / 1 / 02$ & $2 / 1 / 02$ & 62,000 & 0 \\
\hline WNW88 & SY-102 & $2 / 1 / 02$ & $2 / 1 / 02$ & 16,000 & 0 \\
\hline $242-\mathrm{A}$ & LERF & $2 / 2 / 02$ & $2 / 7 / 02$ & 342,000 & 0 \\
\hline $242-\mathrm{A}$ & AW-106 & $2 / 2 / 02$ & $2 / 7 / 02$ & 611,600 & 35 \\
\hline AW-102 & $242-A$ & $2 / 2 / 02$ & $2 / 7 / 02$ & 953,600 & 35 \\
\hline AW-106 & AW-102 & $2 / 7 / 02$ & $2 / 11 / 02$ & 611,500 & 23 \\
\hline $242-\mathrm{A}$ & LERF & $2 / 25 / 02$ & $2 / 28 / 02$ & 220,100 & 0 \\
\hline $242-\mathrm{A}$ & AW-106 & $2 / 25 / 02$ & $2 / 28 / 02$ & 391,300 & 23 \\
\hline AW-102 & $242-\mathrm{A}$ & $2 / 25 / 02$ & $2 / 28 / 02$ & 611,400 & 23 \\
\hline AW-106 & AW-102 & $2 / 28 / 02$ & $3 / 2 / 02$ & 391,200 & 16 \\
\hline WATER & AN-105 & $3 / 1 / 02$ & $3 / 1 / 02$ & 1,000 & 0 \\
\hline WATER & SY-102 & $3 / 1 / 02$ & $3 / 1 / 02$ & 4,000 & 0 \\
\hline WATER & SY-102 & $3 / 1 / 02$ & $3 / 1 / 02$ & 16,000 & 0 \\
\hline WCE88 & AN-105 & $3 / 1 / 02$ & $3 / 1 / 02$ & 2,000 & 0 \\
\hline WCW88 & SY-102 & $3 / 1 / 02$ & $3 / 1 / 02$ & 62,000 & 0 \\
\hline WNW88 & SY-102 & $3 / 1 / 02$ & $3 / 1 / 02$ & 14,000 & 0 \\
\hline $242-\mathrm{A}$ & LERF & $3 / 16 / 02$ & $3 / 18 / 02$ & 140,200 & 0 \\
\hline $242-\mathrm{A}$ & AW-106 & $3 / 16 / 02$ & $3 / 18 / 02$ & 251,100 & 16 \\
\hline AW-102 & 242-A & $3 / 16 / 02$ & $3 / 18 / 02$ & 391,300 & 16 \\
\hline AW-106 & A.W-102 & $3 / 18 / 02$ & $3 / 19 / 02$ & 251,100 & 11 \\
\hline WATER & $\mathrm{AN}-105$ & $4 / 1 / 02$ & $4 / 1 / 02$ & 1,000 & 0 \\
\hline WATER & SY-102 & $4 / 1 / 02$ & $4 / 1 / 02$ & 3,000 & 0 \\
\hline WATER & $S Y-102$ & $4 / 1 / 02$ & $4 / 1 / 02$ & 18,000 & 0 \\
\hline WCE88 & AN-105 & $4 / 1 / 02$ & $4 / 1 / 02$ & 1,000 & 0 \\
\hline WCW88 & SY-102 & $4 / 1 / 02$ & $4 / 1 / 02$ & 71,000 & 0 \\
\hline WNW88 & SY-102 & $4 / 1 / 02$ & $4 / 1 / 02$ & 11,000 & 0 \\
\hline $242-\mathrm{A}$ & LERF & $4 / 2 / 02$ & $4 / 3 / 02$ & 47,155 & 0 \\
\hline $242-\mathrm{A}$ & AW-106 & $4 / 2 / 02$ & $4 / 3 / 02$ & 204,000 & 11 \\
\hline $\mathrm{AW}-102$ & $242-\mathrm{A}$ & $4 / 2 / 02$ & $4 / 3 / 02$ & 251,100 & 11 \\
\hline AN-105 & AW-102 & $4 / 3 / 02$ & $4 / 8 / 02$ & 822,500 & 0 \\
\hline $34 L 87$ & AN-105 & $4 / 8 / 02$ & $4 / 8 / 02$ & 33,200 & 0 \\
\hline AZ-102 & AN-105 & $4 / 8 / 02$ & $4 / 8 / 02$ & 127,700 & 0 \\
\hline TAL88 & AN-105 & $4 / 8 / 02$ & $4 / 8 / 02$ & 23,000 & 0 \\
\hline EVAPF & AN-105 & $4 / 8 / 02$ & $4 / 9 / 02$ & 110,100 & 0 \\
\hline WASH-WATER & $A Z-102$ & $4 / 9 / 02$ & $4 / 11 / 02$ & 213,000 & 0 \\
\hline WATER & SY -102 & $5 / 1 / 02$ & $5 / 1 / 02$ & 0 & 0 \\
\hline WATER & SY-102 & $5 / 1 / 02$ & $5 / 1 / 02$ & 1,000 & 0 \\
\hline WCW88 & SY-102 & $5 / 1 / 02$ & $5 / 1 / 02$ & 17,300 & 0 \\
\hline WNW88 & $5 Y-102$ & $5 / 1 / 02$ & $5 / 1 / 02$ & 6,000 & 0 \\
\hline AZ-101 & P1HLW FEED_TANK & $5 / 17 / 02$ & $5 / 17 / 02$ & 134,300 & 5,079 \\
\hline AZ-102 & AN-105 & $5 / 30 / 02$ & $5 / 31 / 02$ & 210,300 & 0 \\
\hline WASH-WATER & $\mathrm{AZ}-102$ & $6 / 1 / 02$ & $6 / 2 / 02$ & 213,000 & 0 \\
\hline P1HLW_FEED TANK & HLW-CONTRACTOR & $6 / 1 / 02$ & $2 / 21 / 03$ & 134,300 & 5,079 \\
\hline $\mathrm{AP}-106$ & LAW-CONTRACTOR-1 & $6 / 1 / 02$ & $12 / 9 / 05$ & $3,623,000$ & 5,414 \\
\hline AP-108 & LAW-CONTRACTOR-2 & $6 / 1 / 02$ & $2 / 6 / 06$ & $3,759,000$ & 5,424 \\
\hline AZ-102 & AN-105 & $7 / 21 / 02$ & $7 / 22 / 02$ & 211,800 & 0 \\
\hline WASH-WATER & AZ-102 & $7 / 23 / 02$ & $7 / 24 / 02$ & 213,000 & 0 \\
\hline AW-106 & $\mathrm{AN}-104$ & $8 / 15 / 02$ & $8 / 16 / 02$ & 204,000 & 9 \\
\hline $\mathrm{AZ}-102$ & $\mathrm{AN}-105$ & $9 / 11 / 02$ & $9 / 13 / 02$ & 212,500 & 0 \\
\hline WASH-WATER & AZ-102 & $9 / 14 / 02$ & $9 / 14 / 02$ & 110,000 & 0 \\
\hline 34187 & AN-105 & $10 / 1 / 02$ & $10 / 1 / 02$ & 50,000 & 0 \\
\hline
\end{tabular}


HNF-2321

Revision 0

Table A.3-1. Complete Transfer List for Case 3.

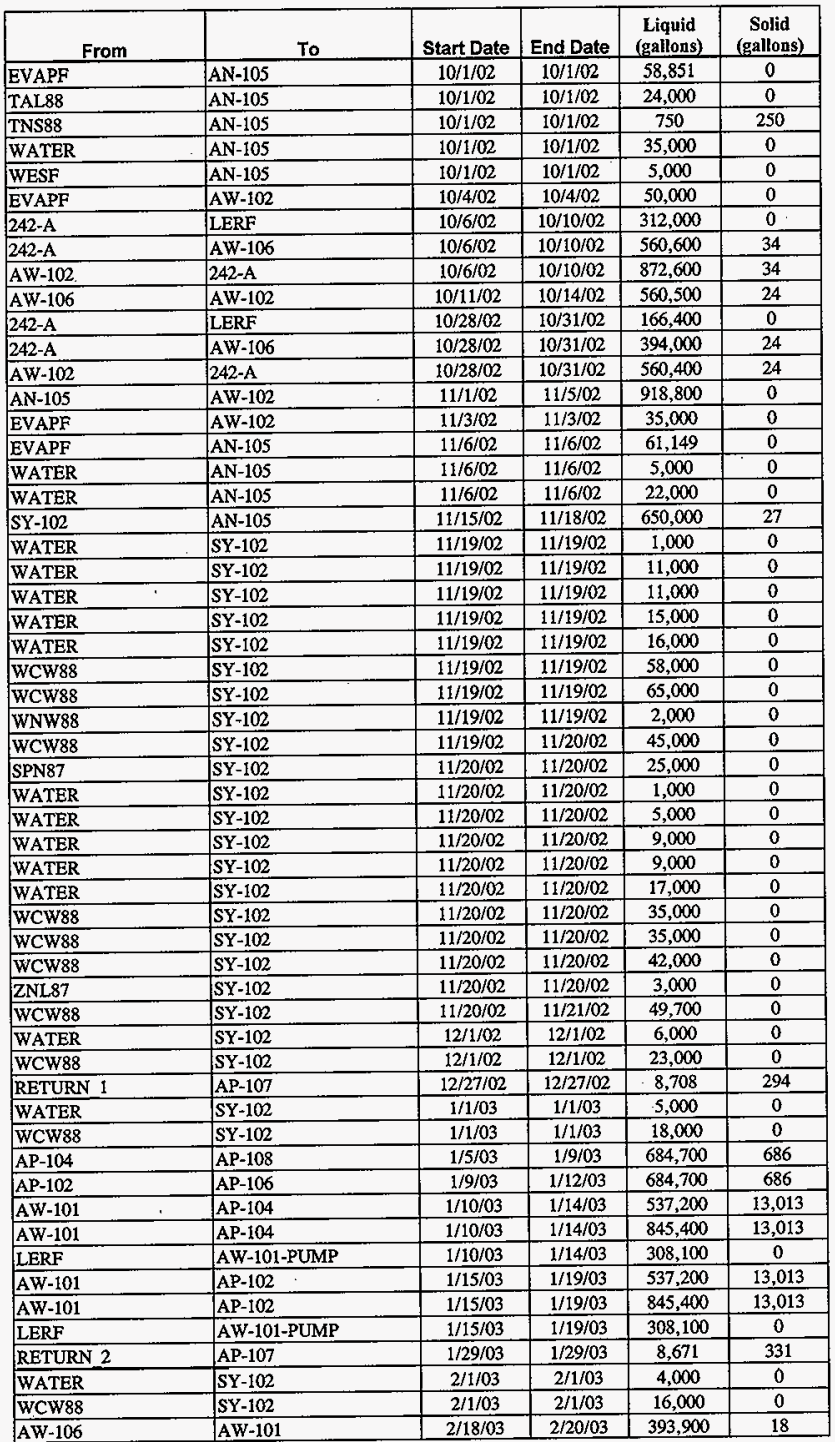


HNF-2321

Revision 0

Table A.3-1. Complete Transfer List for Case 3.

\begin{tabular}{|c|c|c|c|c|c|}
\hline From & To & Start Date & End Date & $\begin{array}{c}\text { Liquid } \\
\text { (gallons) }\end{array}$ & $\begin{array}{c}\text { Solid } \\
\text { (gallons) }\end{array}$ \\
\hline AZ-101 & P1HLW FEED TANK & $.2 / 21 / 03$ & $2 / 21 / 03$ & 134,300 & 5,079 \\
\hline P1HLW FEED TANK & HLW-CONTRACTOR & $2 / 22 / 03$ & $11 / 14 / 03$ & 134,300 & 5,079 \\
\hline $\mathrm{AY}-102$ & AZ-101 & $2 / 26 / 03$ & $3 / 3 / 03$ & 819,600 & 16,032 \\
\hline WATER & SY-102 & $3 / 1 / 03$ & $3 / 1 / 03$ & 4,000 & 0 \\
\hline WCW88 & SY-102 & $3 / 1 / 03$ & $3 / 1 / 03$ & 15,000 & 0 \\
\hline WATER & SY-102 & $4 / 1 / 03$ & $4 / 1 / 03$ & 2,000 & 0 \\
\hline WCW88 & SY-102 & $4 / 1 / 03$ & $4 / 1 / 03$ & 6,000 & 0 \\
\hline $242-\mathrm{A}$ & LERF & $4 / 4 / 03$ & $4 / 9 / 03$ & 342,000 & 0 \\
\hline $242-A$ & AW-106 & $4 / 4 / 03$ & $4 / 9 / 03$ & 612,000 & 33 \\
\hline AW-102 & $242-\mathrm{A}$ & $4 / 4 / 03$ & $4 / 9 / 03$ & 954,000 & 33 \\
\hline AW-106 & AW-102 & $4 / 9 / 03$ & $4 / 13 / 03$ & 611,900 & 22 \\
\hline $242-\mathrm{A}$ & LERF & $4 / 27 / 03$ & $4 / 30 / 03$ & 222,000 & 0 \\
\hline $242-\mathrm{A}$ & AW-106 & $4 / 27 / 03$ & $4 / 30 / 03$ & 389,800 & 22 \\
\hline AW-102 & $242-\mathrm{A}$ & $4 / 27 / 03$ & $4 / 30 / 03$ & 611,800 & 22 \\
\hline AW-106 & AW-102 & $4 / 30 / 03$ & $5 / 2 / 03$ & 389,700 & 14 \\
\hline WATER & SY-102 & $5 / 1 / 03$ & $5 / 1 / 03$ & 1,000 & 0 \\
\hline WCW88 & SY-102 & $5 / 1 / 03$ & $5 / 1 / 03$ & 3,000 & 0 \\
\hline $242-\mathrm{A}$ & LERF & $5 / 16 / 03$ & $5 / 18 / 03$ & 140,700 & 0 \\
\hline $242-\mathrm{A}$ & AW-106 & $5 / 16 / 03$ & $5 / 18 / 03$ & 249,100 & 14 \\
\hline AW-102 & $242-\mathbf{A}$ & $5 / 16 / 03$ & $5 / 18 / 03$ & 389,800 & 14 \\
\hline AW-106 & AW-102 & $5 / 18 / 03$ & $5 / 19 / 03$ & 249,100 & 9 \\
\hline WATER & SY-102 & $6 / 1 / 03$ & $6 / 1 / 03$ & 1,000 & 0 \\
\hline WCW88 & $S Y-102$ & $6 / 1 / 03$ & $6 / 1 / 03$ & 3,000 & 0 \\
\hline $242-\mathrm{A}$ & LERF & $6 / 2 / 03$ & $6 / 3 / 03$ & 90,000 & 0 \\
\hline $242-\mathrm{A}$ & AW-106 & $6 / 2 / 03$ & $6 / 3 / 03$ & 159,100 & 9. \\
\hline AW-102 & $242-\mathrm{A}$ & $6 / 2 / 03$ & $6 / 3 / 03$ & 249,100 & 9 \\
\hline AW-106 & $\mathrm{AW}-102$ & $6 / 3 / 03$ & $6 / 4 / 03$ & 159,100 & 6 \\
\hline AW-102 & AW-106 & $6 / 5 / 03$ & $6 / 6 / 03$ & 159,200 & 6 \\
\hline AN-105 & AW-102 & $6 / 7 / 03$ & $6 / 11 / 03$ & 918,400 & 0 \\
\hline $242-\mathrm{A}$ & LERF & $6 / 27 / 03$ & $7 / 1 / 03$ & 330,000 & 0 \\
\hline $242-\mathrm{A}$ & AW-106 & $6 / 27 / 03$ & $7 / 1 / 03$ & 588,300 & 36 \\
\hline AW-102 & $242-\mathrm{A}$ & $6 / 27 / 03$ & $7 / 1 / 03$ & 918,300 & 36 \\
\hline WATER & SY-102 & $7 / 1 / 03$ & $7 / 1 / 03$ & 1,000 & 0 \\
\hline WCW88 & SY-102 & $7 / 1 / 03$ & $7 / 1 / 03$ & 3,000 & 0 \\
\hline AW-106 & AW-102 & $7 / 2 / 03$ & $7 / 5 / 03$ & 747,400 & 31 \\
\hline $242-\mathrm{A}$ & LERF & $7 / 19 / 03$ & $7 / 23 / 03$ & 267,000 & 0 \\
\hline $242-\mathrm{A}$ & AW-106 & $7 / 19 / 03$ & $7 / 23 / 03$ & 480,300 & 31 \\
\hline AW-102 & 242-A & $7 / 19 / 03$ & $7 / 23 / 03$ & 747,300 & 31 \\
\hline AW-106 & AW-101 & $7 / 23 / 03$ & $7 / 26 / 03$ & 480,300 & 22 \\
\hline RETURN I & AP-107 & $8 / 19 / 03$ & $8 / 19 / 03$ & 8,673 & 330 \\
\hline RETURN 2 & AP-107 & $9 / 28 / 03$ & $9 / 28 / 03$ & 8,670 & 332 \\
\hline AP-104 & AP-108 & $9 / 29 / 03$ & $10 / 3 / 03$ & 740,300 & 1,581 \\
\hline $34 \mathrm{~L} 87$ & $\mathrm{AN}-105$ & $10 / 1 / 03$ & $10 / 1 / 03$ & 7,000 & 0 \\
\hline $34 L 87$ & AN-105 & $10 / 1 / 03$ & $10 / 1 / 03$ & 50,000 & 0 \\
\hline EVAPF & AN-105 & $10 / 1 / 03$ & $10 / 1 / 03$ & 120,000 & 0 \\
\hline SPN87 & SY-102 & $10 / 1 / 03$ & $10 / 1 / 03$ & 25,000 & 0 \\
\hline TAL88 & AN-105 & $10 / 1 / 03$ & $10 / 1 / 03$ & 24,000 & 0 \\
\hline \begin{tabular}{|l|} 
TNS88 \\
\end{tabular} & AN-105 & $10 / 1 / 03$ & $10 / 1 / 03$ & 750 & 250 \\
\hline WATER & AN-105 & $10 / 1 / 03$ & $10 / 1 / 03$ & 35,000 & 0 \\
\hline WESF & AN-105 & $10 / 1 / 03$ & $10 / 1 / 03$ & 5,000 & 0 \\
\hline ZNL87 & SY-102 & $10 / 1 / 03$ & $10 / 1 / 03$ & 3,000 & 0 \\
\hline WATER & AN-105 & $10 / 2 / 03$ & $10 / 2 / 03$ & 3,000 & 0 \\
\hline WATER & $S Y-102$ & $10 / 2 / 03$ & $10 / 2 / 03$ & 5,000 & 0 \\
\hline WATER & AN-105 & $10 / 2 / 03$ & $10 / 2 / 03$ & 5,000 & 0 \\
\hline WATER & AN-105 & $10 / 2 / 03$ & $10 / 2 / 03$ & 22,000 & 0 \\
\hline AP-102 & AP-106 & $10 / 3 / 03$ & $10 / 6 / 03$ & 740,300 & 1,581 \\
\hline
\end{tabular}


HNF-2321

Revision 0

Table A.3-1. Complete Transfer List for Case 3.

\begin{tabular}{|c|c|c|c|c|c|}
\hline From & To & Start Date & End Date & $\begin{array}{c}\text { Liquid } \\
\text { (gallons) }\end{array}$ & $\begin{array}{c}\text { Solid } \\
\text { (gallons) }\end{array}$ \\
\hline $\mathrm{AN}=103$ & AP-104 & $10 / 4 / 03$ & $10 / 7 / 03$ & 436,600 & 28,133 \\
\hline AN-103 & AP-104 & $10 / 4 / 03$ & $10 / 7 / 03$ & 738,300 & 549 \\
\hline LERF & AN-103-PUMP & $10 / 4 / 03$ & $10 / 7 / 03$ & 264,900 & 0 \\
\hline $\mathrm{AN}-103$ & AP-102 & $10 / 8 / 03$ & $10 / 11 / 03$ & 436,600 & 28,133 \\
\hline AN-103 & AP-102 & $10 / 8 / 03$ & $10 / 11 / 03$ & 738,300 & 549 \\
\hline LERF & AN-103-PUMP & $10 / 8 / 03$ & $10 / 11 / 03$ & 264,900 & 0 \\
\hline $\mathrm{AZ}-10 \mathrm{~L}$ & $\mathrm{AN}-105$ & $10 / 28 / 03$ & $10 / 31 / 03$ & 622,700 & 0 \\
\hline WASH-WATER & $A Z-101$ & $10 / 31 / 03$ & $11 / 2 / 03$ & 324,000 & 0 \\
\hline $\mathrm{AN}-105$ & $\mathrm{AW}-102$ & $10 / 31 / 03$ & $11 / 4 / 03$ & 891,500 & 0 \\
\hline EVAPF & $\mathrm{AW}-102$ & $11 / 3 / 03$ & $11 / 3 / 03$ & 35,000 & 0 \\
\hline $\mathrm{AZ}-102$ & P1HLW FEED TANK & $11 / 14 / 03$ & $11 / 14 / 03$ & 123,900 & 5,202 \\
\hline P1HLW FEED TANK & HLW-CONTRACTOR & $11 / 14 / 03$ & $8 / 7 / 04$ & 123,900 & 5,202 \\
\hline $\mathrm{AZ}-101$ & AN-105 & $12 / 21 / 03$ & $12 / 22 / 03$ & 320,000 & 0 \\
\hline WASH-WATER & AZ-101 & $12 / 24 / 03$ & $12 / 25 / 03$ & 324,000 & 0 \\
\hline $\mathrm{AX}-103$ & AN-103 & $1 / 10 / 04$ & $5 / 28 / 04$ & 310,200 & 2,846 \\
\hline RETURN _ & AP-107 & $1 / 22 / 04$ & $1 / 22 / 04$ & 8,672 & 330 \\
\hline $\mathrm{AZ}-101$ & $\mathrm{AN}-105$ & $2 / 12 / 04$ & $2 / 14 / 04$ & 322,300 & 0 \\
\hline RETURN 2 & AP-107 & $2 / 13 / 04$ & $2 / 13 / 04$ & 8,673 & 330 \\
\hline WASH-WATER & AZ-101 & $2 / 15 / 04$ & $2 / 17 / 04$ & 433,600 & 0 \\
\hline $\mathrm{AP}-104$ & AP-108 & $5 / 3 / 04$ & $5 / 7 / 04$ & 753,200 & 1,687 \\
\hline $242-\mathrm{A}$ & LERF & $5 / 4 / 04$ & $5 / 8 / 04$ & 332,500 & 0 \\
\hline $242-\mathrm{A}^{\circ}$ & AW-106 & $5 / 4 / 04$ & $5 / 8 / 04$ & 594,300 & 32 \\
\hline$A W-102$ & $242-\mathrm{A}$ & $5 / 4 / 04$ & $5 / 8 / 04$ & 926,800 & 32 \\
\hline AP-102 & AP-106 & $5 / 7 / 04$ & $5 / 11 / 04$ & 753,200 & 1,687 \\
\hline AW-106 & $\mathrm{AW}-102$ & $5 / 9 / 04$ & $5 / 12 / 04$ & 594,100 & 21 \\
\hline WASH-WATER & AP-102 & $5 / 12 / 04$ & $5 / 12 / 04$ & 118,100 & 0 \\
\hline WASH-WÁTER & AP-104 & $5 / 13 / 04$ & $5 / 13 / 04$ & 118,100 & 0 \\
\hline RETURN 1 & AP-107 & $5 / 18 / 04$ & $5 / 18 / 04$ & 8,676 & 330 \\
\hline RETURN 2 & AP-107 & $5 / 24 / 04$ & $5 / 24 / 04$ & 8,670 & 330 \\
\hline $242-\mathrm{A}$ & LERF. & $5 / 26 / 04$ & $5 / 29 / 04$ & 214,900 & 0 \\
\hline $242-\mathrm{A}$ & AW-105 & $5 / 26 / 04$ & $5 / 29 / 04$ & 379,200 & 21 \\
\hline AW-102 & $242-\mathrm{A}$ & $5 / 26 / 04$ & $5 / 29 / 04$ & 594,100 & 21 \\
\hline $\mathrm{AP}-102$ & AN-105 & $5 / 28 / 04$ & $5 / 29 / 04$ & $209_{2} 500$ & 9,168 \\
\hline C-103 & AN-103 & $5 / 28 / 04$ & $8 / 11 / 04$ & 271,800 & 10,423 \\
\hline AW-106 & $\mathrm{AW}-102$ & $5 / 29 / 04$ & $5 / 31 / 04$ & 379,100 & 14 \\
\hline AP-104 & $\mathrm{AN}-105$ & $5 / 30 / 04$ & $5 / 31 / 04$ & 209,500 & 9,168 \\
\hline WASH-CAUSTIC & AP-102 & $5 / 31 / 04$ & $5 / 31 / 04$ & 103,400 & 0 \\
\hline WASH-CAUSTIC & AP-104 & $6 / 1 / 04$ & $6 / 1 / 04$ & 126,400 & 0 \\
\hline AP-101 & AP-102 & $6 / 2 / 04$ & $6 / 4 / 04$ & 470,100 & 21 \\
\hline AP-101 & AP-102 & $6 / 2 / 04$ & $6 / 4 / 04$ & 521,800 & 21 \\
\hline LERF & AP-101-PUMP & $6 / 2 / 04$ & $6 / 4 / 04$ & 51,711 & 0 \\
\hline AP-101 & AP-104 & $6 / 5 / 04$ & $6 / 8 / 04$ & 574,500 & 26 \\
\hline AP-101 & AP-104 & $6 / 5 / 04$ & $6 / 8 / 04$ & 637,700 & 26 \\
\hline $\begin{array}{l}\text { LERF } \\
\end{array}$ & AP-101-PUMP & $6 / 5 / 04$ & $6 / 8 / 04$ & 63,203 & 0 \\
\hline AW-104 & AP-102 & $6 / 8 / 04$ & $6 / 8 / 04$ & 107.400 & 4 \\
\hline AW-104 & AP-104 & $6 / 9 / 04$ & $6 / 9 / 04$ & 131,200 & 5 \\
\hline $242-\mathrm{A}$ & LERF & $6 / 14 / 04$ & $6 / 16 / 04$ & 138,000 & 0 \\
\hline $242-\mathrm{A}$ & AW-106 & $6 / 14 / 04$ & $6 / 16 / 04$ & 241,200 & 14 \\
\hline $\mathrm{AW}-102$ & $242-A$ & $6 / 14 / 04$ & $6 / 16 / 04$ & 379,200 & 14 \\
\hline AW-106 & AW-102 & $6 / 16 / 04$ & $6 / 17 / 04$ & 241,100 & 9 \\
\hline $242-\mathrm{A}$ & LERF & $7 / 1 / 04$ & $7 / 2 / 04$ & 87,436 & 0 \\
\hline $242-\mathrm{A}$ & AW-106 & $7 / 1 / 04$ & $7 / 2 / 04$ & 153,800 & 9 \\
\hline AW-102 & $242-\mathrm{A}$ & $7 / 1 / 04$ & $7 / 2 / 04$ & 241,200 & 9 \\
\hline AW-106 & AW-102 & $7 / 2 / 04$ & $7 / 3 / 04$ & 153,700 & 6 \\
\hline $\mathrm{AW}-102$ & AW-106 & $7 / 4 / 04$ & $7 / 5 / 04$ & 153,900 & 6 \\
\hline $\mathrm{AN}-105$ & AW-102 & $7 / 6 / 04$ & $7 / 10 / 04$ & 856,100 & 0 \\
\hline
\end{tabular}




\section{$\varepsilon L-V$}

\begin{tabular}{|c|c|c|c|c|c|}
\hline 0 & $6 \varepsilon 0^{\circ} 6 t$ & $\mathrm{SO} / \mathcal{E} / \mathrm{Z}$ & S0/t/Z & Anga7 & $v-\tau+z$ \\
\hline IEE & $1 \angle 9^{\prime} 8$ & So/t/Z & SO/LE/T & $\angle \mathrm{II}^{-} \mathrm{dW}$ & Z N\&กLGA \\
\hline IEE & $8 \angle 9^{\prime} 8$ & $\mathrm{~S}_{0 / \hbar \mathrm{Z} / \mathrm{I}}$ & $\mathrm{s0} / \hbar Z / \mathrm{I}$ & LOI-dV & I N女nLFd \\
\hline $61 \varepsilon^{2} L$ & $00 Z^{\circ} 9 L z$ & $S 0 / \hbar Z / I$ & SO/EZ/I & $\pm 0 I-M V$ & EOI-NV \\
\hline$I \angle t^{\prime} 6$ & $000^{\circ} \angle S E$ & SO/EZ/I & SO/IZ/I & $10 I-\mathrm{dV}$ & EOI-NV \\
\hline $060^{\circ} \varepsilon$ & $00 t^{\prime} 9 \mathrm{II}$ & $50 / \mathrm{IZ/I}$ & $s 0 / 0 z / \pi$ & IOI-NY & EOI-NY \\
\hline EहI'T & $\angle 99^{\prime} Z 5$ & SO/OZ/I & SO/OZ/L & $\varepsilon 0 \mathrm{I}-\mathrm{dV}$ & EOI-NV \\
\hline toI & $\angle 68^{\circ} \varepsilon$ & S0/0z/I & SO/0Z/I & SOI-dV & EOI-NV \\
\hline tor & $968^{\circ} \varepsilon$ & $50 / 02 / 1$ & $S 0 / 0 z / t$ & $\mathrm{EOI}-\mathrm{MV}$ & EOT-NY \\
\hline$\varepsilon \pi c^{2} t$ & $005^{2} 65 !$ & $\$ 0 / 0 Z / I$ & S0/6I/I & toI-NV & E0I-NV \\
\hline$t I$ & $006^{\circ} \mathrm{ZIE}$ & So/8I/I & $\mathrm{SO} / L \mathrm{~L} / \mathrm{L}$ & $20[-M V$ & $90 \mathrm{I}-\mathrm{MV}$ \\
\hline $0 z$ & $006^{4} 98 \mathrm{t}$ & $50 / L \mathrm{I} / \mathrm{I}$ & $\varsigma_{0} / \downarrow T / \tau$ & $\forall-z+z$ & $20 \mathrm{I}-\mathrm{MV}$ \\
\hline 02 & $006^{\circ} \mathrm{ZIE}$ & So/LI/I & $S 0 / t I / I$ & $90 \mathrm{I}-\mathrm{MV}$ & $v-z+z$ \\
\hline 0 & $000^{6} \nabla L I$ & SO/LI/I & SO/tI/I & हथتج I & $\forall-z \mapsto z$ \\
\hline $0 z$ & $008^{6} 98 t$ & t0/IE/ZI & $50 / 62 / 2 \mathrm{I}$ & $20 \mathrm{I}-M \mathrm{MV}$ & $90 \mathrm{I}-\mathrm{MV}$ \\
\hline 62 & $00 t^{\circ} 8 S L$ & $50 / 62 / 2 \mathrm{I}$ & $50 / S Z / 2 I$ & $v-z \not z$ & $201-M V$ \\
\hline $6 z$ & 006987 & 50/6Z/ZI & t0/SZ/ZI & $901-M \mathrm{AV}$ & $\mathrm{v}-2 \nabla c$ \\
\hline 0 & $00 S^{\prime} \mathrm{L} L Z$ & $+0 / 62 / 21$ & t0/SZ/ZI & 걱 & $\mathrm{V}-\mathrm{z} \nabla \mathrm{Z}$ \\
\hline 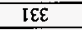 & IL9'8 & $+0 / Z I / Z I$ & t0/ZI/ZI & $20 \mathrm{I}-\mathrm{dV}$ & 2 NQnLE정 \\
\hline $6 z$ & $009^{2} 8 \mathrm{SL}$ & $t 0 /[\mathrm{II} / \mathrm{ZI}$ & tolL/ZI & $20 \mathrm{I}-M \mathrm{VV}$ & 901-MV \\
\hline โย์ & $8 \angle 99^{\prime} 8$ & $t 0 / S / Z \mathrm{I}$ & to/S/ZI & $\angle 0 I^{-} d \mathrm{~V}$ & I N\&nIGy \\
\hline$\nabla \varepsilon$ & $000^{2} \mathrm{It} 6$ & $50 / L / Z I$ & b0/Z/ZI & $v-z+z$ & $201-M V$ \\
\hline$\nabla \mathcal{E}$ & $008^{2}+09$ & $t 0 / L / Z I$ & $\pm 0 / z / Z I$ & $90 \mathrm{I}-\mathrm{MV}$ & $\forall-z \not z$ \\
\hline 0 & $00 z^{\prime} 9 \varepsilon \varepsilon$ & $50 / L / Z I$ & $\hbar 0 / z / z I$ & ज8स 1 & $\forall-z \neq z$ \\
\hline 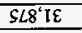 & $005^{\prime} \angle 28$ & $90 / 0 \varepsilon / S$ & †0/0E/II & EOI-NV & $50 \mathrm{t}-\mathrm{D}$ \\
\hline 0 & $000^{\circ} \subseteq \mathcal{E}$ & t0/E/II & †0/E/II & $20 \mathrm{I}^{-M V}$ & GdV $\Lambda$ 目 \\
\hline 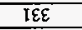 & $1 \angle 9^{\circ} 8$ & t0/EZ/OI & $\$ 0 / \varepsilon Z / 0 I$ & LOt-dV & Z NAnIG \\
\hline IE⿷ & $849^{\circ} 8$ & $\$ 0 / 9 \mathrm{I} / 0 \mathrm{I}$ & $\hbar 0 / 9 \mathrm{I} / 0 \mathrm{I}$ & LOI-dV & I N\&กLF४ \\
\hline 0 & $000^{\prime} 0 \mathrm{~S}$ & $t 0 / t / 0 \mathrm{I}$ & $t 0 / t / 01$ & $20 \mathrm{I}-\mathrm{MV}$ & IdVAG \\
\hline 0 & $000^{c} t$ & $\nabla 0 / \varepsilon / 0 \mathrm{I}$ & $t 0 / \varepsilon / 01$ & SOI-NV & \&ᄏIVM \\
\hline 0 & $000^{\circ} \mathrm{s}$ & $50 / E / 0 I$ & to/z/OI & $20 \mathrm{I}-\mathrm{XS}$ & XGIVM \\
\hline 0 & $000^{2} 88$ & $+0 / 2 / 0 \mathrm{I}$ & $t 0 / z / 0 \mathrm{I}$ & SOI $-M V$ & $8 G I \forall M$ \\
\hline 0 & $000^{6} z z$ & $50 / 2 / 0 \mathrm{I}$ & $t 0 / z / 0 \mathrm{I}$ & SOI-NV & \&GIVM \\
\hline 0 & $000^{\circ} \mathrm{I}$ & $50 / Z / 0 \mathrm{I}$ & $t 0 / 2 / 01$ & SOI-NV & $\mathrm{gHLVM}$ \\
\hline 0 & $000^{\circ} \mathrm{T}$ & $t 0 / 2 / 0 \mathrm{I}$ & $\pm 0 / 2 / 0 \mathrm{I}$ & $z 0 \mathrm{I}-x \mathrm{~S}$ & 8GIVM \\
\hline 0 & $000^{\circ} 00 \mathrm{z}$ & $t 0 / 2 / 0 \mathrm{I}$ & $+0 / \mathrm{t} / 0 \mathrm{I}$ & SOI-MV & 967Iㅓ \\
\hline 0 & $000^{\circ} \varepsilon$ & $50 / \mathrm{L} / 0 \mathrm{l}$ & $+0 / 7 / 01$ & $20 \mathrm{I}-\lambda \mathrm{S}$ & $\angle 87 \mathrm{NZ}$ \\
\hline 0 & $000^{\circ} \mathrm{s}$ & $50 / \mathrm{t} / 0 \mathrm{I}$ & $+0 / / / 0 \mathrm{I}$ & SOI-NV & ISSM \\
\hline 0 & $000^{\circ} \mathrm{SE}$ & $50 / \mathrm{l} / 0 \mathrm{I}$ & $+0 / \mathrm{I} / \mathrm{OI}$ & SOI-NV & GGLVM \\
\hline $0 s z$ & $O S L$ & to/L/OI & $t 0 / \mathrm{L} / 0 \mathrm{I}$ & SOI-NV & 88SNL \\
\hline 0 & $000^{\circ} z z$ & 七0/L/OI & $t 0 / \mathrm{t} / 0 \mathrm{I}$ & SOI-NV & $887 Y 3$ \\
\hline 0 & $000^{\prime} z$ & to/I/OI & $\neq 0 / \mathrm{L} / 0 \mathrm{I}$ & SOI-NV & $88 \mathrm{IVI}$ \\
\hline 0 & $000^{c} \varepsilon \mathcal{Z}$ & to/L/OI & $\pm 0 / \mathrm{L} / 0 \mathrm{~T}$ & $20 \mathrm{I}-\mathrm{XS}$ & $\angle 8 \mathrm{NdS}$ \\
\hline 0 & $000^{\circ} \mathrm{Z}$ & $50 / \mathrm{L} / 0 \mathrm{I}$ & $50 / \mathrm{L} / 0 \mathrm{I}$ & $20 \mathrm{I}-\lambda \mathrm{S}$ & $\angle 8 \mathrm{NdS}$ \\
\hline 0 & $000^{\circ} 0 \mathrm{ZI}$ & $50 / \mathrm{L} / 0 \mathrm{I}$ & t0/I/OI & SOI-NV & 대 $\Lambda$ J \\
\hline 0 & $000^{\circ} 05$ & to/t/OI & $50 / \mathrm{L} / 0 \mathrm{I}$ & SOI-NV & $\angle 8 T \forall \varepsilon$ \\
\hline IEE & $1 \angle 9^{\circ} 8$ & $50 / 2 / 6$ & $50 / 2 / 6$ & $\angle 0 \mathrm{I}-\mathrm{dV}$ & z N\&nlGA \\
\hline TEE & $819^{2} 8$ & t0/LZ/8 & $50 / 9 \mathrm{Z} / 8$ & $\angle 0 \mathrm{I}-\mathrm{dV}$ & I N\&nLat \\
\hline$L I$ & $000^{\circ} 005$ & $t 0 / L I / 8$ & $t 0 / \mathcal{S I} / 8$ & SOI-NV & $S 0 I^{-} M V$ \\
\hline$t \omega L$ & $009^{\circ} 00 \mathrm{I}$ & फ0/0E/LI & $50 / \mathrm{L} \tau / 8$ & E0I-NV & $20 \mathrm{I}-\mathrm{V}$ \\
\hline $20 \tau^{\circ} s$ & $006^{4} \mathrm{EZI}$ & $50 / \mathrm{Z} / \mathrm{S}$ & $\not 0 / 8 / 8$ & \&OLDVYLNOD MTH & XNVI GGGA MTHId \\
\hline $20 z^{\prime} s$ & $006^{E} \mathcal{E} Z$ & $\hbar 0 / 8 / 8$ & $50 / L / 8$ & प्रNVI वGAd MTHId & $20 \pi-z \bar{v}$ \\
\hline $7 Z L^{\prime} 6$ & $00 \mathrm{I}^{\circ} 6 \mathrm{LI}$ & $+50 / 8 / 6$ & $50 / 1 / 8$ & $20 \mathrm{I}-\mathrm{xV}$ & t0I-5 \\
\hline SE & $000^{\circ} 0 S L$ & $50 / 8 \mathrm{I} / \mathrm{L}$ & $\$ 0 / S L / L$ & โ0I-dV & I0I-MV \\
\hline IEE & $1 \angle 9^{4} 8$ & $50 / t / / L$ & $t 0 / t I / L$ & $\angle O T-d V$ & Z NdnLAत्य \\
\hline IE\& & $8 \angle 9^{4} 8$ & b0/L/L & $+0 / L / L$ & $\angle 0 I-d V$ & I NanIGA \\
\hline 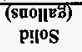 & 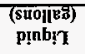 & गеg pus & әlea मfe & OI & uods \\
\hline
\end{tabular}

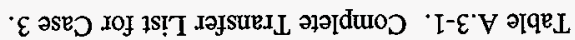


HNF-2321

Revision 0

Table A.3-1. Complete Transfer List for Case 3.

\begin{tabular}{|c|c|c|c|c|c|}
\hline From & To & Start Date & End Date & $\begin{array}{c}\text { Liquid } \\
\text { (gallons) }\end{array}$ & $\begin{array}{c}\text { Solid } \\
\text { (gallons) }\end{array}$ \\
\hline $242-\mathrm{A}$ & AW-106 & $2 / 1 / 05$ & $2 / 3 / 05$ & 263,900 & 14 \\
\hline $\mathrm{AW}-102$ & $242-\mathrm{A}$ & $2 / 1 / 05$ & $2 / 3 / 05$ & 313,000 & 14 \\
\hline \begin{tabular}{|l}
$\mathrm{AW}-106$ \\
\end{tabular} & AW-101 & $2 / 3 / 05$ & $2 / 4 / 05$ & 263,900 & 12 \\
\hline AN-105 & AW-102 & $2 / 3 / 05$ & $2 / 7 / 05$ & 758,600 & 0 \\
\hline AP-104 & AP-108 & $2 / 18 / 05$ & $2 / 22 / 05$ & 894,300 & 1,149 \\
\hline AP-102 & AP-106 & $2 / 22 / 05$ & $2 / 26 / 05$ & 731,500 & 1,136 \\
\hline AY-101 & AP-104 & $2 / 23 / 05$ & $2 / 24 / 05$ & 283,600 & 12 \\
\hline AY-101 & AP-102 & $2 / 27 / 05$ & $2 / 28 / 05$ & 279,700 & 11 \\
\hline$\$ Y-102$ & AN-105 & $3 / 1 / 05$ & $3 / 4 / 05$ & 750,000 & 31 \\
\hline RETURN 1 & AP-107 & $3 / 18 / 05$ & $3 / 18 / 05$ & 8,679 & 331 \\
\hline RETURN 2 & AP-107 & $3 / 27 / 05$ & $3 / 27 / 05$ & 8,682 & 331 \\
\hline $242-\mathrm{A}$ & LERF & $4 / 6 / 05$ & $4 / 10 / 05$ & 272,600 & 0 \\
\hline $242-\mathrm{A}$ & AW-106 & $4 / 6 / 05$ & $4 / 10 / 05$ & 486,100 & 26 \\
\hline AW-102 & $242-A$ & $4 / 6 / 05$ & $4 / 10 / 05$ & 758,700 & 26 \\
\hline AW-106 & AW-102 & $4 / 10 / 05$ & $4 / 12 / 05$ & 486,000 & 17 \\
\hline $242-\mathrm{A}$ & LERF & $4 / 26 / 05$ & $4 / 29 / 05$ & 174,000 & 0 \\
\hline $242-\mathrm{A}$ & AW-106 & $4 / 26 / 05$ & $4 / 29 / 05$ & 312,000 & 17 \\
\hline AW-102 & $242-\mathrm{A}$ & $4 / 26 / 05$ & $4 / 29 / 05$ & 486,000 & 17 \\
\hline AW-106 & AW-102 & $4 / 29 / 05$ & $4 / 30 / 05$ & 311,400 & 11 \\
\hline $\mathrm{AZ}-101$ & P1HLW FEED TANK & $5 / 2 / 05$ & $5 / 2 / 05$ & 127,200 & 3,269 \\
\hline P1HLW FEED TANK & HLW-CONTRACTOR & $5 / 2 / 05$ & $11 / 16 / 05$ & 127,200 & 3,269 \\
\hline RETURN I & $\mathrm{AP}-107$ & $5 / 14 / 05$ & $5 / 14 / 05$ & 8,675 & 330 \\
\hline $242-\mathrm{A}$ & LERF & $5 / 14 / 05$ & $5 / 16 / 05$ & 114,000 & 0 \\
\hline $242-\mathrm{A}$ & AW-106 & $5 / 14 / 05$ & $5 / 16 / 05$ & 197,500 & 11 \\
\hline AW-102 & $242-\mathrm{A}$ & $5 / 14 / 05$ & $5 / 16 / 05$ & 311,500 & 11 \\
\hline AW-106 & AW-102 & $5 / 16 / 05$ & $5 / 17 / 05$ & 197,400 & 7 \\
\hline AW-102 & AW-106 & $5 / 18 / 05$ & $5 / 19 / 05$ & 197,600 & 7 \\
\hline AN-105 & $\mathrm{AW}-102$ & $5 / 19 / 05$ & $5 / 23 / 05$ & 749,700 & 0 \\
\hline RETURN 2 & AP-107 & $5 / 23 / 05$ & $5 / 23 / 05$ & 8,677 & 330 \\
\hline $\mathrm{AN}-103$ & AW-104 & $6 / 1 / 05$ & $6 / 1 / 05$ & 0 & 0 \\
\hline RETURN 1 & AP-107 & $7 / 9 / 05$ & $7 / 10 / 05$ & 8,675 & 330 \\
\hline RETURN 2 & AP-107 & $7 / 19 / 05$ & $7 / 19 / 05$ & 8,677 & 330 \\
\hline AY-102 & AZ-102 & $8 / 1 / 05$ & $8 / 3 / 05$ & 279,400 & 9,360 \\
\hline RETURN 1 & AP-107 & $9 / 4 / 05$ & $9 / 4 / 05$ & 8,675 & 330 \\
\hline $242-\mathrm{A}$ & LERF & $9 / 5 / 05$ & $9 / 8 / 05$ & 268,200 & 0 \\
\hline $242-\mathrm{A}$ & AW-106 & $9 / 5 / 05$ & $9 / 8 / 05$ & 481,500 & 31 \\
\hline AW-102 & $242-\mathrm{A}$ & $9 / 5 / 05$ & $9 / 8 / 05$ & 749,600 & 31 \\
\hline AW-106 & AW-102 & $9 / 9 / 05$ & $9 / 12 / 05$ & 678,900 & 29 \\
\hline RETURN 2 & AP-107 & $9 / 14 / 05$ & $9 / 14 / 05$ & 8,677 & 330 \\
\hline $242-\mathrm{A}$ & LERF & $9 / 26 / 05$ & 9/30/05 & 190,200 & 0 \\
\hline $242-\mathrm{A}$ & AW-106 & $9 / 26 / 05$ & $9 / 30 / 05$ & 488,600 & 29 \\
\hline AW-102 & $242-A$ & $9 / 26 / 05$ & 9/30/05 & 678,800 & 29 \\
\hline AW-106 & AW-101 & $9 / 30 / 05$ & $10 / 2 / 05$ & 488,600 & 23 \\
\hline $34 \mathrm{~L} 87$ & AN-105 & $10 / 1 / 05$ & $10 / 1 / 05$ & 50,000 & 0 \\
\hline EVAPF & AN-105 & $10 / 1 / 05$ & $10 / 1 / 05$ & 120,000 & 0 \\
\hline SPN87 & SY-102 & $10 / 1 / 05$ & $10 / 1 / 05$ & 25,000 & 0 \\
\hline TAL88 & AN-10S & $10 / 1 / 05$ & $10 / 1 / 05$ & 24,000 & 0 \\
\hline TNS88 & AN-105 & $10 / 1 / 05$ & $10 / 1 / 05$ & 750 & 250 \\
\hline WATER & AN-105 & $10 / 1 / 05$ & $10 / 1 / 05$ & 35,000 & 0 \\
\hline WESF & $\mathrm{AN}-105$ & $10 / 1 / 05$ & $10 / 1 / 05$ & 5,000 & 0 \\
\hline ZNL87 & SY-102 & $10 / 1 / 05$ & $10 / 1 / 05$ & 3,000 & 0 \\
\hline WATER & SY-102 & $10 / 2 / 05$ & $10 / 2 / 05$ & 1,000 & 0 \\
\hline WATER & SY-102 & $10 / 2 / 05$ & $10 / 2 / 05$ & 5,000 & 0 \\
\hline WATER & AN-105 & $10 / 2 / 05$ & $10 / 2 / 05$ & 5,000 & 0 \\
\hline WATER & AN-105 & $10 / 2 / 05$ & $10 / 2 / 05$ & 22,000 & 0 \\
\hline RETURN 1 & AP-107 & $10 / 31 / 05$ & $10 / 31 / 05$ & 8,675 & 330 \\
\hline
\end{tabular}


HNF-2321

Revision 0

Table A.3-1. Complete Transfer List for Case 3.

\begin{tabular}{|c|c|c|c|c|c|}
\hline From & To & Start Date & End Date & $\begin{array}{c}\text { Liquid } \\
\text { (gallons) }\end{array}$ & $\begin{array}{c}\text { Solid } \\
\text { (gallons) }\end{array}$ \\
\hline EVAPF & AW-102 & $11 / 3 / 05$ & $11 / 3 / 05$ & 35,000 & 0 \\
\hline RETURN 2 & AP-107 & $11 / 10 / 05$ & $11 / 10 / 05$ & 8,677 & 330 \\
\hline $\mathrm{AZ}-101$ & P1HLW FEED TANK & $11 / 16 / 05$ & $11 / 17 / 05$ & 127,200 & 3,269 \\
\hline P1HLW FEED TANK & HLW-CONTRACTOR & $11 / 17 / 05$ & $6 / 2 / 06$ & 127,200 & 3,269 \\
\hline AP-102 & AP-106 & $12 / 9 / 05$ & $12 / 11 / 05$ & 279,600 & 49 \\
\hline AP-106 & LAW-CONTRACTOR-1 & $12 / 11 / 05$ & $2 / 16 / 06$ & 279,600 & 85 \\
\hline AN-107 & AP-102 & $12 / 12 / 05$ & $12 / 14 / 05$ & 481,700 & 22 \\
\hline AN-107 & AP-102 & $12 / 12 / 05$ & $12 / 14 / 05$ & 554,000 & 22 \\
\hline LERF & AN-107-PUMP & $12 / 12 / 05$ & $12 / 14 / 05$ & 72,259 & 0 \\
\hline $\mathrm{AW}-105$ & $\mathrm{AN}-105$ & $12 / 15 / 05$ & $12 / 17 / 05$ & 415,300 & 14 \\
\hline WATER & SY-102 & $12 / 15 / 05$ & $12 / 17 / 05$ & 426,000 & 0 \\
\hline SY-102 & AW-105 & $1 / 1 / 06$ & $1 / 3 / 06$ & 731,500 & 20,807 \\
\hline RETURN 2 & AP-107 & $1 / 6 / 06$ & $1 / 6 / 06$ & 8,677 & 330 \\
\hline AP-104 & AP-108 & $2 / 6 / 06$ & $2 / 7 / 06$ & 283,600 & 43 \\
\hline AN-107 & AP-104 & $2 / 8 / 06$ & $2 / 10 / 06$ & 481,700 & 22 \\
\hline AN-107 & AP-104 & $2 / 8 / 06$ & $2 / 10 / 06$ & 554,000 & 22 \\
\hline LERF & AN-107-PUMP & $2 / 8 / 06$ & $2 / 10 / 06$ & 72,260 & 0 \\
\hline $\mathrm{AP}-108$ & LAW-CONTRACTOR-2 & $2 / 8 / 06$ & $4 / 16 / 06$ & 283,500 & 73 \\
\hline AP-102 & AP-106 & $2 / 16 / 06$ & $2 / 17 / 06$ & 169,400 & 8 \\
\hline AP-106 & LAW-CONTRACTOR-1 & $2 / 17 / 06$ & $5 / 25 / 09$ & $3,713,000$ & 157. \\
\hline AW-105 & AN-105 & $3 / 1 / 06$ & $3 / 9 / 06$ & 502,600 & 18 \\
\hline AN-105 & $\mathrm{AW}-102$ & $3 / 3 / 06$ & $3 / 7 / 06$ & 865,100 & 0 \\
\hline $\mathrm{AP}-102$ & AP-106 & $3 / 16 / 06$ & $3 / 18 / 06$ & 384,600 & 18 \\
\hline AN-102 & AP-102 & $3 / 19 / 06$ & $3 / 22 / 06$ & 481,700 & 23 \\
\hline AN-102 & AP-102 & $3 / 19 / 06$ & $3 / 22 / 06$ & 770,700 & 23 \\
\hline LERF & AN-102-PUMP & $3 / 19 / 06$ & $3 / 22 / 06$ & 289,000 & 0 \\
\hline $\mathrm{AN}-103$ & AW-105 & $4 / 1 / 06$ & $4 / 1 / 06$ & 161,100 & 4,246 \\
\hline RETURN 1 & AP-107 & $4 / 11 / 06$ & $4 / 11 / 06$ & 8,676 & 325 \\
\hline AW-101 & AW-105 & $4 / 15 / 06$ & $4 / 16 / 06$ & 200,000 & 9 \\
\hline AP-104 & AP-108 & $4 / 16 / 06$ & $4 / 17 / 06$ & 169,400 & 8 \\
\hline AP-108 & LAW-CONTRACTOR-2 & $4 / 17 / 06$ & $5 / 18 / 09$ & $3,448,000$ & 144 \\
\hline AP-104 & AP- 108 & $5 / 14 / 06$ & $5 / 16 / 06$ & 384,600 & 17 \\
\hline AN-102 & AP-104 & $5 / 17 / 06$ & $5 / 20 / 06$ & 481,700 & 23 \\
\hline $\mathrm{AN}-102$ & AP-104 & $5 / 17 / 06$ & $5 / 20 / 06$ & 770,700 & 23 \\
\hline LERF & AN-102-PUMP & $5 / 17 / 06$ & $5 / 20 / 06$ & 289,000 & 0 \\
\hline A-104 & AN-103 & $5 / 30 / 06$ & $9 / 7 / 06$ & 29,827 & 832 \\
\hline $\mathrm{AZ}-101$ & P1HLW FEED TANK & $6 / 2 / 06$ & $6 / 3 / 06$ & 127,200 & 3,269 \\
\hline P1HLW FEED TANK & HLW-CONTRACTOR & $6 / 3 / 06$ & $12 / 18 / 06$ & 127,200 & 3,269 \\
\hline $242-\mathrm{A}$ & LERF & $6 / 20 / 06$ & $6 / 25 / 06$ & 324,000 & 0 \\
\hline $242-\mathrm{A}$ & AW-106 & $6 / 20 / 06$ & $6 / 25 / 06$ & 576,300 & 31 \\
\hline $\mathrm{AW}-102$ & $242-\mathrm{A}$ & $6 / 20 / 06$ & $6 / 25 / 06$ & 900,300 & 31 \\
\hline $\mathrm{AZ}-102$ & AN-105 & $6 / 23 / 06$ & $6 / 24 / 06$ & 159,500 & 0 \\
\hline WASH-WATER & AZ-102 & $6 / 24 / 06$ & $6 / 26 / 06$ & 324,000 & 0 \\
\hline AW-106 & AW-102 & $6 / 25 / 06$ & $6 / 28 / 06$ & 576,200 & 21 \\
\hline $242-\mathrm{A}$ & LERF & $7 / 12 / 06$ & $7 / 15 / 06$ & 208,100 & 0 \\
\hline $242-\mathrm{A}$ & AW-106 & $7 / 12 / 06$ & $7 / 15 / 06$ & 368,100 & 21 \\
\hline AW-102 & $242-\mathrm{A}$ & $7 / 12 / 06$ & $7 / 15 / 06$ & 576,200 & 21 \\
\hline AW-106 & AW-102 & $7 / 15 / 06$ & $7 / 17 / 06$ & 368,000 & 14 \\
\hline AP-102 & AP-106 & $7 / 29 / 06$ & $8 / 2 / 06$ & 770,700 & 23 \\
\hline $242-A$ & LERF & $7 / 31 / 06$ & $8 / 2 / 06$ & 132,000 & 0 \\
\hline $242-\mathrm{A}$ & AW-106 & $7 / 31 / 06$ & $8 / 2 / 06$ & 236,100 & 14 \\
\hline AW-102 & $242-\mathrm{A}$ & $7 / 31 / 06$ & $8 / 2 / 06$ & 368,100 & 14 \\
\hline$A W-106$ & AW-102 & $8 / 2 / 06$ & $8 / 3 / 06$ & 236,000 & 9 \\
\hline AN-106 & $A P-102$ & $8 / 2 / 06$ & $8 / 5 / 06$ & 527,300 & 24 \\
\hline AN-106 & AP-102 & $8 / 2 / 06$ & $8 / 5 / 06$ & 622,200 & 24 \\
\hline LERF & AN-106-PUMP & $8 / 2 / 06$ & $8 / 5 / 06$ & 94,920 & 0 \\
\hline
\end{tabular}


HNF-2321

Revision 0

Table A.3-1. Complete Transfer List for Case 3.

\begin{tabular}{|c|c|c|c|c|c|}
\hline From & To. & Start Date & End Date & $\begin{array}{c}\text { Liquid } \\
\text { (gallons) }\end{array}$ & $\begin{array}{c}\text { Solid } \\
\text { (kallons) }\end{array}$ \\
\hline AZ-102 & AN-105 & $8 / 14 / 06$ & $8 / 15 / 06$ & 320,100 & 0 \\
\hline $242-\mathrm{A}$ & LERF & $8 / 17 / 06$ & $8 / 18 / 06$ & 84,555 & 0 \\
\hline $242-\mathrm{A}$ & AW-106 & $8 / 17 / 06$ & $8 / 18 / 06$ & 151,500 & 9 \\
\hline AW-102 & $242-\mathrm{A}$ & $8 / 17 / 06$ & $8 / 18 / 06$ & 236,100 & 9 \\
\hline WASH-WATER & $A Z-102$ & $8 / 17 / 06$ & $8 / 18 / 06$ & 324,000 & 0 \\
\hline $\mathrm{AW}-106$ & AW-102 & $8 / 18 / 06$ & $8 / 19 / 06$ & 151,500 & 6 \\
\hline AW-102 & AW-106 & $8 / 20 / 06$ & $8 / 21 / 06$ & 151,600 & 6 \\
\hline AN-105 & AW-102 & $8 / 21 / 06$ & $8 / 25 / 06$ & 790,900 & 0 \\
\hline \begin{tabular}{|l} 
C-204 \\
\end{tabular} & AN-103 & $9 / 7 / 06$ & 9/14/06 & 3,775 & 55 \\
\hline C-201 & AN-103 & $9 / 14 / 06$ & 9/20/06 & 2,464 & 59 \\
\hline $\mathrm{AX}-104$ & AN-103 & $9 / 20 / 06$ & $10 / 26 / 06$ & 18,516 & 572 \\
\hline AP-104 & AP-108 & $9 / 26 / 06$ & $9 / 30 / 06$ & 770,700 & 23 \\
\hline $34 \mathrm{~L} 87$ & AN-105 & $10 / 1 / 06$ & $10 / 1 / 06$ & 7,000 & 0 \\
\hline $34 \mathrm{~L} 87$ & AN-105 & $10 / 1 / 06$ & $10 / 1 / 06$ & 50,000 & 0 \\
\hline EVAPF & AN-105 & $10 / 1 / 06$ & $10 / 1 / 06$ & 120,000 & 0 \\
\hline \begin{tabular}{|l|} 
SPN87 \\
\end{tabular} & SY-102 & $10 / 1 / 06$ & $10 / 1 / 06$ & 25,000 & 0 \\
\hline TAL88 & AN-105 & $10 / 1 / 06$ & $10 / 1 / 06$ & 25,000 & 0 \\
\hline \begin{tabular}{|l|} 
TNS88 \\
\end{tabular} & AN-105 & $10 / 1 / 06$ & $10 / 1 / 06$ & 750 & 250 \\
\hline WATER & AN-105 & $10 / 1 / 06$ & 10/1/06 & 35,000 & 0 \\
\hline WESF & AN-105 & $10 / 1 / 06$ & $10 / 1 / 06$ & 5,000 & 0 \\
\hline AN-106 & AP-104 & $10 / 1 / 06$ & $10 / 4 / 06$ & 527,300 & 24 \\
\hline $\mathrm{AN}-106$ & AP-104 & $10 / 1 / 06$ & $10 / 4 / 06$ & 622,200 & 24 \\
\hline LERF & AN-106-PUMP & $10 / 1 / 06$ & $10 / 4 / 06$ & 94,920 & 0 \\
\hline WATER & AN-105 & $10 / 2 / 06$ & $10 / 2 / 06$ & 3,000 & 0 \\
\hline WATER & SY-102 & $10 / 2 / 05$ & $10 / 2 / 06$ & 5,000 & 0 \\
\hline WATER & AN-105 & $10 / 2 / 06$ & $10 / 2 / 06$ & 5,000 & 0 \\
\hline WATER & $A N-105$ & $10 / 2 / 06$ & $10 / 2 / 06$ & 22,000 & 0 \\
\hline LERF & SY-101-PUMP & $10 / 2 / 05$ & $10 / 7 / 06$ & 532,400 & 0 \\
\hline SY-101 & SY-102 & $10 / 2 / 06$ & $10 / 7 / 06$ & 537,800 & 5,486 \\
\hline SY-101 & SY-102 & $10 / 2 / 06$ & $10 / 7 / 06$ & $1,082,000$ & 611 \\
\hline EVAPF & $\mathrm{AW}-102$ & $10 / 4 / 06$ & $10 / 4 / 06$ & 50,000 & 0 \\
\hline AZ-102 & AN-105 & $10 / 6 / 06$ & $10 / 8 / 06$ & 322,700 & 0 \\
\hline \begin{tabular}{|l|} 
WASH-WATER \\
\end{tabular} & AZ-102 & $10 / 9 / 06$ & $10 / 10 / 06$ & 150,500 & 0 \\
\hline SY-102 & AN-102 & $10 / 10 / 06$ & $10 / 13 / 06$ & $1,026,000$ & 2,529 \\
\hline LERF & SY-101-PUMP & $10 / 21 / 06$ & $10 / 26 / 06$ & 532,400 & 0 \\
\hline SY-10t & $S Y-102$ & $10 / 21 / 06$ & $10 / 26 / 06$ & 537,800 & 5,486 \\
\hline$\$ Y-101$ & SY-102 & $10 / 21 / 06$ & $10 / 26 / 06$ & $1,082,000$ & 611 \\
\hline $\mathrm{AX}-102$ & AN-103 & $10 / 26 / 06$ & $2 / 13 / 07$ & 87,597 & 897 \\
\hline EVAPF & AW-102 & $11 / 3 / 06$ & $11 / 3 / 06$ & 35,000 & 0 \\
\hline \begin{tabular}{|l|} 
SY-102 \\
\end{tabular} & AN-107 & $11 / 3 / 06$ & $11 / 6 / 06$ & 983,700 & 807 \\
\hline \begin{tabular}{|l|} 
SY-101 \\
\end{tabular} & SY-102 & $11 / 14 / 06$ & $11 / 14 / 06$ & 0 & 0 \\
\hline $\mathrm{AZ}-101$ & P1HLW FEED TANK & $12 / 18 / 06$ & $12 / 18 / 06$ & 127,200 & 3,269 \\
\hline P1HLW FEED TANK & HLW-CONTRACTOR & $12 / 18 / 06$ & $7 / 4 / 07$ & 127,200 & 3,269 \\
\hline S-103 & SY-101 & $2 / 13 / 07$ & $8 / 7 / 07$ & 712,300 & 1,011 \\
\hline $\mathrm{AX}-101$ & AN-103 & $2 / 13 / 07$ & $10 / 8 / 09$ & $2,149,000$ & 6,072 \\
\hline $242-\mathrm{A}$ & LERF & $2 / 23 / 07$ & $2 / 27 / 07$ & 314,000 & 0 \\
\hline $242-\mathrm{A}$ & AW-106 & $2 / 23 / 07$ & $2 / 27 / 07$ & 561,900 & 31 \\
\hline AW-102 & $242-\mathrm{A}$ & $2 / 23 / 07$ & $2 / 27 / 07$ & 875,900 & 31 \\
\hline AW-106 & AW-102 & $2 / 28 / 07$ & $3 / 3 / 07$ & 713,400 & 27 \\
\hline $242-A$ & LERF & $3 / 17 / 07$ & $3 / 21 / 07$ & 257,500 & 0 \\
\hline $242-A$ & AW-106 & $3 / 17 / 07$ & $3 / 21 / 07$ & 455,800 & 27 \\
\hline AW-102 & $242-\mathrm{A}$ & $3 / 17 / 07$ & $3 / 21 / 07$ & 713,300 & 27 \\
\hline AW-106 & AW-102 & $3 / 21 / 07$ & $3 / 23 / 07$ & 455,700 & 18 \\
\hline AP-102 & AP-106 & $3 / 26 / 07$ & $3 / 29 / 07$ & 622,200 & 24 \\
\hline AN-102 & AP-102 & $3 / 30 / 07$ & $4 / 4 / 07$ & $1,003,000$ & 44 \\
\hline $242-\mathrm{A}$ & LERF & $4 / 6 / 07$ & $4 / 9 / 07$ & 163,300 & 0 \\
\hline
\end{tabular}


HNF-2321

Revision 0

Table A.3-1. Complete Transfer List for Case 3.

\begin{tabular}{|c|c|c|c|c|c|}
\hline From & To & Start Date & End Date & $\begin{array}{c}\text { Liquid } \\
\text { (gallons) }\end{array}$ & $\begin{array}{c}\text { Solid } \\
\text { (gallons) }\end{array}$ \\
\hline 242-A & AW-106 & $4 / 6 / 07$ & $4 / 9 / 07$ & 292,500 & 18 \\
\hline AW-102 & $242-\mathrm{A}$ & $4 / 6 / 07$ & $4 / 9 / 07$ & 455,800 & 18 \\
\hline LERF & SY-103-PUMP & $4 / 7 / 07$ & $4 / 9 / 07$ & 183,500 & 0 \\
\hline SY-103 & SY-102 & $4 / 7 / 07$ & $4 / 9 / 07$ & 350,000 & 9,759 \\
\hline$S Y-103$ & SY-102. & $4 / 7 / 07$ & $4 / 9 / 07$ & 537,500 & 1,449 \\
\hline AW-106 & $A W-102$ & $4 / 9 / 07$ & $4 / 10 / 07$ & 292,500 & 12 \\
\hline SY-102 & AN-102 & $4 / 17 / 07$ & $4 / 19 / 07$ & 777,500 & 1,612 \\
\hline $242-\AA$ & LERF & $4 / 24 / 07$ & $4 / 26 / 07$ & 104,500 & 0 \\
\hline $242-\mathrm{A}$ & AW-106 & $4 / 24 / 07$ & $4 / 26 / 07$ & 188,000 & 12 \\
\hline$\overline{A W}-102$ & 242-A & $4 / 24 / 07$ & $4 / 26 / 07$ & 292,600 & 12 \\
\hline AW-106 & AW-101 & $4 / 26 / 07$ & $4 / 27 / 07$ & 188,000 & 9 \\
\hline AN-103 & AW-105 & $5 / 21 / 07$ & $5 / 22 / 07$ & 134,700 & 2,581 \\
\hline AN-103 & AN-106 & $5 / 22 / 07$ & $5 / 27 / 07$ & 965,600 & 18,496 \\
\hline AP-104 & AP-108 & $5 / 24 / 07$ & $5 / 27 / 07$ & 622,200 & 24 \\
\hline $\mathrm{AN}-107$ & AP-104 & $5 / 28 / 07$ & $6 / 1 / 07$ & 976,700 & 43 \\
\hline LERF & SY-103-PUMP & $6 / 5 / 07$ & $6 / 7 / 07$ & 183,500 & 0 \\
\hline SY-103 & SY-102 & $6 / 5 / 07$ & $6 / 7 / 07$ & 350,000 & 9,759 \\
\hline SY-103 & SY-102 & $6 / 5 / 07$ & $6 / 7 / 07$ & 537,500 & 1,449 \\
\hline SY-102 & AN-107 & $6 / 15 / 07$ & $6 / 16 / 07$ & 537,500 & 1,433 \\
\hline $\mathrm{AZ}-101$ & PIHLW FEED TANK & $7 / 4 / 07$ & $7 / 5 / 07$ & 127,200 & 3,269 \\
\hline P1HLW FEED TANK & HLW-CONTRACTOR & $7 / 5 / 07$ & $1 / 18 / 08$ & 127,200 & 3,269 \\
\hline RETURN 2 & $\mathrm{AP}-107$ & $7 / 6 / 07$ & $7 / 6 / 07$ & 8,670 & 330 \\
\hline S-105 & SY-101 & $8 / 7 / 07$ & $7 / 1 / 11$ & $1,518,000$ & 2,459 \\
\hline SX-113 & SY-103 & $9 / 6 / 07$ & $12 / 12 / 07$ & 10,322 & 399 \\
\hline $34\lfloor, 87$ & AN-105 & $10 / 1 / 07$ & $10 / 1 / 07$ & 50,000 & 0 \\
\hline EVAPF & AN-105 & $10 / 1 / 07$ & $10 / 1 / 07$ & 120,000 & 0 \\
\hline SPN87 & SY-102 & $10 / 1 / 07$ & $10 / 1 / 07$ & 25,000 & 0 \\
\hline TAL88 & AN-105 & $10 / 1 / 07$ & $10 / 1 / 07$ & 25,000 & 0 \\
\hline TNS88 & AN-105 & $10 / 1 / 07$ & $10 / 1 / 07$ & 750 & 250 \\
\hline WATER & AN-105 & $10 / 1 / 07$ & $10 / 1 / 07$ & 35,000 & 0 \\
\hline WESF & AN-105 & $10 / 1 / 07$ & $10 / 1 / 07$ & 5,000 & 0 \\
\hline AN-105 & AW-102 & $10 / 1 / 07$ & $10 / 5 / 07$ & 855,300 & 284 \\
\hline WATER & SY-102 & $10 / 2 / 07$ & $10 / 2 / 07$ & 5,000 & 0 \\
\hline WATER & AN-105 & $10 / 2 / 07$ & $10 / 2 / 07$ & 5,000 & 0 \\
\hline WATER & AN-105 & $10 / 2 / 07$ & $10 / 2 / 07$ & 22,000 & 0 \\
\hline AP-102 & AP-106 & $10 / 30 / 07$ & $11 / 4 / 07$ & $1,003,000$ & 44 \\
\hline EVAPF & AW-102 & $11 / 3 / 07$ & $11 / 3 / 07$ & 35,000 & 0 \\
\hline $\mathrm{AN}-102$ & AP- 102 & $11 / 5 / 07$ & $11 / 8 / 07$ & 763,200 & 34 \\
\hline $\mathrm{SX}-112$ & SY-103 & $12 / 12 / 07$ & $4 / 22 / 08$ & 397,100 & 16,406 \\
\hline $\mathrm{AP}-104$ & AP-108 & $12 / 29 / 07$ & $1 / 3 / 08$ & 976,700 & 43 \\
\hline AN-107 & AP-104 & $1 / 4 / 08$ & $1 / 6 / 08$ & 524,700 & 23 \\
\hline AN-103 & AN-106 & $1 / 10 / 08$ & $1 / 11 / 08$ & 115,100 & 366 \\
\hline $\mathrm{AZ}-102$ & P1HLW FEED TANK & $1 / 18 / 08$ & 1/19/08 & 137,700 & 4,774 \\
\hline P1HLW FEED TANK & HLW-CONTRACTOR & $1 / 19 / 08$ & $12 / 22 / 08$ & 137,700 & 4,774 \\
\hline SY-101 & AN-102 & $2 / 6 / 08$ & $2 / 10 / 08$ & 985,600 . & 1,658 \\
\hline $242-\mathrm{A}$ & LERF & $4 / 3 / 08$ & $4 / 8 / 08$ & 318,000 & 0 \\
\hline $242-\mathrm{A}$ & AW-106 & $4 / 3 / 08$ & $4 / 8 / 08$ & 569,100 & 30 \\
\hline AW-102 & 242-A & $4 / 3 / 08$ & $4 / 8 / 08$ & 887,100 & 30 \\
\hline AN-103 & AN-107 & $4 / 5 / 08$ & $4 / 10 / 08$ & 959,500 & 3,054 \\
\hline AW-106 & $A W-102$ & $4 / 8 / 08$ & $4 / 11 / 08$ & 569,000 & 20 \\
\hline $5 x-110$ & SY-103 & $4 / 22 / 08$ & $8 / 25 / 08$ & 212,700 & 8,909 \\
\hline $242-\mathrm{A}$ & LERF & $4 / 25 / 08$ & $4 / 28 / 08$ & 204,000 & 0 \\
\hline $242-\mathrm{A}$ & AW-106 & $4 / 25 / 08$ & $4 / 28 / 08$ & 365,100 & 20 \\
\hline $\mathrm{AW}-102$ & $242-\mathrm{A}$ & $4 / 25 / 08$ & $4 / 28 / 08$ & 569,100 & 20 \\
\hline AW-106 & AW-102 & $4 / 28 / 08$ & $4 / 30 / 08$ & 365,100 & 13 \\
\hline $242-A$ & LERF & $5 / 14 / 08$ & $5 / 16 / 08$ & 132,000 & 0 \\
\hline
\end{tabular}


HNF-2321

Revision 0

Table A.3-1. Complete Transfer List for Case 3.

\begin{tabular}{|c|c|c|c|c|c|}
\hline From & To & Start Date & End Date & $\begin{array}{c}\text { Liquid } \\
\text { (gallons) }\end{array}$ & $\begin{array}{c}\text { Solid } \\
\text { (gallons) }\end{array}$ \\
\hline $242-\mathrm{A}$ & AW-106 & $5 / 14 / 08$ & $5 / 16 / 08$ & 233,100 & 13 \\
\hline AW-102 & $242-\mathrm{A}$ & $5 / 14 / 08$ & $5 / 16 / 08$ & 365,100 & 13 \\
\hline AW-106 & AW-102 & $5 / 16 / 08$ & $5 / 17 / 08$ & 233,100 & 9 \\
\hline $242-\mathrm{A}$ & LERF & $5 / 31 / 08$ & $6 / 1 / 08$ & 84,000 & 0 \\
\hline 242-A & AW-106 & $5 / 31 / 08$ & $6 / 1 / 08$ & 149,200 & 9 \\
\hline $\mathrm{AW}-102$ & 242-A & $5 / 31 / 08$ & $6 / 1 / 08$ & 233,200 & 9 \\
\hline AW-106 & $\mathrm{AW}-102$ & $6 / 1 / 08$ & $6 / 2 / 08$ & 149,100 & 6 \\
\hline AW-102 & AN-105 & $6 / 3 / 08$ & $6 / 4 / 08$ & 149,200 & 6 \\
\hline SX-115 & SY-103 & $8 / 25 / 08$ & $10 / 26 / 08$ & 42,100 & 1,556 \\
\hline AP-102 & AP-106 & $8 / 27 / 08$ & $8 / 30 / 08$ & 763,200 & 34 \\
\hline $34 \mathrm{~L} 87$ & AN-105 & $10 / 1 / 08$ & $10 / 1 / 08$ & 50,000 & 0 \\
\hline EVAPF & AN-105 & $10 / 1 / 08$ & $10 / 1 / 08$ & 120,000 & 0 \\
\hline SPN87 & SY-102 & $10 / 1 / 08$ & $10 / 1 / 08$ & 25,000 & 0 \\
\hline TAL_88 & $A N-105$ & $10 / 1 / 08$ & $10 / 1 / 08$ & 25,000 & 0 \\
\hline $\begin{array}{l}\text { TNS88 } \\
\end{array}$ & AN-105 & $10 / 1 / 08$ & $10 / 1 / 08$ & 750 & 250 \\
\hline \begin{tabular}{|l|} 
WATER \\
\end{tabular} & AN-105 & $10 / 1 / 08$ & $10 / 1 / 08$ & 35,000 & 0 \\
\hline WESF & AN-105 & $10 / 1 / 08$ & $10 / 1 / 08$ & 5,000 & 0 \\
\hline WATER & SY-102 & $10 / 2 / 08$ & $10 / 2 / 08$ & 5,000 & 0 \\
\hline WATER & AN-105 & $10 / 2 / 08$ & $10 / 2 / 08$ & 5,000 & 0 \\
\hline WATER & AN-105 & $10 / 2 / 08$ & $10 / 2 / 08$ & 22,000 & 0 \\
\hline EVAPF & AW-102 & $10 / 4 / 08$ & $10 / 4 / 08$ & 50,000 & 0 \\
\hline SX-111 & SY-103 & $10 / 26 / 08$ & $8 / 20 / 11$ & 451,500 & 19,767 \\
\hline AP-104 & AP-108 & $11 / 1 / 08$ & $11 / 3 / 08$ & 524,700 & 23 \\
\hline EVAPF & $\mathrm{AW}-102$ & $11 / 3 / 08$ & $11 / 3 / 08$ & 35,000 & 0 \\
\hline AZ-102 & P1HLW FEED TANK & $12 / 22 / 08$ & $12 / 22 / 08$ & 137,700 & 4,774 \\
\hline P1HLW FEED TANK & HLW-CONTRACTOR & $12 / 22 / 08$ & $11 / 25 / 09$ & 137,700 & 4,774 \\
\hline $\mathrm{U}=202$ & UA-2 & $1 / 3 / 09$ & $1 / 10 / 09$ & 27,411 & 1,061 \\
\hline $\mathbf{U}-203$ & UA-2 & $1 / 10 / 09$ & $1 / 16 / 09$ & 13,895 & 537 \\
\hline U-110 & UA-2 & $1 / 16 / 09$ & $3 / 10 / 09$ & 99,290 & 4,252 \\
\hline U-110 & UA-1 & $3 / 10 / 09$ & $5 / 27 / 09$ & 143,800 & 6,160 \\
\hline UA-2 & SY-102 & $3 / 11 / 09$ & $3 / 12 / 09$ & 173,900 & 5,847 \\
\hline RETURN 2 & AP- 107 & $5 / 19 / 09$ & $5 / 19 / 09$ & 2,052 & 78 \\
\hline RETURN 1 & AP- 107 & $5 / 26 / 09$ & $5 / 26 / 09$ & 5,806 & 157 \\
\hline U- 110 & UA-2 & $5 / 27 / 09$ & $5 / 30 / 09$ & 4,829 & 207 \\
\hline UA-1 & SY-102 & $5 / 28 / 09$ & $5 / 29 / 09$ & 186,800 & 6,157 \\
\hline U-101 & UA-2 & $5 / 30 / 09$ & $7 / 30 / 09$ & 27,955 & 668 \\
\hline AP-107 & AZ-101 & $6 / 5 / 09$ & $6 / 6 / 09$ & 277,700 & 9,450 \\
\hline AN-103 & AP-107 & $6 / 15 / 09$ & $6 / 15 / 09$ & 49,018 & 155 \\
\hline AN-102 & AP-107 & $6 / 15 / 09$ & $6 / 20 / 09$ & $1,058,000$ & 9,409 \\
\hline U-104 & UA-2 & $7 / 30 / 09$ & $11 / 9 / 09$ & 50,908 & 1,221 \\
\hline $34 \mathrm{L87}$ & AN-105 & $10 / 1 / 09$ & $10 / 1 / 09$ & 7,000 & 0 \\
\hline 34L87 & AN-105 & $10 / 1 / 09$ & $10 / 1 / 09$ & 50,000 & 0 \\
\hline EVAPF & AN -105 & $10 / 1 / 09$ & 10/1/09 & 120,000 & 0 \\
\hline SPN87 & SY-102 & $10 / 1 / 09$ & $10 / 1 / 09$ & 25,000 & 0 \\
\hline TAL88 & AN-105 & $10 / 1 / 09$ & $10 / 1 / 09$ & 26,000 & 0 \\
\hline TNS88 & AN-105 & $10 / 1 / 09$ & $10 / 1 / 09$ & 750 & 250 \\
\hline WATER & AN-105 & $10 / 1 / 09$ & $10 / 1 / 09$ & 35,000 & 0 \\
\hline WESF & $\mathrm{AN}-105$ & $10 / 1 / 09$ & $10 / 1 / 09$ & 5,000 & 0 \\
\hline WATER & AN-105 & $10 / 2 / 09$ & $10 / 2 / 09$ & 3,000 & 0 \\
\hline WATER & $S Y-102$ & $10 / 2 / 09$ & $10 / 2 / 09$ & 5,000 & 0 \\
\hline WATER & AN-105 & $10 / 2 / 09$ & $10 / 2 / 09$ & 5,000 & 0 \\
\hline WATER & AN-105 & $10 / 2 / 09$ & $10 / 2 / 09$ & 22,000 & 0 \\
\hline $\mathrm{C}-202$ & AN-103 & $10 / 8 / 09$ & $10 / 13 / 09$ & 1,181 & 28 \\
\hline A-105 & AN-103 & $10 / 13 / 09$ & $1 / 5 / 10$ & 50,372 & 1,545 \\
\hline EVAPF & AW-102 & $11 / 3 / 09$ & $11 / 3 / 09$ & 35,000 & 0 \\
\hline U-112 & UA-2 & $11 / 9 / 09$ & $12 / 5 / 09$ & 58,521 & 2,330 \\
\hline
\end{tabular}


HNF-2321

Revision 0

Table A.3-1. Complete Transfer List for Case 3.

\begin{tabular}{|c|c|c|c|c|c|}
\hline From & To & Start Date & End Date & $\begin{array}{c}\text { Liquid } \\
\text { (gallons) }\end{array}$ & $\begin{array}{c}\text { Solid } \\
\text { (gallons) }\end{array}$ \\
\hline UA-2 & SY-102 & $12 / 5 / 09$ & $12 / 6 / 09$ & 154,400 & 4,426 \\
\hline $\mathrm{U}-112$ & UA-1 & $12 / 5 / 09$ & $1 / 17 / 10$ & 102,000 & 4,061 \\
\hline C-203 & AN-103 & $1 / 5 / 10$ & $1 / 12 / 10$ & 6,307 & 150 \\
\hline C-108 & AN-103 & $1 / 12 / 10$ & $3 / 30 / 10$ & 83,934 & 3,840 \\
\hline U-204 & UA-1 & $1 / 17 / 10$ & $1 / 23 / 10$ & 13,004 & 503 \\
\hline $\mathrm{U}-201$ & UA-1 & $1 / 23 / 10$ & $1 / 27 / 10$ & 13,912 & 538 \\
\hline U-201 & UA-2 & $1 / 27 / 10$ & $1 / 30 / 10$ & 13,499 & 522 \\
\hline UA-1 & SY-102 & $1 / 28 / 10$ & $1 / 28 / 10$ & 146,500 & 5,102 \\
\hline U-106 & UA-2 & $1 / 30 / 10$ & $3 / 1 / 10$ & 134,400 & 338 \\
\hline $\mathrm{U}-106$ & UA-1 & $3 / 1 / 10$ & $4 / 4 / 10$ & 149,500 & 376 \\
\hline \begin{tabular}{|l|} 
UA-2 \\
\end{tabular} & SY-102 & $3 / 2 / 10$ & $3 / 3 / 10$ & 151,500 & 862 \\
\hline C-111 & AN-103 & $3 / 30 / 10$ & $6 / 11 / 10$ & 176,500 & 7,080 \\
\hline $\mathrm{U}-106$ & UA-2 & $4 / 4 / 10$ & $5 / 7 / 10$ & 149,500 & 376 \\
\hline $\mathrm{UA-1}$ & SY-102 & $4 / 4 / 10$ & $6 / 25 / 11$ & 152,200 & 379 \\
\hline A-106 & AN-103 & $6 / 11 / 10$ & $8 / 8 / 11$ & 253,300 & $5 ; 796$ \\
\hline $34 \mathrm{~L} 87$ & AN-105 & $10 / 1 / 10$ & $10 / 1 / 10$ & 50,000 & 0 \\
\hline EVAPF & AN-105 & $10 / 1 / 10$ & $10 / 1 / 10$ & 50,260 & 0 \\
\hline \begin{tabular}{|l|} 
SPN87 \\
\end{tabular} & SY-102 & $10 / 1 / 10$ & $10 / 1 / 10$ & 25,000 & 0 \\
\hline TAL88 & AN-105 & $10 / 1 / 10$ & $10 / 1 / 10$ & 26,000 & 0 \\
\hline TNS88 & AN-105 & $10 / 1 / 10$ & $10 / 1 / 10$ & 750 & 250 \\
\hline WATER & AN-105 & $10 / 1 / 10$ & $10 / 1 / 10$ & 25,000 & 0 \\
\hline WATER & AN-105 & $10 / 1 / 10$ & $10 / 1 / 10$ & 35,000 & 0 \\
\hline WESF & AN-105 & $10 / 1 / 10$ & $10 / 1 / 10$ & 5,000 & 0 \\
\hline AN-105 & $\mathrm{AW}-102$ & $10 / 1 / 10$ & $10 / 5 / 10$ & 797,200 & 245 \\
\hline WATER & SY-102 & $10 / 2 / 10$ & $10 / 2 / 10$ & 5,000 & 0 \\
\hline WATER & $\mathrm{AN}-105$ & $10 / 2 / 10$ & $10 / 2 / 10$ & 5,000 & 0 \\
\hline WATER & $\mathrm{AN}-105$ & $10 / 2 / 10$ & $10 / 2 / 10$ & 22,000 & 0 \\
\hline EVAPF & AW-102 & $10 / 4 / 10$ & $10 / 4 / 10$ & 50,000 & 0 \\
\hline EVAPF & AN-105 & $10 / 5 / 10$ & $10 / 5 / 10$ & 69,740 & 0 \\
\hline EVAPF & AW-102 & $11 / 3 / 10$ & $11 / 3 / 10$ & 35,000 & 0 \\
\hline WATER & AN-105 & $1 / 1 / 11$ & $1 / 1 / 11$ & 25,000 & 0 \\
\hline BY-110 & BA-3 & $1 / 3 / 11$ & $2 / 8 / 11$ & 147,400 & 2,633 \\
\hline TX-109 & TA-5 & $1 / 3 / 11$ & $2 / 10 / 11$ & 144,100 & 5,925 \\
\hline TY-101 & $\mathrm{TA}-4$ & $1 / 3 / 11$ & $2 / 22 / 11$ & 146,500 & 3,494 \\
\hline T-106 & TA-6 & $1 / 3 / 11$ & $3 / 2 / 11$ & 74,648 & 2,929 \\
\hline B-112 & BA-1 & $1 / 3 / 11$ & $3 / 9 / 11$ & 75,213 & 1,083 \\
\hline BX-102 & $\mathrm{BA}-2$ & $1 / 3 / 11$ & $4 / 4 / 11$ & 52,548 & 2,443 \\
\hline BY- 110 & $\mathrm{BA}-4$ & $2 / 8 / 11$ & $3 / 16 / 11$ & 147,400 & 2,633 \\
\hline \begin{tabular}{|c|}
$\mathrm{TX}-109$ \\
\end{tabular} & TA-3 & $2 / 10 / 11$ & $3 / 19 / 11$ & 144,100 & 5,925 \\
\hline TY-101 & TA-2 & $2 / 22 / 11$ & 4/13/11 & 146,500 & 3,494 \\
\hline $\mathrm{T}-101$ & TA-6 & $3 / 2 / 11$ & $3 / 25 / 11$ & 61,377 & 2,346 \\
\hline B-111 & $\mathrm{BA}-1$ & $3 / 9 / 11$ & $4 / 9 / 11$ & 72,630 & 2,383 \\
\hline$B X-110$ & BA-5 & $3 / 16 / 11$ & $4 / 21 / 11$ & 147,400 & 2,633 \\
\hline TX-109 & TA-1 & $3 / 19 / 11$ & $4 / 26 / 11$ & 144,100 & 5,925 \\
\hline WATER & $A N-105$ & $4 / 1 / 11$ & $4 / 1 / 11$ & 25,000 & 0 \\
\hline $242-\mathrm{A}$ & LERF & $4 / 4 / 11$ & $4 / 9 / 11$ & 360,000 & 0 \\
\hline $242-\mathrm{A}$ & AW-106 & $4 / 4 / 11$ & $4 / 9 / 11$ & 639,300 & 34 \\
\hline AW-102 & $242-\mathrm{A}$ & $4 / 4 / 11$ & $4 / 9 / 11$ & 999,300 & 34 \\
\hline BX-112 & BA-2 & $4 / 4 / 11$ & $5 / 2 / 11$ & 67,370 & 2,937 \\
\hline B-111 & BA- 6 & $4 / 9 / 11$ & $6 / 11 / 11$ & 145,200 & 4,764 \\
\hline AW-106 & AW-102 & $4 / 10 / 11$ & $4 / 13 / 11$ & 639,200 & 22 \\
\hline $242-\mathrm{A}$ & LERF & $4 / 27 / 11$ & $5 / 1 / 11$ & 228,800 & 0 \\
\hline $242-\mathrm{A}$ & AW-106 & $4 / 27 / 11$ & $5 / 1 / 11$ & 410,500 & 22 \\
\hline AW-102 & $242-A$ & $4 / 27 / 11$ & $5 / 1 / 11$ & 639,300 & 22 \\
\hline AW-106 & AW-102 & $5 / 1 / 11$ & $5 / 3 / 11$ & 410,400 & 15 \\
\hline $242-A$ & LERF & $5 / 17 / 11$ & $5 / 19 / 11$ & 150,000 & 0 \\
\hline
\end{tabular}


HNF-2321

Revision 0

Table A.3-1. Complete Transfer List for Case 3.

\begin{tabular}{|c|c|c|c|c|c|}
\hline From & To & Start Date & End Date & $\begin{array}{c}\text { Liquid } \\
\text { (gallons) }\end{array}$ & $\begin{array}{c}\text { Solid } \\
\text { (gallons) }\end{array}$ \\
\hline $242-A$ & AW-106 & $5 / 17 / 11$ & $5 / 19 / 11$ & 260,500 & 15 \\
\hline AW-102 & $242-\mathrm{A}$ & $5 / 17 / 11$ & $5 / 19 / 11$ & 410,500 & 15 \\
\hline AW-106 & AW-102 & $5 / 19 / 11$ & $5 / 20 / 11$ & 260,400 & 10 \\
\hline AN-102 & AP-102 & $6 / 1 / 11$ & $6 / 1 / 11$ & 49,734 & 439 \\
\hline BA-1 & AP-108 & $6 / 1 / 11$ & $6 / 2 / 11$ & 157,300 & 3,464 \\
\hline $\mathrm{BA}-3$ & AP-104 & $6 / 1 / 11$ & $6 / 2 / 11$ & 155,600 & 2,631 \\
\hline $\mathrm{BA}-4$ & AP-102 & $6 / 1 / 11$ & $6 / 2 / 11$ & 155,600 & 2,631 \\
\hline SY-101 & AN-102 & $6 / 1 / 11$ & $6 / 5 / 11$ & $1,115,000$ & 1,796 \\
\hline AN-103 & AP-106 & $6 / 1 / 11$ & $6 / 6 / 11$ & $1,099,00 \mathrm{x})$ & 17,130 \\
\hline BA-5 & AP-104 & $6 / 2 / 11$ & $6 / 2 / 11$ & 155,600 & 2,631 \\
\hline $\mathrm{BA}-2$ & AP-102 & $6 / 2 / 11$ & $6 / 3 / 11$ & 174,500 & 5,377 \\
\hline BY-110 & $\mathrm{BA}-4$ & $6 / 2 / 11$ & $7 / 8 / 11$ & 147,300 & 2,631 \\
\hline $3 X-112$ & $\mathrm{BA}-3$ & $6 / 2 / 11$ & $8 / 2 / 11$ & 143,600 & 6,262 \\
\hline $242-\mathrm{A}$ & LERF & $6 / 3 / 11$ & $6 / 5 / 11$ & 96,000 & 0 \\
\hline $242-\mathrm{A}$ & $A W-106$ & $6 / 3 / 11$ & $6 / 5 / 11$ & 164,500 & 10 \\
\hline AW-102 & $242-A$ & $6 / 3 / 11$ & $6 / 5 / 11$ & 260,500 & 10 \\
\hline AW-106 & AW-102 & $6 / 5 / 11$ & $6 / 6 / 11$ & 164,500 & 6 \\
\hline AN-102 & AP-102 & $6 / 5 / 11$ & $6 / 9 / 11$ & 727,000 & 1,296 \\
\hline AN-103 & AP-104 & $6 / 6 / 11$ & $6 / 6 / 11$ & 4,514 & 69 \\
\hline AW-102 & AN-105 & $6 / 7 / 11$ & $6 / 8 / 11$ & 164,600 & 6 \\
\hline AN-102 & AP-104 & $6 / 9 / 11$ & $6 / 11 / 11$ & 388,500 & 692 \\
\hline BA-6 & AP-104 & $6 / 11 / 11$ & $6 / 12 / 11$ & 159,400 & 4,762 \\
\hline$S Y-103$ & AN-102 & $6 / 11 / 11$ & $6 / 15 / 11$ & $1,081,000$ & 35,223 \\
\hline B-111 & $\mathrm{BA}-1$ & $6 / 11 / 11$ & $8 / 12 / 11$ & 145,100 & 4,761 \\
\hline AN-102 & AP-104 & $6 / 16 / 11$ & $6 / 17 / 11$ & 234,600 & 7,461 \\
\hline AN-102 & AP-108 & $6 / 17 / 11$ & $6 / 21 / 11$ & 848,400 & 26,963 \\
\hline SY-102 & AN-102 & $6 / 21 / 11$ & $6 / 25 / 11$ & $1,024,000$ & 22,112 \\
\hline AN-102 & AP-108 & $6 / 25 / 11$ & $6 / 25 / 11$ & 78,587 & 1,716 \\
\hline UA-2 & SY-102 & $6 / 25 / 11$ & $6 / 26 / 11$ & 152,200 & 376 \\
\hline $\mathrm{U}-106$ & UA-1 & $6 / 25 / 11$ & $7 / 29 / 11$ & 149,500 & 376 \\
\hline TA-5 & SY-102 & $6 / 26 / 11$ & $6 / 27 / 11$ & 180,300 & 5,922 \\
\hline TA-4 & SY-102 & $6 / 27 / 11$ & $6 / 28 / 11$ & 143,200 & 3,492 \\
\hline TY-101 & TA-5 & $6 / 27 / 11$ & $7 / 18 / 11$ & 59,942 & 1,430 \\
\hline TA-3 & $S Y-102$ & $6 / 28 / 11$ & $6 / 29 / 11$ & 180,300 & 5,922 \\
\hline $\mathrm{TX}-109$ & TA-4 & $6 / 28 / 11$ & $8 / 5 / 11$ & 144,000 & 5,921 \\
\hline TA-6 & SY-102 & $6 / 29 / 11$ & $6 / 30 / 11$ & 154,500 & 5,272 \\
\hline$T-101$ & TA-3 & $6 / 29 / 11$ & $8 / 24 / 11$ & 144,400 & 5,519 \\
\hline WATER & AN-105 & $7 / 1 / 11$ & $7 / 1 / 11$ & 25,000 & 0 \\
\hline BY-110 & BA-5. & $7 / 8 / 11$ & $8 / 13 / 11$ & 147,300 & 2,631 \\
\hline $\mathrm{U}-106$ & UA-2 & $7 / 29 / 11$ & $8 / 13 / 11$ & 66,952 & 168 \\
\hline BX-112 & BA-2 & $8 / 2 / 11$ & $9 / 4 / 11$ & 78,662 & 3,430 \\
\hline TX-109 & TA-6 & $8 / 5 / 11$ & $9 / 11 / 11$ & 144,000 & 5,921 \\
\hline B-111 & BA-6 & $8 / 12 / 11$ & $8 / 13 / 11$ & 719 & 24 \\
\hline $\mathrm{U}-111$ & UA-2 & $8 / 13 / 11$ & $8 / 30 / 11$ & 81,353 & 440 \\
\hline$B Y-110$ & $\mathrm{BA}-6$ & $8 / 13 / 11$ & $9 / 18 / 11$ & 146,500 & 2,618 \\
\hline B-103 & BA-2 & $9 / 4 / 11$ & $9 / 22 / 11$ & 41,507 & 78 \\
\hline
\end{tabular}


Table A.3-2. Phase 1 Low-Activity Waste Feed Staging Transfers for Case 3.

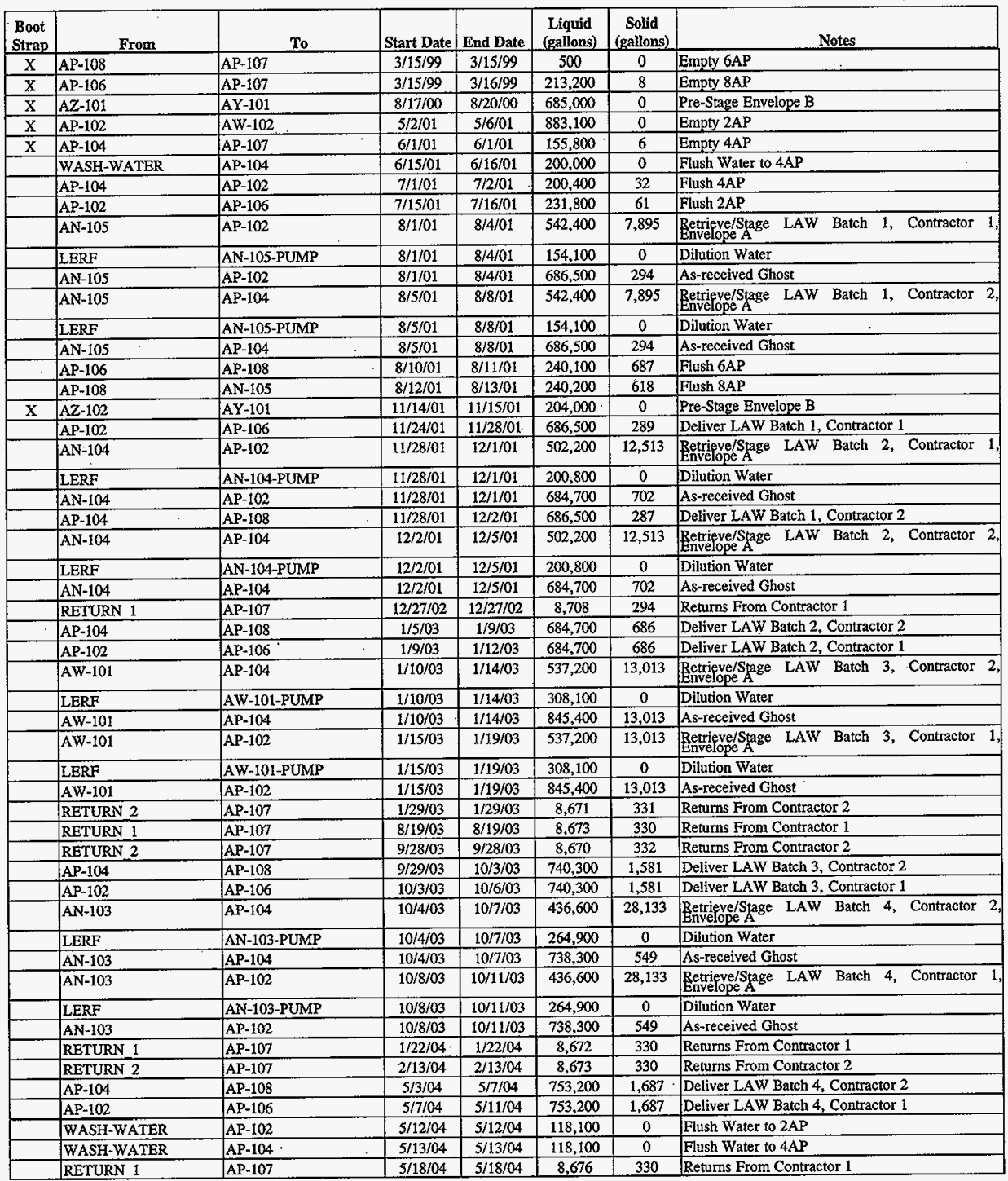


HNF-2321

Revision 0

Table A.3-2. Phase 1 Low-Activity Waste Feed Staging Transfers for Case 3.

\begin{tabular}{|c|c|c|c|c|c|c|c|}
\hline $\begin{array}{c}\text { Boot } \\
\text { Strap }\end{array}$ & From & To & Start Date & End Date & $\begin{array}{c}\text { Liquid } \\
\text { (gallons) }\end{array}$ & $\begin{array}{c}\text { Solid } \\
\text { (gallons) }\end{array}$ & Notes \\
\hline & RETURN 2 & AP-107 & $5 / 24 / 04$ & $5 / 24 / 04$ & 8,670 & 330 & Returns From Contractor 2 \\
\hline & AP-102 & AN-105 & $5 / 28 / 04$ & $5 / 29 / 04$ & 209,500 & 9,168 & Flush 2AP \\
\hline & WASH-CAUSTIC & $A P-102$ & $5 / 31 / 04$ & $5 / 31 / 04$ & 103,400 & 0 & Shim Caustic for Batch 5, Contractor 1 \\
\hline & AP-104 & $A N-105$ & $5 / 30 / 04$ & $5 / 31 / 04$ & 209,500 & 9,168 & Flush 4AP \\
\hline & WASH-CAUSTIC & AP-104 & $6 / 1 / 04$ & $6 / 1 / 04$ & 126,400 & 0 & Shim Caustic for Batch 5, Contractor 2 \\
\hline & AP-101 & AP-102 & $6 / 2 / 04$ & $6 / 4 / 04$ & 470,100 & 21 & $\begin{array}{l}\text { Retrieve/Stage LAW Batch 5, Contractor 1, } \\
\text { Envelope } A\end{array}$ \\
\hline & LERF & AP-101-PUMP & $6 / 2 / 04$ & $6 / 4 / 04$ & 51,711 & 0 & Dilution Water \\
\hline & AP-101 & AP-102 & $6 / 2 / 04$ & $6 / 4 / 04$ & 521,800 & 21 & As-received Ghost \\
\hline & AP-101 & AP-104 & $6 / 5 / 04$ & $6 / 8 / 04$ & 574,500 & 26 & $\begin{array}{l}\text { Retrieve/Stage LAW Batch 5, Contractor } \\
\text { Envelope } A\end{array}$ \\
\hline & LERF & AP-101-PUMP & $6 / 5 / 04$ & $6 / 8 / 04$ & 63,203 & 0 & Dilution Water \\
\hline & AP-101 & AP-104 & $6 / 5 / 04$ & $6 / 8 / 04$ & 637,700 & 26 & As-received Ghost \\
\hline & AW-104 & $\mathrm{AP}-102$ & $6 / 8 / 04$ & $6 / 8 / 04$ & 107,400 & 4 & $\begin{array}{l}\text { Complete Retrieve/Stage LAW Batch 5, Contracton } \\
\text { Envelope A }\end{array}$ \\
\hline & AW-104 & AP-104 & $6 / 9 / 04$ & $6 / 9 / 04$ & 131,200 & 5 & Complete Retrieve/Stage LAW Batch 5, Contractor \\
\hline & RETURN 1 & $\mathrm{AP}-107$ & $7 / 7 / 04$ & $7 / 7 / 04$ & 8,678 & 331 & Reurns From Contractor 1 \\
\hline & RETURN 2 & AP-107 & $7 / 14 / 04$ & $7 / 14 / 04$ & 8,671 & 331 & Returns From Contractor 2 \\
\hline & RETURN 1 & $\mathrm{AP}-107$ & $8 / 26 / 04$ & $8 / 27 / 04$ & 8,678 & 331 & Returns From Contractor 1 \\
\hline & RETURN 2 & AP-107 & $9 / 2 / 04$ & $9 / 2 / 04$ & 8,671 & 331 & Returns From Contractor 2 \\
\hline & RETURN 1 & AP-107 & $10 / 16 / 04$ & $10 / 16 / 04$ & 8,678 & 331 & Returns From Contractor 1 \\
\hline & RETURN 2 & AP-107 & $10 / 23 / 04$ & $10 / 23 / 04$ & 8,671 & 331 & Returns From Contractor 2 \\
\hline & RETURN 1 & AP-107 & $12 / 5 / 04$ & $12 / 5 / 04$ & 8,678 & 331 & Returns From Contractor 1 \\
\hline & RETURN 2 & $A P-107$ & $12 / 12 / 04$ & $12 / 12 / 04$ & 8,671 & 331 & Returns From Contractor 2 \\
\hline & RETURN 1 & AP-107 & $1 / 24 / 05$ & $1 / 24 / 05$ & 8,678 & 331 & Returns From Contractor 1 \\
\hline & RETURN 2 & AP-107 & $1 / 31 / 05$ & $2 / 1 / 05$ & 8,671 & 331 & Renurns From Contractor 2 \\
\hline & AP-104 & AP-108 & $2 / 18 / 05$ & $2 / 22 / 05$ & 894,300 & 1,149 & Deliver LAW Batch 5, Contractor 2 \\
\hline & $A Y-101$ & $\mathrm{AP}-104$ & $2 / 23 / 05$ & $2 / 24 / 05$ & 283,600 & 12 & Stage LAW Batch 6, Contractor 2, Envelope B \\
\hline & AP-102 & AP-106 & $2 / 22 / 05$ & $2 / 26 / 05$ & 731,500 & 1,136 & Detiver LAW Batch 5, Contractor 1 \\
\hline & AY-101 & $\mathrm{AP}-102$ & $2 / 27 / 05$ & $2 / 28 / 05$ & 279,700 & 11 & Stage LAW Batch 6, Contractor 1, Envelope B \\
\hline & RETURN I & AP-107 & $3 / 18 / 05$ & $3 / 18 / 05$ & 8,679 & 331 & Returns From Contractor 1 \\
\hline & RETURN 2 & AP-107 & $3 / 27 / 05$ & $3 / 27 / 05$ & 8,682 & 331 & Returns From Contractor 2 \\
\hline & RETURN I & AP-107 & $5 / 14 / 05$ & $5 / 14 / 05$ & 8,675 & 330 & Returns From Contractor 1 \\
\hline & RETURN 2 & AP-107 & $5 / 23 / 05$ & $5 / 23 / 05$ & 8,677 & 330 & Returns From Contractor 2 \\
\hline & RETURN 1 & AP-107 & $7 / 9 / 05$ & $7 / 10 / 05$ & 8,675 & 330 & Returns From Contractor 1 \\
\hline & RETURN 2 & AP-107 & $7 / 19 / 05$ & $7 / 19 / 05$ & 8,677 & 330 & Returns From Contractor 2 \\
\hline & RETURN 1 & AP-107 & $9 / 4 / 05$ & $9 / 4 / 05$ & 8,675 & 330 & Returns From Contractor 1 \\
\hline & RETURN 2 & AP-107 & $9 / 14 / 05$ & $9 / 14 / 05$ & 8,677 & 330 & Returns From Contractor 2 \\
\hline & RETURN 1 & AP-107 & $10 / 31 / 05$ & $.10 / 31 / 05$ & 8,675 & 330 & Returns From Contractor 1 \\
\hline & RETURN 2 & AP-107 & $11 / 10 / 05$ & $11 / 10 / 05$ & 8,677 & 330 & Returns From Contractor 2 \\
\hline \multirow[t]{6}{*}{$\mathrm{X}$} & SY-102 & AN-105 & $11 / 15 / 02$ & $11 / 18 / 02$ & 650,000 & 27 & Decant Supernate from SY-102 \\
\hline & $A P-106$ & LAW-CONTRACTOR-1 & $6 / 1 / 02$ & $12 / 9 / 05$ & $3,623,000$ & 5,414 & Complete Processing Envelope $\mathrm{A}$, Contractor 1 \\
\hline & $\mathrm{AP}-102$ & $\mathrm{AP}-106$ & $12 / 9 / 05$ & $12 / 11 / 05$ & 279,600 & 49 & Deliver LAW Batch 6 , Contractor 1 \\
\hline & AN-107 & AP-102 & $12 / 12 / 05$ & $12 / 14 / 05$ & 481,700 & 22 & $\begin{array}{l}\text { Retripve/Stage LAW Batch } 7 / 8 \text {, Contractor } 1, \\
\text { Envelope C }\end{array}$ \\
\hline & LERF & AN-107-PUMP & $12 / 12 / 05$ & $12 / 14 / 05$ & 72,259 & 0 & Dilution Water \\
\hline & AN-107 & AP-102 & $12 / 12 / 05$ & $12 / 14 / 05$ & 554,000 & 22 & As-received Ghost \\
\hline $\mathrm{X}$ & WATER & SY-102 & $12 / 15 / 05$ & $12 / 17 / 05$ & 426,000 & 0 & Flush Water for 2SY Solids Cleanout \\
\hline \multirow[t]{9}{*}{$\mathrm{X}$} & SY-102 & AW-105 & $1 / 1 / 06$ & $1 / 3 / 06$ & 731,500 & 20,807 & Cleanout Solids 2SY \\
\hline & RETURN 2 & AP-107 & $1 / 6 / 06$ & $1 / 6 / 06$ & 8,677 & 330 & Returns From Contractor 2 \\
\hline & AP-108 & LAW-CONTRACTOR-2 & $6 / 1 / 02$ & $2 / 6 / 06$ & $3,759,000$ & 5,424 & Complete Processing Envelope A, Contractor 2 \\
\hline & AP-104 & AP-108 & $2 / 6 / 06$ & $2 / 7 / 06$ & 283,600 & 43 & Deliver LAW Batch 6 , Contractor 2 \\
\hline & AN-107 & AP-104 & $2 / 8 / 06$ & $2 / 10 / 06$ & 481,700 & 22 & $\begin{array}{l}\text { Betrieve/Stage LAW Batch } 7 / 8, \text { Contractor } 2, \\
\text { Envelope C }\end{array}$ \\
\hline & LERF & AN-107-PUMP & $2 / 8 / 06$ & $2 / 10 / 06$ & 72,260 & 0 & Dilution Water \\
\hline & AN-107 & AP-104 & $2 / 8 / 06$ & $2 / 10 / 06$ & 554,000 & 22 & As-received Ghost \\
\hline & AP-106 & LAW-CONTRACTOR-1 & $12 / 11 / 05$ & $2 / 16 / 06$ & 279,600 & 85 & Complete Processing Envelope B, Contractor 1 \\
\hline & AP-102 & AP-106 & $2 / 16 / 06$ & $2 / 17 / 06$ & 169,400 & 8 & Deliver LAW Batch 7, Contractor 1 \\
\hline
\end{tabular}


Table A.3-2. Phase 1 Low-Activity Waste Feed Staging Transfers for Case 3.

\begin{tabular}{|c|c|c|c|c|c|c|c|}
\hline $\begin{array}{l}\text { Boot } \\
\text { Strap }\end{array}$ & From & To & Start Date & End Date & $\begin{array}{c}\text { Liquid } \\
\text { (gallons) }\end{array}$ & $\begin{array}{l}\text { Solid } \\
\text { (gallons) }\end{array}$ & Notes \\
\hline & $\mathrm{AP}-102$ & AP-106 & $3 / 16 / 06$ & $3 / 18 / 06$ & 384,600 & 18 & Deliver LAW Batch 8 , Contractor 1 \\
\hline & AN-102 & AP-102 & $3 / 19 / 06$ & $3 / 22 / 06$ & 481,700 & 23 & $\begin{array}{l}\text { Retrieve/Stage LAW Batch 9, Contractor 1, } \\
\text { Envelope C }\end{array}$ \\
\hline & LERF & AN-102-PUMP & $3 / 19 / 06$ & $3 / 22 / 06$ & 289,000 & $\mathbf{0}$ & Dilution Water \\
\hline & AN-102 & AP-102 & $3 / 19 / 06$ & $3 / 22 / 06$ & 770,700 & 23 & As-received Ghost \\
\hline & RETURN 1 & AP-107 & $4 / 11 / 06$ & $4 / 11 / 06$ & 8,676 & 325 & Returns From Contractor 1 \\
\hline & AP-108 & LAW-CONTRACTOR-2 & $2 / 8 / 06$ & $4 / 16 / 06$ & 283,500 & 73 & Complete Processing Envelope B, Contractor 2 \\
\hline & AP-104 & AP-108 & $4 / 16 / 06$ & $4 / 17 / 06$ & 169,400 & 8 & Deliver LAW Batch 7, Contractor 2 \\
\hline & AP-104 & AP-108 & $5 / 14 / 06$ & $5 / 16 / 05$ & 384,600 & 17 & Deliver LAW Batch 8 , Contractor 2 \\
\hline & AN-102 & AP-104 & $5 / 17 / 06$ & $5 / 20 / 06$ & 481,700 & 23 & \begin{tabular}{|l} 
Betrieve/Stage LAW Batch 9, Contractor 2, \\
Envelope
\end{tabular} \\
\hline & LERF & AN-102-PUMP & $5 / 17 / 06$ & $5 / 20 / 06$ & 289,000 & $\mathbf{0}$ & Dilution Water \\
\hline & AN-102 & AP-104 & $5 / 17 / 06$ & $5 / 20 / 06$ & 770,700 & 23 & As-received Ghost \\
\hline & AP-102 & AP-106 & $7 / 29 / 06$ & $8 / 2 / 06$ & 770,700 & 23 & Deliver LAW Batch 9, Contractor 1 \\
\hline & AN-106 & AP-102 & $8 / 2 / 06$ & $8 / 5 / 06$ & 527,300 & 24 & $\begin{array}{l}\text { Retrieve/Stage LAW Batch 10, Contractor } 1 \\
\text { Envelope C }\end{array}$ \\
\hline & LERF & AN-106-PUMP & $8 / 2 / 06$ & $8 / 5 / 06$ & 94,920 & 0 & Dilution Water \\
\hline & AN-106 & AP-102 & $8 / 2 / 06$ & $8 / 5 / 05$ & 622,200 & 24 & As-received Ghost \\
\hline & AP-104 & AP-108 & $9 / 26 / 06$ & $9 / 30 / 06$ & 770,700 & 23 & Deliver LAW Batch 9, Contractor 2 \\
\hline & AN-106 & AP-104 & $10 / 1 / 06$ & $10 / 4 / 06$ & 527,300 & 24 & $\begin{array}{l}\text { Retrieve/Stage LAW Batch } 10, \text { Contractor } 2, \\
\text { Envelope c }\end{array}$ \\
\hline & LERF & AN-106-PUMP & $10 / 1 / 06$ & $10 / 4 / 06$ & 94,920 & 0 & Dilution Water \\
\hline & AN-106 & AP-104 & $10 / 1 / 06$ & $10 / 4 / 06$ & 622,200 & 24 & As-received Ghost \\
\hline & SY-101 & SY-102 & $10 / 2 / 06$ & $10 / 7 / 06$ & 537,800 & 5,486 & Retrieve LAW Batch 11, Envelope C \\
\hline & LERF & SY-101-PUMP & $10 / 2 / 06$ & $10 / 7 / 06$ & 532,400 & 0 & Dilution Water \\
\hline & $S Y-101$ & SY-102 & $10 / 2 / 06$ & $10 / 7 / 06$ & $1,082,000$ & 611 & As-received Ghost \\
\hline & SY-102 & AN-102 & $10 / 10 / 06$ & $10 / 13 / 06$ & $1,026,000$ & 2,529 & Pre-Stage LAW Batch 11, Envelope C \\
\hline & SY-101 & SY-102 & $10 / 21 / 06$ & $10 / 26 / 06$ & 537,800 & 5,486 & Retrieve LAW Batch 11, Envelope C \\
\hline & LERF & SY-101-PUMP & $10 / 21 / 06$ & $10 / 26 / 06$ & 532,400 & 0 & Dilution Water \\
\hline & SY-101 & SY-102 & $10 / 21 / 06$ & $10 / 26 / 06$ & $1,082,000$ & 611 & As-received Ghost \\
\hline & SY-102 & $\mathrm{AN}-107$ & $11 / 3 / 06$ & $11 / 6 / 06$ & 983,700 & 807 & Pre-Stage LAW Batch 11, Envelope C \\
\hline & AP-102 & AP-106 & $3 / 26 / 07$ & $3 / 29 / 07$ & 622,200 & 24 & Deliver LAW Batch 10, Contractor 1 \\
\hline & AN-102 & AP-102 & $3 / 30 / 07$ & $4 / 4 / 07$ & $1,003,000$ & 44 & Stage LAW Batch 11, Contractor 1, Envelope C \\
\hline & SY-103 & SY-102 & $4 / 7 / 07$ & $4 / 9 / 07$ & 350,000 & 9,759 & Retrieve LAW Batch 12, Envelope C \\
\hline & LERF & SY-103-PUMP & $4 / 7 / 07$ & $4 / 9 / 07$ & 183,500 & 0 & Dilution Water \\
\hline & SY-103 & SY-102 & $4 / 7 / 07$ & $4 / 9 / 07$ & 537,500 & 1,449 & As-received Ghost \\
\hline & SY-102 & AN-102 & 4/17/07 & 4/19/07 & 777,500 & 1,612 & Pre-Stage LAW Batch 12, Envelope C \\
\hline & $\mathrm{AP}-104$ & AP-108 & $5 / 24 / 07$ & $5 / 27 / 07$ & 622,200 & 24 & Deliver LAW Batch 10 , Contractor 2 \\
\hline & AN-107 & AP-104: & $5 / 28 / 07$ & $6 / 1 / 07$ & 976,700 & 43 & Stage LAW Batch 11, Contractor 2, Envelope C \\
\hline & SY-103 & SY-102 & $6 / 5 / 07$ & $6 / 7 / 07$ & 350,000 & 9,759 & Retrieve LAW Batch 12, Envelope C \\
\hline & LERF & SY-103-PUMP & $6 / 5 / 07$ & $6 / 7 / 07$ & 183,500 & 0 & Dilution Water \\
\hline & SY-103 & SY-102 & $6 / 5 / 07$ & $6 / 7 / 07$ & 537,500 & 1,449 & As-received Ghost \\
\hline & SY-102 & AN-107 & $6 / 15 / 07$ & $6 / 16 / 07$ & 537,500 & 1,433 & Pre-Stage LAW Batch 12, Envelope C \\
\hline & RETURN 2 & AP-107 & $7 / 6 / 07$ & $7 / 6 / 07$ & 8,670 & 330 & Returns From Contractor 2 \\
\hline & $\mathrm{AP}-102$ & AP-106 & $10 / 30 / 07$ & $11 / 4 / 07$ & $1,003,000$ & 44 & Deliver LAW Batch 11, Contractor 1 \\
\hline & $\mathrm{AN}-102$ & AP-102 & $11 / 5 / 07$ & $11 / 8 / 07$ & 763,200 & 34 & Stage LAW Batch 12, Contractor 1, Envelope C \\
\hline & AP-104 & AP-108 & $12 / 29 / 07$ & $1 / 3 / 08$ & 976,700 & 43 & Deliver LAW Batch 11, Contractor 2 \\
\hline & AN-107 & AP-104 & $1 / 4 / 08$ & $1 / 6 / 08$ & 524,700 & 23 & Stage LAW Batch 12, Contractor 2, Envelope C \\
\hline & $\mathrm{AP}-102$ & AP-106 & $8 / 27 / 08$ & $8 / 30 / 08$ & 763,200 & 34 & Deliver LAW Batch 12, Contractor 1 \\
\hline & AP-104 & AP-108 & $11 / 1 / 08$ & $11 / 3 / 08$ & 524,700 & 23 & Deliver LAW Batch 12, Contractor 2 \\
\hline & AP-108 & LAW-CONTRACTOR-2 & $4 / 17 / 06$ & $5 / 18 / 09$ & $3,448,000$ & 144 & Complete Processing Envelope C, Contractor 1 \\
\hline & RETURN 2 & AP-107 & $5 / 19 / 09$ & $5 / 19 / 09$ & 2,052 & 78 & Returns From Contractor 2 \\
\hline & AP-106 & LAW-CONTRACTOR-1 & $2 / 17 / 06$ & $5 / 25 / 09$ & $3,713,000$ & 157 & Complete Processing Envelope C, Contractor 2 \\
\hline & RETURN 1 & AP-107 & $5 / 26 / 09$ & $5 / 26 / 09$ & 5,806 & 157 & Returns From Contractor 1 \\
\hline $\mathbf{X}$ & AP-107 & AZ-101 & $6 / 5 / 09$ & $6 / 6 / 09$ & 277,700 & 9,450 & Transfer Returns to AZ-101 (Phase $2 \mathrm{HLW}$ Tank) \\
\hline
\end{tabular}


HNF-2321

Revision 0

Table A.3-3. Phase 1 High-Level Waste Feed Staging Transfers for Case 3.

\begin{tabular}{|c|c|c|c|c|c|c|c|}
\hline $\begin{array}{l}\text { Boot } \\
\text { Strap }\end{array}$ & From & To & Start Date & End Date & $\begin{array}{c}\text { Liquid } \\
\text { (gallons) }\end{array}$ & $\begin{array}{c}\text { Solid } \\
\text { (gallons) }\end{array}$ & Notes \\
\hline & AY-102 & C-106 & $7 / 1 / 98$ & $7 / 5 / 98$ & 920,700 & 31 & Sluice "Water" for C-106 Retrieval - Lumped \\
\hline & $\mathrm{C}-106$ & AY-102 & $7 / 15 / 98$ & $11 / 10 / 98$ & 809,000 & 15,169 & C-106 Retrieval (Project W-320) \\
\hline \multirow[t]{8}{*}{$\mathrm{X}$} & AZ-101 & AY-101. & $8 / 17 / 00$ & $8 / 20 / 00$ & 685,000 & 0 & Initial Decant IAZ \\
\hline & WASH-WATER & AZ-101 & $8 / 20 / 00$ & $8 / 21 / 00$ & 146,000 & 0 & First Wash IAZ \\
\hline & AZ-101 & AP-107 & $10 / 9 / 00$ & $10 / 10 / 00$ & 143,200 & 0 & First Decant 1AZ \\
\hline & WASH-WATER & $\overline{A Z-101}$ & $10 / 11 / 00$ & $10 / 12 / 00$ & 146,000 & 0 & Second Wash IAZ \\
\hline & AZ-101 & AP-107 & $11 / 30 / 00$ & $12 / 1 / 00$ & 140,200 & 0 & Second Decant $1 \mathrm{AZ}$ \\
\hline & WASH-WATER & AZ-101 & $12 / 2 / 00$ & $12 / 2 / 00$ & 146,000 & 0 & Third Wash $1 \mathrm{AZ}$ \\
\hline & AZ-101 & $\mathrm{AP}-107$ & $1 / 20 / 01$ & $1 / 21 / 01$ & 142,900 & 0 & Third Decant $1 \mathrm{AZ}$ \\
\hline & WASH-WATER & AZ-101 & $1 / 22 / 01$ & $1 / 23 / 01$ & 138,200 & 0 & Transfer Water Add 1AZ \\
\hline \multirow[t]{14}{*}{$\mathbf{x}$} & AZ-102 & AY-101 & $11 / 14 / 01$ & $11 / 15 / 01$ & 204,000 & 0 & Initial Decant 2AZ \\
\hline & $\mathrm{AZ}-102$ & AP-107 & $11 / 15 / 01$ & $11 / 16 / 01$ & 181,800 & 0 & Complete Initial Decant $2 \mathrm{AZ}$ \\
\hline & WASH-WATER & $\mathrm{AZ}-102$ & $11 / 16 / 01$ & $11 / 17 / 01$ & 213,000 & 0 & First Wash $2 \mathrm{AZ}$ \\
\hline & AZ-101 & P1HLW_FEED_TANK & $5 / 17 / 02$ & $5 / 17 / 02$ & 134,300 & 5,079 & $\begin{array}{l}\text { Beliver } \\
\text { (verall) }\end{array}$ Batch 1 of $1 \mathrm{AZ}$, Envelope D (Batch 1 \\
\hline & AZ-102 & AN-105 & $4 / 8 / 02$ & $4 / 8 / 02$ & 127,700 & 0 & First Decant $2 \mathrm{AZ}$ \\
\hline & WASH-WATER & AZ-102 & $4 / 9 / 02$ & $4 / 11 / 02$ & 213,000 & 0 & Second Wash 2AZ \\
\hline & $A Z-102$ & AN-105 & $5 / 30 / 02$ & $5 / 31 / 02$ & 210,300 & 0 & Second Decant 2AZ \\
\hline & WASH-WATER & AZ-102 & $6 / 1 / 02$ & $6 / 2 / 02$ & 213,000 & 0 & Third Wash $2 \mathrm{AZ}$ \\
\hline & AZ-102 & AN-105 & $7 / 21 / 02$ & $7 / 22 / 02$ & 211,800 & 0 & Third Decant 2AZ \\
\hline & WASH-WATER & $\mathrm{AZ}-102$ & $7 / 23 / 02$ & $7 / 24 / 02$ & 213,000 & 0 & Fourth Wash $2 \mathrm{AZ}$ \\
\hline & AZ-102 & AN-105 & $9 / 11 / 02$ & $9 / 13 / 02$ & 212,500 & 0 & Fourth D \\
\hline & WASH-WATER & $A Z-102$ & $9 / 14 / 02$ & $9 / 14 / 02$ & 110,000 & 0 & Transfer Water Add 2AZ \\
\hline & P1HLW_FEED_TANK & HLW-CONTRACTOR & $6 / 1 / 02$ & $2 / 2 \mathrm{~L} / 03$ & 134,300 & 5,079 & $\begin{array}{l}\text { Process Batch } 1 \text { of } 1 \mathrm{AZ} \text {, Envelope D (Batch } 1 \\
\text { Overali) }\end{array}$ \\
\hline & $A Z-101$ & P1HLW_FEED_TANK & $2 / 21 / 03$ & $2 / 21 / 03$ & 134,300 & 5,079 & Beliver Batch 2 of $1 \mathrm{AZ}$, Envelope $\mathrm{D}$ (Batch 2 \\
\hline \multirow[t]{24}{*}{$\mathrm{x}$} & AY-102 & AZ-101 & $2 / 26 / 03$ & $3 / 3 / 03$ & 819,600 & 16,032 & \begin{tabular}{|lllll} 
Transfer 2AY/6C & Slurry & to & $1 \mathrm{AZ}$ & for \\
Pretreatment
\end{tabular} \\
\hline & AZ-101 & AN-105. & $10 / 28 / 03$ & $10 / 31 / 03$ & 622,700 & 0 & Initial Decant $2 \mathrm{AY} / 6 \mathrm{C}$ Slurry from $1 \mathrm{AZ}$ \\
\hline & WASH-WATER & AZ-101 & $10 / 31 / 03$ & $11 / 2 / 03$ & 324,000 & 0 & First Wash 2AY/6C Slurry in 1AZ \\
\hline & P1HLW_FEED_TANK & HLW-CONTRACTOR & $2 / 22 / 03$ & $11 / 14 / 03$ & 134,300 & 5,079 & $\begin{array}{l}\text { Process Batch } 2 \text { of } 1 \mathrm{AZ} \text {, Envelope D (Batch } 2 \\
\text { Overall) }\end{array}$ \\
\hline & $A Z-102$ & P1HLW_FEED_TANK & $11 / 14 / 03$ & $11 / 14 / 03$ & 123,900 & 5,202 & Beliverl Batch 1 of 2AZ, Envelope D (Batch 3 \\
\hline & AZ-101 & AN-105 & $12 / 21 / 03$ & $12 / 22 / 03$ & 320,000 & 0 & First Decant 2AY/6C Slurry from 1AZ \\
\hline & WASH-WATER & & $12 / 24 / 03$ & $12 / 25 / 03$ & 324,000 & 0 & Second $\mathbf{y}$ \\
\hline & $A Z-101$ & AN-105 & $2 / 12 / 04$ & $2 / 14 / 04$ & 322,300 & 0 & Second Decant 2AY/6C Slurry from 1AZ \\
\hline & WASH-WATER & AZ-101 & $2 / 15 / 04$ & $2 / 17 / 04$ & 433,600 & 0 & Transfer Water Add $1 \mathrm{AZ}$ for $2 \mathrm{AY} / 6 \mathrm{C}$ Slurry \\
\hline & P1HLW_FEED_TANK & HLW-CONTRACTOR & $11 / 14 / 03$ & $8 / 7 / 04$ & 123,900 & 5,202 & $\begin{array}{l}\text { Brocess } \\
\text { overall) }\end{array}$ Batch 1 of $2 A Z$, Envelope D (Batch 3 \\
\hline & AZ-102 & P1HLW_FEED_TANK & $8 / 7 / 04$ & $8 / 8 / 04$ & 123,900 & 5,202 & Beliveral) Batch 2 of $2 A Z$, Envelope D (Batch 4 \\
\hline & C-104 & AY-102 & $8 / 1 / 04$ & 9/8/04 & 279,100 & 9,724 & C-104 Retrieval (Project W-???) \\
\hline & P1HLW_FEED_TANK & HLW-CONTRACTOR & $8 / 8 / 04$ & $5 / 2 / 05$ & 123,900 & 5,202 & $\begin{array}{l}\text { Brocess Batch } 2 \text { of } 2 A Z \text {, Envelope D (Batch } 4 \\
\text { Overail) }\end{array}$ \\
\hline & AZ-101 & PIHLW_FEED_TANK & $5 / 2 / 05$ & $5 / 2 / 05$ & 127,200 & 3,269 & Beliver Batch i of $2 \mathrm{AY} / 6 \mathrm{C}$ from $1 \mathrm{AZ}$, \\
\hline & $\mathrm{AY}-102$ & AZ-102 & $8 / 1 / 05$ & $8 / 3 / 05$ & 279,400 & 9,360 & Transfer $4 \mathrm{C}$ Slurry to $2 \mathrm{AZ}$ for Pretreatment \\
\hline & P1HLW_FEED_TANK & HLW-CONTRACTOR & $5 / 2 / 05$ & $11 / 16 / 05$ & 127,200 & 3,269 & $\begin{array}{l}\text { Eocess Batch } 1 \text { of } 2 \mathrm{AY} / 6 \mathrm{C} \text {, Envelope D } \\
\text { Batch } 5 \text { Bverall) }\end{array}$ \\
\hline & AZ-101 & P1HLW_FEED_TANK & $11 / 16 / 05$ & $11 / 17 / 05$ & 127,200 & 3,269 & $\begin{array}{l}\text { Deliver Batch } 2 \text { of } 2 \mathrm{AY} / 6 \mathrm{C} \text { from } 1 \mathrm{AZ} \text {, } \\
\text { Envelope } \mathrm{D} \text { (Batch } 6 \text { Overal) }\end{array}$ \\
\hline & P1HLW_FEED_TANK & HLW-CONTRACTOR & $11 / 17 / 05$ & $6 / 2 / 06$ & 127,200 & 3,269 & $\begin{array}{l}\text { Process } \\
\text { Batch } 6 \text { Bverall) }\end{array}$ \\
\hline & AZ-101 & P1HLW_FEED_TANK & $6 / 2 / 06$ & $6 / 3 / 06$ & 127,200 & 3,269 & Reliver Batch 3 of $2 \mathrm{AY}(6 \mathrm{C}$ from $\mathrm{IAZ}$, \\
\hline & $A Z-102$ & AN-105 & $6 / 23 / 06$ & $6 / 24 / 06$ & 159,500 & 0 & Initial Decant $4 C$ Slurry from $2 A Z$ \\
\hline & WASH-WATER & AZ-102 & $6 / 24 / 06$ & $6 / 26 / 06$ & 324,000 & 0 & First Wash $4 \mathrm{C}$ Slurry in $2 \mathrm{AZ}$ \\
\hline & AZ-102 & AN-105, & $8 / 14 / 06$ & $8 / 15 / 06$ & & 0 & First Decant $4 \mathrm{C}$ Slurry from $2 \mathrm{AZ}$ \\
\hline & WASH-WATER & AZ-102 & & & & 0 & Second Wash $4 \mathrm{C}$ Slurry in $2 \mathrm{AZ}$ \\
\hline & AZ-102 & AN-105 & $10 / 6 / 06$ & $10 / 8 / 06$ & 322,700 & 0 & Second Decant $4 \mathrm{C}$ Slurry from $2 \mathrm{AZ}$ \\
\hline
\end{tabular}


HNF-2321

Revision 0

Table A.3-3. Phase 1 High-Level Waste Feed Staging Transfers for Case 3.

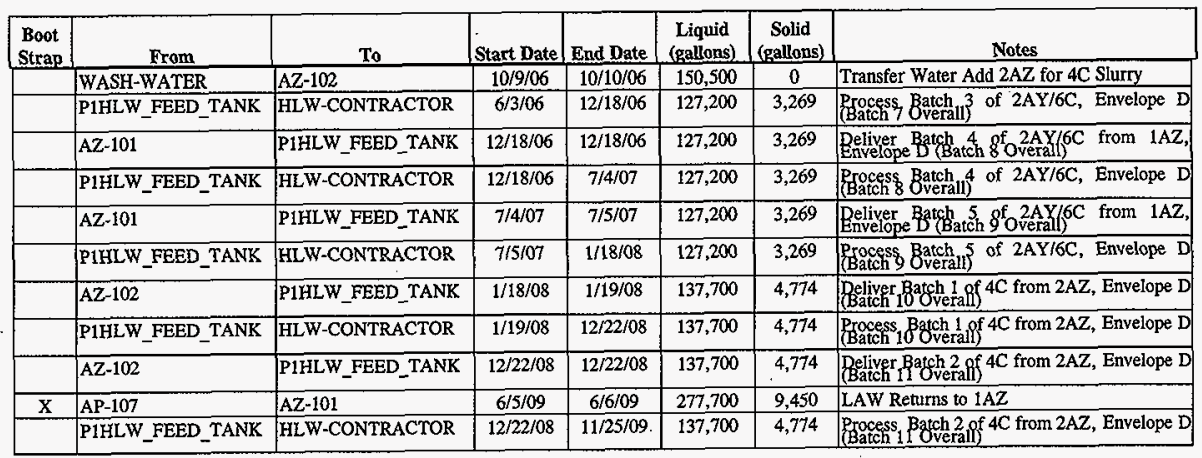


HNF-2321

Revision 0

Table A.4-1. Complete Transfer List for Case 4.

\begin{tabular}{|c|c|c|c|c|c|}
\hline From & To & Start Date & End Date & $\begin{array}{c}\text { Liquid } \\
\text { (gallons) }\end{array}$ & $\begin{array}{c}\text { Solid } \\
\text { (gallons) }\end{array}$ \\
\hline BPTCO & AP-106 & $10 / 1 / 96$ & $10 / 1 / 96$ & 11,000 & 0 \\
\hline $34 \mathrm{LB7}$ & AP-106 & $10 / 1 / 96$ & $10 / 1 / 96$ & 10,000 & 0 \\
\hline EVAPF & AP-106 & $10 / 1 / 96$ & $10 / 1 / 96$ & 10,000 & 0 \\
\hline TAL88 & AP-106 & $10 / 1 / 96$ & $10 / 1 / 96$ & 4,000 & 0 \\
\hline WNE88 & AP-106 & $10 / 1 / 96$ & $10 / 1 / 96$ & 1,000 & 0 \\
\hline \begin{tabular}{|l|} 
TNS88 \\
\end{tabular} & AP-106 & $10 / 1 / 96$ & $10 / 1 / 96$ & 750 & 250 \\
\hline ZNL87 & SY-102 & $10 / 1 / 96$ & $10 / 1 / 96$ & 2,000 & 0 \\
\hline 1NS96 & AW-105 & $10 / 1 / 96$ & $10 / 1 / 96$ & 750 & 250 \\
\hline WATER & SY-102 & $10 / 1 / 96$ & $10 / 1 / 96$ & 5,000 & 0 \\
\hline SPN87 & AP-106 & $10 / 1 / 96$ & $10 / 1 / 96$ & 7,000 & 0 \\
\hline WESF & AP-106 & $10 / 1 / 96$ & $10 / 1 / 96$ & 5,000 & 0 \\
\hline 1FL96 & AW-105 & $10 / 1 / 96$ & $10 / 1 / 96$ & 13,000 & 0 \\
\hline WNW88 & SY-102 & $10 / 1 / 96$ & $10 / 1 / 96$ & 19,000 & 0 \\
\hline WATER & AP-106 & $10 / 2 / 96$ & $10 / 2 / 96$ & 12,000 & 0 \\
\hline $\begin{array}{l}\text { WATER } \\
\end{array}$ & SY-102 & $10 / 2 / 96$ & $10 / 2 / 96$ & 5,000 & 0 \\
\hline WATER & AP-106 & $10 / 2 / 96$ & $10 / 2 / 96$ & 3,000 & 0 \\
\hline WATER & AW-105 & $10 / 2 / 96$ & $10 / 2 / 96$ & 5,000 & 0 \\
\hline WATER & AP-106 & $10 / 2 / 96$ & $10 / 2 / 96$ & 5,000 & 0 \\
\hline WATER & AP-106 & $10 / 2 / 96$ & $10 / 2 / 96$ & 5,000 & 0 \\
\hline WNW88 & SY-102 & $11 / 1 / 96$ & $11 / 1 / 96$ & 14,000 & 0 \\
\hline WATER & $S Y-102$ & $11 / 1 / 96$ & $11 / 1 / 96$ & 3,000 & 0 \\
\hline WNE88 & AP-106 & $11 / 1 / 96$ & $11 / 1 / 96$ & 1,000 & 0 \\
\hline EVAPF & AP-106 & $11 / 1 / 96$ & $11 / 1 / 96$ & 10,000 & 0 \\
\hline BPTCO & AP-106 & $11 / 1 / 96$ & $11 / 1 / 96$ & 10,000 & 0 \\
\hline BPTCO & AP-106 & $12 / 1 / 96$ & $12 / 1 / 96$ & 11,000 & 0 \\
\hline EVAPF & AP-106 & $12 / 1 / 96$ & $12 / 1 / 96$ & 10,000 & 0 \\
\hline WNW88 & $\$ Y-102$ & $12 / 1 / 96$ & $12 / 1 / 96$ & 14,000 & 0 \\
\hline WNE88 & AP-106 & $12 / 1 / 96$ & $12 / 1 / 96$ & 1,000 & 0 \\
\hline WATER & SY-102 & $12 / 1 / 96$ & $12 / 1 / 96$ & 3,000 & 0 \\
\hline WESF & AP-106 & $1 / 1 / 97$ & $1 / 1 / 97$ & 5,000 & 0 \\
\hline WNE88 & AP-106 & $1 / 1 / 97$ & $1 / 1 / 97$ & 1,000 & 0 \\
\hline BPTCO & AP-106 & $1 / 1 / 97$ & $1 / 1 / 97$ & 10,000 & 0 \\
\hline SPN87 & AP-106 & $1 / 1 / 97$ & $1 / 1 / 97$ & 6,000 & 0 \\
\hline WATER & AP-106 & $1 / 1 / 97$ & $1 / 1 / 97$ & 4,000 & 0 \\
\hline $34 \mathrm{~L} 87$ & AP-106 & $1 / 1 / 97$ & $1 / 1 / 97$ & 10,000 & 0 \\
\hline WNW88 & SY-102 & $1 / 1 / 97$ & $1 / 1 / 97$ & 14,000 & 0 \\
\hline TAL88 & AP-106 & $1 / 1 / 97$ & $1 / 1 / 97$ & 4,000 & 0 \\
\hline WATER & SY-102 & $1 / 1 / 97$ & $1 / 1 / 97$ & 4,000 & 0 \\
\hline EVAPF & AP-106 & $1 / 1 / 97$ & 1/1/97 & 10,000 & 0 \\
\hline AW-106 & AP-101 & $1 / 4 / 97$ & $1 / 4 / 97$ & 69,511 & 3 \\
\hline AN-106 & AP-108 & $1 / 11 / 97$ & $1 / 12 / 97$ & 177,000 & 7 \\
\hline WATER & SY-102 & $2 / 1 / 97$ & $2 / 1 / 97$ & 4,000 & 0 \\
\hline WNW88 & SY-102 & $2 / 1 / 97$ & $2 / 1 / 97$ & 14,000 & 0 \\
\hline EVAPF & AP-106 & $2 / 1 / 97$ & $2 / 1 / 97$ & 10,000 & 0 \\
\hline BPTCO & AP-106 & $2 / 1 / 97$ & $2 / 1 / 97$ & 10,000 & 0 \\
\hline EVAPF & AW-102 & $2 / 20 / 97$ & $2 / 20 / 97$ & 50,000 & 0 \\
\hline AP-105 & AW-102 & $2 / 20 / 97$ & $2 / 24 / 97$ & 942,000 & 39 \\
\hline WATER & $\$ Y-102$ & $3 / 1 / 97$ & $3 / 1 / 97$ & 4,000 & 0 \\
\hline WNW88 & SY-102 & $3 / 1 / 97$ & $3 / 1 / 97$ & 16,000 & 0 \\
\hline PXTCO & AP-106 & $3 / 1 / 97$ & $3 / 1 / 97$ & 5,000 & 0 \\
\hline 34L87 & AP-106 & $3 / 1 / 97$ & $3 / 1 / 97$ & 10,000 & 0 \\
\hline EVAPF & AP-106 & $3 / 1 / 97$ & $3 / 1 / 97$ & 10,000 & 0 \\
\hline WATER & AP-106 & $3 / 1 / 97$ & $3 / 1 / 97$ & 5,000 & 0 \\
\hline BPTCO & AP-106 & $3 / 1 / 97$ & $3 / 1 / 97$ & 11,000 & 0 \\
\hline AW-102 & $242-\mathrm{A}$ & $2 / 26 / 97$ & $3 / 2 / 97$ & $1,027,000$ & 42 \\
\hline $242-\mathrm{A}$ & AW-106 & $2 / 26 / 97$ & $3 / 2 / 97$ & 622,000 & 42 \\
\hline
\end{tabular}


HNF-2321

Revision 0

Table A.4-1. Complete Transfer List for Case 4.

\begin{tabular}{|c|c|c|c|c|c|}
\hline From & To & Start Date & End Date & $\begin{array}{c}\text { Liquid } \\
\text { (gallons) }\end{array}$ & $\begin{array}{c}\text { Solid } \\
\text { (gallons) }\end{array}$ \\
\hline $242-\mathrm{A}$ & LERF & $2 / 26 / 97$ & $3 / 2 / 97$ & 405,000 & 0 \\
\hline EVAPF & AW-102 & $3 / 3 / 97$ & $3 / 3 / 97$ & 35,000 & 0 \\
\hline WATER & SY-102 & $4 / 1 / 97$ & $4 / 1 / 97$ & 6,000 & 0 \\
\hline WESF & AP-106 & $4 / 1 / 97$ & $4 / 1 / 97$ & 5,000 & 0 \\
\hline TAL88 & AP-106 & $4 / 1 / 97$ & 4/1/97 & 4,000 & 0 \\
\hline EVAPF & AP-106 & $4 / 1 / 97$ & $4 / 1 / 97$ & 10,000 & 0 \\
\hline WNE88 & AP-106 & $4 / 1 / 97$ & $4 / 1 / 97$ & 1,000 & 0 \\
\hline BPTCO & AP-106 & $4 / 1 / 97$ & $4 / 1 / 97$ & 10,000 & 0 \\
\hline SPN87 & AP-106 & 4/1/97 & $4 / 1 / 97$ & 6,000 & 0 \\
\hline WNW88 & SY-102 & 4/1/97 & $4 / 1 / 97$ & 22,000 & 0 \\
\hline AW-106 & AP-105 & $4 / 25 / 97$ & $4 / 28 / 97$ & 617,000 & 29 \\
\hline AY-101 & AP-108 & $4 / 25 / 97$ & $4 / 29 / 97$ & 809,000 & 30 \\
\hline $\mathrm{BPTCO}$ & AP-106 & $5 / 1 / 97$ & $5 / 1 / 97$ & 11,000 & 0 \\
\hline WATER & AP-106 & $5 / 1 / 97$ & $5 / 1 / 97$ & 4,000 & 0 \\
\hline WNE88 & AP-106 & $5 / 1 / 97$ & $5 / 1 / 97$ & 1,000 & 0 \\
\hline WATER & SY-102 & $5 / 1 / 97$ & $5 / 1 / 97$ & 5,000 & 0 \\
\hline 34L87 & AP-106 & $5 / 1 / 97$ & $5 / 1 / 97$ & 10,000 & 0 \\
\hline EVAPF & AP-106 & $5 / 1 / 97$ & $5 / 1 / 97$ & 10,000 & 0 \\
\hline WNW88 & SY-102 & $5 / 1 / 97$ & $5 / 1 / 97$ & 19,000 & 0 \\
\hline BPTCO & AP-106 & $6 / 1 / 97$ & $6 / 1 / 97$ & 10,000 & 0 \\
\hline WATER & SY-102 & $6 / 1 / 97$ & $6 / 1 / 97$ & 4,000 & 0 \\
\hline WNW88 & SY-102 & $6 / 1 / 97$ & $6 / 1 / 97$ & 16,000 & 0 \\
\hline WNE88 & AP-106 & $6 / 1 / 97$ & $6 / 1 / 97$ & 1,000 & 0 \\
\hline EVAPF & AP-106 & $6 / 1 / 97$ & $6 / 1 / 97$ & 10,000 & 0 \\
\hline WATER & SY-102 & $7 / 1 / 97$ & $7 / 1 / 97$ & 6,000 & 0 \\
\hline $34 L 87$ & AP-106 & $7 / 1 / 97$ & $7 / 1 / 97$ & 10,000 & 0 \\
\hline WNW88 & SY-102 & $7 / 1 / 97$ & $7 / 1 / 97$ & 24,000 & 0 \\
\hline WATER & AN-101 & $7 / 1 / 97$ & $7 / 1 / 97$ & 5,000 & 0 \\
\hline WNE88 & AP-106 & $7 / 1 / 97$ & $7 / 1 / 97$ & 1,000 & 0 \\
\hline TAL88 & AP-106 & $7 / 1 / 97$ & $7 / 1 / 97$ & 4,000 & 0 \\
\hline BPTCO & AP-106 & $7 / 1 / 97$ & $7 / 1 / 97$ & 11,000 & 0 \\
\hline EVAPF & AP-106 & $7 / 1 / 97$ & $7 / 1 / 97$ & 10,000 & 0 \\
\hline WESF & AP-106 & $7 / 1 / 97$ & $7 / 1 / 97$ & 5,000 & 0 \\
\hline WCE 88 & AN-101 & $7 / 1 / 97$ & $7 / 1 / 97$ & 20,000 & 0 \\
\hline WATER & AP-106 & $7 / 1 / 97$ & $7 / 1 / 97$ & 4,000 & 0 \\
\hline SPN87 & AP-106 & $7 / 1 / 97$ & $7 / 1 / 97$ & 6,000 & 0 \\
\hline AN-106 & AP-108 & $7 / 25 / 97$ & $7 / 26 / 97$ & 194,000 & 7 \\
\hline AP-108 & AW-102 & $7 / 25 / 97$ & $7 / 31 / 97$ & $1,198,000$ & 42 \\
\hline WNE88 & A.P-106 & $8 / 1 / 97$ & $8 / 1 / 97$ & 16,000 & 0 \\
\hline WATER & AP-106 & $8 / 1 / 97$ & $8 / 1 / 97$ & 4,000 & 0 \\
\hline EVAPF & AP-106 & $8 / 1 / 97$ & $8 / 1 / 97$ & 10,000 & 0 \\
\hline BPTCO & AP-106 & $8 / 1 / 97$ & $8 / 1 / 97$ & 10,000 & 0 \\
\hline WATER & SY-102 & $8 / 1 / 97$ & $8 / 1 / 97$ & 13,000 & 0 \\
\hline WCE88 & AN-101 & $8 / 1 / 97$ & $8 / 1 / 97$ & 25,000 & 0 \\
\hline WATER & AN-101 & $8 / 1 / 97$ & $8 / 1 / 97$ & 6,000 & 0 \\
\hline $\begin{array}{l}\text { WNW88 } \\
\end{array}$ & SY-102 & $8 / 1 / 97$ & $8 / 1 / 97$ & 51,000 & 0 \\
\hline AW-102 & 242-A & $7 / 28 / 97$ & $8 / 2 / 97$ & $1,198,000$ & 39 \\
\hline $242-\mathrm{A}$ & AW-106 & $7 / 28 / 97$ & $8 / 2 / 97$ & 346,700 & 39 \\
\hline $242-\mathrm{A}$ & LERF & $7 / 28 / 97$ & $8 / 2 / 97$ & 851,300 & 0 \\
\hline EVAPF & AW-102 & $8 / 3 / 97$ & $8 / 3 / 97$ & 25,000 & 0 \\
\hline WATER & AP-107 & $8 / 20 / 97$ & $8 / 20 / 97$ & 35,000 & 0 \\
\hline AW-106 & AN-106 & $8 / 25 / 97$ & $8 / 26 / 97$ & 347,000 & 16 \\
\hline SY-102 & AP-107 & $8 / 25 / 97$ & $8 / 27 / 97$ & 520,000 & 20 \\
\hline WATER & SY-102 & $9 / 1 / 97$ & $9 / 1 / 97$ & 15,000 & 0 \\
\hline WATER & AN-101 & $9 / 1 / 97$ & $9 / 1 / 97$ & 10,000 & 0 \\
\hline WATER & AP-106 & $9 / 1 / 97$ & $9 / 1 / 97$ & 12,000 & 0 \\
\hline
\end{tabular}


HNF-2321

Revision 0

Table A.4-1. Complete Transfer List for Case 4.

\begin{tabular}{|c|c|c|c|c|c|}
\hline From & To & Start Date & End Date & $\begin{array}{c}\text { Liquid } \\
\text { (gallons) }\end{array}$ & $\begin{array}{c}\text { Solid } \\
\text { (gallons) }\end{array}$ \\
\hline EVAPF & AP-106 & $9 / 1 / 97$ & $9 / 1 / 97$ & 10,000 & 0 \\
\hline BPTCO & AP-106 & $9 / 1 / 97$ & $9 / 1 / 97$ & 10,000 & 0 \\
\hline WNW88 & $S Y-102$ & $9 / 1 / 97$ & $9 / 1 / 97$ & 61,000 & 0 \\
\hline WCE88 & AN-101 & $9 / 1 / 97$ & $9 / 1 / 97$ & 40,000 & 0 \\
\hline WNE88 & AP-106 & $9 / 1 / 97$ & 9/1/97 & 46,000 & 0 \\
\hline EVAPF & AP-106 & $10 / 1 / 97$ & $10 / 1 / 97$ & 10,000 & 0 \\
\hline WATER & AP-106 & $10 / 1 / 97$ & $10 / 1 / 97$ & 12,000 & 0 \\
\hline $34 \mathrm{~L} 87$ & AP-106 & $10 / 1 / 97$ & $10 / 1 / 97$ & 7,000 & 0 \\
\hline WATER & AN-101 & $10 / 1 / 97$ & $10 / 1 / 97$ & 10,000 & 0 \\
\hline WESF & AP-106 & $10 / 1 / 97$ & $10 / 1 / 97$ & 5,000 & 0 \\
\hline BPTCO & AP-106 & $10 / 1 / 97$ & $10 / 1 / 97$ & 11,000 & 0 \\
\hline WNE88 & AP-106 & $10 / 1 / 97$ & $10 / 1 / 97$ & 46,000 & 0 \\
\hline TAL 88 & AP-106 & $10 / 1 / 97$ & $10 / 1 / 97$ & 4,000 & 0 \\
\hline ZNL87 & SY-102 & $10 / 1 / 97$ & $10 / 1 / 97$ & 4,000 & 0 \\
\hline PXTCO & AP-106 & $10 / 1 / 97$ & $10 / 1 / 97$ & 5,000 & 0 \\
\hline TNS88 & AP-106 & $10 / 1 / 97$ & $10 / 1 / 97$ & 750 & 250 \\
\hline SPN87 & $S Y-102$ & $10 / 1 / 97$ & $10 / 1 / 97$ & 7,000 & 0 \\
\hline 34187 & AP-106 & $10 / 1 / 97$ & $10 / 1 / 97$ & 10,000 & 0 \\
\hline WCE88 & $A N-101$ & $10 / 1 / 97$ & $10 / 1 / 97$ & 40,000 & 0 \\
\hline WATER & SY-102 & $10 / 1 / 97$ & $10 / 1 / 97$ & 28,000 & 0 \\
\hline WNW88 & SY-102 & $10 / 1 / 97$ & $10 / 1 / 97$ & 111,000 & 0 \\
\hline WATER & SY-102 & $10 / 2 / 97$ & $10 / 2 / 97$ & 5,000 & 0 \\
\hline WATER & SY-102 & $10 / 2 / 97$ & $10 / 2 / 97$ & 1,000 & 0 \\
\hline WATER & AP-106 & $10 / 2 / 97$ & $10 / 2 / 97$ & 5,000 & 0 \\
\hline WASH-CAUSTIC & AN-107 & $10 / 2 / 97$ & $10 / 2 / 97$ & 66,000 & 0 \\
\hline WATER & AP-106 & $10 / 2 / 97$ & $10 / 2 / 97$ & 5,000 & 0 \\
\hline WATER & AP-106 & $10 / 2 / 97$ & $10 / 2 / 97$ & 3,000 & 0 \\
\hline WATER & AP-106 & $10 / 2 / 97$ & $10 / 2 / 97$ & 3,000 & 0 \\
\hline AY -102 & AP-106 & $10 / 25 / 97$ & $10 / 25 / 97$ & 35,999 & 1 \\
\hline WATER & AY-102 & $10 / 26 / 97$ & $10 / 27 / 97$ & 176,000 & 0 \\
\hline EVAPF & AP-106 & $11 / 1 / 97$ & $11 / 1 / 97$ & 10,000 & 0 \\
\hline WATER & AP-106 & $11 / 1 / 97$ & $11 / 1 / 97$ & 12,000 & 0 \\
\hline WATER & AN-101 & $11 / 1 / 97$ & $11 / 1 / 97$ & 10,000 & 0 \\
\hline \begin{tabular}{|l|} 
WNE88 \\
\end{tabular} & AP-106 & $11 / 1 / 97$ & $11 / 1 / 97$ & 46,000 & 0 \\
\hline WCE 88 & AN-101 & $11 / 1 / 97$ & $11 / 1 / 97$ & 40,000 & 0 \\
\hline WATER & SY-102 & $11 / 1 / 97$ & $11 / 1 / 97$ & 30,000 & 0 \\
\hline WNW88 & SY-102 & $11 / 1 / 97$ & $11 / 1 / 97$ & 119,000 & 0 \\
\hline WATER & AN-101 & $12 / 1 / 97$ & $12 / 1 / 97$ & 10,000 & 0 \\
\hline WCE88 & $A N-101$ & $12 / 1 / 97$ & $12 / 1 / 97$ & 40,000 & 0 \\
\hline WATER & SY-102 & $12 / 1 / 97$ & $12 / 1 / 97$ & 33,000 & 0 \\
\hline WATER & AP-106 & $12 / 1 / 97$ & $12 / 1 / 97$ & 11,000 & 0 \\
\hline EVAPF & AP-106 & $12 / 1 / 97$ & $12 / 1 / 97$ & 10,000 & 0 \\
\hline WNE88 & AP-106 & $12 / 1 / 97$ & $12 / 1 / 97$ & 42,000 & 0 \\
\hline WNW88 & SY-102 & $12 / 1 / 97$ & $12 / 1 / 97$ & 131,000 & 0 \\
\hline WATER & AP-107 & $12 / 20 / 97$ & $12 / 20 / 97$ & 35,000 & 0 \\
\hline SY-102 & AP-107 & $12 / 26 / 97$ & $12 / 28 / 97$ & 520,000 & 21 \\
\hline AP-106 & AP-104 & $12 / 25 / 97$ & $12 / 30 / 97$ & 996,600 & 35 \\
\hline SPN87 & SY-102 & 1/L/98 & $1 / 1 / 98$ & 6,000 & 0 \\
\hline BPTCO & AP-106 & $1 / 1 / 98$ & $1 / 1 / 98$ & 11,000 & 0 \\
\hline WATER & $A N-101$ & $1 / 1 / 98$ & $1 / 1 / 98$ & 10,000 & 0 \\
\hline WCE88 & AN-101 & $1 / 1 / 98$ & $1 / 1 / 98$ & 40,000 & 0 \\
\hline WATER & SY-102 & $1 / 1 / 98$ & $1 / 1 / 98$ & 37,000 & 0 \\
\hline WATER & AP-106 & $1 / 1 / 98$ & $1 / 1 / 98$ & 10,000 & 0 \\
\hline TAL88 & AP-106 & $1 / 1 / 98$ & $1 / 1 / 98$ & 4,000 & 0 \\
\hline WATER & AP-106 & $1 / 1 / 98$ & $1 / 1 / 98$ & 4,000 & 0 \\
\hline $34 L 87$ & AP-106 & $1 / 1 / 98$ & $1 / 1 / 98$ & 10,000 & 0 \\
\hline
\end{tabular}


HNF-2321

Revision 0

Table A.4-1. Complete Transfer List for Case 4.

\begin{tabular}{|c|c|c|c|c|c|}
\hline From & To & Start Date & End Date & $\begin{array}{c}\text { Liquid } \\
\text { (gallons) }\end{array}$ & $\begin{array}{c}\text { Solid } \\
\text { (gallons) }\end{array}$ \\
\hline EVAPF & AP-105 & $1 / 1 / 98$ & $1 / 1 / 98$ & 10,000 & 0 \\
\hline WNE88 & AP-106 & $1 / 1 / 98$ & $1 / 1 / 98$ & 38,000 & 0 \\
\hline WNW88 & SY-102 & $1 / 1 / 98$ & $1 / 1 / 98$ & 146,000 & 0 \\
\hline EVAPF & AP-106 & $2 / 1 / 98$ & $2 / 1 / 98$ & 10,000 & 0 \\
\hline WATER & AP-106 & $2 / 1 / 98$ & $2 / 1 / 98$ & 8,000 & 0 \\
\hline WNE88 & AP-106 & $2 / 1 / 98$ & $2 / 1 / 98$ & 33,000 & 0 \\
\hline WATER & SY-102 & $2 / 1 / 98$ & $2 / 1 / 98$ & 35,000 & 0 \\
\hline WCE88 & AN-101 & $2 / 1 / 98$ & $2 / 1 / 98$ & 40,000 & 0 \\
\hline WATER & AN-101 & $2 / 1 / 98$ & $2 / 1 / 98$ & 10,000 & 0 \\
\hline \begin{tabular}{|l} 
WNW88 \\
\end{tabular} & SY-102 & $2 / 1 / 98$ & $2 / 1 / 98$ & 140,000 & 0 \\
\hline $34 \mathrm{~L} 87$ & AP-106 & $3 / 1 / 98$ & $3 / 1 / 98$ & 10,000 & 0 \\
\hline EVAPF & AP-106 & $3 / 1 / 98$ & $3 / 1 / 98$ & 10,000 & 0 \\
\hline WATER & AP-106 & $3 / 1 / 98$ & $3 / 1 / 98$ & 5,000 & 0 \\
\hline WATER & AP-106 & $3 / 1 / 98$ & $3 / 1 / 98$ & 7,000 & 0 \\
\hline BPTCO & AP-106 & $3 / 1 / 98$ & $3 / 1 / 98$ & 11,000 & 0 \\
\hline WATER & SY-102 & $3 / 1 / 98$ & $3 / 1 / 98$ & 31,000 & 0 \\
\hline WNE88 & AP-106 & $3 / 1 / 98$ & $3 / 1 / 98$ & 26,000 & 0 \\
\hline WCE88 & AN-101 & $3 / 1 / 98$ & $3 / 1 / 98$ & 40,000 & 0 \\
\hline WATER & AN-101 & $3 / 1 / 98$ & $3 / 1 / 98$ & 10,000 & 0 \\
\hline WNW88 & SY-102 & $3 / 1 / 98$ & $3 / 1 / 98$ & 124,000 & 0 \\
\hline WATER & AP-104 & $3 / 20 / 98$ & $3 / 20 / 98$ & 35,000 & 0 \\
\hline SY-102 & AP-104 & $3 / 25 / 98$ & $3 / 27 / 98$ & 520,000 & 21 \\
\hline AP-104 & AW-102 & $3 / 25 / 98$ & $3 / 30 / 98$ & 998,000 & 33 \\
\hline EVAPF & $A P-106$ & $4 / 1 / 98$ & $4 / 1 / 98$ & 10,000 & 0 \\
\hline WATER & $\mathrm{AN}-101$ & $4 / 1 / 98$ & $4 / 1 / 98$ & 25,000 & 0 \\
\hline WNE88 & AP-106 & $4 / 1 / 98$ & $4 / 1 / 98$ & 20,000 & 0 \\
\hline \begin{tabular}{|l} 
WATER \\
\end{tabular} & $\mathrm{AP}-106$ & $4 / 1 / 98$ & $4 / 1 / 98$ & 5,000 & 0 \\
\hline TAL88 & AP-106 & $4 / 1 / 98$ & $4 / 1 / 98$ & 4,000 & 0 \\
\hline SPN87 & SY-102 & $4 / 1 / 98$ & $4 / 1 / 98$ & 6,000 & 0 \\
\hline WATER & SY-102 & $4 / 1 / 98$ & $4 / 1 / 98$ & 36,000 & 0 \\
\hline WCE88 & AN-101 & $4 / 1 / 98$ & $4 / 1 / 98$ & 101,000 & 0 \\
\hline AW-102 & $242-\mathrm{A}$ & $3 / 28 / 98$ & $4 / 1 / 98$ & $1,058,000$ & 31 \\
\hline $242-\mathrm{A}$ & AW-106 & $3 / 28 / 98$ & $4 / 1 / 98$ & 180,100 & 31 \\
\hline 242-A & LERF & $3 / 28 / 98$ & $4 / 1 / 98$ & 877,800 & 0 \\
\hline WNW88 & $S Y-102$ & $4 / 1 / 98$ & $4 / 2 / 98$ & 146,000 & 0 \\
\hline $34 \mathrm{~L} 87$ & AP-106 & $5 / 1 / 98$ & $5 / 1 / 98$ & 10,000 & 0 \\
\hline WATER & AP-106 & $5 / 1 / 98$ & $5 / 1 / 98$ & 5,000 & 0 \\
\hline WNE88 & AP-106 & $5 / 1 / 98$ & 5/1/98 & 20,000 & 0 \\
\hline BPTCO & AP-106 & $5 / 1 / 98$ & $5 / 1 / 98$ & 11,000 & 0 \\
\hline WATER & AN-101 & $5 / 1 / 98$ & $5 / 1 / 98$ & 24,000 & 0 \\
\hline WCW88 & SY-102 & $5 / 1 / 98$ & $5 / 1 / 98$ & 19,000 & 0 \\
\hline WATER & SY-102 & $5 / 1 / 98$ & $5 / 1 / 98$ & 5,000 & 0 \\
\hline EVAPF & AP-106 & $5 / 1 / 98$ & $5 / 1 / 98$ & 10,000 & 0 \\
\hline WCE88 & AN-101 & $5 / 1 / 98$ & $5 / 1 / 98$ & 95,000 & 0 \\
\hline WATER & SY-102 & $5 / 1 / 98$ & $5 / 1 / 98$ & 37,000 & 0 \\
\hline WATER & AP-106 & $5 / 1 / 98$ & $5 / 1 / 98$ & 4,000 & 0 \\
\hline WNW88 & $S Y-102$ & $5 / 1 / 98$ & $5 / 2 / 98$ & 147,000 & 0 \\
\hline AP-107 & AW-102 & $5 / 25 / 98$ & $5 / 30 / 98$ & $1,104,000$ & 40 \\
\hline WNE88 & AP-106 & $6 / 1 / 98$ & $6 / 1 / 98$ & 16,000 & 0 \\
\hline EVAPF & A.P-106 & $6 / 1 / 98$ & $6 / 1 / 98$ & 10,000 & 0 \\
\hline WATER & AN-10I & $6 / 1 / 98$ & $6 / 1 / 98$ & 7,000 & 0 \\
\hline WCE 88 & AN-101 & $6 / 1 / 98$ & $6 / 1 / 98$ & 28,000 & 0 \\
\hline WATER & AP-106 & $6 / 1 / 98$ & $6 / 1 / 98$ & 4,000 & 0 \\
\hline WATER & SY-102 & $6 / 1 / 98$ & $6 / 1 / 98$ & 5,000 & 0 \\
\hline WCW88 & SY-102 & $6 / 1 / 98$ & $6 / 1 / 98$ & 19,000 & 0 \\
\hline WATER & SY-102 & $6 / 1 / 98$ & $6 / 1 / 98$ & $.36,000$ & 0 \\
\hline
\end{tabular}


HNF-2321

Revision 0

Table A.4-1. Complete Transfer List for Case 4.

\begin{tabular}{|c|c|c|c|c|c|}
\hline From & To & Start Date & End Date & $\begin{array}{c}\text { Liquid } \\
\text { (gallons) }\end{array}$ & $\begin{array}{c}\text { Solid } \\
\text { (gallons) }\end{array}$ \\
\hline$A W-102$ & $242-A$ & $5 / 28 / 98$ & $6 / 1 / 98$ & $1,104,000$ & 39 \\
\hline $242-\mathrm{A}$ & AW-106 & $5 / 28 / 98$ & $6 / 1 / 98$ & 448,700 & 39 \\
\hline $242-\mathrm{A}$ & LERF & $5 / 28 / 98$ & $6 / 1 / 98$ & 655,200 & 0 \\
\hline WNW88 & SY-102 & $6 / 1 / 98$ & $6 / 2 / 98$ & 142,000 & 0 \\
\hline EVAPF & AW-102 & $6 / 3 / 98$ & $6 / 3 / 98^{\circ}$ & 35,000 & 0 \\
\hline WATER & AP-104 & $6 / 20 / 98$ & $6 / 20 / 98$ & 35,000 & 0 \\
\hline AW-106 & AP-105 & $6 / 25 / 98$ & $6 / 26 / 98$ & 341,000 & 17 \\
\hline SY-102 & AP-104 & $6 / 25 / 98$ & $6 / 27 / 98$ & 520,000 & 21 \\
\hline WATER & SY-102 & $7 / 1 / 98$ & $7 / 1 / 98$ & 3,000 & 0 \\
\hline WCE88 & AN-101 & $7 / 1 / 98$ & $7 / 1 / 98$ & 28,000 & 0 \\
\hline WATER & SY-102 & $7 / 1 / 98$ & $7 / 1 / 98$ & 32,000 & 0 \\
\hline WATER & AN-101 & $7 / 1 / 98$ & $7 / 1 / 98$ & 7,000 & 0 \\
\hline SPN87 & $S Y-102$ & $7 / 1 / 98$ & $7 / 1 / 98$ & 6,000 & 0 \\
\hline WCW88 & SY-102 & $7 / 1 / 98$ & $7 / 1 / 98$ & 13,000 & 0 \\
\hline WNE88 & AP-106 & $7 / 1 / 98$ & $7 / 1 / 98$ & 13,000 & 0 \\
\hline EVAPF & AP-106 & $7 / 1 / 98$ & $7 / 1 / 98$ & 10,000 & 0 \\
\hline $34 \mathrm{~L} 87$ & AP-106 & $7 / 1 / 98$ & $7 / 1 / 98$ & 10,000 & 0 \\
\hline TAL88 & AP-106 & $7 / 1 / 98$ & 7/1/98 & 4,000 & 0 \\
\hline WATER & AP-106 & $7 / 1 / 98$ & $7 / 1 / 98$ & 4,000 & 0 \\
\hline WATER & AP-106 & $7 / 1 / 98$ & $7 / 1 / 98$ & 3,000 & 0 \\
\hline WNW88 & SY-102 & $7 / 1 / 98$ & $7 / 2 / 98$ & 127,000 & 0 \\
\hline$A Y-102$ & C-106 & $7 / 1 / 98$ & $7 / 5 / 98$ & 920,700 & 31 \\
\hline BPTCO & AP-106 & $7 / 11 / 98$ & $7 / 11 / 98$ & 11,000 & 0 \\
\hline WNE88 & AP-106 & $8 / 1 / 98$ & $8 / 1 / 98$ & 7,000 & 0 \\
\hline EVAPF & AP-106 & $8 / 1 / 98$ & $8 / 1 / 98$ & 10,000 & 0 \\
\hline \begin{tabular}{|l} 
WATER \\
\end{tabular} & AP-106 & $8 / 1 / 98$ & $8 / 1 / 98$ & 2,000 & 0 \\
\hline \begin{tabular}{|l} 
WCW8 \\
\end{tabular} & SY-102 & $8 / 1 / 98$ & $8 / 1 / 98$ & 13,000 & 0 \\
\hline WATER & SY-102 & $8 / 1 / 98$ & $8 / 1 / 98$ & 30,000 & 0 \\
\hline WATER & AN-101 & $8 / 1 / 98$ & $8 / 1 / 98$ & 7,000 & 0 \\
\hline WCE88 & AN-101 & $8 / 1 / 98$ & $8 / 1 / 98$ & 28,000 & 0 \\
\hline WATER & SY-102 & $8 / 1 / 98$ & $8 / 1 / 98$ & 3,000 & 0 \\
\hline WNW88 & SY-102 & $8 / 1 / 98$ & $8 / 1 / 98$ & 121,000 & 0 \\
\hline WNE 88 & AP-106 & 9/1/98 & $9 / 1 / 98$ & 7,000 & 0 \\
\hline WATER & AN-101 & 9/1/98 & $9 / 1 / 98$ & 7,000 & 0 \\
\hline EVAPF & AP-106 & 9/1/98 & $9 / 1 / 98$ & 10,000 & 0 \\
\hline WCE88 & AN-IOI & 9/1/98 & $9 / 1 / 98$ & 28,000 & 0 \\
\hline WATER & AP-106 & 9/1/98 & $9 / 1 / 98$ & 2,000 & 0 \\
\hline WATER & SY-102 & $9 / 1 / 98$ & $9 / 1 / 98$ & 2,000 & 0 \\
\hline WCW88 & SY-102 & $9 / 1 / 98$ & $9 / 1 / 98$ & 8,000 & 0 \\
\hline WATER & SY-102 & $9 / 1 / 98$ & $9 / 1 / 98$ & 34,000 & 0 \\
\hline WNW88 & SY-102 & $9 / 1 / 98$ & $9 / 2 / 98$ & 137,000 & 0 \\
\hline WATER & AP-107 & $9 / 20 / 98$ & 9/20/98 & 35,000 & 0 \\
\hline SY-102 & AP-107 & $9 / 25 / 98$ & $9 / 27 / 98$ & 520,000 & 21 \\
\hline AP-101 & AW-103 & $9 / 25 / 98$ & $9 / 28 / 98$ & 625,000 & 27 \\
\hline \begin{tabular}{|l|} 
TNS88 \\
\end{tabular} & AP-106 & $10 / 1 / 98$ & $10 / 1 / 98$ & 750 & 250 \\
\hline WNE88 & AP-106 & $10 / 1 / 98$ & $10 / 1 / 98$ & 3,000 & 0 \\
\hline TAL88 & AP-106 & $10 / 1 / 98$ & $10 / 1 / 98$ & 4,000 & 0 \\
\hline $34 \mathrm{~L} 87$ & AP-106 & $10 / 1 / 98$ & $10 / 1 / 98$ & 10,000 & 0 \\
\hline WATER & AN-101 & $10 / 1 / 98$ & $10 / 1 / 98$ & 7,000 & 0 \\
\hline WESF & AP-106 & $10 / 1 / 98$ & $10 / 1 / 98$ & 5,000 & 0 \\
\hline PXTCO & AP-106 & $10 / 1 / 98$ & $10 / 1 / 98$ & 5,000 & 0 \\
\hline WCE88 & AN-101 & $10 / 1 / 98$ & $10 / 1 / 98$ & 28,000 & 0 \\
\hline WATER & $A P-106$ & $10 / 1 / 98$ & $10 / 1 / 98$ & 1,000 & 0 \\
\hline EVAPF & $A P-106$ & $10 / 1 / 98$ & $10 / 1 / 98$ & 10,000 & 0 \\
\hline WCW88 & SY-102 & $10 / 1 / 98$ & $10 / 1 / 98$ & 8,000 & 0 \\
\hline WATER & SY-102 & $10 / 1 / 98$ & $10 / 1 / 98$ & 30,000 & 0 \\
\hline
\end{tabular}


HNF-2321

Revision 0

Table A.4-1. Complete Transfer List for Case 4.

\begin{tabular}{|c|c|c|c|c|c|}
\hline From & To & Start Date & End Date & $\begin{array}{c}\text { Liquid } \\
\text { (gallons) }\end{array}$ & $\begin{array}{c}\text { Solid } \\
\text { (gallons) }\end{array}$ \\
\hline WATER & SX-102 & $10 / 1 / 98$ & $10 / 1 / 98$ & 2,000 & 0 \\
\hline $\begin{array}{l}\text { SPN87 } \\
\end{array}$ & SY-102 & $10 / 1 / 98$ & $10 / 1 / 98$ & 7,000 & 0 \\
\hline ZNL87 & $S Y-102$ & $10 / 1 / 98$ & $10 / 1 / 98$ & 4,000 & 0 \\
\hline WNW88 & SY-102 & $10 / 1 / 98$ & $10 / 1 / 98$ & 121,000 & 0 \\
\hline WATER & SY-102 & $10 / 2 / 98$ & $10 / 2 / 98$ & 5,000 & 0 \\
\hline WATER & AP- 106 & $10 / 2 / 98$ & $10 / 2 / 98$ & 5,000 & 0 \\
\hline \begin{tabular}{|l} 
WATER \\
\end{tabular} & AP-106 & $10 / 2 / 98$ & $10 / 2 / 98$ & 3,000 & 0 \\
\hline EVAPF & AW-102 & $10 / 4 / 98$ & $10 / 4 / 98$ & 50,000 & 0 \\
\hline AW-102 & AW-106 & $10 / 9 / 98$ & $10 / 9 / 98$ & 150,000 & 6 \\
\hline AP-104 & AW-102 & $10 / 5 / 98$ & $10 / 11 / 98$ & $1,107,000$ & 43 \\
\hline AW-104 & AP-107 & $10 / 9 / 98$ & $10 / 11 / 98$ & 540,000 & 18 \\
\hline AW-104 & AP-104 & $10 / 18 / 98$ & $10 / 18 / 98$ & 164,000 & 6 \\
\hline AP-10I & AW-104 & $10 / 21 / 98$ & $10 / 23 / 98$ & 460,000 & 20 \\
\hline AW-106 & AP-101 & $10 / 29 / 98$ & $10 / 30 / 98$ & 289,000 & 13 \\
\hline EVAPF & AP- 106 & $11 / 1 / 98$ & $11 / 1 / 98$ & 10,000 & 0 \\
\hline WNE8 8 & AP-106 & $11 / 1 / 98$ & $11 / 1 / 98$ & 2,000 & 0 \\
\hline WATER & SY-102 & $11 / 1 / 98$ & $11 / 1 / 98$ & 1,000 & 0 \\
\hline WCW88 & SY-102 & $11 / 1 / 98$ & $11 / 1 / 98$ & 3,000 & 0 \\
\hline WATER & SY-102 & $11 / 1 / 98$ & $11 / 1 / 98$ & 30,000 & 0 \\
\hline WATER & AN-101 & $11 / 1 / 98$ & $11 / 1 / 98$ & 7,000 & 0 \\
\hline WCE 88 & AN-101 & $11 / 1 / 98$ & $11 / 1 / 98$ & 28,000 & 0 \\
\hline WNW88 & SY-102 & $11 / 1 / 98$ & $11 / 1 / 98$ & 120,000 & 0 \\
\hline AW-106 & AW-102 & $11 / 5 / 98$ & $11 / 5 / 98$ & 150,000 & 9 \\
\hline AW-102 & 242-A & $11 / 4 / 98$ & $11 / 9 / 98$ & $1,193,000$ & 46 \\
\hline $242-\mathrm{A}$ & AW-106 & $11 / 4 / 98$ & $11 / 9 / 98$ & 602,900 & 46 \\
\hline $242-A$ & LERF & $11 / 4 / 98$ & $11 / 9 / 98$ & 589,600 & 0 \\
\hline C-106 & AY-102 & $7 / 15 / 98$ & $11 / 10 / 98$ & 809,000 & 15,169 \\
\hline WATER & AN-101 & $12 / 1 / 98$ & $12 / 1 / 98$ & 6,000 & 0 \\
\hline WATER & SY-102 & $12 / 1 / 98$ & $12 / 1 / 98$ & 1,000 & 0 \\
\hline WNE88 & AP-106 & $12 / 1 / 98$ & $12 / 1 / 98$ & 1,000 & 0 \\
\hline EVAPF & AP-106 & $12 / 1 / 98$ & $12 / 1 / 98$ & 10,000 & 0 \\
\hline WCW88 & SY-102 & $12 / 1 / 98$ & $12 / 1 / 98$ & 3,000 & 0 \\
\hline \begin{tabular}{|l|} 
WCE88 \\
\end{tabular} & AN-101 & $12 / 1 / 98$ & $12 / 1 / 98$ & 23,000 & 0 \\
\hline WATER & SY-102 & $12 / 1 / 98$ & $12 / 1 / 98$ & 29,000 & 0 \\
\hline WNW88 & SY-102 & $12 / 1 / 98$ & $12 / 1 / 98$ & 116,000 & 0 \\
\hline WATER & AP-104 & $12 / 20 / 98$ & $12 / 20 / 98$ & 35,000 & 0 \\
\hline SY-102 & AP-104 & $12 / 25 / 98$ & $12 / 27 / 98$ & 520,000 & 21 \\
\hline AW-106 & AP-101 & $12 / 25 / 98$ & $12 / 28 / 98$ & 605,000 & 27 \\
\hline WATER & AN-101 & $1 / 1 / 99$ & $1 / 1 / 99$ & 6,000 & 0 \\
\hline SPN87 & SY-102 & $1 / 1 / 99$ & $1 / 1 / 99$ & 6,000 & 0 \\
\hline WCE 88 & AN-101 & $1 / 1 / 99$ & $1 / 1 / 99$ & 23,000 & 0 \\
\hline WCW88 & SY-102 & $1 / 1 / 99$ & $1 / 1 / 99$ & 23,000 & 0 . \\
\hline WATER & SY-102 & $1 / 1 / 99$ & $1 / 1 / 99$ & 6,000 & 0 \\
\hline TAL88 & AP-106 & $1 / 1 / 99$ & $1 / 1 / 99$ & 4,000 & 0 \\
\hline $34 \mathrm{~L} 87$ & AP-106 & $1 / 1 / 99$ & $1 / 1 / 99$ & 10,000 & 0 \\
\hline EVAPF & AP-106 & $1 / 1 / 99$ & $1 / 1 / 99$ & 10,000 & 0 \\
\hline WATER & $S Y-102$ & $1 / 1 / 99$ & $1 / 1 / 99$ & 25,000 & 0 \\
\hline $\begin{array}{l}\text { WATER } \\
\end{array}$ & AP-106 & $1 / 1 / 99$ & $1 / 1 / 99$ & 4,000 & 0 \\
\hline \begin{tabular}{|l|} 
WNW8 \\
\end{tabular} & SY-102 & $1 / 1 / 99$ & $1 / 1 / 99$ & 102,000 & 0 \\
\hline AP-106 & AP-104 & $1 / 25 / 99$ & $1 / 27 / 99$ & 385,000 & 14 \\
\hline WATER & SY-102 & $2 / 1 / 99$ & $2 / 1 / 99$ & 23,000 & 0 \\
\hline WCE88 & AN-10I & $2 / 1 / 99$ & $2 / 1 / 99$ & 23,000 & 0 \\
\hline WCW88 & SY-102 & $2 / 1 / 99$ & $2 / 1 / 99$ & 45,000 & 0 \\
\hline WATER & AN-101 & $2 / 1 / 99$ & $2 / 1 / 99$ & 6,000 & 0 \\
\hline WATER & SY-102 & $2 / 1 / 99$ & $2 / 1 / 99$ & 11,000 & 0 \\
\hline EVAPF & AP-106 & $2 / 1 / 99$ & $2 / 1 / 99$ & 10,000 & 0 \\
\hline
\end{tabular}


HNF-2321

Revision 0

Table A.4-1. Complete Transfer List for Case 4.

\begin{tabular}{|c|c|c|c|c|c|}
\hline From & To & Start Date & End Date & $\begin{array}{c}\text { Liquid } \\
\text { (gallons) }\end{array}$ & $\begin{array}{c}\text { Solid } \\
\text { (gallons) }\end{array}$ \\
\hline WNW88 & SY-102 & $2 / 1 / 99$ & $2 / 1 / 99$ & 91,000 & 0 \\
\hline WCE88 & AN-101 & $3 / 1 / 99$ & $3 / 1 / 99$ & 17,000 & 0 \\
\hline WATER & SY-102 & $3 / 1 / 99$ & $3 / 1 / 99$ & 21,000 & 0 \\
\hline WATER & AN-101 & $3 / 1 / 99$ & $3 / 1 / 99$ & 4,000 & 0 \\
\hline $34 \mathrm{~L} 87$ & AP-106 & $3 / 1 / 99$ & $3 / 1 / 99$ & 10,000 & 0 \\
\hline EVAPF & AP-106 & $3 / 1 / 99$ & $3 / 1 / 99$ & 10,000 & 0 \\
\hline WATER & SY-102 & $3 / 1 / 99$ & $3 / 1 / 99$ & 17,000 & 0 \\
\hline \begin{tabular}{|l} 
WATER \\
\end{tabular} & AP-106 & $3 / 1 / 99$ & $3 / 1 / 99$ & 5,000 & 0 \\
\hline WCW88 & SY-102 & $3 / 1 / 99$ & $3 / 1 / 99$ & 67,000 & 0 \\
\hline WNW88 & SY-102 & $3 / 1 / 99$ & $3 / 1 / 99$ & 82,000 & 0 \\
\hline AN-101 & AP-103 & $3 / 5 / 99$ & $3 / 10 / 99$ & $1,024,000$ & 0 \\
\hline AP-107 & AW-102 & $3 / 5 / 99$ & $3 / 10 / 99$ & $1,095,000$ & 40 \\
\hline$A W-102$ & $242-A$ & $3 / 8 / 99$ & $3 / 12 / 99$ & $1,095,000$ & 38 \\
\hline $242-\mathrm{A}$ & AW-106 & $3 / 8 / 99$ & $3 / 12 / 99$ & 353,000 & 38 \\
\hline $242-\mathrm{A}$ & LERF & $3 / 8 / 99$ & $3 / 12 / 99$ & 741,900 & 0 \\
\hline WATER & AP-107 & $3 / 20 / 99$ & $3 / 20 / 99$ & 35,000 & 0 \\
\hline SY-102 & AP-107 & $3 / 25 / 99$ & $3 / 27 / 99$ & 520,000 & 21 \\
\hline SPN87 & SX-102 & $4 / 1 / 99$ & $4 / 1 / 99$ & 6.000 & 0 \\
\hline WATER & SY-102 & $4 / 1 / 99$ & $4 / 1 / 99$ & 23,000 & 0 \\
\hline WATER & AP-108 & $4 / 1 / 99$ & $4 / 1 / 99$ & 4,000 & 0 \\
\hline WNW88 & SY-102 & $4 / 1 / 99$ & $4 / 1 / 99$ & 64,000 & 0 \\
\hline \begin{tabular}{|l} 
WATER \\
\end{tabular} & SY-102 & $4 / 1 / 99$ & $4 / 1 / 99$ & 16,000 & 0 \\
\hline TAL88 & AP-106 & $4 / 1 / 99$ & $4 / 1 / 99$ & 4,000 & 0 \\
\hline WCW88 & SY-102 & $4 / 1 / 99$ & $4 / 1 / 99$ & 91,000 & 0 \\
\hline EVAPF & AW-102 & $4 / 2 / 99$ & $4 / 2 / 99$ & 35,000 & 0 \\
\hline EVAPF & AP-106 & $4 / 3 / 99$ & $4 / 3 / 99$ & 10,000 & 0 \\
\hline WCE 88 & AP-108 & $4 / 3 / 99$ & $4 / 3 / 99$ & 17,000 & 0 \\
\hline AW-106 & AP-101 & $4 / 15 / 99$ & $4 / 16 / 99$ & 222,500 & 10 \\
\hline $\mathrm{AW}-106$ & AW-104 & $4 / 25 / 99$ & $4 / 25 / 99$ & 131,100 & 6 \\
\hline WATER & SY-102 & $5 / 1 / 99$ & $5 / 1 / 99$ & 15,000 & 0 \\
\hline WCE88 & AP-108 & $5 / 1 / 99$ & $5 / 1 / 99$ & 17,000 & 0 \\
\hline WATER & AP-108 & $5 / 1 / 99$ & $5 / 1 / 99$ & 4,000 & 0 \\
\hline WATER & SY-102 & $5 / 1 / 99$ & $5 / 1 / 99$ & 21,000 & 0 \\
\hline WNW88 & SY-102 & $5 / 1 / 99$ & $5 / 1 / 99$ & 60,000 & 0 \\
\hline WATER & AP-106 & $5 / 1 / 99$ & $5 / 1 / 99$ & 4,000 & 0 \\
\hline $34 \mathrm{~L} 87$ & AP-106 & $5 / 1 / 99$ & $5 / 1 / 99$ & 10,000 & 0 \\
\hline EVAPF & AP-106 & $5 / 1 / 99$ & $5 / 1 / 99$ & 10,000 & 0 \\
\hline WCW88 & $S Y-102$ & $5 / 1 / 99$ & $5 / 1 / 99$ & 84,000 & 0 \\
\hline WATER & $S Y-102$ & $6 / 1 / 99$ & $6 / 1 / 99$ & 14,000 & 0 \\
\hline WATER & SX-102 & $6 / 1 / 99$ & $6 / 1 / 99$ & 18,000 & 0 \\
\hline WNW88 & SY-102 & $6 / 1 / 99$ & $6 / 1 / 99$ & 56,000 & 0 \\
\hline WATER & AP-108 & $6 / 1 / 99$ & $6 / 1 / 99$ & 4,000 & 0 \\
\hline EVAPF & AP-106 & $6 / 1 / 99$ & $6 / 1 / 99$ & 10,000 & 0 \\
\hline WCE 88 & AP-108 & $6 / 1 / 99$ & $6 / 1 / 99$ & 17,000 & 0 \\
\hline WCW88 & SY-102 & $6 / 1 / 99$ & $6 / 1 / 99$ & 74,000 & 0 \\
\hline WATER & $\mathrm{AP}-107$ & $6 / 20 / 99$ & $6 / 20 / 99$ & 35,000 & 0 \\
\hline SY-102 & AP-107 & $6 / 25 / 99$ & $6 / 27 / 99$ & 520,000 & 22 \\
\hline WCE88 & AP-108 & $7 / 1 / 99$ & $7 / 1 / 99$ & 11,000 & 0 \\
\hline WATER & AP-108 & $7 / 1 / 99$ & $7 / 1 / 99$ & 3,000 & 0 \\
\hline WATER & AP-106 & $7 / 1 / 99$ & $7 / 1 / 99$ & 4,000 & 0 \\
\hline $34 \mathrm{~L} 87$ & AP-106 & $7 / 1 / 99$ & $7 / 1 / 99$ & 10,000 & 0 \\
\hline EVAPF & AP-105 & $7 / 1 / 99$ & $7 / 1 / 99$ & 10,000 & 0 \\
\hline SPN87 & SY-102 & $7 / 1 / 99$ & $7 / 1 / 99$ & 6,000 & 0 \\
\hline WATER & SY-102 & $7 / 1 / 99$ & $7 / 1 / 99$ & 13,000 & 0 \\
\hline TAL88 & AP-106 & $7 / 1 / 99$ & $7 / 1 / 99$ & 4,000 & 0 \\
\hline WATER & SY-102 & $7 / 1 / 99$ & $7 / 1 / 99$ & 16,000 & 0 \\
\hline
\end{tabular}


HNF-2321

Revision 0

Table A.4-1. Complete Transfer List for Case 4.

\begin{tabular}{|c|c|c|c|c|c|}
\hline From & To & Start Date & End Date & $\begin{array}{c}\text { Liquid } \\
\text { (gallons) }\end{array}$ & $\begin{array}{c}\text { Solid } \\
\text { (gallons) }\end{array}$ \\
\hline WNW88 & $S Y-102$ & $7 / 1 / 99$ & $7 / 1 / 99$ & 52,000 & 0 \\
\hline WCW88 & SY-102 & $7 / 1 / 99$ & $7 / 1 / 99$ & 65,000 & 0 \\
\hline AP-103 & AW-102 & $7 / 20 / 99$ & $7 / 25 / 99$ & $1,017,000$ & 44 \\
\hline$A W-102$ & $242-\mathrm{A}$ & $7 / 24 / 99$ & $7 / 28 / 99$ & $1,019,000$ & 41 \\
\hline 242-A. & AW-106 & $7 / 24 / 99$ & $7 / 28 / 99$ & 928,200 & 41 \\
\hline $242-\mathrm{A}$ & LERF & $7 / 24 / 99$ & $7 / 28 / 99$ & 90,768 & 0 \\
\hline WCE8 8 & AP-108 & $8 / 1 / 99$ & $8 / 1 / 99$ & 11,000 & 0 \\
\hline WATER & SY-102 & $8 / 1 / 99$ & $8 / 1 / 99$ & 17,000 & 0 \\
\hline WATER & AP-108 & $8 / 1 / 99$ & $8 / 1 / 99$ & 3,000 & 0 \\
\hline WCW88 & SY-102 & $8 / 1 / 99$ & $8 / 1 / 99$ & 65,000 & 0 \\
\hline WATER & $S Y-102$ & $8 / 1 / 99$ & $8 / 1 / 99$ & 16,000 & 0 \\
\hline EVAPF & AP-106 & $8 / 1 / 99$ & $8 / 1 / 99$ & 10,000 & 0 \\
\hline \begin{tabular}{|l|} 
WNW88 \\
\end{tabular} & SY-102 & $8 / 1 / 99$ & $8 / 1 / 99$ & 69,000 & 0 \\
\hline AW-106 & AN-106 & $8 / 20 / 99$ & $8 / 23 / 99$ & 748,000 & 33 \\
\hline AW-106 & AP-108 & $8 / 25 / 99$ & $8 / 26 / 99$ & 180,000 & 8 \\
\hline WATER & SY-102 & $9 / 1 / 99$ & $9 / 1 / 99$ & 15,000 & 0 \\
\hline WATER & AP-108 & $9 / 1 / 99$ & $9 / 1 / 99$ & 3,000 & 0 \\
\hline WCE88 & AP-108 & $9 / 1 / 99$ & $9 / 1 / 99$ & 11,000 & 0 \\
\hline EVAPF & AP-106 & $9 / 1 / 99$ & $9 / 1 / 99$ & 10,000 & 0 \\
\hline WATER & SY-102 & $9 / 1 / 99$ & $9 / 1 / 99$ & 21,000 & 0 \\
\hline WCW88 & $S Y-102$ & $9 / 1 / 99$ & $9 / 1 / 99$ & 58,000 & 0 \\
\hline WNW88 & SY-102 & $9 / 1 / 99$ & $9 / 1 / 99$ & 85,000 & 0 \\
\hline WATER & AP-104 & $9 / 25 / 99$ & $9 / 25 / 99$ & 35,000 & 0 \\
\hline AW-102 & $\mathrm{AW}-106$ & $9 / 25 / 99$ & 9/25/99 & 99,996 & 4 \\
\hline SY-102 & AP-104 & $9 / 25 / 99$ & $9 / 27 / 99$ & 520,000 & 22 \\
\hline AP-104 & $\mathrm{AW}-102$ & $9 / 24 / 99$ & $9 / 29 / 99$ & $1,104,000$ & 41 \\
\hline 1NS96. & AW-105 & $10 / 1 / 99$ & $10 / 1 / 99$ & 750 & 250 \\
\hline WATER & SY-102 & $10 / 1 / 99$ & $10 / 1 / 99$ & 15,000 & 0 \\
\hline WESF & AP-106 & $10 / 1 / 99$ & $10 / 1 / 99$ & 5,000 & 0 \\
\hline $34 \mathrm{~L} 87$ & AP-106 & $10 / 1 / 99$ & $10 / 1 / 99$ & 10,000 & 0 \\
\hline $1 \mathrm{KL} 96$ & AW-105 & $10 / 1 / 99$ & $10 / 1 / 99$ & 30,000 & 0 \\
\hline WATER & AP-108 & $10 / 1 / 99$ & $10 / 1 / 99$ & 3,000 & 0 \\
\hline WCE88 & AP-108 & $10 / 1 / 99$ & $10 / 1 / 99$ & 11,000 & 0 \\
\hline TNS88 & AP-106 & $10 / 1 / 99$ & $10 / 1 / 99$ & 750 & 250 \\
\hline EVAPF & AP-106 & $10 / 1 / 99$ & $10 / 1 / 99$ & 10,000 & 0 \\
\hline PXTCO & AP-106 & $10 / 1 / 99$ & $10 / 1 / 99$ & 5,000 & 0 \\
\hline TAL88 & AP-106 & $10 / 1 / 99$ & $10 / 1 / 99$ & 6,000 & 0 \\
\hline SPN87 & SY-102 & $10 / 1 / 99$ & $10 / 1 / 99$ & 7,000 & 0 \\
\hline 2NL87 & SY-102 & $10 / 1 / 99$ & $10 / 1 / 99$ & 4,000 & 0 \\
\hline WCW88 & $S Y-102$ & $10 / 1 / 99$ & $10 / 1 / 99$ & 43,000 & 0 \\
\hline 1FL96 & AW-105 & $10 / 1 / 99$ & $10 / 1 / 99$ & 40,000 & 0 \\
\hline WATER & SY-102 & $10 / 1 / 99$ & $10 / 1 / 99$ & 11,000 & 0 \\
\hline WNW88 & SY-102 & $10 / 1 / 99$ & $10 / 1 / 99$ & 62,000 & 0 \\
\hline WATER & AP-106 & $10 / 2 / 99$ & $10 / 2 / 99$ & 5,000 & 0 \\
\hline WATER & AP-106 & $10 / 2 / 99$ & $10 / 2 / 99$ & 5,000 & 0 \\
\hline WATER & $A W-105$ & $10 / 2 / 99$ & $10 / 2 / 99$ & 13,000 & 0 \\
\hline WATER & SY-102 & $10 / 2 / 99$ & $10 / 2 / 99$ & 5,000 & 0 \\
\hline WATER & $\mathrm{AW}-105$ & $10 / 2 / 99$ & $10 / 2 / 99$ & 17,000 & 0 \\
\hline AW-106 & AW-104 & $10 / 20 / 99$ & $10 / 20 / 99$ & 100,200 & 4 \\
\hline \begin{tabular}{|l|} 
AW-102 \\
\end{tabular} & AW-106 & $10 / 25 / 99$ & $10 / 25 / 99$ & 99,996 & 4 \\
\hline AW-106 & AN-101 & $10 / 26 / 99$ & $10 / 26 / 99$ & 159,200 & 6 \\
\hline AW-102 & $242-A$ & $10 / 26 / 99$ & $10 / 30 / 99$ & 937,500 & 37 \\
\hline $242-\mathrm{A}$ & AW-106 & $10 / 26 / 99$ & $10 / 30 / 99$ & 361,400 & 37 \\
\hline $242-\mathrm{A}$ & LERF & $10 / 26 / 99$ & $10 / 30 / 99$ & 576,100 & 0 \\
\hline AP-102 & AP-103 & $10 / 25 / 99$ & $10 / 30 / 99$ & $1,070,000$ & 0 \\
\hline EVAPF & AP-106 & $11 / 1 / 99$ & $11 / 1 / 99$ & 10,000 & 0 \\
\hline
\end{tabular}


HNF-2321

Revision 0

Table A.4-1. Complete Transfer List for Case 4.

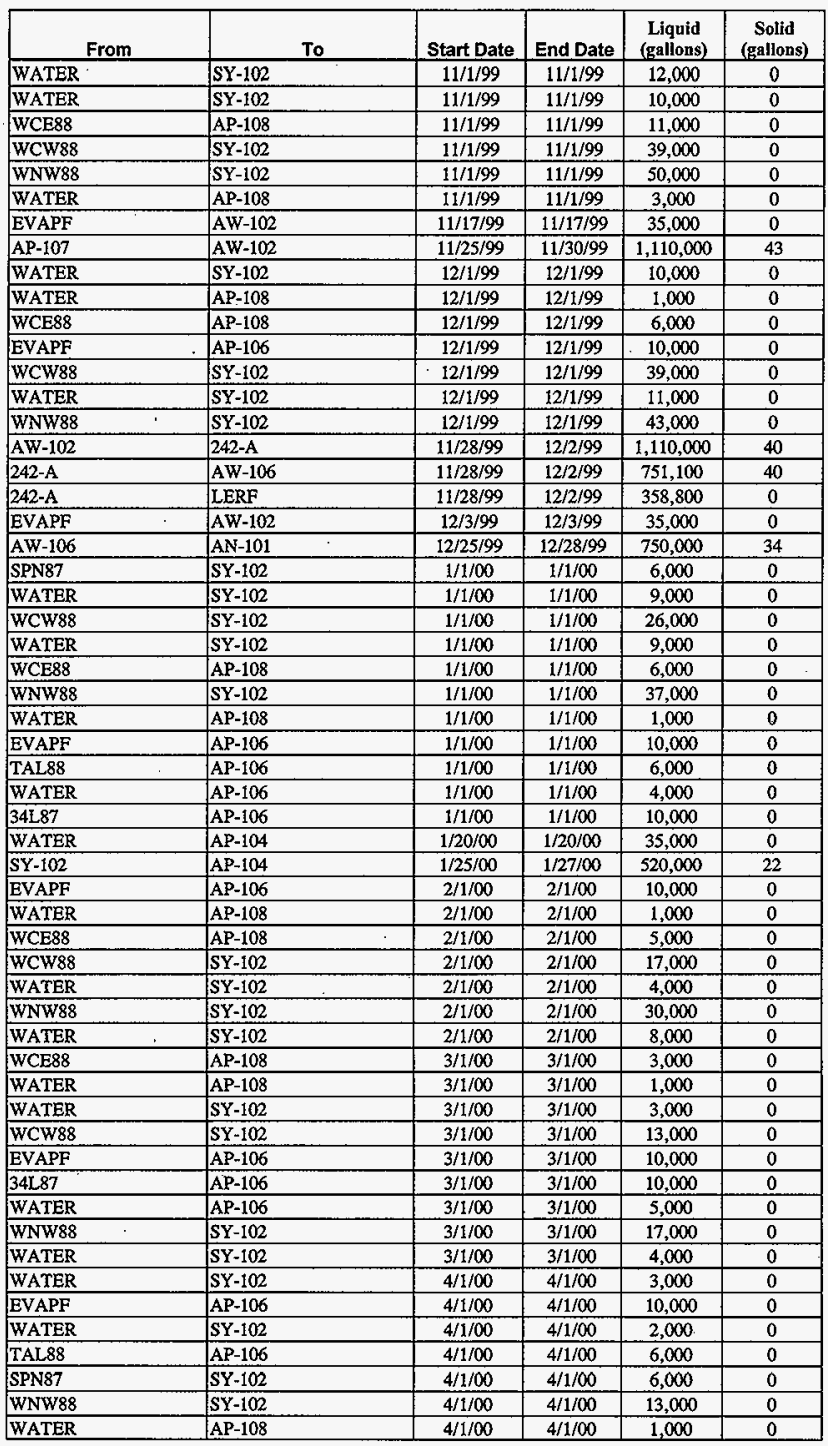


HNF-2321

Revision 0

Table A.4-1. Complete Transfer List for Case 4.

\begin{tabular}{|c|c|c|c|c|c|}
\hline From & To & Start Dafe & End Date & $\begin{array}{c}\text { Liquid } \\
\text { (gallons) }\end{array}$ & $\begin{array}{c}\text { Solid } \\
\text { (gallons) }\end{array}$ \\
\hline WCW88 & $S Y-102$ & $4 / 1 / 00$ & $4 / 1 / 00$ & 9,000 & 0 \\
\hline WCE88 & AP-108 & $4 / 1 / 00$ & $4 / 1 / 00$ & 3,000 & 0 \\
\hline WATER & AP-108 & $5 / 1 / 00$ & $5 / 1 / 00$ & 1,000 & 0 \\
\hline \begin{tabular}{|l} 
WATER \\
\end{tabular} & AP-106 & $5 / 1 / 00$ & $5 / 1 / 00$ & 4,000 & 0 \\
\hline WCE88 & AP-108 & $5 / 1 / 00$ & $5 / 1 / 00$ & 3,000 & 0 \\
\hline $34 \mathrm{~L} 87$ & AP-106 & $5 / 1 / 00$ & $5 / 1 / 00$ & 10,000 & 0 \\
\hline EVAPF & AP-106 & $5 / 1 / 00$ & $5 / 1 / 00$ & 10,000 & 0 \\
\hline WATER & SY-102 & $5 / 1 / 00$ & $5 / 1 / 00$ & 3,000 & 0 \\
\hline WNW88 & SY-102 & $5 / 1 / 00$ & $5 / 1 / 00$ & 11,000 & 0 \\
\hline WCW88 & SY-102 & $5 / 1 / 00$ & $5 / 1 / 00$ & 6,000 & 0 \\
\hline WATER & SY-102 & $5 / 1 / 00$ & $5 / 1 / 00$ & 1,000 & 0 \\
\hline WATER & AP-108 & $6 / 1 / 00$ & $6 / 1 / 00$ & 1,000 & 0 \\
\hline EVAPF & AP-106 & $6 / 1 / 00$ & $6 / 1 / 00$ & 10,000 & 0 \\
\hline WATER & SY-102 & $6 / 1 / 00$ & $6 / 1 / 00$ & 1,000 & 0 \\
\hline WNW88 & SY-102 & $6 / 1 / 00$ & $6 / 1 / 00$ & 4,000 & 0 \\
\hline WCE 88 & AP-108 & $6 / 1 / 00$ & $6 / 1 / 00$ & 3,000 & 0 \\
\hline $34 \mathrm{~L} 87$ & AP-106 & $7 / 1 / 00$ & $7 / 1 / 00$ & 10,000 & 0 \\
\hline WATER & AP-106 & $7 / 1 / 00$ & $7 / 1 / 00$ & 4,000 & 0 \\
\hline SPN87 & SY-102 & $7 / 1 / 00$ & $7 / 1 / 00$ & 6,000 & 0 \\
\hline EVAPF & AP-106 & $7 / 1 / 00$ & $7 / 1 / 00$ & 10,000 & 0 \\
\hline \begin{tabular}{|l|} 
TAL88 \\
\end{tabular} & AP-106 & $7 / 1 / 00$ & $7 / 1 / 00$ & 5,000 & 0 \\
\hline WATER & AP-108 & $7 / 1 / 00$ & $7 / 1 / 00$ & 1,000 & 0 \\
\hline WCE88 & AP-108 & $7 / 1 / 00$ & $7 / 1 / 00$ & 3,000 & 0 \\
\hline WATER & SY-102 & $7 / 1 / 00$ & $7 / 1 / 00$ & 1,000 & 0 \\
\hline \begin{tabular}{|l|} 
WNW88 \\
\end{tabular} & SY-102 & $7 / 1 / 00$ & $7 / 1 / 00$ & 4,000 & 0 \\
\hline AP-104 & $\mathrm{AW}-102$ & $7 / 25 / 00$ & $7 / 30 / 00$ & $1,110,000$ & 43 \\
\hline \begin{tabular}{|l|} 
WCE 88 \\
\end{tabular} & AP-108 & $8 / 1 / 00$ & $8 / 1 / 00$ & 2,000 & 0 \\
\hline EVAPF & AP-106 & $8 / 1 / 00$ & $8 / 1 / 00$ & 10,000 & 0 \\
\hline WNW88 & SY-102 & $8 / 1 / 00$ & $8 / 1 / 00$ & 4,000 & 0 \\
\hline WATER & SY-102 & $8 / 1 / 00$ & $8 / 1 / 00$ & 1,000 & 0 \\
\hline AW-102 & $242-\mathrm{A}$ & $7 / 28 / 00$ & $8 / 2 / 00$ & $1,145,000$ & 39 \\
\hline $242-A$ & AW-106 & $7 / 28 / 00$ & $8 / 2 / 00$ & 802,300 & 39 \\
\hline $242-\mathrm{A}$ & LERF & $7 / 28 / 00$ & $8 / 2 / 00$ & 342,600 & 0 \\
\hline EVAPF & AW-102 & $8 / 3 / 00$ & $8 / 3 / 00$ & 35,000 & 0 \\
\hline$A Z-101$ & $A Y-101$ & $8 / 17 / 00$ & $8 / 20 / 00$ & 685,000 & 0 \\
\hline $\mathrm{AZ}-101$ & AP-107 & $8 / 20 / 00$ & $8 / 20 / 00$ & 0 & 0 \\
\hline WASE-WATER & AZ-101 & $8 / 20 / 00$ & $8 / 21 / 00$ & 146,000 & 0 \\
\hline WCE88 & AP- 108 & $.9 / 1 / 00$ & $9 / 1 / 00$ & 1,000 & 0 \\
\hline EVAPF & AP-106 & $9 / 1 / 00$ & $9 / 1 / 00$ & 10,000 & 0 \\
\hline AW-106 & AP-107 & $9 / 25 / 00$ & $9 / 29 / 00$ & 804,000 & 35 \\
\hline PXTCO & AP-106 & $10 / 1 / 00$ & $10 / 1 / 00$ & 5,000 & 0 \\
\hline \begin{tabular}{|l|} 
WESF \\
\end{tabular} & AP-106 & $10 / 1 / 00$ & $10 / 1 / 00$ & 5,000 & 0 \\
\hline TNS88 & AP-106 & $10 / 1 / 00$ & $10 / 1 / 00$ & 750 & 250 \\
\hline 1NS96 & $A W-105$ & $10 / 1 / 00$ & $10 / 1 / 00$ & 13,502 & 4,498 \\
\hline TAL 88 & AP-106 & $10 / 1 / 00$ & $10 / 1 / 00$ & 23,000 & 0 \\
\hline $34 \mathrm{~L} 87$ & AP-106 & $10 / 1 / 00$ & $10 / 1 / 00$ & 50,000 & 0 \\
\hline $34 \mathrm{~L} 87$ & AP-106 & $10 / 1 / 00$ & $10 / 1 / 00$ & 7,000 & 0 \\
\hline ZNL87 & $5 X-102$ & $10 / 1 / 00$ & $10 / 1 / 00$ & 4,000 & 0 \\
\hline SPN87 & SY-102 & $10 / 1 / 00$ & $10 / 1 / 00$ & 25,000 & 0 \\
\hline EVAPF & AP-106 & $10 / 1 / 00$ & $10 / 1 / 00$ & 120,000 & 0 \\
\hline WASH-WATER & AP-104 & $10 / 1 / 00$ & $10 / 2 / 00$ & 200,000 & 0 \\
\hline WATER & AW-105 & $10 / 2 / 00$ & $10 / 2 / 00$ & $7,000^{\circ}$ & 0 \\
\hline 1KL96 & $\mathrm{AW}-105$ & $10 / 1 / 00$ & $10 / 2 / 00$ & 302,000 & 0 \\
\hline WATER & AW-105 & $10 / 2 / 00$ & $10 / 2 / 00$ & 132,000 & 0 \\
\hline WATER & AP-106 & $10 / 2 / 00$ & $10 / 2 / 00$ & 5,000 & 0 \\
\hline WATER & AP-106 & $10 / 2 / 00$ & $10 / 3 / 00$ & 22,000 & 0 \\
\hline
\end{tabular}


HNF-2321

Revision 0

Table A.4-1. Complete Transfer List for Case 4.

\begin{tabular}{|c|c|c|c|c|c|}
\hline From & To & Start Date & End Date & $\begin{array}{c}\text { Liquid } \\
\text { (gallons) }\end{array}$ & $\begin{array}{c}\text { Solid } \\
\text { (gallons) }\end{array}$ \\
\hline WATER & AP-106 & $10 / 3 / 00$ & $10 / 3 / 00$ & 3,000 & 0 \\
\hline WATER & SY-102 & $10 / 3 / 00$ & $10 / 3 / 00$ & 1,000 & 0 \\
\hline WATER & SY-102 & $10 / 3 / 00$ & $10 / 3 / 00$ & 5,000 & 0 \\
\hline EVAPF & AW-102 & $10 / 4 / 00$ & $10 / 4 / 00$ & 50,000 & 0 \\
\hline AP-106 & AW-102 & $10 / 2 / 00$ & $10 / 4 / 00$ & 474,000 & 16 \\
\hline AP-106 & $\mathrm{AW}-102$ & $10 / 5 / 00$ & $10 / 5 / 00$ & 10,000 & 0 \\
\hline AP-106 & AW-102 & $10 / 6 / 00$ & $10 / 6 / 00$ & 71,998 & 2 \\
\hline AP-106 & AW-102 & $10 / 7 / 00$ & $10 / 7 / 00$ & 180,300 & 6 \\
\hline$A W-105$ & AW-102 & $10 / 6 / 00$ & $10 / 8 / 00$ & 414,000 & 14 \\
\hline AP-106 & AW-102 & $10 / 8 / 00$ & $10 / 8 / 00$ & 0 & 0 \\
\hline AW-105 & AW-102 & $10 / 9 / 00$ & $10 / 10 / 00$ & 213,200 & 7 \\
\hline $\mathrm{AZ}-101$ & AP-106 & $10 / 9 / 00$ & $10 / 10 / 00$ & 143,200 & 0 \\
\hline WASH-WATER & AZ-101 & $10 / 11 / 00$ & $10 / 12 / 00$ & 146,000 & 0 \\
\hline $\mathrm{AW}-102$ & $242-A$ & $10 / 5 / 00$ & $10 / 12 / 00$ & $1,483,000$ & 52 \\
\hline $242-A$ & AW-106 & $10 / 5 / 00$ & $10 / 12 / 00$ & 92,234 & 52 \\
\hline $242-\mathrm{A}$ & LERF & $10 / 5 / 00$ & $10 / 12 / 00$ & $1,391,000$ & 0 \\
\hline AP-104 & AP-102 & $10 / 15 / 00$ & $10 / 16 / 00$ & 200,000 & 1 \\
\hline $\mathrm{AW}-106$ & AP-107 & $10 / 25 / 00$ & $10 / 25 / 00$ & 110,000 & 5 \\
\hline EVAPF & AW-102 & $11 / 3 / 00$ & $11 / 3 / 00$ & 35,000 & 0 \\
\hline $\mathrm{AZ}-101$ & AP-106 & $11 / 30 / 00$ & $12 / 1 / 00$ & 140,200 & 0 \\
\hline WASH-WATER & AZ-101 & $12 / 2 / 00$ & $12 / 2 / 00$ & 146,000 & 0 \\
\hline $\mathrm{AZ}-101$ & AP-106 & $1 / 21 / 01$ & $1 / 21 / 01$ & 142,900 & 0 \\
\hline WASH-WATER & AZ-101 & $1 / 22 / 01$ & $1 / 23 / 01$ & 138,200 & 0 \\
\hline AP-102 & AP-106 & $2 / 15 / 01$ & $2 / 16 / 01$ & 200,500 & 1 \\
\hline $\mathrm{AN}-105$ & AP-102 & $3 / 17 / 01$ & $3 / 20 / 01$ & 542,400 & 7,895 \\
\hline LERF & AN-105-PUMP & $3 / 17 / 01$ & $3 / 20 / 01$ & 154,100 & 0 \\
\hline AN-105 & AP-102 & $3 / 17 / 01$ & $3 / 20 / 01$ & 686,500 & 294 \\
\hline AP-108 & AP-106 & $3 / 22 / 01$ & $3 / 23 / 01$ & 356,500 & 8 \\
\hline AN-105 & AP-104 & $3 / 21 / 01$ & $3 / 24 / 01$ & 542,400 & 7,895 \\
\hline LERF & AN-105-PUMP & $3 / 21 / 01$ & $3 / 24 / 01$ & 154,100 & 0 \\
\hline AN-105 & AP-104 & $3 / 21 / 01$ & $3 / 24 / 01$ & 686,500 & 294 \\
\hline AP-106 & AW-102 & $3 / 23 / 01$ & $3 / 27 / 01$ & 882,400 & 0 \\
\hline WASH-WATER & AP-108 & $4 / 7 / 01$ & $4 / 8 / 01$ & 200,000 & 0 \\
\hline AP-108 & AP-106 & $4 / 21 / 01$ & $4 / 21 / 01$ & 200,000 & 1 \\
\hline WATER & AW-102 & $5 / 1 / 01$ & $5 / 1 / 01$ & 35,000 & 0 \\
\hline EVAPF & $\mathrm{AW}-102$ & $5 / 3 / 01$ & $5 / 3 / 01$ & 35,000 & 0 \\
\hline AW-106 & AW-105 & $7 / 1 / 01$ & $7 / 2 / 01$ & 283,200 & 13 \\
\hline AP-102 & AP-108 & $7 / 10 / 01$ & $7 / 14 / 01$ & 686,500 & 282 \\
\hline AP-107 & $\mathrm{AN}-105$ & $9 / 1 / 01$ & $9 / 4 / 01$ & 914,500 & 40 \\
\hline AW-102 & $242-\mathrm{A}$ & $9 / 25 / 01$ & $9 / 30 / 01$ & 987,300 & 37 \\
\hline $242-\mathrm{A}$ & AW-106 & $9 / 25 / 01$ & $9 / 30 / 01$ & 633,300 & 37 \\
\hline $242-A$ & LERF & 9/25/01 & 9/30/01 & 354,000 & 0 \\
\hline WESF & AP-106 & $10 / 1 / 01$ & $10 / 1 / 01$ & 5,000 & 0 \\
\hline ZNL87 & SY-102 & $10 / 1 / 01$ & $10 / 1 / 01$ & 4,000 & 0 \\
\hline TNS88 & AP-106 & $10 / 1 / 01$ & $10 / 1 / 01$ & 750 & 250 \\
\hline TAL88 & AP-106 & $10 / 1 / 01$ & $10 / 1 / 01$ & 23,000 & 0 \\
\hline \begin{tabular}{|l|} 
SPN87 \\
\end{tabular} & SY-102 & $10 / 1 / 01$ & $10 / 1 / 01$ & 25,000 & 0 \\
\hline $34 \mathrm{~L} 87$ & AP-106 & $10 / 1 / 01$ & $10 / 1 / 01$ & 50,000 & 0 \\
\hline WATER & AP-106 & $10 / 1 / 01$ & $10 / 1 / 01$ & 35,000 & 0 \\
\hline \begin{tabular}{|l} 
EVAPF \\
\end{tabular} & AP-106 & $10 / 1 / 01$ & $10 / 1 / 01$ & 120,000 & 0 \\
\hline WATER & SY-102 & $10 / 2 / 01$ & $10 / 2 / 01$ & 5,000 & 0 \\
\hline WATER & AP-106 & $10 / 2 / 01$ & $10 / 2 / 01$ & 5,000 & 0 \\
\hline WATER & AP-106 & $10 / 2 / 01$ & $10 / 2 / 01$ & 22,000 & 0 \\
\hline AW-106 & $A W-102$ & $9 / 30 / 01$ & $10 / 3 / 01$ & 633,200 & 25 \\
\hline AN-104 & AP-102 & $10 / 1 / 01$ & $10 / 4 / 01$ & 502,200 & 12,513 \\
\hline LERF & AN-104-PUMP & $10 / 1 / 01$ & $10 / 4 / 01$ & 200,800 & 0 \\
\hline
\end{tabular}


HNF-2321

Revision 0

Table A.4-1. Complete Transfer List for Case 4.

\begin{tabular}{|c|c|c|c|c|c|}
\hline From & To & Start Date & End Date & $\begin{array}{c}\text { Liquid } \\
\text { (gallons) }\end{array}$ & $\begin{array}{c}\text { Solid } \\
\text { (gallons) }\end{array}$ \\
\hline AN-104 & AP-102 & $10 / 1 / 01$ & $10 / 4 / 01$ & 684,700 & 702 \\
\hline AW-102 & $242-A$ & $10 / 17 / 01$ & $10 / 20 / 01$ & 633,200 & 25 \\
\hline $242-\mathrm{A}$ & AW-106 & $10 / 17 / 01$ & $10 / 20 / 01$ & 405,200 & 25 \\
\hline $242-A$ & LERF & $10 / 17 / 01$ & $10 / 20 / 01$ & 228,000 & 0 \\
\hline AW-106 & AW-102 & $10 / 20 / 01$ & $10 / 22 / 01$ & 405,200 & 18 \\
\hline EVAPF & AW-102 & $11 / 3 / 01$ & $11 / 3 / 01$ & 35,000 & 0 \\
\hline AW-102 & 242-A & $11 / 5 / 01$ & $11 / 7 / 01$ & 440,300 & 19 \\
\hline $242-A$ & AW-106 & $11 / 5 / 01$ & $11 / 7 / 01$ & 296,100 & 19 \\
\hline $242-\mathrm{A}$ & LERF & $11 / 5 / 01$ & $11 / 7 / 01$ & 144,200 & 0 \\
\hline AZ-102 & $\mathrm{AY}-101$ & $11 / 14 / 01$ & $11 / 15 / 01$ & 204,000 & 0 \\
\hline AZ-102 & AP-107 & $11 / 16 / 01$ & $11 / 16 / 01$ & 181,800 & 0 \\
\hline WASH-WATER , & AZ-102 & $11 / 17 / 01$ & $11 / 18 / 01$ & 213,000 & 0 \\
\hline AZ-102 & AP-106 & $1 / 6 / 02$ & $1 / 6 / 02$ & 127,700 & 0 \\
\hline WASH-WATER & AZ-102 & $1 / 8 / 02$ & $1 / 9 / 02$ & 213,000 & 0 \\
\hline $\mathrm{AZ}-102$ & AP-106 & $2 / 27 / 02$ & $2 / 28 / 02$ & 210,300 & 0 \\
\hline WASH-WATER & $\mathrm{AZ}-102$ & $3 / 1 / 02$ & $3 / 2 / 02$ & 213,000 & 0 \\
\hline AP-106 & AW-102 & $2 / 28 / 02$ & $3 / 4 / 02$ & 897,800 & 0 \\
\hline AZ-102 & AP-106 & $4 / 20 / 02$ & $4 / 21 / 02$ & 211,800 & 0 \\
\hline WASH-WATER & AZ-102 & $4 / 22 / 02$ & $4 / 23 / 02$ & 213,000 & 0 \\
\hline AZ-101 & PLHLW FEED TANK & $5 / 17 / 02$ & $5 / 17 / 02$ & 134,300 & 5,079 \\
\hline$A Z-102$ & AP-106 & $6 / 12 / 02$ & $6 / 13 / 02$ & 212,500 & 0 \\
\hline WASH-WATER & $\mathrm{AZ}-102$ & $6 / 14 / 02$ & $6 / 14 / 02$ & 110,000 & 0 \\
\hline AW-106 & AW-105 & $7 / 1 / 02$ & $7 / 2 / 02$ & 296,100 & 14 \\
\hline AW-102 & $242-\mathrm{A}$ & $9 / 2 / 02$ & $9 / 6 / 02$ & 898,000 & 31 \\
\hline $242-\mathrm{A}$ & AW-106 & $9 / 2 / 02$ & $9 / 6 / 02$ & 574,000 & 31 \\
\hline $242-\mathrm{A}$ & LERF & $9 / 2 / 02$ & $9 / 6 / 02$ & 324,000 & 0 \\
\hline AW-106 & AW-102 & $9 / 7 / 02$ & $9 / 9 / 02$ & 573,900 & 21 \\
\hline AW-102 & $242-\mathrm{A}$ & $9 / 24 / 02$ & $9 / 26 / 02$ & 573,800 & 21 \\
\hline $242-\mathrm{A}$ & AW-106 & $9 / 24 / 02$ & $9 / 26 / 02$ & 367,800 & 21 \\
\hline $242-\mathrm{A}$ & LERRF & $9 / 24 / 02$ & $9 / 26 / 02$ & 206,000 & 0 \\
\hline $\mathrm{AW}-106$ & AW-102 & $9 / 26 / 02$ & $9 / 28 / 02$ & 367,700 & 14 \\
\hline ZNL 87 & SY-102 & $10 / 1 / 02$ & $10 / 1 / 02$ & 3,000 & 0 \\
\hline WESF & AP-106 & $10 / 1 / 02$ & $10 / 1 / 02$ & 5,000 & 0 \\
\hline TNS88 & AP-106 & $10 / 1 / 02$ & $10 / 1 / 02$ & 750 & 250 \\
\hline SPN87 & SY-102 & $10 / 1 / 02$ & $10 / 1 / 02$ & 25,000 & 0 \\
\hline TAL88 & AP-106 & $10 / 1 / 02$ & $10 / 1 / 02$ & 24,000 & 0 \\
\hline WATER & AP-106 & $10 / 1 / 02$ & $10 / 1 / 02$ & 35,000 & 0 \\
\hline $34 \mathrm{~L} 87$ & AP-106 & $10 / 1 / 02$ & $10 / 1 / 02$ & 50,000 & 0 \\
\hline EVAPF & AP-106 & $10 / 1 / 02$ & $10 / 1 / 02$ & 120,000 & 0 \\
\hline WATER & AP-106 & $10 / 2 / 02$ & $10 / 2 / 02$ & 5,000 & 0 \\
\hline WATER & AP-106 & $10 / 2 / 02$ & $10 / 2 / 02$ & 22,000 & 0 \\
\hline WATER & SY-102 & $10 / 2 / 02$ & $10 / 2 / 02$ & 1,000 & 0 \\
\hline WATER & SY-102 & $10 / 2 / 02$ & $10 / 2 / 02$ & 5,000 & 0 \\
\hline EVAPF & AW-102 & $10 / 4 / 02$ & $10 / 4 / 02$ & 50,000 & 0 \\
\hline AW-102 & $242-\mathrm{A}$ & $10 / 12 / 02$ & $10 / 14 / 02$ & 417,800 & 16 \\
\hline $242-A$ & AW-106 & $10 / 12 / 02$ & $10 / 14 / 02$ & 267,800 & 16 \\
\hline $242-\mathrm{A}$ & LERF & $10 / 12 / 02$ & $10 / 14 / 02$ & 150,000 & 0 \\
\hline AW-106 & AW-102 & $10 / 14 / 02$ & $10 / 16 / 02$ & 267,700 & 11 \\
\hline AW-102 & $242-\mathrm{A}$ & $10 / 30 / 02$ & $10 / 31 / 02$ & 267,800 & 10 \\
\hline $242-A$ & AW-106 & $10 / 30 / 02$ & $10 / 31 / 02$ & 171,800 & 10 \\
\hline $242-\mathrm{A}$ & LERF & $10 / 30 / 02$ & $10 / 31 / 02$ & 96,000 & 0 \\
\hline AW-106 & AW -102 & $10 / 31 / 02$ & $11 / 1 / 02$ & 171,800 & 7 \\
\hline EVAPF & AW-102 & $11 / 3 / 02$ & $11 / 3 / 02$ & 35,000 & 0 \\
\hline AW-102 & AP-106 & $11 / 2 / 02$ & $11 / 3 / 02$ & 206,900 & 9 \\
\hline AP-106 & AW-102 & $11 / 3 / 02$ & $11 / 8 / 02$ & 889,900 & 0 \\
\hline AP-104 & AP-108 & $1 / 5 / 03$ & $1 / 9 / 03$ & 686,500 & 282 \\
\hline
\end{tabular}


HNF-2321

Revision 0

Table A.4-1. Complete Transfer List for Case 4.

\begin{tabular}{|c|c|c|c|c|c|}
\hline From & To & Start Date & End Date & $\begin{array}{c}\text { Liquid } \\
\text { (gallons) }\end{array}$ & $\begin{array}{c}\text { Solid } \\
\text { (gallons) }\end{array}$ \\
\hline $\mathrm{AN}-104$ & AP-104 & $1 / 10 / 03$ & $1 / 13 / 03$ & 502,200 & 12,513 \\
\hline LERF & AN-104-PUMP & $1 / 10 / 03$ & $1 / 13 / 03$ & 200,800 & 0 \\
\hline AN-104 & AP-104 & $1 / 10 / 03$ & $1 / 13 / 03$ & 684,700 & 702 \\
\hline$A W-105$ & AN-104 & $2 / 1 / 03$ & $2 / 3 / 03$ & 579,300 & 26 \\
\hline P1HLW FEED TANK & HLW-CONTRACTOR & $6 / 1 / 02$ & $2 / 21 / 03$ & 134,300 & 5,079 \\
\hline AZ-101 & P1HLW FEED TANK & $2 / 21 / 03$ & $2 / 21 / 03$ & 134,300 & 5,079 \\
\hline$A Y-102$ & AZ-101 & $2 / 24 / 03$ & $3 / 1 / 03$ & 819,600 & 16,032 \\
\hline RETURN 2 & AP-107 & $4 / 3 / 03$ & $4 / 3 / 03$ & 8,671 & 331 \\
\hline AW-102 & $242-A$ & $5 / 8 / 03$ & $5 / 12 / 03$ & 889,900 & 31 \\
\hline $242-\mathrm{A}$ & AW-106 & $5 / 8 / 03$ & $5 / 12 / 03$ & 571,900 & 31 \\
\hline $242-\mathrm{A}$ & LERF & $5 / 8 / 03$ & $5 / 12 / 03$ & 318,000 & 0 \\
\hline$A W-106$ & AW-102 & $5 / 13 / 03$ & $5 / 16 / 03$ & 571,700 & 21 \\
\hline AW-102 & $242-\mathrm{A}$ & $5 / 30 / 03$ & $6 / 2 / 03$ & 571,700 & 21 \\
\hline 242-A & AW-106 & $5 / 30 / 03$ & $6 / 2 / 03$ & 367,300 & 21 \\
\hline 242-A & LERF & $5 / 30 / 03$ & $6 / 2 / 03$ & 204,400 & 0 \\
\hline AW-106 & AW-102 & $6 / 2 / 03$ & $6 / 4 / 03$ & 367,200 & 14 \\
\hline$A W-102$ & $242-A$ & $6 / 18 / 03$ & $6 / 20 / 03$ & 367,300 & 14 \\
\hline $242-\mathrm{A}$ & $\mathrm{AW}-106$ & $6 / 18 / 03$ & $6 / 20 / 03$ & 235,300 & 14 \\
\hline $242-\mathrm{A}$ & LERF & $6 / 18 / 03$ & $6 / 20 / 03$ & 132,000 & 0 \\
\hline AW-106 & AW-102 & $6 / 20 / 03$ & $6 / 21 / 03$ & 235,300 & 10 \\
\hline AW-102 & $242-\mathrm{A}$ & $7 / 5 / 03$ & $7 / 6 / 03$ & 235,300 & 10 \\
\hline $242-\mathrm{A}$ & AW-106 & $7 / 5 / 03$ & $7 / 6 / 03$ & 151,300 & 10 \\
\hline $242-\mathrm{A}$ & LERF & $7 / 5 / 03$ & $7 / 6 / 03$ & 84,068 & 0 \\
\hline AW-106 & AW-102 & $7 / 6 / 03$ & $7 / 7 / 03$ & 151,200 & 7 \\
\hline AW-102 & $\mathrm{AP}-106$ & $7 / 8 / 03$ & $7 / 9 / 03$ & 151,400 & 7 \\
\hline AP-102 & AP-108 & $9 / 19 / 03$ & $9 / 22 / 03$ & 684,700 & 686 \\
\hline AW-101 & AP-102 & $9 / 23 / 03$ & $9 / 27 / 03$ & 537,200 & 13,013 \\
\hline LERF & AW-101-PUMP & $9 / 23 / 03$ & $9 / 27 / 03$ & 308,100 & 0 \\
\hline AW-101 & AP-102 & $9 / 23 / 03$ & $9 / 27 / 03$ & 845,400 & 13,013 \\
\hline $34 \mathrm{~L} 87$ & AP-106 & $10 / 1 / 03$ & $10 / 1 / 03$ & 7,000 & 0 \\
\hline TNS88 & AP-106 & $10 / 1 / 03$ & $10 / 1 / 03$ & 750 & 250 \\
\hline TAL88 & AP-106 & $10 / 1 / 03$ & 10/1/03 & $.24,000$ & 0 \\
\hline WESF & AP-106 & $10 / 1 / 03$ & $10 / 1 / 03$ & 5,000 & 0 \\
\hline ZNL87 & SY-102 & $10 / 1 / 03$ & $10 / 1 / 03$ & 3,000 & 0 \\
\hline SPN87 & SY-102 & $10 / 1 / 03$ & $10 / 1 / 03$ & 25,000 & 0 \\
\hline 34187 & $A \mathrm{AP}-106$ & $10 / 1 / 03$ & $10 / 1 / 03$ & 50,000 & 0 \\
\hline WATER & AP-106 & $10 / 1 / 03$ & $10 / 1 / 03$ & 35,000 & 0 \\
\hline EVAPF & AP-106 & $10 / 1 / 03$ & $10 / 1 / 03$ & 120,000 & 0 \\
\hline WATER & AP-106 & $10 / 2 / 03$ & $10 / 2 / 03$ & 3,000 & 0 \\
\hline WATER & AP-106 & $10 / 2 / 03$ & $10 / 2 / 03$ & 22,000 & 0 \\
\hline WATER & AP-106 & $10 / 2 / 03$ & $10 / 2 / 03$ & 5,000 & 0 \\
\hline WATER & $\$ Y-102$ & $10 / 2 / 03$ & $10 / 2 / 03$ & 5,000 & 0 \\
\hline $\mathrm{AZ}-101$ & $A P-106$ & $10 / 28 / 03$ & $10 / 31 / 03$ & 622,700 & 0 \\
\hline WASH-WATER & $A Z-101$ & $10 / 31 / 03$ & $11 / 2 / 03$ & 324,000 & 0 \\
\hline EVAPF & AW-102 & $11 / 3 / 03$ & $11 / 3 / 03$ & 35,000 & 0 \\
\hline AP-106 & AW-102 & $10 / 31 / 03$ & $11 / 5 / 03$ & 918,100 & 0 \\
\hline P1HLW FEED TANK & HLW-CONTRACTOR & $2 / 22 / 03$ & $11 / 14 / 03$ & 134,300 & 5,079 \\
\hline $\mathrm{AZ}-102$ & P1HLW FEED TANK & $11 / 14 / 03$ & $11 / 14 / 03$ & 123,900 & 5,202 \\
\hline $\mathrm{AZ}-101$ & AP-106 & $12 / 21 / 03$ & $12 / 22 / 03$ & 320,000 & 0 \\
\hline WASH-WATER & $\mathrm{AZ}-101$ & $12 / 24 / 03$ & $12 / 25 / 03$ & 324,000 & 0 \\
\hline RETURN 2 & AP-107 & $2 / 2 / 04$ & $2 / 2 / 04$ & 8,670 & 331 \\
\hline AZ-101 & AP-106 & $2 / 12 / 04$ & $2 / 14 / 04$ & 322,300 & 0 \\
\hline WASH-WATER ' & $\mathrm{AZ}-101$ & $2 / 15 / 04$ & $2 / 17 / 04$ & 433,600 & 0 \\
\hline$S X-113$ & SY-103 & $12 / 31 / 03$ & $4 / 6 / 04$ & 10,322 & 399 \\
\hline AW-102 & $242-A$ & $5 / 4 / 04$ & $5 / 9 / 04$ & 953,000 & 34 \\
\hline $242-A$ & AW-106 & $5 / 4 / 04$ & $5 / 9 / 04$ & 611,000 & 34 \\
\hline
\end{tabular}


HNF-2321

Revision 0

Table A.4-1. Complete Transfer List for Case 4.

\begin{tabular}{|c|c|c|c|c|c|}
\hline From & To & Start Date & End Date & $\begin{array}{c}\text { Liquid } \\
\text { (gallons) }\end{array}$ & $\begin{array}{c}\text { Solid } \\
\text { (gallons) }\end{array}$ \\
\hline $242-\mathrm{A}$ & LERF & $5 / 4 / 04$ & $5 / 9 / 04$ & 342,000 & 0 \\
\hline AW-106 & AW-102 & $5 / 9 / 04$ & $5 / 12 / 04$ & 610,900 & 23 \\
\hline AW-102 & $242-\mathrm{A}$ & $5 / 26 / 04$ & $5 / 29 / 04$ & 610,900 & 23 \\
\hline $242-\mathrm{A}$ & AW-106 & $5 / 26 / 04$ & $5 / 29 / 04$ & 390,700 & 23 \\
\hline 242-A & LERF & $5 / 26 / 04$ & $5 / 29 / 04$ & 220,200 & 0 \\
\hline AW-106 & AW-102 & $5 / 29 / 04$ & $5 / 31 / 04$ & 390,600 & 16 \\
\hline AP-104 & AP-108 & $6 / 12 / 04$ & $6 / 15 / 04$ & 684,700 & 686 \\
\hline AW-102 & $242-\mathrm{A}$ & $6 / 14 / 04$ & $6 / 16 / 04$ & 390,700 & 15 \\
\hline 242-A & AW-106 & $6 / 14 / 04$ & $6 / 16 / 04$ & 250,400 & 15 \\
\hline $242-\mathrm{A}$ & LERF & $6 / 14 / 04$ & $6 / 16 / 04$ & 140,300 & 0 \\
\hline AW-106 & AW-102 & $6 / 16 / 04$ & $6 / 17 / 04$ & 250,400 & 11 \\
\hline$A W-101$ & AP-104 & $6 / 16 / 04$ & $6 / 20 / 04$ & 537,200 & 13,013 \\
\hline LERF & AW-101-PUMP & $6 / 16 / 04$ & $6 / 20 / 04$ & 308,100 & 0 \\
\hline AW-101 & AP-104 & $6 / 16 / 04$ & $6 / 20 / 04$ & 845,400 & 13,013 \\
\hline$A W-102$ & $242-A$ & $7 / 1 / 04$ & $7 / 3 / 04$ & 250,400 & 11 \\
\hline 242-A & AW-106 & $7 / 1 / 04$ & $7 / 3 / 04$ & 170,500 & 11 \\
\hline $242-A$ & LERF & $7 / 1 / 04$ & $7 / 3 / 04$ & 79,918 & 0 \\
\hline AN-104 & AW-101 & $7 / 4 / 04$ & $7 / 7 / 04$ & 572,700 & 26 \\
\hline PIHLW FEED TANK & HLW-CONTRACTOR & $11 / 14 / 03$ & $8 / 7 / 04$ & 123,900 & 5,202 \\
\hline $\mathrm{AZ}-102$ & PIHLW FEED_TANK & $8 / 7 / 04$ & $8 / 8 / 04$ & 123,900 & 5,202 \\
\hline RETURN 2 & AP-107 & $8 / 31 / 04$ & $8 / 31 / 04$ & 8,672 & 332 \\
\hline C-104 & $A Y-102$ & $8 / 1 / 04$ & $9 / 8 / 04^{\circ}$ & 279,100 & 9,724 \\
\hline TAL88 & AP-106 & $10 / 1 / 04$ & $10 / 1 / 04$ & 2,000 & 0 \\
\hline WESF & AP-106 & $10 / 1 / 04$ & $10 / 1 / 04$ & 5,000 & 0 \\
\hline TNS88 & AP-106 & $10 / 1 / 04$ & $10 / 1 / 04$ & 750 & 250 \\
\hline ZNL87 & SY-102 & $10 / 1 / 04$ & $10 / 1 / 04$ & 3,000 & 0 \\
\hline $34 \backslash 87$ & AP-106 & $10 / 1 / 04$ & $10 / 1 / 04$ & 50,000 & 0 \\
\hline TAL88 & AP-106 & $10 / 1 / 04$ & $10 / 1 / 04$ & 22,000 & 0 \\
\hline SPN87 & SY-102 & $10 / 1 / 04$ & $10 / 1 / 04$ & 23,000 & 0 \\
\hline WATER & AP-106 & $10 / 1 / 04$ & $10 / 1 / 04$ & 35,000 & 0 \\
\hline SPN87 & SY-102 & $10 / 1 / 04$ & $10 / 1 / 04$ & 2,000 & 0 \\
\hline EVAPF & AP-106 & $10 / 1 / 04$ & $10 / 1 / 04$ & 120,000 & 0 \\
\hline IFL96 & AW-105 & $10 / 1 / 04$ & $10 / 2 / 04$ & 200,000 & 0 \\
\hline WATER & SY-102 & $10 / 2 / 04$ & $10 / 2 / 04$ & 5,000 & 0 \\
\hline WATER & AW-105 & $10 / 2 / 04$ & $10 / 2 / 04$ & 88,000 & 0 \\
\hline WATER & AP-106 & $10 / 2 / 04$ & $10 / 2 / 04$ & 22,000 & 0 \\
\hline WATER & AP-106 & $10 / 2 / 04$ & $10 / 2 / 04$ & 4,000 & 0 \\
\hline WATER & SY'-102 & $10 / 2 / 04$ & $10 / 2 / 04$ & 1,000 & 0 \\
\hline WATER & AP-106 & $10 / 2 / 04$ & $10 / 3 / 04$ & 1,000 & 0 \\
\hline EVAPF & $\mathrm{AW}-102$ & $10 / 4 / 04$ & $10 / 4 / 04$ & 50,000 & 0 \\
\hline AP-106 & $\mathrm{AW}-102$ & $10 / 1 / 04$ & $10 / 5 / 04$ & 917,900 & 251 \\
\hline EVAPF & AW-102 & $11 / 3 / 04$ & $11 / 3 / 04$ & 35,000 & 0 \\
\hline SY-102 & AP-106 & $11 / 15 / 04$ & $11 / 17 / 04$ & 539,800 & 21 \\
\hline $\mathrm{AX}-103$ & AN-104 & $8 / 7 / 04$ & $12 / 24 / 04$ & 310,200 & 2,846 \\
\hline RETURN 2 & AP-107 & $1 / 18 / 05$ & $1 / 18 / 05$ & 8,672 & 332 \\
\hline C-103 & AN-104 & $12 / 24 / 04$ & $3 / 9 / 05$ & 271,800 & 10,423 \\
\hline AP-102 & AP-108 & $3 / 6 / 05$ & $3 / 10 / 05$ & 740,300 & 1,581 \\
\hline WASH-WATER & AP-102 & $3 / 25 / 05$ & $3 / 25 / 05$ & 136,900 & 0 \\
\hline AP-102 & AP-106 & $4 / 8 / 05$ & $4 / 8 / 05$ & 243,100 & 10,337 \\
\hline AW-102 & $242-A$ & $4 / 5 / 05$ & $4 / 10 / 05$ & $1,000,000$ & 34 \\
\hline $242-\mathrm{A}$ & AW-106 & $4 / 5 / 05$ & $4 / 10 / 05$ & 640,000 & 34 \\
\hline $242-\mathrm{A}$ & LERF & $4 / 5 / 05$ & $4 / 10 / 05$ & 360,000 & 0 \\
\hline$A Y-101$ & AP-102 & $4 / 9 / 05$ & $4 / 10 / 05$ & 283,600 & 12 \\
\hline $\mathrm{AW}-106$ & $\mathrm{AW}-102$ & $4 / 11 / 05$ & $4 / 15 / 05$ & 810,400 & 30 \\
\hline P1HLW FEED TANK & HLW-CONTRACTOR & $8 / 8 / 04$ & $5 / 2 / 05$ & 123,900 & 5,202 \\
\hline AZ-101 & P1HLW FEED TANK & $5 / 2 / 05$ & $5 / 2 / 05$ & 127,200 & 3,269 \\
\hline
\end{tabular}


HNF-2321

Revision 0

Table A.4-1. Complete Transfer List for Case 4.

\begin{tabular}{|c|c|c|c|c|c|}
\hline From & To & Start Date & End Date & $\begin{array}{c}\text { Liquid } \\
\text { (gallons) }\end{array}$ & $\begin{array}{c}\text { Solid } \\
\text { (gallons) }\end{array}$ \\
\hline AW-102 & $242-A$ & $4 / 29 / 05$ & $5 / 3 / 05$ & 810,500 & 30 \\
\hline 242-A & AW-106 & $4 / 29 / 05$ & $5 / 3 / 05$ & 519,600 & 30 \\
\hline $242-\mathrm{A}$ & LERF & $4 / 29 / 05$ & $5 / 3 / 05$ & 290,900 & 0 \\
\hline AW-106 & AW-102 & $5 / 3 / 05$ & $5 / 5 / 05$ & 519,500 & 21 \\
\hline $\mathrm{AW}-102$ & 242-A & $5 / 19 / 05$ & $5 / 22 / 05$ & 519,600 & 20 \\
\hline 242-A & $\mathrm{AW}-106$ & $5 / 19 / 05$ & $5 / 22 / 05$ & 333,600 & 20 \\
\hline 242-A & LERF & $5 / 19 / 05$ & $5 / 22 / 05$ & 186,000 & 0 \\
\hline AW-106 & AW-102 & $5 / 22 / 05$ & $5 / 24 / 05$ & 333,600 & 14 \\
\hline RETURN 2 & AP-107 & $6 / 2 / 05$ & $6 / 2 / 05$ & 8,675 & 331 \\
\hline AW-102 & $242-\mathrm{A}^{\prime}$ & $6 / 7 / 05$ & $6 / 8 / 05$ & 333,700 & 14 \\
\hline $242-\mathrm{A}$ & AW-106 & $6 / 7 / 05$ & $6 / 8 / 05$ & 224,900 & 14 \\
\hline $242-\mathrm{A}$ & LERF & $6 / 7 / 05$ & $6 / 8 / 05$ & 108,700 & 0 \\
\hline AW-106 & AW-101 & $6 / 8 / 05$ & $6 / 10 / 05$ & 224,900 & 10 \\
\hline AP-106 & AW-102 & $6 / 9 / 05$ & $6 / 13 / 05$ & 786,000 & 0 \\
\hline $\mathrm{A}-102$ & AN-104 & $3 / 9 / 05$ & $6 / 28 / 05$ & 100,600 & 744 \\
\hline AY-102 & AZ-102 & $8 / 1 / 05$ & $8 / 3 / 05$ & 279,400 & 9,360 \\
\hline AN-104 & AN-105 & $8 / 16 / 05$ & $8 / 17 / 05$ & 197,000 & 5,022 \\
\hline AN-104 & AW-104 & $8 / 17 / 05$ & $8 / 18 / 05$ & 33,797 & 861 \\
\hline AN-104 & AW-103 & $8 / 18 / 05$ & $8 / 18 / 05$ & 3,900 & 99 \\
\hline AN-104 & AP-105 & $8 / 18 / 05$ & $8 / 18 / 05$ & 3,901 & 99 \\
\hline AN-104 & AP-103 & $8 / 18 / 05$ & $8 / 18 / 05$ & 43,402 & 1,104 \\
\hline AN-104 & AP-101 & $8 / 18 / 05$ & $8 / 18 / 05$ & 0 & 0 \\
\hline AN-104 & AN-101 & $8 / 18 / 05$ & $8 / 19 / 05$ & 169,500 & 4,307 \\
\hline AN-104 & AN-106 & $8 / 19 / 05$ & $8 / 19 / 05$ & 3,901 & 99 \\
\hline AN-104 & AW-105 & $8 / 19 / 05$ & $8 / 21 / 05$ & 452.700 & 11,488 \\
\hline TNS88 & AP-106 & $10 / 1 / 05$ & $10 / 1 / 05$ & 750 & 250 \\
\hline WESF & AP-106 & $10 / 1 / 05$ & $10 / 1 / 05$ & 5,000 & 0 \\
\hline ZNL87 & SY -102 & $10 / 1 / 05$ & $10 / 1 / 05$ & 3,000 & 0 \\
\hline TAL88 & AP-106 & $10 / 1 / 05$ & $10 / 1 / 05$ & 24,000 & 0 \\
\hline SPN87 & SY-102 & $10 / 1 / 05$ & $10 / 1 / 05$ & 25,000 & 0 \\
\hline 341,87 & AP-106 & $10 / 1 / 05$ & $10 / 1 / 05$ & 50,000 & 0 \\
\hline WATER & AP-106 & $10 / 1 / 05$ & $10 / 1 / 05$ & 35,000 & 0 \\
\hline EVAPF & AP-106 & $10 / 1 / 05$ & $10 / 1 / 05$ & 120,000 & 0 \\
\hline WATER & SY-102 & $10 / 2 / 05$ & $10 / 2 / 05$ & 1,000 & 0 \\
\hline WATER & AP-106 & $10 / 2 / 05$ & $10 / 2 / 05$ & 5,000 & 0 \\
\hline WATER & SY-102 & $10 / 2 / 05$ & $10 / 2 / 05$ & 5,000 & 0 \\
\hline WATER & AP-106 & $10 / 2 / 05$ & $10 / 2 / 05$ & 22,000 & 0 \\
\hline RETURN 2 & AP-107 & $10 / 10 / 05$ & $10 / 10 / 05$ & 8,679 & 330 \\
\hline $\mathrm{AW}-102$ & 242-A & $10 / 10 / 05$ & $10 / 14 / 05$ & 786,100 & 30 \\
\hline $242-A$ & AW-106 & $10 / 10 / 05$ & $10 / 14 / 05$ & 504,100 & 30 \\
\hline $242-\mathrm{A}$ & LERF & $10 / 10 / 05$ & $10 / 14 / 05$ & 282,000 & 0 \\
\hline AP-104 & $\mathrm{AP}-108$ & $10 / 10 / 05$ & $10 / 14 / 05$ & 740,300 & 1,581 \\
\hline $\mathrm{AW}-106$ & AW-102 & $10 / 14 / 05$ & $10 / 16 / 05$ & 504,000 & 21 \\
\hline WASH-WATER & AP-104 & $10 / 29 / 05$ & $10 / 29 / 05$ & 136,900 & 0 \\
\hline$A W-102$ & $242-\mathrm{A}$ & $10 / 30 / 05$ & $11 / 2 / 05$ & 504,200 & 21 \\
\hline $242-\mathrm{A}$ & AW-106 & $10 / 30 / 05$ & $11 / 2 / 05$ & 324,100 & 21 \\
\hline $242-\mathrm{A}$ & LERF & $10 / 30 / 05$ & $11 / 2 / 05$ & 180,100 & 0 \\
\hline EVAPF & AW-102 & $11 / 3 / 05$ & $11 / 3 / 05$ & 35,000 & 0 \\
\hline AW-106 & AW-101 & $11 / 2 / 05$ & $11 / 4 / 05$ & 318,900 & 15 \\
\hline AP-104 & AP-106 & $11 / 12 / 05$ & $11 / 12 / 05$ & 243,100 & 10,337 \\
\hline AN-107 & AP-104 & $11 / 13 / 05$ & $11 / 15 / 05$ & 481,700 & 22 \\
\hline LERF & AN-107-PUMP & $11 / 13 / 05$ & $11 / 15 / 05$ & 72,259 & 0 \\
\hline AN-107 & $\mathrm{AP}-104$ & $11 / 13 / 05$ & $11 / 15 / 05$ & 554,000 & 22 \\
\hline P1HLW FEED TANK & HLW-CONTRACTOR & $5 / 2 / 05$ & $11 / 16 / 05$ & 127,200 & 3,269 \\
\hline $\mathrm{AZ}-101$ & P1HLW FEED TANK & $11 / 16 / 05$ & $11 / 17 / 05$ & 127,200 & 3,269 \\
\hline RETURN 2 & $A P-107$ & $11 / 29 / 05$ & $11 / 30 / 05$ & 8,677 & 330 \\
\hline
\end{tabular}


HNF-2321

\section{Revision 0}

Table A.4-1. Complete Transfer List for Case 4.

\begin{tabular}{|c|c|c|c|c|c|}
\hline From & To & Start Date & End Date & $\begin{array}{c}\text { Liquid } \\
\text { (gallons) }\end{array}$ & $\begin{array}{c}\text { Solid } \\
\text { (gallons) }\end{array}$ \\
\hline RETURN 2 & AP-107 & $1 / 19 / 06$ & $1 / 19 / 06$ & 8,677 & 330 \\
\hline RETURN 2 & AP-107 & $3 / 10 / 05$ & $3 / 10 / 06$ & 8,677 & 330 \\
\hline RETURN 2 & AP-107 & $4 / 29 / 05$ & $4 / 29 / 06$ & 8,677 & 330 \\
\hline P1HLW FEED TANK & HLW-CONTRACTOR & $11 / 17 / 05$ & $6 / 2 / 06$ & 127,200 & 3,269 \\
\hline$A Z-101$ & PLHLW FEED TANK & $6 / 2 / 06$ & $6 / 3 / 06$ & 127,200 & 3,269 \\
\hline AP-108 & LAW-CONTRACTOR-2 & $6 / 1 / 02$ & $6 / 12 / 06$ & $4,223,000$ & 5,042 \\
\hline AP-102 & AP-108 & $6 / 12 / 05$ & $6 / 13 / 06$ & 277,300 & 552 \\
\hline AN-107 & $\mathrm{AP}-102$ & $6 / 14 / 06$ & $6 / 16 / 06$ & 481,700 & 22 \\
\hline LERF & AN-107-PUMP & $6 / 14 / 06$ & $6 / 16 / 06$ & 72,260 & 0 \\
\hline AN-107 & AP-102 & $6 / 14 / 06$ & $6 / 16 / 06$ & 554,000 & 22 \\
\hline RETURN 2 & AP-107 & $6 / 18 / 06$ & $6 / 18 / 06$ & 8,683 & 330 \\
\hline AZ-102 & AP-106 & $6 / 23 / 06$ & $6 / 24 / 06$ & 159,500 & 0 \\
\hline WASH-WATER & AZ-102 & $6 / 24 / 06$ & $6 / 26 / 06$ & 324,000 & 0 \\
\hline RETURN 2 & AP-107 & $7 / 24 / 06$ & $7 / 24 / 06$ & 8,678 & 328 \\
\hline AP-108 & LAW-CONTRACTOR-2 & $6 / 14 / 06$ & $8 / 12 / 06$ & 277,300 & 555 \\
\hline AP-104 & AP-108 & $8 / 12 / 06$ & $8 / 13 / 06$ & 171,100 & 337 \\
\hline AP-106 & AW-102 & $8 / 16 / 06$ & $8 / 21 / 06$ & 846,800 & 0 \\
\hline AZ-102 & AP-106 & $8 / 14 / 06$ & $8 / 21 / 06$ & 320,100 & 0 \\
\hline WASH-WATER & $A Z-102$ & $8 / 22 / 06$ & $8 / 24 / 06$ & 324,000 & 0 \\
\hline RETURN 2 & AP-107 & $9 / 8 / 06$ & $9 / 8 / 06$ & 8,684 & 329 \\
\hline AP-104 & $\mathrm{AP}-108$ & $9 / 9 / 06$ & $9 / 11 / 06$ & 381,800 & 753 \\
\hline AN-102 & AP-104 & $9 / 12 / 06$ & $9 / 15 / 06$ & 443,500 & 21 \\
\hline LERF. & AN-102-PUMP & $9 / 12 / 06$ & $9 / 15 / 06$ & 266,100 & 0 \\
\hline AN-102 & AP-104 & $9 / 12 / 06$ & $9 / 15 / 06$ & 709,700 & 21 \\
\hline $\mathrm{AN}-104$ & AN-107 & $9 / 14 / 06$ & $9 / 16 / 06$ & 213,900 & 5,416 \\
\hline TNS88 & AP-106 & $10 / 1 / 06$ & $10 / 1 / 06$ & 750 & 250 \\
\hline $34 \mathrm{~L} 87$ & AP-106 & $10 / 1 / 06$ & $10 / 1 / 06$ & 7,000 & 0 \\
\hline WESF & AP-106 & $10 / 1 / 06$ & $10 / 1 / 06$ & 5,000 & 0 \\
\hline TAL88 & AP-106 & $10 / 1 / 06$ & $10 / 1 / 06$ & 25,000 & 0 \\
\hline SPN87 & SY-102 & $10 / 1 / 06$ & $10 / 1 / 06$ & 25,000 & 0 \\
\hline $34 \mathrm{~L} 87$ & AP-106 & $10 / 1 / 06$ & $10 / 1 / 06$ & 50,000 & 0 \\
\hline WATER & AP-106 & $10 / 1 / 06$ & $10 / 1 / 06$ & 35,000 & 0 \\
\hline EVAPF & AP-106 & $10 / 1 / 06$ & $10 / 1 / 06$ & 120,000 & 0 \\
\hline WATER & AP-106 & $10 / 2 / 06$ & $10 / 2 / 06$ & 5,000 & 0 \\
\hline WATER & AP-106 & $10 / 2 / 06$ & $10 / 2 / 06$ & 22,000 & 0 \\
\hline WATER & SY-102 & $10 / 2 / 06$ & $10 / 2 / 06$ & 5,000 & 0 \\
\hline \begin{tabular}{|l} 
WATER \\
\end{tabular} & AP-106 & $10 / 2 / 06$ & $10 / 2 / 06$ & 3,000 & 0 \\
\hline EVAPF & AW-102 & $10 / 4 / 06$ & $10 / 4 / 06$ & 50,000 & 0 \\
\hline $\mathrm{AZ}-102$ & AP-106 & $10 / 12 / 06$ & $10 / 13 / 06$ & 322,700 & 0 \\
\hline WASH-WATER & $A Z-102$ & $10 / 15 / 06$ & $10 / 15 / 06$ & 150,500 & 0 \\
\hline RETURN 2 & AP-107 & $11 / 2 / 06$ & $11 / 2 / 06$ & 8,683 & 330 \\
\hline EVAPF & AW-102 & $11 / 3 / 06$ & $11 / 3 / 06$ & 35,000 & 0 \\
\hline $\mathrm{C}-105$ & AN-104 & $6 / 28 / 05$ & $11 / 13 / 06$ & 827,500 & 31,875 \\
\hline P1HLW FEED TANK & HLW-CONTRACTOR & $6 / 3 / 06$ & $12 / 18 / 06$ & 127,200 & 3,269 \\
\hline \begin{tabular}{|l}
$\mathrm{AZ}-101$ \\
\end{tabular} & P1EILW FEED TANK & $12 / 18 / 06$ & $12 / 18 / 06$ & 127,200 & 3,269 \\
\hline RETURN 2 & AP-107 & $12 / 28 / 06$ & $12 / 28 / 06$ & 8,683 & 330 \\
\hline $\mathrm{AW}-102$ & $242-\mathrm{A}$ & $12 / 26 / 06$ & $12 / 31 / 06$ & 967,000 & 34 \\
\hline $242-\mathrm{A}$ & AW-106 & $12 / 26 / 06$ & $12 / 31 / 06$ & 619,000 & 34 \\
\hline $242-\mathrm{A}$ & LERF & $12 / 26 / 06$ & $12 / 31 / 06$ & 348,000 & 0 \\
\hline AW-106 & $A W-102$ & $12 / 31 / 06$ & $1 / 3 / 07$ & 624,100 & 23 \\
\hline AW-102 & $242-\mathrm{A}$ & $1 / 17 / 07$ & $1 / 20 / 07$ & 624,200 & 23 \\
\hline $242-\mathrm{A}$ & AW-106 & $1 / 17 / 07$ & $1 / 20 / 07$ & 400,200 & 23 \\
\hline $242-\mathrm{A}$ & LERF & $1 / 17 / 07$ & $1 / 20 / 07$ & 224,000 & 0 \\
\hline AW-106 & $\mathrm{AW}-102$ & $1 / 20 / 07$ & $1 / 22 / 07$ & 400,100 & 15 \\
\hline AP-102 & AP-108 & $1 / 20 / 07$ & $1 / 23 / 07$ & 559,200 & 575 \\
\hline $\mathrm{AN}-103$ & AP-102 & $1 / 24 / 07$ & $1 / 27 / 07$ & 436,600 & 28,133 \\
\hline
\end{tabular}


HNF-2321

Revision 0

Table A.4-1. Complete Transfer List for Case 4.

\begin{tabular}{|c|c|c|c|c|c|}
\hline From & To & Start Date & End Date & $\begin{array}{c}\text { Liquid } \\
\text { (gallons) }\end{array}$ & $\begin{array}{c}\text { Solid } \\
\text { (gallons) }\end{array}$ \\
\hline LERF & AN-103-PUMP & $1 / 24 / 07$ & $1 / 27 / 07$ & 264,900 & 0 \\
\hline AN-103 & AP-102 & $1 / 24 / 07$ & $1 / 27 / 07$ & 738,300 & 549 \\
\hline$A W-102$ & $242-\mathrm{A}$ & $2 / 6 / 07$ & $2 / 8 / 07$ & 400,200 & 15 \\
\hline $242-\mathrm{A}$ & AW-106 & $2 / 6 / 07$ & $2 / 8 / 07$ & 256,200 & 15 \\
\hline $242-\mathrm{A}$ & LERF & $2 / 6 / 07$ & $2 / 8 / 07$ & 144,000 & 0 \\
\hline AW-106 & AW-102 & $2 / 8 / 07$ & $2 / 9 / 07$ & 256,200 & 10 \\
\hline A-104 & AN-104 & $11 / 13 / 06$ & $2 / 22 / 07$ & 29,827 & 832. \\
\hline RETURN 2 & AP-107 & $2 / 23 / 07$ & $2 / 24 / 07$ & 8,673 & 330 \\
\hline AW-102 & $242-\mathrm{A}$ & $2 / 23 / 07$ & $2 / 24 / 07$ & 256,300 & 10 \\
\hline $242-\mathrm{A}$ & AW-106 & $2 / 23 / 07$ & $2 / 24 / 07$ & 164,300 & 10 \\
\hline $242-\mathrm{A}$ & LERF & $2 / 23 / 07$ & $2 / 24 / 07$ & 91,975 & 0 \\
\hline$A W-106$ & AW-102 & $2 / 24 / 07$ & $2 / 25 / 07$ & 164,300 & 7 \\
\hline AW-102 & AP-106 & $2 / 26 / 07$ & $2 / 27 / 07$ & 164,400 & 7 \\
\hline C-204 & AN-104 & $2 / 22 / 07$ & $3 / 1 / 07$ & 3,775 & 55 \\
\hline AP-106 & $A W-102$ & $2 / 27 / 07$ & $3 / 3 / 07$ & 776,900 & 0 \\
\hline C-201 & AN-104 & $3 / 1 / 07$ & $3 / 7 / 07$ & 2,464 & 59 \\
\hline $\mathrm{AX}-104$ & AN-104 & $3 / 7 / 07$ & $4 / 12 / 07$ & 18,516 & 572 \\
\hline RETURN 2 & AP-107 & $4 / 23 / 07$ & $4 / 23 / 07$ & 8,672 & 330 \\
\hline RETURN 2 & AP-107 & $6 / 20 / 07$ & $6 / 20 / 07$ & 8,672 & 330 \\
\hline P1HLW FEED TANK & HLW-CONTRACTOR & $12 / 18 / 06$ & $7 / 4 / 07$ & 127,200 & 3,269 \\
\hline AZ-101 & PIHLW FEED TANK & $7 / 4 / 07$ & $7 / 5 / 07$ & 127,200 & 3,269 \\
\hline $\mathrm{AX}-102$ & AN-104 & $4 / 12 / 07$ & $7 / 31 / 07$ & 87,597 & 897 \\
\hline AP-104 & AP-108 & $8 / 3 / 07$ & $8 / 7 / 07$ & 709,600 & 72 \\
\hline $\mathrm{AN}-103$ & AP-104 & $8 / 8 / 07$ & $8 / 11 / 07$ & 436,600 & 28,133 \\
\hline LERF & AN-103-PUMP & $8 / 8 / 07$ & $8 / 11 / 07$ & 264,900 & 0 \\
\hline AN-103 & AP-104 & $8 / 8 / 07$ & $8 / 11 / 07$ & 738,300 & 549 \\
\hline RETURN_2 & AP-107 & $8 / 27 / 07$ & $8 / 27 / 07$ & 8,675 & 330 \\
\hline$A W-102$ & $242-\mathrm{A}$ & $8 / 31 / 07$ & $9 / 4 / 07$ & 776,800 & 28 \\
\hline $242-\mathrm{A}$ & AW-106 & $8 / 31 / 07$ & $9 / 4 / 07$ & 497,600 & 28 \\
\hline $242-\mathrm{A}$ & LERF & $8 / 31 / 07$ & 9/4/07 & 279,200 & 0 \\
\hline AW-106 & AW-102 & $9 / 4 / 07$ & $9 / 7 / 07$ & 497,500 & 19 \\
\hline$\overline{A W-102}$ & $242-\mathrm{A}$ & $9 / 21 / 07$ & 9/23/07 & 497,600 & 19 \\
\hline $242-\mathrm{A}$ & AW-106 & $9 / 21 / 07$ & 9/23/07 & 317,600 & 19 \\
\hline $242-\mathrm{A}$ & LERF & 9/21/07 & 9/23/07 & 180,000 & 0 \\
\hline AW-106 & AW-102 & 9/23/07 & $9 / 25 / 07$ & 317,600 & 13 \\
\hline \begin{tabular}{|l|} 
TNS88 \\
\end{tabular} & AP-106 & $10 / 1 / 07$ & $10 / 1 / 07$ & 750 & 250 \\
\hline WESF & AP-106 & $10 / 1 / 07$ & $10 / 1 / 07$ & 5,000 & 0 \\
\hline SPN87 & $S Y-102$ & $10 / 1 / 07$ & $10 / 1 / 07$ & 25,000 & 0 \\
\hline \begin{tabular}{|l|} 
TAL88 \\
\end{tabular} & AP-106 & $10 / 1 / 07$ & $10 / 1 / 07$ & 25,000 & 0 \\
\hline $34 L 87$ & AP-106 & $10 / 1 / 07$ & $10 / 1 / 07$ & 50,000 & 0 \\
\hline WATER & AP-106 & $10 / 1 / 07$ & $10 / 1 / 07$ & 35,000 & 0 \\
\hline EVAPF & AP-106 & $10 / 1 / 07$ & $10 / 1 / 07$ & 120,000 & 0 \\
\hline WATER & AP-106 & $10 / 2 / 07$ & $10 / 2 / 07$ & 5,000 & 0 \\
\hline WATER & AP-106 & $10 / 2 / 07$ & $10 / 2 / 07$ & 22,000 & 0 \\
\hline WATER & SY-102 & $10 / 2 / 07$ & $10 / 2 / 07$ & 5,000 & 0 \\
\hline AW-102 & $242-A$ & $10 / 9 / 07$ & $10 / 11 / 07$ & 317,600 & 13 \\
\hline $242-\mathrm{A}$ & AW-106 & $10 / 9 / 07$ & $10 / 11 / 07$ & 203,600 & 13 \\
\hline $242-\mathrm{A}$ & LERF & $10 / 9 / 07$ & $10 / 11 / 07$ & 114,000 & 0 \\
\hline$A W-106$ & AW-102 & $10 / 11 / 07$ & $10 / 12 / 07$ & 203,600 & 9 \\
\hline AW-102 & AP-106 & $10 / 13 / 07$ & $10 / 14 / 07$ & 203,800 & 9 \\
\hline EVAPF & $A W-102$ & $11 / 3 / 07$ & $11 / 3 / 07$ & 35,000 & 0 \\
\hline AN-104 & AN-107 & $11 / 4 / 07$ & $11 / 7 / 07$ & 750,600 & 14,520 \\
\hline AN-104 & $\mathrm{AN}-102$ & $11 / 7 / 07$ & 11/9/07 & 349,400 & 6,751 \\
\hline SY-101 & AN-103 & $11 / 9 / 07$ & $11 / 13 / 07$ & $1,105,000$ & 11,018 \\
\hline AN-103 & AN-102 & $11 / 14 / 07$ & $11 / 15 / 07$ & 150,700 & 1,688 \\
\hline RETURN 2 & AP-107 & $12 / 3 / 07$ & $12 / 3 / 07$ & 8.675 & 329 \\
\hline
\end{tabular}


HNF-2321

Revision 0

Table A.4-1. Complete Transfer List for Case 4.

\begin{tabular}{|c|c|c|c|c|c|}
\hline From & To & Start Date & End Date & $\begin{array}{c}\text { Liquid } \\
\text { (gallons) }\end{array}$ & $\begin{array}{c}\text { Solid } \\
\text { (gallons) }\end{array}$ \\
\hline P1HLW FEED TANK & HLW-CONTRACTOR & $7 / 5 / 07$ & $1 / 18 / 08$ & 127,200 & 3,269 \\
\hline$A Z-102$ & PIHLW FEED TANK & $1 / 18 / 08$ & $1 / 19 / 08$ & 137,700 & 4,774 \\
\hline RETURN 2 & AP-107 & $3 / 11 / 08$ & $3 / 11 / 08$ & 8,675 & 329 \\
\hline AP-108 & LAW-CONTRACTOR-2 & $8 / 14 / 06$ & $4 / 10 / 08$ & $1,822,000$ & 1,786 \\
\hline $\mathrm{AP}-102$ & AP-108 & $4 / 10 / 08$ & $4 / 14 / 08$ & 738,300 & 557 \\
\hline$S-103$ & SY-101 & $12 / 31 / 03$ & $5 / 6 / 08$ & 712,300 & 1,011 \\
\hline RETURN 2 & AP-107 & $8 / 17 / 08$ & $8 / 18 / 08$ & 8,672 & 330 \\
\hline TNS88 & AP-106 & $10 / 1 / 08$ & $10 / 1 / 08$ & 750 & 250 \\
\hline WESF & AP-106 & $10 / 1 / 08$ & $10 / 1 / 08$ & 5,000 & 0 \\
\hline TAL88 & AP-106 & $10 / 1 / 08$ & $10 / 1 / 08$ & 25,000 & 0 \\
\hline SPN87 & SY-102 & $10 / 1 / 08$ & $10 / 1 / 08$ & 25,000 & 0 \\
\hline $34 \mathrm{~L} 87$ & AP-106 & $10 / 1 / 08$ & $10 / 1 / 08$ & 50,000 & 0 \\
\hline WATER & AP-106 & $10 / 1 / 08$ & $10 / 1 / 08$ & 35,000 & 0 \\
\hline EVAPF & $\mathrm{AP}-106$ & $10 / 1 / 08$ & $10 / 1 / 08$ & 120,000 & 0 \\
\hline WATER & AP-106 & $10 / 2 / 08$ & $10 / 2 / 08$ & 5,000 & 0 \\
\hline WATER & AP-106 & $10 / 2 / 08$ & $10 / 2 / 08$ & 22,000 & 0 \\
\hline WATER & $5 Y-102$ & $10 / 2 / 08$ & $10 / 2 / 08$ & 5,000 & 0 \\
\hline EVAPF & AW-102 & $10 / 4 / 08$ & $10 / 4 / 08$ & 50,000 & 0 \\
\hline AP-106 & AW-102 & $10 / 1 / 08$ & $10 / 5 / 08$ & 723,500 & 0 \\
\hline EVAPF & $A W-102$ & $11 / 3 / 08$ & $11 / 3 / 08$ & 35,000 & 0 \\
\hline PIHLW FEED TANK & HLW-CONTRACTOR & $1 / 19 / 08$ & $12 / 22 / 08$ & 137,700 & 4,774 \\
\hline $\mathrm{AZ}-102$ & P1HLW FEED TANK & $12 / 22 / 08$ & $12 / 22 / 08$ & 137,700 & 4,774 \\
\hline AP-104 & AP-108 & $1 / 5 / 09$ & $1 / 9 / 09$ & 738,400 & 532 \\
\hline $\mathrm{U}-202$ & UA-2 & $1 / 3 / 09$ & $1 / 10 / 09$ & 27,411 & 1,061 \\
\hline $\mathrm{U}-203$ & UA-2 & $1 / 10 / 09$ & $1 / 16 / 09$ & 13,895 & 537 \\
\hline RETURN 2 & $A P-107$ & $2 / 16 / 09$ & $2 / 16 / 09$ & 8,670 & 331 \\
\hline $\mathrm{U}-110$ & UA-2 & $1 / 16 / 09$ & $3 / 10 / 09$ & 99,290 & 4,252 \\
\hline $\mathrm{UA}-2$ & SY-102 & $3 / 11 / 09$ & $3 / 12 / 09$ & 173,900 & 5,847 \\
\hline AW-102 & $242-\mathrm{A}$ & $4 / 4 / 09$ & $4 / 8 / 09$ & 843,400 & 30 \\
\hline $242-\mathrm{A}$ & AW-106 & $4 / 4 / 09$ & $4 / 8 / 09$ & 540,700 & 30 \\
\hline $242-\mathrm{A}$ & LERF & $4 / 4 / 09$ & $4 / 8 / 09$ & 302,800 & 0 \\
\hline AW-106 & AW-102 & $4 / 9 / 09$ & $4 / 12 / 09$ & 540,500 & 20 \\
\hline AW-102 & $242-\mathrm{A}$ & $4 / 26 / 09$ & $4 / 29 / 09$ & 540,700 & 20 \\
\hline $242-\mathrm{A}$ & AW-106 & $4 / 26 / 09$ & 4/29/09 & 346,600 & 20 \\
\hline $242-\mathrm{A}$ & LERF & $4 / 26 / 09$ & $4 / 29 / 09$ & 194,100 & 0 \\
\hline AW-106 & AW-102 & $4 / 29 / 09$ & $4 / 30 / 09$ & 346,500 & 14 \\
\hline AW-102 & $242-\mathrm{A}$ & $5 / 15 / 09$ & $5 / 16 / 09$ & 346,600 & 14 \\
\hline $242-\mathrm{A}$ & AW-106 & $5 / 15 / 09$ & $5 / 16 / 09$ & 221,100 & 14 \\
\hline $242-\mathrm{A}$ & LERF & $5 / 15 / 09$ & $5 / 16 / 09$ & 125,500 & 0 \\
\hline AW-106 & AW-102 & $5 / 16 / 09$ & $5 / 17 / 09$ & 221,100 & 10 \\
\hline AW-102 & AP-106 & $5 / 19 / 09$ & $5 / 20 / 09$ & 221,200 & 9 \\
\hline \begin{tabular}{|l}
$\mathrm{U}-110$ \\
\end{tabular} & UA-1 & $3 / 10 / 09$ & $5 / 27 / 09$ & 143,800 & 6,160 \\
\hline $\mathrm{UA}-1$ & SY-102 & $5 / 27 / 09$ & $5 / 28 / 09$ & 186,800 & 6,157 \\
\hline $\mathrm{U}-110$ & UA-2 & $5 / 27 / 09$ & $5 / 30 / 09$ & 4,829 & 207 \\
\hline U-101 & UA-2 & $5 / 30 / 09$ & $7 / 29 / 09$ & 27,955 & 668 \\
\hline RETURN 2 & AP-107 & $8 / 17 / 09$ & $8 / 17 / 09$ & 8,670 & 331 \\
\hline TNS88 & AP-106 & $10 / 1 / 09$ & $10 / 1 / 09$ & 750 & 250 \\
\hline WESF & AP-106 & $10 / 1 / 09$ & $10 / 1 / 09$ & 5,000 & 0 \\
\hline SPN87 & SY-102 & $10 / 1 / 09$ & $10 / 1 / 09$ & 25,000 & 0 \\
\hline \begin{tabular}{|l} 
TAL 88 \\
\end{tabular} & AP-106 & $10 / 1 / 09$ & $10 / 1 / 09$ & 26,000 & 0 \\
\hline WATER & AP-106 & $10 / 1 / 09$ & $10 / 1 / 09$ & 35,000 & 0 \\
\hline $34 \mathrm{~L} 87$ & AP-106 & $10 / 1 / 09$ & $10 / 1 / 09$ & 50,000 & 0 \\
\hline $34 \mathrm{~L} 87$ & $\mathrm{AP}-106$ & $10 / 1 / 09$ & $10 / 1 / 09$ & 7,000 & 0 \\
\hline EVAPF & AP-106 & $10 / 1 / 09$ & $10 / 1 / 09$ & 120,000 & 0 \\
\hline \begin{tabular}{|l|} 
WATER \\
\end{tabular} & AP-106 & $10 / 2 / 09$ & $10 / 2 / 09$ & 3,000 & 0 \\
\hline WATER & AP-105 & $10 / 2 / 09$ & $10 / 2 / 09$ & 22,000 & 0 \\
\hline
\end{tabular}


HNF-2321

Revision 0

Table A.4-1. Complete Transfer List for Case 4.

\begin{tabular}{|c|c|c|c|c|c|}
\hline From & To & Start Date & End Date & $\begin{array}{c}\text { Liquid } \\
\text { (gallons) }\end{array}$ & $\begin{array}{c}\text { Solid } \\
\text { (gallons) }\end{array}$ \\
\hline WATER & AP-106 & $10 / 2 / 09$ & $10 / 2 / 09$ & 5,000 & 0 \\
\hline WATER & SY-102 & $10 / 2 / 09$ & $10 / 2 / 09$ & 5,000 & 0 \\
\hline EVAPF & AW-102 & $11 / 3 / 09$ & $11 / 3 / 09$ & 35,000 & 0 \\
\hline U-104. & UA-2 & $7 / 29 / 09$ & $11 / 9 / 09$ & 50,908 & 1,221 \\
\hline P1HLW FEED TANK & HLW-CONTRACTOR & $12 / 22 / 08$ & $11 / 25 / 09$ & 137,700 & 4,774 \\
\hline AP-108 & LAW-CONTRACTOR-2 & $4 / 14 / 08$ & $11 / 28 / 09$ & $1,477,000$ & 1,076 \\
\hline RETURN I & AP-107 & $11 / 29 / 09$ & $11 / 29 / 09$ & 0 & 0 \\
\hline RETURN 2 & AP-107 & $11 / 29 / 09$ & $11 / 29 / 09$ & 4,912 & 188 \\
\hline $\mathrm{U}-112$ & UA-2 & $11 / 9 / 09$ & $12 / 4 / 09$ & 58,521 & 2,330 \\
\hline UA-2 & SY-102 & $12 / 5 / 09$ & $12 / 6 / 09$ & 154,400 & 4,426 \\
\hline AP-107 & AZ-101 & $12 / 9 / 09$ & $12 / 11 / 09$ & 395,500 & 7,596 \\
\hline AN-103 & AP-107 & $12 / 19 / 09$ & $12 / 23 / 09$ & 954,500 & 10,690 \\
\hline AN-104 & AP-107 & $12 / 23 / 09$ & $12 / 24 / 09$ & 150,900 & 481 \\
\hline SY-101 & $\mathrm{AN}-103$ & $12 / 23 / 09$ & $12 / 27 / 09$ & $1,115,000$ & 1,877 \\
\hline$U-112$ & UA-1 & $12 / 4 / 09$ & $1 / 17 / 10$ & 102,000 & 4,061 \\
\hline U-204 & UA-1 & $1 / 17 / 10$ & $1 / 23 / 10$ & 13,004 & 503 \\
\hline U-201 & UA-1 & $1 / 23 / 10$ & $1 / 27 / 10$ & 13,912 & 538 \\
\hline UA-1 & SY-102 & $1 / 27 / 10$ & $1 / 28 / 10$ & 146,500 & 5,102 \\
\hline U-201 & UA-2 & $1 / 27 / 10$ & $1 / 30 / 10$ & 13,499 & 522 \\
\hline U-106 & UA-2 & $1 / 30 / 10$ & $3 / 1 / 10$ & 134,400 & 338 \\
\hline U-106 & UA-1 & $3 / 1 / 10$ & $4 / 4 / 10$ & 149,500 & 376 \\
\hline S-10S & SY-101 & $5 / 6 / 08$ & $7 / 18 / 10$ & $1,518,000$ & 2,459 \\
\hline TNS88 & AP-106 & $10 / 1 / 10$ & $10 / 1 / 10$ & 750 & 250 \\
\hline WESF & AP-106 & $10 / 1 / 10$ & $10 / 1 / 10$ & 5,000 & 0 \\
\hline SPN87 & SY-102 & $10 / 1 / 10$ & $10 / 1 / 10$ & 25,000 & 0 \\
\hline $34 \mathrm{~L} 87$ & AP-106 & $10 / 1 / 10$ & $10 / 1 / 10$ & 50,000 & 0 \\
\hline WATER & AP-106 & $10 / 1 / 10$ & $10 / 1 / 10$ & 35,000 & 0 \\
\hline WATER & AP-106 & $10 / 1 / 10$ & $10 / 1 / 10$ & 25,000 & 0 \\
\hline TAL88 & AP-106 & $10 / 1 / 10$ & $10 / 1 / 10$ & 26,000 & 0 \\
\hline EVAPF & AP-106 & $10 / 1 / 10$ & $10 / 1 / 10$ & 120,000 & 0 \\
\hline WATER & AP-106 & $10 / 2 / 10$ & $10 / 2 / 10$ & 5,000 & 0 \\
\hline WATER & SY-102 & $10 / 2 / 10$ & $10 / 2 / 10$ & 5,000 & 0 \\
\hline WATER & AP-106 & $10 / 2 / 10$ & $10 / 2 / 10$ & 22,000 & 0 \\
\hline EVAPF & AW-102 & $10 / 4 / 10$ & $10 / 4 / 10$ & 50,000 & 0 \\
\hline AP-106 & AW-102 & $10 / 1 / 10$ & $10 / 5 / 10$ & 778,300 & 0 \\
\hline EVAPF & AW-102 & $11 / 3 / 10$ & $11 / 3 / 10$ & 35,000 & 0 \\
\hline WATER & AP-106 & $1 / 1 / 11$ & $1 / 1 / 11$ & 25,000 & 0 \\
\hline BY-110 & $\mathrm{BA}-3$ & $1 / 3 / 11$ & $2 / 8 / 11$ & 147,400 & 2,633 \\
\hline TX-109 & TA-5 & $1 / 3 / 11$ & $2 / 9 / 11$ & 144,100 & 5,925 \\
\hline TY-101 & TA-4 & $1 / 3 / 11$ & $2 / 22 / 11$ & 146,500 & 3,494 \\
\hline T-106 & TA-6 & $1 / 3 / 11$ & $3 / 2 / 11$ & 74,648 & 2,929 \\
\hline$B-112$ & BA-1 & $1 / 3 / 11$ & $3 / 8 / 11$ & 75,213 & 1,083 \\
\hline$B Y-110$ & $\mathrm{BA}-4$ & $2 / 8 / 11$ & $3 / 16 / 11$ & 147,400 & 2,633 \\
\hline TX-109 & TA-3 & $2 / 9 / 11$ & $3 / 19 / 11$ & 144,100 & 5,925 \\
\hline $\mathrm{T}-101$ & TA-6 & $3 / 2 / 11$ & $3 / 25 / 11$ & 61,377 & 2,346 \\
\hline WATER & AP-106 & $4 / 1 / 11$ & $4 / 1 / 11$ & 25,000 & 0 \\
\hline BX-102 & $\mathrm{BA}-2$ & $1 / 3 / 11$ & $4 / 4 / 11$ & 52,548 & 2,443 \\
\hline B-111 & $\mathrm{BA-1}$ & $3 / 8 / 11$ & $4 / 9 / 11$ & 72,630 & 2,383 \\
\hline$A W-102$ & $242-\mathrm{A}$ & $4 / 5 / 11$ & $4 / 9 / 11$ & 898,300 & 32 \\
\hline $242-\mathrm{A}$ & AW-106 & $4 / 5 / 11$ & $4 / 9 / 11$ & 574,300 & 32 \\
\hline $242-A$ & LERF & $4 / 5 / 11$ & $4 / 9 / 11$ & 324,000 & 0 \\
\hline AW-106 & AW-102 & $4 / 10 / 11$ & $4 / 13 / 11$ & 574,100 & 22 \\
\hline TY-101 & TA-2 & $2 / 22 / 11$ & $4 / 13 / 11$ & 146,500 & 3,494 \\
\hline BY-110 & BA-5 & $3 / 16 / 11$ & $4 / 21 / 11$ & 147,400 & 2,633 \\
\hline TX-109 & TA-1 & $3 / 19 / 11$ & $4 / 26 / 11$ & 144,100 & 5,925 \\
\hline$A \bar{W}-102$ & $242-A$ & $4 / 27 / 11$ & $4 / 30 / 11$ & 574,300 & 22 \\
\hline
\end{tabular}


HNF-2321

Revision 0

Table A.4-1. Complete Transfer List for Case 4.

\begin{tabular}{|c|c|c|c|c|c|}
\hline From & To & Start Date & End Date & $\begin{array}{c}\text { Liquid } \\
\text { (gallons) }\end{array}$ & $\begin{array}{c}\text { Solid } \\
\text { (gallons) }\end{array}$ \\
\hline $242-A$ & AW-106 & $4 / 27 / 11$ & $4 / 30 / 11$ & 368,200 & 22 \\
\hline $242-\mathrm{A}$ & LERF & $4 / 27 / 11$ & $4 / 30 / 11$ & 206,100 & 0 \\
\hline AW-106 & AW-102 & $4 / 30 / 11$ & $5 / 1 / 11$ & 368,100 & 15 \\
\hline BX-112 & $\mathrm{BA}-2$ & $4 / 4 / 11$ & $5 / 2 / 11$ & 67,370 & 2,937 \\
\hline \begin{tabular}{|l|}
$\mathrm{AW}-102$ \\
\end{tabular} & $242-\mathrm{A}$ & $5 / 15 / 11$ & $5 / 17 / 11$ & 368,200 & 15 \\
\hline $242-\mathrm{A}$ & AW-106 & $5 / 15 / 11$ & $5 / 17 / 11$ & 236,200 & 15 \\
\hline $242-\mathrm{A}$ & LERF & $5 / 15 / 11$ & $5 / 17 / 11$ & 132,000 & 0 \\
\hline AW-106 & $A W-102$ & $5 / 17 / 11$ & $5 / 19 / 11$ & 236,200 & 10 \\
\hline $\mathrm{BA}-3$ & AP-108 & $6 / 1 / 11$ & $6 / 2 / 11$ & 155,600 & 2,631 \\
\hline$B A-4$ & AP-108 & $6 / 2 / 11$ & $6 / 2 / 11$ & 155,600 & 2,631 \\
\hline$A W-102$ & 242-A & $6 / 2 / 11$ & $6 / 3 / 11$ & 236,200 & 10 \\
\hline $242-\mathrm{A}$ & AW-106 & $6 / 2 / 11$ & $6 / 3 / 11$ & 164,800 & 10 \\
\hline $242-\mathrm{A}$ & LERF & $6 / 2 / 11$ & $6 / 3 / 11$ & 71,427 & 0 \\
\hline $\mathrm{BA}-1$ & AP-108 & $6 / 2 / 11$ & $6 / 3 / 11$ & 157,300 & 3,464 \\
\hline $\mathrm{BA}-5$ & AP-108 & $6 / 3 / 11$ & $6 / 4 / 11$ & 155,600 & 2,631 \\
\hline$B A-2$ & AP-108 & $6 / 4 / 11$ & $6 / 5 / 11$ & 174,500 & 5,377 \\
\hline AN-104 & AP-102 & $6 / 1 / 11$ & $6 / 6 / 11$ & 972,200 & 3,098 \\
\hline AN-103 & AP-104 & $6 / 1 / 11$ & $6 / 6 / 11$ & $1,114,000$ & 2,128 \\
\hline AN-103 & AP-102 & $6 / 6 / 11$ & $6 / 6 / 11$ & 998 & 2 \\
\hline SY-103 & AN-103 & $6 / 6 / 11$ & $6 / 10 / 11$ & $1,084,000$ & 32,737 \\
\hline B-111 & $\mathrm{BA}-6$ & $4 / 9 / 11$ & $6 / 11 / 11$ & 145,200 & 4,764 \\
\hline AN-103 & AP-102 & $6 / 11 / 11$ & $6 / 12 / 11$ & 136,200 & 4,016 \\
\hline BA-6 & AP-108 & $6 / 11 / 11$ & $6 / 12 / 11$ & 159,400 & 4,762 \\
\hline AN-103 & AP-108 & $6 / 12 / 11$ & $6 / 13 / 11$ & 133,100 & 3,925 \\
\hline WATER & AP-106 & $7 / 1 / 11$ & $7 / 1 / 11$ & 25,000 & 0 \\
\hline BY-110 & $\mathrm{BA}-4$ & $6 / 2 / 11$ & $7 / 8 / 11$ & 147,300 & 2,631 \\
\hline $\mathrm{BX}-112$ & $\mathrm{BA}-3$ & $6 / 2 / 11$ & $8 / 2 / 11$ & 143,600 & 6,262 \\
\hline B-111 & BA-1 & $6 / 11 / 11$ & $8 / 12 / 11$ & 145,100 & 4,761 \\
\hline B-111 & BA-6 & $8 / 12 / 11$ & $8 / 13 / 11$ & 719 & 24 \\
\hline BY-110 & $\mathrm{BA}-5$ & $7 / 8 / 11$ & $8 / 13 / 11$ & 147,300 & 2,631 \\
\hline $\mathrm{SX}-112$ & SY-103 & $4 / 6 / 04$ & $8 / 27 / 11$ & 397,100 & 16,406 \\
\hline BX-112 & BA-2 & $8 / 2 / 11$ & $9 / 4 / 11$ & 78,662 & 3,430 \\
\hline BY-110 & $\mathrm{BA}-2$ & $9 / 4 / 11$ & $9 / 14 / 11$ & 40,856 & 730 \\
\hline AX-10I & AN-104 & $7 / 31 / 07$ & $9 / 30 / 11$ & $2,149,000$ & 6,072 \\
\hline
\end{tabular}


Table A.4-2. Phase 1 Low-Activity Waste Feed Staging Transfers for Case 4.

\begin{tabular}{|c|c|c|c|c|c|c|c|}
\hline $\begin{array}{l}\text { Boot } \\
\text { Strap }\end{array}$ & From & To & Start Date & End Date & $\begin{array}{c}\text { Liquid } \\
\text { (gallons) }\end{array}$ & $\begin{array}{c}\text { Solid } \\
\text { (gallons) }\end{array}$ & Notes \\
\hline $\mathrm{X}$ & AP-102 & AP-103 & $10 / 25 / 99$ & $10 / 30 / 99$ & $1,070,000$ & 0 & Empty 2AP \\
\hline $\mathrm{x}$ & AP-104 & AW-102 & $7 / 25 / 00$ & $7 / 30 / 00$ & $1,110,000$ & 43 & Empty 4AP \\
\hline \multirow[t]{7}{*}{$\mathrm{X}$} & AZ-101 & AY-101 & $8 / 17 / 00$ & $8 / 20 / 00$ & 685,000 & 0 & Pre-Stage Envelope B \\
\hline & WASH-WATER & AP-104 & $10 / 1 / 00$ & $10 / 2 / 00$ & 200,000 & 0 & Flush Water to 4AP \\
\hline & AP-104 & $\mathrm{AP}-102$ & $10 / 15 / 00$ & $10 / 16 / 00$ & 200,000 & 1 & Flush 4AP \\
\hline & $A P-102$ & $\mathrm{AP}-106$ & $2 / 15 / 01$ & $2 / 16 / 01$ & 200,500 & 1 & Flush 2AP \\
\hline & AN-105 & AP-102 & $3 / 17 / 01$ & $3 / 20 / 01$ & 542,400 & 7,895 & Retrieve/Stage LAW Batch 1, Envelope A \\
\hline & LERF & AN-105-PUMP & $3 / 17 / 01$ & $3 / 20 / 01$ & 154,100 & 0 & Dilution Water \\
\hline & AN-105 & AP-102 & $3 / 17 / 01$ & $3 / 20 / 01$ & 686,500 & 294 & As-received Ghost \\
\hline \multirow[t]{10}{*}{$\mathrm{x}$} & AP-108 & AP-106 & $3 / 22 / 01$ & $3 / 23 / 01$ & 356,500 & 8 & Empty 8AP \\
\hline & AN-105 & AP-104 & $3 / 21 / 01$ & $3 / 24 / 01$ & 542,400 & 7,895 & Retrieve/Stage LAW Batch 2, Envelope A \\
\hline & LERF & AN-105-PUMP & $3 / 21 / 01$ & $3 / 24 / 01$ & 154,100 & 0 & Dilution Water \\
\hline & AN-105 & AP-104 & $3 / 21 / 01$ & $3 / 24 / 01$ & 686,500 & 294 & As-received Ghost \\
\hline & WASH-WATER & AP-108 & $4 / 7 / 01$ & $4 / 8 / 01$ & 200,000 & 0 & Fiush Water to 8AP \\
\hline & AP-108 & AP-106 & $4 / 21 / 01$ & $4 / 21 / 01$ & 200,000 & 1 & Flush 8AP \\
\hline & AP-102 & AP-108 & $7 / 10 / 01$ & $7 / 14 / 01$ & 686,500 & 282 & Deliver LAW Batch 1 \\
\hline & AN-104 & $\mathrm{AP}-102$ & $10 / 1 / 01$ & $10 / 4 / 01$ & 502,200 & 12,513 & Retrieve/Stage LAW Batch 3, Envelope A \\
\hline & LERF & AN-104-PUMP & $10 / 1 / 01$ & $10 / 4 / 01$ & 200,800 & 0 & Dilution Water \\
\hline & AN-104 & AP-102 & $10 / 1 / 01$ & $10 / 4 / 01$ & 684,700 & 702 & As-received Ghost \\
\hline \multirow[t]{26}{*}{$x$} & $\mathrm{AZ}-102$ & AY-101 & $11 / 14 / 01$ & $11 / 15 / 01$ & 204,000 & 0 & Pre-Stage Envelope B. \\
\hline & AP-104 & AP-108 & $1 / 5 / 03$ & $1 / 9 / 03$ & 686,500 & 282 & Deliver LAW Batch 2 \\
\hline & AN-104 & AP-104 & $1 / 10 / 03$ & $1 / 13 / 03$ & 502,200 & 12,513 & Retrieve/Stage LAW Batch 4, Envelope A \\
\hline & LERF & AN-104-PUMP & $1 / 10 / 03$ & $1 / 13 / 03$ & 200,800 & 0 & Dilution Water \\
\hline & AN-104 & $\mathrm{AP}-104$ & $1 / 10 / 03$ & $1 / 13 / 03$ & 684,700 & 702 & As-received Ghost \\
\hline & RETURN 2 & AP-107 & $4 / 3 / 03$ & $4 / 3 / 03$ & 8,671 & 331 & Returns From Contractor \\
\hline & AP-102 & AP-108 & $9 / 19 / 03$ & $9 / 22 / 03$ & 684,700 & 686 & Deliver LAW Batch 3 \\
\hline & AW-101 & AP-102 & $9 / 23 / 03$ & $9 / 27 / 03$ & 537,200 & 13,013 & Retrieve/Stage LAW Batch 5, Envelope A \\
\hline & LERF & AW-101-PUMP & $9 / 23 / 03$ & $9 / 27 / 03$ & 308,100 & 0 & Dilution Water \\
\hline & AW-101 & AP-102 & $9 / 23 / 03$ & $9 / 27 / 03$ & 845,400 & 13,013 & As-received Ghost \\
\hline & RETURN 2 & AP-107 & $2 / 2 / 04$ & $2 / 2 / 04$ & 8,670 & 331 & Returns From Contractor \\
\hline & AP-104 & $\mathrm{AP}-108$ & $6 / 12 / 04$ & $6 / 15 / 04$ & 684,700 & 686 & Deliver LAW Batch 4 \\
\hline & $\mathrm{AW}-101$ & AP-104 & $6 / 16 / 04$ & $6 / 20 / 04$ & 537,200 & 13,013 & Retrieve/Stage LAW Batch 6, Envelope A \\
\hline & LERF & AW-101-PUMP & $6 / 16 / 04$ & $6 / 20 / 04$ & 308,100 & 0 & Dilution Water \\
\hline & AW-10I & AP-104 & $6 / 16 / 04$ & $6 / 20 / 04$ & 845,400 & 13,013 & As-received Ghost \\
\hline & RETURN 2 & AP-107 & $8 / 31 / 04$ & $8 / 31 / 04$ & 8,672 & 332 & Returns From Contractor \\
\hline & RETURN 2 & AP-107. & $1 / 18 / 05$ & $1 / 18 / 05$ & 8,672 & 332 & Returns From Contractor \\
\hline & AP-102 & AP-108 & $3 / 6 / 05$ & $3 / 10 / 05$ & 740,300 & 1,581 & Deliver LAW Batch 5 \\
\hline & WASH-WATER & $\mathrm{AP}-102$ & $3 / 25 / 05$ & $3 / 25 / 05$ & 136,900 & 0 & Flush Water to 2AP \\
\hline & AP-102 & AP-106 & $4 / 8 / 05$ & $4 / 8 / 05$ & 243,100 & 10,337 & Flush 2AP \\
\hline & AY-101 & AP-102 & $4 / 9 / 05$ & $4 / 10 / 05$ & 283,600 & 12 & Retrieve/Stage LAW Batch 7, Envelope B \\
\hline & RETURN_2 & AP-107 & $6 / 2 / 05$ & $6 / 2 / 05$ & 8,675 & 331 & Returns From Contractor \\
\hline & RETURN_ 2 & AP-107 & $10 / 10 / 05$ & $10 / 10 / 05$ & 8,679 & 330 & Returns From Contractor \\
\hline & AP-104 & AP-108 & $10 / 10 / 05$ & $10 / 14 / 05$ & 740,300 & 1,581 & Deliver LAW Batch 6 \\
\hline & WASH-WATER & AP-104 & $10 / 29 / 05$ & $10 / 29 / 05$ & 136,900 & 0 & Flush Water to 4AP \\
\hline & AP-104 & AP-106 & $11 / 12 / 05$ & $11 / 12 / 05$ & 243,100 & 10,337 & Flush 4AP \\
\hline \multirow[t]{11}{*}{$\cdot$} & AN-107 & AP-104 & $11 / 13 / 05$ & $11 / 15 / 05$ & 481,700 & 22 & Retrieve/Stage LAW Batch $8 / 9$, Envelope C \\
\hline & LERF & AN-107-PUMP & $11 / 13 / 05$ & $11 / 15 / 05$ & 72,259 & 0 & Dilution Water \\
\hline & AN-107 & AP-104 & $11 / 13 / 05$ & $11 / 15 / 05$ & 554,000 & 22 & As-received Ghost \\
\hline & RETURN 2 & AP-107 & $11 / 29 / 05$ & $11 / 30 / 05$ & 8,677 & 330 & Returns From Contractor \\
\hline & RETURN 2 & AP-107 & $1 / 19 / 06$ & $1 / 19 / 06$ & 8,677 & 330 & Returns From Contractor \\
\hline & RETURN 2 & AP-107 & $3 / 10 / 06$ & $3 / 10 / 06$ & 8,677 & 330 & Returns From Contractor \\
\hline & RETURN 2 & AP-107 & $4 / 29 / 06$ & $4 / 29 / 06$ & 8,677 & 330 & Returns From Contractor \\
\hline & AP-108 & LAW-CONTRACTOR-2 & $6 / 1 / 02$ & $6 / 12 / 06$ & $4,223,000$ & 5,042 & Complete Processing Envelope A \\
\hline & AP-102 & AP-108 & $6 / 12 / 06$ & $6 / 13 / 06$ & 277,300 & 552 & Deliver LAW Batch 7 \\
\hline & AN-107 & AP-102 & $6 / 14 / 06$ & $6 / 16 / 06$ & 481,700 & 22 & Retrieve/Stage LAW Batch 10, Envelope C \\
\hline & LERF & AN-107-PUMP & $6 / 14 / 06$ & $6 / 16 / 06$ & 72,260 & 0 & Dilution Water \\
\hline
\end{tabular}


HNF-2321

Revision 0

Table A.4-2. Phase 1 Low-Activity Waste Feed Staging Transfers for Case 4.

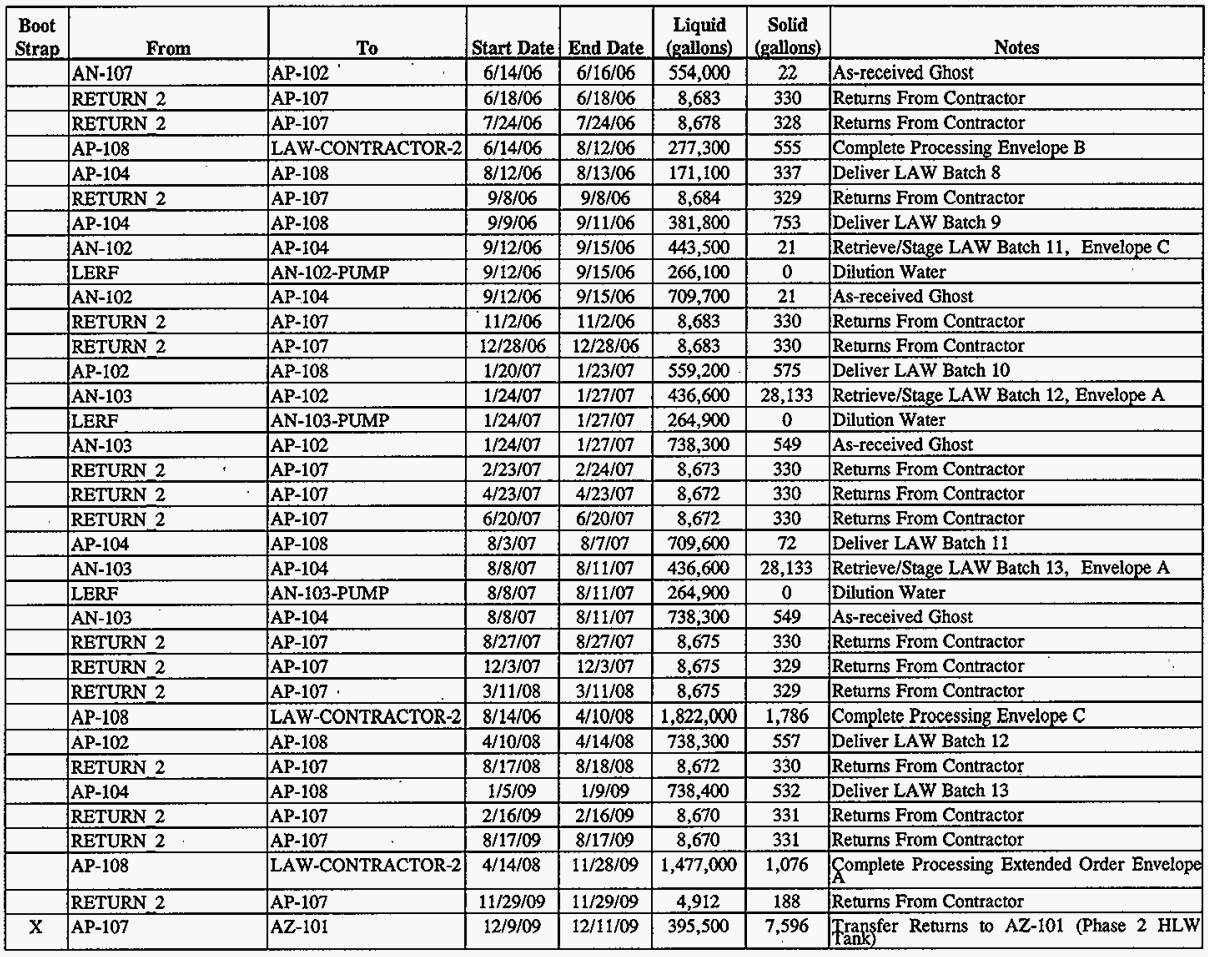


HNF-2321

Revision 0

Table A.4-3. Phase 1 High-Level Waste Feed Staging Transfers for Case 4.

\begin{tabular}{|c|c|c|c|c|c|c|c|}
\hline $\begin{array}{l}\text { Boot } \\
\text { Strap }\end{array}$ & From & To & Start Date & End Date & $\begin{array}{c}\text { Liquid } \\
\text { (gallons) }\end{array}$ & $\begin{array}{c}\text { Solid } \\
\text { (gallons) }\end{array}$ & \begin{tabular}{|c} 
Notes \\
\end{tabular} \\
\hline & AY-102 & C-106 & $7 / 1 / 98$ & $7 / 5 / 98$ & 920,700 & 31 & $\begin{array}{l}\text { Sluice "Water" for C-106 Retrieval - Lumped } \\
\text { (see Below) }\end{array}$ \\
\hline & C-106 & $\mathrm{AX}-102$ & $7 / 15 / 98$ & $11 / 10 / 98$ & 809,000 & 15,169 & C-106 Retrieval (Project W-320) \\
\hline \multirow[t]{8}{*}{$\mathrm{x}$} & AZ-101 & AY-101 & $8 / 17 / 00$ & $8 / 20 / 00$ & 685,000 & 0 & Initial Decant 1AZ \\
\hline & WASH-WATER & AZ-101 & $8 / 20 / 00$ & $8 / 21 / 00$ & 146,000 & 0 & First Wash $1 \mathrm{AZ}$ \\
\hline & AZ-101 & AP-106 & $10 / 9 / 00$ & $10 / 10 / 00$ & 143,200 & 0 & First Decant $1 \mathrm{AZ}$ \\
\hline & WASH-WATER & AZ-10L & $10 / 11 / 00$ & $10 / 12 / 00$ & 146,000 & 0 & Second Wash $1 \mathrm{AZ}$ \\
\hline & AZ-101 & AP-106 & $11 / 30 / 00$ & $12 / 1 / 00$ & 140,200 & 0 & Second Decant 1AZ \\
\hline & WASH-WATER & AZ-101 & $12 / 2 / 00$ & $12 / 2 / 00$ & 146,000 & 0 & Third Wash $1 \mathrm{AZ}$ \\
\hline & $\mathrm{AZ}-101$ & AP-106 & $1 / 21 / 01$ & $1 / 21 / 01$ & 142,900 & $\mathbf{0}$ & Third Decant IAZ \\
\hline & WASH-WATER & AZ-101 & $1 / 22 / 01$ & $1 / 23 / 01$ & 138,200 & 0 & Transfer Water Add 1AZ \\
\hline \multirow[t]{14}{*}{$\mathrm{x}$} & $\mathrm{AZ}-102$ & $\mathrm{AY}-101$ & $11 / 14 / 01$ & $11 / 15 / 01$ & 204,000 & 0 & Initial Decant $2 \mathrm{AZ}$ \\
\hline & AZ-102 & AP-107 & $11 / 16 / 01$ & $11 / 16 / 01$ & 181,800 & $\mathbf{0}$ & Complete Initial Decant $2 \mathrm{AZ}$ \\
\hline & WASH-WATER & $A Z-102$ & $11 / 17 / 01$ & $11 / 18 / 01$ & 213,000 & 0 & First Wash $2 \mathrm{AZ}$ \\
\hline & AZ-102 & AP-106 & $1 / 6 / 02$ & $1 / 6 / 02$ & 127,700 & 0 & First Decant 2AZ \\
\hline & WASH-WATER & AZ-102 & $1 / 8 / 02$ & $1 / 9 / 02$ & 213,000 & 0 & Second Wash $2 \mathrm{AZ}$ \\
\hline & AZ-102 & AP-106 & $2 / 27 / 02$ & $2 / 28 / 02$ & 210,300 & 0 & Second Decant $2 \mathrm{AZ}$ \\
\hline & WASH-WATER & AZ-102' & $3 / 1 / 02$ & $3 / 2 / 02$ & 213,000 & 0 & Third Wash $2 \mathrm{AZ}$ \\
\hline & $\mathrm{AZ}-102$ & AP-106 & $4 / 20 / 02$ & $4 / 21 / 02$ & 211,800 & 0 & Third Decant 2AZ \\
\hline & WASH-WATER & AZ-102 & $4 / 22 / 02$ & $4 / 23 / 02$ & 213,000 & 0 & Fourth Wash $2 \mathrm{AZ}$ \\
\hline & AZ-101 & P1HLW_FEED_TANK & $5 / 17 / 02$ & $5 / 17 / 02$ & 134,300 & 5,079 & $\begin{array}{l}\text { Deliver, Batch } 1 \text { of } 1 \mathrm{AZ} \text {, Envelope D (Batch } \\
\text { Overall) }\end{array}$ \\
\hline & AZ-102 & AP-106 & $6 / 12 / 02$ & $6 / 13 / 02$ & 212,500 & 0 & Fourth Decant $2 \mathrm{AZ}$ \\
\hline & WASH-WATER & AZ-102 & $6 / 14 / 02$ & $6 / 14 / 02$ & 110,000 & 0 & Transfer Water Add 2AZ \\
\hline & P1HLW_FEED_TANK & HLW-CONTRACTOR & $6 / 1 / 02$ & $2 / 21 / 03$ & 134,300 & 5,079 & $\begin{array}{l}\text { Grocess Batch } 1 \text { of } 1 \mathrm{AZ}, \text { Envelope D (Batch } 1 \\
\text { Overal) }\end{array}$ \\
\hline & $A Z-101$ & P1HLW_FEED_TANK & $2 / 21 / 03$ & $2 / 21 / 03$ & 134,300 & 5,079 & $\begin{array}{l}\text { Belivet Batch } 2 \text { of } 1 \mathrm{AZ} \text {, Envelope D (Batch } 2 \\
\text { Bverall) }\end{array}$ \\
\hline \multirow[t]{14}{*}{$\mathrm{x}$} & AY-102 & AZ-101 & $2 / 24 / 03$ & $3 / 1 / 03$ & 819,600 & 16,032 & $\begin{array}{l}\text { Transfer 2AY/6C Slurry to } 1 \mathrm{AZ} \text { for } \\
\text { Pretrealment }\end{array}$ \\
\hline & AZ-101 & AP-106 & $10 / 28 / 03$ & $10 / 31 / 03$ & 622,700 & 0 & Initial Decant $2 \mathrm{AY} / 6 \mathrm{C}$ Slurry from $1 \mathrm{AZ}$ \\
\hline & WASH-WATER & $\mathrm{AZ}-101$ & $10 / 31 / 03$ & $11 / 2 / 03$ & 324,000 & 0 & First Wash 2AY/6C Slurry in 1AZ \\
\hline & P1HLW_FEED_TANK & HLW-CONTRACTOR & $2 / 22 / 03$ & $11 / 14 / 03$ & 134,300 & 5,079 & $\begin{array}{l}\text { Process Batch } 2 \text { of } 1 \mathrm{AZ} \text {, Envelope D (Batch } 2 \\
\text { Overall) }\end{array}$ \\
\hline & AZ-102 & P1HLW_FEED_TANK & $11 / 14 / 03$ & $11 / 14 / 03$ & 123,900 & 5,202 & 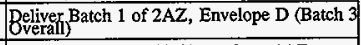 \\
\hline & AZ-101 & AP-106 & $12 / 21 / 03$ & $12 / 22 / 03$ & 320,000 & 0 & First Decant 2AY/6C Slury from 1AZ \\
\hline & WASH-WATER & $A Z-101$ & $12 / 24 / 03$ & $12 / 25 / 03$ & 4,000 & 0 & Second Wash $2 \mathrm{AY} / 6 \mathrm{C}$ Slurry in $1 \mathrm{AZ}$ \\
\hline & AZ-101 & AP-106 & $2 / 12 / 04$ & $2 / 14 / 04$ & 322,300 & 0 & Second Decant 2AY/6C Slurry from $1 \mathrm{AZ}$ \\
\hline & WASH-WATER & $\mathrm{AZ}-101$ & $2 / 15 / 04$ & $2 / 17 / 04$ & 433,600 & 0 & Transfer Water Add 1AZ for 2AY/6C Slurry \\
\hline & PIHLW_FEED_TANK & HLW-CONTRACTOR & $11 / 14 / 03$ & $8 / 7 / 04$ & 123,900 & 5,202 & 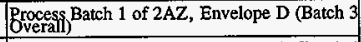 \\
\hline & AZ-102 & P1HLW_FEED_TANK & $8 / 7 / 04$ & $8 / 8 / 04$ & 123,900 & 5,202 & ${ }_{\text {Belierer }}^{\text {Batch } 2 \text { of } 2 A Z \text {, Envelope D (Batch } 4}$ \\
\hline & C-104 & AY-102 & $8 / 1 / 04$ & $9 / 8 / 04$ & 79,100 & 9,724 & C-104 Retrieval (Project W-???) \\
\hline & P1HLW_FEED_TANK & HLW-CONTRACTOR & $8 / 8 / 04$ & $5 / 2 / 05$ & 123,900 & 5,202 & $\begin{array}{l}\text { Process Batch } 2 \text { of } 2 A Z \text {, Envelope D (Batch } 4 \\
\text { Overali) }\end{array}$ \\
\hline & AZ-101 & P1HLW_FEED_TANK & $5 / 2 / 05$ & $5 / 2 / 05$ & 127,200 & 3,269 & 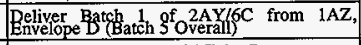 \\
\hline \multirow[t]{10}{*}{$\mathbf{X}$} & AX-102 & $\mathrm{AZ}-102$ & $8 / 1 / 05$ & $8 / 3 / 05$ & 279,400 & 9,360 & Transfer $4 \mathrm{C}$ Slurry to $2 \mathrm{AZ}$ for Pretreatment \\
\hline & PIHLW_FEED_TANK & HLW-CONTRACTOR & $5 / 2 / 05$ & $11 / 16 / 05$ & 127,200 & 3,269 & 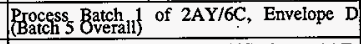 \\
\hline & AZ-101 & PIHLW_FEED_TANK & $11 / 16 / 05$ & $11 / 17 / 05$ & 127,200 & 3,269 & Beliver Batche 2 of $2 \mathrm{AY} / 6 \mathrm{CC}$ from $1 \mathrm{AZ}$, \\
\hline & P1HLW_FEED_TANK & HLW-CONTRACTOR & $11 / 17 / 05$ & $6 / 2 / 06$ & 127,200 & $-3,269$ & $\begin{array}{l}\text { Process Batch } \\
\text { (Batch } 6 \text { b verall) }\end{array}$ \\
\hline & AZ-101 & P1HLW_FEED_TANK & $6 / 2 / 06$ & $6 / 3 / 06$ & 127,200 & 3,269 & $\begin{array}{l}\text { Beliver } \\
\text { Envelope } \mathrm{Btch} 3(\text { Batch } \\
\end{array}$ \\
\hline & AZ-102 & AP-106 & $6 / 23 / 06$ & $6 / 24 / 06$ & 159,500 & 0 & Initial Decant 4C Slurry from $2 \mathrm{AZ}$ \\
\hline & WASH-WATER & AZ-102 & $6 / 24 / 06$ & $6 / 26 / 06$ & 324,000 & 0 & First Wash $4 \mathrm{C}$ Slurry in $2 \mathrm{AZ}$ \\
\hline & $A Z-102$ & AP-106 & $8 / 14 / 06$ & $8 / 21 / 06$ & 320,100 & 0 & First Decant 4C Slurry from $2 \mathrm{AZ}$ \\
\hline & WASH-WATER & AZ-102 & $8 / 22 / 06$ & $8 / 24 / 06$ & 324,000 & 0 & Second Wash $4 \mathrm{C}$ Slurry in $2 \mathrm{AZ}$ \\
\hline & $A Z-102$ & AP-106 & $10 / 12 / 06$ & $10 / 13 / 06$ & 322,700 & 0 & Second Decant $4 \mathrm{C}$ Slurry from $2 \mathrm{AZ}$ \\
\hline
\end{tabular}


HNF-2321

\section{Revision 0}

Table A.4-3. Phase 1 High-Level Waste Feed Staging Transfers for Case 4.

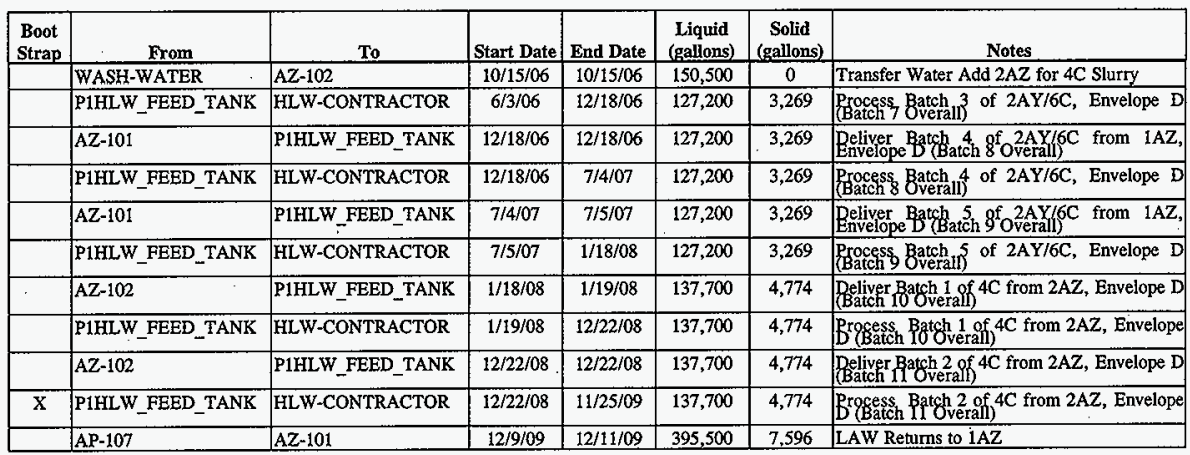


HNF-2321

Revision 0

Table A.5-1. Complete Transfer List for Case 5.

\begin{tabular}{|c|c|c|c|c|c|}
\hline From & To & Start Date & End Date & $\begin{array}{c}\text { Liquid } \\
\text { (gallons) }\end{array}$ & $\begin{array}{c}\text { Solid } \\
\text { (gallons) }\end{array}$ \\
\hline BPTCO & AP-106 & $10 / 1 / 96$ & $10 / 1 / 96$ & 11,000 & 0 \\
\hline $34 \mathrm{~L} 87$ & AP-106 & $10 / 1 / 96$ & $10 / 1 / 96$ & 10,000 & 0 \\
\hline WNE88 & AP-106 & $10 / 1 / 96$ & $10 / 1 / 96$ & 1,000 & 0 \\
\hline TNS88 & AP-106 & $10 / 1 / 96$ & $10 / 1 / 96$ & 750 & 250 \\
\hline TAL88 & AP-106 & $10 / 1 / 96$ & $10 / 1 / 96$ & 4,000 & 0 \\
\hline 1FL96 & AW-10S & $10 / 1 / 96$ & $10 / 1 / 96$ & 13,000 & 0 \\
\hline EVAPF & AP-106 & $10 / 1 / 96$ & $10 / 1 / 96$ & 10,000 & 0 \\
\hline 1NS96 & AW-105 & $10 / 1 / 96$ & $10 / 1 / 96$ & 750 & 250 \\
\hline SPN87 & AP-106 & $10 / 1 / 96$ & $10 / 1 / 96$ & 7,000 & 0 \\
\hline WESF & AP-106 & $10 / 1 / 96$ & $10 / 1 / 96$ & 5,000 & 0 \\
\hline WATER & SY-102 & $10 / 1 / 96$ & $10 / 1 / 96$ & 5,000 & 0 \\
\hline ZNL87 & $S Y-102$ & $10 / 1 / 96$ & $10 / 1 / 96$ & 2,000 & 0 \\
\hline WNW88 & SY-102 & $10 / 1 / 96$ & $10 / 1 / 96$ & 19,000 & 0 \\
\hline WATER & AP-106 & $10 / 2 / 96$ & $10 / 2 / 96$ & 5,000 & 0 \\
\hline WATER & AP-106 & $10 / 2 / 96$ & $10 / 2 / 96$ & 3,000 & 0 \\
\hline WATER & SY-102 & $10 / 2 / 96$ & $10 / 2 / 96$ & 5,000 & 0 \\
\hline WATER & $A W-105$ & $10 / 2 / 96$ & $10 / 2 / 96$ & 5,000 & 0 \\
\hline WATER & AP-106 & $10 / 2 / 96$ & $10 / 2 / 96$ & 5,000 & 0 \\
\hline WATER & AP-106 & $10 / 2 / 96$ & $10 / 2 / 96$ & 12,000 & 0 \\
\hline EVAPF & AP-106 & $11 / 1 / 96$ & $11 / 1 / 96$ & 10,000 & 0 \\
\hline WNE88 & AP-106 & $11 / 1 / 96$ & $11 / 1 / 96$ & 1,000 & 0 \\
\hline BPTCO & AP-106 & $11 / 1 / 96$ & $11 / 1 / 96$ & 10,000 & 0 \\
\hline WATER & $S Y-102$ & $11 / 1 / 96$ & $11 / 1 / 96$ & 3,000 & 0 \\
\hline WNW88 & SY-102 & $11 / 1 / 96$ & $11 / 1 / 96$ & 14,000 & 0 \\
\hline EVAPF & AP-106 & $12 / 1 / 96$ & $12 / 1 / 96$ & 10,000 & 0 \\
\hline WNW88 & SY-102 & $12 / 1 / 96$ & $12 / 1 / 96$ & 14,000 & 0 \\
\hline WATER & SY-102 & $12 / 1 / 96$ & $12 / 1 / 96$ & 3,000 & 0 \\
\hline WNE88 & AP-106 & $12 / 1 / 96$ & $12 / 1 / 96$ & 1,000 & 0 \\
\hline BPTCO & AP-106 & $12 / 1 / 96$ & $12 / 1 / 96$ & 11,000 & 0 \\
\hline EVAPF & AP-106 & $1 / 1 / 97$ & $1 / 1 / 97$ & 10,000 & 0 \\
\hline WNE88 & AP-106 & $1 / 1 / 97$ & $1 / 1 / 97$ & 1,000 & 0 \\
\hline WESF & AP-106 & $1 / 1 / 97$ & $1 / 1 / 97$ & 5,000 & 0 \\
\hline SPN87 & AP-106 & $1 / 1 / 97$ & $1 / 1 / 97$ & 6,000 & 0 \\
\hline WNW88 & SY-102 & $1 / 1 / 97$ & $1 / 1 / 97$ & 14,000 & 0 \\
\hline WATER & SY-102 & $1 / 1 / 97$ & $1 / 1 / 97$ & 4,000 & 0 \\
\hline TAL88 & AP-106 & $1 / 1 / 97$ & $1 / 1 / 97$ & $.4,000$ & 0 \\
\hline BPTCO & AP-106 & $1 / 1 / 97$ & $1 / 1 / 97$ & 10,000 & 0 \\
\hline $34 \mathrm{~L} 87$ & AP-106 & 1/1/97. & $1 / 1 / 97$ & 10,000 & 0 \\
\hline WATER & AP-106 & $1 / 1 / 97$ & $1 / 1 / 97$ & 4,000 & 0 \\
\hline AW-106 & AP-101 & $1 / 4 / 97$ & $1 / 4 / 97$ & 69,511 & 3 \\
\hline AN-106 & AP-108 & $1 / 11 / 97$ & $1 / 12 / 97$ & 177,000 & 7 \\
\hline EVAPF & AP-106 & $2 / 1 / 97$ & $2 / 1 / 97$ & 10,000 & 0 \\
\hline BPTCO & AP-106 & $2 / 1 / 97$ & $2 / 1 / 97$ & 10,000 & 0 \\
\hline WATER & SY-102 & $2 / 1 / 97$ & $2 / 1 / 97$ & 4,000 & 0 \\
\hline WNW88 & SY-102 & $2 / 1 / 97$ & $2 / 1 / 97$ & 14,000 & 0 \\
\hline EVAPF & $A W-102$ & $2 / 20 / 97$ & $2 / 20 / 97$ & 50,000 & 0 \\
\hline AP-105 & AW-102 & $2 / 20 / 97$ & $2 / 24 / 97$ & 942,000 & 39 \\
\hline PXTCO & AP-106 & $3 / 1 / 97$ & $3 / 1 / 97$ & 5,000 & 0 \\
\hline WNW88 & $S Y=102$ & $3 / 1 / 97$ & $3 / 1 / 97$ & 16,000 & 0 \\
\hline WATER & SY-102 & $3 / 1 / 97$ & $3 / 1 / 97$ & 4,000 & 0 \\
\hline WATER & AP-106 & $3 / 1 / 97$ & $3 / 1 / 97$ & 5,000 & 0 \\
\hline $34 \mathrm{~L} 87$ & AP-106 & $3 / 1 / 97$ & $3 / 1 / 97$ & 10,000 & 0 \\
\hline BPTCO & AP-106 & $3 / 1 / 97$ & $3 / 1 / 97$ & 11,000 & 0 \\
\hline EVAPF & AP-106 & $3 / 1 / 97$ & $3 / 1 / 97$ & 10,000 & 0 \\
\hline $\mathrm{AW}-102$ & $242-\mathrm{A}$ & $2 / 26 / 97$ & $3 / 2 / 97$ & $1,027,000$ & 42 \\
\hline $242-\mathrm{A}$ & AW-106 & $2 / 26 / 97$ & $3 / 2 / 97$ & 622,000 & 42 \\
\hline
\end{tabular}


HNF-2321

Revision 0

Table A.5-1. Complete Transfer List for Case 5.

\begin{tabular}{|c|c|c|c|c|c|}
\hline From & To & Start Date & End Date & $\begin{array}{c}\text { Liquid } \\
\text { (gallons) }\end{array}$ & $\begin{array}{c}\text { Solid } \\
\text { (gallons) }\end{array}$ \\
\hline $242-\mathrm{A}$ & LERF & $2 / 26 / 97$ & $3 / 2 / 97$ & 405,000 & 0 \\
\hline EVAPF & AW-102 & $3 / 3 / 97$ & $3 / 3 / 97$ & 35,000 & 0 \\
\hline EVAPF & AP-106 & $4 / 1 / 97$ & $4 / 1 / 97$ & 10,000 & 0 \\
\hline WESF & AP-106 & $4 / 1 / 97$ & $4 / 1 / 97$ & 5,000 & 0 \\
\hline TAL88 & AP- 106 & $4 / 1 / 97$ & $4 / 1 / 97$ & 4,000 & 0 \\
\hline WATER & SY-102 & $4 / 1 / 97$ & $4 / 1 / 97$ & 6,000 & 0 \\
\hline BPTCO & AP-106 & $4 / 1 / 97$ & $4 / 1 / 97$ & 10,000 & 0 \\
\hline WNE88 & AP-106 & $4 / 1 / 97$ & $4 / 1 / 97$ & 1,000 & 0 \\
\hline SPN87 & AP-106 & $4 / 1 / 97$ & $4 / 1 / 97$ & 6,000 & 0 \\
\hline WNW88 & $\$$ SY-102 & $4 / 1 / 97$ & 4/1/97 & 22,000 & 0 \\
\hline AW-106 & AP-105 & $4 / 25 / 97$ & $4 / 28 / 97$ & 617,000 & 29 \\
\hline AY-101 & AP-108 & $4 / 25 / 97$ & $4 / 29 / 97$ & 809,000 & 30 \\
\hline EVAPF & AP-106 & $5 / 1 / 97$ & $5 / 1 / 97$ & 10,000 & 0 \\
\hline $34 \mathrm{~L} 87$ & AP-106 & $5 / 1 / 97$ & $5 / 1 / 97$ & 10,000 & 0 \\
\hline WNE88 & AP-106 & $5 / 1 / 97$ & $5 / 1 / 97$ & 1,000 & 0 \\
\hline BPTCO & AP-106 & $5 / 1 / 97$ & $5 / 1 / 97$ & 11,000 & 0 \\
\hline WATER & AP-106 & $5 / 1 / 97$ & $5 / 1 / 97$ & 4,000 & 0 \\
\hline WNW88 & SY-102 & $5 / 1 / 97$ & $5 / 1 / 97$ & 19,000 & 0 \\
\hline WATER & SY-102 & $5 / 1 / 97$ & $5 / 1 / 97$ & 5,000 & 0 \\
\hline BPTCO & AP-106 & $6 / 1 / 97$ & $6 / 1 / 97$ & 10,000 & 0 \\
\hline WATER & SY-102 & $6 / 1 / 97$ & $6 / 1 / 97$ & 4,000 & 0 \\
\hline WNW88 & SY-102 & $6 / 1 / 97$ & $6 / 1 / 97$ & 16,000 & 0 \\
\hline WNE88 & AP-106 & $6 / 1 / 97$ & $6 / 1 / 97$ & 1,000 & 0 \\
\hline EVAPF & AP-106 & $6 / 1 / 97$ & $6 / 1 / 97$ & 10,000 & 0 \\
\hline $34 \mathrm{~L} 87$ & AP-106 & $7 / 1 / 97$ & $7 / 1 / 97$ & 10,000 & 0 \\
\hline WESF & AP-106 & $7 / 1 / 97$ & $7 / 1 / 97$ & 5,000 & 0 \\
\hline WATER & AN-101 & $7 / 1 / 97$ & $7 / 1 / 97$ & 5,000 & 0 \\
\hline \begin{tabular}{|l|} 
WNE88 \\
\end{tabular} & AP-106 & $7 / 1 / 97$ & $7 / 1 / 97$ & 1,000 & 0 \\
\hline EVAPF & AP-106 & $7 / 1 / 97$ & $7 / 1 / 97$ & 10,000 & 0 \\
\hline WNW88 & SY-102 & $7 / 1 / 97$ & $7 / 1 / 97$ & 24,000 & 0 \\
\hline WATER & SY-102 & $7 / 1 / 97$ & $7 / 1 / 97$ & 6,000 & 0 \\
\hline WATER & AP-106 & $7 / 1 / 97$ & $7 / 1 / 97$ & 4,000 & 0 \\
\hline SPN87 & AP-106 & $7 / 1 / 97$ & $7 / 1 / 97$ & 6,000 & 0 \\
\hline \begin{tabular}{|l|} 
TAL88 \\
\end{tabular} & AP-106 & $7 / 1 / 97$ & $7 / 1 / 97$ & 4,000 & 0 \\
\hline BPTCO & AP-106 & $7 / 1 / 97$ & $7 / 1 / 97$ & 11,000 & 0 \\
\hline \begin{tabular}{|l} 
WCE88 \\
\end{tabular} & $\mathrm{AN}-101$ & $7 / 1 / 97$ & $7 / 1 / 97$ & 20,000 & 0 \\
\hline AN-106 & AP-108 & $7 / 25 / 97$ & $7 / 26 / 97$ & 194,000 & 7 \\
\hline AP-108 & AW-102 & $7 / 25 / 97$ & $7 / 31 / 97$ & $1,198,000$ & 42 \\
\hline EVAPF & AP-106 & $8 / 1 / 97$ & $8 / 1 / 97$ & 10,000 & 0 \\
\hline WATER & AP-106 & $8 / 1 / 97$ & $8 / 1 / 97$ & 4,000 & 0 \\
\hline WATER & SY-102 & $8 / 1 / 97$ & $8 / 1 / 97$ & 13,000 & 0 \\
\hline WCE88 & AN-101 & $8 / 1 / 97$ & $8 / 1 / 97$ & 25,000 & 0 \\
\hline WATER & $\mathrm{AN}-101$ & $8 / 1 / 97$ & $8 / 1 / 97$ & 6,000 & 0 \\
\hline BPTCO & AP-106 & $8 / 1 / 97$ & $8 / 1 / 97$ & 10,000 & 0 \\
\hline WNE88 & AP-106 & $8 / 1 / 97$ & $8 / 1 / 97$ & 16,000 & 0 \\
\hline WNW88 & SY-102 & $8 / 1 / 97$ & $8 / 1 / 97$ & 51,000 & 0 \\
\hline AW-102 & $242-A$ & $7 / 28 / 97$ & $8 / 2 / 97$ & $1,198,000$ & 39 \\
\hline $242-\mathrm{A}$ & AW-106 & $7 / 28 / 97$ & $8 / 2 / 97$ & 346,700 & 39 \\
\hline $242-\mathrm{A}$ & LERF & $7 / 28 / 97$ & $8 / 2 / 97$ & 851,300 & 0 \\
\hline EVAPF & $\mathrm{AW}-102$ & $8 / 3 / 97$ & $8 / 3 / 97$ & 25,000 & 0 \\
\hline WATER & AP-107 & $8 / 20 / 97$ & $8 / 20 / 97$ & 35,000 & 0 \\
\hline AW-106 & AN-106 & $8 / 25 / 97$ & $8 / 26 / 97$ & 347,000 & 16 \\
\hline SY-102 & AP-107 & $8 / 25 / 97$ & $8 / 27 / 97$ & 520,000 & 20 \\
\hline WATER & AN-101 & $9 / 1 / 97$ & $9 / 1 / 97$ & 10,000 & 0 \\
\hline EVAPF & AP-106 & $9 / 1 / 97$ & $9 / 1 / 97$ & 10,000 & 0 \\
\hline WATER & SY-102 & $9 / 1 / 97$ & 9/1/97 & 15,000 & 0 \\
\hline
\end{tabular}


Table A.5-1. Complete Transfer List for Case 5.

\begin{tabular}{|c|c|c|c|c|c|}
\hline From & To & Start Date & End Date & $\begin{array}{c}\text { Liquid } \\
\text { (gallons) }\end{array}$ & $\begin{array}{c}\text { Solid } \\
\text { (gallons) }\end{array}$ \\
\hline WATER & AP-106 & $9 / 1 / 97$ & 9/1/97 & 12,000 & 0 \\
\hline BPTCO & AP-106 & $9 / 1 / 97$ & 9/1/97 & 10,000 & 0 \\
\hline WNW88 & SY-102 & $9 / 1 / 97$ & $9 / 1 / 97$ & 61,000 & 0 \\
\hline WNE88 & AP-106 & $9 / 1 / 97$ & $9 / 1 / 97$ & 46,000 & 0 \\
\hline WCE88 & AN-101 & $9 / 1 / 97$ & $9 / 1 / 97$ & 40,000 & 0 \\
\hline WATER & AP-106 & $10 / 1 / 97$ & $10 / 1 / 97$ & 12,000 & 0 \\
\hline WESF & AP-106 & $10 / 1 / 97$ & $10 / 1 / 97$ & 5,000 & 0 \\
\hline WATER & AN-101 & $10 / 1 / 97$ & $10 / 1 / 97$ & 10,000 & 0 \\
\hline TAL88 & AP-106 & $10 / 1 / 97$ & $10 / 1 / 97$ & 4,000 & 0 \\
\hline $34 L 87$ & $\mathrm{AP}-106$ & $10 / 1 / 97$ & $10 / 1 / 97$ & 10,000 & 0 \\
\hline BPTCO & AP-106 & $10 / 1 / 97$ & $10 / 1 / 97$ & 11,000 & 0 \\
\hline EVAPF & AP-106 & $10 / 1 / 97$ & $10 / 1 / 97$ & 10,000 & 0 \\
\hline TNS88 & AP-106 & $10 / 1 / 97$ & $10 / 1 / 97$ & 750 & 250 \\
\hline $34 \mathrm{~L} 87$ & AP-106 & $10 / 1 / 97$ & $10 / 1 / 97$ & 7,000 & 0 \\
\hline PXTCO & AP-106 & $10 / 1 / 97$ & $10 / 1 / 97$ & 5,000 & 0 \\
\hline SPN87 & $S Y-102$ & $10 / 1 / 97$ & $10 / 1 / 97$ & 7,000 & 0 \\
\hline ZNL87 & SY-102 & $10 / 1 / 97$ & $10 / 1 / 97$ & 4,000 & $0^{\circ}$ \\
\hline WNE88 & AP-106 & $10 / 1 / 97$ & $10 / 1 / 97$ & 46,000 & 0 \\
\hline WATER & SY-102 & $10 / 1 / 97$ & $10 / 1 / 97$ & 28,000 & 0 \\
\hline WCE88 & AN-101 & $10 / 1 / 97$ & $10 / 1 / 97$ & 40,000 & 0 \\
\hline WNW88 & SY-102 & $10 / 1 / 97$ & $10 / 1 / 97$ & 111,000 & 0 \\
\hline \begin{tabular}{|l} 
WATER \\
\end{tabular} & AP-106 & $10 / 2 / 97$ & $10 / 2 / 97$ & 5,000 & 0 \\
\hline WATER & AP-106 & $10 / 2 / 97$ & $10 / 2 / 97$ & 5,000 & 0 \\
\hline WATER & SY-102 & $10 / 2 / 97$ & $10 / 2 / 97$ & 5,000 & 0 \\
\hline WASH-CAUSTIC & $A N-107$ & $10 / 2 / 97$ & $10 / 2 / 97$ & 66,000 & 0 \\
\hline WATER & SY-102 & $10 / 2 / 97$ & $10 / 2 / 97$ & 1,000 & 0 \\
\hline WATER & AP-106 & $10 / 2 / 97$ & $10 / 2 / 97$ & 3,000 & 0 \\
\hline WATER & $\mathrm{AP}-106$ & $10 / 2 / 97$ & $10 / 2 / 97$ & 3,000 & 0 \\
\hline AY-102 & AP-106 & $10 / 25 / 97$ & $10 / 25 / 97$ & 35,999 & 1 \\
\hline WATER & AY-102 & $10 / 26 / 97$ & $10 / 27 / 97$ & 176,000 & 0 \\
\hline EVAPF & AP-106 & $11 / 1 / 97$ & $11 / 1 / 97$ & 10,000 & 0 \\
\hline WATER & AP-106 & $11 / 1 / 97$ & $11 / 1 / 97$ & 12,000 & 0 \\
\hline WNE88 & AP-106 & $11 / 1 / 97$ & $11 / 1 / 97$ & 46,000 & 0 \\
\hline WATER & SY-102 & $11 / 1 / 97$ & $11 / 1 / 97$ & 30,000 & 0 \\
\hline WATER & AN-101 & $11 / 1 / 97$ & $11 / 1 / 97$ & 10,000 & 0 \\
\hline WCE88 & AN-101 & $11 / 1 / 97$ & $11 / 1 / 97$ & 40,000 & 0 \\
\hline WNW88 & SY-102 & $11 / 1 / 97$ & $11 / 1 / 97$ & 119,000 & 0 \\
\hline EVAPF & AP-106 & $12 / 1 / 97$ & $12 / 1 / 97$ & 10,000 & 0 \\
\hline WATER & AP-106 & $12 / 1 / 97$ & $12 / 1 / 97$ & 11,000 & 0 \\
\hline WNE88 & AP-106 & $12 / 1 / 97$ & $12 / 1 / 97$ & 42,000 & 0 \\
\hline WATER & SX-102 & $12 / 1 / 97$ & $12 / 1 / 97$ & 33,000 & 0 \\
\hline WATER & AN-101 & 12/1/97 & $12 / 1 / 97$ & 10,000 & 0 \\
\hline WCE88 & AN-101 & $12 / 1 / 97$ & $12 / 1 / 97$ & 40,000 & 0 \\
\hline WNW88 & SY-102 & $12 / 1 / 97$ & $12 / 1 / 97$ & 131,000 & 0 \\
\hline WATER & AP-107 & $12 / 20 / 97$ & $12 / 20 / 97$ & 35,000 & 0 \\
\hline \begin{tabular}{|l|} 
SY-102 \\
\end{tabular} & $\mathrm{AP}-107$ & $12 / 26 / 97$ & $12 / 28 / 97$ & 520,000 & 21 \\
\hline AP-106 & AP-104 & $12 / 25 / 97$ & $12 / 30 / 97$ & 996,600 & 35 \\
\hline $34 \mathrm{~L} 87$ & AP-106 & $1 / 1 / 98$ & $1 / 1 / 98$ & 10,000 & 0 \\
\hline TAL88 & AP-106 & $1 / 1 / 98$ & $1 / 1 / 98$ & 4,000 & 0 \\
\hline WATER & AP-106 & $1 / 1 / 98$ & $1 / 1 / 98$ & 10,000 & 0 \\
\hline WATER & AP-106 & $1 / 1 / 98$ & $1 / 1 / 98$ & 4,000 & 0 \\
\hline EVAPF & AP-106 & $1 / 1 / 98$ & $1 / 1 / 98$ & 10,000 & 0 \\
\hline SPN87 & SY-102 & $1 / 1 / 98$ & $1 / 1 / 98$ & 6,000 & 0 \\
\hline BPTCO & AP-106 & $1 / 1 / 98$ & $1 / 1 / 98$ & 11,000 & 0 \\
\hline WATER & SY-102 & 1/1/98 & $1 / 1 / 98$ & 37,000 & 0 \\
\hline WNE88 & AP-106 & $1 / 1 / 98$ & $1 / 1 / 98$ & 38.000 & 0 \\
\hline
\end{tabular}


HNF-2321

Revision 0

Table A.5-1. Complete Transfer List for Case 5.

\begin{tabular}{|c|c|c|c|c|c|}
\hline From & To & Start Date & End Date & $\begin{array}{c}\text { Liquid } \\
\text { (gallons) }\end{array}$ & $\begin{array}{c}\text { Solid } \\
\text { (gallons) }\end{array}$ \\
\hline WCE88 & AN-101 & $1 / 1 / 98$ & $1 / 1 / 98$ & 40,000 & 0 \\
\hline WATER & AN-101 & $1 / 1 / 98$ & $1 / 1 / 98$ & 10,000 & 0 \\
\hline WNW88 & SY-102 & $1 / 1 / 98$ & $1 / 2 / 98$ & 146,000 & 0 \\
\hline WATER & SY-102 & $2 / 1 / 98$ & $2 / 1 / 98$ & 35,000 & 0 \\
\hline WATER & AN-101 & $2 / 1 / 98$ & $2 / 1 / 98$ & 10,000 & 0 \\
\hline EVAPF & AP-106 & $2 / 1 / 98$ & $2 / 1 / 98$ & 10,000 & 0 \\
\hline WNE88 & AP-106 & $2 / 1 / 98$ & $2 / 1 / 98$ & $33,0,00$ & 0 \\
\hline WATER & AP-106 & $2 / 1 / 98$ & $2 / 1 / 98$ & 8,000 & 0 \\
\hline WCE88 & AN-101 & $2 / 1 / 98$ & $2 / 1 / 98$ & 40,000 & 0 \\
\hline WNW88 & SY-102 & $2 / 1 / 98$ & $2 / 2 / 98$ & 140,000 & 0 \\
\hline BPTCO & AP-106 & $3 / 1 / 98$ & $3 / 1 / 98$ & 11,000 & 0 \\
\hline WATER & AP-106 & $3 / 1 / 98$ & $3 / 1 / 98$ & 5,000 & 0 \\
\hline EVAPF & AP-106 & $3 / 1 / 98$ & $3 / 1 / 98$ & 10,000 & 0 \\
\hline $34 \mathrm{~L} 87$ & AP-106 & $3 / 1 / 98$ & $3 / 1 / 98$ & 10,000 & 0 \\
\hline WNE88 & AP-106 & $3 / 1 / 98$ & $3 / 1 / 98$ & 26,000 & 0 \\
\hline WATER & AP.-106 & $3 / 1 / 98$ & $3 / 1 / 98$ & 7,000 & 0 \\
\hline WATER & AN-101 & $3 / 1 / 98$ & $3 / 1 / 98$ & 10,000 & 0 \\
\hline WATER & SY-102 & $3 / 1 / 98$ & $3 / 1 / 98$ & 31,000 & 0 \\
\hline WCE 88 & AN-101 & $3 / 1 / 98$ & $3 / 1 / 98$ & 40,000 & 0 \\
\hline WNW88 & SY-102 & $3 / 1 / 98$ & $3 / 1 / 98$ & 124,000 & 0 \\
\hline WATER & AP-104 & $3 / 20 / 98$ & $3 / 20 / 98$ & 35,000 & 0 \\
\hline SY-102 & AP-104 & $3 / 25 / 98$ & $3 / 27 / 98$ & 520,000 & 21 \\
\hline AP-104 & $A W-102$ & $3 / 25 / 98$ & $3 / 30 / 98$ & 998,000 & 33 \\
\hline SPN87 & SY-102 & $4 / 1 / 98$ & $4 / 1 / 98$ & 6,000 & 0 \\
\hline WNE88 & AP-106 & $4 / 1 / 98$ & $4 / 1 / 98$ & 20,000 & 0 \\
\hline WATER & SY-102 & $4 / 1 / 98$ & $4 / 1 / 98$ & 36,000 & 0 \\
\hline WATER & AN-101 & $4 / 1 / 98$ & $4 / 1 / 98$ & 25,000 & 0 \\
\hline WATER & AP-106 & $4 / 1 / 98$ & $4 / 1 / 98$ & 5,000 & 0 \\
\hline TAL88 & $\mathrm{AP}-106$ & $4 / 1 / 98$ & $4 / 1 / 98$ & 4,000 & 0 \\
\hline EVAPF & AP-106 & $4 / 1 / 98$ & $4 / 1 / 98$ & 10,000 & 0 \\
\hline WCE 88 & AN-101 & $4 / 1 / 98$ & $4 / 1 / 98$ & 101,000 & 0 \\
\hline AW-102 & $242-\mathrm{A}$ & $3 / 28 / 98$ & $4 / 1 / 98$ & $1,058,000$ & 31 \\
\hline 242-A & $A W-106$ & $3 / 28 / 98$ & $4 / 1 / 98$ & 180,100 & 31 \\
\hline $242-\mathrm{A}$ & LERF & $3 / 28 / 98$ & $4 / 1 / 98$ & 877,800 & 0 \\
\hline WNW88 & SY-102 & $4 / 1 / 98$ & $4 / 1 / 98$ & 146,000 & 0 \\
\hline $34 \mathrm{~L} 87$ & AP-106 & $5 / 1 / 98$ & $5 / 1 / 98$ & 10,000 & 0 \\
\hline BPTCO & AP-106 & $5 / 1 / 98$ & $5 / 1 / 98$ & 11,000 & 0 \\
\hline WATER & AP-106 & $5 / 1 / 98$ & $5 / 1 / 98$ & 4,000 & 0 \\
\hline EVAPF & AP-106 & $5 / 1 / 98$ & $5 / 1 / 98$ & 10,000 & 0 \\
\hline WATER & AP-106 & $5 / 1 / 98$ & $5 / 1 / 98$ & 5,000 & 0 \\
\hline WNE88 & AP- 106 & $5 / 1 / 98$ & $5 / 1 / 98$ & 20,000 & 0 \\
\hline WATER & AN-101 & $5 / 1 / 98$ & $5 / 1 / 98$ & 24,000 & 0 \\
\hline WCW88 & $S Y-102$ & $5 / 1 / 98$ & $5 / 1 / 98$ & 19.000 & 0 \\
\hline WCE 88 & AN-101 & $5 / 1 / 98$ & $5 / 1 / 98$ & 95,000 & 0 \\
\hline WATER & SY-102 & $5 / 1 / 98$ & $5 / 1 / 98$ & 37,000 & 0 \\
\hline WATER & $S Y-102$ & $5 / 1 / 98$ & $5 / 1 / 98$ & 5,000 & 0 \\
\hline WNW88 & SY-102 & $5 / 1 / 98$ & $5 / 1 / 98$ & 147,000 & 0 \\
\hline AP-107 & AW-102 & $5 / 25 / 98$ & $5 / 30 / 98$ & $1,104,000$ & 40 \\
\hline WATER & $\mathrm{AN}-101$ & $6 / 1 / 98$ & $6 / 1 / 98$ & 7,000 & 0 \\
\hline WCE 88 & AN-101 & $6 / 1 / 98$ & $6 / 1 / 98$ & 28,000 & 0 \\
\hline WCW88 & $S Y-102$ & $6 / 1 / 98$ & $6 / 1 / 98$ & 19,000 & 0 \\
\hline EVAPF & AP-106 & $6 / 1 / 98$ & $6 / 1 / 98$ & 10,000 & 0 \\
\hline WNE88 & AP-106 & $6 / 1 / 98$ & $6 / 1 / 98$ & 16,000 & 0 \\
\hline WATER & SY-102 & $6 / 1 / 98$ & $6 / 1 / 98$ & 36,000 & 0 \\
\hline WATER & AP-106 & $6 / 1 / 98$ & $6 / 1 / 98$ & 4,000 & 0 \\
\hline WATER & SY-102 & $6 / 1 / 98$ & $6 / 1 / 98$ & 5,000 & 0 \\
\hline
\end{tabular}


HNF-2321

Revision 0

Table A.5-1. Complete Transfer List for Case 5.

\begin{tabular}{|c|c|c|c|c|c|}
\hline From & To & Start Date & End Date & $\begin{array}{c}\text { Liquid } \\
\text { (gallons) }\end{array}$ & $\begin{array}{c}\text { Solid } \\
\text { (gallons) }\end{array}$ \\
\hline $\mathrm{AW}-102$ & $242-A$ & $5 / 28 / 98$ & $6 / 1 / 98$ & $1,104,000$ & 39 \\
\hline $242-\mathrm{A}$ & AW-106 & $5 / 28 / 98$ & $6 / 1 / 98$ & 448,700 & 39 \\
\hline $242-\mathrm{A}$ & LERF & $5 / 28 / 98$ & $6 / 1 / 98$ & 655,200 & 0 \\
\hline WNW88 & $S Y-102$ & $6 / 1 / 98$ & $6 / 2 / 98$ & 142,000 & 0 \\
\hline EVAPF & AW-102 & $6 / 3 / 98$ & $6 / 3 / 98$ & 35,000 & 0 \\
\hline WATER & AP-104 & $6 / 20 / 98$ & $6 / 20 / 98$ & 35,000 & 0 \\
\hline AW-106 & AP-105 & $6 / 25 / 98$ & $6 / 26 / 98$ & 341,000 & 17 \\
\hline SY-102 & AP-104 & $6 / 25 / 98$ & $6 / 27 / 98$ & 520,000 & 21 \\
\hline EVAPF & AP-106 & $7 / 1 / 98$ & $7 / 1 / 98$ & 10,000 & 0 \\
\hline \begin{tabular}{|l} 
WNE88 \\
\end{tabular} & AP-106 & $7 / 1 / 98$ & $7 / 1 / 98$ & 13,000 & 0 \\
\hline WCE88 & AN-101 & $7 / 1 / 98$ & $7 / 1 / 98$ & 28,000 & 0 \\
\hline WATER & SY-102 & $7 / 1 / 98$ & $7 / 1 / 98$ & 32,000 & 0 \\
\hline WATER & AN-101 & 7/1/98 & $7 / 1 / 98$ & 7,000 & 0 \\
\hline WATER & SY-102 & $7 / 1 / 98$ & $7 / 1 / 98$ & 3,000 & 0 \\
\hline SPN87 & SY-102 & $7 / 1 / 98$ & $7 / 1 / 98$ & 6,000 & 0 \\
\hline WCW88 & SY-102 & $.7 / 1 / 98$ & $7 / 1 / 98$ & 13,000 & 0 \\
\hline WATER & AP-106 & $7 / 1 / 98$ & $7 / 1 / 98$ & 4,000 & 0 \\
\hline $34 \mathrm{~L} 87$ & AP-106 & $7 / 1 / 98$ & $7 / 1 / 98$ & 10,000 & 0 \\
\hline TAL88 & AP-106 & $7 / 1 / 98$ & $7 / 1 / 98$ & 4,000 & 0 \\
\hline \begin{tabular}{|l} 
WATER \\
\end{tabular} & AP-106 & $7 / 1 / 98$ & $7 / 1 / 98$ & 3,000 & 0 \\
\hline WNW88 & SY-102 & $7 / 1 / 98$ & $7 / 2 / 98$ & 127,000 & 0 \\
\hline AY-102 & C-106 & $7 / 1 / 98$ & $7 / 5 / 98$ & 920,700 & 31 \\
\hline BPTCO & AP-106 & $7 / 11 / 98$ & $7 / 11 / 98$ & 11,000 & 0 \\
\hline WATER & $\mathrm{AN}-101$ & $8 / 1 / 98$ & $8 / 1 / 98$ & 7,000 & 0 \\
\hline EVAPF & AP-106 & $8 / 1 / 98$ & $8 / 1 / 98$ & 10,000 & 0 \\
\hline WCE 88 & AN-101 & $8 / 1 / 98$ & $8 / 1 / 98$ & 28,000 & 0 \\
\hline WATER & SY-102 & $8 / 1 / 98$ & $8 / 1 / 98$ & 30,000 & 0 \\
\hline WATER & AP-106 & $8 / 1 / 98$ & $8 / 1 / 98$ & 2,000 & 0 \\
\hline WNE88 & AP-106 & $8 / 1 / 98$ & $8 / 1 / 98$ & 7,000 & 0 \\
\hline WCW88 & $S Y-102$ & $8 / 1 / 98$ & $8 / 1 / 98$ & 13,000 & 0 \\
\hline WATER & SY-102 & $8 / 1 / 98$ & $8 / 1 / 98$ & 3,000 & 0 \\
\hline WNW88 & SY-102 & $8 / 1 / 98$ & $8 / 2 / 98$ & 121,000 & 0 \\
\hline WCW88 & SY-102 & $9 / 1 / 98$ & 9/1/98 & 8,000 & 0 \\
\hline WNE8 8 & AP-106 & $9 / 1 / 98$ & $9 / 1 / 98$ & 7,000 & 0 \\
\hline WATER & AP-106 & 9/1/98 & $9 / 1 / 98$ & 2,000 & 0 \\
\hline WATER & SY-102 & $9 / 1 / 98$ & $9 / 1 / 98$ & 34,000 & 0 \\
\hline WATER & SY-102 & $9 / 1 / 98$ & $9 / 1 / 98$ & 2,000 & 0 \\
\hline EVAPF & AP-106 & $9 / 1 / 98$ & $9 / 1 / 98$ & 10,000 & 0 \\
\hline WATER & AN-101 & $9 / 1 / 98$ & $9 / 1 / 98$ & $7,000^{\circ}$ & 0 \\
\hline WCE 88 & AN-101 & $9 / 1 / 98$ & 9/1/98 & 28,000 & 0 \\
\hline WNW88 & SY-102 & $9 / 1 / 98$ & $9 / 1 / 98$ & 137,000 & 0 \\
\hline WATER & AP-107 & $9 / 20 / 98$ & $9 / 20 / 98$ & 35,000 & 0 \\
\hline \begin{tabular}{|l|} 
SY-102 \\
\end{tabular} & AP-107 & $9 / 25 / 98$ & $9 / 27 / 98$ & 520,000 & 21 \\
\hline$A P-10 \mathrm{~L}$ & AW-103 & $9 / 25 / 98$ & $9 / 28 / 98$ & 625,000 & 27 \\
\hline ZNL87 & SY-102 & $10 / 1 / 98$ & $10 / 1 / 98$ & 4,000 & 0 \\
\hline 34L87 & AP-106 & $10 / 1 / 98$ & $.10 / 1 / 98$ & 10,000 & 0 \\
\hline PXTCO & AP-106 & $10 / 1 / 98$ & $10 / 1 / 98$ & 5,000 & 0 \\
\hline EVAPF & AP-106 & $10 / 1 / 98$ & $10 / 1 / 98$ & 10,000 & 0 \\
\hline TAL88 & AP-106 & $10 / 1 / 98$ & $10 / 1 / 98$ & 4,000 & 0 \\
\hline WNE88 & AP-106 & $10 / 1 / 98$ & $10 / 1 / 98$ & 3,000 & 0 \\
\hline WESF & AP-106 & $10 / 1 / 98$ & $10 / 1 / 98$ & 5,000 & 0 \\
\hline TNS88 & AP-106 & $10 / 1 / 98$ & $10 / 1 / 98$ & 750 & 250 \\
\hline WCE88 & AN-101 & $10 / 1 / 98$ & $10 / 1 / 98$ & 28,000 & 0 \\
\hline WATER & SY-102 & $10 / 1 / 98$ & $10 / 1 / 98$ & 30,000 & 0 \\
\hline WATER & AP-106 & $10 / 1 / 98$ & $10 / 1 / 98$ & 1,000 & 0 \\
\hline WATER & AN-101 & $10 / 1 / 98$ & $10 / 1 / 98$ & 7,000 & 0 \\
\hline
\end{tabular}


HNF-2321

Revision 0

Table A.5-1. Complete Transfer List for Case 5.

\begin{tabular}{|c|c|c|c|c|c|}
\hline From & To & Start Date & End Date & $\begin{array}{c}\text { Liquid } \\
\text { (gallons) }\end{array}$ & $\begin{array}{c}\begin{array}{c}\text { Solid } \\
\text { (gallons) }\end{array} \\
\end{array}$ \\
\hline WCW88 & SY-102 & $10 / 1 / 98$ & $10 / 1 / 98$ & 8,000 & 0 \\
\hline WATER & SY-102 & $10 / 1 / 98$ & $10 / 1 / 98$ & 2,000 & 0 \\
\hline SPN87 & SY-102 & $10 / 1 / 98$ & $10 / 1 / 98$ & 7,000 & 0 \\
\hline WNW88 & SY-102 & $10 / 1 / 98$ & $10 / 1 / 98$ & 121,000 & 0 \\
\hline WATER & AP-106 & $10 / 2 / 98$ & $10 / 2 / 98$ & 3,000 & 0 \\
\hline \begin{tabular}{|l} 
WATER \\
\end{tabular} & AP-106 & $10 / 2 / 98$ & $10 / 2 / 98$ & 5,000 & 0 \\
\hline WATER & SY-102 & $10 / 2 / 98$ & $10 / 2 / 98$ & 5,000 & 0 \\
\hline EVAPF & AW-102 & $10 / 4 / 98$ & $10 / 4 / 98$ & 50,000 & 0 \\
\hline AW-102 & AW-106 & $10 / 9 / 98$ & $10 / 9 / 98$ & 150,000 & 6 \\
\hline AP-104 & AW-102 & $10 / 5 / 98$ & $10 / 11 / 98$ & $1,107,000$ & 43 \\
\hline AW-104 & AP-107 & $10 / 9 / 98$ & $10 / 11 / 98$ & 540,000 & 18 \\
\hline AW-104 & AP-104 & $10 / 18 / 98$ & $10 / 18 / 98$ & 164,000 & 6 \\
\hline AP-101 & AW-104 & $10 / 21 / 98$ & $10 / 23 / 98$ & 460,000 & 20 \\
\hline AW-106 & AP-101 & $10 / 29 / 98$ & $10 / 30 / 98$ & 289,000 & 13 \\
\hline WCW88 & SY-102 & $11 / 1 / 98$ & $11 / 1 / 98$ & 3,000 & 0 \\
\hline WATER & SY-102 & $11 / 1 / 98$ & 11/1/98 & 30,000 & 0 \\
\hline \begin{tabular}{|l|} 
WATER \\
\end{tabular} & AN-10L & $11 / 1 / 98$ & 11/1/98 & 7,000 & 0 \\
\hline WNE88 & AP-106 & $11 / 1 / 98$ & $11 / 1 / 98$ & 2,000 & 0 \\
\hline WCE88 & AN-101 & $11 / 1 / 98$ & 11/1/98 & 28,000 & 0 \\
\hline \begin{tabular}{|l} 
WATER \\
\end{tabular} & SY-102 & $11 / 1 / 98$ & $11 / 1 / 98$ & 1,000 & 0 \\
\hline EVAPF & AP-106 & $11 / 1 / 98$ & 11/1/98 & 10,000 & 0 \\
\hline \begin{tabular}{|l} 
WNW88 \\
\end{tabular} & SY-102 & $11 / 1 / 98$ & 11/1/98 & 120,000 & 0 \\
\hline AW-106 & AW-102 & $11 / 5 / 98$ & $11 / 5 / 98$ & 150,000 & 9 \\
\hline AW-102 & $242-\mathrm{A}$ & $11 / 4 / 98$ & $11 / 9 / 98$ & $1,193,000$ & 46 \\
\hline $242-\mathrm{A}$ & AW-106 & $11 / 4 / 98$ & $11 / 9 / 98$ & 602,900 & 46 \\
\hline $242-\mathrm{A}$ & LERF & $11 / 4 / 98$ & $11 / 9 / 98$ & 589,600 & 0 \\
\hline $\mathrm{C}-106$ & AY-102 & $7 / 15 / 98$ & $11 / 10 / 98$ & 809,000 & 15,169 \\
\hline WCW88 & $S Y-102$ & $12 / 1 / 98$ & $12 / 1 / 98$ & 3,000 & 0 \\
\hline WATER & SY-102 & $12 / 1 / 98$ & $12 / 1 / 98$ & 29,000 & 0 \\
\hline WATER & AN-101 & $12 / 1 / 98$ & $12 / 1 / 98$ & 6,000 & 0 \\
\hline WATER & SY-102 & $12 / 1 / 98$ & $12 / 1 / 98$ & 1,000 & 0 \\
\hline WNE88 & AP-106 & $12 / 1 / 98$ & $12 / 1 / 98$ & 1,000 & 0 \\
\hline EVAPF & AP-106 & $12 / 1 / 98$ & $12 / 1 / 98$ & 10,000 & 0 \\
\hline WCE88 & $\mathrm{AN}-101$ & $12 / 1 / 98$ & $12 / 1 / 98$ & 23,000 & 0 \\
\hline WNW88 & SY-102 & $12 / 1 / 98$ & $12 / 1 / 98$ & 116,000 & 0 \\
\hline WATER & $\mathrm{AP}-104$ & $12 / 20 / 98$ & $12 / 20 / 98$ & 35,000 & 0 \\
\hline SY-102 & AP-104 & $12 / 25 / 98$ & $12 / 27 / 98$ & 520,000 & 21 \\
\hline AW-106 & AP-101 & $12 / 25 / 98$ & $12 / 28 / 98$ & 605,000 & 27 \\
\hline \begin{tabular}{|l} 
WATER \\
\end{tabular} & SY-102 & $1 / 1 / 99$ & $1 / 1 / 99$ & 6,000 & 0 \\
\hline SPN87 & SY-102 & $1 / 1 / 99$ & $1 / 1 / 99$ & 6,000 & 0 \\
\hline TAL88 & AP-106 & $1 / 1 / 99$ & $1 / 1 / 99$ & 4,000 & 0 \\
\hline WATER & AP-106 & $1 / 1 / 99$ & $1 / 1 / 99$ & 4,000 & 0 \\
\hline $34 \mathrm{~L} 87$ & AP-106 & $1 / 1 / 99$ & $1 / 1 / 99$ & 10,000 & 0 \\
\hline WATER & AN-101 & $1 / 1 / 99$ & $1 / 1 / 99$ & 6,000 & 0 \\
\hline EVAPF & AP-106 & $1 / 1 / 99$ & $1 / 1 / 99$ & 10,000 & 0 \\
\hline WCW88 & SY-102 & $1 / 1 / 99$ & $1 / 1 / 99$ & 23,000 & 0 \\
\hline WATER & SY-102 & 1/1/99 & $1 / 1 / 99$ & 25,000 & 0 \\
\hline WCE88 & AN-101 & $1 / 1 / 99$ & $1 / 1 / 99$ & 23,000 & 0 \\
\hline \begin{tabular}{|l} 
WNW88 \\
\end{tabular} & SY-102 & $1 / 1 / 99$ & $1 / 1 / 99$ & 102,000 & 0 \\
\hline AP-106 & AP-104 & $1 / 25 / 99$ & $1 / 27 / 99$ & 385,000 & 14 \\
\hline WATER & AN-101 & $2 / 1 / 99$ & $2 / 1 / 99$ & 6,000 & 0 \\
\hline EVAPF & AP-106 & $2 / 1 / 99$ & $2 / 1 / 99$ & 10,000 & 0 \\
\hline WCE88 & AN-101 & $2 / 1 / 99$ & $2 / 1 / 99$ & 23,000 & 0 \\
\hline WATER & $S Y-102$ & $2 / 1 / 99$ & $2 / 1 / 99$ & 11,000 & 0 \\
\hline WCW88 & SY-102 & $2 / 1 / 99$ & $2 / 1 / 99$ & 45,000 & 0 \\
\hline WATER & SY-102 & $2 / 1 / 99$ & $2 / 1 / 99$ & 23,000 & 0 \\
\hline
\end{tabular}


HNF-2321

Revision 0

Table A.5-1. Complete Transfer List for Case 5.

\begin{tabular}{|c|c|c|c|c|c|}
\hline From & To & Start Date & End Date & $\begin{array}{c}\text { Liquid } \\
\text { (gallons) }\end{array}$ & $\begin{array}{c}\text { Solid } \\
\text { (gallons) }\end{array}$ \\
\hline WNW88 & $S Y=102$ & $2 / 1 / 99$ & $2 / 1 / 99$ & 91,000 & 0 \\
\hline WATER & SY-102 & $3 / 1 / 99$ & $3 / 1 / 99$ & 17,000 & 0 \\
\hline 34 L87 & AP-106 & $3 / 1 / 99$ & $3 / 1 / 99$ & 10,000 & 0 \\
\hline WCW88 & SY-102 & $3 / 1 / 99$ & $3 / 1 / 99$ & 67,000 & 0 \\
\hline WATER & SY-102 & $3 / 1 / 99$ & $3 / 1 / 99$ & 21,000 & 0 \\
\hline WATER & AP-106 & $3 / 1 / 99$ & $3 / 1 / 99$ & 5,000 & 0 \\
\hline EVAPF & AP-106 & $3 / 1 / 99$ & $3 / 1 / 99$ & 10,000 & 0 \\
\hline WATER & AN-101 & $3 / 1 / 99$ & $3 / 1 / 99$ & 4,000 & 0 \\
\hline WCE88 & AN-101 & $3 / 1 / 99$ & $3 / 1 / 99$ & 17,000 & 0 \\
\hline WNW88 & $S Y-102$ & $3 / 1 / 99$ & $3 / 1 / 99$ & 82,000 & 0 \\
\hline $\mathrm{AN}-101$ & AP-103 & $3 / 5 / 99$ & $3 / 10 / 99$ & $1,024,000$ & 0 \\
\hline AP-107 & AW-102 & $3 / 5 / 99$ & $3 / 10 / 99$ & $1,095,000$ & 40 \\
\hline AW-102 & $242-\mathrm{A}$ & $3 / 8 / 99$ & $3 / 12 / 99$ & $1,095,000$ & 38 \\
\hline $242-\mathrm{A}$ & AW-106 & $3 / 8 / 99$ & $3 / 12 / 99$ & 353,000 & 38 \\
\hline $242-\mathrm{A}$ & LERF & $3 / 8 / 99$ & $3 / 12 / 99$ & 741,900 & 0 \\
\hline AP-108 & AP-106 & $3 / 15 / 99$ & $3 / 15 / 99$ & 500 & 0 \\
\hline WATER & AP-107 & $3 / 20 / 99$ & $3 / 20 / 99$ & 35,000 & 0 \\
\hline SY-102 & AP-107 & $3 / 25 / 99$ & $3 / 27 / 99$ & 520,000 & 21 \\
\hline SPN87 & SY-102 & $4 / 1 / 99$ & $4 / 1 / 99$ & 6,000 & 0 \\
\hline WATER & AP-106 & $4 / 1 / 99$ & $4 / 1 / 99$ & 4,000 & 0 \\
\hline WATER & SY-102 & $4 / 1 / 99$ & 4/1/99 & 23,000 & 0 \\
\hline WATER & SY-102 & $4 / 1 / 99$ & $4 / 1 / 99$ & 16,000 & 0 \\
\hline TAL88 & AP-106 & $4 / 1 / 99$ & $4 / 1 / 99$ & 4,000 & 0 \\
\hline WNW88 & SY-102 & $4 / 1 / 99$ & $4 / 1 / 99$ & 64,000 & 0 \\
\hline \begin{tabular}{|l} 
WCW88 \\
\end{tabular} & SY-102 & $4 / 1 / 99$ & $4 / 1 / 99$ & 91,000 & 0 \\
\hline EVAPF & AW-102 & $4 / 2 / 99$ & $4 / 2 / 99$ & 35,000 & 0 \\
\hline EVAPF & AP-106 & $4 / 3 / 99$ & $4 / 3 / 99$ & 10,000 & 0 \\
\hline WCE88 & AP-106 & $4 / 3 / 99$ & $4 / 3 / 99$ & 17,000 & 0 \\
\hline AP-104 & AW-102 & $4 / 3 / 99$ & $4 / 7 / 99$ & 917,400 & 0 \\
\hline WATER & AP-106 & $5 / 1 / 99$ & $5 / 1 / 99$ & 4,000 & 0 \\
\hline WATER & SY-102 & $5 / 1 / 99$ & $5 / 1 / 99$ & 15,000 & 0 \\
\hline $34\lfloor 87$ & AP-106 & $5 / 1 / 99$ & $5 / 1 / 99$ & 10,000 & 0 \\
\hline WATER & AP-106 & $5 / 1 / 99$ & $5 / 1 / 99$ & 4,000 & 0 \\
\hline EVAPF & AP-106 & $5 / 1 / 99$ & $5 / 1 / 99$ & 10,000 & 0 \\
\hline WCE88 & AP-106 & $5 / 1 / 99$ & $5 / 1 / 99$ & 17,000 & 0 \\
\hline WATER & SY-102 & $5 / 1 / 99$ & $5 / 1 / 99$ & 21,000 & 0 \\
\hline WNW88 & SY-102 & $5 / 1 / 99$ & $5 / 1 / 99$ & 60,000 & 0 \\
\hline WCW88 & SY-102 & $5 / 1 / 99$ & $5 / 1 / 99$ & 84,000 & 0 \\
\hline AW-106 & AN-101 & $5 / 15 / 99$ & $5 / 16 / 99$ & 353,600 & 16 \\
\hline WATER & SY-102 & $6 / 1 / 99$ & $6 / 1 / 99$ & 18,000 & 0 \\
\hline WATER & SY-102 & $6 / 1 / 99$ & $6 / 1 / 99$ & 14,000 & 0 \\
\hline \begin{tabular}{|l|} 
WNW88 \\
\end{tabular} & SY-102 & $6 / 1 / 99$ & $6 / 1 / 99$ & 56,000 & 0 \\
\hline WATER & AP-106 & $6 / 1 / 99$ & $6 / 1 / 99$ & 4,000 & 0 \\
\hline EVAPF & AP-106 & $6 / 1 / 99$ & $6 / 1 / 99$ & 10,000 & 0 \\
\hline WCW88 & SY-102 & $6 / 1 / 99$ & $6 / 1 / 99$ & 74,000 & 0 \\
\hline WCE 88 & AP-106 & $6 / 1 / 99$ & $6 / 1 / 99$ & 17,000 & 0 \\
\hline AP-107 & AP-106 & $6 / 15 / 99$ & $6 / 17 / 99$ & 555,500 & 21 \\
\hline \begin{tabular}{|l} 
WATER \\
\end{tabular} & AP-107 & $6 / 20 / 99$ & $6 / 20 / 99$ & 35,000 & 0 \\
\hline TAL88 & AP-106 & $7 / 1 / 99$ & $7 / 1 / 99$ & 4,000 & 0 \\
\hline EVAPF & AP-106 & $7 / 1 / 99$ & $7 / 1 / 99$ & 10,000 & 0 \\
\hline WCE88 & AP-106 & $7 / 1 / 99$ & 7/1/99 & 11,000 & 0 \\
\hline WATER & AP-106 & $7 / 1 / 99$ & $7 / 1 / 99$ & 3,000 & 0 \\
\hline WATER & AP-106 & $7 / 1 / 99$ & $7 / 1 / 99$ & 4,000 & 0 \\
\hline WATER & SY-102 & $7 / 1 / 99$ & $7 / 1 / 99$ & 13,000 & 0 \\
\hline SPN87 & SY-102 & $7 / 1 / 99$ & $7 / 1 / 99$ & 6,000 & 0 \\
\hline $34 \mathrm{~L} 87$ & AP-106 & $7 / 1 / 99$ & $7 / 1 / 99$ & 10,000 & 0 \\
\hline
\end{tabular}


HNF-2321

Revision 0

Table A.5-1. Complete Transfer List for Case 5.

\begin{tabular}{|c|c|c|c|c|c|}
\hline From & To & Start Date & End Date & $\begin{array}{c}\text { Liquid } \\
\text { (gallons) }\end{array}$ & $\begin{array}{c}\text { Solid } \\
\text { (gallons) }\end{array}$ \\
\hline WCW88 & $\$ Y-102$ & $7 / 1 / 99$ & $7 / 1 / 99$ & 65,000 & 0 \\
\hline WATER & SY-102 & $7 / 1 / 99$ & $7 / 1 / 99$ & 16,000 & 0 \\
\hline WNW88 & $S Y-102$ & $7 / 1 / 99$ & $7 / 1 / 99$ & 52,000 & 0 \\
\hline SY-102 & AP-107 & $7 / 15 / 99$ & $7 / 19 / 99$ & 964,500 & 40 \\
\hline EVAPF & AP-106 & $8 / 1 / 99$ & $8 / 1 / 99$ & 10,000 & 0 \\
\hline WCE88 & AP-106 & $8 / 1 / 99$ & $8 / 1 / 99$ & 11,000 & 0 \\
\hline WATER & SY-102 & $8 / 1 / 99$ & $8 / 1 / 99$ & 17,000 & 0 \\
\hline WATER & AP-106 & $8 / 1 / 99$ & $8 / 1 / 99$ & 3,000 & 0 \\
\hline WCW88 & SY-102 & $8 / 1 / 99$ & $8 / 1 / 99$ & 65,000 & 0 \\
\hline WATER & SY-102 & $8 / 1 / 99$ & $8 / 1 / 99$ & 16,000 & 0 \\
\hline WNW88 & SY-102 & $8 / 1 / 99$ & $8 / 1 / 99$ & 69,000 & 0 \\
\hline AW-102 & $242-\mathrm{A}$ & $8 / 1 / 99$ & $8 / 6 / 99$ & 952,400 & 36 \\
\hline $242-\mathrm{A}$ & AW-106 & $8 / 1 / 99$ & $8 / 6 / 99$ & 610,400 & 36 \\
\hline $242-\mathrm{A}$ & LERF & $8 / 1 / 99$ & $8 / 6 / 99$ & 342,000 & 0 \\
\hline AW-106 & AW-102 & $8 / 6 / 99$ & $8 / 9 / 99$ & 610,300 & 25 \\
\hline AW-102 & $242-\mathrm{A}$ & $8 / 23 / 99$ & $8 / 27 / 99$ & 610,300 & 25 \\
\hline 242-A & AW-106 & $8 / 23 / 99$ & $8 / 27 / 99$ & 391,800 & 25 \\
\hline $242-A$ & LERF & $8 / 23 / 99$ & $8 / 27 / 99$ & 218,500 & 0 \\
\hline AW-106 & AW-102 & $8 / 27 / 99$ & $8 / 29 / 99$ & 391,700 & 17 \\
\hline WATER & SY-102 & $9 / 1 / 99$ & $9 / 1 / 99$ & 21,000 & 0 \\
\hline WATER & AP-106 & 9/1/99 & $9 / 1 / 99$ & 3,000 & 0 \\
\hline W'CE88 & AP-106 & $9 / 1 / 99$ & $9 / 1 / 99$ & 11,000 & 0 \\
\hline WATER & SY-102 & $9 / 1 / 99$ & $9 / 1 / 99$ & 15,000 & 0 \\
\hline EVAPF & AP-106 & 9/1/99 & $9 / 1 / 99$ & 10,000 & 0 \\
\hline \begin{tabular}{|l} 
WCW88 \\
\end{tabular} & SY-102 & $9 / 1 / 99$ & $9 / 1 / 99$ & 58,000 & 0 \\
\hline WNW88 & SY-102 & $9 / 1 / 99$ & $9 / 1 / 99$ & 85,000 & 0 \\
\hline AW-102 & 242-A & $9 / 12 / 99$ & $9 / 14 / 99$ & 391,800 & 17 \\
\hline $242-\mathrm{A}$ & AW-106 & $9 / 12 / 99$ & $9 / 14 / 99$ & 320,300 & 17. \\
\hline $242-\mathrm{A}$ & LERF & $9 / 12 / 99$ & $9 / 14 / 99$ & 71,534 & 0 \\
\hline AP-106 & AW-102 & $9 / 14 / 99$ & $9 / 19 / 99$ & 917,500 & 0 \\
\hline WATER & AP-106 & 9/20/99 & $9 / 20 / 99$ & 35,000 & 0 \\
\hline WATER & AP-106 & $9 / 20 / 99$ & $9 / 20 / 99$ & 35,000 & 0 \\
\hline 1NS96 & AW-105 & $10 / 1 / 99$ & $10 / 1 / 99$ & 750 & 250 \\
\hline WATER & SY-102 & $10 / 1 / 99$ & $10 / 1 / 99$ & 15,000 & 0 \\
\hline SPN87 & SY-102 & $10 / 1 / 99$ & $10 / 1 / 99$ & 7,000 & 0 \\
\hline 1KL96 & AW-105 & $10 / 1 / 99$ & $10 / 1 / 99$ & 30,000 & 0 \\
\hline WATER & SY-102 & $10 / 1 / 99$ & $10 / 1 / 99$ & 11,000 & 0 \\
\hline TNS88 & AP-106 & $10 / 1 / 99$ & $10 / 1 / 99$ & 750 & 250 \\
\hline WESF & AP-106 & $10 / 1 / 99$ & $10 / 1 / 99$ & 5,000 & 0 \\
\hline WCE88 & AP-106 & $10 / 1 / 99$ & $10 / 1 / 99$ & 11,000 & 0 \\
\hline WCW88 & SY-102 & $10 / 1 / 99$ & $10 / 1 / 99$ & 43,000 & 0 \\
\hline 1FL96 & AW-105 & $10 / 1 / 99$ & $10 / 1 / 99$ & 40,000 & 0 \\
\hline WATER & AP-106 & $10 / 1 / 99$ & $10 / 1 / 99$ & 3,000 & 0 \\
\hline \begin{tabular}{|l|} 
ZNL87 \\
\end{tabular} & SY-102 & $10 / 1 / 99$ & $10 / 1 / 99$ & 4,000 & 0 \\
\hline TAL88 & AP-106 & $10 / 1 / 99$ & $10 / 1 / 99$ & 6,000 & 0 \\
\hline PXTCO & AP-106 & $10 / 1 / 99$ & $10 / 1 / 99$ & 5,000 & 0 \\
\hline EVAPF & AP-106 & $10 / 1 / 99$ & $10 / 1 / 99$ & 10,000 & 0 \\
\hline $34 L 87$ & AP-106 & $10 / 1 / 99$ & $10 / 1 / 99$ & 10,000 & 0 \\
\hline WNW88 & SY-102 & $10 / 1 / 99$ & $10 / 1 / 99$ & 62,000 & 0 \\
\hline \begin{tabular}{|l} 
WATER \\
\end{tabular} & SY-102 & $10 / 2 / 99$ & $10 / 2 / 99$ & 5,000 & 0 \\
\hline WATER & AW-105 & $10 / 2 / 99$ & $10 / 2 / 99$ & 17,000 & 0 \\
\hline WATER & AP-106 & $10 / 2 / 99$ & $10 / 2 / 99$ & 5,000 & 0 \\
\hline WATER & AW-105 & $10 / 2 / 99$ & $10 / 2 / 99$ & 13,000 & 0 \\
\hline WATER & AP-106 & $10 / 2 / 99$ & $10 / 2 / 99$ & 5,000 & 0 \\
\hline AW-106 & AN-101 & $10 / 1 / 99$ & $10 / 2 / 99$ & 320,300 & 15 \\
\hline WATER & SY-102 & $11 / 1 / 99$ & $11 / 1 / 99$ & 12,000 & 0 \\
\hline
\end{tabular}


HNF-2321

Revision 0

Table A.5-1. Complete Transfer List for Case 5.

\begin{tabular}{|c|c|c|c|c|c|}
\hline From & To & Start Date & End Date & $\begin{array}{c}\text { Liquid } \\
\text { (gallons) }\end{array}$ & $\begin{array}{c}\text { Solid } \\
\text { (gallons) }\end{array}$ \\
\hline WATER & SY-102 & $11 / 1 / 99$ & $11 / 1 / 99$ & 10,000 & 0 \\
\hline EVAPF & AP-106 & $11 / 1 / 99$ & $11 / 1 / 99$ & 10,000 & 0 \\
\hline WCW88 & SY-102 & $11 / 1 / 99$ & $11 / 1 / 99$ & 39,000 & 0 \\
\hline WNW88 & SY-102 & $11 / 1 / 99$ & $11 / 1 / 99$ & 50,000 & $\overline{0}$ \\
\hline WATER & AP-106 & $11 / 1 / 99$ & $11 / 1 / 99$ & 3,000 & 0 \\
\hline WCE88 & AP-106 & $11 / 1 / 99$ & $11 / 1 / 99$ & 11,000 & 0 \\
\hline EVAPF & AW-102 & $11 / 17 / 99$ & $11 / 17 / 99$ & 35,000 & 0 \\
\hline WATER & SY-102 & $12 / 1 / 99$ & $12 / 1 / 99$ & 10,000 & 0 \\
\hline WCE88 & AP-106 & $12 / 1 / 99$ & $12 / 1 / 99$ & 6,000 & 0 \\
\hline WATER & AP-106 & $12 / 1 / 99$ & $12 / 1 / 99$ & 1,000 & 0 \\
\hline EVAPF & AP-106 & $12 / 1 / 99$ & $12 / 1 / 99$ & 10,000 & 0 \\
\hline WNW88 & SY-102 & $12 / 1 / 99$ & $12 / 1 / 99$ & 43,000 & 0 \\
\hline WATER & SY-102 & $12 / 1 / 99$ & $12 / 1 / 99$ & 11,000 & 0 \\
\hline WCW88 & $S Y-102$ & $12 / 1 / 99$ & $12 / 1 / 99$ & 39,000 & 0 \\
\hline EVAPF & $\mathrm{AW}-102$ & $12 / 3 / 99$ & $12 / 3 / 99$ & 35,000 & 0 \\
\hline WATER & SY-102 & $1 / 1 / 00$ & $1 / 1 / 00$ & 9,000 & 0 \\
\hline WATER & SY-102 & $1 / 1 / 00$ & $1 / 1 / 00$ & 9,000 & 0 \\
\hline SPN87 & SY-102 & $1 / 1 / 00$ & $1 / 1 / 00$ & 6,000 & 0 \\
\hline EVAPF & AP-106 & $1 / 1 / 00$ & $1 / 1 / 00$ & 10,000 & 0 \\
\hline WCE 88 & AP-106 & $1 / 1 / 00$ & $1 / 1 / 00$ & 6,000 & 0 \\
\hline WCW88 & SY-102 & $1 / 1 / 00$ & $1 / 1 / 00$ & 26,000 & 0 \\
\hline WATER & AP-106 & $1 / 1 / 00$ & $1 / 1 / 00$ & 1,000 & 0 \\
\hline $34 \mathrm{~L} 87$ & AP-106 & $1 / 1 / 00$ & $1 / 1 / 00$ & 10,000 & 0 \\
\hline WNW88 & SY-102 & $1 / 1 / 00$ & $1 / 1 / 00$ & 37,000 & 0 \\
\hline WATER & AP-106 & $1 / 1 / 00$ & $1 / 1 / 00$ & 4,000 & 0 \\
\hline TAL88 & AP-106 & $1 / 1 / 00$ & $1 / 1 / 00$ & 6,000 & 0 \\
\hline WATER & AP-106 & $1 / 20 / 00$ & $1 / 20 / 00$ & 35,000 & 0 \\
\hline WATER & AP-106 & $1 / 20 / 00$ & $1 / 20 / 00$ & 35,000 & 0 \\
\hline WATER & SY-102 & $2 / 1 / 00$ & $2 / 1 / 00$ & 4,000 & 0 \\
\hline \begin{tabular}{|l} 
WATER \\
\end{tabular} & SY-102 & $2 / 1 / 00$ & $2 / 1 / 00$ & 8,000 & 0 \\
\hline WCE88 & AP-106 & $2 / 1 / 00$ & $2 / 1 / 00$ & 5,000 & 0 \\
\hline EVAPF & AP-106 & $2 / 1 / 00$ & $2 / 1 / 00$ & 10,000 & 0 \\
\hline WNW88 & SY-102 & $2 / 1 / 00$ & $2 / 1 / 00$ & 30,000 & 0 \\
\hline WCW88 & SY-102 & $2 / 1 / 00$ & $2 / 1 / 00$ & 17,000 & 0 \\
\hline WATER & AP-106 & $2 / 1 / 00$ & $2 / 1 / 00$ & 1,000 & 0 \\
\hline AW-105 & AP-106 & $2 / 15 / 00$ & $2 / 16 / 00$ & 226,200 & 8 \\
\hline WATER & SY-102 & $3 / 1 / 00$ & $3 / 1 / 00$ & 3,000 & 0 \\
\hline EVAPF & AP-106 & $3 / 1 / 00$ & $3 / 1 / 00$ & 10,000 & 0 \\
\hline WATER & AP-106 & $3 / 1 / 00$ & $3 / 1 / 00$ & 5,000 & 0 \\
\hline $34 L 87$ & AP-106 & $3 / 1 / 00$ & $3 / 1 / 00$ & 10,000 & 0 \\
\hline WATER & AP-106 & $3 / 1 / 00$ & $3 / 1 / 00$ & 1,000 & 0 \\
\hline \begin{tabular}{|l|} 
WCE 88 \\
\end{tabular} & AP-106 & $3 / 1 / 00$ & $3 / 1 / 00$ & 3,000 & 0 \\
\hline WCW88 & SY-102 & $3 / 1 / 00$ & $3 / 1 / 00$ & 13,000 & 0 \\
\hline WATER & SY-102 & $3 / 1 / 00$ & $3 / 1 / 00$ & 4,000 & 0 \\
\hline WNW88 & SY-102 & $3 / 1 / 00$ & $3 / 1 / 00$ & 17,000 & 0 \\
\hline SY-102 & AW-105 & $3 / 15 / 00$ & $3 / 18 / 00$ & 750,000 & 31 \\
\hline AW-102 & $242-A$ & $3 / 18 / 00$ & $3 / 23 / 00$ & 987,600 & 39 \\
\hline $242-\mathrm{A}$ & AW-106 & $3 / 18 / 00$ & $3 / 23 / 00$ & 633,600 & 39 \\
\hline $242-\mathrm{A}$ & LERF & $3 / 18 / 00$ & $3 / 23 / 00$ & 354,000 & 0 \\
\hline AW-106 & AW-102 & $3 / 23 / 00$ & $3 / 26 / 00$ & 633,500 & 27 \\
\hline \begin{tabular}{|l} 
WATER \\
\end{tabular} & AP-106 & $4 / 1 / 00$ & $4 / 1 / 00$ & 1,000 & 0 \\
\hline WCE88 & AP-106 & $4 / 1 / 00$ & $4 / 1 / 00$ & 3,000 & 0 \\
\hline EVAPF & AP-106 & $4 / 1 / 00$ & $4 / 1 / 00$ & 10,000 & 0 \\
\hline TAL88 & AP-106 & $4 / 1 / 00$ & $4 / 1 / 00$ & 6,000 & 0 \\
\hline SPN87 & SY-102 & $4 / 1 / 00$ & $4 / 1 / 00$ & 6,000 & 0 \\
\hline WATER & SY-102 & $4 / 1 / 00$ & $4 / 1 / 00$ & 2,000 & 0 \\
\hline
\end{tabular}


Table A.5-1. Complete Transfer List for Case 5.

\begin{tabular}{|c|c|c|c|c|c|}
\hline From & To & Start Date & End Date & $\begin{array}{c}\text { Liquid } \\
\text { (gallons) }\end{array}$ & $\begin{array}{c}\text { Solid } \\
\text { (gallons) }\end{array}$ \\
\hline WNW88 & SY-102 & $4 / 1 / 00$ & $4 / 1 / 00$ & 13,000 & 0 \\
\hline WATER & SY-102 & $4 / 1 / 00$ & $4 / 1 / 00$ & 3,000 & 0 \\
\hline WCW88 & SY-102 & $4 / 1 / 00$ & $4 / 1 / 00$ & 9,000 & 0 \\
\hline$A W-102$ & $242-\mathrm{A}$ & $4 / 9 / 00$ & $4 / 13 / 00$ & 633,500 & 27 \\
\hline 242-A & AW-106 & $4 / 9 / 00$ & $4 / 13 / 00$ & 424,100 & 27 \\
\hline $242-A$ & LERF & $4 / 9 / 00$ & $4 / 13 / 00$ & 209,400 & 0 \\
\hline WATER & SY-102 & $5 / 1 / 00$ & $5 / 1 / 00$ & 3,000 & 0 \\
\hline WATER & AP-106 & $5 / 1 / 00$ & $5 / 1 / 00$ & 4,000 & 0 \\
\hline $34 \mathrm{~L} 87$ & AP-106 & $5 / 1 / 00$ & $5 / 1 / 00$ & 10,000 & 0 \\
\hline WATER & SY-102 & $5 / 1 / 00$ & $5 / 1 / 00$ & 1,000 & 0 \\
\hline WNW88 & SY-102 & $5 / 1 / 00$ & $5 / 1 / 00$ & 11,000 & 0 \\
\hline WCW88 & SY-102 & $5 / 1 / 00$ & $5 / 1 / 00$ & 6,000 & 0 \\
\hline WATER & AP-106 & $5 / 1 / 00$ & $5 / 1 / 00$ & 1,000 & 0 \\
\hline WCE88 & AP-106 & $5 / 1 / 00$ & $5 / 1 / 00$ & 3,000 & 0 \\
\hline EVAPF & AP-106 & $5 / 1 / 00$ & $5 / 1 / 00$ & 10,000 & 0 \\
\hline AP-103 & AW-102 & $5 / 2 / 00$ & $5 / 6 / 00$ & 917,600 & 0 \\
\hline AW-106 & AN-10I & $5 / 15 / 00$ & $5 / 17 / 00$ & 409,100 & 19 \\
\hline $\mathrm{AW}-102$ & $242-A$ & $5 / 21 / 00$ & $5 / 26 / 00$ & 917,900 & 39 \\
\hline $242-\mathrm{A}$ & AW-106 & $5 / 21 / 00$ & $5 / 26 / 00$ & 666,500 & 39 \\
\hline $242-\mathrm{A}$ & LERF & $5 / 21 / 00$ & $5 / 26 / 00$ & 251,300 & 0 \\
\hline \begin{tabular}{|l} 
WNW88 \\
\end{tabular} & SY-102 & $6 / 1 / 00$ & $6 / 1 / 00$ & 4,000 & 0 \\
\hline WATER & SY-102 & $6 / 1 / 00$ & $6 / 1 / 00$ & 1,000 & 0 \\
\hline EVAPF & AP-106 & $6 / 1 / 00$ & $6 / 1 / 00$ & 10,000 & 0 \\
\hline WCE88 & AP-106 & $6 / 1 / 00$ & $6 / 1 / 00$ & 3,000 & 0 \\
\hline WATER & AP-106 & $6 / 1 / 00$ & $6 / 1 / 00$ & 1,000 & 0 \\
\hline AP-103 & AP-106 & $6 / 1 / 00$ & $6 / 1 / 00$ & 99,463 & 4 \\
\hline AP-102 & AP-103 & $6 / 15 / 00$ & $6 / 20 / 00$ & $1,070,000$ & 0 \\
\hline WCE88 & AP-106 & $7 / 1 / 00$ & $7 / 1 / 00$ & 3,000 & 0 \\
\hline WATER & AP-106 & $7 / 1 / 00$ & $7 / 1 / 00$ & 1,000 & 0 \\
\hline EVAPF & AP-106 & $7 / 1 / 00$ & $7 / 1 / 00$ & 10,000 & 0 \\
\hline TAL88 & AP-106 & $7 / 1 / 00$ & $7 / 1 / 00$ & 5,000 & 0 \\
\hline SPN87 & $S Y-102$ & $7 / 1 / 00$ & $7 / 1 / 00$ & 6,000 & 0 \\
\hline 34L87 & $A P-106$ & $7 / 1 / 00$ & $7 / 1 / 00$ & 10,000 & 0 \\
\hline WATER & AP-106 & $7 / 1 / 00$ & $7 / 1 / 00$ & 4,000 & 0 \\
\hline \begin{tabular}{|l|} 
WATER \\
\end{tabular} & SY-102 & $7 / 1 / 00$ & $7 / 1 / 00$ & 1,000 & 0 \\
\hline WNW88 & SY-102 & $7 / 1 / 00$ & $7 / 1 / 00$ & 4,000 & 0 \\
\hline AP-107 & AW-102 & $7 / 2 / 00$ & $7 / 6 / 00$ & 917,900 & 0 \\
\hline AW-106 & AN-106 & $7 / 10 / 00$ & $7 / 13 / 00$ & 681,400 & 32 \\
\hline AW-102 & $242-\mathrm{A}$ & $7 / 21 / 00$ & $7 / 26 / 00$ & 918,100 & 38 \\
\hline $242-\mathrm{A}$ & AW-106 & $7 / 21 / 00$ & $7 / 26 / 00$ & 590,000 & 38 \\
\hline $242-A$ & LERF & $7 / 21 / 00$ & $7 / 26 / 00$ & 328,100 & 0 \\
\hline \begin{tabular}{|l} 
EVAPF \\
\end{tabular} & AP-106 & $8 / 1 / 00$ & $8 / 1 / 00$ & 10,000 & 0 \\
\hline WATER & SY-102 & $8 / 1 / 00$ & $8 / 1 / 00$ & 1,000 & 0 \\
\hline WCE88 & AP-106 & $8 / 1 / 00$ & $8 / 1 / 00$ & 2,000 & 0 \\
\hline WNW88 & SY-102 & $8 / 1 / 00$ & $8 / 1 / 00$ & 4,000 & 0 \\
\hline EVAPF & AW-102 & $8 / 3 / 00$ & $8 / 3 / 00$ & 35,000 & 0 \\
\hline $\mathrm{AZ}-101$ & AY-101 & $8 / 17 / 00$ & $8 / 20 / 00$ & 685,000 & 0 \\
\hline $\mathrm{AZ}-101$ & AP-107 & $8 / 20 / 00$ & $8 / 20 / 00$ & 0 & 0 \\
\hline WASH-WATER & $A Z-101$ & $8 / 20 / 00$ & $8 / 21 / 00$ & 146,000 & 0 \\
\hline EVAPF & AP-106 & $9 / 1 / 00$ & $9 / 1 / 00$ & 10,000 & 0 \\
\hline WCE 88 & AP-106 & $9 / 1 / 00$ & $9 / 1 / 00$ & 1,000 & 0 \\
\hline AW-10S & AP-106 & $9 / 1 / 00$ & $9 / 3 / 00$ & 500,000 & 21 \\
\hline AP-106 & AW-102 & $9 / 1 / 00$ & $9 / 5 / 00$ & 883,200 & 2 \\
\hline ZNL87 & SY-102 & $10 / 1 / 00$ & $10 / 1 / 00$ & 4,000 & 0 \\
\hline \begin{tabular}{|l|} 
TNS88 \\
\end{tabular} & AP-106 & $10 / 1 / 00$ & $10 / 1 / 00$ & 750 & 250 \\
\hline 1NS96 & AW-10S & $10 / 1 / 00$ & $10 / 1 / 00$ & 13,502 & 4,498 \\
\hline
\end{tabular}


HNF-2321

Revision 0

Table A.5-1. Complete Transfer List for Case 5.

\begin{tabular}{|c|c|c|c|c|c|}
\hline From & To & Start Date & End Date & $\begin{array}{c}\text { Liquid } \\
\text { (gallons) }\end{array}$ & $\begin{array}{c}\text { Solid } \\
\text { (gallons) }\end{array}$ \\
\hline TAL88 & AP-106 & $10 / 1 / 00$ & $10 / 1 / 00$ & 23,000 & 0 \\
\hline $34 \mathrm{~L} 87$ & AP-106 & $10 / 1 / 00$ & $10 / 1 / 00$ & 50,000 & 0 \\
\hline 34L87 & AP-106 & $10 / 1 / 00$ & $10 / 1 / 00$ & 7,000 & 0 \\
\hline WESF & AP-106 & $10 / 1 / 00$ & $10 / 1 / 00$ & 5,000 & 0 \\
\hline PXTCO & AP-106 & $10 / 1 / 00$ & $10 / 1 / 00$ & 5,000 & 0 \\
\hline SPN87 & SY-102 & $10 / 1 / 00$ & $10 / 1 / 00$ & 25,000 & 0 \\
\hline EVAPF & AP-106 & $10 / 1 / 00$ & $10 / 1 / 00$ & 120,000 & 0 \\
\hline \begin{tabular}{|l} 
WATER \\
\end{tabular} & SY-102 & $10 / 2 / 00$ & $10 / 2 / 00$ & 1,000 & 0 \\
\hline WATER & SY-102 & $10 / 2 / 00$ & $10 / 2 / 00$ & 5,000 & 0 \\
\hline \begin{tabular}{|l} 
WATER \\
\end{tabular} & AP-106 & $10 / 2 / 00$ & $10 / 2 / 00$ & 3,000 & 0 \\
\hline WATER & AP-106 & $10 / 2 / 00$ & $10 / 2 / 00$ & 22,000 & 0 \\
\hline $1 \mathrm{KL} 96$ & AW-105 & $10 / 1 / 00$ & $10 / 2 / 00$ & 302,000 & 0 \\
\hline WATER & AW-105 & $10 / 2 / 00$ & $10 / 2 / 00$ & 7,000 & 0 \\
\hline WATER & $A W-105$ & $10 / 2 / 00$ & $10 / 3 / 00$ & 132,000 & 0 \\
\hline WATER & AP-106 & $10 / 3 / 00$ & $10 / 3 / 00$ & 5,000 & 0 \\
\hline AW-106 & AP-107 & $10 / 1 / 100$ & $10 / 4 / 00$ & 589,900 & 27 \\
\hline EVAPF & AW-102 & $10 / 4 / 00$ & $10 / 4 / 00$ & 50,000 & 0 \\
\hline $\mathrm{AZ}-101$ & AP-106 & $10 / 9 / 00$ & $10 / 10 / 00$ & 143,200 & 0 \\
\hline WASH-WATER & AZ-101 & $10 / 11 / 00$ & $10 / 12 / 00$ & 146,000 & 0 \\
\hline EVAPF & $\mathrm{AW}-102$ & $11 / 3 / 00$ & $11 / 3 / 00$ & 35,000 & 0 \\
\hline AW-102 & $242-\mathrm{A}$ & $3 / 5 / 01$ & $3 / 10 / 01$ & $1,003,000$ & 38 \\
\hline $242-\mathrm{A}$ & AW-106 & $3 / 5 / 01$ & $3 / 10 / 01$ & 643,300 & 38 \\
\hline $242-\mathrm{A}$ & LERF & $3 / 5 / 01$ & $3 / 10 / 01$ & 360,000 & 0 \\
\hline AW-106 & AW-102 & $3 / 11 / 01$ & $3 / 15 / 01$ & 643,200 & 26 \\
\hline AW-102 & $242-\mathrm{A}$ & $3 / 29 / 01$ & $4 / 1 / 01$ & 643,200 & 26 \\
\hline $242-\mathrm{A}$ & AW-106 & $3 / 29 / 01$ & $4 / 1 / 01$ & 412,800 & 26 \\
\hline $242-A$ & LERF & $3 / 29 / 01$ & $4 / 1 / 01$ & 230,400 & 0 \\
\hline AW-106 & AW-102 & $4 / 1 / 01$ & $4 / 3 / 01$ & 412,700 & 18 \\
\hline AW-102 & $242-\mathrm{A}$ & $4 / 17 / 01$ & $4 / 19 / 01$ & 412,800 & 18 \\
\hline $242-\mathrm{A}$ & AW-106 & $4 / 17 / 01$ & $4 / 19 / 01$ & 305,100 & 18 \\
\hline $242-\mathrm{A}$ & LERF & $4 / 17 / 01$ & $4 / 19 / 01$ & 107,700 & 0 \\
\hline \begin{tabular}{|l} 
AP-106 \\
\end{tabular} & AW-102 & $4 / 20 / 01$ & $4 / 24 / 01$ & 775,900 & 0 \\
\hline$A Z-101$ & AP-106 & $4 / 24 / 01$ & $4 / 25 / 01$ & 140,200 & 0 \\
\hline WASH-WATER & $A Z-101$ & $4 / 26 / 01$ & $4 / 26 / 01$ & 146,000 & 0 \\
\hline WATER & AW-102 & $5 / 1 / 01$ & $5 / 1 / 01$ & 35,000 & 0 \\
\hline EVAPF & $\mathrm{AW}-102$ & $5 / 3 / 01$ & $5 / 3 / 01$ & 35,000 & 0 \\
\hline AW-102 & $242-\mathrm{A}$ & $5 / 9 / 01$ & $5 / 13 / 01$ & 846,000 & 31 \\
\hline $242-\mathrm{A}$ & AW-106 & $5 / 9 / 01$ & $5 / 13 / 01$ & $541,500^{\circ}$ & 31 \\
\hline $242-\mathrm{A}$ & LERF & $5 / 9 / 01$ & $5 / 13 / 01$ & 304,500 & 0 \\
\hline AW-106 & AW-102 & $5 / 14 / 01$ & $5 / 18 / 01$ & 846,500 & 35 \\
\hline AW-102 & 242-A & $6 / 1 / 01$ & $6 / 6 / 01$ & 846,300 & 35 \\
\hline $242-\mathrm{A}$ & AW-106 & $6 / 1 / 01$ & $6 / 6 / 01$ & 543,800 & 35 \\
\hline 242-A & LERF & $6 / 1 / 01$ & $6 / 6 / 01$ & 302,500 & 0 \\
\hline AZ-101 & AP-106 & $6 / 15 / 01$ & $6 / 15 / 01$ & 142,900 & 0 \\
\hline WASH-WATER & $\mathrm{AZ}-101$ & $6 / 16 / 01$ & $6 / 17 / 01$ & 138,200 & 0 \\
\hline AW-106 & AP-107 & $7 / 1 / 01$ & $7 / 3 / 01$ & 444,900 & 20 \\
\hline AW-105 & $\mathrm{AW}-102$ & $7 / 2 / 01$ & $7 / 5 / 01$ & 655,900 & 0 \\
\hline $\mathrm{AW}-102$ & $242-A$ & $7 / 20 / 01$ & $7 / 24 / 01$ & 656,200 & 24 \\
\hline $242-\mathrm{A}$ & AW-106 & $7 / 20 / 01$ & $7 / 24 / 01$ & 421,400 & 24 \\
\hline $242-\mathrm{A}$ & LERF & $7 / 20 / 01$ & $7 / 24 / 01$ & 234,800 & 0 \\
\hline $\mathrm{AW}-106$ & AW-102 & $7 / 24 / 01$ & $7 / 27 / 01$ & 520,100 & 21 \\
\hline AW-102 & $242-\mathrm{A}$ & $8 / 10 / 01$ & $8 / 12 / 01$ & 520,100 & 21 \\
\hline $242-\mathrm{A}$ & AW-106 & $8 / 10 / 01$ & $8 / 12 / 01$ & 334,100 & 21 \\
\hline $242-\mathrm{A}$ & LERF & $8 / 10 / 01$ & $8 / 12 / 01$ & 186,000 & 0 \\
\hline AW-106 & AW-102 & $8 / 12 / 01$ & $8 / 14 / 01$ & 334,000 & 14 \\
\hline$A W-102$ & $242-\mathrm{A}$ & $8 / 28 / 01$ & $8 / 30 / 01$ & 334,100 & 14 \\
\hline
\end{tabular}


Table A.5-1. Complete Transfer List for Case 5.

\begin{tabular}{|c|c|c|c|c|c|}
\hline From & To & Start Date & End Date & $\begin{array}{c}\text { Liquid } \\
\text { (gallons) }\end{array}$ & $\begin{array}{c}\text { Solid } \\
\text { (gallons) }\end{array}$ \\
\hline $242-A$ & AW-106 & $8 / 28 / 01$ & $8 / 30 / 01$ & 242,700 & 14 \\
\hline $242-\mathrm{A}$ & LERF & $8 / 28 / 01$ & $8 / 30 / 01$ & 91,401 & 0 \\
\hline ZNL87 & SY-102 & $10 / 1 / 01$ & $10 / 1 / 01$ & 4,000 & 0 \\
\hline TNS88 & AP-106 & $10 / 1 / 01$ & $10 / 1 / 01$ & 750 & 250 \\
\hline WESF & AP-106 & $10 / 1 / 01$ & $10 / 1 / 01$ & 5,000 & 0 \\
\hline SPN87 & SY-102 & $10 / 1 / 01$ & $10 / 1 / 01$ & 25,000 & 0 \\
\hline TAL88 & AP-106: & $10 / 1 / 01$ & $10 / 1 / 01$ & 23,000 & 0 \\
\hline WATER & AP-106 & $10 / 1 / 01$ & $10 / 1 / 01$ & 35,000 & 0 \\
\hline $34 \mathrm{~L} 87$ & AP-106 & $10 / 1 / 01$ & $10 / 1 / 01$ & 50,000 & 0 \\
\hline EVAPF & AP-106 & $10 / 1 / 01$ & $10 / 1 / 01$ & 120,000 & 0 \\
\hline WASH-WATER & AP-104 & $10 / 1 / 01$ & $10 / 2 / 01$ & 200,000 & 0 \\
\hline WATER & SY-102 & $10 / 2 / 01$ & $10 / 2 / 01$ & 5,000 & 0 \\
\hline WATER & AP-106 & $10 / 2 / 01$ & $10 / 2 / 01$ & 22,000 & 0 \\
\hline WATER & AP-106 & $10 / 2 / 01$ & $10 / 2 / 01$ & 5,000 & 0 \\
\hline AW-106 & AW-104 & $10 / 15 / 01$ & $10 / 16 / 01$ & 242,700 & 11 \\
\hline AP-104 & AP-102 & $10 / 15 / 01$ & $10 / 17 / 01$ & 386,500 & 39 \\
\hline AP-102 & AP-106 & $11 / 1 / 01$ & $11 / 3 / 01$ & 386,500 & 37 \\
\hline EVAPF & AW-102 & $11 / 3 / 01$ & $11 / 3 / 01$ & 35,000 & 0 \\
\hline AP-106 & $A W-102$ & $11 / 3 / 01$ & $11 / 7 / 01$ & 918,500 & 270 \\
\hline AZ-102 & AY-101 & $11 / 14 / 01$ & $11 / 15 / 01$ & 204,000 & 0 \\
\hline$A Z-102$ & AP-107 & $11 / 15 / 01$ & $11 / 16 / 01$ & 0 & 0 \\
\hline WASH-WATER & $A Z-102$ & $11 / 16 / 01$ & $11 / 17 / 01$ & 213,000 & 0 \\
\hline AN-105 & AP-102 & $11 / 16 / 01$ & $11 / 19 / 01$ & 542,400 & 7,895 \\
\hline LERF & AN-10S-PUMP & $11 / 16 / 01$ & $11 / 19 / 01$ & 154,100 & 0 \\
\hline AN-105 & AP-102 & $11 / 16 / 01$ & $11 / 19 / 01$ & 686,500 & 294 \\
\hline AP-108 & AP-106 & $11 / 21 / 01$ & $11 / 21 / 01$ & 0 & 0 \\
\hline AN-105 & $\mathrm{AP}-104$ & $11 / 20 / 01$ & $11 / 23 / 01$ & 542,400 & 7,895 \\
\hline LERF & AN-105-PUMP & $11 / 20 / 01$ & $11 / 23 / 01$ & 154,100 & 0 \\
\hline AN-105 & AP-104 & $11 / 20 / 01$ & $11 / 23 / 01$ & 686,500 & 294 \\
\hline WASH-WATER & AP-108 & $12 / 6 / 01$ & $12 / 7 / 01$ & 200,000 & 0 \\
\hline AP-108 & AP-106 & $12 / 20 / 01$ & $12 / 20 / 01$ & 200,000 & 1 \\
\hline AP-107 & AN-105 & $12 / 20 / 01$ & $12 / 23 / 01$ & $1,116,000$ & 87 \\
\hline $\mathrm{AZ}-102$ & AP-106 & $1 / 5 / 02$ & $1 / 6 / 02$ & 317,500 & 0 \\
\hline WASH-WATER & $A Z-102$ & $1 / 8 / 02$ & $1 / 9 / 02$ & 213,000 & 0 \\
\hline $\mathrm{AZ}-102$ & AP-106 & $2 / 27 / 02$ & $2 / 28 / 02$ & 205,900 & 0 \\
\hline WASH-WATER & $\mathrm{AZ}-102$ & $3 / 1 / 02$ & $3 / 2 / 02$ & 213,000 & 0 \\
\hline $\mathrm{AP}-102$ & $\mathrm{AP}-108$ & $3 / 11 / 02$ & $3 / 15 / 02$ & 686,500 & 285 \\
\hline AN-104 & AP-102 & $3 / 16 / 02$ & $3 / 19 / 02$ & 502,200 & 12,513 \\
\hline LERF & AN-104-PUMP & $3 / 16 / 02$ & $3 / 19 / 02$ & 200,800 & 0 \\
\hline AN-104 & AP-102 & $3 / 16 / 02$ & $3 / 19 / 02$ & 684,700 & 702 \\
\hline$A Z-102$ & AP-106 & $4 / 20 / 02$ & $4 / 21 / 02$ & 209,900 & 0 \\
\hline WASH-WATER & $A Z-102$ & $4 / 22 / 02$ & $4 / 23 / 02$ & 213,000 & 0 \\
\hline AW-102 & $242-\mathrm{A}$ & $5 / 7 / 02$ & $5 / 12 / 02$ & 950,600 & 34 \\
\hline $242-\mathrm{A}$ & AW-106 & $5 / 7 / 02$ & $5 / 12 / 02$ & 608,600 & 34 \\
\hline $242-\mathrm{A}$ & LERF & $5 / 7 / 02$ & $5 / 12 / 02$ & 342,000 & 0 \\
\hline AW-106 & $A W-102$ & $5 / 12 / 02$ & $5 / 15 / 02$ & 608,500 & 22 \\
\hline $\mathrm{AZ}-101$ & PIHLW FEED TANK & $5 / 17 / 02$ & $5 / 17 / 02$ & 134,300 & 5,079 \\
\hline AW-102 & $242-A$ & $5 / 30 / 02$ & $6 / 2 / 02$ & 608,600 & 22 \\
\hline $242-\mathrm{A}$ & AW-106 & $5 / 30 / 02$ & $6 / 2 / 02$ & 389,800 & 22 \\
\hline $242-\mathrm{A}$ & LEERF & $5 / 30 / 02$ & $6 / 2 / 02$ & 218,800 & 0 \\
\hline AW-106 & AW-102 & $6 / 2 / 02$ & $6 / 4 / 02$ & 389,700 & 15 \\
\hline AW-102 & $242-\mathrm{A}$ & $6 / 18 / 02$ & $6 / 20 / 02$ & 389,800 & 15 \\
\hline $242-A$ & AW-106 & $6 / 18 / 02$ & $6 / 20 / 02$ & 249,700 & 15 \\
\hline $242-\mathrm{A}$ & LERF & $6 / 18 / 02$ & $6 / 20 / 02$ & 140,100 & 0 \\
\hline$A W-106$ & $\mathrm{AW}-102$ & $6 / 20 / 02$ & $6 / 21 / 02$ & 249,600 & 10 \\
\hline AW-102 & 242-A & $7 / 5 / 02$ & $7 / 6 / 02$ & 249,700 & 10 \\
\hline
\end{tabular}


HNF-2321

Revision 0

Table A.5-1. Complete Transfer List for Case 5.

\begin{tabular}{|c|c|c|c|c|c|}
\hline From & To & Start Date & End Date & $\begin{array}{c}\text { Liquid } \\
\text { (gallons) }\end{array}$ & $\begin{array}{c}\text { Solid } \\
\text { (gallogs) }\end{array}$ \\
\hline $242-\mathrm{A}$ & $\mathrm{AW}-106$ & $7 / 5 / 02$ & $7 / 6 / 02$ & 159,700 & 10 \\
\hline $242-\mathrm{A}$ & LERF & $7 / 5 / 02$ & $7 / 6 / 02$ & 90,000 & 0 \\
\hline AW-106 & AW-102 & $7 / 6 / 02$ & $7 / 7 / 02$ & 159,700 & 7 \\
\hline $\mathrm{AW}-102$ & $A W-106$ & $7 / 8 / 02$ & $7 / 9 / 02$ & 159,800 & 7 \\
\hline AP-106 & AW-102 & $7 / 10 / 02$ & $7 / 15 / 02$ & 916,000 & 0 \\
\hline $\mathrm{AZ}-102$ & AP-106 & $7 / 15 / 02$ & $7 / 16 / 02$ & 211,600 & 0 \\
\hline WASH-WATER & AZ-102 & $7 / 17 / 02$ & $7 / 18 / 02$ & 118,000 & 0 \\
\hline WESF & AP-106 & $10 / 1 / 02$ & $10 / 1 / 02$ & 5,000 & 0 \\
\hline ZNL87 & SY-102 & $10 / 1 / 02$ & $10 / 1 / 02$ & 3,000 & 0 \\
\hline TNS88 & AP-106 & $10 / 1 / 02$ & $10 / 1 / 02$ & 750 & 250 \\
\hline SPN87 & SY-102 & $10 / 1 / 02$ & $10 / 1 / 02$ & 25,000 & 0 \\
\hline TAL88 & AP-106 & $10 / 1 / 02$ & $10 / 1 / 02$ & 24,000 & 0 \\
\hline WATER & AP-106 & $10 / 1 / 02$ & $10 / 1 / 02$ & 35,000 & 0 \\
\hline 34L87 & AP-106 & $10 / 1 / 02$ & $10 / 1 / 02$ & 50,000 & 0 \\
\hline EVAPF & AP-106 & $10 / 1 / 02$ & $10 / 1 / 02$ & 120,000 & 0 \\
\hline WATER & AP-106 & $10 / 2 / 02$ & $10 / 2 / 02$ & 22,000 & 0 \\
\hline WATER & SY-102 & $10 / 2 / 02$ & $10 / 2 / 02$ & 5,000 & 0 \\
\hline WATER & $A P-106$ & $10 / 2 / 02$ & $10 / 2 / 02$ & 5,000 & 0 \\
\hline WATER & SY-102 & $10 / 2 / 02$ & $10 / 2 / 02$ & 1,000 & 0 \\
\hline EVAPF & AW-102 & $10 / 4 / 02$ & $10 / 4 / 02$ & 50,000 & 0 \\
\hline $\mathrm{AW}-102$ & $242-\mathrm{A}$ & $10 / 23 / 02$ & $10 / 28 / 02$ & 966,000 & 34 \\
\hline $242-\mathrm{A}$ & AW-106 & $10 / 23 / 02$ & $10 / 28 / 02$ & 618,000 & 34 \\
\hline $242-\mathrm{A}$ & LERF & $10 / 23 / 02$ & $10 / 28 / 02$ & 348,000 & 0 \\
\hline AW-106 & $\mathrm{AW}-102$ & $10 / 28 / 02$ & $11 / 1 / 02$ & 777,700 & 30 \\
\hline EVAPF & AW-102 & $11 / 3 / 02$ & $11 / 3 / 02$ & 35,000 & 0 \\
\hline$A W-102$ & $242-\mathrm{A}$ & $11 / 15 / 02$ & $11 / 20 / 02$ & 812,800 & 31 \\
\hline $242=\mathrm{A}$ & AW-106 & $11 / 15 / 02$ & $11 / 20 / 02$ & 520,600 & 31 \\
\hline $242-\mathrm{A}$ & LERF & $11 / 15 / 02$ & $11 / 20 / 02$ & 292,100 & 0 \\
\hline AW-106 & AW-102 & $11 / 20 / 02$ & $11 / 22 / 02$ & 520,500 & 21 \\
\hline AW-102 & $242-\mathrm{A}$ & $12 / 6 / 02$ & $12 / 9 / 02$ & 520,700 & 21 \\
\hline $242-\mathbf{A}$ & AW-106 & $12 / 6 / 02$ & $12 / 9 / 02$ & 334,700 & 21 \\
\hline $242-\mathrm{A}$ & LERF & $12 / 6 / 02$ & $12 / 9 / 02$ & 186,000 & 0 \\
\hline $\mathrm{AW}-106$ & AW-102 & $12 / 9 / 02$ & $12 / 10 / 02$ & 334,600 & 15 \\
\hline$A W-102$ & $242-\mathrm{A}$ & $12 / 25 / 02$ & $12 / 26 / 02$ & 334,700 & 15 \\
\hline 242-A & AW-106 & $12 / 25 / 02$ & $12 / 26 / 02$ & 262,400 & 15 \\
\hline $242-\mathrm{A}$ & LERF & $12 / 25 / 02$ & $12 / 26 / 02$ & 72,297 & 0 \\
\hline AP-104 & AP-108 & $1 / 5 / 03$ & $1 / 9 / 03$ & 686,500 & 285 \\
\hline AN-104 & AP-104 & $1 / 9 / 03$ & $1 / 12 / 03$ & 502,200 & 12,513 \\
\hline LERF & AN-104-PUMP & $1 / 9 / 03$ & 1/12/03 & 200,800 & 0 \\
\hline AN-104 & AP-104 & $1 / 9 / 03$ & $1 / 12 / 03$ & 684,700 & 702 \\
\hline $\mathrm{AW}-106$ & AN-104 & $2 / 11 / 03$ & $2 / 13 / 03$ & 262,400 & 12 \\
\hline P1HLW FEED TANK & HLW-CONTRACTOR & $6 / 1 / 02$ & $2 / 21 / 03$ & 134,300 & 5,079 \\
\hline $\mathrm{AZ}-101$ & PIFLW FEED TANK & $2 / 21 / 03$ & $2 / 21 / 03$ & 134,300 & 5,079 \\
\hline $\mathrm{AY}-102$ & $A Z-101$ & $2 / 23 / 03$ & $2 / 27 / 03$ & 819,600 & 16,032 \\
\hline RETURN 2 & AP-107 & $3 / 31 / 03$ & $\cdot 3 / 31 / 03$ & 8,671 & 331 \\
\hline$\overline{\mathrm{AP}}-102$ & AP-108 & $9 / 20 / 03$ & $9 / 23 / 03$ & 684,700 & 686 \\
\hline AW-101 & AP-102 & $9 / 24 / 03$ & $9 / 28 / 03$ & 537,200 & 13,013 \\
\hline LERF & AW-101-PUMP & $9 / 24 / 03$ & $9 / 28 / 03$ & 308,100 & 0 \\
\hline AW-101 & AP-102 & $9 / 24 / 03$ & $9 / 28 / 03$ & 845,400 & 13,013 \\
\hline $34 \mathrm{~L} 87$ & AP-106 & $10 / 1 / 03$ & $10 / 1 / 03$ & 7,000 & 0 \\
\hline TNS88 & AP-106 & $10 / 1 / 03$ & $10 / 1 / 03$ & 750 & 250 \\
\hline WESF & AP-106 & $10 / 1 / 03$ & $10 / 1 / 03$ & 5,000 & 0 \\
\hline ZNL87 & SY-102 & $10 / 1 / 03$ & $10 / 1 / 03$ & 3,000 & 0 \\
\hline SPN87 & SY-102 & $10 / 1 / 03$ & $10 / 1 / 03$ & 25,000 & 0 \\
\hline TAL88 & AP-106 & $10 / 1 / 03$ & $10 / 1 / 03$ & 24,000 & 0 \\
\hline WATER & AP-106 & $10 / 1 / 03$ & $10 / 1 / 03$ & 35,000 & 0 \\
\hline
\end{tabular}


Table A.5-1. Complete Transfer List for Case 5.

\begin{tabular}{|c|c|c|c|c|c|}
\hline From & To & Start Date & End Date & $\begin{array}{c}\text { Liquid } \\
\text { (gallons) }\end{array}$ & $\begin{array}{c}\text { Solid } \\
\text { (gallons) }\end{array}$ \\
\hline $34 \mathrm{~L} 87$ & AP-106 & $10 / 1 / 03$ & $10 / 1 / 03$ & 50,000 & 0 \\
\hline EVAPF & AP-106 & $10 / 1 / 03$ & $10 / 1 / 03$ & 120,000 & 0 \\
\hline WATER & AP-106 & $10 / 2 / 03$ & $10 / 2 / 03$ & $.22,000$ & 0 \\
\hline WATER & SY-102 & $10 / 2 / 03$ & $10 / 2 / 03$ & 5,000 & 0 \\
\hline WATER & AP-106 & $10 / 2 / 03$ & $10 / 2 / 03$ & 3,000 & 0 \\
\hline WATER & AP-106 & $10 / 2 / 03$ & $10 / 2 / 03$ & 5,000 & 0 \\
\hline$A Z-101$ & AP-106 & $10 / 3 / 03$ & $10 / 5 / 03$ & 326,500 & 0 \\
\hline$A Z-101$ & AW-105 & $10 / 5 / 03$ & $10 / 6 / 03$ & 296,200 & 0 \\
\hline WASH-WATER & $A Z-101$ & $10 / 6 / 03$ & $10 / 8 / 03$ & 324,000 & 0 \\
\hline AP-106 & AW-102 & $10 / 5 / 03$ & $10 / 9 / 03$ & 916,300 & 0 \\
\hline AW-105 & AP-106 & $10 / 10 / 03$ & $10 / 12 / 03$ & 296,200 & 0 \\
\hline EVAPF & $\mathrm{AW}-102$ & $11 / 3 / 03$ & $11 / 3 / 03$ & 35,000 & 0 \\
\hline P1HLW FEED TANK & HLW-CONTRACTOR & $2 / 22 / 03$ & $11 / 14 / 03$ & 134,300 & 5,079 \\
\hline$A Z-102$ & P1HLW FEED TANK & $11 / 14 / 03$ & $11 / 14 / 03$ & 127,500 & 5,216 \\
\hline AZ-101 & AP-106 & $11 / 26 / 03$ & $11 / 28 / 03$ & 320,000 & 0 \\
\hline WASH-WATER & AZ-101 & $11 / 29 / 03$ & $12 / 1 / 03$ & 324,000 & 0 \\
\hline RETURN 2 & AP-107 & $1 / 28 / 04$ & $1 / 28 / 04$ & 8,671 & 331 \\
\hline $\mathrm{SX}-113$ & SY-103 & $12 / 31 / 03$ & $4 / 6 / 04$ & 10,322 & 399 \\
\hline$A W-102$ & $242-\mathrm{A}$ & $4 / 8 / 04$ & $4 / 12 / 04$ & 951,400 & 33 \\
\hline $242-\mathrm{A}$ & AW-106 & $4 / 8 / 04$ & $4 / 12 / 04$ & 609,400 & 33 \\
\hline $242-\mathrm{A}$ & LERF & $4 / 8 / 04$ & $4 / 12 / 04$ & 342,000 & 0 \\
\hline AW-106 & AW-102 & $4 / 13 / 04$ & $4 / 16 / 04$ & 609,300 & 22 \\
\hline AW-102 & $242-\mathrm{A}$ & $4 / 30 / 04$ & $5 / 3 / 04$ & 609,400 & 22 \\
\hline $242-\mathrm{A}$ & AW-106 & $4 / 30 / 04$ & $5 / 3 / 04$ & 389,500 & 22 \\
\hline $242-\mathrm{A}$ & LERF & $4 / 30 / 04$ & $5 / 3 / 04$ & 220,000 & 0 \\
\hline$A W-106$ & AW-102 & $5 / 3 / 04$ & $5 / 5 / 04$ & 389,400 & 14 \\
\hline$A W-102$ & $242-\mathrm{A}$ & $5 / 19 / 04$ & $5 / 21 / 04$ & 389,500 & 14 \\
\hline $242-\mathrm{A}$ & AW-106 & $5 / 19 / 04$ & $5 / 21 / 04$ & 249,100 & 14 \\
\hline $242-\mathrm{A}$ & LERF & $5 / 19 / 04$ & $5 / 21 / 04$ & 140,400 & 0 \\
\hline AW-106 & $\mathrm{AW}-\mathrm{102}$ & $5 / 21 / 04$ & $5 / 22 / 04$ & 249,000 & 9 \\
\hline $\mathrm{AW}-102$ & $242-\mathrm{A}$ & $6 / 5 / 04$ & $6 / 7 / 04$ & 249,100 & 9 \\
\hline $242-\mathrm{A}$ & AW-106 & $6 / 5 / 04$ & $6 / 7 / 04$ & 159,100 & 9 \\
\hline $242-\mathrm{A}$ & LERF & $6 / 5 / 04$ & $6 / 7 / 04$ & 90,000 & 0 \\
\hline AW-106 & $A W-102$ & $6 / 7 / 04$ & $6 / 7 / 04$ & 159,100 & 6 \\
\hline AW-102 & AW-106 & $6 / 9 / 04$ & $6 / 10 / 04$ & 159,200 & 6 \\
\hline AP-106 & $\mathrm{AW}-102$ & $6 / 10 / 04$ & $6 / 14 / 04$ & 794,200 & 0 \\
\hline AZ-10I & AP-106 & $6 / 14 / 04$ & $6 / 16 / 04$ & 322,300 & 0 \\
\hline $\mathrm{AP}-104$ & AP-108 & $6 / 13 / 04$ & $6 / 16 / 04$ & 684,700 & 686 \\
\hline WASH-WATER & AZ-101 & $6 / 17 / 04$ & $6 / 19 / 04$ & 433,600 & 0 \\
\hline AW-101 & AP-104 & $6 / 17 / 04$ & $6 / 21 / 04$ & 537,200 & 13,013 \\
\hline LERF & AW-101-PUMP & $6 / 17 / 04$ & $6 / 21 / 04$ & 308,100 & 0 \\
\hline AW-101 & AP-104 & $6 / 17 / 04$ & $6 / 21 / 04$ & 845,400 & 13,013 \\
\hline$A W-102$ & $242-\mathrm{A}$ & $6 / 29 / 04$ & $7 / 3 / 04$ & 794,200 & 27 \\
\hline $242-\mathrm{A}$ & AW-106 & $6 / 29 / 04$ & $7 / 3 / 04$ & 508,000 & 27 \\
\hline $242-A$ & LERF & $6 / 29 / 04$ & $7 / 3 / 04$ & 286,200 & 0 \\
\hline AN-104 & AW-101 & $7 / 5 / 04$ & $7 / 6 / 04$ & 256,000 & 12 \\
\hline$A W-106$ & AW-102 & $7 / 4 / 04$ & $7 / 7 / 04$ & 667,100 & 24 \\
\hline AW-102 & $242-\mathrm{A}$ & $7 / 22 / 04$ & $7 / 25 / 04$ & 667,100 & 24 \\
\hline $242-\mathrm{A}$ & AW-106 & $7 / 22 / 04$ & $7 / 25 / 04$ & 427,100 & 24 \\
\hline $242 \div \mathrm{A}$ & LERF & $7 / 22 / 04$ & $7 / 25 / 04$ & 240,000 & 0 \\
\hline AW-106 & AW-102 & $7 / 25 / 04$ & $7 / 27 / 04$ & 427,100 & 16 \\
\hline P1HLW FEED TANK & HLW-CONTRACTOR & $11 / 14 / 03$ & $8 / 9 / 04$ & 127,500 & 5,216 \\
\hline $\mathrm{AZ}-102$ & P1HLW FEED_TANK & $8 / 9 / 04$ & $8 / 10 / 04$ & 127,500 & 5,216 \\
\hline $\mathrm{AW}-102$ & $242-\mathrm{A}$ & $8 / 10 / 04$ & $8 / 12 / 04$ & 427,200 & 16 \\
\hline $242-\mathbf{A}$ & AW-106 & $8 / 10 / 04$ & $8 / 12 / 04$ & 272,400 & 16 \\
\hline $242-A$ & LERF & $8 / 10 / 04$ & $8 / 12 / 04$ & 154,700 & 0 \\
\hline
\end{tabular}


HNF-2321

Revision 0

Table A.5-1. Complete Transfer List for Case 5.

\begin{tabular}{|c|c|c|c|c|c|}
\hline From & To & Start Date & End Date & $\begin{array}{c}\text { Liquid } \\
\text { (gallons) }\end{array}$ & $\begin{array}{c}\text { Solid } \\
\text { (gallons) }\end{array}$ \\
\hline$A W-106$ & AW-102 & $8 / 12 / 04$ & $8 / 14 / 04$ & 272,400 & 11 \\
\hline RETURN 2 & AP-107 & $8 / 29 / 04$ & $8 / 29 / 04$ & 8,673 & 332 \\
\hline $\mathrm{AW}-102$ & $242-\mathrm{A}$ & $8 / 28 / 04$ & $8 / 29 / 04$ & 272,400 & 11 \\
\hline $242-\mathrm{A}$ & AW-106 & $8 / 28 / 04$ & $8 / 29 / 04$ & 174,600 & 11 \\
\hline $242-\mathrm{A}$ & LERF & $8 / 28 / 04$ & $8 / 29 / 04$ & 97,828 & 0 \\
\hline AW-106 & AW-102 & $8 / 29 / 04$ & $8 / 30 / 04$ & 174,600 & 8 \\
\hline AW-102 & AP-106 & $8 / 31 / 04$ & $9 / 1 / 04$ & 174,700 & 7 \\
\hline C-104 & AY-102 & $8 / 1 / 04$ & $9 / 8 / 04$ & 279,100 & 9,724 \\
\hline WESF & AP-106 & $10 / 1 / 04$ & $10 / 1 / 04$ & 5,000 & 0 \\
\hline TNS88 & AP-106 & $10 / 1 / 04$ & $10 / 1 / 04$ & 750 & 250 \\
\hline ZNNL87 & SY-102 & $10 / 1 / 04$ & $10 / 1 / 04$ & 3,000 & 0. \\
\hline SPN87 & $5 x-102$ & $10 / 1 / 04$ & $10 / 1 / 04$ & 2,000 & 0 \\
\hline TAL88 & AP-106 & $10 / 1 / 04$ & $10 / \mathrm{L} / 04$ & 22,000 & 0 \\
\hline WATER & AP-106 & $10 / 1 / 04$ & $10 / 1 / 04$ & 35,000 & 0 \\
\hline $34 \mathrm{~L} 87$ & AP-106 & $10 / 1 / 04$ & $10 / 1 / 04$ & 50,000 & 0 \\
\hline SPN87 & SY-102 & $10 / 1 / 04$ & $10 / 1 / 04$ & 23,000 & 0 \\
\hline TAL88 & AP-106 & $10 / 1 / 04$ & $10 / 1 / 04$ & 2,000 & 0 \\
\hline EVAPF & AP-106 & $10 / 1 / 04$ & $10 / 1 / 04$ & 120,000 & 0 \\
\hline 1FL96 & AW-105 & $10 / 1 / 04$ & $10 / 2 / 04$ & 200,000 & 0 \\
\hline WATER & AP-106 & $10 / 2 / 04$ & $10 / 2 / 04$ & 22,000 & 0 \\
\hline WATER & AP-106 & $10 / 2 / 04$ & $10 / 2 / 04$ & 4,000 & 0 \\
\hline WATER & SY-102 & $10 / 2 / 04$ & $10 / 2 / 04$ & 1,000 & 0 \\
\hline WATER & AW-105 & $10 / 2 / 04$ & $10 / 2 / 04$ & 88,000 & 0 \\
\hline WATER & SY-102 & $10 / 2 / 04$ & $10 / 3 / 04$ & 5,000 & 0 \\
\hline WATER & AP-106 & $10 / 3 / 04$ & $10 / 3 / 04$ & 1,000 & 0 \\
\hline EVAPF & $\mathrm{AW}-102$ & $10 / 4 / 04$ & $10 / 4 / 04$ & 50,000 & 0 \\
\hline EVAPF & AW-102 & $11 / 3 / 04$ & $11 / 3 / 04$ & 35,000 & 0 \\
\hline $\mathrm{AX}-103$ & AN-104 & $8 / 7 / 04$ & $12 / 24 / 04$ & 310,200 & 2,846 \\
\hline RETURN 2 & AP-107 & $1 / 16 / 05$ & $1 / 16 / 05$ & 8,670 & 332 \\
\hline $\mathrm{C}-103$ & AN-104 & $12 / 24 / 04$ & $3 / 9 / 05$ & 271,800 & 10,423 \\
\hline AP-102 & AP-108 & $3 / 7 / 05$ & $3 / 11 / 05$ & 740,300 & 1,581 \\
\hline WASH-WATER & AP-102 & $3 / 26 / 05$ & $3 / 26 / 05$ & 136,900 & 0 \\
\hline AP-102 & AP-106 & $4 / 9 / 05$ & $4 / 9 / 05$ & 243,100 & 10,337 \\
\hline AY-101 & AP-102 & $4 / 10 / 05$ & $4 / 11 / 05$ & 283,600 & 12 \\
\hline AP-106 & AW-102 & $4 / 9 / 05$ & $4 / 13 / 05$ & 832,200 & 0 \\
\hline P1HLW FEED TANK & HLW-CONTRACTOR & $8 / 10 / 04$ & $5 / 6 / 05$ & 127,500 & 5,216 \\
\hline$A Z-101$ & P1HLW FEED TANK & $5 / 6 / 05$ & $5 / 7 / 05$ & 127,200 & 3,269 \\
\hline RETURN 2 & AP-107 & $5 / 31 / 05$ & $5 / 31 / 05$ & 8,672 & 331 \\
\hline $\mathrm{A}-102$ & AN-104 & $3 / 9 / 05$ & $6 / 28 / 05$ & 100,600 & 744 \\
\hline AP-106 & AW-102 & $7 / 15 / 05$ & $7 / 15 / 05$ & 53,172 & 2 \\
\hline AY-102 & $A Z-102$ & $8 / 1 / 05$ & $8 / 3 / 05$ & 279,400 & 9,360 \\
\hline AN-104 & AN-105 & $8 / 16 / 05$ & $8 / 16 / 05$ & 0 & 0 \\
\hline SY-102 & AP-106 & $8 / 15 / 05$ & $8 / 16 / 05$ & 385,200 & 15 \\
\hline AN-104 & AW-104 & $8 / 16 / 05$ & $8 / 17 / 05$ & 22,739 & 580 \\
\hline $\mathrm{AN}-104$ & AW-103 & $8 / 17 / 05$ & $8 / 17 / 05$ & 3,900 & 99 \\
\hline $\mathrm{AN}-104$ & AP-105 & $8 / 17 / 05$ & $8 / 17 / 05$ & 3,901 & 99 \\
\hline AN-104 & AP-103 & $8 / 17 / 05$ & $8 / 17 / 05$ & 42,517 & 1,082 \\
\hline AN-104 & AP-101 & $8 / 17 / 05$ & $8 / 18 / 05$ & 217,000 & 5,521 \\
\hline $\mathrm{AN}-104$ & AN-101 & $8 / 18 / 05$ & $8 / 18 / 05$ & 0 & 0 \\
\hline AN-104 & AN-106 & $8 / 18 / 05$ & $8 / 19 / 05$ & 68,852 & 1.750 \\
\hline AN-104 & AW-105 & $8 / 19 / 05$ & $8 / 21 / 05$ & 457,500 & 11,620 \\
\hline TNS88 & AP-106 & $10 / 1 / 05$ & $10 / 1 / 05$ & 750 & 250 \\
\hline WESF & AP-106 & $10 / 1 / 05$ & $10 / 1 / 05$ & 5,000 & 0 \\
\hline ZNL87 & SY-102 & $10 / 1 / 05$ & $10 / 1 / 05$ & 3,000 & 0 \\
\hline TAL88 & AP-106 & $10 / 1 / 05$ & $10 / 1 / 05$ & 24,000 & 0 \\
\hline SPN87 & SY-102 & $10 / 1 / 05$ & $10 / 1 / 05$ & 25,000 & 0 \\
\hline
\end{tabular}


HNF-2321

Revision 0

Table A.5-1. Complete Transfer List for Case 5.

\begin{tabular}{|c|c|c|c|c|c|}
\hline From & To & Start Date & End Date & $\begin{array}{c}\text { Liquid } \\
\text { (gallons) }\end{array}$ & $\begin{array}{c}\text { Solid } \\
\text { (gallons) }\end{array}$ \\
\hline WATER & AP-106 & $10 / 1 / 05$ & $10 / 1 / 05$ & 35,000 & 0 \\
\hline $34 \mathrm{~L} 87$ & AP-106 & $10 / 1 / 05$ & $10 / 1 / 05$ & 50,000 & 0 \\
\hline EVAPF & AP-106 & $10 / 1 / 05$ & $10 / 1 / 05$ & 120,000 & 0 \\
\hline WATER & SY-102 & $10 / 2 / 05$ & $10 / 2 / 05$ & 1,000 & 0 \\
\hline WATER & AP-106 & $10 / 2 / 05$ & $10 / 2 / 05$ & 22,000 & 0 \\
\hline WATER & AP-106 & $10 / 2 / 05$ & $10 / 2 / 05$ & 5,000 & 0 \\
\hline WATER & SY-102 & $10 / 2 / 05$ & $10 / 2 / 05$ & 5,000 & 0 \\
\hline RETURN 2 & AP-107 & $10 / 9 / 05$ & $10 / 9 / 05$ & 8,672 & 330 \\
\hline AP-104 & AP-108 & $10 / 11 / 05$ & $10 / 15 / 05$ & 740,300 & 1,581 \\
\hline AW-102 & $242-\mathrm{A}$ & $10 / 11 / 05$ & $10 / 16 / 05$ & 970,300 & 35 \\
\hline $242-\mathrm{A}$ & AW-106 & $10 / 11 / 05$ & $10 / 16 / 05$ & 622,300 & 35 \\
\hline $242-\mathrm{A}$ & LERF & $10 / 11 / 05$ & $10 / 16 / 05$ & 348,000 & 0 \\
\hline AW-106 & AW-102 & $10 / 16 / 05$ & $10 / 19 / 05$ & 622,200 & 24 \\
\hline WASH-WATER & AP-104 & $10 / 30 / 05$ & $10 / 30 / 05$ & 136,900 & 0 \\
\hline EVAPF & AW-102 & $11 / 3 / 05$ & $11 / 3 / 05$ & 35,000 & 0 \\
\hline AW-102 & $242-A$ & $11 / 2 / 05$ & $11 / 5 / 05$ & 622,400 & 24 \\
\hline $242-\mathrm{A}$ & AW-106 & $11 / 2 / 05$ & $11 / 5 / 05$ & 399,800 & 24 \\
\hline $242-\mathrm{A}$ & LERF & $11 / 2 / 05$ & $11 / 5 / 05$ & 222,500 & 0 \\
\hline AW-106 & $\mathrm{AW}-102$ & $11 / 5 / 05$ & $11 / 7 / 05$ & 399,500 & 16 \\
\hline AP-104 & AP-106 & $11 / 13 / 05$ & $11 / 13 / 05$ & 243,100 & 10,337 \\
\hline AN-107 & AP-104 & $11 / 14 / 05$ & $11 / 16 / 05$ & 481,700 & 22 \\
\hline LERF & AN-107-PUMP & $11 / 14 / 05$ & $11 / 16 / 05$ & 72,259 & 0 \\
\hline AN-107 & AP-104 & $11 / 14 / 05$ & $11 / 16 / 05$ & 554,000 & 22 \\
\hline PLHLW FEED TANK & HLW-CONTRACTOR & $5 / 7 / 05$ & $11 / 21 / 05$ & 127,200 & 3,269 \\
\hline$A Z-101$ & PIHLW FEED TANK & $11 / 21 / 05$ & $11 / 21 / 05$ & 127,200 & 3,269 \\
\hline AW-102 & $242-\mathrm{A}$ & $11 / 22 / 05$ & $11 / 24 / 05$ & 434,600 & 17 \\
\hline $242-\mathrm{A}$ & AW-106 & $11 / 22 / 05$ & $11 / 24 / 05$ & 278,600 & 17 \\
\hline $242-\mathrm{A}$ & LERF & $11 / 22 / 05$ & $11 / 24 / 05$ & 156,000 & 0 \\
\hline $\mathrm{AW}-106$ & AW-102 & $11 / 24 / 05$ & $11 / 25 / 05$ & 278,600 & 12 \\
\hline RETURN_2 & AP-107 & $11 / 29 / 05$ & $11 / 29 / 05$ & 8,673 & 330 \\
\hline AW-102 & $242-\mathrm{A}$ & $12 / 9 / 05$ & $12 / 11 / 05$ & 278,600 & 12 \\
\hline $242-\mathrm{A}$ & AW-106 & $12 / 9 / 05$ & $12 / 11 / 05$ & 202,100 & 12 \\
\hline $242-A$ & LERF & $12 / 9 / 05$ & $12 / 11 / 05$ & 76,590 & 0 \\
\hline AW-106 & AW-101 & $12 / 11 / 05$ & $12 / 12 / 05$ & 202,000 & 9 \\
\hline AP-106 & AW-102 & $12 / 12 / 05$ & $12 / 16 / 05$ & 775,500 & 0 \\
\hline RETURN 2 & AP-107 & $1 / 18 / 06$ & $1 / 18 / 06$ & 8,677 & 330 \\
\hline RETURN 2 & AP-107 & $3 / 10 / 06$ & $3 / 10 / 06$ & 8,677 & 330 \\
\hline RETURN 2 & AP-107 & $4 / 29 / 06$ & $4 / 29 / 06$ & 8,677 & 330 \\
\hline AW-102 & $242-\mathrm{A}$ & $5 / 17 / 06$ & $5 / 21 / 06$ & 775,600 & 29 \\
\hline $242-\mathrm{A}$ & AW-105 & $5 / 17 / 06$ & $5 / 21 / 06$ & 497,600 & 29 \\
\hline $242-\mathrm{A}$ & LERF & $5 / 17 / 06$ & $5 / 21 / 06$ & 278,000 & 0 \\
\hline AW-106 & $\mathrm{AW}-102$ & $5 / 21 / 06$ & $5 / 23 / 06$ & 497,500 & 20 \\
\hline P1HLW FEED TANK & HLW-CONTRACTOR & $11 / 21 / 05$ & $6 / 7 / 06$ & 127,200 & 3,269 \\
\hline $\mathrm{AZ}-101$ & P1HLW FEED_TANK & $6 / 7 / 06$ & $6 / 8 / 06$ & 127,200 & 3,269 \\
\hline $\mathrm{AW}-102$ & $242-\mathrm{A}$ & $6 / 6 / 06$ & $6 / 9 / 06$ & 497,600 & 20 \\
\hline $242-\mathrm{A}$ & AW-106 & $6 / 6 / 06$ & $6 / 9 / 06$ & 318,100 & 20 \\
\hline $242-A$ & LERF & $6 / 6 / 06$ & $6 / 9 / 06$ & 179,500 & 0 \\
\hline AW-106 & AW-102 & $6 / 9 / 06$ & $6 / 10 / 06$ & 318,100 & 14 \\
\hline AP-108 & LAW-CONTRACTOR-2 & $6 / 1 / 02$ & $6 / 13 / 06$ & $4,223,000$ & 5,047 \\
\hline AP-102 & AP-108 & $6 / 13 / 06$ & $6 / 14 / 06$ & 277,300 & 552 \\
\hline AN-107 & AP-102 & $6 / 15 / 06$ & $6 / 17 / 06$ & 481,700 & 22 \\
\hline LERF & AN-107-PUMP & $6 / 15 / 06$ & $6 / 17 / 06$ & 72,260 & 0 \\
\hline AN-107 & AP-102 & $6 / 15 / 06$ & $6 / 17 / 06$ & 554,000 & 22 \\
\hline RETURN 2 & AP-107 & $6 / 18 / 06$ & $6 / 18 / 06$ & 8,676 & 330 \\
\hline AW-102 & $242-\mathrm{A}$ & $6 / 25 / 06$ & $6 / 26 / 06$ & 318,100 & 14 \\
\hline $242-\mathrm{A}$ & AW-106 & $6 / 25 / 06$ & $6 / 26 / 06$ & 222,300 & 14 \\
\hline
\end{tabular}


HNF-2321

Revision 0

Table A.5-1. Complete Transfer List for Case 5.

\begin{tabular}{|c|c|c|c|c|c|}
\hline From & To & Start Date & End Date & $\begin{array}{c}\text { Liquid } \\
\text { (gallons) }\end{array}$ & $\begin{array}{c}\text { Solid } \\
\text { (gallons) }\end{array}$ \\
\hline $242-A$ & LERF & $6 / 25 / 06$ & $6 / 26 / 06$ & 95,841 & 0 \\
\hline AW-106 & AW-101 & $6 / 26 / 06$ & $6 / 27 / 06$ & 222,300 & 10 \\
\hline $\mathrm{AZ}-102$ & AP-106 & $6 / 27 / 06$ & $6 / 28 / 06$ & 159,800 & 0 \\
\hline WASH-WATER & $\mathrm{AZ}-102$ & $6 / 28 / 06$ & $6 / 29 / 06$ & 324,000 & 0 \\
\hline RETURN 2 & AP-107 & $7 / 24 / 06$ & $7 / 24 / 06$ & 8,678 & 328 \\
\hline AP-108 & LAW-CONTRACTOR-2 & $6 / 15 / 06$ & $8 / 13 / 06$ & 277,300 & 555 \\
\hline AP-104 & AP-108 & $8 / 13 / 06$ & $8 / 14 / 06$ & 171,100 & 337 \\
\hline AZ-102 & AP-106 & $8 / 17 / 06$ & $8 / 19 / 06$ & 320,100 & 0 \\
\hline WASH-WATER & $A Z-102$ & $8 / 20 / 06$ & $8 / 22 / 06$ & 324,000 & 0 \\
\hline RETURN 2 & AP-107 & $9 / 7 / 06$ & $9 / 7 / 06$ & 8,673 & 329 \\
\hline AP-104 & AP-108 & $9 / 10 / 06$ & $9 / 12 / 06$ & 381,800 & 753 \\
\hline AN-102 & AP-104 & $9 / 13 / 06$ & $9 / 16 / 06$ & 443,500 & 21 \\
\hline LERF & AN-102-PUMP & $9 / 13 / 06$ & $9 / 16 / 06$ & 266,100 & 0 \\
\hline AN-102 & AP-104 & $9 / 13 / 06$ & $9 / 16 / 06$ & 709,700 & 21 \\
\hline AN-104 & AN-107. & $9 / 15 / 06$ & $9 / 17 / 06$ & 305,600 & 7,751 \\
\hline $34 \mathrm{~L} 87$ & AP-106 & $10 / 1 / 06$ & $10 / 1 / 06$ & 7,000 & 0 \\
\hline TNS88 & AP-106 & $10 / 1 / 06$ & $10 / 1 / 06$ & 750 & 250 \\
\hline WESF & AP-106 & $10 / 1 / 06$ & 10/1/06. & 5,000 & 0 \\
\hline TAL88 & AP-106 & $10 / 1 / 06$ & $10 / 1 / 06$ & 25,000 & 0 \\
\hline SPN87 & SY-102 & $10 / 1 / 06$ & $10 / 1 / 06$ & 25,000 & 0 \\
\hline 34 L87 & AP-106 & $10 / 1 / 06$ & $10 / 1 / 06$ & 50,000 & 0 \\
\hline WATER & AP-106 & $10 / 1 / 06$ & $10 / 1 / 06$ & 35,000 & 0 \\
\hline EVAPF & AP-106 & $10 / 1 / 06$ & $10 / 1 / 06$ & 120,000 & 0 \\
\hline WATER & AP-106 & $10 / 2 / 06$ & $10 / 2 / 06$ & 3,000 & 0 \\
\hline WATER & AP-106 & $10 / 2 / 06$ & $10 / 2 / 06$ & 22,000 & 0 \\
\hline WATER & AP-106 & $10 / 2 / 06$ & $10 / 2 / 06$ & 5,000 & 0 \\
\hline WATER & SY-102 & $10 / 2 / 06$ & $10 / 2 / 06$ & 5,000 & 0 \\
\hline EVAPF & AW-102 & $10 / 4 / 06$ & $10 / 4 / 06$ & 50,000 & 0 \\
\hline AP-106 & AW-102 & $10 / 1 / 06$ & $10 / 5 / 06$ & 749,800 & 0 \\
\hline$A Z-102$ & AP-106 & $10 / 10 / 06$ & $10 / 12 / 06$ & 322,700 & 0 \\
\hline WASH-WATER & AZ-102 & $10 / 13 / 06$ & $10 / 14 / 06$ & 150,200 & 0 \\
\hline RETURN 2 & AP-107 & $11 / 2 / 06$ & $11 / 2 / 06$ & 8,683 & 330 \\
\hline EVAPF & AW-102 & $11 / 3 / 06$ & $11 / 3 / 06$ & 35,000 & 0 \\
\hline $\mathrm{C}-105$ & AN-104 & $6 / 28 / 05$ & $11 / 15 / 06$ & 827,500 & 31,875 \\
\hline P1HLW FEED TANK & HLW-CONTRACTOR & $6 / 8 / 06$ & $12 / 22 / 06$ & 127,200 & 3,269 \\
\hline $\mathrm{AZ}-101$ & P1HLW FEED TANK & $12 / 22 / 06$ & $12 / 23 / 06$ & 127,200 & 3,269 \\
\hline RETURN 2 & AP-107 & $12 / 28 / 06$ & $12 / 28 / 06$ & 8,683 & 330 \\
\hline AP-102 & AP-108 & $1 / 21 / 07$ & $1 / 24 / 07$ & 559,200 & 575 \\
\hline AN-103 & AP-102 & $1 / 25 / 07$ & $1 / 28 / 07$ & 436,600 & 28,133 \\
\hline LERF & AN-103-PUMP & $1 / 25 / 07$ & $1 / 28 / 07$ & 264,900 & 0 \\
\hline AN-103 & AP-102 & $1 / 25 / 07$ & $1 / 28 / 07$ & 738,300 & 549 \\
\hline RETURN 2 & AP-107 & $2 / 23 / 07$ & $2 / 23 / 07$ & 8,670 & 330 \\
\hline $\mathrm{A}-104$ & AN-104 & $11 / 15 / 06$ & $2 / 24 / 07$ & 29,827 & 832 \\
\hline C-204 & AN-104 & $2 / 24 / 07$ & $3 / 2 / 07$ & 3,775 & 55 \\
\hline C-201 & $\mathrm{AN}-104$ & $3 / 2 / 07$ & $3 / 8 / 07$ & 2,464 & 59 \\
\hline AW-102 & $242-\mathrm{A}$ & $4 / 4 / 07$ & $4 / 8 / 07$ & 834,900 & 29 \\
\hline $242-A$ & AW-106 & $4 / 4 / 07$ & $4 / 8 / 07$ & 534,900 & 29 \\
\hline $242-\mathrm{A}$ & LERF & $4 / 4 / 07$ & $4 / 8 / 07$ & 300,000 & 0 \\
\hline AW-106 & AW-102 & $4 / 9 / 07$ & $4 / 12 / 07$ & 534,800 & 19 \\
\hline $\mathrm{AX}-104$ & AN-104 & $3 / 8 / 07$ & $4 / 13 / 07$ & 18,516 & 572 \\
\hline RETURN 2 & AP-107 & $4 / 22 / 07$ & $4 / 22 / 07$ & 8,672 & 330 \\
\hline AW-102 & $242-\mathrm{A}$ & $4 / 26 / 07$ & $4 / 29 / 07$ & 534,900 & 19 \\
\hline $242-A$ & AW-106 & $4 / 26 / 07$ & $4 / 29 / 07$ & 342,900 & 19 \\
\hline $242-\mathrm{A}$ & LERF & $4 / 26 / 07$ & $4 / 29 / 07$ & 192,000 & 0 \\
\hline AW-106 & AW-102 & $4 / 29 / 07$ & $4 / 30 / 07$ & 342,800 & 13 \\
\hline $\mathrm{AW}-102$ & $242-\mathrm{A}$ & $5 / 15 / 07$ & $5 / 16 / 07$ & 342,900 & 13 \\
\hline
\end{tabular}


HNF-2321

Revision 0

Table A.5-1. Complete Transfer List for Case 5.

\begin{tabular}{|c|c|c|c|c|c|}
\hline From & To & Start Date & End Date & $\begin{array}{c}\text { Liquid } \\
\text { (gallons) }\end{array}$ & $\begin{array}{c}\text { Solid } \\
\text { (gallons) }\end{array}$ \\
\hline $242-\mathrm{A}$ & AW-106 & $5 / 15 / 07$ & $5 / 16 / 07$ & 217,700 & 13 \\
\hline $242-\mathrm{A}$ & LERF & $5 / 15 / 07$ & $5 / 16 / 07$ & 125,200 & 0 \\
\hline AW-106 & AW-102 & $5 / 16 / 07$ & $5 / 17 / 07$ & 217,700 & 8 \\
\hline AW-102 & AP-106 & $5 / 18 / 07$ & $5 / 20 / 07$ & 217,800 & 8 \\
\hline RETURN 2 & AP-107 & $6 / 19 / 07$ & $6 / 19 / 07$ & 8,672 & 330 \\
\hline P1HLW FEED TANK & HLW-CONTRACTOR & $12 / 23 / 06$ & $7 / 9 / 07$ & 127,200 & 3,269 \\
\hline $\mathrm{AZ-101}$ & PIHLW FEED TANK & $7 / 9 / 07$ & $7 / 9 / 07$ & 127,200 & 3,269 \\
\hline AX-102 & AN-104 & $4 / 13 / 07$ & $8 / 1 / 07$ & 87,597 & 897 \\
\hline AP-104 & AP-108 & $8 / 4 / 07$ & $8 / 8 / 07$ & 709,600 & 72 \\
\hline AN-103 & AP-104 & $8 / 9 / 07$ & $8 / 12 / 07$ & 436,600 & 28,133 \\
\hline LERF & AN-103-PUMP & $8 / 9 / 07$ & $8 / 12 / 07$ & 264,900 & 0 \\
\hline AN-103 & AP-104 & $8 / 9 / 07$ & $8 / 12 / 07$ & 738,300 & 549 \\
\hline RETURN 2 & AP-107 & $8 / 25 / 07$ & $8 / 25 / 07$ & 8,676 & 330 \\
\hline WESF & AP-106 & $10 / 1 / 07$ & $10 / 1 / 07$ & 5,000 & 0 \\
\hline TNS88 & AP-106 & $10 / 1 / 07$ & $10 / 1 / 07$ & 750 & 250 \\
\hline TAL88 & AP-106 & $10 / 1 / 07$ & $10 / 1 / 07$ & 25,000 & 0 \\
\hline SPN87 & SY-102 & $10 / 1 / 07$ & $10 / 1 / 07$ & 25,000 & 0 \\
\hline $34 \mathrm{~L} 87$ & AP-106 & $10 / 1 / 07$ & $10 / 1 / 07$ & 50,000 & 0 \\
\hline WATER & AP-106 & $10 / 1 / 07$ & $10 / 1 / 07$ & 35,000 & 0 \\
\hline EVAPF & AP-106 & $10 / 1 / 07$ & $10 / 1 / 07$ & 120,000 & 0 \\
\hline WATER & AP-106 & $10 / 2 / 07$ & $10 / 2 / 07$ & 5,000 & 0 \\
\hline WATER & SY-102 & $10 / 2 / 07$ & $10 / 2 / 07$ & 5,000 & 0 \\
\hline WATER & AP-106 & $10 / 2 / 07$ & $10 / 2 / 07$ & 22,000 & 0 \\
\hline AP-106 & AW-102 & $10 / 1 / 07$ & $10 / 5 / 07$ & 803,200 & 274 \\
\hline EVAPF & $A W-102$ & $11 / 3 / 07$ & $11 / 3 / 07$ & 35,000 & 0 \\
\hline AN-104 & $\mathrm{AN}-107$ & $11 / 5 / 07$ & $11 / 8 / 07$ & 658,300 & 12,731 \\
\hline AN-104 & AN-102 & $11 / 8 / 07$ & $11 / 11 / 07$ & 441,700 & 8,533 \\
\hline SY-101 & $\mathrm{AN}-103$ & $11 / 10 / 07$ & $11 / 14 / 07$ & $1,105,000$ & 11,018 \\
\hline AN-103 & AN-102 & $11 / 15 / 07$ & $11 / 15 / 07$ & 57,642 & 646 \\
\hline RETURN_2 & AP-107 & $12 / 2 / 07$ & $12 / 2 / 07$ & 8,675 & 329 \\
\hline P1HLW FEED TANK & HLW-CONTRACTOR & $7 / 9 / 07$ & $1 / 23 / 08$ & 127,200 & 3,269 \\
\hline $\mathrm{AZ}-102$ & PIHLW FEED TANK & $1 / 23 / 08$ & $1 / 24 / 08$ & 137,400 & 4,760 \\
\hline RETURN 2 & AP-107 & $3 / 9 / 08$ & $3 / 9 / 08$ & 8,675 & 329 \\
\hline AW-102 & $242-\mathrm{A}$ & $4 / 3 / 08$ & $4 / 7 / 08$ & 835,100 & 29 \\
\hline $242-\mathrm{A}$ & AW-106 & $4 / 3 / 08$ & $4 / 7 / 08$ & 535,100 & 29 \\
\hline $242-\mathrm{A}$ & LERF & $4 / 3 / 08$ & $4 / 7 / 08$ & 300,000 & 0 \\
\hline AW-106 & AW-102 & $4 / 8 / 08$ & $4 / 11 / 08$ & 535,000 & 19 \\
\hline AP-108 & LAW-CONTRACTOR-2 & $8 / 15 / 06$ & $4 / 11 / 08$ & $1,822,000$ & 1,786 \\
\hline AP-102 & $\mathrm{AP}-108$ & $4 / 11 / 08$ & $4 / 15 / 08$ & 738,300 & 557 \\
\hline AW-102 & $242-\mathrm{A}$ & $4 / 25 / 08$ & $4 / 28 / 08$ & 535,100 & 19 \\
\hline $242-\mathrm{A}$ & AW-106 & $4 / 25 / 08$ & $4 / 28 / 08$ & 343,100 & 19 \\
\hline $242-\mathrm{A}$ & LERF & $4 / 25 / 08$ & $4 / 28 / 08$ & 192,000 & 0 \\
\hline AW-106 & $\mathrm{AW}-102$ & $4 / 28 / 08$ & $4 / 29 / 08$ & 343,100 & 13 \\
\hline S-103 & $S Y-101$ & $12 / 31 / 03$ & $5 / 7 / 08$ & 712,300 & 1,011 \\
\hline AW-102 & 242-A & $5 / 13 / 08$ & $5 / 15 / 08$ & 343,100 & 13 \\
\hline $242-\mathrm{A}$ & AW-106 & $5 / 13 / 08$ & $5 / 15 / 08$ & 218,000 & 13 \\
\hline $242-\mathrm{A}$ & LERF & $5 / 13 / 08$ & $5 / 15 / 08$ & 125,100 & 0 \\
\hline AW-106 & AW-102 & $5 / 15 / 08$ & $5 / 16 / 08$ & 218,000 & 9 \\
\hline AW-102 & AP-106 & $5 / 17 / 08$ & $5 / 18 / 08$ & 218,100 & 8 \\
\hline RETURN 2 & AP-107 & $8 / 14 / 08$ & $8 / 14 / 08$ & 8,673 & 330 \\
\hline WESF & AP-106 & $10 / 1 / 08$ & $10 / 1 / 08$ & 5,000 & 0 \\
\hline TNS88 & AP-106 & $10 / 1 / 08$ & $10 / 1 / 08$ & 750 & 250 \\
\hline \begin{tabular}{|l|} 
SPN87 \\
\end{tabular} & SY-102 & $10 / 1 / 08$ & $10 / 1 / 08$ & 25,000 & 0 \\
\hline TAL88 & AP-106 & $10 / 1 / 08$ & $10 / 1 / 08$ & 25,000 & 0 \\
\hline WATER & AP-106 & $10 / 1 / 08$ & $10 / 1 / 08$ & 35,000 & 0 \\
\hline $34 \mathrm{~L} 87$ & $\longdiv { \mathrm { AP } - 1 0 6 }$ & $10 / 1 / 08$ & $10 / 1 / 08$ & 50,000 & 0 \\
\hline
\end{tabular}


HNF-2321

Revision 0

Table A.5-1. Complete Transfer List for Case 5.

\begin{tabular}{|c|c|c|c|c|c|}
\hline From & To & Start Date & End Date & $\begin{array}{c}\text { Liquid } \\
\text { (gallons) }\end{array}$ & $\begin{array}{c}\text { Solid } \\
\text { (gallous) }\end{array}$ \\
\hline EVAPF & AP-106 & $10 / 1 / 08$ & $10 / 1 / 08$ & 120,000 & 0 \\
\hline WATER & AP-106 & $10 / 2 / 08$ & $10 / 2 / 08$ & 22,000 & 0 \\
\hline WATER & $\$ Y-102$ & $10 / 2 / 08$ & $10 / 2 / 08$ & 5,000 & 0 \\
\hline WATER & AP-106 & $10 / 2 / 08$ & $10 / 2 / 08$ & 5,000 & 0 \\
\hline EVAPF & AW-102 & $10 / 4 / 08$ & $10 / 4 / 08$ & 50,000 & 0 \\
\hline EVAPF & AW-102 & $11 / 3 / 08$ & $11 / 3 / 08$ & 35,000 & 0 \\
\hline PIHLW FEED TANK & HLW-CONTRACTOR & $1 / 24 / 08$ & $12 / 26 / 08$ & 137,400 & 4,760 \\
\hline AZ-102 & PIHLW FEED TANK & $12 / 26 / 08$ & $12 / 27 / 08$ & 137,400 & 4,760 \\
\hline $\mathrm{U}-202$ & UA-2 & $1 / 3 / 09$ & $1 / 10 / 09$ & 27,411 & 1,061 \\
\hline AP-104 & AP-108 & $1 / 6 / 09$ & $1 / 10 / 09$ & 738,400 & 532 \\
\hline U-203 & UA-2 & $1 / 10 / 09$ & $1 / 16 / 09$ & 13,895 & 537 \\
\hline RETURN 2 & AP-107 & $2 / 12 / 09$ & $2 / 12 / 09$ & $8,670^{\circ}$ & 331 \\
\hline $\mathrm{U}-110$ & UA-2 & $1 / 16 / 09$ & $3 / 10 / 09$ & 99,290 & 4,252 \\
\hline UA-2 & $S Y-102$ & $3 / 11 / 09$ & $3 / 12 / 09$ & 173,900 & 5,847 \\
\hline $\mathrm{U}-110$ & UA-1 & $3 / 10 / 09$ & $5 / 27 / 09$ & 143,800 & 6,160 \\
\hline UA-1 & SY-102 & $5 / 28 / 09$ & $5 / 29 / 09$ & 186,800 & 6,157 \\
\hline U-110 & UA-2 & $5 / 27 / 09$ & $5 / 30 / 09$ & 4,829 & 207 \\
\hline $\mathrm{U}-101$ & UA-2 & $5 / 30 / 09$ & $7 / 30 / 09$ & 27,955 & 668 \\
\hline RETURN_2 & AP-107 & $8 / 13 / 09$ & $8 / 13 / 09$ & 8,670 & 331 \\
\hline TNS88 & AP-106 & $10 / 1 / 09$ & $10 / 1 / 09$ & 750 & 250 \\
\hline WESF & AP-106 & $10 / 1 / 09$ & $10 / 1 / 09$ & 5,000 & 0 \\
\hline TAL88 & AP-106 & $10 / 1 / 09$ & $10 / 1 / 09$ & 26,000 & 0 \\
\hline $34 \mathrm{L87}$ & AP-106 & $10 / 1 / 09$ & $10 / 1 / 09$ & 50,000 & 0 \\
\hline WATER & AP-106 & $10 / 1 / 09$ & $10 / 1 / 09$ & 35,000 & 0 \\
\hline $34 \mathrm{~L} 87$ & AP-106 & $10 / 1 / 09$ & $10 / 1 / 09$ & 7,000 & 0 \\
\hline \begin{tabular}{|l} 
SPN87 \\
\end{tabular} & SY-102 & $10 / 1 / 09$ & $10 / 1 / 09$ & 25,000 & 0 \\
\hline EVAPF & AP-106 & $10 / 1 / 09$ & $10 / 1 / 09$ & 120,000 & 0 \\
\hline WATER & AP-106 & $10 / 2 / 09$ & $10 / 2 / 09$ & 5,000 & 0 \\
\hline \begin{tabular}{|l} 
WATER \\
\end{tabular} & AP-106 & $10 / 2 / 09$ & $10 / 2 / 09$ & 3,000 & 0 \\
\hline WATER & SY-102 & $10 / 2 / 09$ & $10 / 2 / 09$ & 5,000 & 0 \\
\hline WATER & AP-106 & $10 / 2 / 09$ & $10 / 2 / 09$ & 22,000 & 0 \\
\hline AP-106 & AW-102 & $10 / 1 / 09$ & $10 / 5 / 09$ & 749,000 & 0 \\
\hline EVAPF & AW-102 & $11 / 3 / 09$ & $11 / 3 / 09$ & 35,000 & 0 \\
\hline $\mathrm{U}-104$ & UA-2 & $7 / 30 / 09$ & $11 / 9 / 09$ & 50,908 & 1,221 \\
\hline PIHLW FEED TANK & HLW-CONTRACTOR & $12 / 27 / 08$ & $11 / 29 / 09$ & 137,400 & 4,760 \\
\hline AP-108 & LAW-CONTRACTOR-2 & $4 / 15 / 08$ & $11 / 29 / 09$ & $1,477,000$ & 1,076 \\
\hline RETURN 1 & AP-107 & $11 / 30 / 09$ & $11 / 30 / 09$ & 0 & 0 \\
\hline RETURN 2 & AP-107 & $11 / 30 / 09$ & $11 / 30 / 09$ & 5,146 & 196 \\
\hline $\mathrm{U}-112$ & UA-2 & $11 / 9 / 09$ & $12 / 5 / 09$ & 58,521 & 2,330 \\
\hline UA-2 & $S Y-102$ & $12 / 5 / 09$ & $12 / 6 / 09$ & 154,400 & 4,426 \\
\hline AP-107 & AZ-101 & $12 / 10 / 09$ & $12 / 11 / 09$ & 214,200 & 7,208 \\
\hline AN-103 & AP-107 & $12 / 20 / 09$ & $12 / 25 / 09$ & $1,047,000$ & 11,732 \\
\hline AN-104 & AP-107 & $12 / 25 / 09$ & $12 / 25 / 09$ & 57,106 & 182 \\
\hline SY-101 & AN-103 & $12 / 25 / 09$ & $12 / 29 / 09$ & $1,115,000$ & 1,877 \\
\hline $\mathrm{U}-112$ & UA-1 & $12 / 5 / 09$ & $1 / 17 / 10$ & 102,000 & 4,061 \\
\hline $\mathrm{U}=204$ & UA-1 & $1 / 17 / 10$ & $1 / 23 / 10$ & 13,004 & 503 \\
\hline U-201 & UA-1 & $1 / 23 / 10$ & $1 / 27 / 10$ & 13,912 & 538 \\
\hline UA-1 & SX-102 & $1 / 28 / 10$ & $1 / 28 / 10$ & 146,500 & 5,102 \\
\hline U-201 & UA-2 & $1 / 27 / 10$ & $1 / 30 / 10$ & 13,499 & 522 \\
\hline U-106 & UA-2 & $1 / 30 / 10$ & $3 / 1 / 10$ & 134,400 & 338 \\
\hline U-106 & UA-1 & $3 / 1 / 10$ & $4 / 4 / 10$ & 149,500 & 376 \\
\hline AW-102 & $242-\mathrm{A}$ & $4 / 4 / 10$ & $4 / 9 / 10$ & 868,900 & 30 \\
\hline $242-\mathrm{A}$ & AW-106 & $4 / 4 / 10$ & $4 / 9 / 10$ & 556,900 & 30 \\
\hline $242-\mathrm{A}$ & LERR & $4 / 4 / 10$ & $4 / 9 / 10$ & 312,000 & 0 \\
\hline AW-106 & AW-102 & $4 / 9 / 10$ & $4 / 12 / 10$ & 556,800 & 20 \\
\hline AW-102 & $242-\mathrm{A}$ & $4 / 26 / 10$ & $4 / 29 / 10$ & 556,900 & 20 \\
\hline
\end{tabular}


HNF-2321

Revision 0

Table A.5-1. Complete Transfer List for Case 5.

\begin{tabular}{|c|c|c|c|c|c|}
\hline From & To & Start Date & End Date & $\begin{array}{c}\text { Liquid } \\
\text { (gallons) }\end{array}$ & $\begin{array}{c}\text { Solid } \\
\text { (galions) }\end{array}$ \\
\hline 242-A & $A W-106$ & $4 / 26 / 10$ & $4 / 29 / 10$ & 356,900 & 20 \\
\hline $242-\mathrm{A}$ & LERF & $4 / 26 / 10$ & $4 / 29 / 10$ & 200,000 & 0 \\
\hline AW-106 & AW-102 & $4 / 29 / 10$ & $5 / 1 / 10$ & 356,800 & 13 \\
\hline$A W-102$ & 242-A & $5 / 15 / 10$ & $5 / 17 / 10$ & 356,900 & 13 \\
\hline $242-\mathrm{A}$ & AW-106 & $5 / 15 / 10$ & $5 / 17 / 10$ & 228,000 & 13 \\
\hline $242-\mathrm{A}$ & LERF & $5 / 15 / 10$ & $5 / 17 / 10$ & 128,900 & 0 \\
\hline AW-106 & AW-102 & $5 / 17 / 10$ & $5 / 18 / 10$ & 228,000 & 9 \\
\hline AW-102 & $242-\mathrm{A}$ & $6 / 1 / 10$ & $6 / 2 / 10$ & 228,000 & 9 \\
\hline $242-\mathrm{A}$ & AW-106 & $6 / 1 / 10$ & $6 / 2 / 10$ & 144,600 & 9 \\
\hline $242-\mathrm{A}$ & LERF & $6 / 1 / 10$ & $6 / 2 / 10$ & 83,442 & 0 \\
\hline AW-106 & AW-102 & $6 / 2 / 10$ & $6 / 3 / 10$ & 144,600 & 6 \\
\hline AW-102 & AP-106 & $6 / 4 / 10$ & $6 / 5 / 10$ & 144,700 & 6 \\
\hline S-105 & SY-101 & $5 / 7 / 08$ & $7 / 19 / 10$ & $1,518,000$ & 2,459 \\
\hline TNS88 & AP-106 & $10 / 1 / 10$ & $10 / 1 / 10$ & 750 & 250 \\
\hline TAL88 & AP-106 & $10 / 1 / 10$ & $10 / 1 / 10$ & 26,000 & 0 \\
\hline WATER & AP-106 & $10 / 1 / 10$ & $10 / 1 / 10$ & 25,000 & 0 \\
\hline WESF & AP-106 & $10 / 1 / 10$ & $10 / 1 / 10$ & 5,000 & 0 \\
\hline \begin{tabular}{|l|} 
SPN87 \\
\end{tabular} & $\$ Y-102$ & $10 / 1 / 10$ & $10 / 1 / 10$ & 25,000 & 0 \\
\hline WATER & AP-106 & $10 / 1 / 10$ & $10 / 1 / 10$ & 35,000 & 0 \\
\hline $34 \mathrm{~L} 87$ & AP-106 & $10 / 1 / 10$ & $10 / 1 / 10$ & 50,000 & 0 \\
\hline EVAPF & AP-106 & $10 / 1 / 10$ & $10 / 1 / 10$ & 120,000 & 0 \\
\hline WATER & AP-106 & $10 / 2 / 10$ & $10 / 2 / 10$ & 5,000 & 0 \\
\hline WATER & SY-102 & $10 / 2 / 10$ & $10 / 2 / 10$ & 5,000 & 0 \\
\hline \begin{tabular}{|l} 
WATER \\
\end{tabular} & AP-106 & $10 / 2 / 10$ & $10 / 2 / 10$ & 22,000 & 0 \\
\hline EVAPF & AW-102 & $10 / 4 / 10$ & $10 / 4 / 10$ & 50,000 & 0 \\
\hline EVAPF & AW-102 & $11 / 3 / 10$ & $11 / 3 / 10$ & 35,000 & 0 \\
\hline WATER & AP-106 & $1 / 1 / 11$ & $1 / 1 / 11$ & 25,000 & 0 \\
\hline BY-110 & $\mathrm{BA}-3$ & $1 / 3 / 11$ & $2 / 8 / 11$ & 147,400 & 2,633 \\
\hline TX-109 & TA-5 & $1 / 3 / 11$ & $2 / 10 / 11$ & 144,100 & 5,925 \\
\hline TY-101 & TA-4 & $1 / 3 / 11$ & $2 / 22 / 11$ & 146,500 & 3,494 \\
\hline \begin{tabular}{|l|} 
T-106 \\
\end{tabular} & TA-6 & $1 / 3 / 11$ & $3 / 2 / 11$ & 74,648 & 2,929 \\
\hline B-112 & $\mathrm{BA}-1$ & $1 / 3 / 11$ & $3 / 9 / 11$ & 75,213 & 1,083 \\
\hline $\mathrm{BY}-110$ & BA-4 & $2 / 8 / 11$ & $3 / 16 / 11$ & 147,400 & 2,633 \\
\hline TX-109 & $\mathrm{TA}-3$ & $2 / 10 / 11$ & $3 / 19 / 11$ & 144,100 & 5,925 \\
\hline \begin{tabular}{|l|} 
T-101 \\
\end{tabular} & TA-6 & $3 / 2 / 11$ & $3 / 25 / 11$ & 61,377 & 2,346 \\
\hline WATER & AP-106 & $4 / 1 / 11$ & $4 / 1 / 11$ & 25,000 & 0 \\
\hline $\mathrm{BX}-102$ & $\mathrm{BA}-2$ & $1 / 3 / 11$ & $4 / 4 / 11$ & 52,548 & 2,443 \\
\hline B-111 & BA-1 & $3 / 9 / 11$ & $4 / 9 / 11$ & 72,630 & 2,383 \\
\hline TY-101 & TA-2 & $2 / 22 / 11$ & $4 / 13 / 11$ & 146,500 & 3,494 \\
\hline BY-110 & BA-5 & $3 / 16 / 11$ & $4 / 21 / 11$ & 147,400 & 2,633 \\
\hline TX-109 & TA-1 & $3 / 19 / 11$ & $4 / 26 / 11$ & 144,100 & 5,925 \\
\hline $\mathrm{BX}-112$ & $\mathrm{BA}-2$ & $4 / 4 / 11$ & $5 / 2 / 11$ & 67,370 & 2,937 \\
\hline BA-3 & AP-108 & $6 / 1 / 11$ & $6 / 2 / 11$ & 155,600 & 2,631 \\
\hline $\mathrm{BA}-4$ & AP-108 & $6 / 2 / 11$ & $6 / 2 / 11$ & 155,600 & 2,631 \\
\hline $\mathrm{BA}-1$ & AP-108 & $6 / 2 / 11$ & $6 / 3 / 11$ & 157,300 & 3,464 \\
\hline BA-5 & AP-108 & $6 / 3 / 11$ & $6 / 4 / 11$ & 155,600 & 2,631 \\
\hline BA-2 & AP-108 & $6 / 4 / 11$ & $6 / 5 / 11$ & 174,500 & 5,377 \\
\hline AN-104 & AP-102 & $6 / 1 / 11$ & $6 / 6 / 11$ & $1,066,000$ & 3,397 \\
\hline $\mathrm{AN}-103$ & AP-104 & $6 / 1 / 11$ & $6 / 6 / 11$ & $1,114,000$ & 2,128 \\
\hline AN-103 & AP-102 & $6 / 6 / 11$ & $6 / 6 / 11$ & 998 & 2 \\
\hline SY-103 & AN-103 & $6 / 6 / 11$ & $6 / 10 / 11$ & $1,084,000$ & 32,737 \\
\hline B-111 & $\mathrm{BA}-6$ & $4 / 9 / 11$ & $6 / 11 / 11$ & 145,200 & 4,764 \\
\hline AN-103 & AP-102 & $6 / 11 / 11$ & $6 / 11 / 11$ & 44,789 & 1,321 \\
\hline AN-103 & AP-108 & $6 / 11 / 11$ & $6 / 13 / 11$ & 292,600 & 8,627 \\
\hline WATER & AP-106 & $7 / 1 / 11$ & $7 / 1 / 11$ & 25,000 & 0 \\
\hline BY-110 & $\mathrm{BA}-4$ & $6 / 2 / 11$ & $7 / 8 / 11$ & 147,300 & 2,631 \\
\hline
\end{tabular}


HNF-2321

Revision 0

Täble A.5-1. Complete Transfer List for Case 5.

\begin{tabular}{|l|l|c|c|c|c|}
\hline \multicolumn{1}{|c|}{ From } & To & Start Date & End Date & $\begin{array}{c}\text { Liquid } \\
\text { (gallons) }\end{array}$ & $\begin{array}{c}\text { Solid } \\
\text { (gallons) }\end{array}$ \\
\hline BX-112 & BA-3 & $6 / 2 / 11$ & $8 / 2 / 11$ & 143,600 & 6,262 \\
\hline B-111 & BA-1 & $6 / 11 / 11$ & $8 / 12 / 11$ & 145,100 & 4,761 \\
\hline BY-110 & BA-5 & $7 / 8 / 11$ & $8 / 13 / 11$ & 147,300 & 2,631 \\
\hline SX-112 & SY-103 & $4 / 6 / 04$ & $8 / 27 / 11$ & 397,100 & 16,406 \\
\hline BX-112 & BA-2 & $8 / 2 / 11$ & $9 / 4 / 11$ & 78,662 & 3,430 \\
\hline B-111 & BA-2 & $9 / 4 / 11$ & $9 / 4 / 11$ & 719 & 24 \\
\hline BY-110 & BA-2 & $9 / 4 / 11$ & $9 / 14 / 11$ & 40,093 & 716 \\
\hline
\end{tabular}


HNF-2321

Revision 0

Table A.5-2. Phase 1 Low-Activity Waste Feed Staging Transfers for Case 5.

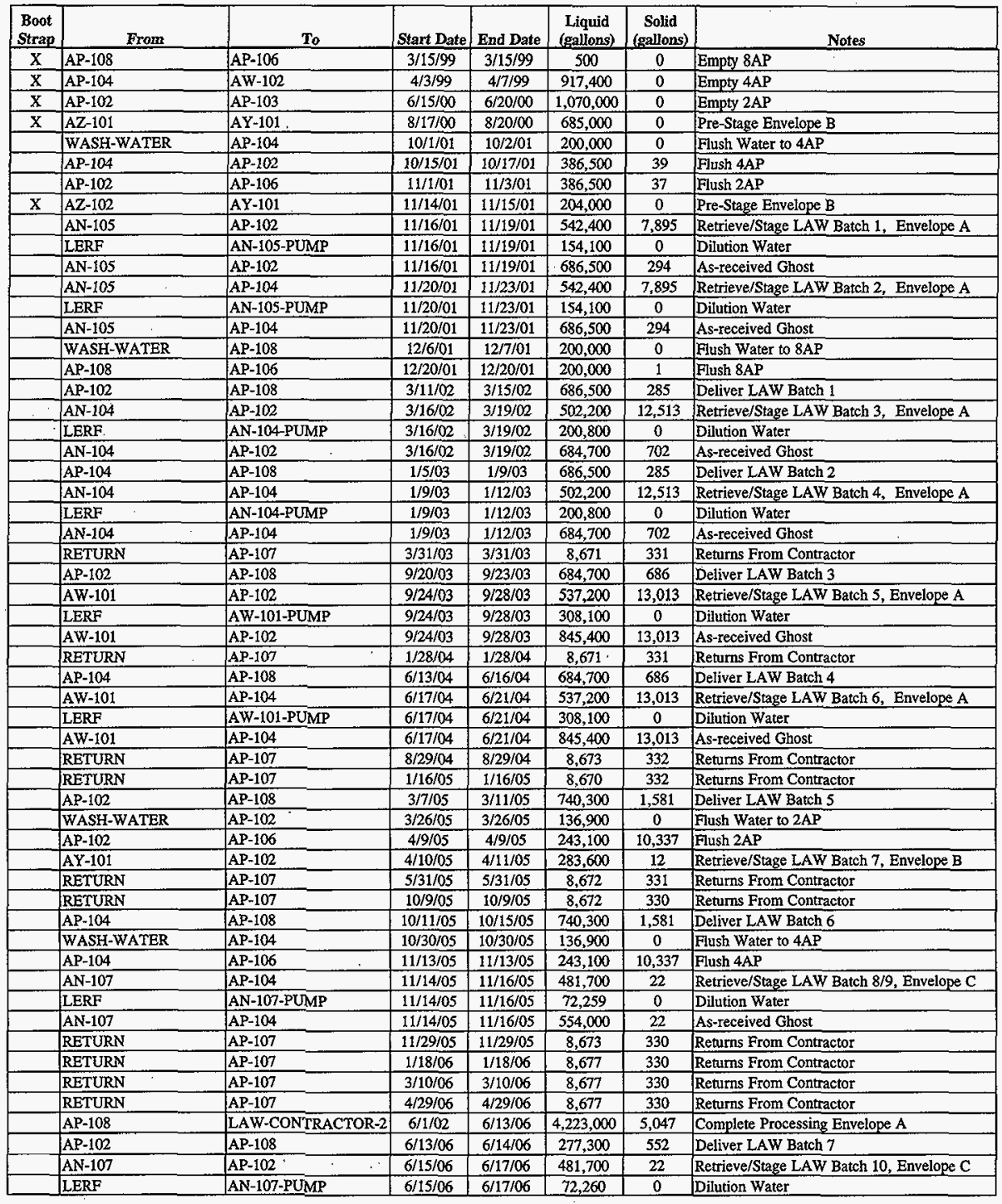


HNF-2321

Revision 0

Table A.5-2. Phase 1 Low-Activity Waste Feed Staging Transfers for Case 5.

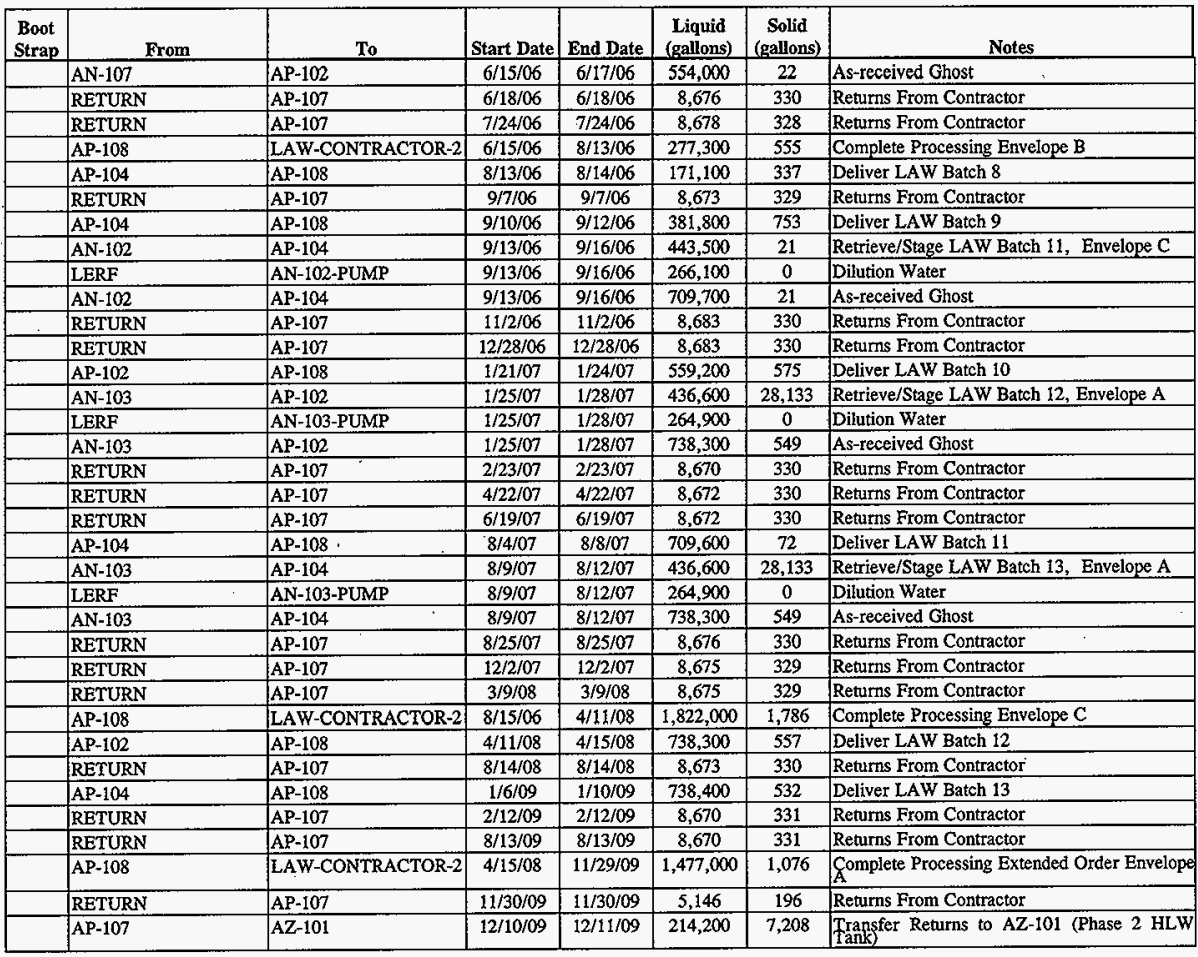


HNF-2321

Revision 0

Table A.5-3. Phase 1 High-Level Waste Feed Staging Transfers for Case 5.

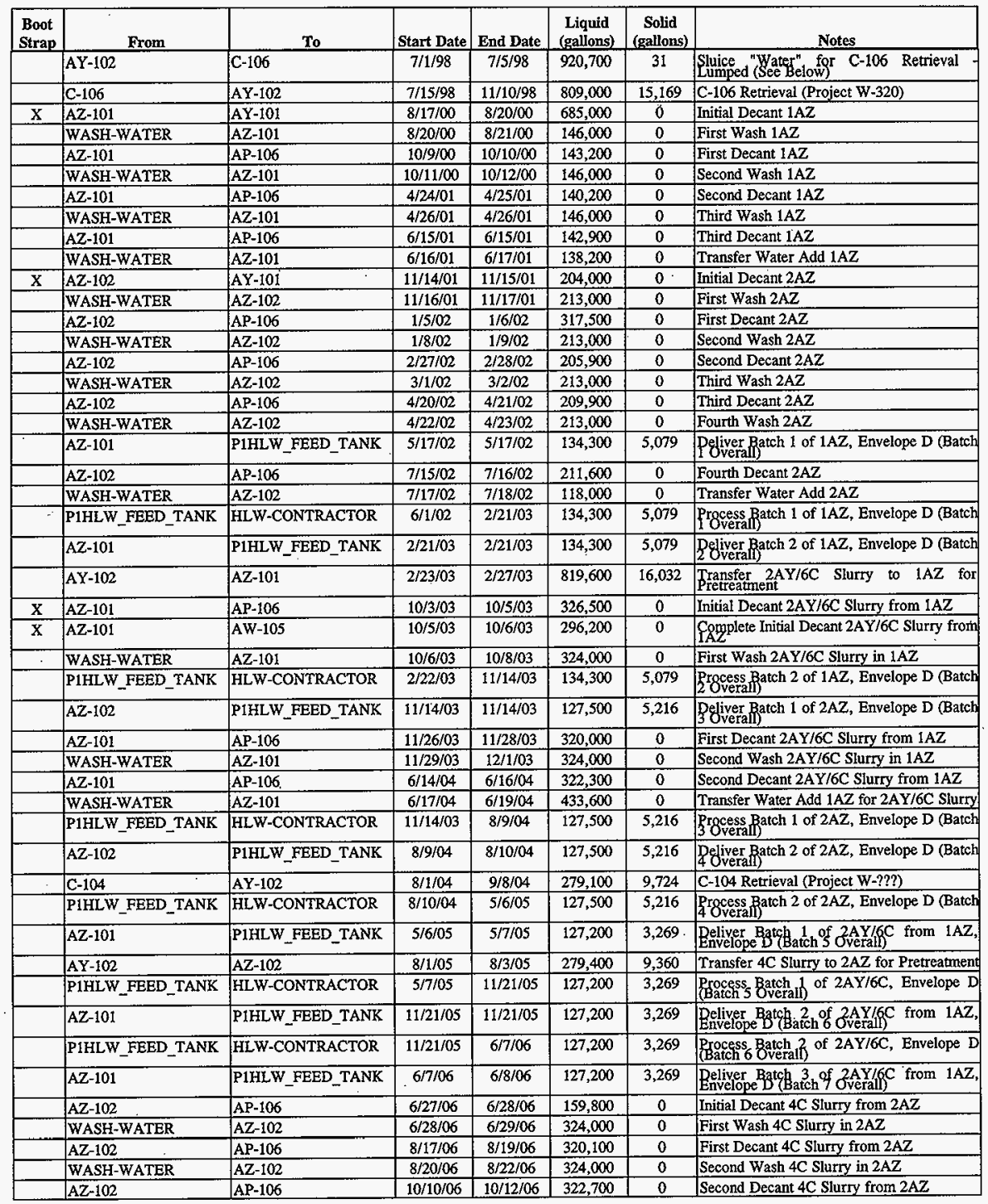


HNF-2321

Revision 0

Table A.5-3. Phase 1 High-Level Waste Feed Staging Transfers for Case 5.

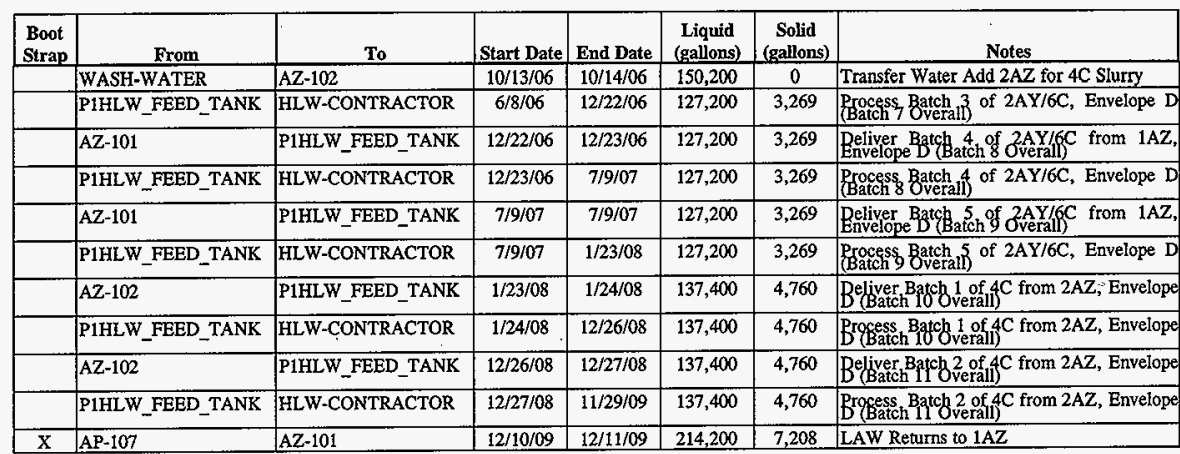


HNF-2321

Revision 0

Table A.6-1. Complete Transfer List for Case 6.

\begin{tabular}{|c|c|c|c|c|c|}
\hline From & To & Start Date & End Date & $\begin{array}{c}\text { Liquid } \\
\text { (gallons) }\end{array}$ & $\begin{array}{c}\text { Solid } \\
\text { (gallons) }\end{array}$ \\
\hline WATER & SY-102 & $10 / 1 / 96$ & $10 / 1 / 96$ & 5,000 & 0 \\
\hline 1NS96 & $\mathrm{AW}-105$ & $10 / 1 / 96$ & $10 / 1 / 96$ & 750 & 250 \\
\hline ZNL87 & SY-102 & $10 / 1 / 96$ & $10 / 1 / 96$ & 2,000 & 0 \\
\hline TAL88 & AP-106 & $10 / 1 / 96$ & $10 / 1 / 96$ & 4,000 & 0 \\
\hline 1FL96 & AW-105 & $10 / 1 / 96$ & $10 / 1 / 96$ & 13,000 & 0 \\
\hline TNS88 & AP-106 & $10 / 1 / 96$ & $10 / 1 / 96$ & 750 & 250 \\
\hline WESF & AP-106 & $10 / 1 / 96$ & $10 / 1 / 96$ & 5,000 & 0 \\
\hline SPN87 & AP-106 & $10 / 1 / 96$ & $10 / 1 / 96$ & 7,000 & 0 \\
\hline BPTCO & AP-106 & $10 / 1 / 96$ & $10 / 1 / 96$ & 11,000 & 0 \\
\hline EVAPF & AP-106 & $10 / 1 / 96$ & $10 / 1 / 96$ & 10,000 & 0 \\
\hline $34 \mathrm{~L} 87$ & AP-106 & $10 / 1 / 96$ & $10 / 1 / 96$ & 10,000 & 0 \\
\hline WNW88 & SY-102 & $10 / 1 / 96$ & $10 / 1 / 96$ & 19,000 & 0 \\
\hline WATER & AP-106 & $10 / 2 / 96$ & $10 / 2 / 96$ & 12,000 & 0 \\
\hline WATER & AW-105 & $10 / 2 / 96$ & $10 / 2 / 96$ & 5,000 & 0 \\
\hline WATER & SY-102 & $10 / 2 / 96$ & $10 / 2 / 96$ & 5,000 & 0 \\
\hline WATER & AP-106 & $10 / 2 / 96$ & $10 / 2 / 96$ & 3,000 & 0 \\
\hline WATER & AP-106 & $10 / 2 / 96$ & $10 / 2 / 96$ & 5,000 & 0 \\
\hline WATER & AP-106 & $10 / 2 / 96$ & $10 / 2 / 96$ & 5,000 & 0 \\
\hline WATER & SY-102 & $11 / 1 / 96$ & $11 / 1 / 96$ & 3,000 & 0 \\
\hline WNW88 & SY-102 & $11 / 1 / 96$ & $11 / 1 / 96$ & 14,000 & 0 \\
\hline EVAPF & AP-106 & $11 / 1 / 96$ & $11 / 1 / 96$ & 10,000 & 0 \\
\hline BPTCO & AP-106 & 11/1/96 & $11 / 1 / 96$ & 10,000 & 0 \\
\hline WATER & SY-102 & $12 / 1 / 96$ & $12 / 1 / 96$ & 3,000 & 0 \\
\hline WNW88 & SY-102 & $12 / 1 / 96$ & $12 / 1 / 96$ & 14,000 & 0 \\
\hline EVAPF & AP-106 & $12 / 1 / 96$ & $12 / 1 / 96$ & 10,000 & 0 \\
\hline BPTCO & AP-106 & $12 / 1 / 96$ & $12 / 1 / 96$ & 11,000 & 0 \\
\hline WESF & AP-106 & $1 / 1 / 97$ & $1 / 1 / 97$ & 5,000 & 0 \\
\hline \begin{tabular}{|l|} 
TAL88 \\
\end{tabular} & AP-106 & $1 / 1 / 97$ & $1 / 1 / 97$ & 4,000 & 0 \\
\hline WATER & SY-102 & $1 / 1 / 97$ & $1 / 1 / 97$ & 4,000 & 0 \\
\hline WNW88 & SY-102 & $1 / 1 / 97$ & $1 / 1 / 97$ & 14,000 & 0 \\
\hline BPTCO & AP-106 & $1 / 1 / 97$ & $1 / 1 / 97$ & 10,000 & 0 \\
\hline SPN87 & AP-106 & $1 / 1 / 97$ & $1 / 1 / 97$ & 6,000 & 0 \\
\hline EVAPF & AP-106 & $1 / 1 / 97$ & $1 / 1 / 97$ & 10,000 & 0 \\
\hline $34 \mathrm{~L} 87$ & AP-106 & $1 / 1 / 97$ & $1 / 1 / 97$ & 10,000 & 0 \\
\hline AW-106 & AP-101 & $1 / 4 / 97$ & $1 / 4 / 97$ & 69,511 & 3 \\
\hline $\mathrm{AN}-106$ & AP-108 & $1 / 11 / 97$ & $1 / 12 / 97$ & 177,000 & 7 \\
\hline WATER & SY-102 & $2 / 1 / 97$ & $2 / 1 / 97$ & 4,000 & 0 \\
\hline WNW88 & SY-102 & $2 / 1 / 97$ & $2 / 1 / 97$ & 14,000 & 0 \\
\hline EVAPF & AP-106 & $2 / 1 / 97$ & $2 / 1 / 97$ & 10,000 & 0 \\
\hline BPTCO & AP-106 & $2 / 1 / 97$ & $2 / 1 / 97$ & 10,000 & 0 \\
\hline EVAPF & AW-102 & $2 / 20 / 97$ & $2 / 20 / 97$ & 50,000 & 0 \\
\hline AP-105 & AW-102 & $2 / 20 / 97$ & $2 / 24 / 97$ & 942,000 & 39 \\
\hline WATER & AP-106 & $3 / 1 / 97$ & $3 / 1 / 97$ & 5,000 & 0 \\
\hline WATER & SY-102 & $3 / 1 / 97$ & $3 / 1 / 97$ & 4,000 & 0 \\
\hline WNW88 & SY-102 & $3 / 1 / 97$ & $3 / 1 / 97$ & 16,000 & 0 \\
\hline BPTCO & AP-106 & $3 / 1 / 97$ & $3 / 1 / 97$ & 11,000 & 0 \\
\hline PXTCO & AP-106 & $3 / 1 / 97$ & $3 / 1 / 97$ & 5,000 & 0 \\
\hline EVAPF & AP-106 & $3 / 1 / 97$ & $3 / 1 / 97$ & 10,000 & 0 \\
\hline $34 \mathrm{~L} 87$ & AP-106 & $3 / 1 / 97$ & $3 / 1 / 97$ & 10,000 & 0 \\
\hline AW-102 & $242-\mathrm{A}$ & $2 / 26 / 97$ & $3 / 2 / 97$ & $1,027,000$ & 42 \\
\hline $242-\mathrm{A}$ & AW-106 & $2 / 26 / 97$ & $3 / 2 / 97$ & 622,000 & 42 \\
\hline $242-\mathrm{A}$ & LERF & $2 / 26 / 97$ & $3 / 2 / 97$ & 405,000 & 0 \\
\hline EVAPF & AW-102 & $3 / 3 / 97$ & $3 / 3 / 97$ & 35,000 & 0 \\
\hline \begin{tabular}{|l|} 
WESF \\
\end{tabular} & AP-106 & $4 / 1 / 97$ & $4 / 1 / 97$ & 5,000 & 0 \\
\hline WATER & SY-102 & $4 / 1 / 97$ & $4 / 1 / 97$ & 6,000 & 0 \\
\hline EVAPF & AP-106 & $4 / 1 / 97$ & $4 / 1 / 97$ & 10,000 & 0 \\
\hline
\end{tabular}


HNF-2321

Revision 0

Table A.6-1. Complete Transfer List for Case 6.

\begin{tabular}{|c|c|c|c|c|c|}
\hline From & To & Start Date & End Date & $\begin{array}{c}\text { Liquid } \\
\text { (gallons) }\end{array}$ & $\begin{array}{c}\text { Solid } \\
\text { (gallons) }\end{array}$ \\
\hline TAL88 & AP-106 & $4 / 1 / 97$ & \begin{tabular}{|l|l|}
$4 / 97$ \\
\end{tabular} & 4,000 & 0 \\
\hline SPN87 & AP-106 & $4 / 1 / 97$ & $4 / 1 / 97$ & 6,000 & 0 \\
\hline BPTCO & AP-106 & $4 / 1 / 97$ & $4 / 1 / 97$ & 10,000 & 0 \\
\hline WNW88 & SY-102 & $4 / 1 / 97$ & $4 / 1 / 97$ & 22,000 & 0 \\
\hline AW-106 & $\mathrm{AP}-105$ & $4 / 25 / 97$ & $4 / 28 / 97$ & 617,000 & 29 \\
\hline$A Y-101$ & AP-108 & $4 / 25 / 97$ & $4 / 29 / 97$ & 809,000 & 30 \\
\hline WATER & SY-102 & $5 / 1 / 97$ & $5 / 1 / 97$ & 5,000 & 0 \\
\hline WNW88 & SY-102 & $5 / 1 / 97$ & $5 / 1 / 97$ & 19,000 & 0 \\
\hline WATER & AP-106 & $5 / 1 / 97$ & $5 / 1 / 97$ & 4,000 & 0 \\
\hline EVAPF & AP-106 & $5 / 1 / 97$ & $5 / 1 / 97$ & 10,000 & 0 \\
\hline BPTCO & AP-106 & $5 / 1 / 97$ & $5 / 1 / 97$ & 11,000 & 0 \\
\hline $34 \mathrm{~L} 87$ & AP-106 & $5 / 1 / 97$ & $5 / 1 / 97$ & 10,000 & 0 \\
\hline EVAPF & $\mathrm{AP}-106$ & $6 / 1 / 97$ & $6 / 1 / 97$ & 10,000 & 0 \\
\hline BPTCO & AP-106 & $6 / 1 / 97$ & $6 / 1 / 97$ & 10,000 & 0 \\
\hline \begin{tabular}{|l} 
WNW88 \\
\end{tabular} & SY-102 & $6 / 1 / 97$ & $6 / 1 / 97$ & 16,000 & 0 \\
\hline WATER & SY-102 & $6 / 1 / 97$ & $6 / 1 / 97$ & 4,000 & 0 \\
\hline TAL88 & AP-106 & $7 / 1 / 97$ & $7 / 1 / 97$ & 4,000 & 0 \\
\hline SPN87 & AP-106 & $7 / 1 / 97$ & $7 / 1 / 97$ & 6,000 & 0 \\
\hline WESF & AP-106 & $7 / 1 / 97$ & $7 / 1 / 97$ & 5,000 & 0 \\
\hline WATER & $S Y-102$ & $7 / 1 / 97$ & $7 / 1 / 97$ & 6,000 & 0 \\
\hline EVAPF & AP-106 & $7 / 1 / 97$ & $7 / 1 / 97$ & 10,000 & 0 \\
\hline $34 \mathrm{~L} 87$ & AP-106 & $7 / 1 / 97$ & $7 / 1 / 97$ & 10,000 & 0 \\
\hline BPTCO & AP-106 & $7 / 1 / 97$ & $7 / 1 / 97$ & 11,000 & 0 \\
\hline WNW88 & SY-102 & $7 / 1 / 97$ & $7 / 1 / 97$ & 24,000 & 0 \\
\hline WCE88 & AN-101 & $7 / 1 / 97$ & $7 / 1 / 97$ & 20,000 & 0 \\
\hline WATER & AN-101 & $7 / 1 / 97$ & $7 / 1 / 97$ & 5,000 & 0 \\
\hline AN-106 & AP-108 & $7 / 25 / 97$ & $7 / 26 / 97$ & 194,000 & 7 \\
\hline AP-108 & AW-102 & $7 / 25 / 97$ & $7 / 31 / 97$ & $1,198,000$ & 42 \\
\hline WATER & SY-102 & $8 / 1 / 97$ & $8 / 1 / 97$ & 13,000 & 0 \\
\hline EVAPF & $\mathrm{AP}-106$ & $8 / 1 / 97$ & $8 / 1 / 97$ & 10,000 & 0 \\
\hline WCE 88 & AN-101 & $8 / 1 / 97$ & $8 / 1 / 97$ & 25,000 & 0 \\
\hline WATER & AN-101 & $8 / 1 / 97$ & $8 / 1 / 97$ & 6,000 & 0 \\
\hline BPTCO & AP-106 & $8 / 1 / 97$ & $8 / 1 / 97$ & 10,000 & 0 \\
\hline WNW88 & SY-102 & $8 / 1 / 97$ & $8 / 1 / 97$ & 51,000 & 0 \\
\hline $\mathrm{AW}-102$ & $242-\mathrm{A}$ & $7 / 28 / 97$ & $8 / 2 / 97$ & $1,198,000$ & 39 \\
\hline $242-\mathrm{A}$ & AW-106 & $7 / 28 / 97$ & $8 / 2 / 97$ & 346,700 & 39 \\
\hline $242-\mathrm{A}$ & LERF & $7 / 28 / 97$ & $8 / 2 / 97$ & 851,300 & 0 \\
\hline EVAPF & AW-102 & $8 / 3 / 97$ & $8 / 3 / 97$ & 25,000 & 0 \\
\hline WATER & AP-107 & $8 / 20 / 97$ & $8 / 20 / 97$ & 35,000 & 0 \\
\hline AW-106 & AN-106 & $8 / 25 / 97$ & $8 / 26 / 97$ & 347,000 & 16 \\
\hline SY-102 & AP-107 & $8 / 25 / 97$ & $8 / 27 / 97$ & 520,000 & 20 \\
\hline EVAPF & AP-106 & $9 / 1 / 97$ & $9 / 1 / 97$ & 10,000 & 0 \\
\hline WATER & SY-102 & $9 / 1 / 97$ & $9 / 1 / 97$ & 15,000 & 0 \\
\hline WATER & AN-101 & $9 / 1 / 97$ & $9 / 1 / 97$ & 10,000 & 0 \\
\hline BPTCO & AP-106 & $9 / 1 / 97$ & 9/1/97 & 10,000 & 0 \\
\hline WNW88 & $5 Y-102$ & $9 / 1 / 97$ & $9 / 1 / 97$ & 61,000 & 0 \\
\hline WCE 88 & AN-101 & $9 / 1 / 97$ & $9 / 1 / 97$ & 40,000 & 0 \\
\hline SPN87 & SY-102 & $10 / 1 / 97$ & $10 / 1 / 97$ & 7,000 & 0 \\
\hline WESF & AP-106 & $10 / 1 / 97$ & $10 / 1 / 97$ & 5,000 & 0 \\
\hline WATER & SY-102 & $10 / 1 / 97$ & $10 / 1 / 97$ & 4,000 & 0 \\
\hline ZNL87 & SY-102 & $10 / 1 / 97$ & $10 / 1 / 97$ & 4,000 & 0 \\
\hline TAL88 & AP-106 & $10 / 1 / 97$ & $10 / 1 / 97$ & 4,000 & 0 \\
\hline PXTCO & AP-106 & $10 / 1 / 97$ & $10 / 1 / 97$ & 5,000 & 0 \\
\hline WNW88 & SY-102 & $10 / 1 / 97$ & $10 / 1 / 97$ & 18,000 & 0 \\
\hline WATER & AP-106 & $10 / 1 / 97$ & $10 / 1 / 97$ & 1,000 & 0 \\
\hline TNS88 & AP-106 & $10 / 1 / 97$ & $10 / 1 / 97$ & 750 & 250 \\
\hline
\end{tabular}


HNF-2321

Revision 0

Table A.6-1. Complete Transfer List for Case 6.

\begin{tabular}{|c|c|c|c|c|c|}
\hline From & To & Start Date & End Date & $\begin{array}{c}\text { Liquid } \\
\text { (gallons) }\end{array}$ & $\begin{array}{c}\text { Solid } \\
\text { (gallons) }\end{array}$ \\
\hline WNE88 & AP-106 & $10 / 1 / 97$ & $10 / 1 / 97$ & 1,000 & 0 \\
\hline EVAPF & AP-106 & $10 / 1 / 97$ & $10 / 1 / 97$ & 10,000 & 0 \\
\hline WATER & AN-10I & $10 / 1 / 97$ & $10 / 1 / 97$ & 2,000 & 0 \\
\hline WCE88 & $\mathrm{AN}-101$ & $10 / 1 / 97$ & $10 / 1 / 97$ & 7,000 & 0 \\
\hline $34 \mathrm{~L} 87$ & AP-106 & $10 / 1 / 97$ & $10 / 1 / 97$ & 7,000 & 0 \\
\hline $34 \mathrm{~L} 87$ & AP-106 & $10 / 1 / 97$ & $10 / 1 / 97$ & 10,000 & 0 \\
\hline BPTCO & AP-106 & $10 / 1 / 97$ & $10 / 1 / 97$ & 11,000 & 0 \\
\hline WATER & AP-106 & $10 / 2 / 97$ & $10 / 2 / 97$ & 3,000 & 0 \\
\hline WATER & SY-102 & $10 / 2 / 97$ & $10 / 2 / 97$ & 5,000 & 0 \\
\hline WATER & SY-102 & $10 / 2 / 97$ & $10 / 2 / 97$ & 1,000 & 0 \\
\hline WASH-CAUSTIC, & $\mathrm{AN}-107$ & $10 / 2 / 97$ & $10 / 2 / 97$ & 66,000 & 0 \\
\hline WATER & $\mathrm{AP}-106$ & $10 / 2 / 97$ & $10 / 2 / 97$ & 3,000 & 0 \\
\hline WATER & $\mathrm{AP}-106$ & $10 / 2 / 97$ & $10 / 2 / 97$ & 5,000 & 0 \\
\hline WATER & AP-106 & $10 / 2 / 97$ & $10 / 2 / 97$ & 5,000 & 0 \\
\hline $\mathrm{AY}-102$ & AP-106 & $10 / 25 / 97$ & $10 / 25 / 97$ & 35,999 & 1 \\
\hline WATER & AY-102 & $10 / 26 / 97$ & $10 / 27 / 97$ & 176,000 & 0 \\
\hline WATER & SY-102 & $11 / 1 / 97$ & $11 / 1 / 97$ & 4,000 & 0 \\
\hline WNW88 & $S Y-102$ & $11 / 1 / 97$ & $11 / 1 / 97$ & 16,000 & 0 \\
\hline WATER & AP-106 & $11 / 1 / 97$ & $11 / 1 / 97$ & 1,000 & 0 \\
\hline WNE88 & AP-106 & $11 / 1 / 97$ & $11 / 1 / 97$ & 1,000 & 0 \\
\hline EVAPF & AP-106 & $11 / 1 / 97$ & $11 / 1 / 97$ & 10,000 & 0 \\
\hline WCE88 & AN-101 & $11 / 1 / 97$ & $11 / 1 / 97$ & 20,000 & 0 \\
\hline WATER & AN-101 & $11 / 1 / 97$ & $11 / 1 / 97$ & 5,000 & 0 \\
\hline EVAPF & AP-106 & $12 / 1 / 97$ & $12 / 1 / 97$ & 10,000 & 0 \\
\hline WNE 88 & AP-106 & $12 / 1 / 97$ & $12 / 1 / 97$ & 1,000 & 0 \\
\hline WATER & AP-106 & $12 / 1 / 97$ & $12 / 1 / 97$ & 1,000 & 0 \\
\hline WNW88 & SY-102 & $12 / 1 / 97$ & $12 / 1 / 97$ & 14,000 & 0 \\
\hline WCE88 & AN-101 & $12 / 1 / 97$ & $12 / 1 / 97$ & 20,000 & 0 \\
\hline WATER & AN-101 & $12 / 1 / 97$ & $12 / 1 / 97$ & 5,000 & 0 \\
\hline WATER & SY-102 & $12 / 1 / 97$ & $12 / 1 / 97$ & 3,000 & 0 \\
\hline WATER & AP-107 & $12 / 20 / 97$ & $12 / 20 / 97$ & 35,000 & 0 \\
\hline SY-102 & AP-107 & $12 / 26 / 97$ & $12 / 27 / 97$ & 351,700 & 14 \\
\hline AP-106 & AP-104 & $12 / 25 / 97$ & $12 / 28 / 97$ & 739,400 & 25 \\
\hline SPN87 & SY-102 & $1 / 1 / 98$ & $1 / 1 / 98$ & 6,000 & 0 \\
\hline WATER & AP-106 & $1 / 1 / 98$ & $1 / 1 / 98$ & 4,000 & 0 \\
\hline WATER & SY-102 & $1 / 1 / 98$ & $1 / 1 / 98$ & 4,000 & 0 \\
\hline WNW88 & SY-102 & $1 / 1 / 98$ & $1 / 1 / 98$ & 14,000 & 0 \\
\hline EVAPF & AP-106 & $1 / 1 / 98$ & $1 / 1 / 98$ & 10,000 & 0 \\
\hline WATER & AP-106 & $1 / 1 / 98$ & 1/1/98 & 1,000 & 0 \\
\hline TAL88 & AP-106 & $1 / 1 / 98$ & $1 / 1 / 98$ & 4,000 & 0 \\
\hline WNE88 & AP-106 & $1 / 1 / 98$ & 1/1/98 & 1,000 & 0 \\
\hline BPTCO & AP-106 & $1 / 1 / 98$ & $1 / 1 / 98$ & 11,000 & 0 \\
\hline WATER & $\mathrm{AN}-101$ & $1 / 1 / 98$ & $1 / 1 / 98$ & 5,000 & 0 \\
\hline $34 \mathrm{~L} 87$ & AP-106 & $1 / 1 / 98$ & $1 / 1 / 98$ & 10,000 & 0 \\
\hline WCE88 & AN-101 & $1 / 1 / 98$ & $1 / 1 / 98$ & 20,000 & 0 \\
\hline WATER & SY-102 & $2 / 1 / 98$ & $2 / 1 / 98$ & 4,000 & 0 \\
\hline WNW88 & SY-102 & $2 / 1 / 98$ & $2 / 1 / 98$ & 14,000 & 0 \\
\hline WATER & AP-106 & $2 / 1 / 98$ & $2 / 1 / 98$ & 1,000 & 0 \\
\hline WNE88 & AP-106 & $2 / 1 / 98$ & $2 / 1 / 98$ & 1,000 & 0 \\
\hline EVAPF & AP-106 & $2 / 1 / 98$ & $2 / 1 / 98$ & 10,000 & 0 \\
\hline WATER & AN-101 & $2 / 1 / 98$ & $2 / 1 / 98$ & 5,000 & 0 \\
\hline WCE88 & AN-101 & $2 / 1 / 98$ & $2 / 1 / 98$ & 20,000 & 0 \\
\hline WATER & AP-106 & $3 / 1 / 98$ & $3 / 1 / 98$ & 1,000 & 0 \\
\hline WNE88 & AP-106 & $3 / 1 / 98$ & $3 / 1 / 98$ & 1,000 & 0 \\
\hline \begin{tabular}{|l} 
WATER \\
\end{tabular} & AP-106 & $3 / 1 / 98$ & $3 / 1 / 98$ & 5,000 & 0 \\
\hline WATER & SY-102 & $3 / 1 / 98$ & $3 / 1 / 98$ & 3,000 & 0 \\
\hline
\end{tabular}


HNF-2321

Revision 0

Table A.6-1. Complete Transfer List for Case 6.

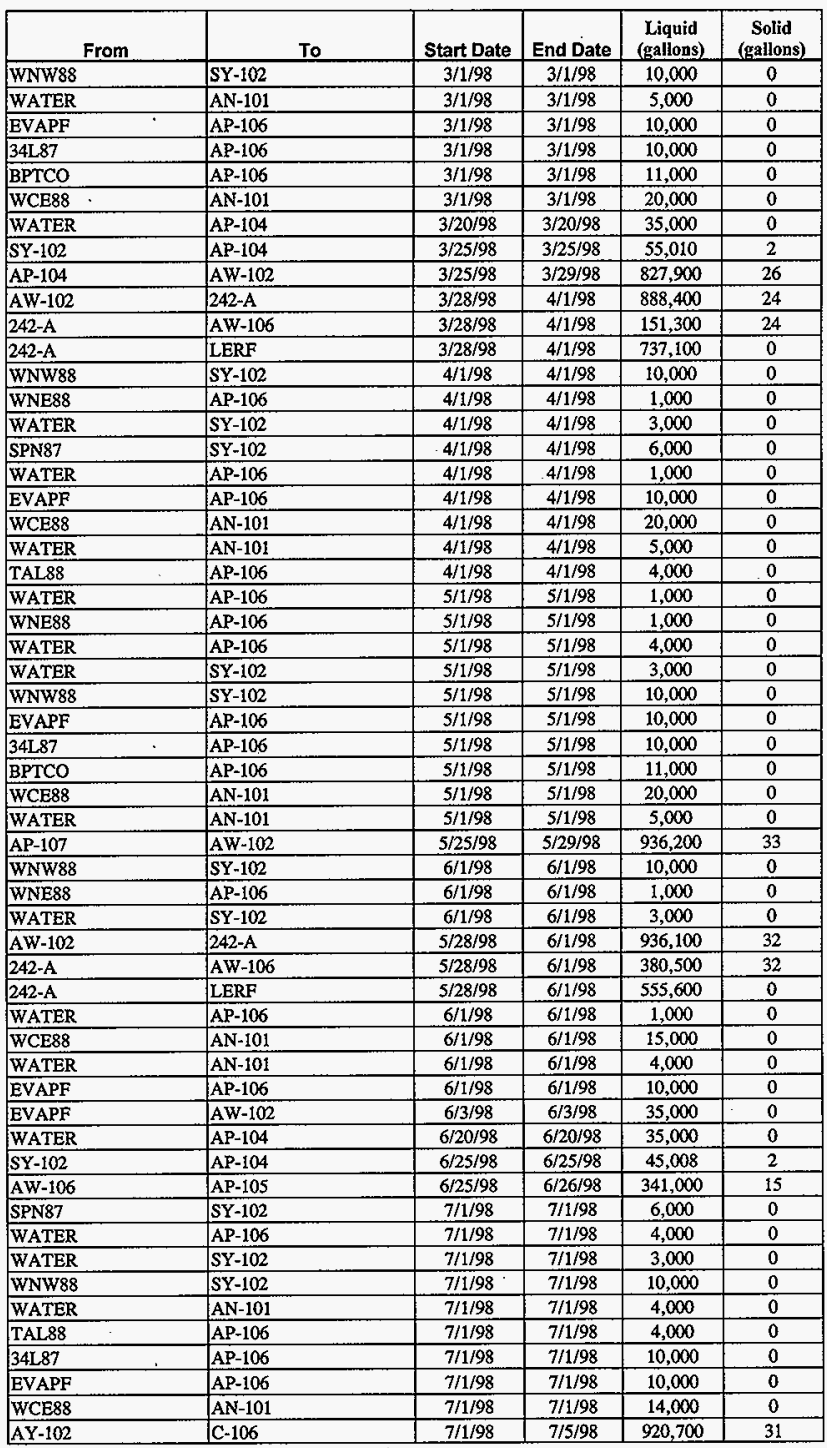


HNF-2321

Revision 0

Table A.6-1. Complete Transfer List for Case 6.

\begin{tabular}{|c|c|c|c|c|c|}
\hline From & To & Start Date & End Date & $\begin{array}{c}\text { Liquid } \\
\text { (gallons) }\end{array}$ & $\begin{array}{c}\text { Solid } \\
\text { (gallons) }\end{array}$ \\
\hline BPTCO & AP-106 & $7 / 11 / 98$ & $7 / 11 / 98$ & 11,000 & 0 \\
\hline WCE88 & AN-101 & $8 / 1 / 98$ & $8 / 1 / 98$ & 14,000 & 0 \\
\hline EVAPF & AP-106 & $8 / 1 / 98$ & $8 / 1 / 98$ & 10,000 & 0 \\
\hline WNW88 & SY-102 & $8 / 1 / 98$ & $8 / 1 / 98$ & 10,000 & 0 \\
\hline WATER & $S Y-102$ & $8 / 1 / 98$ & $8 / 1 / 98$ & 3,000 & 0 \\
\hline WATER & AN-101 & $8 / 1 / 98$ & $8 / 1 / 98$ & 4,000 & 0 \\
\hline WATER & AP-106 & $9 / 1 / 98$ & $9 / 1 / 98$ & 5,000 & 0 \\
\hline WATER & SY-102 & $9 / 1 / 98$ & $9 / 1 / 98$ & 2,000 & 0 \\
\hline WNW88 & SY-102 & $9 / 1 / 98$ & $9 / 1 / 98$ & 6,000 & 0 \\
\hline WATER & AN-101 & 9/1/98 & 9/1/98 & 4,000 & 0 \\
\hline EVAPF & AP-106 & $9 / 1 / 98$ & 9/1/98 & 10,000 & 0 \\
\hline WCE88 & $\mathrm{AN}-101$ & $9 / 1 / 98$ & 9/1/98 & 14,000 & 0 \\
\hline WNE88 & AP-106 & $9 / 1 / 98$ & $9 / 1 / 98$ & 22,000 & 0 \\
\hline WATER & AP-107 & $9 / 20 / 98$ & $9 / 20 / 98$ & 35,000 & 0 \\
\hline$S Y-102$ & AP-107 & 9/25/98 & $9 / 25 / 98$ & 40,007 & 2 \\
\hline $\mathrm{AP}-101$ & AW-103 & $9 / 25 / 98$ & $9 / 28 / 98$ & 625,000 & 27 \\
\hline WESF & AP-106 & $10 / 1 / 98$ & $10 / 1 / 98$ & 5,000 & 0 \\
\hline WATER & AP-106 & $10 / 1 / 98$ & $10 / 1 / 98$ & 5,000 & 0 \\
\hline WNW88 & SY-102 & $10 / 1 / 98$ & $10 / 1 / 98$ & 6,000 & 0 \\
\hline SPN87 & SY-102 & $10 / 1 / 98$ & $10 / 1 / 98$ & 7,000 & 0 \\
\hline WNE88 & AP-106 & $10 / 1 / 98$ & $10 / 1 / 98$ & 22,000 & 0 \\
\hline WATER & SY-102 & $10 / 1 / 98$ & $10 / 1 / 98$ & 2,000 & 0 \\
\hline ZNL87 & SY-102 & $10 / 1 / 98$ & $10 / 1 / 98$ & 4,000 & 0 \\
\hline $34 \mathrm{~L} 87$ & AP-106 & $10 / 1 / 98$ & $10 / 1 / 98$ & 10,000 & 0 \\
\hline WCE88 & AN-101 & $10 / 1 / 98$ & $10 / 1 / 98$ & 14,000 & 0 \\
\hline TNS88 & AP-106 & $10 / 1 / 98$ & $10 / 1 / 98$ & 750 & 250 \\
\hline TAL88 & AP-106 & $10 / 1 / 98$ & $10 / 1 / 98$ & 4,000 & 0 \\
\hline EVAPF & AP-106 & $10 / 1 / 98$ & $10 / 1 / 98$ & 10,000 & 0 \\
\hline PXTCO & AP-106 & $10 / 1 / 98$ & $10 / 1 / 98$ & 5,000 & 0 \\
\hline WATER & $\mathrm{AN}-101$ & $10 / 1 / 98$ & $10 / 1 / 98$ & 4,000 & 0 \\
\hline WATER & AP-106 & $10 / 2 / 98$ & $10 / 2 / 98$ & 3,000 & 0 \\
\hline WATER & AP-106 & $10 / 2 / 98$ & $10 / 2 / 98$ & 5,000 & 0 \\
\hline WATER & SY-102 & $10 / 2 / 98$ & $10 / 2 / 98$ & 5,000 & 0 \\
\hline EVAPF & $\mathrm{AW}-102$ & $10 / 4 / 98$ & $10 / 4 / 98$ & 50,000 & 0 \\
\hline AP-104 & $\mathrm{AW}-102$ & $10 / 5 / 98$ & $10 / 5 / 98$ & 80,008 & 2 \\
\hline AW-102 & AW-106 & $10 / 9 / 98$ & $10 / 9 / 98$ & 150,000 & 5 \\
\hline AW-104 & AP-107 & $10 / 9 / 98$ & $10 / 11 / 98$ & 540,000 & 18 \\
\hline AW-104 & AP-104 & $10 / 18 / 98$ & $10 / 18 / 98$ & 164,000 & 6 \\
\hline AP-101 & AW-104 & $10 / 21 / 98$ & $10 / 23 / 98$ & 460,000 & 20 \\
\hline AW-106 & AP-101 & $10 / 29 / 98$ & $10 / 30 / 98$ & 289,000 & 12 \\
\hline WNW88 & SY-102 & $11 / 1 / 98$ & $11 / 1 / 98$ & 6,000 & 0 \\
\hline WATER & SY-102 & $11 / 1 / 98$ & $11 / 1 / 98$ & 2,000 & 0 \\
\hline WNE88 & AP-106 & $11 / 1 / 98$ & $11 / 1 / 98$ & 22,000 & 0 \\
\hline WATER & AP-106 & $11 / 1 / 98$ & $11 / 1 / 98$ & 5,000 & 0 \\
\hline WCE 88 & AN-101 & $11 / 1 / 98$ & $11 / 1 / 98$ & 14,000 & 0 \\
\hline WATER & AN-101 & $11 / 1 / 98$ & $11 / 1 / 98$ & 4,000 & 0 \\
\hline EVAPF & AP-106 & $11 / 1 / 98$ & $11 / 1 / 98$ & 10,000 & 0 \\
\hline AW-106 & AW-102 & $11 / 5 / 98$ & $11 / 5 / 98$ & 55,857 & 2 \\
\hline $\mathrm{C}-106$ & AY-102 & $7 / 15 / 98$ & $11 / 10 / 98$ & 809,000 & 15,169 \\
\hline WNW88 & SY-102 & $12 / 1 / 98$ & $12 / 1 / 98$ & 6,000 & 0 \\
\hline EVAPF & AP-106 & $12 / 1 / 98$ & $12 / 1 / 98$ & 10,000 & 0 \\
\hline WATER & SY-102 & $12 / 1 / 98$ & $12 / 1 / 98$ & 2,000 & 0 \\
\hline WATER & AP-106 & $12 / 1 / 98$ & $12 / 1 / 98$ & 7,000 & 0 \\
\hline WNE88 & AP-106 & $12 / 1 / 98$ & $12 / 1 / 98$ & 29,000 & 0 \\
\hline WATER & AN-101 & $12 / 1 / 98$ & $12 / 1 / 98$ & 4,000 & 0 \\
\hline WCE88 & AN-101 & $12 / 1 / 98$ & $12 / 1 / 98$ & 14,000 & 0 \\
\hline
\end{tabular}


HNF-2321

Revision 0

Table A.6-1. Complete Transfer List for Case 6.

\begin{tabular}{|c|c|c|c|c|c|}
\hline From & To & Start Date & End Date & $\begin{array}{c}\text { Liquid } \\
\text { (gallons) }\end{array}$ & $\begin{array}{c}\text { Solid } \\
\text { (gallons) }\end{array}$ \\
\hline WATER & AP-104 & $12 / 20 / 98$ & $12 / 20 / 98$ & 35,000 & 0 \\
\hline AW-106 & AP-101 & $12 / 25 / 98$ & $12 / 25 / 98$ & 0 & 0 \\
\hline SY-102 & AP-104 & $12 / 25 / 98$ & $12 / 25 / 98$ & 40,007 & 2 \\
\hline SPN87 & SY-102 & $1 / 1 / 99$ & $1 / 1 / 99$ & 6,000 & 0 \\
\hline WATER & AP-106 & $1 / 1 / 99$ & $1 / 1 / 99$ & 4,000 & 0 \\
\hline WATER & $\$ Y-102$ & $1 / 1 / 99$ & $1 / 1 / 99$ & 2,000 & 0 \\
\hline \begin{tabular}{|l} 
WNW88 \\
\end{tabular} & SY-102 & $1 / 1 / 99$ & $1 / 1 / 99$ & 6,000 & 0 \\
\hline TAL88 & AP-106 & $1 / 1 / 99$ & $1 / 1 / 99$ & 4,000 & 0 \\
\hline WATER & AP-106 & $1 / 1 / 99$ & $1 / 1 / 99$ & 10,000 & 0 \\
\hline EVAPF & AP-106 & $1 / 1 / 99$ & $1 / 1 / 99$ & 10,000 & 0 \\
\hline WATER & AN-101 & $1 / 1 / 99$ & $1 / 1 / 99$ & 4,000 & 0 \\
\hline 34 L87 & AP-106 & $1 / 1 / 99$ & $1 / 1 / 99$ & 10,000 & 0 \\
\hline WCE88 & AN-101 & $1 / 1 / 99$ & $1 / 1 / 99$ & 14,000 & 0 \\
\hline WNE88 & AP-106 & $1 / 1 / 99$ & $1 / 1 / 99$ & 39,000 & 0 \\
\hline AP-106 & AP-104 & $1 / 25 / 99$ & $1 / 27 / 99$ & 385,000 & 14 \\
\hline WATER & SY-102 & $2 / 1 / 99$ & $2 / 1 / 99$ & 2,000 & 0 \\
\hline WATER & AP-106 & $2 / 1 / 99$ & $2 / 1 / 99$ & 10,000 & 0 \\
\hline WNW88 & SY-102 & 2/1/99 & $2 / 1 / 99$ & 6,000 & 0 \\
\hline WATER & AN-101 & $2 / 1 / 99$ & $2 / 1 / 99$ & 7,000 & 0 \\
\hline EVAPF & AP-106 & $2 / 1 / 99$ & $2 / 1 / 99$ & 10,000 & 0 \\
\hline WCE88 & AN-101 & $2 / 1 / 99$ & $2 / 1 / 99$ & 28,000 & 0 \\
\hline WNE88 & AP-106 & $2 / 1 / 99$ & $2 / 1 / 99$ & 39,000 & 0 \\
\hline WATER & AP-106 & $3 / 1 / 99$ & $3 / 1 / 99$ & 5,000 & 0 \\
\hline WATER & $S \bar{Y}-102$ & $3 / 1 / 99$ & $3 / 1 / 99$ & 2,000 & 0 \\
\hline WNW88 & SY-102 & $3 / 1 / 99$ & $3 / 1 / 99$ & 6,000 & 0 \\
\hline EVAPF & AP-106 & $3 / 1 / 99$ & $3 / 1 / 99$ & 10,000 & 0 \\
\hline WATER & AP-106 & $3 / 1 / 99$ & $3 / 1 / 99$ & 9,000 & 0 \\
\hline $34 \mathrm{~L} 87$ & AP-106 & $3 / 1 / 99$ & $3 / 1 / 99$ & 10,000 & 0 \\
\hline WATER & AN-101 & $3 / 1 / 99$ & $3 / 1 / 99$ & 7,000 & 0 \\
\hline WNE88 & AP-106 & $3 / 1 / 99$ & $3 / 1 / 99$ & 36,000 & 0 \\
\hline WCE88 & AN-101 & $3 / 1 / 99$ & $3 / 1 / 99$ & 28,000 & 0 \\
\hline$A N-101$ & AP-103 & $3 / 5 / 99$ & $3 / 8 / 99$ & 593,500 & 0 \\
\hline \begin{tabular}{|l|} 
AP-107 \\
\end{tabular} & $A W-102$ & $3 / 5 / 99$ & $3 / 8 / 99$ & 615,000 & 20 \\
\hline AW-102 & $242-\mathrm{A}$ & $3 / 8 / 99$ & $3 / 11 / 99$ & 685,900 & 19 \\
\hline $242-\mathrm{A}$ & AW-106 & $3 / 8 / 99$ & $3 / 11 / 99$ & 221,100 & 19 \\
\hline $242-\mathrm{A}$ & LERF & $3 / 8 / 99$ & $3 / 11 / 99$ & 464,700 & 0 \\
\hline AP-108 & AP-106 & $3 / 15 / 99$ & $3 / 15 / 99$ & 500 & 0 \\
\hline WATER & AP-107 & $3 / 20 / 99$ & $3 / 20 / 99$ & 35,000 & 0 \\
\hline SY-102 & AP-107 & $3 / 25 / 99$ & $3 / 25 / 99$ & 30,006 & 1 \\
\hline WATER & AP-106 & $4 / 1 / 99$ & $4 / 1 / 99$ & 4,000 & 0 \\
\hline WATER & AP-106 & $4 / 1 / 99$ & $4 / 1 / 99$ & 7,000 & 0 \\
\hline WNW88 & $S Y-102$ & $4 / 1 / 99$ & $4 / 1 / 99$ & 10,000 & 0 \\
\hline WATER & SY-102 & $4 / 1 / 99$ & $4 / 1 / 99$ & 2,000 & 0 \\
\hline SPN87 & SY-102 & $4 / 1 / 99$ & $4 / 1 / 99$ & 6,000 & 0 \\
\hline WCE 88 & AN-101 & $4 / 1 / 99$ & $4 / 1 / 99$ & 28,000 & 0 \\
\hline WATER & AN-101 & $4 / 1 / 99$ & $4 / 1 / 99$ & 7,000 & 0 \\
\hline TAL88 & AP-106 & $4 / 1 / 99$ & $4 / 1 / 99$ & 4,000 & 0 \\
\hline WNE88 & AP-106 & $4 / 1 / 99$ & $4 / 1 / 99$ & 31,000 & 0 \\
\hline EVAPF & AW-102 & $4 / 2 / 99$ & $4 / 2 / 99$ & 35,000 & 0 \\
\hline EVAPF & AP-106 & $4 / 3 / 99$ & $4 / 3 / 99$ & 10,000 & 0 \\
\hline WCE88 & AP-106 & $4 / 3 / 99$ & $4 / 3 / 99$ & 17,000 & 0 \\
\hline AP-103 & AP-106 & $4 / 1 / 99$ & $4 / 3 / 99$ & 586,800 & 25 \\
\hline$A P-106$ & AW-102 & $4 / 3 / 99$ & $4 / 8 / 99$ & 873,100 & 0 \\
\hline AW-106 & AP-10I & $4 / 15 / 99$ & $4 / 16 / 99$ & 221,000 & 8 \\
\hline WATER & SY-102 & $5 / 1 / 99$ & $5 / 1 / 99$ & 5,000 & 0 \\
\hline WCE88 & AP-106 & $5 / 1 / 99$ & $5 / 1 / 99$ & 17,000 & 0 \\
\hline
\end{tabular}


HNF-2321

Revision 0

Table A.6-1. Complete Transfer List for Case 6.

\begin{tabular}{|c|c|c|c|c|c|}
\hline From & To & Start Date & End Date & $\begin{array}{c}\text { Liquid } \\
\text { (gallons) }\end{array}$ & $\begin{array}{c}\text { Solid } \\
\text { (gallons) }\end{array}$ \\
\hline WNW88 & SY-102 & $5 / 1 / 99$ & $5 / 1 / 99$ & 20,000 & 0 \\
\hline WATER & AP-106 & $5 / 1 / 99$ & $5 / 1 / 99$ & 7,000 & 0 \\
\hline WATER & AP-106 & $5 / 1 / 99$ & $5 / 1 / 99$ & 4,000 & 0 \\
\hline WATER & AP-106 & $5 / 1 / 99$ & $5 / 1 / 99$ & 4,000 & 0 \\
\hline 34L87 & AP-106 & $5 / 1 / 99$ & $5 / 1 / 99$ & 10,000 & 0 \\
\hline EVAPF & AP-106 & $5 / 1 / 99$ & $5 / 1 / 99$ & 10,000 & 0 \\
\hline WNE88 & AP-106 & $5 / 1 / 99$ & $5 / 1 / 99$ & 31,000 & 0 \\
\hline WATER & AN-101 & $5 / 1 / 99$ & $5 / 1 / 99$ & 7,000 & 0 \\
\hline WCE88 & AN-101 & $5 / 1 / 99$ & $5 / 1 / 99$ & 28,000 & 0 \\
\hline AP-102 & AP-103 & $5 / 1 / 99$ & $5 / 6 / 99$ & $1,070,000$ & 0 \\
\hline WATER & SY-102 & $6 / 1 / 99$ & $6 / 1 / 99$ & 10,000 & 0 \\
\hline WCE88 & AP-106 & $6 / 1 / 99$ & $6 / 1 / 99$ & 17,000 & 0 \\
\hline WNE88 & AP-106 & $6 / 1 / 99$ & $6 / 1 / 99$ & 26,000 & 0 \\
\hline WATER & AP-106 & $6 / 1 / 99$ & $6 / 1 / 99$ & 4,000 & 0 \\
\hline WNW88 & SY-102 & $6 / 1 / 99$ & $6 / 1 / 99$ & 39,000 & 0 \\
\hline WATER & AP-106 & $6 / 1 / 99$ & $6 / 1 / 99$ & 6,000 & 0 \\
\hline EVAPF & AP-106 & $6 / 1 / 99$ & $6 / 1 / 99$ & 10,000 & 0 \\
\hline WCE 88 & AN-101 & $6 / 1 / 99$ & $6 / 1 / 99$ & 28,000 & 0 \\
\hline WATER & AN-101 & $6 / 1 / 99$ & $6 / 1 / 99$ & 7,000 & 0 \\
\hline WATER & AP- 107 & $6 / 20 / 99$ & $6 / 20 / 99$ & 35,000 & 0 \\
\hline WATER & AP- 106 & $7 / 1 / 99$ & $7 / 1 / 99$ & 3,000 & 0 \\
\hline WATER & AP-106 & $7 / 1 / 99$ & $7 / 1 / 99$ & 4,000 & 0 \\
\hline WATER & AP-106 & $7 / 1 / 99$ & $7 / 1 / 99$ & 5,000 & 0 \\
\hline WATER & SY-102 & $7 / 1 / 99$ & $7 / 1 / 99$ & 14,000 & 0 \\
\hline WCE88 & AP-106 & $7 / 1 / 99$ & $7 / 1 / 99$ & 11,000 & 0 \\
\hline SPN87 & SY-102 & $7 / 1 / 99$ & $7 / 1 / 99$ & 6,000 & 0 \\
\hline WATER & AN-101 & $7 / 1 / 99$ & $7 / 1 / 99$ & 7,000 & 0 \\
\hline TAL88 & AP-106 & $7 / 1 / 99$ & $7 / 1 / 99$ & 4,000 & 0 \\
\hline $34 \mathrm{~L} 87$ & AP-105 & $7 / 1 / 99$ & $7 / 1 / 99$ & 10,000 & 0 \\
\hline EVAPF & AP-106 & $7 / 1 / 99$ & $7 / 1 / 99$ & 10,000 & 0 \\
\hline WCE88 & AN-101 & $7 / 1 / 99$ & $7 / 1 / 99$ & 28,000 & 0 \\
\hline WNE88 & AP-106 & $7 / 1 / 99$ & $7 / 1 / 99$ & 20,000 & 0 \\
\hline WNW88 & SY-102 & $7 / 1 / 99$ & $7 / 1 / 99$ & 57,000 & 0 \\
\hline WCE 88 & AP-106 & $8 / 1 / 99$ & $8 / 1 / 99$ & 11,000 & 0 \\
\hline WATER & SY-102 & $8 / 1 / 99$ & $8 / 1 / 99$ & 20,000 & 0 \\
\hline WATER & AP-106 & $8 / 1 / 99$ & $8 / 1 / 99$ & 3,000 & 0 \\
\hline WATER & AP-106 & $8 / 1 / 99$ & $8 / 1 / 99$ & 3,000 & 0 \\
\hline EVAPF & AP-105 & $8 / 1 / 99$ & $8 / 1 / 99$ & 10,000 & 0 \\
\hline WNE88 & AP-106 & $8 / 1 / 99$ & $8 / 1 / 99$ & 13,000 & 0 \\
\hline WNW88 & SY-102 & $8 / 1 / 99$ & $8 / 1 / 99$ & 82,000 & 0 \\
\hline WATER & AN-101 & $8 / 1 / 99$ & $8 / 1 / 99$ & 7,000 & 0 \\
\hline WCE 88 & AN-101 & $8 / 1 / 99$ & $8 / 1 / 99$ & 28,000 & 0 \\
\hline WCE88 & A.P-106 & $9 / 1 / 99$ & $9 / 1 / 99$ & 11,000 & 0 \\
\hline WCE88 & AN-10I & $9 / 1 / 99$ & $9 / 1 / 99$ & 23,000 & 0 \\
\hline WATER & SY-102 & $9 / 1 / 99$ & $9 / 1 / 99$ & 19,000 & 0 \\
\hline \begin{tabular}{|l} 
WATER \\
\end{tabular} & AP-106 & $9 / 1 / 99$ & $9 / 1 / 99$ & 3,000 & 0 \\
\hline WATER & AP-106 & $9 / 1 / 99$ & $9 / 1 / 99$ & 2,000 & 0 \\
\hline WNW88 & SY-102 & $9 / 1 / 99$ & $9 / 1 / 99$ & 76,000 & 0 \\
\hline WATER & AN-101 & $9 / 1 / 99$ & $9 / 1 / 99$ & 6,000 & 0 \\
\hline WNE88 & AP-106 & $9 / 1 / 99$ & $9 / 1 / 99$ & 13,000 & 0 \\
\hline EVAPF & A.P-106 & $9 / 1 / 99$ & $9 / 1 / 99$ & 10,000 & 0 \\
\hline \begin{tabular}{|l} 
WATER \\
\end{tabular} & AP-106 & $9 / 25 / 99$ & $9 / 25 / 99$ & 35,000 & 0 \\
\hline ZNL87 & SY-102 & $10 / 1 / 99$ & $10 / 1 / 99$ & 4,000 & 0 \\
\hline WCE88 & AP-106 & $10 / 1 / 99$ & $10 / 1 / 99$ & 11,000 & 0 \\
\hline WATER & AP-106 & $10 / 1 / 99$ & $10 / 1 / 99$ & 3,000 & 0 \\
\hline INS96 & AW-105 & $10 / 1 / 99$ & $10 / 1 / 99$ & 750 & 250 \\
\hline
\end{tabular}


HNF-2321

Revision 0

Table A.6-1. Complete Transfer List for Case 6.

\begin{tabular}{|c|c|c|c|c|c|}
\hline From & To & Start Date & End Date & $\begin{array}{c}\text { Liquid } \\
\text { (gallons) }\end{array}$ & $\begin{array}{c}\text { Solid } \\
\text { (gallons) }\end{array}$ \\
\hline IKL96 & AW-105 & $10 / 1 / 99$ & $10 / 1 / 99$ & 30,000 & 0 \\
\hline SPN87 & SY-102 & $10 / 1 / 99$ & $10 / 1 / 99$ & 7,000 & 0 \\
\hline EVAPF & AP-106 & $10 / 1 / 99$ & $10 / 1 / 99$ & 10,000 & 0 \\
\hline PXTCO & AP-106 & $10 / 1 / 99$ & $10 / 1 / 99$ & 5,000 & 0 \\
\hline 34 L87 & AP-106 & $10 / 1 / 99$ & $10 / 1 / 99$ & 10,000 & 0 \\
\hline 1 FL96 & AW-105 & $10 / 1 / 99$ & $10 / 1 / 99$ & 40,000 & 0 \\
\hline WATER & $S Y=102$ & $10 / 1 / 99$ & $10 / 1 / 99$ & 20,000 & 0 \\
\hline WCE 88 & AN-101 & $10 / 1 / 99$ & $10 / 1 / 99$ & 23,000 & 0 \\
\hline WATER & AN-101 & $10 / 1 / 99$ & $10 / 1 / 99$ & 6,000 & 0 \\
\hline WESF & AP-106 & $10 / 1 / 99$ & $10 / 1 / 99$ & 5,000 & 0 \\
\hline WNW88 & SY -102 & $10 / 1 / 99$ & $10 / 1 / 99$ & 79,000 & 0 \\
\hline WATER & AP-106 & $10 / 1 / 99$ & $10 / 1 / 99$ & 2,000 & 0 \\
\hline TAL88 & AP-106 & $10 / 1 / 99$ & $10 / 1 / 99$ & 6,000 & 0 \\
\hline WNE88 & AP-106 & $10 / 1 / 99$ & $10 / 1 / 99$ & 10,000 & 0 \\
\hline TNS88 & AP-106 & $10 / 1 / 99$ & $10 / 1 / 99$ & 750 & 250 \\
\hline WATER & SY-102 & $10 / 2 / 99$ & $10 / 2 / 99$ & 5,000 & 0 \\
\hline WATER & AP-106 & $10 / 2 / 99$ & $10 / 2 / 99$ & 5,000 & 0 \\
\hline WATER & AP-106 & $10 / 2 / 99$ & $10 / 2 / 99$ & 5,000 & 0 \\
\hline WATER & AW-105 & $10 / 2 / 99$ & $10 / 2 / 99$ & 17,000 & 0 \\
\hline WATER & AW-105 & $10 / 2 / 99$ & $10 / 2 / 99$ & 13,000 & 0 \\
\hline$A W-102$ & $242-A$ & $10 / 6 / 99$ & $10 / 11 / 99$ & 908,000 & 37 \\
\hline $242-\mathrm{A}$ & AW-106 & $10 / 6 / 99$ & $10 / 11 / 99$ & 583,700 & 37 \\
\hline $242-\mathrm{A}$ & LERF & $10 / 6 / 99$ & $10 / 11 / 99$ & 324,400 & 0 \\
\hline AW-106 & AW-102 & $10 / 11 / 99$ & $10 / 14 / 99$ & 583,600 & 26 \\
\hline AW-102 & $242-\mathrm{A}$ & $10 / 28 / 99$ & $10 / 31 / 99$ & 583,600 & 26 \\
\hline $242-A$ & AW-106 & $10 / 28 / 99$ & $10 / 31 / 99$ & 481,800 & 26 \\
\hline $242-\mathrm{A}$ & LERF & $10 / 28 / 99$ & $10 / 31 / 99$ & 101,800 & 0 \\
\hline WATER & SY-102 & $11 / 1 / 99$ & $11 / 1 / 99$ & 23,000 & 0 \\
\hline WATER & AP-106 & $11 / 1 / 99$ & $11 / 1 / 99$ & 3,000 & 0 \\
\hline WATER & AP-106 & $11 / 1 / 99$ & $11 / 1 / 99$ & 2,000 & 0 \\
\hline WCE 88 & AP-106 & $11 / 1 / 99$ & $11 / 1 / 99$ & 11,000 & 0 \\
\hline WATER & AN-101 & $11 / 1 / 99$ & $11 / 1 / 99$ & 6,000 & 0 \\
\hline WNE88 & AP-106 & $11 / 1 / 99$ & $11 / 1 / 99$ & 10,000 & 0 \\
\hline EVAPF & AP-106 & $11 / 1 / 99$ & $11 / 1 / 99$ & 10,000 & 0 \\
\hline WNW88 & SY-102 & $11 / 1 / 99$ & $11 / 1 / 99$ & 93,000 & 0 \\
\hline WCE88 & AN-101 & $11 / 1 / 99$ & $11 / 1 / 99$ & 23,000 & 0 \\
\hline EVAPF & AW-102 & $11 / 17 / 99$ & $11 / 17 / 99$ & 35,000 & 0 \\
\hline AP-104 & AP-106 & $11 / 15 / 99$ & $11 / 17 / 99$ & 500,000 & 17 \\
\hline AP-106 & $\mathrm{AW}-102$ & $11 / 17 / 99$ & $11 / 21 / 99$ & 882,400 & 0 \\
\hline WCE88 & AP-106 & $12 / 1 / 99$ & $12 / 1 / 99$ & 6,000 & 0 \\
\hline WATER & AP-106 & $12 / 1 / 99$ & $12 / 1 / 99$ & 1,000 & 0 \\
\hline WATER & SY-102 & $12 / 1 / 99$ & $12 / 1 / 99$ & 26,000 & 0 \\
\hline WCE88 & AN-101 & $12 / 1 / 99$ & $12 / 1 / 99$ & 17,000 & 0 \\
\hline WATER & AN-101 & $12 / 1 / 99$ & $12 / 1 / 99$ & 4,000 & 0 \\
\hline WATER & AP-106 & $12 / 1 / 99$ & $12 / 1 / 99$ & 1,000 & 0 \\
\hline WNE88 & AP-106 & $12 / 1 / 99$ & $12 / 1 / 99$ & 3,000 & 0 \\
\hline EVAPF & AP-106 & $12 / 1 / 99$ & $12 / 1 / 99$ & 10,000 & 0 \\
\hline WNW88 & SY-102 & $12 / 1 / 99$ & $12 / 1 / 99$ & 105,000 & 0 \\
\hline EVAPF & AW-102 & $12 / 3 / 99$ & $12 / 3 / 99$ & 35,000 & 0 \\
\hline AW-106 & AN-106 & $12 / 1 / 99$ & $12 / 3 / 99$ & 481,700 & 22 \\
\hline WCE88 & AP-106 & $1 / 1 / 00$ & $1 / 1 / 00$ & 6,000 & 0 \\
\hline WATER & AP-106 & $1 / 1 / 00$ & $1 / 1 / 00$ & 1,000 & 0 \\
\hline WATER & AP-106 & $1 / 1 / 00$ & $1 / 1 / 00$ & 4,000 & 0 \\
\hline SPN87 & SY-102 & $1 / 1 / 00$ & $1 / 1 / 00$ & 6,000 & 0 \\
\hline WATER & $S Y-102$ & $1 / 1 / 00$ & $1 / 1 / 00$ & 24,000 & 0 \\
\hline WATER & AN-10I & $1 / 1 / 00$ & $1 / 1 / 00$ & 4,000 & 0 \\
\hline
\end{tabular}


HNF-2321

Revision 0

Table A.6-1. Complete Transfer List for Case 6.

\begin{tabular}{|c|c|c|c|c|c|}
\hline From & To & Start Date & End Date & $\begin{array}{c}\text { Liquid } \\
\text { (gallons) }\end{array}$ & $\begin{array}{c}\text { Solid } \\
\text { (gallons) }\end{array}$ \\
\hline 34L87 & AP-106 & $1 / 1 / 00$ & $1 / 1 / 00$ & 10,000 & 0 \\
\hline WNW88 & SY -102 & $1 / 1 / 00$ & $1 / 1 / 00$ & 98,000 & 0 \\
\hline WCE88 & AN-101 & $1 / 1 / 00$ & $1 / 1 / 00$ & 17,000 & 0 \\
\hline WATER & AP-106 & $1 / 1 / 00$ & $1 / 1 / 00$ & 1,000 & 0 \\
\hline WNE88 & AP-106 & $1 / 1 / 00$ & $1 / 1 / 00$ & 3,000 & 0 \\
\hline EVAPF & AP-106 & $1 / 1 / 00$ & 1/1/00 & 10,000 & 0 \\
\hline TAL88 & AP-106 & $1 / 1 / 00$ & $1 / 1 / 00$ & 6,000 & 0 \\
\hline SY-102 & AP-107 & $1 / 15 / 00$ & $1 / 18 / 00$ & 856,200 & 35 \\
\hline WATER & AP-106 & $1 / 20 / 00$ & $1 / 20 / 00$ & 35,000 & 0 \\
\hline WCE 88 & AP-106 & $2 / 1 / 00$ & $2 / 1 / 00$ & 5,000 & 0 \\
\hline WATER & SY-102 & $2 / 1 / 00$ & $2 / 1 / 00$ & 22,000 & 0 \\
\hline WATER & AP-106 & $2 / 1 / 00$ & $2 / 1 / 00$ & 1,000 & 0 \\
\hline WNE88 & AP-106 & $2 / 1 / 00$ & $2 / 1 / 00$ & 3,000 & 0 \\
\hline WATER & AP-106 & $2 / 1 / 00$ & $2 / 1 / 00$ & 1,000 & 0 \\
\hline EVAPF & $\mathrm{AP}-106$ & $2 / 1 / 00$ & $2 / 1 / 00$ & 10,000 & 0 \\
\hline WCE88 & AN-101 & $2 / 1 / 00$ & $2 / 1 / 00$ & 17,000 & 0 \\
\hline WATER & $\mathrm{AN}-101$ & $2 / 1 / 00$ & $2 / 1 / 00$ & 4,000 & 0 \\
\hline WNW88 & SY-102 & $2 / 1 / 00$ & $2 / 1 / 00$ & 90,000 & 0 \\
\hline AP-104 & AP-107 & $2 / 15 / 00$ & $2 / 15 / 00$ & 124,000 & 4 \\
\hline WCE88 & AP-106 & $3 / 1 / 00$ & $3 / 1 / 00$ & 3,000 & 0 \\
\hline WATER & SY-102 & $3 / 1 / 00$ & $3 / 1 / 00$ & 21,000 & 0 \\
\hline WATER & AP-106 & $3 / 1 / 00$ & $3 / 1 / 00$ & 1,000 & 0 \\
\hline WATER & AP-106 & $3 / 1 / 00$ & $3 / 1 / 00$ & 1,000 & 0 \\
\hline WNE88 & AP-106 & $3 / 1 / 00$ & $3 / 1 / 00$ & 3,000 & 0 \\
\hline WNW88 & SY-102 & $3 / 1 / 00$ & $3 / 1 / 00$ & 82,000 & 0 \\
\hline WATER & AP-106 & $3 / 1 / 00$ & $3 / 1 / 00$ & 5,000 & 0 \\
\hline WATER & AN-101 & $3 / 1 / 00$ & $3 / 1 / 00$ & 4,000 & 0 \\
\hline EVAPF & AP-106 & $3 / 1 / 00$ & $3 / 1 / 00$ & 10,000 & 0 \\
\hline $34 \mathrm{~L} 87$ & AP-106 & $3 / 1 / 00$ & $3 / 1 / 00$ & 10,000 & 0 \\
\hline WCE88 & AN-101 & $3 / 1 / 00$ & $3 / 1 / 00$ & 17,000 & 0 \\
\hline WCE88 & AP-106 & $4 / 1 / 00$ & $4 / 1 / 00$ & 3,000 & 0 \\
\hline WATER & SY-102 & $4 / 1 / 00$ & $4 / 1 / 00$ & 20,000 & 0 \\
\hline WATER & AP-106 & $4 / 1 / 00$ & $4 / 1 / 00$ & 1,000 & 0 \\
\hline \begin{tabular}{|l|} 
SPN87 \\
\end{tabular} & SY-102 & $4 / 1 / 00$ & $4 / 1 / 00$ & 6,000 & 0 \\
\hline TAL88 & AP-106 & $4 / 1 / 00$ & $4 / 1 / 00$ & 6,000 & 0 \\
\hline EVAPF & AP-106 & $4 / 1 / 00$ & $4 / 1 / 00$ & 10,000 & 0 \\
\hline WNW88 & SY-102 & $4 / 1 / 00$ & $4 / 1 / 00$ & 79,000 & 0 \\
\hline WATER & $A N-101$ & $4 / 1 / 00$ & $4 / 1 / 00$ & 20,000 & 0 \\
\hline WCE88 & $\mathrm{AN}-101$ & $4 / 1 / 00$ & $4 / 1 / 00$ & 81,000 & 0 \\
\hline WCE88 & AP-106 & $5 / 1 / 00$ & $5 / 1 / 00$ & 3,000 & 0 \\
\hline WATER & $\$ Y-102$ & $5 / 1 / 00$ & $5 / 1 / 00$ & 22,000 & 0 \\
\hline WATER & AP-106 & $5 / 1 / 00$ & $5 / 1 / 00$ & 4,000 & 0 \\
\hline WATER & AP-106 & $5 / 1 / 00$ & $5 / 1 / 00$ & 1,000 & 0 \\
\hline EVAPF & AP-106 & $5 / 1 / 00$ & $5 / 1 / 00$ & 10,000 & 0 \\
\hline $34 \mathrm{~L} 87$ & AP-106 & $5 / 1 / 00$ & $5 / 1 / 00$ & 10,000 & 0 \\
\hline WNW88 & SY-102 & $5 / 1 / 00$ & $5 / 1 / 00$ & 88,000 & 0 \\
\hline WATER & AN-101 & $5 / 1 / 00$ & $5 / 1 / 00$ & 20,000 & 0 \\
\hline WCE88 & AN-101 & $5 / 1 / 00$ & $5 / 1 / 00$ & 81,000 & 0 \\
\hline AW-102 & $242-\mathrm{A}$ & $5 / 21 / 00$ & $5 / 25 / 00$ & 952,700 & 35 \\
\hline $242-\mathrm{A}$ & AW-106 & $5 / 21 / 00$ & $5 / 25 / 00$ & 610,700 & 35 \\
\hline $242-\mathrm{A}$ & LERF & $5 / 21 / 00$ & $5 / 25 / 00$ & 342,000 & 0 \\
\hline AW-106 & AW-102 & $5 / 26 / 00$ & $5 / 29 / 00$ & 610,500 & 24 \\
\hline WCE88 & AP-106 & $6 / 1 / 00$ & $6 / 1 / 00$ & 3,000 & 0 \\
\hline WATER & SY-102 & $6 / 1 / 00$ & $6 / 1 / 00$ & 23,000 & 0 \\
\hline WCE8 8 & AN-101 & $6 / 1 / 00$ & $6 / 1 / 00$ & 14,000 & 0 \\
\hline WATER & AN-101 & $6 / 1 / 00$ & $6 / 1 / 00$ & 4,000 & 0 \\
\hline
\end{tabular}


HNF-2321

Revision 0

Table A.6-1. Complete Transfer List for Case 6.

\begin{tabular}{|c|c|c|c|c|c|}
\hline From & $\therefore \quad$ To & Start Date & End Date & $\begin{array}{c}\text { Liquid } \\
\text { (gallons) }\end{array}$ & $\begin{array}{c}\text { Solid } \\
\text { (gallons) }\end{array}$ \\
\hline WATER & AP-106 & $6 / 1 / 00$ & $6 / 1 / 00$ & 1,000 & 0 \\
\hline EVAPF & AP-106 & $6 / 1 / 00$ & $6 / 1 / 00$ & 10,000 & 0 \\
\hline WNW88 & SY-102 & $6 / 1 / 00$ & $6 / 1 / 00$ & 91,000 & 0 \\
\hline AW-102 & $242-\mathrm{A}$ & $6 / 12 / 00$ & $6 / 15 / 00$ & 610,500 & 24 \\
\hline $242-A$ & AW-106 & $6 / 12 / 00$ & $6 / 15 / 00$ & 391,400 & 24 \\
\hline $242-A$ & LERF & $6 / 12 / 00$ & $6 / 15 / 00$ & 219,100 & 0 \\
\hline$A W-106$ & AW-102 & $6 / 15 / 00$ & $6 / 17 / 00$ & 391,300 & 17 \\
\hline SPN87 & $S Y-102$ & $7 / 1 / 00$ & $7 / 1 / 00$ & 6,000 & 0 \\
\hline WCE88 & AP-106 & $7 / 1 / 00$ & $7 / 1 / 00$ & 3,000 & 0 \\
\hline WATER & SY-102 & $7 / 1 / 00$ & $7 / 1 / 00$ & 25,000 & 0 \\
\hline WATER & AP-106 & $7 / 1 / 00$ & $7 / 1 / 00$ & 4,000 & 0 \\
\hline TAL 88 & AP-106 & $7 / 1 / 00$ & $7 / 1 / 00$ & 5,000 & 0 \\
\hline WATER & AP-106 & $7 / 1 / 00$ & $7 / 1 / 00$ & 1,000 & 0 \\
\hline \begin{tabular}{|l|} 
WCE 88 \\
\end{tabular} & AN-101 & $7 / 1 / 00$ & $7 / 1 / 00$ & 14,000 & 0 \\
\hline \begin{tabular}{|l} 
WATER \\
\end{tabular} & AN-101 & $7 / 1 / 00$ & $7 / 1 / 00$ & 4,000 & 0 \\
\hline EVAPF & AP-106 & $7 / 1 / 00$ & $7 / 1 / 00$ & 10,000 & 0 \\
\hline $34 L 87$ & AP-106 & $7 / 1 / 00$ & $7 / 1 / 00$ & 10,000 & 0 \\
\hline WNW88 & SY-102 & $7 / 1 / 00$ & $7 / 1 / 00$ & 98,000 & 0 \\
\hline AW-102 & $242-\mathrm{A}$ & $7 / 1 / 00$ & $7 / 3 / 00$ & 391,400 & 17 \\
\hline $242-\mathrm{A}$ & AW-106 & $7 / 1 / 00$ & $7 / 3 / 00$ & 265,200 & 17 \\
\hline $242-\mathrm{A}$ & LERF & $7 / 1 / 00$ & $7 / 3 / 00$ & 126,200 & 0 \\
\hline AP-107 & AW-102 & $7 / 4 / 00$ & $7 / 8 / 00$ & 917,700 & 0 \\
\hline WATER & SY-102 & $8 / 1 / 00$ & $8 / 1 / 00$ & 5,000 & 0 \\
\hline WCE8 8 & AN-101 & $8 / 1 / 00$ & $8 / 1 / 00$ & 14,000 & 0 \\
\hline WCW88 & SY-102 & $8 / 1 / 00$ & $8 / 1 / 00$ & 19,000 & 0 \\
\hline WATER & AN-101 & $8 / 1 / 00$ & $8 / 1 / 00$ & 4,000 & 0 \\
\hline EVAPF & AP-106 & $8 / 1 / 00$ & $8 / 1 / 00$ & 10,000 & 0 \\
\hline WCE88 & AP-106 & $8 / 1 / 00$ & $8 / 1 / 00$ & 2,000 & 0 \\
\hline WATER & SY-102 & $8 / 1 / 00$ & $8 / 1 / 00$ & 24,000 & 0 \\
\hline WNW88 & SY-102 & $8 / 1 / 00$ & $8 / 1 / 00$ & 93,000 & 0 \\
\hline AW-106 & AN-106 & $8 / 1 / 00$ & $8 / 2 / 00$ & 265,100 & 12 \\
\hline EVAPF & AW-102 & $8 / 3 / 00$ & $8 / 3 / 00$ & 35,000 & 0 \\
\hline AP-101 & AP-106 & $8 / 15 / 00$ & $8 / 17 / 00$ & 510,000 & 20 \\
\hline AZ-101 & AY-101 & $8 / 17 / 00$ & $8 / 20 / 00$ & 685,000 & 0 \\
\hline AZ-101 & AP-107 & $8 / 20 / 00$ & $8 / 20 / 00$ & 0 & 0 \\
\hline WASH-WATER & AZ-101 & $8 / 20 / 00$ & $8 / 21 / 00$ & 146,000 & 0 \\
\hline $\mathrm{AW}-102$ & $242-\mathrm{A}$ & $8 / 18 / 00$ & $8 / 23 / 00$ & 952,900 & 37 \\
\hline $242-\mathrm{A}$ & AW-106 & $8 / 18 / 00$ & $8 / 23 / 00$ & 610,900 & 37 \\
\hline $242-\mathrm{A}$ & LERF & $8 / 18 / 00$ & $8 / 23 / 00$ & 342,000 & 0 \\
\hline AW-106 & AW-102 & $8 / 23 / 00$ & $8 / 27 / 00$ & 610,800 & 26 \\
\hline WATER & SY-102 & $9 / 1 / 00$ & $9 / 1 / 00$ & 5,000 & 0 \\
\hline WCW88 & SY-102 & $9 / 1 / 00$ & $9 / 1 / 00$ & 19,000 & 0 \\
\hline WCE 88 & AP-106 & $9 / 1 / 00$ & $9 / 1 / 00$ & 1,000 & 0 \\
\hline WATER & SY-102 & $9 / 1 / 00$ & $9 / 1 / 00$ & 22,000 & 0 \\
\hline WATER & AN-101 & $9 / 1 / 00$ & $9 / 1 / 00$ & 4,000 & 0 \\
\hline EVAPF & AP-106 & $9 / 1 / 00$ & $9 / 1 / 00$ & 10,000 & 0 \\
\hline \begin{tabular}{|l|} 
WCE 88 \\
\end{tabular} & AN-101 & $9 / 1 / 00$ & $9 / 1 / 00$ & 14,000 & 0 \\
\hline WNW88 & SY-102 & $9 / 1 / 00$ & $9 / 1 / 00$ & 88,000 & 0 \\
\hline AW-102 & $242-\mathrm{A}$ & $9 / 10 / 00$ & $9 / 13 / 00$ & 610,700 & 26 \\
\hline $242-\mathrm{A}$ & AW-106 & $9 / 10 / 00$ & $9 / 13 / 00$ & 422,300 & 26 \\
\hline $242-\mathrm{A}$ & LERF & $9 / 10 / 00$ & $9 / 13 / 00$ & 188,400 & 0 \\
\hline AP-106 & $A W-102$ & $9 / 13 / 00$ & $9 / 17 / 00$ & 828,300 & 0 \\
\hline SY-102 & AP-107 & $9 / 15 / 00$ & $9 / 19 / 00$ & 900,000 & 37 \\
\hline ZNL87 & SY-102 & $10 / 1 / 00$ & $10 / 1 / 00$ & 4,000 & 0 \\
\hline \begin{tabular}{|l} 
WCW88 \\
\end{tabular} & SY-102 & $10 / 1 / 00$ & $10 / 1 / 00$ & 13,000 & 0 \\
\hline WATER & SY-102 & $10 / 1 / 00$ & $10 / 1 / 00$ & 3,000 & 0 \\
\hline
\end{tabular}


HNF-2321

Revision 0

Table A.6-1. Complete Transfer List for Case 6.

\begin{tabular}{|c|c|c|c|c|c|}
\hline From & To & Start Date & End Date & $\begin{array}{c}\text { Liquid } \\
\text { (gallons) }\end{array}$ & $\begin{array}{c}\text { Solid } \\
\text { (gallons) }\end{array}$ \\
\hline WNW88 & SY-102 & $10 / 1 / 00$ & $10 / 1 / 00$ & 26,000 & 0 \\
\hline SPN87 & $5 Y-102$ & $10 / 1 / 00$ & $10 / 1 / 00$ & 25,000 & 0 \\
\hline 1NS96 & AW-105 & $10 / 1 / 00$ & $10 / 1 / 00$ & 13,502 & 4,498 \\
\hline PXTCO & AP-106 & $.10 / 1 / 00$ & $10 / 1 / 00$ & 5,000 & 0 \\
\hline TNS88 & AP-106 & $10 / 1 / 00$ & $10 / 1 / 00$ & 750 & 250 \\
\hline WESF & AP-106 & $10 / 1 / 00$ & $10 / 1 / 00$ & 5,000 & 0 \\
\hline TAL88 & AP-106 & $10 / 1 / 00$ & $10 / 1 / 00$ & 23,000 & 0 \\
\hline WATER & SY-102 & $10 / 1 / 00$ & $10 / 1 / 00$ & 103,000 & 0 \\
\hline WATER & AN-101 & $10 / 1 / 00$ & $10 / 1 / 00$ & 2,000 & 0 \\
\hline WCE 88 & AN-10I & $10 / 1 / 00$ & $10 / 1 / 00$ & 9,000 & 0 \\
\hline $34 \mathrm{~L} 87$ & AP-106 & $10 / 1 / 00$ & $10 / 2 / 00$ & 50,000 & 0 \\
\hline $34 \mathrm{~L} 87$ & AP-106 & $10 / 2 / 00$ & $10 / 2 / 00$ & 7,000 & 0 \\
\hline WATER & SY-102 & $10 / 2 / 00$ & $10 / 2 / 00$ & 1,000 & 0 \\
\hline WATER & SY-102 & $10 / 2 / 00$ & $10 / 2 / 00$ & 5,000 & 0 \\
\hline EVAPF & AP-106 & $10 / 1 / 00$ & $10 / 2 / 00$ & 120,000 & 0 \\
\hline 1 KL96 & AW-105 & $10 / 1 / 00$ & $10 / 2 / 00$ & 302,000 & 0 \\
\hline WATER & AW-105 & $10 / 2 / 00$ & $10 / 2 / 00$ & 132,000 & 0 \\
\hline WATER & AW-105 & $10 / 2 / 00$ & $10 / 3 / 00$ & 7,000 & 0 \\
\hline WATER & AP-106 & $10 / 3 / 00$ & $10 / 3 / 00$ & 5,000 & 0 \\
\hline WATER & AP-106 & $10 / 3 / 00$ & $10 / 3 / 00$ & 3,000 & 0 \\
\hline WATER & AP-106 & $10 / 3 / 00$ & $10 / 3 / 00$ & 22,000 & 0 \\
\hline EVAPF & AW-102 & $10 / 4 / 00$ & $10 / 4 / 00$ & 50,000 & 0 \\
\hline AZ-101 & AP-106 & $10 / 9 / 00$ & $10 / 10 / 00$ & 143,200 & 0 \\
\hline WASH-WATER & AZ-101 & $10 / 11 / 00$ & $10 / 12 / 00$ & 146,000 & 0 \\
\hline AW-106 & AP-101 & $10 / 15 / 00$ & $10 / 17 / 00$ & 422,200 & 20 \\
\hline WCW88 & SY-102 & $11 / 1 / 00$ & $11 / 1 / 00$ & 13,000 & 0 \\
\hline WCE 88 & AN-101 & $11 / 1 / 00$ & $11 / 1 / 00$ & 9,000 & 0 \\
\hline WATER & SY-102 & $11 / 1 / 00$ & $11 / 1 / 00$ & 28,000 & 0 \\
\hline WATER & SY-102 & $11 / 1 / 00$ & $11 / 1 / 00$ & 3,000 & 0 \\
\hline WATER & AN-101 & $11 / 1 / 00$ & $11 / 1 / 00$ & 2,000 & 0 \\
\hline WNW88 & SY-102 & $11 / 1 / 00$ & $11 / 1 / 00$ & 111,000 & 0 \\
\hline EVAPF & $A W-102$ & $11 / 3 / 00$ & $11 / 3 / 00$ & 35,000 & $\overline{0}$ \\
\hline AZ-101 & AP-106 & $11 / 30 / 00$ & $12 / 1 / 00$ & 140,200 & 0 \\
\hline WCW88 & SY-102 & $12 / 1 / 00$ & $12 / 1 / 00$ & 8,000 & 0 \\
\hline WATER & AN-101 & $12 / 1 / 00$ & $12 / 1 / 00$ & 2,000 & 0 \\
\hline WCE 88 & AN-101 & $12 / 1 / 00$ & $12 / 1 / 00$ & 9.000 & 0 \\
\hline WATER & SY-102 & $12 / 1 / 00$ & $12 / 1 / 00$ & 2,000 & 0 \\
\hline WATER & SY-102 & $12 / 1 / 00$ & $12 / 1 / 00$ & 25,000 & 0 \\
\hline WNW88 & SY-102 & $12 / 1 / 00$ & $12 / 1 / 00$ & 99,000 & 0 \\
\hline WASH-WATER & AZ-101 & $12 / 2 / 00$ & $12 / 2 / 00$ & 146,000 & 0 \\
\hline WCW88 & SY-102 & $1 / 1 / 01$ & $1 / 1 / 01$ & 8,000 & 0 \\
\hline WATER & SY-10' & $1 / 1 / 01$ & $1 / 1 / 01$ & 2,000 & 0 \\
\hline WATER & AN-101 & $1 / 1 / 01$ & $1 / 1 / 01$ & 2,000 & 0 \\
\hline WCE8 8 & AN-101 & $1 / 1 / 01$ & $1 / 1 / 01$ & 9,000 & 0 \\
\hline WATER & SY-102 & $1 / 1 / 01$ & $1 / 1 / 01$ & 26,000 & 0 \\
\hline WNW 88 & SY-102 & $1 / 1 / 01$ & $1 / 1 / 01$ & 105,000 & 0 \\
\hline $\mathrm{AZ}-101$ & AP-106 & $1 / 20 / 01$ & $1 / 21 / 01$ & 142,900 & 0 \\
\hline WASH-WATER & AZ-101 & $1 / 22 / 01$ & $1 / 23 / 01$ & 138,200 & 0 \\
\hline WATER & $5 Y-102$ & $2 / 1 / 01$ & $2 / 1 / 01$ & 1,000 & 0 \\
\hline WCW88 & SY-102 & $2 / 1 / 01$ & $2 / 1 / 01$ & 3,000 & 0 \\
\hline WCE88 & AN-101 & $2 / 1 / 01$ & $2 / 1 / 01$ & 9,000 & 0 \\
\hline WATER & SY-102 & $2 / 1 / 01$ & $2 / 1 / 01$ & 23,000 & 0 \\
\hline WATER & AN-101 & $2 / 1 / 01$ & $2 / 1 / 01$ & 2,000 & 0 \\
\hline WNW88 & $S Y-102$ & $2 / 1 / 01$ & $2 / 1 / 01$ & 91,000 & 0 \\
\hline \begin{tabular}{|l|} 
WCW88 \\
\end{tabular} & SY-102 & $3 / 1 / 01$ & $3 / 1 / 01$ & 2,000 & 0 \\
\hline WATER & SY-102 & $3 / 1 / 01$ & $3 / 1 / 01$ & 1,000 & 0 \\
\hline
\end{tabular}


HNF-2321

Revision 0

Table A.6-1. Complete Transfer List for Case 6.

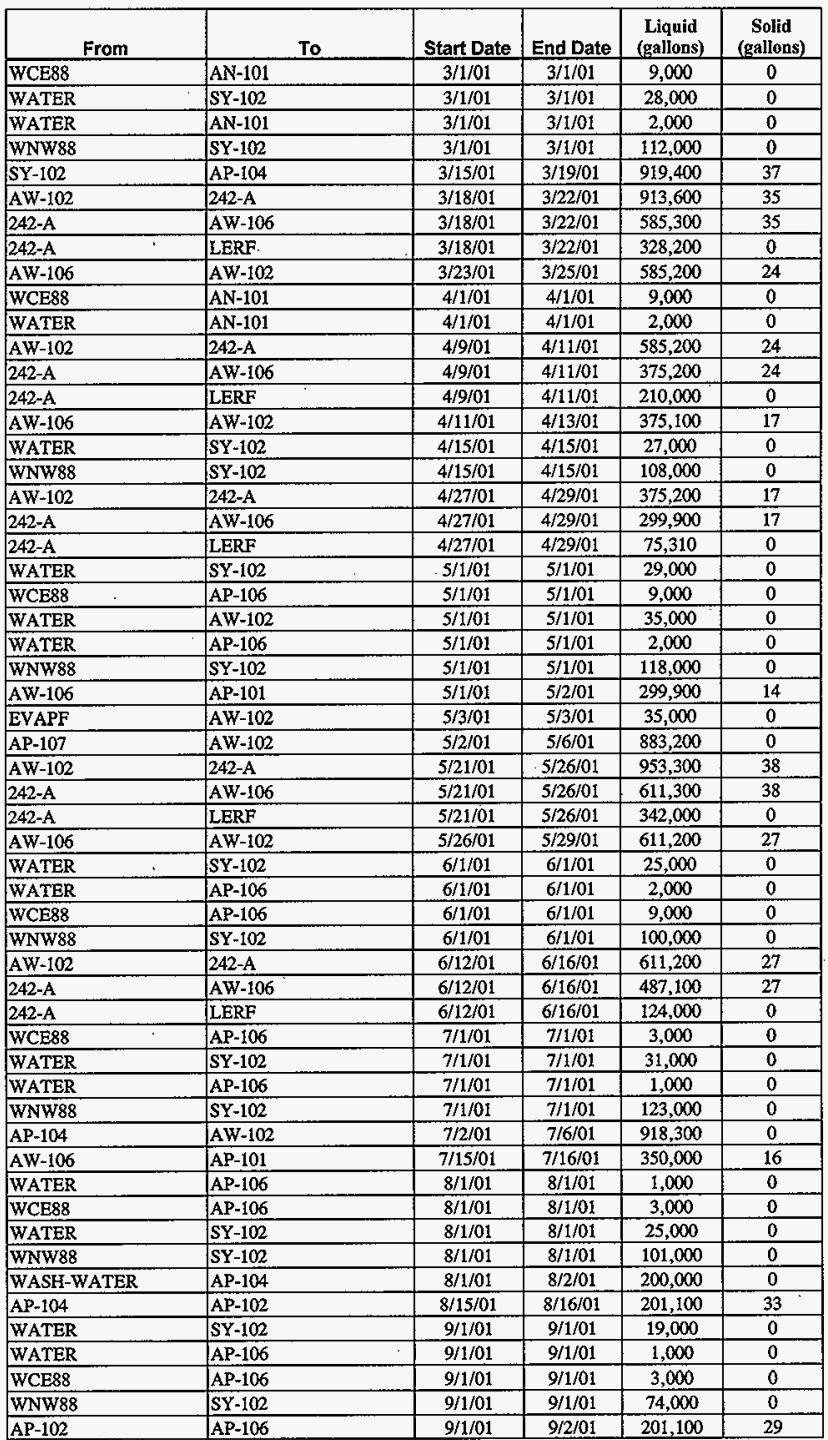


HNF-2321

Revision 0

Table A.6-1. Complete Transfer List for Case 6.

\begin{tabular}{|c|c|c|c|c|c|}
\hline From & To & Start Date & End Date & $\begin{array}{c}\text { Liquid } \\
\text { (gallons) }\end{array}$ & $\begin{array}{c}\text { Solid } \\
\text { (gallons) }\end{array}$ \\
\hline AN-105 & AP-102 & $9 / 15 / 01$ & $9 / 18 / 01$ & 542,400 & 7,895 \\
\hline LERF & AN-105-PUMP & 9/15/01 & $9 / 18 / 01$ & 154,100 & 0 \\
\hline AN-105 & AP-102 & $9 / 15 / 01$ & $9 / 18 / 01$ & 686,500 & 294 \\
\hline $\mathrm{AP}-108$ & AP-106 & $9 / 20 / 01$ & $9 / 20 / 01$ & 0 & 0 \\
\hline AN-105 & AP-104 & $9 / 19 / 01$ & $9 / 22 / 01$ & 542,400 & 7,895 \\
\hline LERF & AN-105-PUMP & $9 / 19 / 01$ & $9 / 22 / 01$ & 154,100 & 0 \\
\hline AN-105 & AP-104 & $9 / 19 / 01$ & $9 / 22 / 01$ & 686,500 & 294 \\
\hline $\mathrm{AW}-102$ & $242-\mathrm{A}$ & $9 / 20 / 01$ & $9 / 25 / 01$ & 918,600 & 37 \\
\hline $242-\mathrm{A}$ & AW-106 & $9 / 20 / 01$ & $9 / 25 / 01$ & 588,900 & 37 \\
\hline $242-\mathrm{A}$ & LERF & $9 / 20 / 01$ & $9 / 25 / 01$ & 329,700 & 0 \\
\hline AW-106 & $A W-102$ & $9 / 25 / 01$ & $9 / 29 / 01$ & 725,900 & 32 \\
\hline ZNL87 & SY-102 & $10 / 1 / 01$ & $10 / 1 / 01$ & 4,000 & 0 \\
\hline WESF & AP-106 & $10 / 1 / 01$ & $10 / 1 / 01$ & 5,000 & 0 \\
\hline WCW88 & SY-102 & $10 / 1 / 01$ & $10 / 1 / 01$ & 23,000 & 0 \\
\hline SPN87 & SY-102 & 10/1/01 & $10 / 1 / 01$ & 25,000 & 0 \\
\hline WATER & $\mathrm{AP}-106$ & $10 / 1 / 01$ & $10 / 1 / 01$ & 35,000 & 0 \\
\hline WNW88 & SY-102 & $10 / 1 / 01$ & $10 / 1 / 01$ & 62,000 & 0 \\
\hline WATER & SY-102 & $10 / 1 / 01$ & $10 / 1 / 01$ & 6,000 & 0 \\
\hline WATER & SY-102 & $10 / 1 / 01$ & $10 / 1 / 01$ & 16,000 & 0 \\
\hline WATER & AP-106 & $10 / 1 / 01$ & $10 / 1 / 01$ & 1,000 & 0 \\
\hline WCE 88 & AP-106 & $10 / 1 / 01$ & $10 / 1 / 01$ & 3,000 & 0 \\
\hline \begin{tabular}{|l|} 
TNS88 \\
\end{tabular} & AP-106 & $10 / 1 / 01$ & $10 / 1 / 01$ & 750 & 250 \\
\hline TAL88 & AP-106 & $10 / 1 / 01$ & $10 / 1 / 01$ & 23,000 & 0 \\
\hline 34187 & AP-106 & $10 / 1 / 01$ & $10 / 1 / 01$ & 50,000 & 0 \\
\hline EVAPF & AP-106 & $10 / 1 / 01$ & $10 / 1 / 01$ & 83,495 & 0 \\
\hline WATER & SY-102 & $10 / 2 / 01$ & $10 / 2 / 01$ & 5,000 & 0 \\
\hline WASH-WATER & AP-108 & $10 / 5 / 01$ & $10 / 6 / 01$ & 200,000 & 0 \\
\hline $\mathrm{AW}-102$ & $242-\mathrm{A}$ & $10 / 13 / 01$ & $10 / 17 / 01$ & 725,800 & 32 \\
\hline $242-\mathrm{A}$ & AW-106 & $10 / 13 / 01$ & $10 / 17 / 01$ & 591,000 & 32 \\
\hline $242-\mathrm{A}$ & LERF & $10 / 13 / 01$ & $10 / 17 / 01$ & 134,800 & 0 \\
\hline SY-102 & AP-107 & $10 / 15 / 01$ & $10 / 19 / 01$ & 800,000 & 33 \\
\hline AP-106 & AW-102 & $10 / 17 / 01$ & $10 / 21 / 01$ & 918,500 & 0 \\
\hline WATER & $\mathrm{AP}-106$ & $10 / 22 / 01$ & $10 / 22 / 01$ & 5,000 & 0 \\
\hline WATER & AP-106 & $10 / 22 / 01$ & $10 / 22 / 01$ & 22,000 & 0 \\
\hline EVAPF & AP-106 & $10 / 22 / 01$ & $10 / 23 / 01$ & 36,505 & 0 \\
\hline WATER & SY-102 & 11/1/01 & $11 / 1 / 01$ & 12,000 & 0 \\
\hline WCW88 & SY-102 & $11 / 1 / 01$ & $11 / 1 / 01$ & 23,000 & 0 \\
\hline $\begin{array}{l}\text { WATER } \\
\end{array}$ & AP-106 & $11 / 1 / 01$ & $11 / 1 / 01$ & 1,000 & 0 \\
\hline WNW88 & SY.102 & $11 / 1 / 01$ & $11 / 1 / 01$ & 47,000 & 0 \\
\hline WATER & SY-102 & $11 / 1 / 01$ & $11 / 1 / 01$ & 6,000 & 0 \\
\hline WCE88 & AP-106 & $11 / 1 / 01$ & $11 / 1 / 01$ & 3,000 & 0 \\
\hline EVAPF & $\mathrm{AW}-102$ & $11 / 3 / 01$ & $11 / 3 / 01$ & 35,000 & 0 \\
\hline AP-108 & AP-106 & $11 / 5 / 01$ & $11 / 5 / 01$ & 200,000 & 1 \\
\hline AP-107 & AN-105 & $11 / 5 / 01$ & $11 / 5 / 01$ & 979,200 & 107 \\
\hline AZ-102 & $\mathrm{AY}-10 \mathrm{~L}$ & $11 / 14 / 01$ & $11 / 15 / 01$ & 204,000 & 0 \\
\hline AZ-102 & AP-107 & $11 / 15 / 01$ & $11 / 16 / 01$ & 181,800 & 0 \\
\hline AW-106 & AN-101 & $11 / 15 / 01$ & $11 / 17 / 01$ & 400,000 & 19 \\
\hline \begin{tabular}{|l} 
WASH-WATER \\
\end{tabular} & AZ-102 & $11 / 16 / 01$ & $11 / 17 / 01$ & 213,000 & 0 \\
\hline \begin{tabular}{|l} 
WATER \\
\end{tabular} & AP-106 & $12 / 1 / 01$ & $12 / 1 / 01$ & 1,000 & 0 \\
\hline WCE88 & AP-106 & $12 / 1 / 01$ & $12 / 1 / 01$ & 3,000 & 0 \\
\hline WATER & SY-102 & $12 / 1 / 01$ & $12 / 1 / 01$ & 12,000 & 0 \\
\hline WCW88 & SY-102 & $12 / 1 / 01$ & $12 / 1 / 01$ & 46,000 & 0 \\
\hline \begin{tabular}{|l} 
WNW88. \\
\end{tabular} & SY-102 & $12 / 1 / 01$ & $12 / 1 / 01$ & 37,000 & 0 \\
\hline WATER & SY-102 & $12 / 1 / 01$ & $12 / 1 / 01$ & 9,000 & 0 \\
\hline AW-106 & AW-104 & $12 / 15 / 01$ & $12 / 16 / 01$ & 191,000 & 9 \\
\hline WCE88 & AP-106 & $1 / 1 / 02$ & $1 / 1 / 02$ & 3,000 & 0 \\
\hline
\end{tabular}


HNF-2321

Revision 0

Table A.6-1. Complete Transfer List for Case 6.

\begin{tabular}{|c|c|c|c|c|c|}
\hline From & To & Start Date & End Date & $\begin{array}{c}\text { Liquid } \\
\text { (gallons) }\end{array}$ & $\begin{array}{c}\text { Solid } \\
\text { (gallons) }\end{array}$ \\
\hline WATER & SY-102 & $1 / 1 / 02$ & $1 / 1 / 02$ & 8,000 & 0 \\
\hline WATER & AP-106 & $1 / 1 / 02$ & $1 / 1 / 02$ & 1,000 & 0 \\
\hline WNW88 & SY-102 & $1 / 1 / 02$ & $1 / 1 / 02$ & 32,000 & 0 \\
\hline WATER & SY-102 & $1 / 1 / 02$ & $1 / 1 / 02$ & 10,000 & 0 \\
\hline WCW88 & SY-102 & $1 / 1 / 02$ & $1 / 1 / 02$ & 39,000 & 0 \\
\hline $\mathrm{AZ}-102$ & AP-106 & $1 / 6 / 02$ & $1 / 6 / 02$ & 127,700 & 0 \\
\hline WASH-WATER & AZ-102 & $1 / 7 / 02$ & $1 / 8 / 02$ & 213,000 & 0 \\
\hline AP-102 & AP-108 & $1 / 8 / 02$ & $1 / 12 / 02$ & 686,500 & 286 \\
\hline AN-104 & AP-102 & $1 / 12 / 02$ & $1 / 15 / 02$ & 502,200 & 12,513 \\
\hline LERF & AN-104-PUMP & $1 / 12 / 02$ & $1 / 15 / 02$ & 200,800 & 0 \\
\hline AN-104 & AP-102 & $1 / 12 / 02$ & $1 / 15 / 02$ & 684,700 & 702 \\
\hline WNW88 & SY-102 & $2 / 1 / 02$ & $2 / 1 / 02$ & 16,000 & 0 \\
\hline WATER & SY-102 & $2 / 1 / 02$ & $2 / 1 / 02$ & 4,000 & 0 \\
\hline WCE 88 & AP-106 & $2 / 1 / 02$ & $2 / 1 / 02$ & 2,000 & 0 \\
\hline WATER & SY-102 & $2 / 1 / 02$ & $2 / 1 / 02$ & 16,000 & 0 \\
\hline WATER & AP-106 & $2 / 1 / 02$ & $2 / 1 / 02$ & 1,000 & 0 \\
\hline WCW88 & SY-102 & $2 / 1 / 02$ & $2 / 1 / 02$ & 62,000 & 0 \\
\hline AZ-102 & AP-106 & $2 / 27 / 02$ & $2 / 28 / 02$ & 210,300 & 0 \\
\hline WNW88 & SY-102 & $3 / 1 / 02$ & $3 / 1 / 02$ & 14,000 & 0 \\
\hline WATER & SY-102 & $3 / 1 / 02$ & $3 / 1 / 02$ & 4,000 & 0 \\
\hline WCE88 & AP-106 & $3 / 1 / 02$ & $3 / 1 / 02$ & 2,000 & 0 \\
\hline WATER & SY-102 & $3 / 1 / 02$ & $3 / 1 / 02$ & 16.000 & 0 \\
\hline WATER & AP-106 & $3 / 1 / 02$ & $3 / 1 / 02$ & 1,000 & 0 \\
\hline WCW88 & SY-102 & $3 / 1 / 02$ & $3 / 1 / 02$ & 62,000 & 0 \\
\hline WASH-WATER & AZ-102 & $3 / 1 / 02$ & $3 / 2 / 02$ & 213,000 & 0 \\
\hline WNW88 & SY-102 & $4 / 1 / 02$ & $4 / 1 / 02$ & 11,000 & 0 \\
\hline WATER & SY-102 & $4 / 1 / 02$ & $4 / 1 / 02$ & 18,000 & 0 \\
\hline WATER & $\mathrm{SY}-102$ & $4 / 1 / 02$ & $4 / 1 / 02$ & 3,000 & 0 \\
\hline WATER & AP-106 & $4 / 1 / 02$ & $4 / 1 / 02$ & 1,000 & 0 \\
\hline WCE 88 & AP-106 & $4 / 1 / 02$ & $4 / 1 / 02$ & 1,000 & 0 \\
\hline WCW88 & $S Y-102$ & $4 / 1 / 02$ & $4 / 1 / 02$ & 71,000 & 0 \\
\hline$A W-102$ & $242-\mathrm{A}$ & $4 / 4 / 02$ & $4 / 9 / 02$ & 953,800 & 34 \\
\hline $242-\mathrm{A}$ & AW-106 & $4 / 4 / 02$ & $4 / 9 / 02$ & 611,800 & 34 \\
\hline $242-\mathrm{A}$ & LERF & $4 / 4 / 02$ & $4 / 9 / 02$ & 342,000 & 0 \\
\hline AW-106 & AW-102 & $4 / 9 / 02$ & $4 / 13 / 02$ & 611,700 & 23 \\
\hline AW-102 & 242-A & $4 / 27 / 02$ & $4 / 30 / 02$ & 611,600 & 22 \\
\hline $242-\mathrm{A}$ & AW-106 & $4 / 27 / 02$ & $4 / 30 / 02$ & 390,500 & 22 \\
\hline $242-\mathrm{A}$ & LERF & $4 / 27 / 02$ & $4 / 30 / 02$ & 221,100 & 0 \\
\hline WNW88 & SY'-102 & $5 / 1 / 02$ & $5 / 1 / 02$ & 6,000 & 0 \\
\hline WATER & SY-102 & $5 / 1 / 02$ & $5 / 1 / 02$ & 1,000 & 0 \\
\hline WATER & SY -102 & $5 / 1 / 02$ & $5 / 1 / 02$ & 17,000 & 0 \\
\hline WCW88 & SY-102 & $5 / 1 / 02$ & $5 / 1 / 02$ & 67,000 & 0 \\
\hline AW-106 & AW-102 & $4 / 30 / 02$ & $5 / 2 / 02$ & 390,400 & 15 \\
\hline$A Z-101$ & P1HLW FEED TANK & $5 / 17 / 02$ & $5 / 17 / 02$ & 134,300 & 5,079 \\
\hline AW-102 & $242-\mathrm{A}$ & $5 / 16 / 02$ & $5 / 18 / 02$ & 390,500 & 15 \\
\hline $242-A$ & AW-106 & $5 / 16 / 02$ & $5 / 18 / 02$ & 250,000 & 15 \\
\hline $242-\mathrm{A}$ & LERF & $5 / 16 / 02$ & $5 / 18 / 02$ & 140,600 & 0 \\
\hline AW-106 & AW-102 & $5 / 18 / 02$ & $5 / 19 / 02$ & 249,900 & 10 \\
\hline WNW88 & $S Y-102$ & $6 / 1 / 02$ & $6 / 1 / 02$ & 2,000 & 0 \\
\hline WATER & SY-102 & $6 / 1 / 02$ & $6 / 1 / 02$ & 1,000 & 0 \\
\hline WATER & SY-102 & $6 / 1 / 02$ & $6 / 1 / 02$ & 16,000 & 0 \\
\hline WCW88 & SY-102 & $6 / 1 / 02$ & $6 / 1 / 02$ & 65,000 & 0 \\
\hline AW-102 & $242-\mathrm{A}$ & $\cdot 6 / 2 / 02$ & $6 / 3 / 02$ & 250,000 & 10 \\
\hline $242-A$ & AW-106 & $6 / 2 / 02$ & $6 / 3 / 02$ & 160,000 & 10 \\
\hline $242-A$ & LERF & $6 / 2 / 02$ & $6 / 3 / 02$ & 90,000 & 0 \\
\hline$A W-106$ & AW-102 & $6 / 3 / 02$ & $6 / 4 / 02$ & 160,000 & 7 \\
\hline
\end{tabular}


HNF-2321

Revision 0

Table A.6-1. Complete Transfer List for Case 6.

\begin{tabular}{|c|c|c|c|c|c|}
\hline From & To & Start Date & End Date & $\begin{array}{c}\text { Liquid } \\
\text { (gallons) }\end{array}$ & $\begin{array}{c}\text { Solid } \\
\text { (gallons) }\end{array}$ \\
\hline AW-102 & AW-106 & $6 / 5 / 02$ & $6 / 6 / 02$ & 160,100 & 7 \\
\hline AP-106 & AW-102 & $6 / 7 / 02$ & $6 / 11 / 02$ & 800,400 & 0 \\
\hline$A Z-102$ & AP-106 & $6 / 11 / 02$ & $6 / 12 / 02$ & 211,800 & 0 \\
\hline WASH-WATER & AZ-102 & $6 / 13 / 02$ & $6 / 14 / 02$ & 213,000 & 0 \\
\hline SY-102 & AP-106 & $6 / 15 / 02$ & $6 / 16 / 02$ & 300,000 & 13 \\
\hline WATER & SY-102 & $7 / 1 / 02$ & $7 / 1 / 02$ & 15,000 & 0 \\
\hline WCW88 & SY-102 & $7 / 1 / 02$ & $7 / 1 / 02$ & 58,000 & 0 \\
\hline WATER & $S Y-102$ & $8 / 1 / 02$ & $8 / 1 / 02$ & 11,000 & 0 \\
\hline WCW88 & SY-102 & $8 / 1 / 02$ & $8 / 1 / 02$ & 45,000 & 0 \\
\hline $\mathrm{AZ}-102$ & AP-106 & $8 / 2 / 02$ & $8 / 3 / 02$ & 212,500 & 0 \\
\hline WASH-WATER & AZ-102 & $8 / 4 / 02$ & $8 / 5 / 02$ & 110,000 & 0 \\
\hline WATER & SY-102 & $9 / 1 / 02$ & $9 / 1 / 02$ & 11,000 & 0 \\
\hline WCW88 & SY-102 & $9 / 1 / 02$ & $9 / 1 / 02$ & 42,000 & 0 \\
\hline ZNL87 & $S Y-102$ & $10 / 1 / 02$ & $10 / 1 / 02$ & 3,000 & 0 \\
\hline WATER & SY-102 & $10 / 1 / 02$ & $10 / 1 / 02$ & 9,000 & 0 \\
\hline SPN87 & SY-102 & $10 / 1 / 02$ & $10 / 1 / 02$ & 25,000 & 0 \\
\hline WESF & AP-106 & $10 / 1 / 02$ & $10 / 1 / 02$ & 5,000 & 0 \\
\hline TNS88 & AP-106 & $10 / 1 / 02$ & $10 / 1 / 02$ & 750 & 250 \\
\hline WCW88 & SY-102 & $10 / 1 / 02$ & $10 / 1 / 02$ & 35,000 & 0 \\
\hline TAL88 & AP-106 & $10 / 1 / 02$ & $10 / 1 / 02$ & 24,000 & 0 \\
\hline WATER & AP-106 & $10 / 1 / 02$ & $10 / 1 / 02$ & 35,000 & 0 \\
\hline $34 \mathrm{~L} 87$ & AP-106 & $10 / 1 / 02$ & $10 / 1 / 02$ & 50,000 & 0 \\
\hline EVAPF & AP-106 & $10 / 1 / 02$ & $10 / 1 / 02$ & 120,000 & 0 \\
\hline WATER & SY-102 & $10 / 2 / 02$ & $10 / 2 / 02$ & 1,000 & 0 \\
\hline WATER & $S Y-102$ & $10 / 2 / 02$ & $10 / 2 / 02$ & 5,000 & 0 \\
\hline WATER & AP-106 & $10 / 2 / 02$ & $10 / 2 / 02$ & 5,000 & 0 \\
\hline WATER & AP-106 & $10 / 2 / 02$ & $10 / 2 / 02$ & 22,000 & 0 \\
\hline EVAPF & AW-102 & $10 / 4 / 02$ & $10 / 4 / 02$ & 50,000 & 0 \\
\hline AW-102 & $242-\mathrm{A}$ & $10 / 3 / 02$ & $10 / 7 / 02$ & 800,400 & 28 \\
\hline 242-A & AW-106 & $10 / 3 / 02$ & $10 / 7 / 02$ & $512 ; 400$ & 28 \\
\hline 242-A & LERF & $10 / 3 / 02$ & $10 / 7 / 02$ & 288,000 & 0 \\
\hline AW-106 & AW-102 & $10 / 8 / 02$ & $10 / 11 / 02$ & 672,300 & 25 \\
\hline AW-102 & $242-\mathrm{A}$ & $10 / 25 / 02$ & $10 / 29 / 02$ & 722,200 & 27 \\
\hline $242-\mathrm{A}$ & AW-106 & $10 / 25 / 02$ & $10 / 29 / 02$ & 464,200 & 27 \\
\hline $242-A$ & LERF & $10 / 25 / 02$ & $10 / 29 / 02$ & 258,000 & 0 \\
\hline AW-106 & AW-102 & $10 / 29 / 02$ & $10 / 31 / 02$ & 464,100 & 18 \\
\hline WATER & SY-102 & $11 / 1 / 02$ & $11 / 1 / 02$ & 9,000 & 0 \\
\hline WCW88 & SY-102 & $11 / 1 / 02$ & $11 / 1 / 02$ & 35,000 & 0 \\
\hline EVAPF & AW-102 & $11 / 3 / 02$ & $11 / 3 / 02$ & 35,000 & 0 \\
\hline AW-102 & $242-\mathrm{A}$ & $11 / 14 / 02$ & $11 / 17 / 02$ & 499,200 & 20 \\
\hline $242-\mathrm{A}$ & AW-106 & $11 / 14 / 02$ & $11 / 17 / 02$ & 319,200 & 20 \\
\hline $242-\mathrm{A}$ & LERF & $11 / 14 / 02$ & $11 / 17 / 02$ & 180,000 & 0 \\
\hline AW-106 & \begin{tabular}{|l|}
$A W-102$ \\
\end{tabular} & $11 / 17 / 02$ & $11 / 18 / 02$ & 319,200 & 13 \\
\hline WATER & SY-102 & $12 / 1 / 02$ & $12 / 1 / 02$ & 6,000 & 0 \\
\hline WCW88 & SY-102 & $12 / 1 / 02$ & $12 / 1 / 02$ & 23,000 & 0 \\
\hline AW-102 & 242-A & $12 / 3 / 02$ & $12 / 4 / 02$ & 319.200 & 13 \\
\hline $242-\mathrm{A}$ & AW-106 & $12 / 3 / 02$ & $12 / 4 / 02$ & 208,400 & 13 \\
\hline $242-\mathrm{A}$ & LERF & $12 / 3 / 02$ & $12 / 4 / 02$ & 110,800 & 0 \\
\hline AP-106 & AW-102 & $12 / 5 / 02$ & $12 / 9 / 02$ & 919,100 & 0 \\
\hline WATER & $S Y-102$ & $1 / 1 / 03$ & $1 / 1 / 03$ & 5,000 & 0 \\
\hline WCW88 & SY-102 & $1 / 1 / 03$ & $1 / 1 / 03$ & 18,000 & 0 \\
\hline AP-104 & AP-108 & $1 / 4 / 03$ & $1 / 8 / 03$ & 686,500 & 287 \\
\hline AN-104 & AP-104 & $1 / 9 / 03$ & $1 / 12 / 03$ & 502,200 & 12,513 \\
\hline LERF & AN-104-PUMP & $1 / 9 / 03$ & $1 / 12 / 03$ & 200,800 & 0 \\
\hline AN-104 & AP-104 & $1 / 9 / 03$ & $1 / 12 / 03$ & 684,700 & 702 \\
\hline WATER & SY-102 & $2 / 1 / 03$ & $2 / 1 / 03$ & 4,000 & 0 \\
\hline
\end{tabular}


HNF-2321

Revision 0

Table A.6-1. Complete Transfer List for Case 6.

\begin{tabular}{|c|c|c|c|c|c|}
\hline From & To & Start Date & End Date & $\begin{array}{c}\text { Liquid } \\
\text { (gallons) }\end{array}$ & $\begin{array}{c}\text { Solid } \\
\text { (gallons) }\end{array}$ \\
\hline WCW88 & $\$ Y-102$ & $2 / 1 / 03$ & $2 / 1 / 03$ & 16,000 & 0 \\
\hline AW-106 & AN-104 & $2 / 15 / 03$ & $2 / 16 / 03$ & 208,400 & 10 \\
\hline P1HLW FEED TANK & HLW-CONTRACTOR & $6 / 1 / 02$ & $2 / 21 / 03$ & 134,300 & 5,079 \\
\hline AZ-101 & P1HLW FEED TANK & $2 / 21 / 03$ & $2 / 21 / 03$ & 134,300 & 5,079 \\
\hline WATER & SY-102 & $3 / 1 / 03$ & $3 / 1 / 03$ & 4,000 & 0 \\
\hline WCW88 & SY-102 & $3 / 1 / 03$ & $3 / 1 / 03$ & 15,000 & 0 \\
\hline$A Y-102$ & AZ-101 & $2 / 26 / 03$ & $3 / 2 / 03$ & 819,600 & 16,032 \\
\hline RETURN 2 & $A P-107$ & $3 / 28 / 03$ & $3 / 28 / 03$ & 8,669 & 331 \\
\hline WATER & SY-102 & $4 / 1 / 03$ & $4 / 1 / 03$ & 2,000 & 0 \\
\hline WCW88 & SY-102 & $4 / 1 / 03$ & $4 / 1 / 03$ & 6,000 & 0 \\
\hline AW-102 & $242-\mathrm{A}$ & $4 / 5 / 03$ & $4 / 10 / 03$ & 919,200 & 34 \\
\hline $242-\mathrm{A}$ & AW-106 & $4 / 5 / 03$ & $4 / 10 / 03$ & 589,200 & 34 \\
\hline $242-\mathrm{A}$ & LERF & $4 / 5 / 03$ & $4 / 10 / 03$ & 330,000 & 0 \\
\hline AW-106 & AW-102 & $4 / 10 / 03$ & $4 / 13 / 03$ & 589,100 & 23 \\
\hline $\mathrm{AW}-102$ & $242-\mathrm{A}$ & $4 / 28 / 03$ & $5 / 1 / 03$ & 589,000 & 23 \\
\hline $242-\mathrm{A}$ & AW-106 & $4 / 28 / 03$ & $5 / 1 / 03$ & 378,400 & 23 \\
\hline $242-\mathrm{A}$ & LERF & $4 / 28 / 03$ & $5 / 1 / 03$ & 210,600 & 0 \\
\hline WATER & SY-102 & $5 / 1 / 03$ & $5 / 1 / 03$ & 1,000 & 0 \\
\hline WCW88 & $\$ Y-102$ & $5 / 1 / 03$ & $5 / 1 / 03$ & 3,000 & 0 \\
\hline AW-106 & AW-102 & $5 / 1 / 03$ & $5 / 2 / 03$ & 378,300 & 16 \\
\hline$A W-102$ & 242-A & $5 / 17 / 03$ & $5 / 18 / 03$ & 378,400 & 15 \\
\hline $242-\mathrm{A}$ & AW-106 & $5 / 17 / 03$ & $5 / 18 / 03$ & 242,600 & 15 \\
\hline $242-\mathrm{A}$ & LERF & $5 / 17 / 03$ & $5 / 18 / 03$ & 135,800 & 0 \\
\hline AW-106 & AW-102 & $5 / 18 / 03$ & $5 / 20 / 03$ & 242,600 & 11 \\
\hline WATER & SY-102 & $6 / 1 / 03$ & $6 / 1 / 03$ & 1,000 & 0 \\
\hline WCW88 & SY-102 & $6 / 1 / 03$ & $6 / 1 / 03$ & 2,361 & 0 \\
\hline AW-102 & $242-\mathrm{A}$ & $6 / 3 / 03$ & $6 / 4 / 03$ & 242,600 & 11 \\
\hline $242-A$ & AW-106 & $6 / 3 / 03$ & $6 / 4 / 03$ & 201,200 & 11 \\
\hline $242-\mathrm{A}$ & LERF & $6 / 3 / 03$ & $6 / 4 / 03$ & 41,478 & 0 \\
\hline AW-106 & AN-104 & $6 / 4 / 03$ & $6 / 5 / 03$ & 201,100 & 9 \\
\hline AN-105 & AW-102 & $6 / 5 / 03$ & $6 / 9 / 03$ & 919,300 & 0 \\
\hline SY-102 & AP-106 & $6 / 15 / 03$ & $6 / 19 / 03$ & 984,800 & 42 \\
\hline WCW88 & SY-102 & $6 / 20 / 03$ & $6 / 20 / 03$ & 639 & 0 \\
\hline AW-102 & $242-A$ & $6 / 24 / 03$ & $6 / 29 / 03$ & 919,400 & 38 \\
\hline $242-\mathrm{A}$ & AW-106 & $6 / 24 / 03$ & $6 / 29 / 03$ & 590,200 & 38 \\
\hline $242-A$ & LERF & $6 / 24 / 03$ & $6 / 29 / 03$ & 329,200 & 0 \\
\hline WATER & SY-102 & $7 / 1 / 03$ & $7 / 1 / 03$ & 1,000 & 0 \\
\hline WCW88 & SY-102 & $7 / 1 / 03$ & $7 / 1 / 03$ & 3,000 & 0 \\
\hline AW-106 & AN-104 & $6 / 29 / 03$ & $7 / 2 / 03$ & 590,100 & 27 \\
\hline AP-106 & $\mathrm{AW}-102$ & $6 / 29 / 03$ & $7 / 4 / 03$ & 919,400 & 0 \\
\hline AN-104 & AN-105 & $8 / 15 / 03$ & $8 / 20 / 03$ & 993,200 & 45 \\
\hline AP-102 & AP-108 & $9 / 17 / 03$ & $9 / 21 / 03$ & 684,700 & 686 \\
\hline AW-101 & AP- 102 & $9 / 22 / 03$ & $9 / 26 / 03$ & 537,200 & 13,013 \\
\hline LERF & AW-101-PUMP & $9 / 22 / 03$ & $9 / 26 / 03$ & 308,100 & 0 \\
\hline AW-101 & AP-102 & $9 / 22 / 03$ & $9 / 26 / 03$ & 845,400 & 13,013 \\
\hline WESF & AP-106 & $10 / 1 / 03$ & $10 / 1 / 03$ & 5,000 & 0 \\
\hline ZNL87 & SY-102 & $10 / 1 / 03$ & $10 / 1 / 03$ & 3,000 & 0 \\
\hline TNS88 & AP-106 & $10 / 1 / 03$ & $10 / 1 / 03$ & 750 & 250 \\
\hline SPN87 & SY-102 & $10 / 1 / 03$ & $10 / 1 / 03$ & 25,000 & 0 \\
\hline WATER & AP-106 & $10 / 1 / 03$ & $10 / 1 / 03$ & 35,000 & 0 \\
\hline $34 \mathrm{~L} 87$ & AP-106 & $10 / 1 / 03$ & $10 / 1 / 03$ & 50,000 & 0 \\
\hline $34 \mathrm{~L} 87$ & AP-106 & $10 / 1 / 03$ & $10 / 1 / 03$ & 7,000 & 0 \\
\hline TAL 88 & AP-106 & $10 / 1 / 03$ & $10 / 1 / 03$ & 24,000 & 0 \\
\hline EVAPF & AP-106 & $10 / 1 / 03$ & $10 / 1 / 03$ & 120,000 & 0 \\
\hline WATER & SY-102 & $10 / 2 / 03$ & $10 / 2 / 03$ & 5,000 & 0 \\
\hline WATER & AP-106 & $10 / 2 / 03$ & $10 / 2 / 03$ & 3,000 & 0 \\
\hline
\end{tabular}


HNF-2321

Revision 0

Table A.6-1. Complete Transfer List for Case 6.

\begin{tabular}{|c|c|c|c|c|c|}
\hline From & To & Start Date & End Date & $\begin{array}{c}\text { Liquid } \\
\text { (gallons) }\end{array}$ & $\begin{array}{c}\text { Solid } \\
\text { (gallons) }\end{array}$ \\
\hline WATER & AP-106 & $10 / 2 / 03$ & $10 / 2 / 03$ & 22,000 & 0 \\
\hline WATER & AP-106 & $10 / 2 / 03$ & $10 / 2 / 03$ & 5,000 & 0 \\
\hline AZ-101 & AP-106 & $10 / 28 / 03$ & $11 / 1 / 03$ & 622,700 & 0 \\
\hline WASH-WATER & AZ-101 & $11 / 1 / 03$ & $11 / 2 / 03$ & 324,000 & 0 \\
\hline EVAPF & AW-102 & $11 / 3 / 03$ & $11 / 3 / 03$ & 35,000 & 0 \\
\hline P1HLW FEED_TANK & HLW-CONTRACTOR & $2 / 22 / 03$ & $11 / 14 / 03$ & 134,300 & 5,079 \\
\hline $\mathrm{AZ}-102$ & P1HLW FEED TANK & $11 / 14 / 03$ & $11 / 14 / 03$ & 123,900 & 5,202 \\
\hline AW-102 & $242-\mathrm{A}$ & $1 / 1 / 04$ & $1 / 6 / 04$ & 954,600 & 40 \\
\hline $242-\mathrm{A}$ & AW-106 & $1 / 1 / 04$ & $1 / 6 / 04$ & 613,300 & 40 \\
\hline $242-\mathrm{A}$ & LERF & $1 / 1 / 04$ & $1 / 6 / 04$ & 341,300 & 0 \\
\hline $\mathrm{AW}-106$ & AN-104 & $1 / 6 / 04$ & $1 / 9 / 04$ & 613,200 & 28 \\
\hline AP-106 & AW-102 & $1 / 7 / 04$ & $1 / 11 / 04$ & 919,600 & 0 \\
\hline $\mathrm{AZ}-101$ & AP-106 & $1 / 11 / 04$ & $1 / 13 / 04$ & 320,000 & 0 \\
\hline WASH-WATER & $A Z-101$ & $1 / 14 / 04$ & $1 / 16 / 04$ & 324,000 & 0 \\
\hline RETURN 2 & AP-107 & $1 / 23 / 04$ & $1 / 23 / 04$ & 8,669 & 331 \\
\hline AZ-10I & AP-106 & $3 / 5 / 04$ & $3 / 6 / 04$ & 322,300 & 0 \\
\hline WASH-WATER & AZ-101 & $3 / 8 / 04$ & $3 / 10 / 04$ & 433,600 & .0 \\
\hline$\$ \mathrm{XX}-113$ & SY-103 & $12 / 31 / 03$ & $4 / 6 / 04$ & 10,322 & 399 \\
\hline $\mathrm{AW}-102$ & $242-A$ & $5 / 4 / 04$ & $5 / 9 / 04$ & 919,900 & 33 \\
\hline $242-\mathrm{A}$ & AW-106 & $5 / 4 / 04$ & $5 / 9 / 04$ & 589,900 & 33 \\
\hline 242-A & LERF & $5 / 4 / 04$ & $5 / 9 / 04$ & 330,000 & 0 \\
\hline AW-106 & $A W-102$ & $5 / 9 / 04$ & $5 / 12 / 04$ & 589,700 & 22 \\
\hline $\mathrm{AW}-102$ & $242-\mathrm{A}$ & $5 / 27 / 04$ & $5 / 30 / 04$ & 589,700 & 22 \\
\hline $242-\mathrm{A}$ & AW-106 & $5 / 27 / 04$ & $5 / 30 / 04$ & 378,500 & 22 \\
\hline $242-\mathrm{A}$ & LERF & $5 / 27 / 04$ & $5 / 30 / 04$ & 211,200 & 0 \\
\hline AW-106 & $\mathrm{AW}-102$ & $5 / 30 / 04$ & $5 / 31 / 04$ & 378,400 & 15 \\
\hline AP-104 & AP-108 & $6 / 11 / 04$ & $6 / 14 / 04$ & 684,700 & 686 \\
\hline $\mathrm{AW}-102$ & $242-\mathrm{A}$ & $6 / 15 / 04$ & $6 / 16 / 04$ & 378,500 & 15 \\
\hline $242-\mathrm{A}$ & AW-106 & $6 / 15 / 04$ & $6 / 16 / 04$ & 241,600 & 15 \\
\hline $242-\mathrm{A}$ & LERF & $6 / 15 / 04$ & $6 / 16 / 04$ & 136,900 & 0 \\
\hline AW-106 & $\mathrm{AW}-102$ & $6 / 16 / 04$ & $6 / 18 / 04$ & 241,600 & 10 \\
\hline AW-101 & AP-104 & $6 / 15 / 04$ & $6 / 19 / 04$ & 537,200 & 13,013 \\
\hline LERF & AW-101-PUMP & $6 / 15 / 04$ & $6 / 19 / 04$ & 308,100 & 0 \\
\hline AW-101 & AP-104 & $6 / 15 / 04$ & $6 / 19 / 04$ & 845,400 & 13,013 \\
\hline $\mathrm{AW}-102$ & $242-\mathrm{A}$ & $7 / 2 / 04$ & $7 / 3 / 04$ & 241,600 & 10 \\
\hline $242-\mathrm{A}$ & AW-106 & $7 / 2 / 04$ & $7 / 3 / 04$ & 158,800 & 10 \\
\hline $242-\mathrm{A}$ & LERF & $7 / 2 / 04$ & $7 / 3 / 04$ & 82,837 & 0 \\
\hline AN-104 & AW-101 & $7 / 3 / 04$ & $7 / 6 / 04$ & 613,200 & 28 \\
\hline AW-10S & AW-102 & $7 / 16 / 04$ & $7 / 19 / 04$ & 626,900 & 0 \\
\hline $\mathrm{AW}-102$ & $242-\mathrm{A}$ & $8 / 3 / 04$ & $8 / 6 / 04$ & 627,000 & 21 \\
\hline $242-\mathrm{A}$ & AW-106 & $8 / 3 / 04$ & $8 / 6 / 04$ & 399,800 & 21 \\
\hline $242-\mathrm{A}$ & LERF & $8 / 3 / 04$ & $8 / 6 / 04$ & 227,200 & 0 \\
\hline P1HLW FEED TANK & HLW-CONTRACTOR & $11 / 14 / 03$ & $8 / 7 / 04$ & 123,900 & 5,202 \\
\hline $\mathrm{AZ}-102$ & P1HLW FEED TANK & $8 / 7 / 04$ & $8 / 8 / 04$ & 123,900 & 5,202 \\
\hline AW-106 & AW-102 & $8 / 7 / 04$ & $8 / 10 / 04$ & 558,500 & 21 \\
\hline RETURN 2 & AP-107 & $8 / 25 / 04$ & $8 / 25 / 04$ & 8,673 & 332 \\
\hline AW-102 & $242-\mathrm{A}$ & $8 / 24 / 04$ & $8 / 27 / 04$ & 558,400 & 21 \\
\hline 242-A & AW-106 & $8 / 24 / 04$ & $8 / 27 / 04$ & 357,700 & 21 \\
\hline $242-\mathrm{A}$ & LERF & $8 / 24 / 04$ & $8 / 27 / 04$ & 200,700 & 0 \\
\hline AW-106 & AW-102 & $8 / 27 / 04$ & $8 / 29 / 04$ & 357,600 & 14 \\
\hline C-104 & $\mathrm{AY}-102$ & $8 / 1 / 04$ & $9 / 8 / 04$ & 279,100 & 9,724 \\
\hline AW-102 & $242-A$ & $9 / 12 / 04$ & $9 / 14 / 04$ & 357,700 & 14 \\
\hline $242-\mathrm{A}$ & AW-106 & $9 / 12 / 04$ & $9 / 14 / 04$ & 229,200 & 14 \\
\hline $242-\mathrm{A}$ & LERF & $9 / 12 / 04$ & $9 / 14 / 04$ & 128,500 & 0 \\
\hline AW-106 & AW-102 & $9 / 14 / 04$ & $9 / 15 / 04$ & 229,200 & 10 \\
\hline $\mathrm{AW}-102$ & $242-\mathrm{A}$ & $9 / 29 / 04$ & $9 / 30 / 04$ & 229,300 & 10 \\
\hline
\end{tabular}


HNF-2321

Revision 0

Table A.6-1. Complete Transfer List for Case 6.

\begin{tabular}{|c|c|c|c|c|c|}
\hline From & To & Start Date & End Date & $\begin{array}{c}\text { Liquid } \\
\text { (gallons) }\end{array}$ & $\begin{array}{c}\text { Solid } \\
\text { (gallons) }\end{array}$ \\
\hline $242-A$ & AW-106 & $9 / 29 / 04$ & $9 / 30 / 04$ & 163,700 & 10 \\
\hline $242-\mathrm{A}$ & LERF & 9/29/04 & $9 / 30 / 04$ & 65,596 & 0 \\
\hline ZNL87 & SY-102 & $10 / 1 / 04$ & $10 / 1 / 04$ & 3,000 & 0 \\
\hline WESF & AP-106 & $10 / 1 / 04$ & $10 / 1 / 04$ & 5,000 & 0 \\
\hline SPN87 & SY-102 & $10 / 1 / 04$ & $10 / 1 / 04$ & 2,000 & 0 \\
\hline TN\$88 & AP-106 & $10 / 1 / 04$ & $10 / 1 / 04$ & 750 & 250 \\
\hline TAL88 & AP-106 & $10 / 1 / 04$ & $10 / 1 / 04$ & 2,000 & 0 \\
\hline WATER & AP-106 & $10 / 1 / 04$ & $10 / 1 / 04$ & 35,000 & 0 \\
\hline SPN87 & SY-102 & $10 / 1 / 04$ & $10 / 1 / 04$ & 23,000 & 0 \\
\hline $34 \mathrm{~L} 87$ & AP-106 & $10 / 1 / 04$ & $10 / 1 / 04$ & 50,000 & 0 \\
\hline TAL 88 & AP-106 & $10 / 1 / 04$ & $10 / 1 / 04$ & 22,000 & 0 \\
\hline EVAPF & AP-106 & $10 / 1 / 04$ & $10 / 1 / 04$ & 120,000 & 0 \\
\hline IFL96 & AW-105 & $10 / 1 / 04$ & $10 / 2 / 04$ & 200,000 & 0 \\
\hline WATER & $S Y-102$ & $10 / 2 / 04$ & $10 / 2 / 04$ & 1,000 & 0 \\
\hline WATER & AP-106 & $10 / 2 / 04$ & $10 / 2 / 04$ & 22,000 & 0 \\
\hline WATER & AW-105 & $10 / 2 / 04$ & $10 / 2 / 04$ & 88,000 & 0 \\
\hline WATER & AP-106 & $10 / 2 / 04$ & $10 / 2 / 04$ & 1,000 & 0 \\
\hline WATER & SY-102 & $.10 / 2 / 04$ & $10 / 3 / 04$ & 5,000 & 0 \\
\hline WATER & AP-106 & $10 / 3 / 04$ & $10 / 3 / 04$ & 4,000 & 0 \\
\hline EVAPF & AW-102 & $10 / 4 / 04$ & $10 / 4 / 04$ & 50,000 & 0 \\
\hline AP-106 & AW-102 & $10 / 1 / 04$ & $10 / 5 / 04$ & 919,900 & 266 \\
\hline EVAPF & $A W-102$ & $11 / 3 / 04$ & $11 / 3 / 04$ & 35,000 & 0 \\
\hline AX-103 & AN-104 & $8 / 7 / 04$ & $12 / 24 / 04$ & 310,200 & 2,846 \\
\hline RETURN 2 & AP-107 & $1 / 12 / 05$ & $1 / 12 / 05$ & 8,671 & 332 \\
\hline AW-101 & AW-105 & $2 / 15 / 05$ & $2 / 16 / 05$ & 200,000 & 9 \\
\hline $\mathrm{C}-103$ & AN-104 & $12 / 24 / 04$ & $3 / 9 / 05$ & 271,800 & 10,423 \\
\hline AP-102 & AP-108 & $3 / 5 / 05$ & $3 / 9 / 05$ & 740,300 & 1,581 \\
\hline WASH-WATER & AP-102 & $3 / 24 / 05$ & $3 / 24 / 05$ & 136,900 & 0 \\
\hline AP-102 & AP-106 & $4 / 7 / 05$ & $4 / 7 / 65$ & 243,100 & 10,337 \\
\hline AY-101 & AP-102 & $4 / 8 / 05$ & $4 / 9 / 05$ & 283,600 & 12 \\
\hline $\mathrm{AW}-102$ & $242-\mathrm{A}$ & $4 / 5 / 05$ & $4 / 10 / 05$ & $1,002,000$ & 34 \\
\hline $242-\mathrm{A}$ & AW-106 & $4 / 5 / 05$ & $4 / 10 / 05$ & 641,900 & 34 \\
\hline $242-\mathrm{A}$ & LERF & $4 / 5 / 05$ & $4 / 10 / 05$ & 360,000 & 0 \\
\hline AW-106 & AW-102 & $4 / 11 / 05$ & $4 / 15 / 05$ & 805,500 & 30 \\
\hline PLHLW FEED TANK & HLW-CONTRACTOR & $8 / 8 / 04$ & $5 / 2 / 05$ & 123,900 & 5,202 \\
\hline $\mathrm{AZ}-10 \mathrm{I}$ & P1HLW FEED TANK & $5 / 2 / 05$ & $5 / 2 / 05$ & 127,200 & 3,269 \\
\hline AW-102 & $242-\mathrm{A}$ & $4 / 29 / 05$ & $5 / 3 / 05$ & 805,600 & 30 \\
\hline $242-\mathrm{A}$ & AW-106 & $4 / 29 / 05$ & $5 / 3 / 05$ & 517,600 & 30 \\
\hline $242-A$ & LERF & $4 / 29 / 05$ & $5 / 3 / 05$ & 288,000 & 0 \\
\hline AW-106 & AW-102 & $5 / 3 / 05$ & $5 / 5 / 05$ & 517,500 & 20 \\
\hline$A W-102$ & $242-A$ & $5 / 19 / 05$ & $5 / 22 / 05$ & 517,600 & 20 \\
\hline $242-\mathrm{A}$ & AW-106 & $5 / 19 / 05$ & $5 / 22 / 05$ & 331,600 & 20 \\
\hline $242-\mathrm{A}$ & LERF & $5 / 19 / 05$ & $5 / 22 / 05$ & 186,000 & 0 \\
\hline AW-106 & AW-102 & $5 / 22 / 05$ & $5 / 24 / 05$ & 331,500 & 14 \\
\hline RETURN 2 & AP-107 & $5 / 26 / 05$ & $5 / 26 / 05$ & 8,672 & 331 \\
\hline$A W-102$ & $242-\mathrm{A}$ & $6 / 7 / 05$ & $6 / 8 / 05$ & 331,600 & 14 \\
\hline $242-\mathrm{A}$ & AW-106 & $6 / 7 / 05$ & $6 / 8 / 05$ & 217,000 & 14 \\
\hline $242-\mathrm{A}$ & LERF & $6 / 7 / 05$ & $6 / 8 / 05$ & 114,600 & 0 \\
\hline AW-106 & AW-101 & $6 / 8 / 05$ & $6 / 9 / 05$ & 216,900 & 10 \\
\hline A-102 & AN-104 & $3 / 9 / 05$ & $6 / 28 / 05$ & 100,600 & 744 \\
\hline AY-102 & AZ-102 & $8 / 1 / 05$ & $8 / 2 / 05$ & 279,400 & 9,360 \\
\hline AN-104 & AN-105 & $8 / 16 / 05$ & $8 / 17 / 05$ & 61,736 & 1,574 \\
\hline AN-104 & $A W-104$ & $8 / 17 / 05$ & $8 / 17 / 05$ & 73,164 & 1,865 \\
\hline AN-104 & AW-103 & $8 / 17 / 05$ & $8 / 17 / 05$ & 3,900 & 99 \\
\hline AN-104 & AP-105 & $8 / 17 / 05$ & $8 / 17 / 05$ & 3,901 & 99 \\
\hline AN-104 & AP-101 & $8 / 17 / 05$ & $8 / 17 / 05$ & 43,245 & 1,101 \\
\hline
\end{tabular}


HNF-2321

Revision 0

Table A.6-1. Complete Transfer List for Case 6.

\begin{tabular}{|c|c|c|c|c|c|}
\hline From & To & Start Date & End Date & $\begin{array}{c}\text { Liquid } \\
\text { (gallons) }\end{array}$ & $\begin{array}{c}\text { Solid } \\
\text { (gallons) }\end{array}$ \\
\hline AN-104 & AN-101 & $8 / 17 / 05$ & $8 / 18 / 05$ & 19,016 & 484 \\
\hline $\mathrm{AN}-104$ & AN-106 & $8 / 18 / 05$ & $8 / 18 / 05$ & 4,957 & 126 \\
\hline AN-104 & AP-103 & $8 / 18 / 05$ & $8 / 18 / 05$ & 42,715 & 1,085 \\
\hline ZNL87 & SY-102 & $10 / 1 / 05$ & $10 / \mathrm{t} / 05$ & 3,000 & 0 \\
\hline WESF & AP-106 & $10 / 1 / 05$ & $10 / 1 / 05$ & 5,000 & 0 \\
\hline TNS88 & AP-106 & $10 / 1 / 05$ & $10 / 1 / 05$ & 750 & 250 \\
\hline SPN87 & SY-102 & $10 / 1 / 05$ & $10 / 1 / 05$ & 25,000 & 0 \\
\hline TAL88 & AP-106 & $10 / 1 / 05$ & $10 / 1 / 05$ & 24,000 & 0 \\
\hline WATER & AP-106 & $10 / 1 / 05$ & $10 / 1 / 05$ & 35,000 & 0 \\
\hline $34 \mathrm{~L} 87$ & AP-106 & $10 / 1 / 05$ & $10 / 1 / 05$ & 50,000 & 0 \\
\hline EVAPF & AP-106 & $10 / 1 / 05$ & $10 / 1 / 05$ & 120,000 & 0 \\
\hline WATER & SY-102 & $10 / 2 / 05$ & $10 / 2 / 05$ & 1,000 & 0 \\
\hline WATER & SY-102 & $10 / 2 / 05$ & $10 / 2 / 05$ & 5,000 & 0 \\
\hline WATER & AP-106 & $10 / 2 / 05$ & $10 / 2 / 05$ & 5,000 & 0 \\
\hline WATER & AP-106 & $10 / 2 / 05$ & $10 / 2 / 05$ & 22,000 & 0 \\
\hline RETURN 2 & AP- 107 & $10 / 4 / 05$ & $10 / 4 / 05$ & 8,674 & 330 \\
\hline AP-104 & AP-108 & $10 / 9 / 05$ & $10 / 13 / 05$ & 740,300 & 1,581 \\
\hline WASHI-WATER & AP-104 & $10 / 27 / 05$ & $10 / 27 / 05$ & 136,900 & 0 \\
\hline EVAPF & AW-102 & $11 / 3 / 05$ & $11 / 3 / 05$ & 35,000 & 0 \\
\hline AP-104 & AP-106 & $11 / 10 / 05$ & $11 / 10 / 05$ & 243,100 & 10,337 \\
\hline AN-107 & AP-104 & $11 / 11 / 05$ & $11 / 13 / 05$ & 481,700 & 22 \\
\hline LERF & AN-107-PUMP & $11 / 11 / 05$ & $11 / 13 / 05$ & 72,259 & 0 \\
\hline AN-107 & AP-104 & $11 / 11 / 05$ & $11 / 13 / 05$ & 554,000 & 22 \\
\hline P1HLW FEED TANK & HLW-CONTRACTOR & $5 / 2 / 05$ & $11 / 16 / 05$ & 127,200 & 3,269 \\
\hline AZ-101 & P1HLW FEED TANK & $11 / 16 / 05$ & $11 / 17 / 05$ & 127,200 & 3,269 \\
\hline RETURN 2 & AP-107 & $11 / 26 / 05$ & $11 / 26 / 05$ & 8,683 & 330 \\
\hline$A W-105$ & AP-106 & $12 / 15 / 05$ & $12 / 16 / 05$ & 200,000 & 8 \\
\hline AP-106 & $\mathrm{AW}-102$ & $12 / 16 / 05$ & $12 / 20 / 05$ & 804,800 & 0 \\
\hline RETURN 2 2 & AP-107 & $1 / 15 / 06$ & $1 / 16 / 06$ & 8,679 & 330 \\
\hline RETURN 2 & $\mathrm{AP}-107$ & $3 / 7 / 06$ & $3 / 7 / 06$ & 8,679 & 330 \\
\hline RETURN 2 & AP-107 & $4 / 26 / 06$ & $4 / 26 / 06$ & 8,679 & 330 \\
\hline AN-104 & $\mathrm{AW}-105$ & $5 / 30 / 06$ & $6 / 1 / 06$ & 452,500 & 11,491 \\
\hline P1HLW FEED TANK & HLW-CONTRACTOR & $11 / 17 / 05$ & $6 / 2 / 06$ & 127,200 & 3,269 \\
\hline AZ-101 & PIHLWW FEED TANK & $6 / 2 / 06$ & $6 / 3 / 06$ & 127,200 & 3,269 \\
\hline AP-108 & LAW-CONTRACTOR-2 & $6 / 1 / 02$ & $6 / 11 / 06$ & $4,223,000$ & 5,051 \\
\hline AP-102 & AP-108 & $6 / 11 / 06$ & $6 / 12 / 06$ & 277,300 & 552 \\
\hline AN-107 & AP-102 & $6 / 13 / 06$ & $6 / 15 / 06$ & 481,700 & 22 \\
\hline LERF & AN-107-PUMP & $6 / 13 / 06$ & $6 / 15 / 06$ & 72,260 & 0 \\
\hline AN-107 & AP-102 & $6 / 13 / 06$ & $6 / 15 / 06$ & 554,000 & 22 \\
\hline RETURN 2 & $\mathrm{AP}-107$ & $6 / 15 / 06$ & $6 / 15 / 06$ & 8,686 & 330 \\
\hline $\mathrm{AW}-102$ & $242-\mathrm{A}$ & $6 / 19 / 06$ & $6 / 23 / 06$ & 839,900 & 31 \\
\hline $242-\mathrm{A}$ & AW-106 & $6 / 19 / 06$ & $6 / 23 / 06$ & 539,900 & 31 \\
\hline $242-\mathrm{A}$ & LERF & $6 / 19 / 06$ & $6 / 23 / 06$ & 300,000 & 0 \\
\hline$\overline{\mathrm{AZ}}-102$ & AP-106 & $6 / 23 / 06$ & $6 / 23 / 06$ & 159,500 & 0 \\
\hline WASH-WATER & AZ-102 & $6 / 24 / 06$ & $6 / 25 / 06$ & 324,000 & 0 \\
\hline AW-106 & AW-102 & $6 / 24 / 06$ & $6 / 27 / 06$ & 539,800 & 21 \\
\hline AW-102 & $242-\mathrm{A}$ & $7 / 11 / 06$ & $7 / 13 / 06$ & 540,000 & 21 \\
\hline $242-\mathrm{A}$ & AW-106 & $7 / 11 / 06$ & $7 / 13 / 06$ & 346,600 & 21 \\
\hline $242-\mathrm{A}$ & LERF & $7 / 11 / 06$ & $7 / 13 / 06$ & 193,400 & 0 \\
\hline $\mathrm{AW}-106$ & AW-102 & $7 / 13 / 06$ & $7 / 15 / 06$ & 346,500 & 15 \\
\hline RETURN 2 & AP-107 & $7 / 21 / 06$ & $7 / 21 / 06$ & 8,678 & 328 \\
\hline AW-102 & $242-\mathrm{A}$ & $7 / 29 / 06$ & $7 / 31 / 06$ & 346,600 & 14 \\
\hline $242-\mathrm{A}$ & AW-106 & $7 / 29 / 06$ & $7 / 31 / 06$ & 222,700 & 14 \\
\hline $242-\mathrm{A}$ & LERF & $7 / 29 / 06$ & $7 / 31 / 06$ & 124,000 & 0 \\
\hline AW-106 & AW-101 & $7 / 31 / 06$ & $8 / 1 / 06$ & 222,600 & 10 \\
\hline AP-108 & LAW-CONTRACTOR-2 & $6 / 12 / 06$ & $8 / 11 / 06$ & 277,300 & 555 \\
\hline
\end{tabular}


HNF-2321

Revision 0

Table A.6-1. Complete Transfer List for Case 6.

\begin{tabular}{|c|c|c|c|c|c|}
\hline From & To & Start Date & End Date & $\begin{array}{c}\text { Liquid } \\
\text { (gallons) }\end{array}$ & $\begin{array}{c}\text { Solid } \\
\text { (gallons) }\end{array}$ \\
\hline AP-104 & AP-108 & $8 / 11 / 06$ & $8 / 12 / 06$ & 171,100 & 337 \\
\hline AZ-102 & AP-106 & $8 / 13 / 06$ & $8 / 15 / 06$ & 320,100 & .0 \\
\hline WASH-WATER & $\mathrm{AZ}-102$ & $8 / 16 / 06$ & $8 / 18 / 06$ & 324,000 & 0 \\
\hline RETURN_2 & AP-107 & $9 / 4 / 06$ & $9 / 4 / 06$. & 8,678 & 329 \\
\hline AP-104 & AP-108 & $9 / 8 / 06$ & $9 / 10 / 06$ & 381,800 & 753 \\
\hline AN-102 & AP-104 & $9 / 11 / 06$ & $9 / 14 / 06$ & 443,500 & 21 \\
\hline LERF & AN-102-PUMP & $9 / 11 / 06$ & $9 / 14 / 06$ & 266,100 & 0 \\
\hline $\mathrm{AN}-102$ & AP-104 & $9 / 11 / 06$ & $9 / 14 / 06$ & 709,700 & 21 \\
\hline AN-104 & AN-107 & $9 / 13 / 06$ & 9/16/06 & 416,900 & 10,577 \\
\hline WESF & AP-106 & $10 / 1 / 06$ & $10 / 1 / 06$ & 5,000 & 0 \\
\hline TNS88 & AP-106 & $10 / 1 / 06$ & $10 / 1 / 06$ & 750 & 250 \\
\hline $34 \mathrm{~L} 87$ & AP-106 & $10 / 1 / 06$ & $10 / 1 / 06$ & 7,000 & 0 \\
\hline SPN87 & SY-102 & $10 / 1 / 06$ & $10 / 1 / 06$ & 25,000 & 0 \\
\hline WATER & AP-106 & $10 / 1 / 06$ & $10 / 1 / 06$ & 35,000 & 0 \\
\hline TAL88 & AP-106 & $10 / 1 / 06$ & $10 / 1 / 06$ & 25,000 & 0 \\
\hline 34 L87 & AP-106 & $10 / 1 / 06$ & $10 / 1 / 06$ & 50,000 & 0 \\
\hline EVAPF & AP-106 & $10 / 1 / 06$ & $10 / 1 / 06$ & 120,000 & 0 \\
\hline WATER & SY-102 & $10 / 2 / 06$ & $10 / 2 / 06$ & 5,000 & 0 \\
\hline WATER & AP-106 & $10 / 2 / 06$ & $10 / 2 / 06$ & 3,000 & 0 \\
\hline WATER & AP-106 & $10 / 2 / 06$ & $10 / 2 / 06$ & 5,000 & 0 \\
\hline WATER & AP-106 & $10 / 2 / 06$ & $10 / 2 / 06$ & 22,000 & 0 \\
\hline EVAPF & AW-102 & $10 / 4 / 06$ & $10 / 4 / 06$ & 50,000 & 0 \\
\hline AP-106 & AW-102 & $10 / 1 / 06$ & $10 / 5 / 06$ & 749,400 & 0 \\
\hline AZ-102 & AP-106 & $10 / 6 / 06$ & $10 / 8 / 06$ & 322,700 & 0 \\
\hline WASH-WATER & AZ-102 & $10 / 9 / 06$ & $10 / 9 / 06$ & 150,500 & 0 \\
\hline RETURN 2 & AP-107 & $10 / 30 / 06$ & $10 / 30 / 06$ & 8,670 & 330 \\
\hline EVAPF & AW-102 & $11 / 3 / 06$ & $11 / 3 / 06$ & 35,000 & 0 \\
\hline $\mathrm{C}-105$ & AN-104 & $6 / 28 / 05$ & $11 / 13 / 06$ & 827,500 & 31,875 \\
\hline P1HLW FEED TANK & HLW-CONTRACTOR & $6 / 3 / 06$ & $12 / 18 / 06$ & 127,200 & 3,269 \\
\hline$A Z-101$ & P1HLW FEED TANK & $12 / 18 / 06$ & $12 / 18 / 06$ & 127,200 & 3,269 \\
\hline RETURN 2 & AP-107 & $12 / 25 / 06$ & $12 / 25 / 06$ & 8,683 & 330 \\
\hline AP-102 & AP-108 & $1 / 19 / 07$ & $1 / 21 / 07$ & 559,200 & 575 \\
\hline AN-103 & AP-102 & $1 / 22 / 07$ & $1 / 25 / 07$ & 436,600 & 28,133 \\
\hline LERF & AN-103-PUMP & $1 / 22 / 07$ & $1 / 25 / 07$ & 264,900 & 0 \\
\hline AN-103 & AP-102 & $1 / 22 / 07$ & $1 / 25 / 07$ & 738,300 & 549 \\
\hline RETURN_2 & AP-107 & $2 / 20 / 07$ & $2 / 20 / 07$ & 8,676 & 330 \\
\hline A-104 & $\mathrm{AN}-104$ & $11 / 13 / 06$ & $2 / 22 / 07$ & 29,827 & 832 \\
\hline C-204 & AN-104 & $2 / 22 / 07$ & $3 / 1 / 07$ & 3,775 & 55 \\
\hline C-201 & $\mathrm{AN}-104$ & $3 / 1 / 07$ & $3 / 7 / 07$ & 2,464 & 59 \\
\hline AW-102 & $242-\mathrm{A}$ & $4 / 4 / 07$ & $4 / 9 / 07$ & 834,500 & 29 \\
\hline $242-\mathrm{A}$ & AW-106 & $4 / 4 / 07$ & $4 / 9 / 07$ & 534,500 & 29 \\
\hline $242-\mathrm{A}$ & LERF & $4 / 4 / 07$ & $4 / 9 / 07$ & 300,000 & 0 \\
\hline AX-104 & AN-104 & $3 / 7 / 07$ & $4 / 12 / 07$ & 18,516 & 572 \\
\hline AW-106 & AW-102 & $4 / 9 / 07$ & $4 / 12 / 07$ & 534,400 & 19 \\
\hline RETURN 2 & AP-107 & $4 / 19 / 07$ & $4 / 19 / 07$ & 8,672 & 330 \\
\hline $\mathrm{AW}-102$ & $242-\mathrm{A}$ & $4 / 26 / 07$ & $4 / 29 / 07$ & 534,500 & 19 \\
\hline $242-\mathrm{A}$ & AW-106 & $4 / 26 / 07$ & $4 / 29 / 07$ & 342,500 & 19 \\
\hline $242-\mathrm{A}$ & LERF & $4 / 26 / 07$ & $4 / 29 / 07$ & 192,000 & 0 \\
\hline AW-106 & AW-102 & $4 / 29 / 07$ & $5 / 1 / 07$ & 342,500 & 13 \\
\hline $\mathrm{AW}-102$ & $242-A$ & $5 / 15 / 07$ & $5 / 16 / 07$ & 342,600 & 13 \\
\hline $242-\mathrm{A}$ & AW-106 & $5 / 15 / 07$ & $5 / 16 / 07$ & 217,600 & 13 \\
\hline $242-\mathrm{A}$ & LERF & $5 / 15 / 07$ & $5 / 16 / 07$ & 125,000 & 0 \\
\hline \begin{tabular}{|l|} 
AW-106 \\
\end{tabular} & AW-102 & $5 / 16 / 07$ & $5 / 17 / 07$ & 217,500 & 8 \\
\hline AW-102 & AP-106 & $5 / 19 / 07$ & $5 / 20 / 07$ & 217,700 & 8 \\
\hline RETURN 2 & AP-107 & $6 / 16 / 07$ & $6 / 16 / 07$ & 8,672 & 330 \\
\hline P1HLW FEED TANK & HLW-CONTRACTOR & $12 / 18 / 06$ & $7 / 4 / 07$ & 127,200 & 3,269 \\
\hline
\end{tabular}


HNF-2321

Revision 0

Table A.6-1. Complete Transfer List for Case 6.

\begin{tabular}{|c|c|c|c|c|c|}
\hline From & To & Start Date & End Date & $\begin{array}{c}\text { Liquid } \\
\text { (gallons) }\end{array}$ & $\begin{array}{c}\text { Solid } \\
\text { (gallons) }\end{array}$ \\
\hline AZ-101 & P1HLW FEED TANK & $7 / 4 / 07$ & $7 / 5 / 07$ & 127,200 & 3,269 \\
\hline $\mathrm{AX}-102$ & AN-104 & $4 / 12 / 07$ & $7 / 31 / 07$ & 87,597 & 897 \\
\hline AP-104 & AP-108 & $8 / 2 / 07$ & $8 / 6 / 07$ & 709,600 & 72 \\
\hline $\mathrm{AN}-103$ & AP-104 & $8 / 7 / 07$ & $8 / 10 / 07$ & 436,600 & 28,133 \\
\hline LERF & AN-103-PUMP & $8 / 7 / 07$ & $8 / 10 / 07$ & 264,900 & 0 \\
\hline$A N-103$ & AP-104 & $8 / 7 / 07$ & $8 / 10 / 07$ & 738,300 & 549 \\
\hline RETURN 2 & AP-107 & $8 / 22 / 07$ & $8 / 22 / 07$ & 8,675 & 330 \\
\hline WESF & AP-106 & $10 / 1 / 07$ & $10 / 1 / 07$ & 5,000 & 0 \\
\hline TNS88 & AP-106 & $10 / 1 / 07$ & $10 / 1 / 07$ & 750 & 250 \\
\hline SPN87 & SY-102 & $10 / 1 / 07$ & $10 / 1 / 07$ & 25,000 & 0 \\
\hline TAL88 & AP-106 & $10 / 1 / 07$ & $10 / 1 / 07$ & 25,000 & 0 \\
\hline WATER & AP-106 & $10 / 1 / 07$ & $10 / 1 / 07$ & 35,000 & 0 \\
\hline $34 \mathrm{~L} 87$ & AP-106 & $10 / 1 / 07$ & $10 / 1 / 07$ & 50,000 & 0 \\
\hline EVAPF & AP-106 & $10 / 1 / 07$ & $10 / 1 / 07$ & 120,000 & 0 \\
\hline WATER & SY-102 & $10 / 2 / 07$ & $10 / 2 / 07$ & 5,000 & 0 \\
\hline WATER & AP-106 & $10 / 2 / 07$ & $10 / 2 / 07$ & 5,000 & 0 \\
\hline WATER & AP-106 & $10 / 2 / 07$ & $10 / 2 / 07$ & 22,000 & 0 \\
\hline AP-106 & $\mathrm{AW}-102$ & $10 / 1 / 07$ & $10 / 5 / 07$ & 800,200 & 0 \\
\hline EVAPF & AW-102 & $11 / 3 / 07$ & $11 / 3 / 07$ & 35,000 & 0 \\
\hline AN-104 & AN-107 & $11 / 4 / 07$ & $11 / 6 / 07$ & 546,400 & 10,567 \\
\hline AN-104 & $\mathrm{AN}-102$ & $11 / 6 / 07$ & $11 / 9 / 07$ & 498,900 & 9,640 \\
\hline SY-101 & AN-I03 & $11 / 8 / 07$ & $11 / 12 / 07$ & $1,105,000$ & 11,018 \\
\hline RETURN 2 & AP-107 & $11 / 28 / 07$ & $11 / 28 / 07$ & 8,675 & 329 \\
\hline P1HLW FEED TANK & HLW-CONTRACTOR & $7 / 5 / 07$ & $1 / 18 / 08$ & 127,200 & 3,269 \\
\hline $\mathrm{AZ}-102$ & P1HLW FEED TANK & $1 / 18 / 08$ & $1 / 19 / 08$ & 137,700 & 4,774 \\
\hline RETURN 2 & AP-107 & $3 / 5 / 08$ & $3 / 5 / 08$ & 8,675 & 329 \\
\hline AW-102 & $242-\mathrm{A}$ & $4 / 4 / 08$ & $4 / 8 / 08$ & 835,200 & 29 \\
\hline $242-\mathrm{A}$ & AW-106 & $4 / 4 / 08$ & $4 / 8 / 08$ & 535,200 & 29 \\
\hline $242-\mathrm{A}$ & LERF & $4 / 4 / 08$ & $4 / 8 / 08$ & 300,000 & 0 \\
\hline AP-108 & LAW-CONTRACTOR-2 & $8 / 12 / 06$ & $4 / 9 / 08$ & $1,822,000$ & 1,786 \\
\hline AW-106 & AW-102 & $4 / 9 / 08$ & $4 / 11 / 08$ & 535,100 & 19 \\
\hline AP-102 & AP-108 & 4/9/08 & $4 / 13 / 08$ & 738,300 & 557 \\
\hline $\mathrm{AW}-102$ & $242-\mathrm{A}$ & $4 / 25 / 08$ & $4 / 28 / 08$ & 535,200 & 19 \\
\hline $242-\mathrm{A}$ & AW-106 & $4 / 25 / 08$ & $4 / 28 / 08$ & 343,200 & 19 \\
\hline $242-\mathrm{A}$ & LERF & $4 / 25 / 08$ & $4 / 28 / 08$ & 192,000 & 0 \\
\hline AW-106 & $\mathrm{AW}-102$ & $4 / 28 / 08$ & $4 / 30 / 08$ & 343,100 & 13 \\
\hline S-103 & SY-101 & $12 / 31 / 03$ & $5 / 5 / 08$ & 712,300 & 1,011 \\
\hline $\mathrm{AW}-102$ & $242-\mathrm{A}$ & $5 / 14 / 08$ & $5 / 16 / 08$ & 343,200 & 13 \\
\hline $242-\mathrm{A}$ & AW-106 & $5 / 14 / 08$ & $5 / 16 / 08$ & 218,000 & 13 \\
\hline $242-\mathrm{A}$ & LERF & $5 / 14 / 08$ & $5 / 16 / 08$ & 125,200 & 0 \\
\hline AW-106 & AW-102 & $5 / 16 / 08$ & $5 / 17 / 08$ & 217,900 & 8 \\
\hline$A W-102$ & AP-106 & $5 / 18 / 08$ & $5 / 19 / 08$ & 218,100 & 8 \\
\hline RETURN 2 & AP-107 & $8 / 9 / 08$ & $8 / 9 / 08$ & 8,672 & 330 \\
\hline WESF & AP-106 & $10 / 1 / 08$ & $10 / 1 / 08$ & 5,000 & 0 \\
\hline TNS88 & AP-106 & $10 / 1 / 08$ & $10 / 1 / 08$ & 750 & 250 \\
\hline SPN87 & SY-102 & $10 / 1 / 08$ & $10 / 1 / 08$ & 25,000 & 0 \\
\hline TAL88 & AP-106 & $10 / 1 / 08$ & $10 / 1 / 08$ & 25,000 & 0 \\
\hline WATER & AP-106 & $10 / 1 / 08$ & $10 / 1 / 08$ & 35,000 & 0 \\
\hline $34 \mathrm{~L} 87$ & AP-106 & $10 / 1 / 08$ & $10 / 1 / 08$ & 50,000 & 0 \\
\hline EVAPF & AP-106 & $10 / 1 / 08$ & $10 / 1 / 08$ & 120,000 & 0 \\
\hline WATER & SY-102 & $10 / 2 / 08$ & $10 / 2 / 08$ & 5,000 & 0 \\
\hline WATER & AP-106 & $10 / 2 / 08$ & $10 / 2 / 08$ & 5,000 & 0 \\
\hline WATER & AP-106 & $10 / 2 / 08$ & $10 / 2 / 08$ & 22,000 & 0 \\
\hline EVAPF & $\mathrm{AW}-102$ & $10 / 4 / 08$ & $10 / 4 / 08$ & 50,000 & 0 \\
\hline EVAPF & $\mathrm{AW}-102$ & $11 / 3 / 08$ & $11 / 3 / 08$ & 35,000 & 0 \\
\hline P1HLW FEED TANK & HLW-CONTRACTOR & $1 / 19 / 08$ & $12 / 22 / 08$ & 137,700 & 4,774 \\
\hline
\end{tabular}


HNF-2321

Revision 0

Table A.6-1. Complete Transfer List for Case 6.

\begin{tabular}{|c|c|c|c|c|c|}
\hline From & To & Start Date & End Date & $\begin{array}{c}\text { Liquid } \\
\text { (gallons) }\end{array}$ & $\begin{array}{c}\text { Solid } \\
\text { (gallons) }\end{array}$ \\
\hline $\mathrm{AZ}-102$ & P1HLW FEED TANK & $12 / 22 / 08$ & $12 / 22 / 08$ & 137,700 & 4,774 \\
\hline$\overline{A P}-104$ & AP-108 & $1 / 4 / 09$ & $1 / 8 / 09$ & 738,400 & 532 \\
\hline $\mathrm{U}-202$ & UA-2 & $1 / 3 / 09$ & $1 / 10 / 09$ & 27,411 & 1,061 \\
\hline $\mathrm{U}-203$ & UA-2 & $1 / 10 / 09$ & $1 / 16 / 09$ & 13,895 & 537 \\
\hline RETURN 2 & AP-107 & $2 / 7 / 09$ & $2 / 7 / 09$ & 8,670 & 331 \\
\hline U-110 & UA-2 & $1 / 16 / 09$ & $3 / 10 / 09$ & 99,290 & 4,252 \\
\hline UA-2 & SY-102 & $3 / 11 / 09$ & $3 / 12 / 09$ & 173,900 & 5,847 \\
\hline $\mathrm{U}-110$ & UA-1 & $3 / 10 / 09$ & $5 / 27 / 09$ & 143,800 & 6,160 \\
\hline UA-1 & SY-102 & $5 / 28 / 09$ & $5 / 29 / 09$ & 186,800 & 6,157 \\
\hline $\mathrm{U}-110$ & UA-2 & $5 / 27 / 09$ & $5 / 30 / 09$ & 4,829 & 207 \\
\hline $\mathrm{U}-101$ & UA-2 & $5 / 30 / 09$ & $7 / 30 / 09$ & 27,955 & 668 \\
\hline RETURN 2 & AP- 107 & $8 / 8 / 09$ & $8 / 8 / 09$ & 8,670 & 331 \\
\hline TNS88 & AP-106 & $10 / 1 / 09$ & $10 / 1 / 09$ & 750 & 250 \\
\hline WESF & AP-106 & $10 / 1 / 09$ & $10 / 1 / 09$ & 5,000 & 0 \\
\hline 34L.87 & AP-106 & $10 / 1 / 09$ & $10 / 1 / 09$ & 7,000 & 0 \\
\hline SPN87 & SY-102 & $10 / 1 / 09$ & $10 / 1 / 09$ & 25,000 & 0 \\
\hline WATER & AP-106 & $10 / 1 / 09$ & $10 / 1 / 09$ & 35,000 & 0 \\
\hline TAL88 & AP-106 & $10 / 1 / 09$ & $10 / 1 / 09$ & 26,000 & 0 \\
\hline $34 \mathrm{~L} 87$ & AP-106 & $10 / 1 / 09$ & $10 / 1 / 09$ & 50,000 & 0 \\
\hline EVAPF & AP-106 & $10 / 1 / 09$ & $10 / 1 / 09$ & 120,000 & 0 \\
\hline WATER & SY-102 & $10 / 2 / 09$ & $10 / 2 / 09$ & 5,000 & 0 \\
\hline WATER & AP-106 & $10 / 2 / 09$ & $10 / 2 / 09$ & 22,000 & 0 \\
\hline WATER & AP-106 & $10 / 2 / 09$ & $10 / 2 / 09$ & 5,000 & 0 \\
\hline WATER & AP-106 & $10 / 2 / 09$ & $10 / 2 / 09$ & 3,000 & 0 \\
\hline AP-106 & AW-102 & $10 / 1 / 09$ & $10 / 5 / 09$ & 748,900 & 0 \\
\hline EVAPF & AW-102 & $11 / 3 / 09$ & $11 / 3 / 09$ & 35,000 & 0 \\
\hline $\mathrm{U}-104$ & UA-2 & $7 / 30 / 09$ & $11 / 9 / 09$ & 50,908 & 1,221 \\
\hline PLHLW FEED TANK & HLW-CONTRACTOR & $12 / 22 / 08$ & $11 / 25 / 09$ & 137,700 & 4,774 \\
\hline AP-108 & LAW-CONTRACTOR-2 & $4 / 13 / 08$ & $11 / 27 / 09$ & $1,477,000$ & 1,076 \\
\hline RETURN 1 & AP-107 & $11 / 28 / 09$ & $11 / 28 / 09$ & 0 & 0 \\
\hline RETURN 2 & AP-107 & $11 / 28 / 09$ & $11 / 28 / 09$ & 5,272 & 201 \\
\hline $\mathrm{U}-112$ & UA-2 & $11 / 9 / 09$ & $12 / 5 / 09$ & 58,521 & 2,330 \\
\hline $\mathrm{UA}-2$ & SY-102 & $12 / 5 / 09$ & $12 / 6 / 09$ & 154,400 & 4,426 \\
\hline AP-107 & AZ-101 & $12 / 8 / 09$ & $12 / 10 / 09$ & 395,800 & 7,587 \\
\hline AN-103 & AP-107 & $12 / 18 / 09$ & $12 / 23 / 09$ & $1,104,000$ & 12,373 \\
\hline SY-103 & AN-103 & $12 / 23 / 09$ & $12 / 27 / 09$ & $1,083,000$ & 32,723 \\
\hline $\mathrm{U}-112$ & UA-1 & $12 / 5 / 09$ & $1 / 17 / 10$ & 102,000 & 4,061 \\
\hline $\mathrm{U}-204$ & UA-1 & $1 / 17 / 10$ & $1 / 23 / 10$ & 13,004 & 503 \\
\hline U-201 & UA-1 & $1 / 23 / 10$ & $1 / 27 / 10$ & 13,912 & 538 \\
\hline $\mathrm{U}-201$ & UA-2 & $1 / 27 / 10$ & $1 / 30 / 10$ & 13,499 & 522 \\
\hline $\mathrm{U}-106$ & UA-2 & $1 / 30 / 10$ & $3 / 1 / 10$ & 134,400 & 338 \\
\hline $5 \mathrm{X}-112$ & $S Y-103$ & $4 / 6 / 04$ & $3 / 15 / 10$ & 397,100 & 16,406 \\
\hline $\mathrm{AW}-102$ & $242-A$ & $4 / 4 / 10$ & $4 / 9 / 10$ & 868,800 & 30 \\
\hline $242-A$ & AW-106 & $4 / 4 / 10$ & $4 / 9 / 10$ & 556,800 & 30 \\
\hline $242-\mathrm{A}$ & LERF & $4 / 4 / 10$ & $4 / 9 / 10$ & 312,000 & 0 \\
\hline AW-106 & $\mathrm{AW}-102$ & $4 / 9 / 10$ & $4 / 12 / 10$ & 556,700 & 20 \\
\hline AW-102 & $242-A$ & $4 / 26 / 10$ & $4 / 29 / 10$ & 556,900 & 20 \\
\hline $242-\mathrm{A}$ & AW-106 & $4 / 26 / 10$ & $4 / 29 / 10$ & 356,900 & 20 \\
\hline $242-\mathrm{A}$ & LERF & $4 / 26 / 10$ & $4 / 29 / 10$ & 200,000 & 0 \\
\hline AW-106 & AW-102 & $4 / 29 / 10$ & $5 / 1 / 10$ & 356,800 & 13 \\
\hline AW-102 & $242-\mathrm{A}$ & $5 / 15 / 10$ & $5 / 17 / 10$ & 356,900 & 13 \\
\hline $242-\mathrm{A}$ & AW-106 & $5 / 15 / 10$ & $5 / 17 / 10$ & 228,000 & 13 \\
\hline $242-\mathrm{A}$ & LERF & $5 / 15 / 10$ & $5 / 17 / 10$ & 128,900 & 0 \\
\hline AW-106 & AW-102 & $5 / 17 / 10$ & $5 / 18 / 10$ & 227,900 & 9 \\
\hline AW-102 & $242-A$ & $6 / 1 / 10$ & $6 / 2 / 10$ & 228,000 & 9 \\
\hline 242-A & AW-106 & $6 / 1 / 10$ & $6 / 2 / 10$ & 144,500 & 9 \\
\hline
\end{tabular}


HNF-2321

Revision 0

Table A.6-1. Complete Transfer List for Case 6.

\begin{tabular}{|c|c|c|c|c|c|}
\hline From & To & Start Date & End Date & $\begin{array}{c}\text { Liquid } \\
\text { (gallons) }\end{array}$ & $\begin{array}{c}\text { Solid } \\
\text { (gallons) }\end{array}$ \\
\hline $242-\mathrm{A}$ & LERF & $6 / 1 / 10$ & $6 / 2 / 10$ & 83,532 & 0 \\
\hline AW-106 & AW-102 & $6 / 2 / 10$ & $6 / 3 / 10$ & 144,400 & 6 \\
\hline AW-102 & AP-106 & $6 / 4 / 10$ & $6 / 5 / 10$ & 144,600 & 6 \\
\hline SX-110 & SY-103 & $3 / 15 / 10$ & $7 / 18 / 10$ & 212,700 & 8,909 \\
\hline SX-115 & $5 X-103$ & $7 / 18 / 10$ & $9 / 18 / 10$ & 42,100 & 1,556 \\
\hline SPN87 & SY-102 & $10 / 1 / 10$ & $10 / 1 / 10$ & 25,000 & 0 \\
\hline WATER & AP-106 & $10 / 1 / 10$ & $10 / 1 / 10$ & 25,000 & 0 \\
\hline \begin{tabular}{|l} 
WESF \\
\end{tabular} & AP-106 & $10 / 1 / 10$ & $10 / 1 / 10$ & 5,000 & 0 \\
\hline TNS88 & AP-106 & $10 / 1 / 10$ & $10 / 1 / 10$ & 750 & 250 \\
\hline \begin{tabular}{|l|} 
TAL 88 \\
\end{tabular} & AP-106 & $10 / 1 / 10$ & $10 / 1 / 10$ & 26,000 & 0 \\
\hline WATER & AP-105 & $10 / 1 / 10$ & $10 / 1 / 10$ & 35,000 & 0 \\
\hline $34 \mathrm{~L} 87$ & AP-105 & $10 / 1 / 10$ & $10 / 1 / 10$ & 50,000 & 0 \\
\hline EVAPF & AP-106 & $10 / 1 / 10$ & $10 / 1 / 10$ & 120,000 & 0 \\
\hline WATER & SX-102 & $10 / 2 / 10$ & $10 / 2 / 10$ & 5,000 & 0 \\
\hline WATER & AP-106 & $10 / 2 / 10$ & $10 / 2 / 10$ & 22,000 & 0 \\
\hline WATER & AP-106 & $10 / 2 / 10$ & $10 / 2 / 10$ & 5,000 & 0 \\
\hline EVAPF & AW-102 & $10 / 4 / 10$ & $10 / 4 / 10$ & 50,000 & 0 \\
\hline EVAPF & AW-102 & $11 / 3 / 10$ & $11 / 3 / 10$ & 35,000 & 0 \\
\hline WATER & AP-106 & U/1/11 & $1 / 1 / 11$ & 25,000 & 0 \\
\hline BY-110 & BA-3 & $1 / 3 / 11$ & $2 / 8 / 11$ & 147,400 & 2,633 \\
\hline $7 X-109$ & TA-5 & $\mathrm{t} / 3 / 11$ & $2 / 10 / 11$ & 144,100 & 5,925 \\
\hline SX-11! & SY-103 & $9 / 18 / 10$ & $2 / 10 / 11$ & 451,500 & 19,767 \\
\hline TX-101 & $\mathrm{TA}-4$ & $\mathrm{~L} / 3 / 11$ & $2 / 22 / 11$ & 146,500 & 3,494 \\
\hline $\mathrm{T}-106$ & TA-6 & $1 / 3 / 11$ & $3 / 2 / 11$ & 74,648 & 2,929 \\
\hline B-112 & $\mathrm{BA}-1$ & $1 / 3 / 11$ & $3 / 9 / 11$ & 75,213 & 1,083 \\
\hline BY-110 & $\mathrm{BA}-4$ & $2 / 8 / 11$ & $3 / 16 / 11$ & 147,400 & 2,633 \\
\hline$T X-109$ & TA-3 & $2 / 10 / 11$ & $3 / 19 / 11$ & 144,100 & 5,925 \\
\hline T-101 & TA-6 & $3 / 2 / 11$ & $3 / 25 / 11$ & 61,377 & 2,346 \\
\hline WATER & AP-106 & $4 / 1 / 11$ & $4 / 1 / 11$ & 25,000 & 0 \\
\hline BX-102 & $\mathrm{BA}-2$ & $1 / 3 / 11$ & $4 / 4 / 11$ & 52,548 & 2,443 \\
\hline B-111 & BA-1 & $3 / 9 / 11$ & $4 / 9 / 11$ & 72,630 & 2,383 \\
\hline TY-101 & TA-2 & $2 / 22 / 11$ & $4 / 13 / 11$ & 146,500 & 3,494 \\
\hline BY-110 & BA-5 & $3 / 16 / 11$ & $4 / 21 / 11$ & 147,400 & 2,633 \\
\hline TX-109 & TA-1 & $3 / 19 / 11$ & $4 / 26 / 11$ & 144,100 & 5,925 \\
\hline $\mathrm{BX}-112$ & BA-2 & $4 / 4 / 11$ & $5 / 2 / 11$ & 67,370 & 2,937 \\
\hline AN-104 & AP-104 & $6 / 1 / 11$ & $6 / 1 / 11$ & 55,264 & 1,061 \\
\hline BA-3 & AP-108 & $6 / 1 / 11$ & $6 / 2 / 11$ & 155,600 & 2,631 \\
\hline$\overline{\mathrm{BA}-4}$ & AP-104 & $6 / 1 / 11$ & $6 / 2 / 11$ & 155,600 & 2,631 \\
\hline $\mathrm{BA}-1$ & AP-108 & $6 / 2 / 11$ & $6 / 2 / 11$ & 157,300 & 3,464 \\
\hline $\mathrm{BA}-5$ & AP-104 & $6 / 2 / 11$ & $6 / 3 / 11$ & 155,600 & 2,631 \\
\hline$\overline{B A-2}$ & AP-108 & $6 / 2 / 11$ & $6 / 3 / 11$ & 174,500 & 5,377 \\
\hline$A N-103$ & AP-102 & $6 / 1 / 11$ & $6 / 6 / 11$ & $1,084,000$ & 32,225 \\
\hline AN-103 & AP-104 & $6 / 6 / 11$ & $6 / 6 / 11$ & 972 & 28 \\
\hline SY-101 & AN-103 & $6 / 6 / 11$ & $6 / 10 / 11$ & $1,115,000$ & 1,877 \\
\hline B-111 & $\mathrm{BA}-6$ & $4 / 9 / 11$ & $6 / 11 / 11$ & 145,200 & 4,764 \\
\hline BA-6 & AP-108 & $6 / 11 / 11$ & $6 / 12 / 11$ & 159,400 & 4,762 \\
\hline $\mathrm{AN}-103$ & AP-104 & $6 / 11 / 11$ & $6 / 14 / 11$ & 741,100 & 1,724 \\
\hline AN-103 & AP-108 & $6 / 14 / 11$ & $6 / 16 / 11$ & 373,800 & 869 \\
\hline SY-102 & AN-103 & $6 / 16 / 11$ & $6 / 20 / 11$ & $1,004,000$ & 42,735 \\
\hline UA-1 & SY-102 & $1 / 28 / 10$ & $6 / 20 / 11$ & 146,500 & 5,102 \\
\hline AN-103 & AP-108 & $6 / 20 / 11$ & $6 / 20 / 11$ & 75,598 & 3,136 \\
\hline UA-2 & SY-102 & $6 / 20 / 11$ & $6 / 21 / 11$ & 151,500 & 862 \\
\hline $\mathrm{TA}-5$ & SY-102 & $6 / 21 / 11$ & $6 / 22 / 11$ & 180,300 & 5,922 \\
\hline $\mathrm{TA}-4$ & SY-102 & $6 / 22 / 11$ & $6 / 23 / 11$ & 143,200 & 3,492 \\
\hline TA-3 & SY-102 & $6 / 23 / 11$ & $6 / 24 / 11$ & 180,300 & 5,922 \\
\hline TA-6 & SY-102 & $6 / 24 / 11$ & $6 / 25 / 11$ & 154,500 & 5,272 \\
\hline
\end{tabular}


HNF-2321

Revision 0

Table A.6-1. Complete Transfer List for Case 6.

\begin{tabular}{|c|c|c|c|c|c|}
\hline From & To & Start Date & End Date & $\begin{array}{c}\text { Liquid } \\
\text { (gallons) }\end{array}$ & $\begin{array}{c}\text { Solid } \\
\text { (gallons) }\end{array}$ \\
\hline TA-2 & SY-102 & $6 / 25 / 11$ & $6 / 25 / 11$ & 143,200 & 3,492 \\
\hline WATER & AP-106 & $7 / 1 / 11$ & $7 / 1 / 11$ & 25,000 & 0 \\
\hline$B Y-110$ & $\mathrm{BA}-4$ & $6 / 2 / 11$ & $7 / 8 / 11$ & 147,300 & 2,631 \\
\hline TY-101 & TA-5 & $6 / 22 / 11$ & $7 / 13 / 11$ & 59,942 & 1,430 \\
\hline $\mathrm{U}-106$ & UA-1 & $6 / 20 / 11$ & $7 / 24 / 11$ & 149,500 & 376 \\
\hline TX-109 & $\mathrm{TA}-4$ & $6 / 23 / 11$ & $7 / 31 / 11$ & 144,000 & 5,921 \\
\hline $\mathrm{BX}-112$ & BA-3 & $6 / 2 / 11$ & $8 / 2 / 11$ & 143,600 & 6,262 \\
\hline$B-111$ & BA-1 & $6 / 11 / 11$ & $8 / 12 / 11$ & 145,100 & 4,761 \\
\hline $8-111$ & BA-6 & $8 / 12 / 11$ & $8 / 13 / 11$ & 719 & 24 \\
\hline BY-110 & BA-5 & $7 / 8 / 11$ & $8 / 13 / 11$ & 147,300 & 2,631 \\
\hline $\mathrm{T}-101$ & TA-3 & $6 / 24 / 11$ & $8 / 19 / 11$ & 144,400 & 5,519 \\
\hline U-106 & UA-2 & $7 / 24 / 11$ & $8 / 26 / 11$ & 149,500 & 376 \\
\hline T-101 & TA-2 & $8 / 19 / 11$ & $9 / 1 / 11$ & 34,122 & 1,304 \\
\hline BX-112 & $\mathrm{BA}-2$ & $8 / 2 / 11$ & $9 / 4 / 11$ & 78,662 & 3,430 \\
\hline TX-109 & TA-6 & $7 / 31 / 11$ & $9 / 6 / 11$ & 144,000 & 5,921 \\
\hline BY-110 & BA-2 & $9 / 4 / 11$ & $9 / 14 / 11$ & 40,856 & 730 \\
\hline
\end{tabular}


HNF-2321

Revision 0

Table A.6-2. Phase 1 Low-Activity Waste Feed Staging Transfers for Case 6.

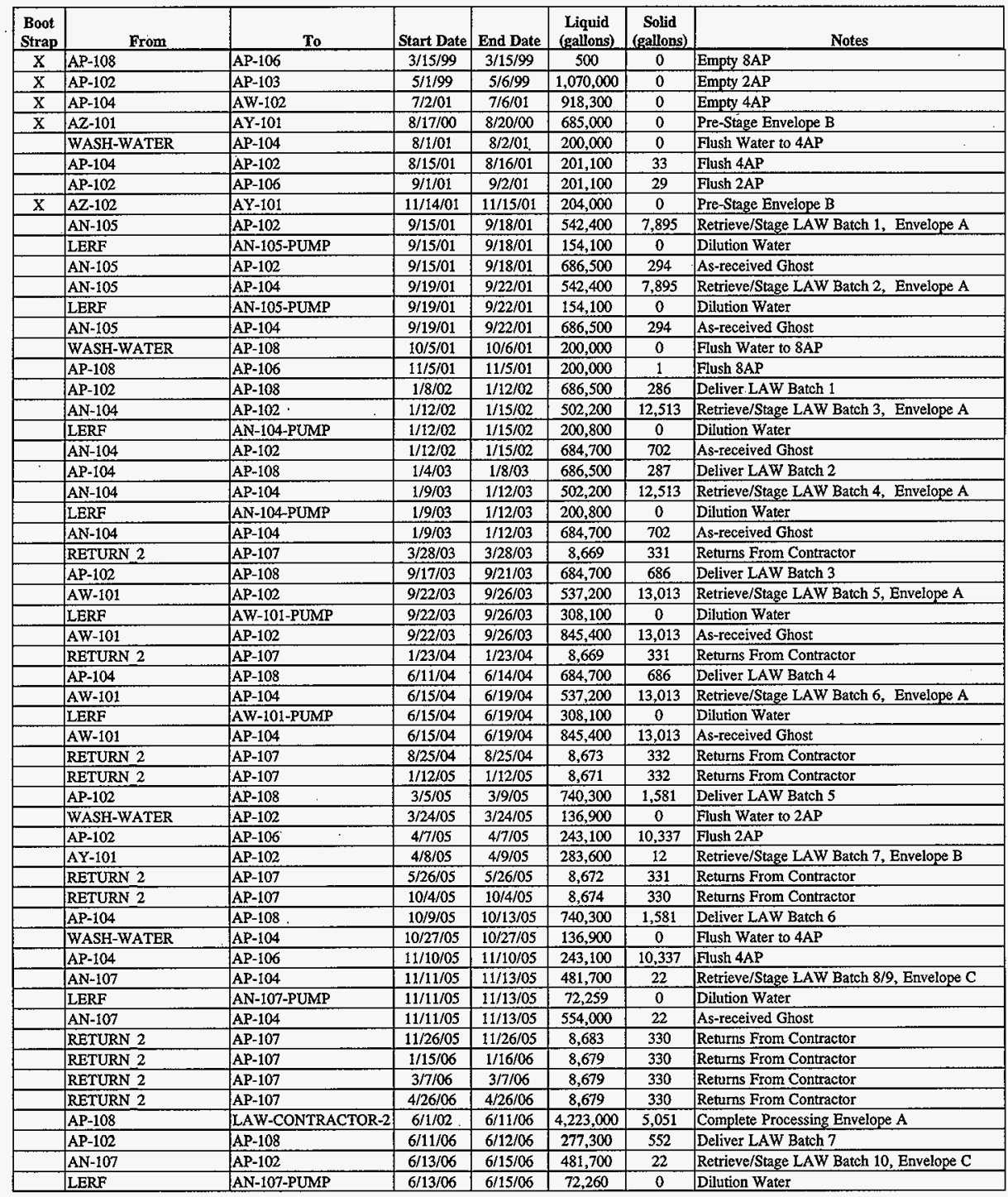


HNF-2321

Revision 0

Table A.6-2. Phase 1 Low-Activity Waste Feed Staging Transfers for Case 6.

\begin{tabular}{|c|c|c|c|c|c|c|c|}
\hline $\begin{array}{l}\text { Boot } \\
\text { Strap }\end{array}$ & From & $T_{0}$ & Start Date & End Date & $\begin{array}{c}\text { Liquid } \\
\text { (gallons) }\end{array}$ & $\begin{array}{c}\text { Solid } \\
\text { (galions) }\end{array}$ & Notes \\
\hline & AN-107 & AP-102 & $6 / 13 / 06$ & $6 / 15 / 06$ & 554,000 & 22 & As-received Ghost \\
\hline & RETURN 2 & AP-107 & $6 / 15 / 06$ & $6 / 15 / 06$ & 8,686 & 330 & Returns From Contractor \\
\hline & RETURN 2 & AP-107 & $7 / 21 / 06$ & $7 / 21 / 06$ & 8,678 & 328 & Returns From Contractor \\
\hline & AP-108 & LAW-CONTRACTOR-2 & $6 / 12 / 06$ & $8 / 11 / 06$ & 277,300 & 555 & Complete Processing Envelope B \\
\hline & AP-104 & AP-108 & $8 / 11 / 06$ & $8 / 12 / 06$ & 171,100 & 337 & Deliver LAW Batch 8 \\
\hline & RETURN 2 & AP-107 & $9 / 4 / 06$ & $9 / 4 / 06$ & 8,678 & 329 & Returns From Contractor \\
\hline & AP-104 & AP-108 & $9 / 8 / 06$ & 9/10/06 & 381,800 & 753 & Deliver LAW Batch 9 \\
\hline & $\mathrm{AN}-102$ & AP-104 & $9 / 11 / 06$ & $9 / 14 / 06$ & 443,500 & 21 & Retrieve/Stage LAW Batch 11, Envelope C \\
\hline & LERF & AN-102-PUMMP & $9 / 11 / 06$ & $9 / 14 / 06$ & 266,100 & 0 & Dilution Water \\
\hline & $\mathrm{AN}-102$ & AP-104 & $9 / 11 / 06$ & $9 / 14 / 06$ & 709,700 & 21 & As-received Ghost \\
\hline & RETURN 2 & AP-107 & $10 / 30 / 06$ & $10 / 30 / 06$ & 8,670 & 330 & Returns From Contractor \\
\hline & RETURN 2 & AP-107 & $12 / 25 / 06$ & $12 / 25 / 06$ & 8,683 & 330 & Returns From Contractor \\
\hline & AP-102 & AP-108 & $1 / 19 / 07$ & $1 / 21 / 07$ & 559,200 & 575 & Deliver LAW Batch 10 \\
\hline & AN-103 & AP-102 & $1 / 22 / 07$ & $1 / 25 / 07$ & 436,600 & 28,133 & Retrieve/Stage LAW Batch 12, Envelope A \\
\hline & LERF & AN-103-PUMP & $1 / 22 / 07$ & $1 / 25 / 07$ & 264,900 & 0 & Dilution Water \\
\hline & $\mathrm{AN}-103$ & AP-102 & $1 / 22 / 07$ & $1 / 25 / 07$ & 738,300 & 549 & As-received Ghost \\
\hline & RETURN 2 & AP-107 & $2 / 20 / 07$ & $2 / 20 / 07$ & 8,676 & 330 & Returns From Contractor \\
\hline & RETURN 2 & AP-107 & $4 / 19 / 07$ & $4 / 19 / 07$ & 8,672 & 330 & Returns From Contractor \\
\hline & RETURN 2 & AP-107 & $6 / 16 / 07$ & $6 / 16 / 07$ & 8,672 & 330 & Returns From Contractor \\
\hline & AP-104 & AP-108 & $8 / 2 / 07$ & $8 / 6 / 07$ & 709,600 & 72 & Deliver LAW Batch 11 \\
\hline & AN-103 & AP-104 & $8 / 7 / 07$ & $8 / 10 / 07$ & 436,600 & 28,133 & Retrieve/Stage LAW Batch 13, Envelope A \\
\hline & LERF & AN-103-PUMP & $8 / 7 / 07$ & $8 / 10 / 07$ & 264,900 & 0 & Dilution Water \\
\hline & AN-103 & AP-104 & $8 / 7 / 07$ & $8 / 10 / 07$ & 738,300 & 549 & As-received Ghost \\
\hline & RETURN 2 & AP-107 & $8 / 22 / 07$ & $8 / 22 / 07$ & 8,675 & 330 & Returns From Contractor \\
\hline & RETURN 2 & AP-107 & $11 / 28 / 07$ & $11 / 28 / 07$ & 8,675 & 329 & Returns From Contractor \\
\hline & RETURN 2 & AP-107 & $3 / 5 / 08$ & $3 / 5 / 08$ & 8,675 & 329 & Returns From Contractor \\
\hline & AP-108 & LAW-CONTRACTOR-2 & $8 / 12 / 06$ & $4 / 9 / 08$ & $1,822,000$ & 1,786 & Complete Processing Envelope C \\
\hline & AP-102 & AP-108 & $4 / 9 / 08$ & $4 / 13 / 08$ & 738,300 & 557 & Deliver LAW Batch 12 \\
\hline & RETURN 2 & AP-107 & $8 / 9 / 08$ & $8 / 9 / 08$ & 8,672 & 330 & Returns From Contractor \\
\hline & AP-104 & AP-108 & $1 / 4 / 09$ & $1 / 8 / 09$ & 738,400 & 532 & Deliver LAW Batch 13 \\
\hline & RETURN 2 & AP-107 & $2 / 7 / 09$ & $2 / 7 / 09$ & 8,670 & 331 & Returns From Contractor \\
\hline & RETURN_ 2 & AP-107 & $8 / 8 / 09$ & $8 / 8 / 09$ & 8,670 & 331 & Returns From Contractor \\
\hline & AP-108 & LAW-CONTRACTOR-2 & $4 / 13 / 08$ & $11 / 27 / 09$ & $1,477,000$ & 1,076 & Complete Processing Extended Order Envelope \\
\hline & RETURN 2 & AP-107 & $11 / 28 / 09$ & $11 / 28 / 09$ & 5,272 & 201 & Returns From Contractor \\
\hline $\mathrm{X}$ & AP -107 & AZ-101 & $12 / 8 / 09$ & $12 / 10 / 09$ & 395,800 & 7,587 & Transfer Returns to AZ-101 (Phase $2 \mathrm{HLW}$ \\
\hline
\end{tabular}


HNF-2321

Revision 0

Table A.6-3. Phase 1 High-Level Waste Feed Staging Transfers for Case 6.

\begin{tabular}{|c|c|c|c|c|c|c|c|}
\hline $\begin{array}{l}\text { Boot } \\
\text { Strap }\end{array}$ & From & To & Start Date & End Date & $\begin{array}{c}\text { Liquid } \\
\text { (gallons) }\end{array}$ & $\begin{array}{c}\text { Solid } \\
\text { (gallons) }\end{array}$ & Notes \\
\hline & AY-102 & $\mathrm{C}-106$ & $7 / 1 / 98$ & $7 / 5 / 98$ & 920,700 & 31 & $\begin{array}{l}\text { Sluice "Water" for C-106 Retrieval - Lumped } \\
\text { (See Below) }\end{array}$ \\
\hline & C-106 & AY-102 & $7 / 15 / 98$ & $11 / 10 / 98$ & 809,000 & 15,169 & C-106 Retrieval (Project W-320) \\
\hline \multirow[t]{8}{*}{$x$} & AZ-101 & AP-107 & $8 / 20 / 00$ & $8 / 20 / 00$ & 0 & 0 & Initial Decant $1 \mathrm{AZ}$ \\
\hline & WASH-WATER & AZ-101 & $8 / 20 / 00$ & $8 / 21 / 00$ & 146,000 & 0 & First Wash 1AZ \\
\hline & AZ-101 & AP-106 & $10 / 9 / 00$ & $10 / 10 / 00$ & 143,200 & 0 & First Decant $1 \mathrm{AZ}$ \\
\hline & WASH-WATER & AZ-101 & $10 / 11 / 00$ & $10 / 12 / 00$ & 146,000 & 0 & Second Wash 1AZ \\
\hline & AZ-101 & AP-106 & $11 / 30 / 00$ & $12 / 1 / 00$ & 140,200 & 0 & Second Decant $1 \mathrm{AZ}$ \\
\hline & WASH-WATER & $\mathrm{AZZ}-101$ & $12 / 2 / 00$ & $12 / 2 / 00$ & 146,000 & 0 & Third Wash LAZ \\
\hline & AZ-101 & AP-106 & $1 / 20 / 01$ & $1 / 21 / 01$ & 142,900 & 0 & Third Decant 1AZ \\
\hline & WASH-WATER & AZ-101 & $1 / 22 / 01$ & $1 / 23 / 01$ & 138,200 & 0 & Transfer Water Add $1 \mathrm{AZ}$ \\
\hline $\mathrm{x}$ & $A Z-102$ & AY-101 & $11 / 14 / 01$ & $11 / 15 / 01$ & 204,000 & 0 & Initial Decant $2 \mathrm{AZ}$ \\
\hline \multirow[t]{13}{*}{$\mathrm{x}$} & AZ-102 & AP-107 & $11 / 15 / 01$ & $11 / 16 / 01$ & 181,800 & 0 & Complete Initial Decant $2 \mathrm{AZ}$ \\
\hline & WASH-WATER & AZ-102 & $11 / 16 / 01$ & $11 / 17 / 01$ & 213,000 & 0 & First Wash 2AZ \\
\hline & AZ-102 & AP-106 & $1 / 6 / 02$ & $1 / 6 / 02$ & 127,700 & 0 & First Decant 2AZ \\
\hline & WASH-WATER & $\mathrm{AZ}-102$ & $1 / 7 / 02$ & $1 / 8 / 02$ & 213,000 & 0 & Second Wash $2 \mathrm{AZ}$ \\
\hline & AZ-102 & AP-106 & $2 / 27 / 02$ & $2 / 28 / 02$ & 210,300 & 0 & Second Decant 2AZ \\
\hline & WASH-WATER & AZ-102 & $3 / 1 / 02$ & $3 / 2 / 02$ & 213,000 & 0 & Third Wash $2 \mathrm{AZ}$ \\
\hline & AZ-101 & P1HLW_FEED_TANK & $5 / 17 / 02$ & $5 / 17 / 02$ & 134,300 & 5,079 & $\begin{array}{l}\text { Beliver Batch } 1 \text { of } 1 \mathrm{AZ} \text {, Envelope D (Batch } 1 \\
\text { 8verall) }\end{array}$ \\
\hline & AZ-102 & AP-106 & $6 / 11 / 02$ & $6 / 12 / 02$ & 211,800 & 0 & Third Decant 2AZ \\
\hline & WASYI-WATER & AZ-102 & $6 / 13 / 02$ & $6 / 14 / 02$ & 213,000 & 0 & Fourth Wash $2 \mathrm{AZ}$ \\
\hline & AZ-102 & AP-106 & $8 / 2 / 02$ & $8 / 3 / 02$ & 212,500 & 0 & Fourth Decant $2 \mathrm{AZ}$ \\
\hline & WASH-WATER & AZ-102 & $8 / 4 / 02$ & $8 / 5 / 02$ & 110,000 & 0 & Transfer Water Add 2AZ \\
\hline & PIHLW_FEED_TANK & HLW-CONTRACTOR & $6 / 1 / 02$ & $2 / 21 / 03$ & 134,300 & 5,079 & $\begin{array}{l}\text { Process Batch } 1 \text { of } 1 \mathrm{AZ} \text {, Envelope D (Batch } 1 \\
\text { Overall) }\end{array}$ \\
\hline & AZ-101 & P1HLW_FEED_TANK & $2 / 21 / 03$ & $2 / 21 / 03$ & 134,300 & 5,079 & $\begin{array}{l}\text { Beliver Batch } 2 \text { of } 1 \mathrm{AZ} \text {, Envelope D (Batch } 2 \\
\text { Overal) }\end{array}$ \\
\hline \multirow[t]{24}{*}{$\mathrm{x}$} & $A Y-102$ & AZ-101 & $2 / 26 / 03$ & $3 / 2 / 03$ & 819,600 & 16,032 & \begin{tabular}{|l} 
Transfer 2AY/6C Slurry to \\
rretreatment
\end{tabular} \\
\hline & AZ-101 & AP-106 & $10 / 28 / 03$ & $11 / 1 / 03$ & 622,700 & 0 & Initial Decant $2 \mathrm{AY} / 6 \mathrm{C}$ Slurry from $1 \mathrm{AZ}$ \\
\hline & WASH-WATER & $\mathrm{AZ}-101$ & $11 / 1 / 03$ & $11 / 2 / 03$ & 324,000 & 0 & First Wash 2AY/6C Slurry in 1AZ \\
\hline & P1HLW_FEED_TANK & HLW-CONTRACTOR & $2 / 22 / 03$ & $11 / 14 / 03$ & 134,300 & 5,079 & $\begin{array}{l}\text { Process } \\
\text { Overal) }\end{array}$ Batch 2 of 1AZ, Envelope D (Batch 2 \\
\hline & AZ-102 & P1HLW_FEED_TANK & $11 / 14 / 03$ & $11 / 14 / 03$ & 123,900 & 5,202 & Qeliver Batch 1 of 2AZ, Envelope D (Batch 3 \\
\hline & AZ-101 & AP-106 & $1 / 11 / 04$ & $1 / 13 / 04$ & 320,000 & 0 & First Decant $2 \mathrm{AY} / 6 \mathrm{C}$ Slurry from $1 \mathrm{AZ}$ \\
\hline & WASH-WATER & $\mathrm{AZ}-101$ & $1 / 14 / 04$ & $1 / 16 / 04$ & 324,000 & 0 & Second Wash $2 \mathrm{AY} / 6 \mathrm{C}$ Slurry in $1 \mathrm{AZ}$ \\
\hline & AZ-101 & AP-106 & $3 / 5 / 04$ & $3 / 6 / 04$ & 322,300 & 0 & Second Decant $2 \mathrm{AY} / 6 \mathrm{C}$ Slurry from $1 \mathrm{AZ}$ \\
\hline & WASH-WATER & AZ-101 & $3 / 8 / 04$ & $3 / 10 / 04$ & 433,600 & 0 & Transfer Water Add $1 \mathrm{AZ}$ for $2 \mathrm{AX} / 6 \mathrm{C}$ Slurry \\
\hline & P1HLW_FEED_TANK & HLW-CONTRACTOR & $11 / 14 / 03$ & $8 / 7 / 04$ & 123,900 & 5,202 & $\begin{array}{l}\text { Process Batch } 1 \text { of 2AZ, Envelope D (Batch } 3 \\
\text { Overal) }\end{array}$ \\
\hline & AZ-102 & P1HLW_FEED_TANK & $8 / 7 / 04$ & $8 / 8 / 04$ & 123,900 & 5,202 & $\begin{array}{l}\text { Beliver } \\
\text { Beral) } \\
\text { Batch } 2 \text { of } 2 A Z \text {, Envelope D (Batch } 4\end{array}$ \\
\hline & $\mathrm{C}-104$ & $A Y-102$ & $8 / 1 / 04$ & 9/8/04 & 279,100 & 9,724 & C-104 Retrieval (Project W-???) \\
\hline & P1HLW_FEED_TANK & HLW-CONTRACTOR & $8 / 8 / 04$ & $5 / 2 / 05$ & 123,900 & 5,202 & $\begin{array}{l}\text { Process } \\
\text { Overall) }\end{array}$ atch 2 of $2 A Z$, Envelope D (Batch 4 \\
\hline & AZ-101 & P1HLW_FEED_TANK & $5 / 2 / 05$ & $5 / 2 / 05$ & 127,200 & 3,269 & 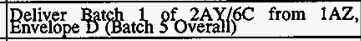 \\
\hline & AY-102 & AZ-102 & $8 / 1 / 05$ & $8 / 2 / 05$ & 279,400 & 9,360 & Transfer 4C Slurry to $2 \mathrm{AZ}$ for Pretreatment \\
\hline & P1HLW_FEED_TANK & HLW-CONTRACTOR & $5 / 2 / 05$ & $11 / 16 / 05$ & 127,200 & 3,269 & $\begin{array}{l}\text { Process Batch } 1 \text { of } 2 \mathrm{AY} / 6 \mathrm{C} \text {, Envelope D } \\
\text { (Batch } 5 \text { Overall) }\end{array}$ \\
\hline & AZ-101 & P1HLW_FEED_TANK & $11 / 16 / 05$ & $11 / 17 / 05$ & 127,200 & 3,269 & 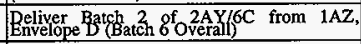 \\
\hline & P1HLW_FEED_TANK & HLW-CONTRACTOR & $11 / 17 / 05$ & $6 / 2 / 06$ & 127,200 & 3,269 & $\begin{array}{l}\text { Process } \\
\text { Batch } 6 \text { Bverall) }\end{array}$ of $2 \mathrm{AY} / 6 \mathrm{C}$, Envelope $\mathrm{D}$ \\
\hline & $\mathrm{AZ}-101$ & P1HLW_FEED_TANK & $6 / 2 / 06$ & $6 / 3 / 06$ & 127,200 & 3,269 & 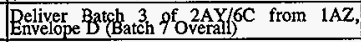 \\
\hline & AZ-102 & AP-106 & $6 / 23 / 06$ & $6 / 23 / 06$ & 159,500 & 0 & Initial Decant 4C Slurry from $2 \mathrm{AZ}$ \\
\hline & WASH-WATER & $A Z-102$ & $6 / 24 / 06$ & $6 / 25 / 06$ & 324,000 & 0 & First Wash $4 C$ Slurry in $2 A Z$ \\
\hline & AZ-102 & AP-106 & $8 / 13 / 06$ & $8 / 15 / 06$ & 320,100 & 0 & First Decant 4C Slurry from $2 \mathrm{AZ}$ \\
\hline & WASH-WATER & $A Z-102$ & & & & 0 & Second Wash 4C Slurry in $2 \mathrm{AZ}$ \\
\hline & $A Z-102$ & AP-106 & $10 / 6 / 06$ & $10 / 8 / 06$ & 322,700 & 0 & Second Decant $4 C$ Slurry from $2 A Z$ \\
\hline
\end{tabular}


HNF-2321

Revision 0

Table A.6-3. Phase 1 High-Level Waste Feed Staging Transfers for Case 6.

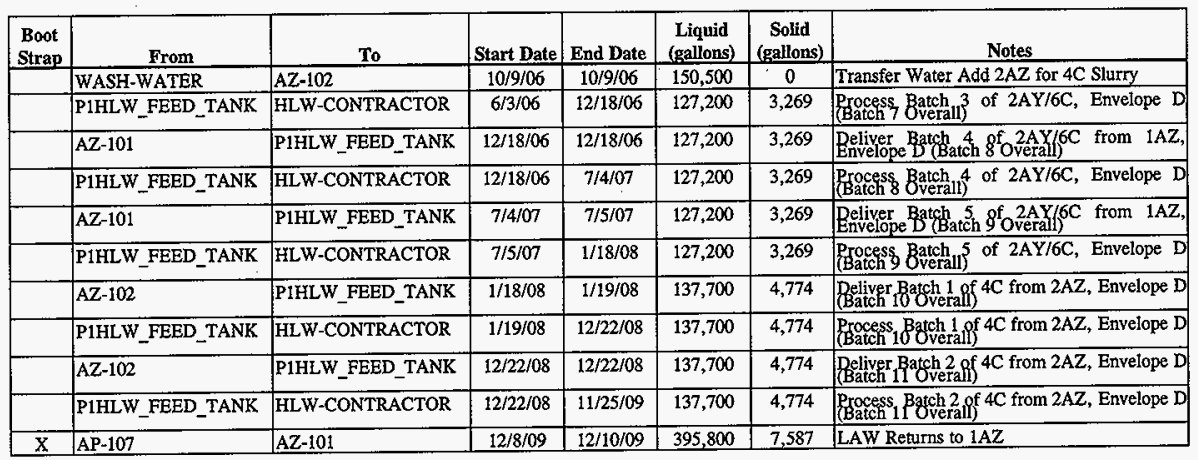




\begin{tabular}{|c|c|c|c|c|c|}
\hline \multicolumn{6}{|c|}{ DISTRIBUTION SHEET } \\
\hline \multirow{2}{*}{$\begin{array}{l}\text { To } \\
\text { Distribution }\end{array}$} & \multirow{2}{*}{\multicolumn{3}{|c|}{$\begin{array}{l}\text { From } \\
\text { E. J. Slaathaug }\end{array}$}} & \multicolumn{2}{|l|}{ Page 1 of 1} \\
\hline & & & & \multicolumn{2}{|l|}{ Date $5 / 4 / 98$} \\
\hline \multicolumn{4}{|c|}{ Project Title/Work Order } & \multicolumn{2}{|c|}{ EDT No. 622710} \\
\hline \multicolumn{4}{|c|}{$\begin{array}{l}\text { Trade Study for the Feed Tank Fill Status Issue for Low-Activity } \\
\text { Waste Feed Issue 19D, HNF-2321, Rev. } 0\end{array}$} & \multicolumn{2}{|c|}{ ECN No. } \\
\hline Name & MSIN & $\begin{array}{c}\text { Text } \\
\text { With All } \\
\text { Attach. }\end{array}$ & Text Only & $\begin{array}{l}\text { Attach./ } \\
\text { Appendix } \\
\text { Only }\end{array}$ & $\begin{array}{l}\text { EDT/ECN } \\
\text { Only }\end{array}$ \\
\hline $\begin{array}{l}\text { Central Files } \\
\text { DIMC - 2- } \\
\text { DOE Reading Room }\end{array}$ & $\begin{array}{l}\mathrm{BI}-07 \\
\mathrm{H} 6-15 \\
\mathrm{H} 2-53\end{array}$ & $\begin{array}{l}x \\
X \\
X\end{array}$ & & & \\
\hline $\begin{array}{l}\text { S. K. Baker } \\
\text { H. L. Boston } \\
\text { R. Carreon } \\
\text { P. J. Certa } \\
\text { J. D. Galbraith } \\
\text { J. S. Garfield } \\
\text { R. A. Kirkbride } \\
\text { I. G. Papp } \\
\text { R. W. Powell } \\
\text { G. M. Ramin } \\
\text { E. J. Slaathaug } \\
\text { J. N. Strode } \\
\text { R. L. Treat }\end{array}$ & $\begin{array}{l}H 5-49 \\
G 3-21 \\
A 0-21 \\
H 5-61 \\
H 5-49 \\
H 5-49 \\
H 5-27 \\
H 5-49 \\
H 5-03 \\
B 4-56 \\
H 5-49 \\
R 2-11 \\
H 5-03\end{array}$ & $\times(6$ & $\begin{array}{r}X \\
X \\
X \\
X \\
X \\
X \\
X \\
X \\
X \\
\text { copies) } \\
X \\
X\end{array}$ & & \\
\hline
\end{tabular}

